

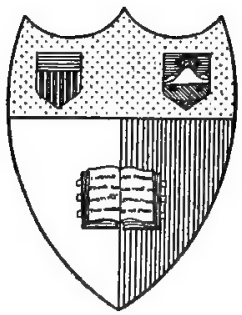

\section{O4nenell Mlnumeraity Mihrary}

\#thara, Arw jork

THE GIFT OF

University of Nebraska

\section{RETURN TO}

\section{ALBERT R. MANN LIBRARY}

ITHACA, N. Y. 


\section{Cornell University Library \\ QK 172.N36}

Botanical survey of Nebraska.Conducted b

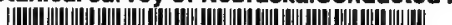

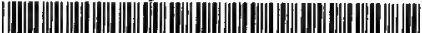

3 $1924001690563 \mathrm{mam}$ 


\section{Cornell University Library}

The original of this book is in the Cornell University Library.

There are no known copyright restrictions in the United States on the use of the text. 
UNIVERSITY OF NEBRASKA,

\section{Botanical Survey of Nebraska.}

Conducted by the Botanical Seminar.

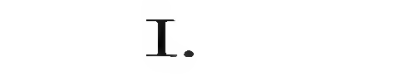

\section{Preliminary :}

\section{The Plan and Scope of the Survey.}


Q

172

N36

A. 377543 


\title{
BOTANICAL SURVEY OF NEBRASKA.
}

\author{
IONDUCTHD BY THE
}

Botanical Seminar of the University.

\section{ADVISORY COMMITTEE:}

Hon. Chas. H. Morrill, President of the Board of Regents.

James H. Canfield, Chancellor of the University.

Charles E. Bessey, Professor of Botany.

\section{MEMBERS OF THE SURVEY :}

JAtED G. SMITH, M.A.

Roscoe Pound, M.A. Albert F. Woods, M.A. Herbert Marsland, B.Se. Per A. Rydberg, B.Sc. Fred Clements.

BOTANICAL ADVISOR:

Charles E. Bessey, Ph.D.

SECRETARY AND TREASURER:

ALbFrt F. WOODS.

HERBARITM COMMITTEE :

Albert F. Woons.

Per A. Rydberg.

Fred Climents .

EDITORIAL COMMITTEE:

Roscoe Podnd.

A trber' F. Woons.

Herbert Marsland. 



\section{PRELIMINARY: THE PLAN AND SCOPE OF THE SURVEY.}

The object of this, the first publication of the Botanical Survey of Nebraska, is introductory only. It is intended to serve as a preface and introduction to the future publications of the Survey.

Although the general plan of the Survey was agreed upon in June of this year, and no little collecting done in accordance with it in July and August, the Survey was not formally organized till August 24. At that time the organization set forth on the preceding page was effected. As there indicated, the Survey will be entirely under the control of the Seminar. The Seminar selects the members and assigns the work, and its members will bear the entire expense of the Survey. But in the conduct of it they will be advised and assisted by the gentlemen who have kindly consented to act as the advisory committee, and in botanical and scientific matters they will rely largely on the advice and assistance of Dr. Bessey.

In entering upon the Survey, the Seminar is fully aware of the difficulties which must necessarily beset such an undertaking when conducted by private means and enterprise. But if there are difficulties attending the conduct of the Survey by private enterprise, there are also undoubted advantages. The memabers need never fear to do purely scientific work, they need not spend their time in strengthening their official rather than their scientific position, and they need not be distracted from more important matters by the burden of continually demonstrating to doubting Thomases the practical nature of their undertaking.

No apology for the undertaking of such a survey is needed. The changes which are taking place in the flora of the state have already been noted by Mr. Webber in the preface to his catalogue. The rapid settlement of the western portions of the state is undoubtedly accelerating these changes, and requires that those regions be examined at once, while the native flora is intact. The 
number of collectors and persons interested in botany in the state is increasing, and they demand that the local flora-for the determination of which, as regards the Anthophytes, two manuals are required in many parts of the state-be systematically set before them, and that the lower plants of the state be made accessible to them. At present, but a limiter number can form any acquaintance with the larger part of the plant life of the state. Mr. Webber's catalogue and the supplements to it have been of very great assistance to botanists and collectors in Nebraska. But they are not enough, and, besides, are far from representing the whole flora of the state, as is shown by the additions made almost daily. To continue to add to Mr. Webber's catalogue in a haphazard way will needlessly and indefinitely postpone the complete presentation of our flora which is desirable. Systematic botanical exploration of the state will bring such a catalogue much sooner, make it a far better one, and cannot fail to develop many things of practical as well as scientific importance.

It would be greatly to the public. interest to have a state Natural History Survey. But the time when such a survey ean be conducted with putlic funds seems far distant. 'The next best thing is a private survey. This the Seminar has undertaken for the botanical part, believing that the time when such a survey should be had is at hancl.

It is the intention of the Seminar to make a thorough and complete survey of the state, extending over several years and covering all forms of plant life. Those parts of the state which are less known will be carefully explored, and, as far as possible, complete collections will be made there. Distinct cases have been provided in the Herbarium of the University for the Survey Herbarium, and several collections of fair size and no little importance have been made already. The plan is to make the survey Herbarium a complete representation of the flora of the state, to iuclicate there, as far as possible, the distribution of each species, to determine the floras of the various regions of the state, and to provide for the botanists of the state a herbarium for the study 
of the local flora as well as complete and accurate lists and catalogues, and monographs of the more difficult groups. At the close of the Survey the Herbarimm will be presented to the University. And during the progress of the Survey it will be accessible to all persons, prepared to use it, who wish to study the plants of Nebraska or any grou, of them.

In addition to the reports, catalogues of local floras and monographs of particular groups, principally of the lower plauts, will be published. Several such monographs are now in prepuration, and more are contemplated. These will make possible a more thorough acquaintance with the plant life of the state by the public generally, and, it is hoped, enable the public schools of the state to do much which at present they cannot do for lack of the numerous and expensive books which such work requires. But the Seminar will aim to make the work of the Survey scientific rather than popular.

While the Survey is essentially a private undertaking, conducted by a private organization and carried on with private means, the members of the Seminar recognize that their connection with the State University, most of them being graduate students at that institution, pats them under obligation to the public. They will endeavor, therefore, to give such practical direction to the Survey as will be consistent with a purely scientific aim. The grasses of the state, the trees and woody plants, injurious and beneficial fungi and their observed effects, and other matters of interest to Agriculturist and Horticulturist, will receive special notice. But the bulletins of the Agricultural Experiment Station are the place for the most of such work, especially the popular side of it. The Survey must often treat them on an equality with matters of little economic or purely scientific importance.

With no intention of being unduly radical, the Seminar will endeavor to have the publications of the Survey fully represent the most recent development of Botany in all directions. The Survey is intended for scientific purposes, and its publications for scientific eyes, primarily. Furthermore the Seminar cannot 
assent to the doctrine that the public are too weak to stand a lranght of modern scientifie results, unless strongly diluted with ancient and untenable ideas, no longer held even by those who continue to put them off upon others. 


\section{Botanical Survey of Nebraska.}

Conducted by the Botanical Seminar.

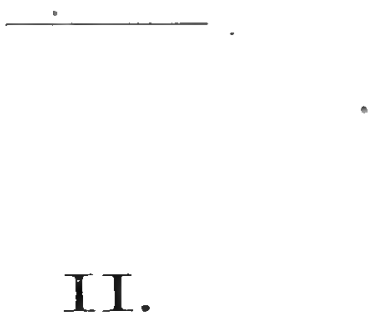

\section{Report on Collections Made in 1892.}

Lincoln, Nebraska, U. S. A.

Published by the Seminar. 1803 .

(Distributed April 15, 1893.) 



\section{CONTENTS.}

1. Flora of the Sand Hill Region of Sheridan and Cherry counties, and List of Plants Collected on a Journey through the Sand Fills in July and August, 1892, by Jared G. Smith and Roscoe Pound.

2. Notes on the Canon Flora of Sioux county, with List of Plants Collected in July and August, 1892, by A. F. Woods.

3. Miscellaneous Additions to the Flora of the State and New or Noteworthy Species from Various Localities. 


\section{NOTE.}

In this report, in addition to full lists of the two principal collections made during the past year, a list is given of all additions to the reported flora of Nebraska up to January 1st, 1893, as far as possible. A great deal of material remains to be examined, and many things which should appear in this report must be deferred for the reason that the publication has already been held back much longer than was intended.

The thanks of the Seminar are due to the gentlemen who have collected for the Survey in different parts of the state. Rev. J. M. Bates, of Valentine, collected many of the species from northwestern Nebraska. Dr. H. Hapeman, of Minden, collected the algæ reported from that place. Mr. D. A. Saunders, of the Botanical Departmənt of the University, collected many of the species reported from Lancaster county, and determined most of the algx. Messrs. W. H. Skinner and A. T. Bell, of the Crete High School, collected the Erysiphece reported.

The members of the Seminar wish to remind collectors that no species can be reported unless a specimen is deposited in the . herbarium of the Survey. 
I.

Flora of the Sand Hill Region of Sheridan and Cherry Counties axd List of Plants Collected on a Journey Through the Sand

Hills ix Jult and August, 1892, By Jared G. Smith and Roscoe Pound.

On July 6th we started from Alliance, and between the 6th and 8th we traversed on foot the sand hill region of Sheridan and Cherry counties, emerging at Johnstown in Brown county. From Johnstown we went to O'Neill, following, at no great distance, the Fremont, Elkhorn, \& Missouri Valley railroad. We drove across country from O'Neill to Lincoln, arriving there August 6th.

The greater part of the collecting was done in the sand hills and in the lake region of Cherry county.

GENERAL FEATURES OF THE REGION TRAVERSED.

The main group of the sand hills of western Nebraska extends from the $103 d$ meridian, midway between the North Platte and the Niobrara, eastward to the 98 th meridian. It extends southward to the 41 st parallel, and has for its northern boundary the Niobrara. In passing into the sand hills from the old lake basin around Alliance there is a perceptible fall. One does not go up, but goes down; and there is a general slope towards the east, indicated by the fact that in the wet valleys the lake or pond is generally at the eastern end. We met with no exception.

The sand hills are a region free from drainage. The surface water, instead of flowing off in the river channels, collects in the valleys and forms countless lakes or ponds without outlet. The valleys are, as a rule, parallel, and have a general east and west trend. They are not strictly continuous, being separated by low ridges or groups of low hills. But the passage is always easy from one to the next, while getting over the side of the valley is difficult and sometimes well nigh impossible. 
Between these more or less pa.allel valley's are ranges of hills of nearly pure sand, rising from one hundred to five or six huudred feet above the center of the ralley. In some cases the valleys are bounded on north and south by high, steep hills; oftener the sides rise gradually through a succession of hills of increasing size. The hills bear all manner of fantastic shapes, showing their formation to be due to winds rather than to water. The upper slopes are abrupt and the ridge is narrow, and a characteristic mark is the great pits or craters blown out of their sides or tops. These "blow-outs" are usually on the southwest face of a hill, though there are many exceptions.

We came upon two streams. The North Loup rises in a marshy region in west-central Cherry county around Brush Lake. Where we first saw it, it was very cold and swift and about as deep as it was wide ( 2 to $3 \mathrm{ft}$.) It flows. constantly and rapidly increasing in size, through a valley bounded by high, steep sand hills. Sometimes the valley is broad and level, but as a rule it is narrow, and often a perfect gorge. The stream has a limestone bottom, and not far from its source has innumerable falls-. some of them quite large. The Gordon is a clear stream, more sluggish than the other, but swift compared with streams in the eastern part of the state. It was quite wide and deep where we came upon it, and flowed through a wide, swampy valley, bounded by high sand hills.

In eastem Cherry county there is a region of permanent lakes, of which the largest, Dad's Lake, seemed to be about 5 to 6 miles long and 1 to $1_{4}^{3}$ miles wide. Beyond this fine body of water there is a network of lakes, of which Pelican Lake is the largest. From a hill above this lake twenty-four are visible.

In Sheridan county and western Cherry county the valleys were, roughly speaking, of two kinds-wet valleys and dry valleys. In some seasons valleys are dry that are wet at other times, but some- valley's appear to be permanently dry. They are usually quite long and the sides are provokingly steep and high. The wet valleys vary in length from 2 or 3 to 15 miles and are 
somewhat narww. Siome of then are beautiful as seen from a hill-top, and some of the hest are oceupied by ranches. Between the valleys in the passes are often a large number of small pouds.

It is commonly said by stockmen and others interested in the region that the sand hills were entirely bare of vegetation at a comparatively recent date and have only commencerl to be grassed over since the days of the Indian and the buffalo. This is doubtful. Wr have accounts of the sand hills written in the early part of this century which give the salient features of the landscape about as they appear to-day. Furthermore we have access to collections of plauts gathered in the sand hills from 1839 to $\mathbf{1 8 5 8}$, and these collections agree in species and type with those collected in more recent years. There seems to be neither increase nor diminution in the number of species. The region is one where physical conditions may vary greatly in a term of years. We were told by stockmen who had been in the hills a long time that the lakes sometimes disappear during periods of drouth, and that one year a crop of hay may be cut where, the year before, there was a fine body of water. We saw one case in point. In one valley the bottom for a long distance was white with the dried remains of a species of Charc But two or three insignificant ponds remained, and they were full of the living plant. If one sees the sand hill region for the first time when bare of vegetation in winter or early spring or after the drying out of July and August, he may easily get the idea that they have never been grassed over. When the freshening up comes after the rains, he may conclude that they are becoming turfed over for the first time.

\section{FLORA OF THE SAND HILL REGION.}

There are, roughly speaking, three floras in the region. The hills are sparsely covered with the typical sand hill species. The dry valleys and the upper parts of the wot valleys farthest from the lake have a characteristic flora scarcely different from that of the prairies of eastern Nebraska. It is evidently the prairie flora of the surrounding regions mixed with a few individuals which 
have crept down from the hills. The wet valleys, especially around the lakes, have a flora of their own, and it is in this flora that there is the most localization of species. The species peculiar to the sand hills and blow-outs and the prairie species of the dry valleys are common to the entire region, from the point where we entered the sand hills east of Alliance to the sand hills near $\mathrm{Ne}$ ligh in Antelope county. We also found the sanse sand hill species-no more and no less-on a sandy bluff near Loretto in Boone county. The species peculiar to the wet valleys change as one passes from west to east, only two or three being found throughout the region. A species is frequently confined to isolated patches or widely separated lake-basins.

The following species are to be found in every blow-out throughout the region: Stipa comata, Oryzopsis cuspidata, Redfieldia flexuosa, Tradescantia vurginica, Eriogonum anmum, Oenothera serrulatc, Prunus pumila, Lathyrus sp. (ornatus?), Astragalus pictus var. filifolius, Hymenopappus filifolius. Not only are these species in every blow-out, but they are on every hill. Besides these, the most common sand hill inhabitants are: Andropogon scoparius, A. hallii, Muhlenbergia pungens, Abronia fragrans, Rosa virginiana var. arkansana, $R$. fendleri, Acerutes vividiflora, Erigeron divergens, Haplopappus spinulosus, Chrysopsis villosa, and Laciniaria squar"rosa var. intermedic.

Few species are common to all the wet valleys or to all the wet valleys of a particular locality. The most widely distributed are: Sisyrinchium angustifolium, Salix longifolia, Glycyrrrhiza lepidota Amorpha fruticosa, Utriculavire vulyaris, Lobelia spirata, and Rudbeckia hirta.

The dry valley flora includes about the same species as the prairie flora of Lancaster county. In addition there are Haplopappus spinulosus and Cnicus undulatus. Prunus demissa was found in a very peculiar dry valley in Sheridan county. It is common on the banks of the North Loup and in wet valleys in the lake region of eastern Cherry county. 
THE PRAIRTE FLORA OF BOX BUT'TE COUNTY.

The prairie in Box Butte county was covered with Agropymum glaucum and Stipa comata, the former, apparently, predominating on lower or waste ground, the latter on high ground. Bulbilis: drectyloides was found in small patches, and Oryzopsis ruspidrutr, a typical sand hill species, had wandeled out along the trail seven or eight miles from the hills.

The most striking flowers of the prairie were Tradescrutia virginica, Erysimum asperum, Lupinus plattensis, Spiesir Inmberti, Astragalus adsurgens, and Thelesper'ma filifolium, all very common. Pentstemon albidus, Astragalus mollissimus, and Eriyeron pumilus were also common near Alliance. As one approaches the sand hills, these disappear. Monarda citviodor becomes very common on the prairie, and Phlox douglesii and Polygala alba on the hill-sides.

Several of 'the typical "sand hill plants had wandered out on the prairie and were found within a few miles of the Sheridan county line. Stipa comata Tradescantia virginica, Plilox douglasii, and Plantrgo patagonica var. gnuphalioides were common to the prairies of Box Butte county and the sand hills.

NOTES ON THE FLORA OF BROWN, ROCK, HOLT, AND ANTELOPE COUNTIES.

We saw only the worst parts of Sheridan and Cherry counties. But on leaving the lake region, we came at once upon the best part of Brown county. We left the lake region of Cherry county through a wet valley over thirty miles in length, in which, for the last few miles, flows a brauch of Plum creek. This valley had most of the characteristics of the ordinary wet valley, but the soil was better, and in the last third of the valley it was not so marshy, and the hills at the sides were not so completely "of pure sand. The commonest plant in this valley was Froelichic floridana. Besides a large number of wet valley species, we found as we progressed the ordinary prairie flora. Soon after entering Brown county, we came quite suddenly upon a new region. One of the 
first things that attracted our notice was a small clump of Popullus moniliferce on the hanks of a small stream. We hacl not seen a tree or woody plant larger than a dwarf willow since the day we started.

The prairie in Brown county was yellow in every direction with Rudberkia columncris and in some fields it had the appearance of being cultivated. Other common plants were Oenotheres serrulata, (). rhombipetrala, (). albienulis. and Lacinianir scariosa.

IVe did not see the best portion of Rock county. The part we traversed resembled, on a smaller scale, the region at the head of the North Loup. For the most part we were in wet valleys, full of small ponds and swamps, aud bounded by low saud hills with occasional blow-outs. Lilium philadelphicum and Lobelia spicata were common.

We entered Holt county southwest of Stuart, and passed through low, wet prairies covered with a luxuriant growth of grass. The whole region is devoted to hay-making, and seems excellently adapted to it.

In the part of Antelope county we passed through we found ourselves again in the sand hills. The well known sand hill species-especially Androporyon hallii-were everywhere. In addition there were Calamovilfa longifolir and Cycloloma platyphyllum in great abundance.

LIST OF PLANTS COLLECTED, AND NOTES.

(Species designated by a * not previously reported.)

Laciniaria squarrosa (J.) Hill rar. intermedia. (Lindl.) DC.

Cherry county, July 23. (137) Sand hills throughont the region.

Lrreiniaria serniosa (L.) Hill.

Cherry county, July 27. Dry valleys, and prairie in Brown county.

Laciniarir puluctertu (Hook.) OK.

Dry valleys, wet valley Cherry county, July 27. 
Lacinirivic pycnostechya (Michx.j OK.

Wet valley, Cherry county, July '־ ' also wet valleys, Rock county, July 30.

Chrysopsis villosa (Pursh) Nutt.

Cherry county, July 27. Sand hills throughout the region. Haplopappus spinulosus (Pursh) DC,

Sheridan county, July 11. (66) Sand hills throughout the region.

Solidcugo speciosa Nutt. var. rigidliuscula Torr. \& Gray.

Dry valleys in Sheridan and western Cherry counties. Aster salicifolins Lam.

In canon, Long Pine, July 29.

Erigeron divergens Torr. \& (Yray.

Cherry county, July 20. (250) Sand hills throughout the region.

*Erigeron ramosus (Walt.) BSP, var. beyrichii (Fisch. \& Mey.) Torr. \& Gray.

In wet valley near Hannah's ranch, Cherry county, July 27. (154)

Erigeron pumilus Nutt.

Box Butte county, July 6. (155)

Antennaria plantaginifolia (L.) Hook.

Cherry county, July 14. (60) Prairies, Box Butte county, and dry valleys throughout the region.

Rulbeckicu augustifolia (DC.) Benth. \& Hook.

Dry valleys, western Cherry county.

Rudbeckia columueris Pursh.

Cherry county, July 1t. (146) In wet valleys, central

Cherry county; prairies, Brown county.

Ritlbeckia columnaris Pursh var. pulcherrima (DC.) Torr. \& Gray.

LaPorte's ranch, Cherr'y county, July 23. (147) Rutbeckia hirta $\mathrm{L}$.

Sheridan county, July 1:3. (14.j) Wet valleys throughout the region. 
IIclicullens anmun: L.

TVaste places around a rauch, Cherry county, July 26.

Helicuthus petiolaris Nutt.

Box Butte county, July 7.

Hrlicuthus rigielus (Cass.) Desf.

Cherry county, July 26. (71) Sand hills, and dry valleys throughout the region.

Thelesperma grecile Gray.

Cherry county, July 18. (1 141$)$ Sand hills, western and central Cherry county.

Thelesperma filifolizm Gray.

Prairies, Box Butte county, July (i. (112)

Hymenopappus filifolius Hook.

Box Butte county, July 7. (.) S) Sand hills throughout the region.

Crepis runcinete (James) Torr. \& Gray.

In wet valley, Sheridan county, July $9 . \quad$ (15̆2) Artemisia cancudensis Michx.

Brown county, July 29. (65) (Lower leaves sparsely silky canescent. Farther south than its usual range. S.) Artemisia ludoviciuma Nutt.

Cherry county, July 26. (62) Dry valleys throughout the region.

* Senerio auveus L. var. borectis Torr. \& Gray.

Saud hills, eastern sheridau county, July 9. (61) (Agrees with specimens in Herb. Engelm. collected by Hayden, "Loup Fork, July 24," 181'-, and named hy Dr. Gray. This is much farther south than its usual range, and it is probably one of the "buffalo disseminated plants." See Bot. Gaz. XVII, 321. S.)

Cuicus undululus (Nutt.) Gray.

Dry valley, Sheridan county, July 21, (6i3).

Cnicus undulrtus (Nutt.) Gray var. comescens (Nutt.) Gray.

Prairies, eastern edge of Box Butte county, July 7 . (ijt)

Saud hills throughout the region. 
Troximon cuspidatum Pursh.

Dry valleys, Sheridan county.

Lactuca ludoviciana (Nutt.) DC.

Wet valley, Cherry county, July $26 . \quad(68,70)$

Lactuca pulchella (Nutt.) DC.

Cherry county, July 15. (69) Valleys throughout Cherry county.

Lygodesmia juncea (Pursh) Don.

Cherry county, July 19. (36) Sand hills and blow-outs throughout the region. (The length of the upper leaves approaches $L$. rostrata $L$. juncea. Gr. But it is 3-5 flowered, corymbosely branched, flowers terminal on the upper branches. I consider it an intermediate form between juncea and rostrata. S.)

Symphoricarpos occidentalis (R.Br.) Hook.

Cherry county, July 23. (54) Valleys here and there throughout the region.

Galium trifidum L.

In thicket of Prunus demissa, falls of the North Loup, Cherry county, July 22. (193)

Specularia perfoliata (L.) A.DC.

Box Butte county, July 7.

Campanula aparinoides Pursh.

Along the North Loup and in wet valleys in the lake region, Cherry county, July 26.

Lobelia spicata Lam.

Cherry county, July 19. (37) Wet valleys throughout the region.

Acerates viridiflora (Raf.) Ell.

Cherry county, July 15. (134) Sand hills throughout the region.

Acerates viridiflora (Raf.) Ell, var. linearis Gr.

Cherry county, July 23. (134 a) Sand hills, Cherry county. 
Asclepias verticillata L.

Dry valley, Cherry county, July 23.

Asclepias stenophylla Gray.

Sand hills. Sheridan county, July 12, Cherry county, July 23. (136)

Asclepias arenaria Torr.

Cherry countv, July 20. (133) Sand hills, western and central Cherry county.

Asclepias speciosa Torr.

Cherry county, July 19. (132) Sand hills and dry valleys,

Sheridan county, and western Cherry county.

Asclepias incarnata L.

Wet valleys at head of North Loup and in ${ }_{\Xi}^{7}$ lake region, Cherry county, July 20, 26. (131)

Apocynum cannabinum L.

Cherry county, July 19. (140) Dry valleys, Sheridan county and western Cherry county.

Plantago eriopoda Torr.

In wet valley, Sheridan county, July 9.

Plantago patagonica Jacq. var. gnaphalioides (Nutt.) Gray.

Prairies, Box Butte county, July 6. (76) Dry valleys throughout the region.

Verbena hastata L.

Wet valley, Cherry county, July 23.

Verbena stricta Vent.

Valleys here and there in Cherry county.

V'erbena bracteosa Michx.

Box Butte county, July 6; Cherry county, July 24.

Wet valleys near the Gordon and in the lake region.

Lycopus sinuatus Ell.

Wet valleys near the Gordon and in the lake region, July 24. (83)

Koellia virginiana (L.) OK.

Brown county, July 29. (160) 
Hedeoma hispida Pursh.

Box Butte county, July 6.

Monarda citriodora Cerv.

Prairie, Box Butte county, July 7.

Stachys palustris L.

Cherry county, July 15. (56) Wet valleys, eastern Sheridan county, and lake region of Cherry county.

Tencrium occidentale Gray.

Valley of Dad's Lake, July 26.

Utricularia vulgaris $\mathrm{L}$.

Cherry county, July 15. (72) In the Gordon, July 24; wet valleys throughout the region.

Pentstemon gracilis Nutt.

Wet valleys, western Cherry county, July 14.

Pentstemon albidus Nutt.

Prairies, Box Butte county, July 6.

Pentstemon caeruleus Nutt.

Box Butte county, July 7.

Pentstemon grandiflorus Nutt.

Prairie, Brown county, July 29.

Mimulus glabratus HBK. var. jamesii (Torr. \& Gray) Gray.

Margin of cold spring in wet valley at Dye's ranch, Cherry county, July 19.

Monniera rotundifolia Michx.

In dried-up pond, Cherry county, July 15.

Veronica peregrina $\mathrm{L}$.

Box Butte county, July 6. Wet valley at head' of North Loup, Cherry county, July 19. (158)

Gratiola virginiana $\mathrm{L}$.

Wet valleys near the head of the North Loup, Cherry county, July 20. (176)

Physalis mollis Nutt. var. cinerascens Gray.

Dry valley, Sheridan county, July 12. (78) (Apparently the same as specimens in Herb. Univ. collected by Mr. 
Rydberg in Scott's Bluff county. The typical mollis is a much smaller leaved plant of the Texas-Arizona region. S.) Physalis virginiana Mill.

Dry valleys, Sheridan county, July 12. (150)

Physalis lanceolata Michx.

Dry valleys, Sheridan county, July 12.

Lappula redowskii (Hornem.) Greene.

Box Butte county, July 6. (169)

*Krynitzkia jamesii (Torr.) Gray.

Box Butte county, July 7. (170)

Krynitzkia fendleri Gray.

Dry valley, Sheridan county, July 9.

Lithospermum angustifolium Michx.

Box Butte county, July 7. (171)

Lithospermum carolinense (Walt.) McM.

Sheridan county, July 11. (80)

Onosmodium carolinianum (Lam.) DU. var. molle (Michx.) Gray.

Dry valley, Sheridan county, July 9.

Ellisia nyctelea L.

Box Butte county, July 7. (73) (Macrocalyx Trew, does not seem available, as it. was not applied in a binomial name. P.)

Phlox douglasii Hook.

Box Butte county, July 7. (74) Sand hills, Sheridan county, and western Cherry county.

Gilia linearis (Nutt.) Gray.

Box Butte county, July 7.

Ipomoea leptophylla Torr.

Brown county, July 29.

Cuscuta arvensis Beyrich?

On Polygonum sp., not in flower, Joy's ranch, Sheridan county, July 10. (205)

Steironema lanceolatum (Walt.). Gray.

Wet valleys, lake region of Cherry county, July 26. 
Comandra pallida A.DC.

Sand hills, Sheridan county, July 12, western Cherry county,

$$
\text { July 15. (255) }
$$

Lupinus plattensis Wats.

Box Butte county, July 7.

Lotus americanus (Nutt.) Bisch.

Cherry county, July 19. (42) Wet valleys at head of

North Loup, and in lake region.

Psoralen campestris Nutt.

Dry valley, Sheridan county, July 9.

Psoralea lanceolata Pursh.

Sheridan county, July 9. (46) Sand hills and blow outs throughout the region.

Psoralea incana Nutt.

Box Butte county, July 7.

Psoralea esculenta Pursh.

Dry valleys Sheridan county, July 12. (44)

Amorpha canescens Nutt.

Sheridan county, July 13. (43) Dry valleys throughout

- the region.

Amorpha fruticosa $\mathrm{L}$.

Banks of the North Loup; wet valleys throughout the region.

Kuhniastera purpurea (Vent.) MacM.

Brown county, July 28. (38) Dry valleys throughout the region.

Kuhniastera candida (Willd.) OK.

With the preceding. (39)

Kuhniastera villosa (Nutt.) OK.

Sand hills and dry valleys throughout the region.

Astragalus mollissimus Torr.

Box Butte county, July 6. (48)

Astragalus caryocarpus Ker.

Dry valley, Sheridan county, July 13. (50) 
Astragalus microlobus Gray.

Box Butte county, July 7.

Astragalus adsurgens Pall.

Box Butte county, July 6.

Spiesia lamberti (Pursh) OK.

Box Butte county, July 6.

Glycyrrhiza lepidota Nutt.

Cherry county, July 26. region.

(52) Wet valleys throughout the

Iathyrus sp. (ornatus Nutt?)

Sand hills and blow-outs throughout the region, very common. In fruit only.

Prunus demissa Walpers.

Dry valley, Sheridan county, July 12; Banks of North Loup, Cherry county, July 21, Brown county, July 29. Wet valleys in the lake region.

Geum strictum Ait.

Falls of the North Loup, Cherry county, July 21.

Potentilla arguta Pursh.

Cherry county, July 22. (94) Wet valleys at head of North Loup and along the Gordon.

* Potentilla pennsylvanica L. var. strigosa Pursh.

Dry valleys, Sheridan county, July 12. Potentilla norvegica $\mathbf{L}$.

Wet valley, Cherry county, July 21. Agrimonia eupatoria L.

In canon, Long Pine, July 29. Rosa fendleri Crepin.

Sheridan county, July 9. (88) Sand hills, Sheridan county, and western and central Cherry county.

Rosa virginiana Mill. var. arkansana (Porter) Best.

Dry valleys, Sheridan county, July 12, Cherry county, JulK 23. (87) Sand hills throughout Cherry county. Amelanchier alnifolia Nutt.

In canon, Long Pine, July 29. 
Ammannia coccinea Rottb.

Cherry county, July 26. (89) A peculiar form, found once in a wet valley in the lake region.

Lythrum alatum L.

Valley of Dad's Lake, Cherry county, July 26.

Oenothera albicaulis Nutt.

Brown county, July 28.

Oenothera coronopifolia Torr. \& Gray.

Box Butte county, July 6.

Oenothera rhombipetala Nutt.

Sheridan county, July 12. (214) Dry valleys and sand hills throughout the region; prairies, Brown county.

Oenothera serrulata Nutt.

Sheridan county, July 12. (215) Prairies, Box Butte county, July 7, Brown county, July 28. Blow-outs and sand hills throughout the region. In the sand hills it has a different aspect-low, very woody, spreading, much branched, flowers dark yellow. On the prairie it is erect, simple, slightly wondy at base only, flowers light yellow. Gaura coccinea Nutt.

Box Butte county, July 6.

Mamillaria vivipara (Nutt.) Haw.

Cherry county, July 15. (216) Prairies, Box Butte county, and sand hills and dry valleys throughout the region.

Opuntia rafinesquii Engelm.

Cherry county, July 15. (217) Sand hills throughout the region.

Opuntia fragilis (Nutt.) Haw.

Joy's ranch, Sheridan county, July 10. (218) Sand hills, Sheridan county.

*Cornus pubescens Nutt.

Liong Pine, July 29.

(183) (Has both appressed and silky hairs on the same leaves, as in C. baileyi Coulter \& Evans, but the stone is that of $C$. pubescens. It may possibly be a hybrid between $C$. pubescens and C. stolonifera Michxs.) 
Cicuta virosa L. var. maculata (L.) Coulter \& Rose.

Wet valley, LaPorte's ranch, Cherry county, July 22. (196)

Euphorbia petaloidea Engelm.

Sheridan county, July 11. (96) Blow-outs, Sheridan county, and Western Cherry county.

Euphorbia hexagona Nutt.

Sand hills, Antelope county, August 2.

Croton texensis Muell.

Sand hills, Sheridan county, July 9.

Polygala verticillata L.

Cherry county, July 21. (93) Dry valleys throughout the region.

Polygala alba Nutt.

Sandy hillsides, eastern Box Butte county, July 7.

Polygala sanguinea L.

Wet valley, Rock county, July 30.

Rhus radicans $\mathrm{L}$.

Banks of the North Loup, July 23. Wet valleys throughout the region.

Linum sulcatum Riddell.

Prairies, Box Butte county, July 7. (283) Sand hills and blow-outs, Sheridan county, and western Cherry county. Oxalis stricta $\mathrm{L}$.

Pullman, Cherry county, July 20.

Malveopsis coccinea (Nutt.) OK.

Box Butte county, July 6.

Cleome serrulata Pursh.

Rock county, July 28. (163) Waste places around ranches (only) in eastern Cherry county.

Cristatella jamesii Torr. \& Gray.

Side of canon, in sandy soil, Brown county, July 29. Lesquerella argentea (Pursh) MacM.

Box Butte county, July 7. 
Erysimum asperum DC.

Box Butte county, July 7.

Argemone platyceras Link \& Otto.

Prairies, Box Butte county, July 7. (161) Sand hills throughout the region.

Nymphaea advena Solander.

In lake, Hannah's ranch, Cherry county (lake region), July 27. (168)

Anemone cylindrica Gray.

Dry valley, Cherry county, July 15.

Ranunculus aquatilis L. var. trichophyllus Chaix.

Ponds in wet valleys at the head of the North Loup, July

19, in the North Loup, July 20, in the Gordon, July 24. (181)

Ranunculus cymbalaria Pursh.

Wet valley, Wilson's ranch, Sheridan county, July 11. (182)

Delphinium azureum Michx.

Cherry county, July 23. (179) Dry valleys throughout the region.

Paronychia jamesii Torr. \& Gray.

Sandy hillsides, Box 'Butte cọunty, July 7. (189)

Silene antirrhina $\mathrm{L}$.

Dry valleys, Cherry county, July 20.

Cerastium nutans Raf.

Prairies, Box Butte county, July 7, wet valleys at the head of the North Loup, Cherry county, July 20. (187)

Arenaria lateriflorı I.

In canon, Long Pine, July 29.

Lychnis drummondii Wats.

Dry valley, Sheridan county, July 12.

Allionia angustifolia (Nutt.) OK.

Box Butte county, July 6.

Allionia hirsuta Pursh.

Dry valley, Sheridan county; July 12, valley of Pelican Lake,

Cherry county, July 26. (143) 
Abronia frograns Nutt.

Sand hills, Sheridan county, July 79.

Froelichia floridana (Nutt.) Moq.

Wet valley, Cherry county, July 27.

Cycloloma platyphyllum (Michx.) Moq.

Dry valley, Cherry county, July 19. (99) Sand hills and waste places around Neligh, Antelope county, August 2. Chenopodium leptophyllum Nutt.

Dry valley, Sheridan county, July 9.

Eriogonum annuum $\mathrm{Nutt.}$

Sheridan county, July 11. (199) Sand hills and blow-outs throughout the region.

Polygonum emersum (Michx.) Britton.

Cherry county, July 19. (200) Wet valleys throughout

Cherry county.

Polygonum amplibium $\mathbf{L}$.

In lake, Hannah's ranch, lake region of Cherry county, July 26. (200)

Polygonum harherightii Gray.

Wet valleys, lake region, July 26. (201)

Rumex venosus Pursh.

Box Butte county, July 7, Sheridan county, July 9.

Sand hills throughout Sheridan county.

Rumex persiccirioides L.

Antelope county, August 2. (204)

Salix longifolia Muhl.

Sheridan county, July 11. (208) Small pools in passes between the valleys and in the sand hills throughout the region.

Salix cordatu Mubl. var. vestita Anders.

Cherry county, July 22. (209) Wet valleys at the head of the North Loup and in the lake region.

Salix tristis Ait.

Wet valleys, Cherry county and Brown county, July 27. (280) 
Habenaria leucophaea (Nutt.) Gray.

Cherry county, July 19. (219) Wet valleys at the head of the North Loup and in the lake region.

Sisyrinchium angustifolium Mill.

Joy's ranch, Sheridan county, July 10. (223) Wet valleys throughout the region.

Yucca glauca Fraser.

Sheridan county, July 11. (222) Sand hills, Sheridan county, and western Cherry county.

*Allium reticulctum Fraser.

Sandy hillsides, Box Butte county, July 7; sand hills, westexn Sheridan county. (225)

Lilium philadelphicum .L.

Wet valleys at the head of the North Loup, July 20; wet valley, Rock county near Newport, July 30. (220)

Juncus bufonius $\mathbf{L}$.

Wet valley, Oxyoke ranch, Sheridan county, July 17. (191) Juncus marginatus Rostk.

Around lake, Hannah's ranch, July 27.

Juncus nodosus L.

Valley of Pelican Lake, Cherry county, July 26.

Juncus nodosus L. var. megacephalus Torr.

Valley of Pelican Lake, July 26. (240) Grew near the lake in a different part of the valley from the preceding, and in much greater abundance.

* Juncus longistylis Torr. \& Gray.

Wet valleys, Sheridan county, July 9.

Juncus balticus Deth.

With the preceding.

Commelina virginica $\mathrm{L}$.

Sides of sand hills around wet valley, Oxyoke ranch, Cherry county (just beyond Sheridan county line), July 14. (222)

Tradescantia virginica $\mathrm{L}$.

Prairies, Box Butte county, July 6, (221) Sand hills and 
blow-outs throughout the region; one of the commonest blow-out inhabitants.

Panicum virgatum $\mathrm{L}$.

Dry valley, Cherry county, July 21. (106)

Panicum dichotomum $\mathrm{L}$.

Sheridan county, July 12. (123)

Spartina gracilis Trin.

Wet valley, Sheridan county, July 9.

Spartina cynosuroides (L.) Willd.

Wet valleys at head of North Loup, Cherry county, July 19. (130)

Andropogon scoparius Michx.

Sand bills throughout the region.

Andropogon hallii Hack.

Antelope county, August 2. (206) Sand hills from eastern

Cherry county eastward.

Alopecurus geniculatus L.

Box Butte county, July 12.

Stipa comata Trin. \& Rupr.

Prairies, Box Butte county, July 6. (125) - Sand hills throughout the region.

Oryzopsis cuspidata (Nutt.) Benth.

Box Butte county, July 7. (114) Sand hills, especially blow outs, Sheridan county and western Cherry county. (Stipa membranacea Pursh; O. membranacea (Pursh) Vasey. But as there was already a Stipa membranacea L.-F'estuca uniglumis Sol.-Pursh's name is hardly available. P.)

Oryzopsis micrantha (Trin. \& Rupr.) Thurber.

In canon, Long Pine, July 29. (101)

Muhlenbergia pungens Thurb.

Blow-outs and sand hills throughout the region. Phleum pratense $\mathrm{L}$.

In canon, Brown county, July 29. Sporobolus airoides (Steud.) Torr.

Joy's ranch, Sheridan county, July 10. 
Sporobolus cryptandrus (Torr.) Gray.

Cherry county, July 21. (107)

Sporobolus cuspidatus (Torr.) Scrib.

Cherry county, July 14. (109)

Agrostis hiemalis (Walt.) BSP.

Dry valley, Sheridan county, July 9.

Calamovilfa longifolia (Hook.) Hack.

Antelope county, August 2. (120)

Calamagrostis stricta Trin.

Sheridan county, July $11 . \quad$ (252)

Bouteloua oligostachya (Nutt.) Torr.

Broad fields in the valley of the North Loup, Cherry county, July 24. (105) These broad places in the valley of the North Loup are miniature prairies with all the characteristics of dry valleys. The narrow places in the valley are wet and marshy.

Bouteloua hirsuta Lag.

Sand hills, valley of the Gordon, July 24.

Bulbilis dactyloides (Nutt.) Raf.

Prairies, Box Butte county, July 7. (113) Dry valleys,

Sheridan county.

Phragmites vulgaris (Lam.) BSP.

Dad's Lake, July 26.

Munroa squarrosa Torr.

In blow-out near the forks of the North Loup, Cherry county, July 21.

Koeleria cristata (L.) Pers.

Box Butte county, July 7 ; Holt county, July 31. (121)

Eatonia obtusata (Michx.) Gray.

Shallow pond, Oxyoke ranch, Sheridan county, July 13. (110)

Eatonia obtusata (Michx.) Gray var. robusta Vasey.

Cherry county, July 22. (100)

Catabrosa aquatica (L.) Beauv.

Margins of cold spring in wet valley, Dye's ranch, Cherry county, July 19. (104) 
Errogrostis multiflora (Forsk.) Aschs.

Brown county, July 28. (102)

Distichlis spicatc (I.) Gieone var. stricta Thurber.

Low prairie, Box Butte county, July 7; wet valley (alkaline)

Sheridan county, July $9 . \quad$ (116)

Poa compressa $\mathbf{L}$.

Sheridan county, July 9.

Redfieldia flexuosa (Thurber) Vasey.

Antelope county, August 1. (207) Blow-outs throughout

the region.

Festuca octoflore Walt.

Box Butte county, July 7.

Festuca ovina L.

Box Butte county, July 7.

Bromus ciliatus $\mathrm{L}$.

Cherry county, July 22. (108)

Agropyrum glaucum (Desf.) R. \& S. var. occidentale Vasey \& Scribner.

Box Butte county, July 6.

Hordeum jubatum I.

Dry valleys, Sheridan county, July 9.

Elymus canadensis L.

Wet valleys at head of North Loup and in central Cherry county, July 19, 23.

Cyperus aristatus Rottb.

Sheridan county, July 12; Cherry county, July 19.

Wet valleys throughout the region.

Scirpus lacuster L.

Cherry county, July 27. (238) Wet valleys at head of North Loup and in the lake region Grows very tall, often twelve feet.

Carex douglasii Boott.

Sheridan county, July 12; valley of Pelican Lake, Cherry county, July 26.

Typha latifolia L.

Wet valleys along the North Loup, Cherry county, July 20. 
Sparganium eurycarpum Engelm.

Wet valleys at head of North Loup and near the falls, July

20. (224)

Lemna minor $\mathrm{L}$.

In old channel of the North Loup, Cherry county, July 2*2. (272)

Lemua trisulca $\mathrm{L}$.

Wet valleys near the head of the North Loup in small ponds.

July 19; Marsh Lake, July 26. (260)

Alisma plantago L.

Wet valleys near the North Loup, July 20, in the North fork, July 2 ..

Sagittaria variabilis Engelm. var. angustifolia Gray.

In the north fork of the North Loup, July 21.

* Sagittaria variabilis Engelm. var. diversifolia Gray.

Small pond in wet valley, Oxyoke ranch, Sheridan county, July 13. (235)

Triglochin maritimum L.

Wet valley, Sheridan county, July 9. (226)

Potamogeton natans $\mathbf{L}$.

In lake, Hannah's ranch, Cherry county, July 27.

Potamogeton pectinctus L.

With the preceding (229)

Potcmogeton heterophyllus Schreb. var. graminifolius Gray.

Ponds along the North Loup, Cherry county, July 20. (234)

* Vaias flexilis (Willd.) Rostk \& Schm.

With the preceding (231)

Ruppia occidentalis Wats.

Small pond in a wet valley, Sheridan county, July 10 . (230)

Selaginella rupestiris (L.) Spreng.

High prairie, Brown county, July 28. (271) .

Marsilic vestita Hook. \& Grev.

Small ponds, Box Butte county, July 7. 
Aspidium thelypteris (L.) Sw.

Wet valleys at the head of the North Loup, July 19; falls of the North Loup, July 2:. (278)

Onoclea sensibilis L.

With the preceding (277)

Cystopteris fragilis (L.) Bernh.

Canons, Long Pine, July 29.

Marchantia polymorpha $\mathrm{L}$.

Banks of tine North Loup at the falls, July 22.

Chara fragilis Desv.

In small lake, Cherry county, July 19.

Chara contraria A. Br.

Ponds in wet valleys, Cherry county, July 17, 18.

* Chara foetida A. Br. var. longibracteata A. Br.

Ponds in wet valleys, Sheridan county, July 12, 13.

Chara coronata A. Br.

In a small lake, Cherry county, July 18.

Simblum rubescens Gerrard.

Sand hills, Cherry county, July 15.

Aecidium compositarum Mart. var. helianthi Burrill.

On Helianthus rigidus, Cherry county, July 19.

Aecidium compositırum Mart. var. lygodesmiae Webber.

On Lygodesmia junceu, Sheridan county, July 9.

Aecidium compositarum Mart. var. liatridis Webber.

On Laciniaria squariosa var. intermedia, Sheridan county, July 13.

Aecidium jamesianum Pk.

On Asclepices speciosa, Cherry county, July 19. Asclepias arenaria, Cherry county, July 15.

(7) On

*Aecidium chenopodii-fruticosi DC.

On Chenopodium leptophyllum, Sheridan.county, July 9. (Does not entirely agree with Ae. ellisii Tracy \& Gall. It agrees well with specimens of Ae. chenopodii-fruticosi in Mycotheca Univer'salis No. 1028. Ae. ellisii and Ae. chenopodii-fruticosi are not very unlike. P.) 
Phragmidium subcorticium (Schrank) Wint.

On Rosa virginirua var. arkansana, Sheridan county, July 12.

* Phragmidium potentillae (Pers.) Karst.

On Fotentilla pennsylvanica var. strigosa, Sheridan county, July 1?. (15)

Gymnosporangium clarariforme (Jacq.) Rees.

Spermagones on Amelunchier alnifolia, Long Pine, July 29.

Puccinia helianthi Schw.

On Helianthus annuus, Cherry county, July 25.

Puccinic rubigo-vera (DC.) Wint.

On wheat in a small pateh near a ranch, Cherry county, July

25.

* Puccinia troximontis Pk.

On Troximon cuspidatum Sheridan county, July 12.

Hardly distinct enough from $P$. hieracii (Schum.) Mart.

See Ellis N.A.F. no. 145ั2. P.)

* Puccivia physalidis Pl.

On Physalis virginirana, Sheridan county, July 12.

Paccinia thesii (Desv.) Chaill.

I. On Comandra pallida, Cherry county, July 15.

(Fide Webber in Catalngue, the aecidium of this species. It agrees sufficiently with Ae. pustulatum Curt. P.)

* Puccinia solidaginis $\mathrm{Pk}$.

On Solidago speciosa var. rigilliuscula, Cherry county, July 15. (18)

Uromyces trifolii (A. \& S.) Wint. f. glycyrrhizae E \&. E.

On Glycyrrhiza lepidota, Sheridan county, July 8. Uromyces fabae (Pers.) DBy.

On Lathyrus sp. (ornatus?), Cherry county, July 14. Melampsora farinosa (Pers.) Schroet.

On Sulix tristis, Cherry county, July 27. Exoascus prumi Fkl.

On Prunus pumila, Sheriảan county, July 12. 
Septoria pruni Ell.

On Prunus demissa, Cherry county, Jaly 26.

* Septoria lobeticue Pk.

On Lobelia spicata, Cherry county, July 26.

Kellermannia yuccigena E. \& E.

On dead leaves of Yucca glauca, Sheridan county, July 11.

Cercospora teucrii E. \& $\mathrm{K}$.

On Teucrium sp. (occidentale?), Cherry county, July 26.

Cercospora pentstemonis E. \& $\mathrm{K}$.

On Pentstemon grandiflorus, Brown county, July 29. (281) Cercospora symphoricarpi E. \& E.

On Symphoricarpos occidentalis, Sheridan county, July 11. (17)

* Ramularia decipiens E. \&. E.

On Rumex venosus, Box Butte county, July 7.

Marsonia martinii Sacc. \& Ell.

On Quercus macrocarpa, Long Pine, July 29.

Coleochaete orbicularis Pringsh.

On Lemna trisulca, Cherry county, July 19.

Coleochaete irregularis Pringsh.

On Chara contraria, Cherry county, July 19.

* Bulbochaete mirabilis Wittr.

On Chara foetida var. longibracteatı, Sheridan county, July 12. (267)

* Oedogonium stagnale $\mathrm{Kg}$.

In shallow pond in wet valley, Sheridan county, July 11. 269)

Nostoc commune Vauch.

On the ground and in shallow ponds in a pass between two wet valleys, Cherry county, July 15. (66) Forms dense masses of considerable size. 
II.

Notes on the Canon flora of Sioux County, With List of Plants ColLeCted in JUly ANd August, 189:, BY A. F. Woods.

Sioux county is the northwest corner county of Nebraska. It is drained to the northeast by Hat creek with its tributaries, the Long Branch, Sand, Dry, Sowbelly, Warbonnet, Squaw, and Antelope. These tributaries radiate from the principal stream in such a way as to present the appearance of a fan. The line of the divide between Hut creek valley and the White river country begins at the northeast corner of the county and passes southwest, almost directly towards the southwest corner of the county, to near Andrews, on the Fremont, Elkhorn and Missouri Valley railroad. From this point the general trend of the canon country is slightly northwest. Hat creek basin is several hundred feet below the level of the table land to the south.

The creeks have their sources in canons, which generally have the same name as the creek. In most cases the canons proper are cut and divided by many smaller side canons, sometimes very narrow and deep, having a rivulet at the bottom, fed by a spring at the head of the canon and by smaller springs at the side.

In nearly all cases the sides of the canons are covered with a rather dense growth of pine-Pinus ponderosa var. scopulorum. Juniperus virginiana occurs here also. Higber up, along the buttes, a prostrate form of Juniperus communis is very common. Lower down, the monotony of the pine forest is broken by the general intermingling of Ulmus americana (comparatively large trees, sometimes two or three feet in diameter), Acer negundo, Populus monilifera, P. tremuloides, Ostrya virginiana, Hraxinus viridis, Betula occidentalis, and Acer glabıum. The latter is usually a small tree, but in Squaw canon I found an individual about four inches in diameter and from fifteen to eighteen feet bigh. P(ırthenocissus quinquefolia and Humulus lupulus often by twining in and out among the underbrush and trees make dense jungles, difficult to penetrate. 
Along the brooks in the wider canons is a dense growth of willow, mostly Salix nigra. Lepargyraea argentea is often seen along the upper edges of the draws further out in the valley. Everywhere in the canons is the smooth gooseberry, Ribes oxycanthoides, which has very large and fine fruit. $R$. cereum, $R$. floridum, and $R$. aureum are also quite common. Along the upper parts of the canons, and in open places, Rhus canadensis var. trilobata grows in dense patches, and everywhere, on high and low ground, in shade and in sun, in dry and in damp places, is Rhus radicans. For some reason, perhaps on account of its firmer leaves, it does not appear to be as poisonous as the ivy found in the eastern part of the state. Prunus demissa and Amelcnchier alnifolic a common on the sides of the canons. Along the sides of the buttes and well up the sides of the canons is the little Symphoricarpos racemosus var. pauciflorus, and extending down into the canons is the larger $S$. occidentalis.

Among the most conspicuous of the herbaceous plants on the sides and tops of the buttes and canons are Pentstemon glaber, $P$. gracilis, Campanula rolundifolia, Calochortus nuttallii and $C$. gunnisonii, and Zygadenus elegans. All of these extend well down into the canons. Phacelia circinata and Gilia iberidifolia are confined to the upper edges of the canons and sides of the buttes. Here are found a large number of prairie species, extending down into the valley, or so-called bad land region. Down in the damper portions of the camons, among many others, are Habenaria hyperborea, H. bracteata, Coralorrhiza multiflora and C. striatc, and Spiranthes romanzoffiana. Pterospora andromedea is common in the narrower canons, under the pines.

LIST OF PLANTS COLLECTED.

(Species designated by a * not previously reported.)

Eupatorium purpureum L. Squaw canon.

Chrysopsis villosa Nutt. var. hispida Gray. Squaw butte. (346) Haplopappus nuttallii Torr. \& Gray. Squaw butte. 
Haplopoppus spimulosus (Push) DC. Sipuaw canon. (337) Sulidugo missouriensis Nutt. var. momlunu Gray. Słuaw canon. (34j)

Tounsendia grandiflorm Nutt. Squaw buttr. (:311)

Eriferon glubellus. Nutt. var. mollis (iray. Syuaw canon. (34.i) Erigeron pumilus Nitt. Siquaw butte. (:H4)

Rullusline angustifolin (DC.) Beuth. \& Hook. Buttes. . (35̃) Rulbeckic columur is Pursh. Buttes near Squaw canon. (3.jt) Rulbechicu columnaris Parsh var. pulcherrima (DC.) Torr \& Gray. With the preceling. (353)

Helicunthus ammus L. Squaw canon. (350)

Helianthns rigidus (Caws) Desv. Fields arvound Squaw butte. $(3+2)$

Hymenoperpus filifolius Hook. Squaw canon. (35j) Lrimella recullis (Pursh) Nutt. Squaw butte. (310) Crepis gleuce Torr. \& Gray. Squaw camon. (35.5)

Arlillec millefolium L. Squaw canou. (3+3)

Cnicus undulctus (Nutt.) Gray. Bad lands near Squaw butte. $(3+8)$

Lretucu concudensis L. Squaw canon. (359)

Lachea pulchella (Nutt.) DC. Fields around Squaw canon. $(3 \pm 2)$

Lygodesmia juncer (Pursh) Don. In valleys. (\$56) Slephanomric minor. DC. Bad lands. (357) Symphoricurpos occilentalis (R.Br.) Hook. Squaw canon. (312) Symphoricurpos recemosus Michx. var. panciflorus Robbins Squaw butte. $(417)$

Galium aparine L. Squaw canon. (318) Galium borcale L. Squaw canon. (31T) Galium triflorum Michx. Squaw canon. (316) Campanula rotumlifolic L. Squaw canon. $\quad(3 \geq 0)$ Asclepires speciose 'Torr. Squaw canon. (360) Asclepias errticillate L. var. mmila Gray. Squaw canon. (365) Fraximus viridis Michx. Squaw canon. (313) 
Plcuntago patagonica Jacq. var. gnaphalioiles (Nutt.) Gray. Lowlands near canous. (3y)

Phrymc leptostachya L. Squaw canon. (314)

Menthc canculensis L. var. boreculis (Michx.) Wood. Squaw canon. (323)

Vleckir focnicula (Pursh) Raf. Squaw canon. (32t)

Aplyllon ludovicianum (Nutt.) Gray. Buttes near Squaw canon. (335)

Orthocarpus luteus Nutt. Squaw canon. (331)

Teronica americana Schw. In spring, Squaw canon.

Pentstemon glaber Pursh. Buttes near Squaw canon.

Pentstemon gracilis Nutt. With the preceding. (333)

Physalis lanceolata Mivbx. Around Squaw canon.

$(330)$

Krynitzkic glomeratce (DC.) Gray. Buttes. (325)

Krynitzkir jamesii (Torr.) Gray. With the preceding. (326)

Phacelic circinata Jacq. Buttes near Squaw canon. (327)

Gilia ibericifolia Benth. Buttes near Squaw canon. (328)

Steironema cilialum (L.) Raf. Canons. (374)

Pirola secunda L. Squaw and Warbonnet canons. (319)

* Plerospora undromedea Nutt. Squaw and Warbonnet canons. (309)

Comandra pallida A.DC. Squaw canon. (364)

Thermopsis rhombifolic Richardson. Upper edges of canons. $(+10)$

Lupinus plattensis Wats. Squaw prairie. (403)

Psoralea incana Nutt. With the preceding. (404)

Psoralea digitata Nutt. Prairies. (405)

Psoralea esculen/a Pursh. Prairies. (412)

Psoralea lanceolata Pursh. Squaw butte. (408)

Psoralea tenuiftonca Pursh. Squaw butte. (407)

Kunnicstera candida (Willd.) OK. Prairies. (414)

Kuhniastera purpurea (Vent.) MacM. With the preceding. $(402)$

Astragalus gracilis Nutt. Squaw butte. (406)

Astragalus hypoglottis L. Squaw butte. (416) 
Astraguhus multiflorus Gray. Bad lands. (413)

Astragalus pichs Gray var. filifolius Gray. Squaw butte. $( \pm 15)$

Spiesia lamberti (Pursh) OK. Squaw prairie. (401)

Gl!cyrihiza lopillota Pursh. Squaw canon. (409)

Prumus demissa Walp. Squaw and Warbonnet canons. (389)

Geum album Gmelin. Squaw canon. (391)

Frrgaria vesca L. Squaw canon. (B90)

Potentilla arguta Pursh. Squaw canon. (392)

Agrimonic eupctoria L. Common in canons. Hat creek. (383) Amelanchier clnifolic Nutt. Squaw canon. (394)

Epilobium adenocculon Haussk. Squaw canon. (321)

Oenothera caespitosa Nutt. Buttes near Squaw canon. (375)

Oenothera servulcite Nutt. With the preceding. (377)

Gaurce cocciner. Nutt. Squaw butte. (379)

Ribes rumeum Pursh. Warbonnet canon. (398)

Ribes cereum Dougl. Near Warbonnet canon. (397)

Ribes floridum L'Her. Squaw canon. (400)

* Ribes oxyccunthoirles L. Common in all canons. (.396)

Sedum stenopetalum Pursh. Buttes. (367)

Cornus pubescens Nutt. Squaw canon. (387)

Sanicula marylandica L. var. canculensis (L.) Torr. Squaw canon. (385)

Musenium tenuifolium Nutt. Bad lands. (388)

Osmorrhizu longistylis (Torr.) DC. Squaw canon. (387)

Euphorbia petaloidea Engelm. Buttes. (370)

Euphorbia montrna Engelm. Buttes. (372)

Polygala alba Nutt. Prairie about Squaw canon.

Acer glabrum Torr. Squaw canon. (378)

Rhus aromatica Ait. var. trilobata (Nutt.) Gray. Squaw canon. (399)

Rluus radicans L. Everywhere throughout the region.

Malveopsis coccinea (Nutt.) OK. Squaw butte. (380)

Viola conadensis L. Squaw canon. (376)

Nasturtium officinale R.Br. Squaw canon. (3.)3) 
Lesquerella argented (Pursh) MacM. Bad lands.

Argemone platyceras Link \& Otto. Squaw canon. (35:2)

Thalictin purpurcuscens L. Squaw canon. (373)

* Actceer spicrela L. var. corgute Torr. Squaw canon.

Berheris repens Lindl. Siquaw butte. (395)

Paronyclia jomesii Torr. \& Gray. Squaw canon.

Arenaria franklinii Dougl. Squaw butte. (361)

Allionic hirsufc (Nutt.) Pursh. Squaw canon. (336)

Allionire nyctergin'el Michx. var. oblongifolic Gray. Squaw canon. $(33: 32)$

Abronic frorgrans Nutt. Prairies near Squaw canon. (371)

Eviogonum ammum Nutt. Buttes and highlands. (368)

Eviogonum flucum Nintt. Buttes. (3ib)

* Eriogonum jemersii Benth. Barl lands. (362)

Rumex renosus Pursh. Fields and canons. (36t)

Humulus lupulus L. Warbonnet canou. (311)

Parielcuia permsylvenicu Mubl. Damp places in canons. (322)

Spircuthes romenzoffience Cham. Squaw canon. (3(1)

Habenavia brarteata R.Br. Squaw cauon. (306)

* Ilabenuria hyperborea R. Br. Squaw canon. (305)

Coralorrhiza multiflore Nutt. Warbonnet canon. (307)

* Coralornhiza striater Lindl. Warbonnet canou. (300)

Ýcer glcuen Fraser. Squaw canon. (30t)

Calochortus muttallii Torr. \& Gray. Squaw canon. (301)

Calochortus gumisonii Wats. Squaw canon. (302)

Zygadmus elegans Pursh. Squaw canon. (30:3)

Di:porum trachyspermm (Wats.) Benth. \& Hook. Squaw canon. (310)

Spartina rynosuroides (L.) Willd. Creek banks in canons. $(4: 3: 2)$

Phleum pratense L. Canons. (421)

(1)yropsis cuspidata (Nutt.) Benth. Prairies. (418)

Agrostis asperifolic Triu. Warbonnet canon. (43j) Determined by F. L. Scribner.

Agroslis excuralu Trin. Canous. (437) 
C'ulumovilfu longifolice (Hook.) Hack. Bad lands. (433)

Calumagrostis stricta Trin. Prairies around canons. (420)

Boutelona oligostachya (Nutt.) Torr. Valleys and bad langls $(4 \cdot 2)$

Bulbilis dactyloides (Nutt.) Raf. Prairies and bad lands. (128) Koeleriu cristata (L.) Pers. Prairies and edge of bad lands.

(4'2t)

Eatonia obtusata (Michx) Gray. Marbonnet canon. (436)

Distichlis spicata (L.) Greene var. stricta Thurber. Lige of bad lands. (4:2:3)

Panicularia nereata (Willd.) OK. Canons. (427)

Bromus ciliatus L. var. purgans Gray. Canons. (430)

Agropyrum glaucum (Desf.) R. \& s. var. occidentale Vasey \& Scribner. Prairies. (43t)

Agropyrum repens (L.) Beauv. Common everywbere. (425)

Hordeum jubutum L. Common everywhere. (422)

Elymus sitanion Schult. Bad lands. (4?!)

Elymus striatus Willd. Canons. (426)

Botrychium virginiumum (L.) Sw. Squaw canon. (4tt)

Cystopteris fragilis (L.) Bermh. Squaw canon. ( $13 ! 1)$

Woodsia oregona Eaton Squaw canon. (4t0)

Equisetum arvense L. Squaw canon. (4+7)

Equisetum levigatum Braun. Squaw canon. (445)

Equisetum robustum Braun. Squaw canon. (447)

Timmia megapolitana Hedw. Squaw canon. (438)

* Psathyrella fulvipes Mont. Sandy ground, Squaw canon. (500)

* Deconica bullacea Bull. Squaw canon. (อ02)

* Psilocybe corneipes Fr. On ground, Squaw canon. (501) Stropharia semiglobata Batsch. On horse dung, Squaw canon. (503)

* Inoeybe lannginosa Bull. On ground, Squaw canon. (504)

* Volvaria viscosa Clements n.sp. Warbonnet canon. (505)

Pileus fleshy, campanulate-convex, smooth, very viscous, fulvous-ochraceous; stipe prominently bulbous, nearly 
equal above, solid, smooth, ochraceous; volva ample, lobed, concolorous; lamellae touching, brown; spores ovoidellipsoid, dilutely flesh colored, with a large locule, 8x̃ $\mu$. Pileus $6 \mathrm{~cm}$. wide; stipe $6 \mathrm{~cm}$. long, at base $1 \frac{1}{2} \mathrm{~cm}$. wide, above $\underset{2}{1} \mathrm{~cm}$. Related to V. primulina Cooke \& Massee. C.

* Mycena acuto-conica Clements n.sp. In sand, Squaw canon. (506)

Pileus slightly membranaceous, persistently conical, acute, viscous, smooth, bright jellow; stipe long, equal, smooth, yellowish-brown; lamellae free, linear, deep ochraceons when dry; spores oblong-eiliptical, 12x7-S $\mu$. Pileus $2 \frac{1}{2}$ $\mathrm{cm}$. wide by $3 \mathrm{~cm}$. high. Stipe $6-7 \mathrm{~cm}$. long. Distinguished by its persistently conical, bright yellow pileus, and by the free ochraceous gills. O.

Aecirlium clematidis DC. On Clemalis ligusticifolia.

Aecidium pentstemonis Schw. On Pentstemon glaber.

* Puccinia ancachoreta Ell. \& Hark. On Calochorlus gunnisonii. $(451)$

Puccinia menthae Pers. On Monarda fistulosa.

Cronatium asclepiadeum (Willd.) Fr. var. thesii Berk. On Conandra pallida. (45t)

Melampsora farinosa (Pers.) Schroet. On Salix tristis. (402) * Helvella infula Schaeff. In sand, Warbonnet cauon. (507)

* Ilelvella sulcata Afzel. In wet sand, Squaw canon. (j08) * Septoria argophylla E. \& K. On Psoralea incana. (457)

* Septorir grossulariae (Lib.) Westd. On Ribes anrerum. (456) * Septoria irregularis Pk. On Rhus ralicans. (453)

* Septoria podagraniae Lasch. On Osmormhiza longistylis. (458)

*Dothidea collecta (Schw.) Ell. On twigs of Celastrus scandens, Squaw cawon. (510)

*Hypoxylou rrustaceum (Sow.) Nits. On twigs, Squaw canon. (511) 
III.

Miscellaneous Addtrions to the Florl of the State, and New or Noteworthy Species From Various Localities.

COMPOSITE.

Helenium nudiflor'm Nutt. Lincoln.

VIOLACE

Frola sagitteta Ait. Fremont. (513)

RANUNCULACEE.

Caltha palustris L. Norfolk. (õ14)

\section{SALICACE}

Populus acuminata Rydberg, in Bulletin Torrey Botanical Club XX., 4i;. Scotts Bluff county. (515)

(A new poplar, nearly related to the Balm of Gilead, $P$. balsamiferc, and the Black Cottonwood, P. angustifolia, but distinguished by its leaves, which are green on both sides, long petioled, cuneate at the base, and with a long acumination. R.)

GRAMINE正.

Paspalum leve Michx. Ashland. (כ̃16)

Cinna arundinaces L. Lineoln. (517)

FILICES.

Asplenium filix-foemina (L.) Bernh. Long Pine. (441)

BRYACE正.

Physcomitrium hookeri Hampe. Valentine. (518)

AGAIIICINE无.

Punaeolus campanulatus L. On horse dung, Lincoln. (519)

Coprinus gramulosus Clements $\mathbf{n}$. sp.

Pileus membranaceous, ovoid-oblong, at length campanulate, closely radiate-sulcate, furfuraceous, pale yellowishbrown, umbo prominent, trauslucent, flavo-fuscous, at first covered with more or less persistent coarse brown gran- 
ules; stipe white, hollow, equal, everywhere persistently pruinose; lamellae ventricose, brown, finally black, touching, at length free; spores ohlong-elliptical, brownish-purple, 1-2. guttulate, 8-10xวั-6 \%..

Pileus $2 \frac{1}{2} \mathrm{~cm}$. wide and high. Stipe $3 \mathrm{~cm} . \mathrm{x} 4 \mathrm{~mm}$.

On wet ground, in greenhouse, Lincoln. (5:0)

Coprimus lagopides Karst. In flower bed, Lincoln. (521)

Coprinus mycenopsis Karst. At base of stumps, Lincoln. (52:) Psathyra obtusata Fr. On damp ground, Lincoln. (523)

Psathyra subnuda Karst. On wet ground, Lincoln. (524)

Psathyra helobia Kalchbr. On wet earth in greenhouse, Lincoln. (525)

Psathyra fulkii Weinm. On ground, Lincoln. (526)

Psathyra schulzeri Quel. On ground, Ashlaud. (527)

Psilocybe hebes Fr. On ground, Lincoln. (528)

Psilocybe comta Fr. In grass, Lincoln. (529)

Psilocybe cernua Vahl. In grass, Lincoln. (う30)

Psilocybe uda Pers. On muddy ground, Lincoln. (531)

Paxillus chrysophyllus Trog. On pine railroad tie, Lincoln. (532)

Galera pubescens Gill. On ground, Valentine.

Galera tener Schaeff. In grass, Lincoln. . (53t)

Naucoria centunculus Fr. On railroad ties, Lincoln. (535)

Hebeloma latericolor Mont. Wet ground at base of stumps Wa. bash. (536)

Inocybe tuberosa. Clements n.sp.

Pileus expanded, squamose, fleshy, deep brown; stipe tuberous, equal above, gilvous, lamellae rather few, adnexed, deep brown; spores obtuse, ovoid-elliptical, apiculate at one end, l-guttate, $6 \times 4$,'.

Pileus $3 \mathrm{~cm}$. wide. Stipe $4 \mathrm{~cm}$. long; at base $10 \mathrm{~mm}$. above $7 \mathrm{~mm}$. wide. Related to $I$ insequens Britz. Lincoln. (537) 
Pholiote sperciosa Clements n.sp.

Pileus fleshy, plano-convex, squamose, umbonate, dirtywhite; stipe fistulose, thick, equal, white; annulus small, white; gills free, crowded, soot colored; spores umber, ovoid, 1-2-guttate, 5x31, $\%$ Pileus $4-6 \mathrm{~cm}$. wide; stipe 5 cm. long. Related to $P$. gibberosa Fr.

On ground, in woods, Wabash. (5:38)

Pholiota praecox Pers. var. minor. Batl.

In grass, Lincoln. (539)

Marasmius acicularis B. \& C. On dead wood, Valentine. (540) Marasmius rotula (Scop.) Fr. On tree trunks, among moss, Elmwood, Lincoln. (541)

Russula emetica Fr. On ground, Milford. (512)

Pleurolus limpidoides Karst. On decaying stumps, Lincoln. (543)

Tricholoma impolitum Lasch. In wet saw dust, Lincoln. (544)

Tricholoma favillare Fr. On ground in greenhouse, Lincoln. $(545)$

Tricholoma georgii Fr. On ground, Lincoln. (546)

Lepiota avellanea Clements n.sp:

Pileus fleshy, dry, plane, drab-colored, cuticle lacerate toward the margin, forming appressed brown scales; stipe somewhat hollow, bulbous, brownish-fibrillose; annulus small, fleshy, concolorous, fixed, inferior; lamellae remote, attached to an indistinct collar, cream-colored, becoming reddish with age; spores irregularly ovate, acute at one end, $8-10 \times 5-6$, ,

Pileus $5 \mathrm{~cm}$. wide, stipe $4 \mathrm{~cm}$. long by $8 \mathrm{~mm}$. wide. On ground in greenhouse, Lincoln. (547)

Lepiota naucina Fr. Damp ground in woods, Elmwood.

Lepiota implana Berk. On ground, Lincoln.

Lepiota boudieri Bres. On ground, Lincoln.

HYMENOGASTRACE 正.

Phlyctospora fusca Corda. Lincoln. 


\section{UREDINE AE.}

Puccinice cladophita Pk. II. and III. on Stephenomerien miror, Scott's Bluff county. ( $\left.\because i^{\circ}\right)$

Puccinia hicracii (Schum.) Mart. On Cnicus umlulatus, Scott's Bluff county. ( $(\div 7)$

Puccinia nicrosperme B. \& C. On Lobelia syphititica, Lincoln.

( $\left.10 t^{2}\right)$ Not previously reported from eastern Nebraska. Uromyces caryophyllimus (Schrank.) Schroat. On Dianthus sinensis in greenhouse, Lincoln. (1127) Determined by Dr. Bessey.

\section{PEZIZE.E.}

Sclerotinia tuba Batsch? Lincoln. (5.59)

(As the specimens correspond to the very meager descriptions of S. tuba, I have referred them to that species provisionally. I append a description of them: Cups infundibuliform, arising from a black sclerotium, $1 \mathrm{~cm}$. in diameter, soot-black, smooth, thin; stipe long, black, coriaceous, striate when dry, $4 \mathrm{~cm}$. hy $4-5 \mathrm{~mm}$., gradually widening into the cup; asci cylindrical, 8-spored, $200 \times 16$ \%. Cups $2 \frac{1}{2}-3 \mathrm{~cm}$. wide by $2.2 \mathrm{~cm}$. high. C.)

Helotium sulfurellum E. \& E. On dead limbs, Elmwood, Syracuse. (.53)

Neotticlla culichroa Boud. On earth in flower pot, Lincoln. (5j4) Lachnea laeticolor Karst. On fallen leaves, Lincoln. (5jJ)

Lachner fissilis Sacc. \& Cke. On wet boards in greenhouse, Lincoln. (5).5i)

Humaria rutiluns Fr. On grouud with moss. Lincoln. (5ீ37) Peziza sepiatra Cke. On earth in greenhouso, Lincoln. (568) Pezizer resiculosa Bull. var. minor Sace. On wet wood in greenhonse, Lincoln. (jtil)

Geopyxis carbonaria A. \& S. On damp ground in woods, Lincoln. (5it)

Geopyxis palliclula C. \& $\mathrm{P}$. On wet sand and wood in greenhouse, Lincoln. (571) 
Geopyxis Imberculosu Sace. \& Cke. On damp ground in hot-bed, Lincoln. (57:)

SPHAEROL'AIDEA.

Septoria verbena: Rob. On Verbena hastrta, Lincoln. MELANCONIE.E.

Glocosporium muscu'um Cke. \& Massee. On bananas, Lincoln. (อ̃73)

(G. lagemurum (Pass.) Sace. var. musarum E. \& E., in Journ. of Mycol. V. 15.j. The latter described by Ellis and Everhart in 1859 , from specimens collected by me appear's to be the same as $G$. musarnm described, from specimens collected in Australia, in Grevillea XVI. 3. (1587) Conidia long-ellipsoid, rounded or sometimes pointed, $7 \%-11 \times 3- \pm \mu$. As is said by Ellis and Everhart l. c., scarcely differs from G. lagenarium. P.)

DEMATICEA.

Cercospora brunkii Ell. \& Gal. On Pelargonium sp.(cultivated) Lincoln. (1126) Determined by Dr. Bessey. DOTHIDIACE.E.

Dothider, collecta (Schw.) Ell. On decaying stems, Lincoln. (574) HYPOCREACER.

Megalonectria caespitosa Speg. On block of wood supporting a south-Mexican orchia in greenhouse, Lincoln. (1125) This species was discovered by Spegazini in Brazil on fragments of wood, and does not seem to have been reported since. The block on which it was growing was an ordinary one picked up about the greenhouse, and it seems probable that the orchid was respousible for the presence of the fungus.

SPHARIICE臣.

Phomatospona berkleyi Sace. On stems of Srmbucus conartensis, Saltillo. (575) 
Trichosphaeria pullllriseta ( $\mathrm{Pl}$.) Ell. On herbaceous stems, Saltillo. (576)

Hypoxylon perforctum (Schw.) Sacc. On twigs of Acer negundo, Saltillo. (567)

\section{ERYSIPHER.}

Erysiphe cirhoracearum DC. OnVerbena bracteosa, Crete (1091); on Ambrosia artemisifolia, Crete (1094); on Tragopogon porrifolius, Crete. (1099)

Erysiphe communis (Wallr.) Fr. On Oenothera biennis, Crete (1095); on Thalictrum purpurascons, Crete (1098); on Ampricarpaca comosa, Crete. (1100)

Erysiphe galeopsidis DC. On Scutellaria lateriflora, Crete. (1081); on Stachys palustris, Crete. (1085)

Microsphaera elevata Burrill. On Catalpa speciosa, Crete. (1115)

Microsphaera quercina Schw. On Quercus macrocarpa, Crete. (1117)

Microsphaera russellii Clinton. On Oxalis stricta, Crete. (1114)

Uncinula circinata $\mathrm{Pk}$. On Acer saccharinum, Crete. (1104) Uncinula necator (Schw.) Burrill. On Vitis riparia, Crete (1103); on Vitis sp. (cult'd) Crete. (1107)

Phyllactinia suffulta (Reb.) Sacc. On Fraxinus viridis, Crete. (1120)

Sphaerotheca castagnei Lév. On Bidlens frondosa, Lincoln (1023), Crete (1102); on Bidens levis, Crete.

CLADOPHORACE...

Chaetophora pisiformis (Roth.) Ag. Minden. (578)

ULOTRICHACEN.

Conferva affinis $\mathrm{Kg}$. Lineoln. (579)

Microspora abbreviata (Rabh.) Lagerh. Lincoln. (580) 
DESMIDIACEA.

Sphaerozosma filiforme Rabh. Minden. (581)

Pleurotaenium nodulosum DBy. Minden. (582)

Cosmarium leve Rabh. var. septentrionale Wolle. Minden. (583)

Cosmurium granatum Bréb. Minden. (58t)

Cosmarium subcrenatum Hantzsch. Minden. (585)

Cosmarium tinctum Rabh. Minden. (586)

Staurastrum crenulatum (Delp.) Naeg. Minden. (587.)

DIATOMACE $/$.

Navicula tabellaria Kg. Minden. (588)

Navicula cuspidata Kg. Minden. (589)

Navicula gibba $\mathrm{Kg}$. Minden. (590)

Navicula viridula $\mathrm{Kg}$. Minden. (591)

Navicula viridula $\mathrm{Kg}$. var. minor $\mathrm{Kg}$. Minden. (593)

Navicula tenela Bréb. Minden. (592)

Stauroneis smithii Grun. Minden. (594)

Pleurosigma kuetzingii Grun. Minden. (595)

Amphiprora alata Ehrb. Minden. (596)

Cymbella turgidula A.Schm. Minden. (597)

Amphora libyca Ehrb. Minden. (598)

Gomphonema olivaceum Ehrb. Minden. (丂99.)

Gomphonema lagenula $\mathrm{Kg}$. Minden. (600)

Gomphonema intricatum $\mathrm{Kg}$. Minden. (601)

Gomphonema parvulum Kg. Minden. (602)

Nitzschia coarctata Grun. Minden. (603)

Nitzschia gracilis Hantzsch. Minden. (604)

Nitzschia palea (Kg.) W. Sm. Minden. (605)

Suriraya saxonica Auersw. Minden. (606)

Suriraya spiralis Kg. Minden. (607)

Suriraya norvegica Eulenst. Minden. (608)

Suriraya euglypta Ehrb. Minden. (609)

Fragilaria entomon Ehrb. Minden. (610)

Fragilaria capucina Rabh. Minden. (611) 
Fragilaria mutabilis Grun. Minden. (612)

Eunotic parallela Ehrb. Minden. (613)

Eunotia major Rabh. Minden. (61t)

Cymatopleura elliptica W.Sm. Minden. (615)

PALMELLACE $\notin$.

Pediastrum tetras (Ehrb.) Ralfs. Lincoln.

BACTERIACE A.

Beggiatoa arachnoidea (.Ag.) Rabh. Lincoln.

Leptothrix calcicola $\mathrm{Kg}$. Lincoln. (1i18)

NOSTOCACEAS.

Sphaerozygu smithii Thwaites. Lincoln. (619)

Cylindrospermum limnicola $\mathrm{Kg}$. Lincoln. (625)

OSCILLARIACE AD.

Oscillaria violacea Wallr. Lincoln. (620)

Oscillaria antliaria Juerg. Lincoln. (621)

Oscillaria tenerrima $\mathrm{Kg}$. Lincoln. (622)

CHROOCOCCACEE.

Chroococcus rufescens (Bréb.) Naeg. Lincoln.

Gloeocapsa arenaria Rabh. Lincoln. (624). 
UNIVERSITY OF NEBRASKA.

\section{Botanical Survey of Nebraska.}

Conducted by the Botanical Seminar.

\section{III.}

\section{REPORT FOR 1893.}

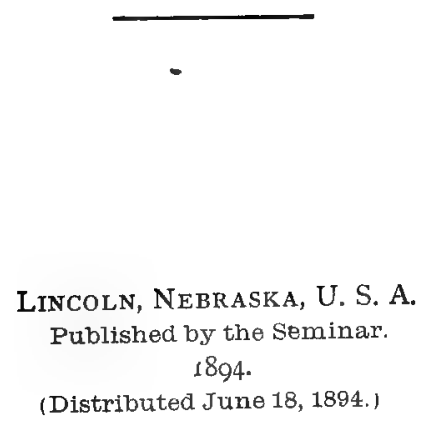





\section{CONTENTS.}

Note.

Additions to the Reported Flora of Nebraska made during

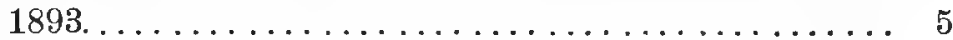

A Revision of the Nomenclature of the Nebraska Polypetalæ,

by P. A. Rydberg ..................... 20

Preliminary List of Botanical Expeditions made in Nebraska,

by Frederick E. Clements .................. 39

Bibliography of the Flora of Nebraska, by Roscoe Pound ... 43 


\section{NOTE.}

The principal collections made during the year 1893 were three. Messrs. Woods and Saunders collected in the Republican valley from the Colorado line eastward, and also on the Little Blue. Mr. Rydberg spent the entire summer in the sandhill region, principally along the Dismal river. Mr. Clements spent the entire summer in northeastern Nebraska, beginning at Emerson, in Dakota county, and collecting along the Missouri and Niobrara to Meadville, in Brown county. The last two collections were made possible by the United States Department of Agriculture which undertook them jointly with the Survey.

The thanks of the Seminar are also due to Rev. J. M. Bates, of Valentine, Dr. H. Hapeman, of Minden, and to Mr. C. A. Turrell, Mr. Chas. Engberg, and Misses Cora F. Smith and Louise Lee, stndents in the Department of Botany at the University, for collections or additions to the reported flora. 
additions to the Reported Flora of Nebrasea Made During 1893.

*Indicates new host only.

*Phytomyxa leguminosarum (Frank) Schroeter.

On Falcata comosa, Cushman. (4044)

On Dalea dalea, Bellevue. (3225)

Lampropedia littoralis (Oerst.) DeToni and Trevisan.

Stagnant water in salt marshes, Lincoln. (3380)

Merismopedia convoluta Breb.

South Bend. (3376)

Scytonema cinereum Menegh.

Lincoln. (3381)

Scytonema hoffmanii Ag.

Lincoln. (3382)

Stigeoclonium tenue Kuetz.

Lincoln. (3377)

Hormiscia flaccida nitens (Menegh.) Hansg.

In Greenhouse, Lincoln. (3378)

Closterium acuminatum Kuetz.

Lincoln. (3383)

Closterium lanceolatum Kuetz.

Lincoln. (3384)

Cosmarium gottlandicum Wittr.

Lincoln. (3385)

Cosmarium ralfsii Breb.

South Bend. (3386)

Staurastrum pseudopachyrinchium Wolle.

South Bend. (3387)

Spirogyra grevillicina (Hass.) Kuetz.

Lincoln. (3379) 
Spirogyra tenuissima (Hass.) Kuetz.

Lincoln. (3390)

Spirogyra varians (Hass.) Kuetz.

Lincoln. (3391)

Vaucheria aversa Hass.

Lincoln. (3388)

Vaucheria terrestris Lyngb.

Minden. (3389)

Vaucheria tuberosa A. Br.

Saltillo. (3392)

* Peronospora parasitica (Pers.) DeBary.

On Roripa palustris. Bellevue. (3108)

Plasmopara halstedii (Farlow) Berlese \& DeToni.

On Silphium perfoliatum, Lincoln. (3178)

Batrachospermum gelatinosum (L.) Woods.

Bellevue. (3393)

Chantransia violacea Kuetz.

Bellevue. (3394)

*Sphaerotheca pannosa (Wallr.) Lev.

On Rosa multiflora (cultivated), Lincoln. (4000)

* Erysiphe cichoracearum DC.

On Helianthus decapetalus, Lincoln. (3168)

Nitschkia tristis (Pers.) E. \& E.

On decaying branches, Saltillo.

Fracchiaen heterogenea Sacc.

On log, Lincoln. (3270)

Coronophora annexa (Nits.) Fkl.

On dead limbs, Saltillo. (3268)

Diatrype albo-pruinosa (Schw.) Cooke.

On dead oak limbs, Saltillo. (3269)

Hypoxylon colliculosum (Schw.) Berk.

On log, Cedar Bluffs. (3274)

Hypoxylon marginatum (Schw.) Berk.

On dead limbs, Saltillo. (3396) 
Hypoxylon stigmateum Cooke.

On oak, Lincoln. (3271)

Nummularia repanda (Fr.) Nits.

On oak, Saltillo. (3275).

Nummularia bulliardii Tul.

On dead limbs, Saltillo. (3397)

Chaetomium bostrichodes Zopf.

On rotting cloth, Lincoln. (3398)

Trichosphaeria socia Sacc.

On dead limbs; Saltillo.

Didymosphaeria accedens Sacc.

On dead twigs, Saltillo.

Amphisphaeria pseudo-umbrinc Sacc.

On Ostrya ostrya, Cedar Bluffs.

Valsaria insitiva (Cesati) De Not.

On Gleditsia triacanthos, Saltillo.

(3239)

Valsaria foedans (Karst.) Sacc.

On decaying twigs, Saltillo.

Melanomma cupularis Clements, n.sp.

Perithecia generally forming a thin crust, sometimes scattered, superficial, globose, varying to disciform, finally cupulate, slightly roughened, black, small, $250-300 \mu$; ostiolum inconspicuous; asci lacking; spores oblong, straight, or curved; ends obtuse, tri-septate; end cells truncate-globose, hyaline; interior cells oblong fuscous, uniguttate, 25-28x5-6 $\mu$. On stump, Saltillo. (325j)

Pleospora saccardiana Roum.

On Fraxinus viridis, Saltillo.

Cucurbitaria kelseyi E. \& E.

On dead branches, Cedar Bluffs.

Ophiobolus fulgidus (C. \& P.) Sacc.

On dead sunflower stalks, Saltillo.

Gloniopsis australis (Duby) Sacc.

On oak, Saltillo (3272) 
Hysterographium variabile (C. \& P.) Sacc.

On decorticated stump, Saltillo.

Hysterographium mori (Schw.) Rehm.

On twigs Saltillo.

(3395)

Peziza rivularis Clements, n.sp.

Sessile, carnose, explanate or slightly concave, margin strongly raised; hymenium umber, without blâckish brown, minutely verrucose; asci cylindrical, not becoming blue at tip with $\operatorname{iodin} \theta, 250 \times 20 \mu$; paraphyses few, hyaline, granular, 5-6 $\mu$; sporidia broadly ellipsoid, monostichous, with two large guttae (10 $\mu$ in diameter), 25$26 \times 15-16 \mu$.

On immersed branches in streams, Meadville.

Discina venosa rabenhorstii Cooke.

On wet branches, Meadville.

(2990)

Sarcoscypha coccinea (Jacq.) Fr.

On dead limbs, Lincoln.

Lachnea theloboloides Alb. \& Schw.

On bark, Saltillo.

(3266)

Ciboria tabacina Ell. \& Holw.

On dead limbs, Saltillo.

(4153)

Mollisia cinerea canella Karst.

On dead limbs, South Bend.

Mollisia stictella Sacc. \& Speg.

On bark, Saltillo. (3262)

(2991)

richopeziza subochracea (C. \& P.) Sacc.

On sunflower stalks, Saltillo.

(3277)

Cenangium populneum (Pers.) Rehm.

On Quercus rubra, Bellevue.

Schyzoxylon bagnisianum Speg.

On decaying twigs, Saltillo.

(2996)

(3261)

Lecanidion atratum (Hedw.) Rabh.

On decorticated ash treos, Saltillo.

(3260) 
Blitrydium megalosporum Cloments, n.sp.

Ascoma patellate, sessile, carbonaceous, black, $\frac{1}{2}$ to $1 \frac{1}{2} \mathrm{~mm}$; disc black, plane, or strongly convex, margin more or less convex; asci long clavate, nearly sessile, 8-spored, 140$175 \times 30-40 \mu$; spores elliptical oblong, brownish yellow, attenuated both ways but obtusely rounded at the ends, inequilateral, with 12-15 transverse septa and 5-7 longitudinal interrupted septa, becoming fenestrate muriform, distichous, not constricted, 50-72×20-25 $\mu$; paraphyses colorless, clavate, furcate above.

On decorticated twigs of Fraxinus viridis Saltillo.

Puccinia angustata $\mathrm{Pk}$.

On Cyperus esculentus, Kennedy.

On Scirpus atrovirens, Saltillo. (3296)

*Puccinia caricis (Schum.) Rebent.

On Cyper'us houghtonii, Thomas county. (3317)

*Puccinia helianthi Schw.

On Heliopsis scubra, Bellevue. (3208)

Puccinia lygodesmice E. \& E.

On Lygodesnia juncea, Arapahoe.

* Puccinia petalostemonis Farlow.

I. and II. on Kuhniastera purpurea, Lincoln. (3360, 3090) *Puccinia silphii Schw.

On Silphium perfoliatum, Lincoln. (3176)

Puccinia porri (Sow.) Winter.

I. on Allium mutabile, Lincoln.

*Puccinia pimpinellae (Strauss) Lk.

III. on Peucedanum foeniculaceum, South Bend.

$(3352)$

*Aecidium compositarum Mart.

On Rudbeckia angustifolia, Lincoln.

$(3096)$

*Aecidium clematidis DC.

On Clematis virginiana, Lincoln.

*Aecidium jamesianum $\mathrm{Pk}$.

On Asclepias tuberosa, Lincoln. 
Aecidium phrymae Halst.

On Phryma leptostachya, Thomas county. (3303)

* Aecidium oxalidis Thuem.

On Oxalis stricta, Lincoln.

(3363)

Tilletia montana E. \& E.

On Redfieldia flexuosa, Hooker county. (4055)

Tilletia tritio (Bjerk.) Winter.

On wheat from the Republican valley. (3267) Spores reticulate.

Phoma pustulata Sacc.

On dead maple branches, Saltillo. (3136)

Sphacropsis gleditschiicola Cooke.

On Gleditsic tricucanthos, Saltillo. (3006)

Sphrecronema longirostris Clements, n.sp.

Perithecium innate, with a long, stout, cylindrical beak, breaking through the cortex, black, globose, cespitose 1$1 \frac{1}{2} \mathrm{~mm}$. diam.; spores fusiform, elongate, slightly curved, 2-nucleate, $20 \times 3 \mu$.

In living branches of Cratcogus tomentosa, Saltillo. Cytospora hyalosperma Fr.

On Acer negundo., Saltillo.

Phlyctaena arcuata Berk.

On Datura stramonium, Lincoln.

* Septoria verbence Rob.

On Verbena bracteosa, Cushman.

Nelanconium magnum (Grev.) Berk.

On Hicoric sp., Saltillo.

Trimmatostroma americana Thuom.

On Acer negundo, Saltillo.

Pestrulozzic monorhincha Sacc.

(3012)

On Cleditsia triacanthos, Saltillo.

I'estalozzir pezizoides DeNot.

(3007)

On Vitis riparia, Saltillo. 
Monilia penicillata E. \& E.

On rotton pine wood, Kennedy. (3334)

Botrytis (Polyactis) doryphora Pound \& Clements, n.sp.

Mycelium white, cobwebby, at length more-compact and darker; filaments greatly elongated, nearly erect, septate, sparely branched above; branches arising at right angles to filament, short, strict, with a large terminal hastate enlargement also found on short lateral branches; coridia elliptical, white, in dense capitula, $5 \times 2 \frac{1}{2} \mu$.

On leaves and stems of Pelargonium, Lincoln.

Arthobotrys superba oligospora (Fres.) Coemans.

On Pleurotus ostreatus, Lincoln.

Trichothecium obovatum (Berk.) Sacc.

On Cucurbitaria morbosa, Saltillo.

Diplocladium penicillioides Sacc.

On decaying fungi, Lincoln.

$(3010)$

(3002)

Heterosporium didymosporum Clements, n.sp.

Effuse, dark green; hyphae very long, flexuose, sparingly branched, fuscous, septate, $200 \times 6 \mu$, conidia oblong-cylindrical or obovate, rounded at both ends, rarely aculeate, olivaceous, 1, rarely 2-3-septate, strongly constricted at the septum, 20-25x10-12 $\mu$.

On decaying osage orange, Lincoln. (3335)

Helminthosporium macrocarpum caudatum B. \& Br.

On dead limbs, Saltillo. (3005)

Tubercularia pircuniae Speg.

On Hickory bark, Saltillo. (4201)

Hymenula fruticola Pound \& Clements n.sp.

Sporodochia superficial, ellipsoid or oblong, often confluent, pale reddish, farinose gelatinous, disposed in lines on the angles of the capsules; sporophore simple, rarely furcate, short, 30-45x4 $\mu$; spores ellipsoid or ovoid, spherical, hyaline, 6-8x5-6 $\mu$.

On fruits of Salix longifolia, Stevens Creek. (4202) 
Fusarium luteum Clements, n.sp.

Innate or erumpent, hard, convex or plane, golden yellow hyphae short, simple, continuous; conidia falcate, curved, acute at both ends, always 3-septate, hyaline, $32-39 \times 4-5 \mu$.

In decaying wood, Lincoln. (4203)

Fusarium rhizogenum Pound \& Clements, n.sp.

Mycelium superficial, 1-2 mm. in diameter, dense, conver, white, then yellow; filaments densly aggregated, ascending, septate, sparingly branched; conidia at the ends of the branches, oblong, rounded at the ends, hyaline, 1-septate, $70 \times 4 \mu$.

On roots of seedling apple, Lincoln. (3013)

Phlyctospora sclerodermoides Clements, n.sp.

Subterranean, depressed globose, nearly smooth, brown; peridium thick, coriaceous, radicate, gleba firm, chestnut colored; spores crowded, globose, brown, covered with more or less reticulate papilla which are $2 \frac{1}{2}-3 \frac{1}{2} \mu$ long, involved in an indefinite hyaline mucuous, $18-25 \mu$ in diameter.

Peridium $3 \frac{1}{2} \mathrm{~cm}$. wide by $2 \frac{1}{2} \mathrm{~cm}$. high. In cultivated soil, Lincoln. (4204)

Lepiota spectabilis Clements, n.sp.

Solitary; pileus carnose, membranaceous, densely covered with silky fibrillae, or pulverulent, dilutely sulphur colored, radiately striate or sulcate toward the margin, disc depressed, smooth, yellow, $5 \mathrm{~mm}$. in diameter; stipe slender, strongly incrassate at the base, furnished above with an annulus with minute scales, glabrous below, concolorous; annulus straw colored, inferior, fixed; lamellae crowded, narrow, dilutely yellow, remote; spores ovoid 5-6 $\mu$ diameter, 1-guttate.

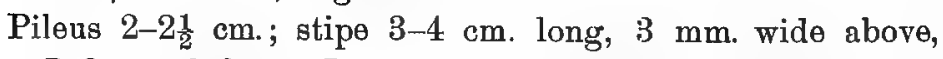
7-8 $\mathrm{mm}$. below. In greenhouse, Lincoln.

Agaricus haemorrhoidarius Kalch.

(3227)

On ground, Lincoln.

(3.28) 
Flammula alnicola Fr.

On stumps, Lincoln. (4207)

Galera striatula Clements, n.sp.

Pileus membranaceous, campanulate, convex, silky atomaceous, soot brown, strongly striate to the umbo, umbo smooth, glabrous, brown; stipe elongate, equal, white, then rufescent, glabrous; lamellae free, remote, affixed to a collar, narrow, ochraceous rubiginous; spores ellipsoid, eguttulate, $12-15 \times 7-8 \mu$.

Pileus $1 \frac{1}{2}-2 \mathrm{~cm}$. ; stipe $9-10 \mathrm{~cm}$. by $2 \mathrm{~mm}$.

On moist ground, Lincoln. (3322)

Psilocybe gillettii Karst.

Franklin. (3159)

Collybia loripes $\mathrm{Fr}$.

Franklin.

(3258)

Psathyrella sulcata Clements, n.sp.

Pileus campanulate, at length convex expanded, gray, verging into black, pellucid, deeply radiately sulcate, umbo smooth, yellow; stipe smooth, shining, semi-pellucid, slender, fistulose, white above, rufescent below; lamellae adnexed, slightly ventricose, cinereous, edge black, at length everywhere brownish black; spores ovate, apiculate at one end, fuscous, or brownish purple, 8-10×5-6 $\mu$ Pileus 1-2 $\frac{1}{2} \mathrm{~cm}$; stipe 4-6 cm. x 1-2 om.

On ground, Lincoln. (4208)

Coprinus atramentarius (Bull.) Fr.

On ground, Kennedy. (4211)

Coprinus picaceus (Bull.) Fr.

On ground, Hastings. (4206)

Coprinus velox Godey.

On ground, Lincoln. (4209)

Polyporus varius $\mathrm{Fr}$.

On wood, Lincoln. (4210) 
Poria contigua Fr.

On dead limbs, Lincoln. (3344)

Craterellus sinuosus crispus (Fr.) Massee.

Lincoln. (3343)

Corticium lacteum Fr.

On bark, Lincoln.

Corticium pellicula Karst.

On bark, Lincoln.

Corticium roseolum Masseo.

On wood, Lincoln. (4198)

Coniophora umbrina (A, \& S.) Fr.

On bark, Lincoln. (3342)

Nitella translucens (Pers.) Ag.

York. (3370)

Nitella translucens form confervoides Thuill.

York. (3369)

Chara crassicaulis Schleich.

Haigler (3366, 3367), Pine Ridge.

Chara evoluta Allen.

Sheridan county, part of material reported as $C$. foetida

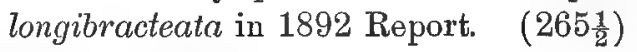

Chara sejuncta $\mathrm{A}$. Br.

Minden. (3365)

Pottia truncata Fuern.

Saltillo. (3364)

Iypnum aduncum Hedw.

Decatur. (3351)

Dryopteris cristata (I.) Gray.

South Dismal River, Hooker Co. (4101)

Potamogeton heterophyllus Schreb.

Kennedy. (4101)

Potamogeton interruptus Kit.

Thedford. (4102) 
Potamogeton perfoliatus richurdsonii Arn.

Bennet Lake, Grant county. (4103)

Potamogeton pusillus L.

Thedford. (4104)

Naias guadalupensis (Spreng.) Morong.

Whitman. (4105)

Sagittaria arifolia J. G. Smith in lit.

Hooker county. (4106)

Paspalum setaceum ciliatifolium (Michx) Vasey.

Mullen. (4107)

Panicum dichotomum villosum Vasey.

Plum Creek. (2913)

Sporobulus filiformis (Thurber) Rydberg. S. depauperalus filiformis Thurber, S. gracillimus Scribner.

Thedford. (4108)

Poa fendleriana (Steudel) Vasey.

Thedford. (4109)

Scolochloa festucacer (Willd.) Lk.

Whitman. (4110)

Agropyrum caninum unilaterale (Cassidy) Vasey.

Grant county. (4114)

Hordeum pusillum Nutt.

Thedford. (4114) Some of the Nebraska specimens named H. nodosum belong here-perhaps all. (Rydberg).

Elymus macounii Vasey.

Grant county. (4113)

Cyperus houghtonii Torr.

Natick. (4115)

Carex beckii Boott.

Long Pine. (4132)

Carex filiformis lanuginosa (Michx.) B.S.P.

Thedford. (4116)

Carex limosa $\mathrm{L}$.

Kennedy. (4133) 
Carex pseudocyperus americana Hochst.

Grant county. (4120)

Carex scoparia Schkur.

Thedford. (4117)

Scirpus lacustris occidentalis Watson.

Thomas and Hooker counties." (4121)

Lemna gibba L.

Plummer Ford, Thomas county. (4130)

Lemna perpusilla Torr.

Plummer Ford, Thomas county.

Heteranthera dubia, (Jacq.) Morong.

Kearney. (2998)

Juncus alpinus insignis, Fries.

Pishelville. (2247)

Leptorchis loesilii (L.) MacM.

Thedford. (4134)

Salix cordata angustifolia (Pursh) Anders.

Thomas and Hooker counties. (4118)

Boehmeria cylindrica (L.) Willd.

Halsey (4122), Fremont (3348), Pishelville (2768), Endicott, (2169)

Polygonum litorale Lk.

Mullen. (4136)

Polygonum punctatum leptostachyum (Meisn.) Small.

Thomas, Hooker, and Grant counties. (4136)

Chenopodium rubrum $\mathbf{L}$.

Grant county. (4129)

Salsola kali tragus Oeder.

Aten (2646), Republican City. (2102) All localities heretofore given for $S$. kali should be transferred to the var. tragus. All material in the Survey Herbarium is to be referred to the variety. 
Acnida tamariscina (Nutt.) Gray.

- Thomas county. 4138. This has been confounded with $A$. tuberculata Moq. which is common in eastern Nebraska. (Rydberg.)

Talinum calycinum Engelm.

Specimens collected in Nebraska, probably at Fort Robinson, by Dr. Wilcox are in the National Herbarium at Washington. (Rydberg.)

Arenaria michauxii Hook.f.

Franklin. (2089)

Ranunculus multifidus terrestris Gray.

Fremont. (3349)

Ranunculus septentrionalis Poir.

Emerson. (2510)

Sanguinaria canadensis L.

Bellevue. (B116)

Cardamine hirsuta $\mathrm{L}$.

Grand Rapids.

(2851)

Lepidium draba L.

Wymore. (3393) Introduced.

Crataegus coccinea $\mathrm{L}$.

Forks of Dismal River, Hooker county. (4128)

Crataegus coccinea macracantha Dudley.

Grand Rapids. (2850)

Geum japonicum Thurber.

Natick. (4123)

Amorpha nana Nutt.

Aten. (2653)

Psoralea cuspidata Pursh.

Grand Rapids. (2946)

Euphorbia heterophylla graminifolia (Michx.) Engelm.

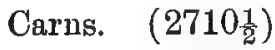

Euphorbia serpens H. B. K.

Lincoln. (3375) 
Hypericum sphaerocarpum Michx.

Richardson county, July 22, 1873. In the Aughey Collection in the herbarium of the University, labeled Lysimachic stricta. (Rydberg.)

Didiplis linearis Raf.

Lincoln. (4139)

Ludwigia alternifolia $\mathrm{L}$.

Endicott, (2047)

Stenosiphon virgatus Spach.

Franklin. (20こ2)

Hippurus vulgaris $\mathrm{L}$.

Whitman. (4124)

Myriophyllum spicatum $\mathrm{L}$.

Grant county.

Aralia nudicaulis $\mathbf{L}$.

Shaded bluffs of the Missouri throughout northeastern Nebraska. (2548)

Sium cicutifolium Gmelin.

Grant county.

$(4127)$

Foeniculum officinale $\mathbf{L}$.

Pishelville. (2754)

Gentiana andrewsii Griseb.

Mullen (4126), Fremout (3350), Minden.

Asclepias jamesii Torr.

Haigler. (2057)

Cuscuta coryli Eng.

On Salix sp., and Aster sp., Hooker county (4125), on Salix longifolia, Chelsea. (2808)

Gilia inconspicua Dougl.

Wabash. (3358)

Lithospermum arvense $\mathrm{L}$.

Lincoln. (4095)

Verbena hastata $x$ stricta Rydberg. (V. puniculata $x$ stricta Engelm?) Hooker county. (4132) 
Agastache scrophularifolia (Willd.) OK. ,

Bellevue. (3128)

Koellia lancelata (Pursh) OK.

Valleys of Middle Loup and South Dismal. (4133)

Pedicularis lanceolata Michx.

Bellevue. (3123)

Datura tatula $\mathbf{L}$.

Thedford, escaped. (4140)

Lobelia spicata hirtella Gray.

Thedford (4134). Perhaps the majority of specimens of L. spicata reported from the state belong to this variety.

(Rydberg).

Aster junceus Ait?

Thedford. (4139)

Aster oblongifolius rigidulus Gray.

Long Pine Canon. (29+1)

Aster umbellatus pubens Gray.

Halsey. (4135)

Helianthus petiolaris patens (Lehm).

Grant county. (4139)

Helianthus strumosus $\mathbf{L}$.

Long Pine. (2895)

Coreopsis involucrata Nutt.

Endicott. (2080)

Bidens connata comosa Gray,

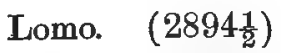

Gaillardia aristata Pursh.

Franklin. (2078)

Polypteris hookeriana Gray.

Haigler. (2064) 
SUMMARY.

Number of species reported in Webber's Catalogue (1889) . 1890 Additions in Webber's appendix to his catalogue, (March

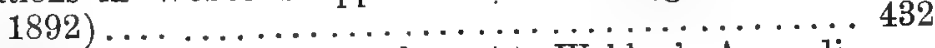
Additions in Dr. Bessey's supplement to Webber's Appendix

(June 1892)....................... 170 Additions in Report of the Survey for 1892 (1893)... 159 Less new hosts only .............. 13

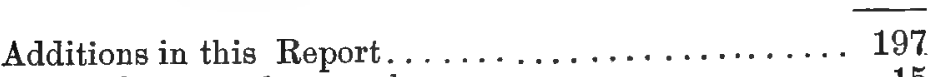

Less new hosts only.............. 15

Total number of species reported for the state.....2820

\section{a Revision of the Nomenolatore of the Nebraska Poly petalae.}

BY P. A. RYDBERG. *

Herewith I submit an attempt at a revision of the nomenclature of the reported Polypetalae of Nebraska. I say an attempt, as I know full well that the list is not perfect. Owing to the fact that my time has been limited and that the otherwise very good botanical library of the University lacks some of the older foreign works in which original descriptions of certain of our plants are to be found, I have not been able to do the work as I had wished. The original description of a species is especially necessary in this work, as American botanists have been in the habit of citing nomina nuda in the same manner in which they cite names accompanied by descriptions. Professor Greene's reprint of Fraser's catalogue has saved me from doing as Professor MacMillan has done in a number of cases in his Metaspermae of the Minnesota valley, namely, accepting nomina nuda.

\footnotetext{
* Read belore the Seminar, February 17, 1894.
} 
I have tried to follow as rigidly as possible the Paris Code, the Rochester Rules of the Botanical Club of the A. A. A. S., and the amendments male thereto at the Madison meeting, although I wish to record my strongest protest against one of the latter, as it introduces some most inelegant nomenclature. In work of this kind the one indispensible book is, of course, Dr. Kuntze's Revisio Generum Plantarum. This book, also, has caused the most changes in (or rather restorations of) generic names. Many corrections of the nomenclature of generic names have been made from the writings of Professor Greene and Dr. Britton; one (Leptoglottis for Schrankia) I have made myself. The sources from which I have derived corrections of specific names are too diverse to be mentioned here. The two books most often referred to and from which, perhaps, I have had the. most help are Watson's Index and MacMillan's Metaspermae. At the same time I have found Professor Greene correct in stating that the former is not exact, and that the latter will be useful to "those who know how to use" it.

As a basis I have taken the sixth edition of Gray's Manual, as it is most commonly used and latest published manual, and have changed those names which do not conform to recent views. Those not changed may be regarded as in accordance with them except as to the citation of authors. In the case of our western species not given in Gray's Manual, the corrections are made from Coulter's Manual of the Rocky Mountain Region. In cases where the two books disagree and no correction is made, the name in Gray's Manual should be regarded as the correct one. Thus, Coulter has Anemone decapetala* and the genus Vesicaria. Gray has Anemone caroliniana and Lesquerella. The latter two should be used.

My list contains 130 names, which is about thirty per cent of the reported Polypetalae of the state. Not all of these changes, however, are due to the requirements of correct nomen-

\footnotetext{
"The true A. decapetala may be found in the southern part of the Rocky Mountain region, but not in Nebrasku. Coulter's $\boldsymbol{A}$. decapetala may include both.
} 
clature, several being required by different limitation of genera or species. It is quite likely also that some necessary changes have been overlooked, and, on the other hand, that some changes may not stand.

The first name given is the corrected name, the last one the name used in the manuals. In many cases $I$ have found it necessary to interpolate either the binomial under which the plant was first described or such synonyms as will show the derivation of the several names.

Pulsatilla hirsutissima (Pursh) Britton. Contrib. Herb. Columbia Coll. 23,217 (1891), first published as Clematis hirsutissima Pursh. Fl. Am. Sept..385. (1814)

Anemone patens var. nuttalliana Gray Man. Ed. 536.

Dr. Britton as well as the best European authorities, consider Pulsatilla a genus distinct from Anemone.

Anemone quinquefolia L. Spec. Pl. 541. (1753)

Anemone nemorosa American authors, not Linne.

Anemone canadensis L. Syst. Ed. 12, III., app. 231.

Anemone pennsylvanica L. Mant. II. 247. (1771)

Syndesmon thalictroides (L.) Hoffmangg. Flora XV., part II.,

Intell. Bl. No. IV., 34. (1832)

Anemone thalictroides L. Spec. Pl. 542. (1753)

Anemonella thalictroides Spach. Hist.Veg. VII., 240.

(1839)

This plant has been referred to Thalictrum as well as to Anemone. It is generally regarded as the type of a distinct genus, and should have the older generic name-Syndesmon.

Ranunculis lacustris Beck \& Tracy, Eaton's Man. Ed. 3,423. (1822)

Ranunculus multifidus Pursh. Fl. Am. Sept. 736 (1814), which name is preoccupied by $R$. multificlus Forskal Fl. Egypt, 102. (1755)

Ranunculus lacustris terrestris (Gray) Mac M., Met., Minn. Valloy 217; (1892) 
Ranunculus multifidus terrestris Gray Man. Ed. 5, 41.

Ranunculus ovalis Ruf. Journ. Bot., 268. (1814)

Rconunculus rhomboideus Goldie Edinb. Phil. Journ. VI., 329.

(1822)

Ranunculus macounii Britton Contrib. Herb. Columbia Coll. No. 30, 3. (1892)

Ranunculus hispidus Hook. Fl. Bor. Am. I, 19. (1830), not

R. hispidus, Michx. Fl. N. Am. I., 321. (1803)

Ranunculus ranunculinus ( $\mathrm{Nutt}$.)

Cyrtorrhynca ranunculina Nutt. in Torry \& Gray's Fl. N. Am. I., 26. (1838)

Ranunculus nuttallii Gray, Proc. Acad. Phil., 56.

Delphinium carolinianum Walt. Fl. Car., 155. (1788)

Delphinium azureum Michx. Fl. N. Am. I., 314. (1803)

Delphinium urceolatum Jacq. Icon. Pl. Par., I., 101. (1871), and Collect. I., 153. (1786)

Delphinium exaltatum Ait. Hort. Kew. II., 244. (1789)

Leontice thalictroides L. Spec. Pl., $312 . \quad$ (175̃3)

Caulophyllum thalictroides Michx. Fl. N. Am. I., 205. (1803)

Recent authors are of the opinion that our species is not different enough from the other species of Leontice to warrant the establishment of a separate genus.

Castalia tuberosa (Paine) Greene Bull, Torr. Bot. Club XV., 84. (1888)

Nymphaea tuberosa Paine Cat. Pl. Oneida, 184. (1864)

Nymphaen reniformis DC. Syst. II., 55 (1821), not N. reniformis Walt. Fl. Car. 155. (1788)

The genus Castalia was separated from Nymphaea by Salisbury in 1805. Ignoring this, Smith in 1808 made the genus Nuphar, including therein the species loft in Nymphrea by Salisbury, and used the latter name for those species for which Salisbury founded Castalia. The next change must follow: 
Nymphaea advena Solander in Ait. Hort. Kew. II., 226.

Nuphar advena Ait. f. Hort. Kew. III., 295. (1811)

Neckeria aurea (Michx.) Pfeiffer Bot. Zeit. XV., 649.

(1789)

Corydalis aurea Willd. Enum. 740. (1809)

The genus Neckeria was established by Scopoli in 1777,

Corydalis by DeCandolle in $\mathbf{1 8 0 5 .}$

Neckeria aurea occidentalis (Gray.)

Corydalis aurea occidentalis Gray Pl. Fend. 6. (1849)

Neckeria micrantha (Engelm.) MacM. Met. Minn. Vall. 255.

(1892)

Corydalis aurea micrantha Engelm. in Gray Man. Ed. 5, 62. (1868)

Corydalis micrantha Gray Man. Ed. 6, 61.

Neckeria curvisiliqua (Gray).

Corydalis aurea curvisiliqua Gray, Proc. Am. Phil. 57. (1863)

Corydalis curvisiliqua Engelm. Gray Man. Ed. 5, 62. (1868) Bicuculla cucullaria (L.)

Fumaria cucullaria L. Spec.`Pl. 699.

Dicentra“cucullaria DC. Syst. I., 108.

Bicuculla Adans. was published in 1763, Dicentra Bernh. in 1833.

Biculla canadensis (Goldie).

Corydalis canadensis Goldie Edin.Phil. Journ.V., 330. (1822)

Dicentra canadensis DC. l.c.

Lepidium incisum Roth Nov. Cat. I., 224 (1797-1806), not $L$. incisum M.v. Riebst. Fl. T. \& C. II., 98 (1808), is, according to Dr. Kuntze, Rev. Gen. Pl. I., 35, the same as

Lepidium intermedium Gray Pl. Wright, II., 15.

Bursa bursa-pastoris (L.)

(1852)

Thlaspi bursa-pastoris L. Spec. Pl. 647.

Capsella bursa-pastoris Moonch. Meth. 271.

This is a good illustration of what a strict following of the rules of the Botanical Club of theA. A. A. S. will lead to. Bursapastoris is the oldest specific name. It is agreed generally that the species should not be included in the genus 
Thlaspi, and the oldest generic name after 1753 is Bursa, used by Wiggers in 1780. Capsella Med. appeared first in 1792. Bursa must therefore be the generic name, and bursa-pastoris the specific name. The amendment to the Rochester rules made at Madison in striking out a part of §III, was, in my opinion, not a wise one. The nomenclature which results is often, to say the least, inelegant and uncouth. Apios apios and Phragmites phragmites are in plain English. comparable to "dog dog" or "cat cat." Bursa bursa-pastoris translated is "Shepherd's purse purse."

Lesquerella argentea (Pursh) MacM. Met. Minn. Vall. 263. (1892)

Myagrum argenteum Pursh Pl. Am. 434.

Alyssum ludovicianum Nutt. Gen. II., 63.

Lesquerella ludoviciana Wats. Proc. Am. Acad. A. S. XXIII., 254. (1888)

Coulterina didymocarpa (Hook.) OK. Rev. Gen. I., 431. (1891) Vesicaria didymocarpa Hook. Fl. Bor. Am. I., 48. (1833) Physaria didymocarpa Gray, Gen. I., 162. (1848)

The name Physaria cannot be used as there is a genus Physarium Persoon (1795) among the fungi which is far older.

Roripa nasturtium (L.) Rusby Mem. Torr. Bot. Club III., no 3,

5. (1893)

Sisymbrium nasturtium L. Spec. Pl. II., 657. (1753)

Nasturtium officinale R. Br. Hort. Kew. Ed. 2, IV., 110. (1812)

Roripa Scopoli was published in 1760; Nasturtium Robert Brown in 1812 .

Roripa palustris (L.) Greene Man. Bay Reg. Bot. 21.

Sisymbrium palustre L. Spec. Pl. 657. (1753)

Nasturtium palustre DC. Syst. II, 191. (1821) 
Roripa palustris hispida (Desv.)

Brachylobus hispidus Desv. Journ. III., 183. (1814)

Nasturtium palustre hispidum Fisch. \& Mej. Ind. Sem.

Petr. III., 41. (1838)

Roripa obtusa (Nutt.)

Nasturtium obtusum Nutt. T. \& Gr. Fl. I., 74.

Roripa sessiliflora (Nutt.)

Nasturtium sessiliflorum Nutt. T. \& Gr. Fl. I., 73.

Roripa curvisiliqua (Nutt.)

Nasturtium curvisiliqua Nutt. l.c.

Roripa sinuata (Nutt.)

Nasturtium sinuatum Nutt. l.c.

Roripa armoracin. (L.)

Cochlearia armoracia L. Spec. Pl. 648.

Nasturtium armoracia Fries Fl. Scand. (1835)

Sisymbrium pinnatum (Walt.) Greene Bull. Cal. Acad. II. (1887)

Erysimum pinnatum Walt. Fl. Car. 174.

Sisymbrium canescens Nutt. Gen. II., 71. (1818)

Stanleya pinnata (Pursh.)

Cleoma pinnata Pursh Fl. Am. 739. (1814)

Stanleya pinnatifida Nutt. Gen. II., 71. (1818)

Arabis brachycarpa (Torr. \& Gr.)

Turritis brachycarpa Torr. \& Gr. Fl. I., 79. (1838)

Arabis drummondii Gray Man. Ed. 5, 69. (1868) not Graham. Arabis confinis Wats. Proc. Am. Acad. A. \& S. XXII.,. 466. (1887)

Arabis glabra (L.) Weinman Cat. Dorp. 18.

(1810)

Turritis glabra L. Spec. Pl. 666. (1753)

Arabis perfoliata Lam. Dict. I., 219.

Cardamine laciniata (Muhl.) Wood Bot. and Flor. 38.

Dentaria laciniata Muhl. in Willd Sp. Pl. III., 479.

Dentaria is included in Cardamine by the best authoritios. 
Jacksonia trifolinta Raf. Med. Rep. 352. (1808)

Cleome dodecandra Michx. Fl. Am. II., 32. (1803) not Linne.

Polanisia graveolens Raf. Journ. Phys. 98. (1819)

Jacksonia trachysperma (Torr. \& Gr.) Greene Pittonia II., 17.).

(1891)

Polanisia trachysperma 'Torr. \& Gr. Fl. I., 122.

C'eome serrulata Pursh. FI. Am. 441. (1814)

Cleome integrifoliu T. \& G. Fl. I., $122 . \quad$ (1838)

Helianthemum majus (L.) B. S. P. Cat., N. Y. (1888)

Lechea major L. Spec. Pl. $90 . \quad$ (1753)

Helianthemum canadense Michx. Fl. Am. Bor. I., 308.

Viola palmata obliqua (Hill) Hitcheoek Fl. Ames 487.

$(1803)$

Viola obliqua Hill Hort. Kew. 316. (1768)

Viola cucullata Ait. Hort. Kew. III., 288. (1789)

Viola palmata cucullata Gray Bot. Gaz. XI., 254. (1386)

Agrostemma githago L. Spec. Pl. 435. (1753)

Lychnis githago Lam. Encyc. III., 643.

This is now generally held distinct from Lychnis.

Arenaria franklinii hookeri (Nutt.)

Arencria hookeri Nutt. in T. \& Gr. Fl. I., 178. (1838)

Arenaria franklinii minor Hook. \& Arn. Bot. Beachy, 326.

(1841)

Anychic cancadensis (L.) B. S. P. Cat., N. Y. (1888)

Queria canadensis L. Spec. Pl., 90. (1753)

Queria capillacea Nutt. Gen. I., 159. (1818)

Anychia capillacea DO. Prodr. III., 369. (1828)

Elodes virginica (L.) Nutt. Gen. II., 17. (1818)

Hypericum virginicum L. Spec. Pl. Ed. 2, 110t. (1761)

Hypericum campanulatum Walt. Fl. Car., 191. (1788)

Elodes campanulata Pursh Fl., 379. (1814)

Hıbiscus laevis Scop. Del. Flor. III., 35̃. (1788)

Hibiscus militaris Cav. Diss., 352. (1790)

Abutilon abutilon (L.)

Sida abutilon L. Spec. Pl., 685: (1753)

Abutilon avicennae Gaertn. Fr. II., 251. (1800) 
Mclveopsis coccinea (Nutt.) OK. Rev. Gen., 72.

(1891)

Malva coccinea, Nutt. Fraser's Cat. (1813)

Malvastrun coccineum Gray P1. Fend., 21. (1849)

Malveopsis Presl. was published in 1844; Malvastrum Gray in 1849.

Impatiens biflora Walt. Fl. Car., 219.

Impatiens fulva Nutt. Gen. I., 146.

Parthenocissus quinquefolia (L.) Planchon Monogr. Ampelid.

I., 488. (1887)

Hedera quinquefolia L. Spec. Pl., 292. (1753)

Ampelopsis quinquefolia Michx. Fl. I., 160. (1803)

According to Planchon the name Ampelopsis belongs to the

genus Cissus of Gray's Manuel Ed. 6. No true Cissus

Linne is found in the region of the manual.

Vitis americana Marsh.

Vitis vinifera americana Marsh. Arb. 166. (1785)

Vitis aestivalis Michx. Fl. II., 230. (1803)

Acer saccharinum L. Spec. Pl. 1055. (1753)

Acer dasycarpum Ehrh. Beitr. IV., 24.

The sugar maple, $A$. Saccharinum Wang., which does not grow in Nebraska, becomes $A$. saccharum Marsh:

Acer negundo L. Spec. Pl. 266. (1753)

Negundo aceroides Moench. Meth. 334.

Rhus radicans L. Spec. Pl. 266. (1753)

Rhus toxicodendron L. Spec. P1. l. c.

Both are published on the same page, but $R$. radicans first. Rhus trilobata Nutt. in Torr. \& Gr. Fl. N. A. L, 219. (1838)

Rhus canadensis trilobata Gray Man. Ed. 6, 119. (1889)

This is a good species.

Lotus americanus (Nutt.) Bisch. Hort. Heid. (1839)

Trigonella americana Nutt. Gen. II., 120. (1818)

Hosackia purshiana Benth. in Bot. Reg. 1257.

This must be included in the genus Lotus. The oldest name is Lotus sericeus Pursh (1814), which, however, is preoccupied by Lotus sericeus DC. Cat. Mons.

(1813) 
Medicago arabica (L.)

Medicago polymorpha e. arabica L. Spec. Pl. 280.

Medicago maculata Willd. Sp. Pl. 1412. (1800)

As the plant is not a native of Arabia, Willdenow thought the name inappropriate. Hence the change.

Psoralea digitata Nutt. Torr. \& Gray Fl. N. A. I., 300.

Psoralea campestris Nutt. 1. c.

The latter is only a form of the former, and scarcely deserved a varietal name.

Amorpha nana Nutt. Fraser's Cát. (1813)

Amorpha microphylla Pursh Fl. 466. (1814)

There has been an idea generally prevailing that the $A$. nana of Fraser's Catalogue and that of Nuttall's Genera are not the same. The causes of this idea seem to have been that Dr. Gray found in Lambert's Herbarium under this name a variety of $A$. fruticosa (var. angustifolia Pursh), and that the same variety has been cultivated in England and figured in the botanical magazines under this name. Mr. Fraser was a nurseryman, not a botanist, and Fraser's 'Catalogue is known to have been prepared by Nuttall. It may be that Fraser had both $A$. nance and $A$. fruticosa angustifolia in the nursery and a mis. take of labels was made or a mistake might even have been made when the plants were shipped from America to England. It is evident enough that the $A$. nana of Fraser's Catalogue and that of Nuttall's Genera are the same; first, because the descriptions agree perfectly; second, because A. nana in Fraser's catalogue is said to be "collected near the Mandan towns 1,600 miles up the Missouri," which is, as far as I know, far outside of the range of $A$. fruticosc angustifolia, but about the center of that of A. microphylla Pursh; third, because Nuttall in his Genera expressly says " $A$. nana T. N. (Thomas Nuttall) in Fraser's Catal. 1813," and also says that it is the same as 
A. microphylla Pursh II., 466. Which should be regarded as the strongest evidence-specimens found in a foreign herbarium and plants sent out from a foreign nursery on the one hand, or on the other the author's own words in print, strenghthened by those of Pursh, who states that his $A$. microphylla is the same as $A$. nana of Fraser's Catalogue? The $A$. nana of Fraser's nursery may be whatever it please.

Dalea dalea (I.) Mac M. Torr. Bull. XIX.

Psoralea dalea L. Spec. $\mathrm{Pl}_{1}, 764 . \quad$ (1753)

Dalea alepecuroides Willd. Spec. PI. III., 1336. (1803)

Dalea eneneandra Nutt. Fraser's Cat. (1813)

Dalea laxiflora Pursh Fl. 741. (1814)

Kuhniastera compacta (Spreng.) OK. Rev. Gen., 192.

Dalea compacta Spreng. Syst., 327. (1826)

Petalostemon macrostachyus Torr. Ann. Lyc., N. Y., 176. (1828)

Kuhniastera Lam. was founded in 1789, Petalostemon Michx. in 1803.

Kuhniastera purpurea (Vent.) Mac M. Met. Minn. Vall., 329. (1892)

Dalea purpurea Vent. Hort. Cels., 40. (1800)

Petalostemon violaceus Michx. Fl. II., 50. (1803)

Kuhniastera candida (Michx.; OK. Rev. Gen. 19ะ. (1891)

Petalostemon candidus Michx. Fl. II., 49. (1803) Kuhniastera multiflora (Nutt.) OK. l.c.

Petalostemon multiflorus Nutt. in Journ. Acad. Philad. VII., 92.

Kuhniastera villosa (Nutt.) OK. l.c.

Petalostemon villosus Nutt. Gen. II., 85. (1818)

Astragalus ceramicus Sheldon Minn. Bot. Stud. No. 9, 19. (1894)

Phaca picta Gray Pl. Fend. 37. (1849)

Astragalus pictus Gray Proc. Am. Acad. VI., 214 (1866), not A. pictus Steud. (1840), nor A. pictus Boiss (1853). 
Astragalus ceramicus longifolius (Pur'sh)

Psoralea longifolia Pursh Fl. Am. Sept. II., 741. (181t), not A. longifolius Lam. Enc. Meth. I., $322 . \quad$ (1783)

The name longifolius, which is the first specific name, can not be used as a specific name in the genus Astragalus, but may well be used as a varietal name under A. ceramicus. In fact it is the only one to be permitted. Mr. Sheldon in naming the varieties $A$. ceramicus jonesii and A. ceramicus imperfectus violated articles 57 and 58 of the Paris Code.

Astragalus carolinianus L. Spec. Pl. 757. (1753)

Astragalus canadensis L. l.c., but lower on the page. Astragalus crassicarpus Nutt. Fraser's Cat. (1813)

Astragalus caryocarpus Ker. Bot. Reg. II., 174.

Astragalus viridis (Nutt.)

Kentrophyta montana Nutt. Torr. \& Gray Fl. I., 353. (1838), $\operatorname{not} A$. montanus $\mathrm{L}$.

Kentrophyta viridis Nutt. I.c.

Astragalus kentrophyta Gray Proc. Am. Phil., 60. (1863) dstragalus gilviflorus Sheldon Minn. Bot. Stud. No. 9, ¿1. (1894)

Astragalus triphyllus Pursh Fl Am. Sept. II., 740 (1814), not A. triphyllus Pallas Astr., 68. (1800)

This species has received two other names, but neither can be used in the genus Astragalus.

Astragalus spatulatus Sheldon Minn. Bot. Stud. No. 9, 22. (1894)

Homalobus caespitosus Nutt. in Torr. \& Gray Fl. N. A. I., 352. (1838)

Astragalus ccespitosus Gray Proc. Am. Acad. VI., 230 (1864) not A. caespitosus Pallas Astr. 70. (1800)

This species has received two other names, both preoccupied. Spiesia lambertii Pursh OK. Rev. Gen. I., 207. (1891)

Oxytropis lambertii Pursh Fl. 740. (1814)

Spiesia Necker was founded in 1790, Oxytropis DC. in 1802. 
Spiesia lambertii sericea (Nutt.)

Oxytropis sericea Nutt. Torr. \& Gray FI. I., 339.

Oxytropis lambertii sericea Gray Coult. Man. 71.

Spiesia multiceps (Nutt.) OK. 1.c.

Oxytropis multiceps Nutt. Torr. \& Gray Fl, I., 341.

Spiesia inflata (Hook.)

Oxytropis arctica inflata Hook. Fl. Bor. Aì. I., 146.

Oxytropis podocarpa Gray Proc. Am. Acad. VI., 234. (1863)

Meibomia canadensis (L.) OK. Rev. Gen. I., 195. (1891)

Hedysarum canadense L. Spec. Pl., 749. (17533)

Desmodium canadense DC. Prodr. I1., 328. (1825)

Meibomia was used by Fabricius in 1763 , Desmodium Desv. was established in 1813.

Meibomia canescens (L.) OK. 1.c.

Hedysarum canescens L. Spec. Pl. 748. (1753)

Desmodium canescens DC. Prodr. II., 328.

Meibomia grandiflora (Walt.) OK. 1.c., 196.

Hedysarum grandiftorum Walt. Fl. Car., 185.

Hedysarum acuminatum Michx. Fl. II., 72. (1803)

Desmodium acuminatum DC. Prodr. II., 329.

Meibomia dillenii (Darlingt) OK. 1.c., 195.

Desmodium dillenii Darlington Fl. Cestr. 414.

Meibomia rigida Ell. OK. 1.c., 198.

Hedysarum paniculatum L. Spec. Pl., 749.

Desmodium panioulatum DC. Prodr. II., 329. (1825).

Meibomia illinoensis (Gray) OK. l.c.

Desmodium illinoense Gray Proc. Am. Acad. VIII., 289. (1870)

Lespedeza frutescens (Willd.) Ell. Sk., 206.

Hedysarum frutescens Willd. Sp. Pl. III., 1193. (1802)

Lespedeza capitata Michx. Fl. Am. II., 71. (1803)

Lithyrus decaphyllus Pursh Fl. Am., 471. (1841)

Lathyrus polymorphus Nutt. Gen. II, 96. 
Fulcata comosa (L.) OK. Rev. Gen. I., 182.

Glycine comosa L. Spec. Pl., 754.

Glycine monoica L. Spec. Pl. Ed. 2, 1023.

(1753)

Amphicarprea monoica Nutt. Gen. II., 113.

(1761)

(1818)

- Falcata Gmelin was established in 1791, Amphicarpa Ell. in September, 1818. The latter was changed into Amphicarpaect by DeCandolle in 1825. The two latter names are also antedated by Amphicarpum Raf. (January, 1818). which is something else.

Falcata pitcheri (T. \& Gr.) OK. l.c.

Amphicarpaen pitcheri T. \& Gr. Fl. N. A., I., 292. Apios apios (L.) McM. Bull. Torr. Bot. Club XIX.

(1892)

- Glycine apios L. Spec. Pl. 753. (1753)

Apios tuberosa Moench Meth. 165. (1794)

Phaseolus polystachyus (L.) B. S. P. Cat. N. Y.

(1888)

Dolichos polystachyus L. Spec. Pl. 726.

Phaseolus perennis Walt. Fl. Car. 182.

Gymnocladus dioicus (L.) Koch Dendr. I., 5.

-Guilandina dioica L. Spec. Pl. 381.

(1753)

Gymnocladus canadensis Lam. Dict. I., 33: (1783)

Acuania illinoensis (Michx.) OK. Rev. Gen. I., 158.

(1891)

Mimosa illinoensis Michx. Fl. II., 254. (1803)

Acacia brachyloba Willd. Sp. Pl. VI., 1071. (1805)

Desmanthus brachylobus Benth. Hook. Journ. Bot. VI., 358, Leptoglottis intsia (Walt.)

Mimosa intsia Walt. Fl. Car. 252. (1788)

Schrankia uncinata Willd. Spec. Pl. IV., 1042. (1805)

Leptoglottis nuttallii DC. Mem. Leg. 451.

(125)

The genus Schrankia of Willdenow (1805) is antedated by Schrankia Med. Uster. N. Annal. I., 42, and used by Moench in Meth. 263 (1794). As the name Schrankia cannot be used, the only available generic name is Leptoglottis DC., although the generic characters given are not good. 
Opulaster opulifolius (L.) OK. Rev. Gen. 949.

Spircea opulifolia L. Spec. Pl. 489.

Physocarpus opulifolius Maxim.

Opulaster Med. was established on this species in 1799 , Physacarpus Camp. as a section of Spiraea in 1824. . Rosa virginiana Miller Dict. (1768)

Rosa blanda Ait. Hort. Kew. II., 202. (1789)

Rosa virginiana arkansana (Porter) IMacM. Met. Minn. Vall. 304, (1892).

Rosa arkansana Porter Fl. Col. 38.

Fragaria vesca americana Porter Bull. Torr. Bot. Club XVII., 15. $(1890)$

Fragaria vesca Auct. Am., not Linne.

The American variety differs somewhat from the European, especially in its thinner and smoother leaves, but I do not think sufficiently to justify a new specios. Dr. Britton in Bull. Torr Bot. Club 1892, page 222, makes of it a species and points out as a distinctive character that the achenes are superficial, "which are scarcely or not at all imbedded in the ovoid fruit." But so they are in the European $F$. vesca. DeCandolle and other European botanists use this very character to distinguish $F$. vesca from $F$. elatior and $F$. virginiana.

Geum canadense Jacq. Hort. Vind. II., 82. (1772)

Not Geum canadense Murr. Con. Goott. V., 34.

Geum album Gmelin Syst. II., 861. (1791)

Potentilla gracilis chrysantha (Lehm.)

Potentilla chrysantha Lehm. Hook. Fl. Bor. Am. I., 193. (1833), not P. chrysantha Trev., which is older.

Potentilla rigida Nutt. Journ. Acad. Philad. VII., 20 (1833)

Not $P$. rigida Wall., which is older.

Potentilla gracilis rigida Wats. Rev. Potentilla 557.

If this is to be regarded as a variety of $P$. grucilis, which I believe it should, the varietal name must be chrysantha. 
But if it should be raised to the rank of a species, its name must be $P$ uxttallii Lehm Iud. Sem. h. Hand. Add. 12 (1852), as the other two are not available as specific names.

Sunyuisorba sanguisorba (L.)

Poterium sanguisorba L. Spec. Pl. 99t. (1753)

The two genera are now united, even by Dr. Gray. But he chose the latter name. Sanguisorba is found on page 116 of the Species Plantarum, Poterium on page 994.

Agrimonic striata Michx. Fl. Am. I., 287.

Ayrimonia eupatoria Auct. Am.

Dr. Britton in Bull. Torr. Bot. Club XVIII., 367 (1891) has pointed out that our common Ayrimonia is not the A. eupatoria of Europe.

Pirus coroncuria iowensis Wood Class Book Rev. Ed.,333. (1868) Pirus iowensis Bailey Am. Garden XII., 473. (1891)

Pirus coronaria L. of Gray's Manual, as far as Nebraska and other western specimens are concerned.

Crutcuegus mollis (T. \& Gr.) Scheele Linnaea XXI., 569. (1847)

Crataegus subvillosa Torr. P. R. Rep. IV., 86. Cralciegus coccinea mollis T. \& Gr. Fl. I., 465.

This is generally accepted as a good species.

Ribes rubrum albinervium (Michx.) Mac M. Met. Minn. Vall., 279. (1892)

Ribes albinervium Michx. Fl. I., 110. (1803)

Ribes rubrum subglandulosum Maxim Bull. Acad. Pet. 19, 261. (1878)

Myriophyllum pinnatum (Walt.) B. S. P. Cat., N. Y.

Potamogeton pinnatum Walt. Fl Car., 90. (1788)

Myriophyllum scabratum Michx. Fl. II., 190. (1803)

Callitriche palustris L. Spec. Pl., 4. (1753)

Callitriche verna L. Spec Pl. Ed. 2,6. (1761) 
Oenothera albicaulis Pursh Fl., 733 (1814), not O. albicaulis Nutt. Gen. (1818)

Oenother a pinnatifida Nutt. Gen. I., 245. (1818)

As O. albicaulis Nutt. in Fraser's catalogue is but a nomen. nudum, it has no standing. Hence O. albicaulis Pursh is the first published species bearing that name, which should be restored, though apt to cause some confusion.

Oenother a pallida Lindl. Bot. Reg. $1 \pm$ t. 1142. (1830)

Oenothera albicaulis Nutt. Gen. I., 245 (1818), which name

is preoccupied by the foregoing.

Epilobium angustifolium L. Spec. Pl. 347 (1753) not Lam.

Epilobium spicatum Lam. Fl. Franc. 1077. (1778)

This is corrected in Gray's manual, but not in Coulter's. Dr. Trelease in his revision of Epilobium changes it back to $E$. spicatum, as he believes, as did Lamarck, that $E$. angustifolium $\mathrm{L}$. is the same as $E$. dodonei Vill. ' It would have been strange if Linne had regarded this as the typical $E$. angustifolium, as the species is comparatively rare in Europe and not found at all in the native land of Linne. What is most common in Sweden is a narrower leaved form of $E$. angustifolium L., E. spicatum Lam. This is Linne's E. angustifolium a. It grades into the broader leaved form which is common here, Linne's $E$. angustifolium b, E. angustifolium latum DC. in Prodr. Linne's $E$. angustifolium $\mathrm{c}$ is $E$. dodone $\mathrm{Vill}$. and $E$. angustifolium Lam.

Mentzelia decapetala (Pursh).

Bartonia decapetala Pursh in Sims Bot. Mag. t 1487 (between 1810 and 1813)

Bartonic ornata Pursh Fl. 327.

Mentzelia ornata T. \& Gr. Fl.

Cactus viviparus Nutt. Gen. I., 295.

Mamillaria vivipara Haw. Syn. Pl. Succ. Supp. 72.

Cuctus mamillaris, the type of the genus Mamillaria, Haw., is the first of the two species in Linne's Species Plantarum . 
belonging to that section of the genus Cactus $\mathbf{L}$. which has been regarded by Linne and others as representing the typical cacti. The name Cactus should therefore be retained for this group. The name Mamillaria Haw. is also, according to Dr. Kuntze, antedated by Mamillarir Stackh. (1809)

Cactus missouriensis (Sweet) OK. Rev. Gen. I., 259.

Mamillaria missouriensis Sweet Hort. Brit. 171. (1827)

Micrampelis lobalc (Michx.) Greene Pittonia II., 128.

Sicyos lobatu Michx. Fl. II, 217. (1803)

Micrampelis echinata Raf. Med. Repos. N. Y. V., 352.

Echinocystis lobata T. \& Gr. Fl. N. A. I., 542. (1838)

Micrampelis was established some thirty-two years before Echinocystis.

Osmorrhiza claytonii (Michx.) B. S. P. Cat. N. Y.

Myrrhis claylonii Michx. Fl. I., 170. (1803)

Osmorrhiza brevistylis DC. Prodr. IV., 232.

Osmorrhiza aristata (Thunb.)

Chaerophyllum aristatum Thunb. Fl. Japon. 119.

Osmorrhiza longistylis DC. I.c.

Adorium tenuifolium (Nutt.) OK. Rev. Gen. 264. (1891)

Musenium tenuifolium Nutt. in Torr. \& Gray FI. I., 642. (1840)

Adorium Raf. was published in 1825, Musenium T. \& G.r. in 1840 .

Adorium divaricatum (Pursh).

Sesili divaricatum Pursh Fl. 732. (1814)

Musenium divaricatum Nutt. l.c. (1840)

Dr. Kuntze writes $A$. lucidum, his name being based on Sesili lucidum Nutt. in Fraser's Catalogue, which is a

- mere nomen nudum. 
Peucedanum graveolens Wats. King's Rep. V., 128. (1871)

Peucedanum kingii Wats. Proc. Am. Acad. XXII., 474. (1887)

J. B. Davy, in Erythea II., 48 (1894), points out that Bentham \& Hooker in Gen. Pl. I., 919 (1867), did not give Anethmum graveolens L. the name Peucedamum graveolens, but only indicated that it should be included in that genus. The name Peucedanum graveolens was given to the latter species by Baillon, Hișt. d. Pl. VII., 97 (1880), some nine years later than the publication of $P$. graveolens Watson.

Cymopterus acaulis (Pursh)

Selinum accule Pursh Fl. 732. (1814)

Thapsia glomerata Nutt. Gen. I., 18t. (1818)

Cymopterus glomeratus Raf. Journ. Phys. 100. (1819)

Sanicula canadensis L. Spec. Pl. 235. (1753)

Sanicula menilandica canadensis Torr. Fl. U. S. 302, (1829)

As S. canadensis and S. marilandica are both on the same page of the Species Plantarum, but S. cancadensis is first, the latter must keep its name. If one is to be regarded as a variety of the other, as it generally is, $S$. marilandica must take the place of variety; hence

Sanicula canadensis marilandica (L.)

Sanicula marilandica L. Spec. Pl., 235. (1753)

Deeringia canadensis (L.) OK. Rev. Gen., 266. (1891)

Sison canadense L. Spec. Pl., 252. (1753)

Cryptotuenia canadensis DC. Mem. Umb., 42. (1829)

Decringia Adanson was published in 1763, Cryptotaenia DC. in 1829.

Berula erecta (Huds.) Coville Bot. Death Vall. Exp., 115. (1893)

Sium erectum Hudson Fl. Aug., 103. (1762)

Berula angustifolia Koch in Mert. \& Koch Deut. Fl. II., 455. (1826) 
Cicuta virosa maculata Coulter \& Rose Rev. N. A. Umbel., 130. (1888)

Cicuta maculata L. Spec. Pl., 256. (17ต̃3)

This is reduced to a variety by Conlter and Rose.

Cornus candirlissima Marsl. Arb., $35 . \quad$ (178i)

Cornus stricla Lan. Diet. II., 116 (1786) and

Cornus premiculata L'Her. Corn. 9 t. $5 . \quad$ (1788)

A Preiliminary List of the Botanical Expeditions in NeBR.SKA, 1803-1893.

BY FREDERICR E. CLEMENTS.

The object of the subjoined list is to furnish easy and ready reference to botanical field work done within Nebraska as now bounded, and also to aid in the survey of the stata by showing in what region work has already been done and by affording some hint as to the nature and extent of this work, the disposition of collections, etc. The present list does not pretend to completeness by any means. It simply purports to be a fairly full and accurate resume of the present status of our knowledge on this subject and is published partly for reasons given above and partly in the hope of obtaining further information through outside channels. This preliminary list is intended merely to catalogue such botanical excursions as have assumed the proportions of expeditions and is in no degree a measure of all the field work done in the state.

I. Lewis, Meriwether, and Clark, William. (1803-1806)

Along the Missouri river from the southeast corner of the state to the mouth of the Niobrara, with short excursions into the state. Collections made on the outward journey lost; those made on the return identified by Frederick Pursh and new species described in his Flora Americae Septentrionalis. (1814) 
II. Nuttall, Thomas, and Bradbury, John. (1808)

General route, up the Missouri; collected in Nebraska at the mouth of the Platte, Council Bluffs (old Council Bluffs on the Nebraska side), Blackbird, and the lower valley of the L'Eau qui Court.

Collection in possession of the Academy of Natural Sciences of Philadelphia. Descriptions of new species in Fraser's Catalogue (1813) and Nuttall's Genera of North American Plants. (1818)

III. Nicollet, I. N. (1838-39)

Along the Missouri river with extensive excursions into the eastern part of the state. Collections made by Charles Geyer and identified by Dr. John Torrey. List in Nicollet's Report (1843); collection preserved in the Herbarium of Columbia College, New York.

IV. Fremont, John O. (1842-44)

(1842) Entered Nebraska at about the point where the Little Blue leaves the state, followed up that river to its head, came upon the Platte at Kearney, and followed the South Platte out of the state.

Extensive collections throughout the valley of the Little Blue and of the Platte; identified by Dr. John Torrey; some descriptions published in Torrey \& Gray's Flora of North America, and list published in Fremont's Report (1845); preserved in the Herbarium of Columbia College, New York.

(1844) Passed through the southwest corner of the state along the Republican. Greater part of the collections lost.

V. Stansbury, Howard. (1849)

Entered the state in the southeast corner, followed the Little Blue, reached the Platte at Kearney, and followed the North Platte out of the state.

Collections identified by Dr. John Torrey, in the Herbarium of Columbia College; list in Stansbury's report.

(1855) 
VI. WARREN, Gouverneur K. (1855-57)

(1855) From the Middle Keya Paha nearly due south through the Loup valley to Ft. Kearney. A mere military reconnoisance without important botanical results.

(1856-57) From Sioux City southwestward through the Elkhorn valley, thence northwest through the Loup valley to Ft. Laramie. Return through the Niobrara valley to the Missouri.

Collections mostly identified by Dr. George Engelmann preserved in the Engelmann Herbarium at St. Louis. List in Warren's Report. (1858)

VII. Bessey, Charles E. (1887)

Along F. E. \&. M. V. R. R. from Long Pine to Ft. Robinson. List in Webber's Catalogue. (1890) Collection in the Herbarium of the University of Nebraska.

VIII. Bessey, Charles E. (1889)

Followed survey of route of B. \& M. R. R. from Alliance to Pine Ridge. List in Webber's Catalogue. Collection in the Herbarium of the University of Nebraska.

IX. Sмгтн, Jared G. (1889)

From Alliance to Camp Clarke. Collection in the Survey Herbarium.

X. WebBer, Herbert J. (1889)

In valley of the Dismal river. List in Webber's Catalogue. Collection in the Survey Herbarium.

XI. Webber, Herbert J. (1889)

Along the bluffs of the Missouri from Rulo to Nebraska City. List in Webber's Catalogue. Collection in the Survey Herbarium.

XII. Williams, Thomas A." (1891)

In Hat Creek basin and the White River valley. Collection in Mr. Williams' herbarium, a few specimens in the Survey Herbarium. Additions published in Webber's Appendix. (1892) 
Xitil. Rydberg, Per A.

From Kearney along B. \& M. R. R. to the Colorado line; thence along Lodge Pole creek and the Sonth Platte to Wyoming. Collections in the National Herbarium at Washington and in the Survey Herbarium. Additions published in Webber's Appendix (1892), and Dr. Bessey's Supplement. (1892)

XIV. Woods, Albert F. (1892)

Hat Creek basin. Collection in the Survey Herbarium.

List in Report of the Survey for 1892.

XV. Smith, Jared G., and Pound, Roscoe. (1892)

From Alliance to O'Neill through the sand hills and lake region; thence to Columbus. Collection in the Survey Herbarium. List in report of the survey for 1892.

XVI. Rydberg, Per A. '(1893)

Sand hills of central Nebraska and Dismal River valley. Collections in the National Herbarium at Washington and in the Survey Herbarium. Additions published in this report.

XVII. Woons, Albert F., and SArunders, DeAlton. (1893)

From Haigler to Superior along the Republican, thence to Endicott and Fairbury on the Little Blue. Collection in the Survey Herbarium. Additions published in this report.

XVIit. Clements, Frederick E. (1893)

From Emerson along the Missouri and Niobrara to Western Brown county. Collections in the National Herbarium at Washington and in the Survey Herbarium. Additions published in this report.

In addition, nention should be made of the collections made by Rev. J. M. Bates along the Elkhorn and Niobrara between Ewing and Ft. Robinson, and by Dr. H. Hapemau in Kearuey and Adams counties. 


\section{Bibliography of the Flora of Nebraska.}

BY ROSCOE POUND.

The following list contains such books or articles as deal with the flora of Nebraska or with the flora of localities within the state, and such other articles as have to do with collections made in Nebraska, or describe new species from the state, or are otherwise of local interest.

A few words seem to be required with reference to Professor Aughey's Catalogue and his sketches of the Flora of Nebraska, enumerated below, as they have deceived many persons in times past. In the catalogue he enumerates 2,034 species, of which 1,671 are Anthophytes, 49 Pteridophytes, 163 Bryophytes, 90 Algæ, and 61 Lichens. No fungi are catalogued. Elsewhere he states that 2,043 species are known to exist in Nebraska. These, however, are only estimates. Professor Aughey was primarily a geologist. His heart was in geological work, and the bulk of what time he had after the labors of the class room was devoted to it. His herbarium is now in the Herbarium of the University and shows what he actually accomplished as regards the flora of the state. It consists of about 500 specimens, representing less than 200 species, all Anthophytes or Pteridophytes, and almost all from the south-eastern portion of the state. Very few, therefore, of the species reported in the catalogue are represented, and a comparison has shown (as was noted by Mr. Webber in the inintroduction to his catalogue) that the list was based almost entirely upon the range of species as given in other books. In other words, its purpose evidently was to inform the collectors of the state of what they might expect to find in its borders. At that early day, with the manifold duties of his chair, then undivided, with no adequate library facilities, few helpers, and a large unexplored region before him, perhaps nothing more could 
be expected. At any rate it must be said that subsequent researches have failed to confirm his estimates, and that, though some of the eastern plants he listed are now begiuning to make their way into the state at a distance of nearly twenty years, his catalogue is substantially unreliable. It seems necessary to say this because many species are to be found cited as occurring in Nebraska by writers who have depended on his catalogue, which in fact have never been collected in the state.

Aughey, Samuel. Catalogue of the Flora of Nebraska 1875.

Sketches in the Physical Geography and Geology of $\mathrm{Ne}$ braska (1880). [Contains: chapter VII. General Flora of Nebraska; VIII. Forest Trees and Shrubs of Nebraska with Notes on their Distribution; IX. The Wild Fruits of $\mathrm{Ne}$ braska; X. Wild Grasses.]

See Curley.

Bates, J. M. The Grasses of Northwestern Nebraska. In Report of the Botanist in Rep. Nebr. St. Board of Agr. 1891. (1892)

Bell, A. T. The Slime Moulds (Myxomycetes) of Crete. In Publ. Nebr. Acad. Sci. II. (1892)

Berkeley, M. J. Notices of North American Fungi.

Grevillea, 1876, pp. 93 and 141. [Describes two new black fungi from Nebraska, collected by Hayden. No. 881, and No. 969.]

Bessey, C. E. Ruppia maritima I. in Nebraska. Am. Nat., . 1886, p. 1052.

Grasses and Forage Plants of Nebraska. In Rep. Nebr. St. Agr. Soc., 1886. (1887)

The Eastward Extension of Pinus ponderosa Dougl. var. scopulornm. Am. Nat., 1887, p. 927.

The Westward Extension of the Black Walnut. Ibid., p. 928. The Grass-Flora of the Nebraska Plains Am. Nat., 1888, p. 171. 
Grasses and Forage Plants of Nebraska. Second Report. In Riep. Nebr. St. Board Agr., 1887. (1888).

Natural Horticultural Regions of Nebraska. In Rep. Nebr. St. Hort. Soc., 1887-8. '(1888) [Gives a list of the trees and woody plants of the state and their distribution.]

A few notable weeds of the Nebraska Plains. Am. Nat., December, 1888.

Report on the Grasses and Forage Plants of Nebraska. In Rep. Nebr. St. Board of Agr., 1888. (1889)

Two Big-rooted Plants of the Plains. Am. Nat., 1889, p. 174. The Flora of the Upper Niobrara. Am. Nat., 1889, p. 537. Report of the Botanist on the Grasses and Forage Plants of Nebraska. In Rep. Nebr. St. Board of Agr., 1889. (1890) [Contains: Grasses of Central Nebraska by H. J. Webber; Grasses of Northwestern Nebraska, by H. J. Webber; Grasses of Box Butte and Cheyenne counties, by J. G. Smith; List of Grasses Exhibited at State Fair, 1888, showing the distribution of many species.]

The Bearberry in Central Nebraska. Am. Nat., 1891, p. 1130. The Native Trees of Nebraska. In Nebraska Farmer, 1891, page 537. [Gives a list with distribution.]

The Native Shrubs of Nebraska. In Nebraska Farmer 1891, p. 590. [List with distribution.]

Preliminary Report on the Native Trees and Shrubs of Nebraska. Bull Agr. Exp. Sta., Nebr., Vol. IV, No. 4. (1891)

Sixth Annual Report of the Botanist. In Rep. Nebr. St. Board Agr., 1891. Also in Contrib. Bot. Dept. Univ. Neb., n. s., II. (1892) [Contains: Grasses Exhibited at State Fair, 1891, showing distribution; List of Weeds of Nebraska; Preliminary List of the Grasses of Nebraska; Grasses of Northwestern Nebraska, by J. M. Bates; Grasses of Southwestern Nebraska. ] 
Second Edition of Webber's Appendix to the Catalogue of the Flora of Nebraska, with a Supplementary List of Recently Reportod Spocies. In Contrib. Bot. Dept., Univ. Neb., n. s., III. (1892)

Sccoud Report upon the Native Trees and Shrulss of Nebraska. In Rep. Neb. Hort. Soc., 1892. Also in Contrib. Bot. Dept. Univ. Neb., n. s., I. (1892)

Seventh Anural Report of the Botanist. In Rep. Neb. St. Board Agr., 1892. Also in Contrib. Bot. Dept. Univ. Neb. n. s., V. (1893) [Contains: List of Weeds of Nebraska; List of Grasses Exhibited at the State Fair, 1892, showing distribution; Preliminary Description of the Native and Introduced Grasses of Neliraska.]

Botanical Survey of Nebraska.-Report on Collections Made in 1892. (1893) [Contains Flora of the Sand Hill Regrion of Sheridan and Cherry Counties, and List of Plants Collected on a Journey Through the Sand Hills in July and August, 1892, by J. G. Smith and Roscoe Pound; Notes on the Canyon Flora of Sioux County, with List of Plauts Collected in July and August, 1892, by A. F. Woods; Miscellaneous Additions to the Flora of the State and New or Noteworthy Spocies from Various Localities.]

Coulter, John M. Maunal of the Botany (Phacenogamice and Plevidoplytu) of the Rocky Mountain Regrion, from New Mexico to the British Boundary. 1885. [Covers western Nebraska to the hundredth meridian. ]

Curley, Edwin A. Nebraska: its Advantages, Resources, and Drawbacks, 1876. [Contains chapter XXIV., The Wild Fruits of Nebraska, by S. Aughey, in which is also a sketch of the "General Botany of Nebraska."]

Ellis, J. B., and Everhart, B. M. New Species of Fungi from Various Localities. Journ. Myc. IV., 97. (1888)

New Specios of Hyphomycetous Fungi. Journ. Myc. V., 68. (1889) 
New and Rare species of Norll Imerican Fungi. Jonrn. Myc. V., 145. (1889)

New Species of Fungi from Various Localities. In Proc. Acad. Nat. Sci. Philad., 1891. [In each of the foreguing" articles new species from Nebraska are described.]

Fremont, John C. Report of the Exploring Expedition to the Rocky Mountrins in the Year 1842, and to Oregon aud North California in the Years 18t3-4t. (18-15) [Contains: Catalogue of Plants Collected by Lieutenant Fremont in his Expedition to the Rocky Mountains. By John Torrey.]

Gray, Asa. Manual of the Botany of Northern United States, Including the District East of the Mississippi and North of North Carolina and Tennessee. Sixth Edition Revised and Extended Westward to the Hundredth Meridian by Sereno Watson and John M. Coulter, 1890. [This edition (sixth) connects with Coulter's Manual and includes the Flora of eastern and central Nebraska.]

Morgan, A. P. The Genus Geaster. Am. Nat., 1887, p. 1026. [Describes two new species from Nebraska.]

Nicollet, I. N. Report Intended to Illustrate a Map of the $\mathbf{H y -}$ drographical Basin of the Upper Mississippi River, $18+3$. [Contains: Catalogue of Plants Collected by Mr. Charles Geyer, under the Direction of Mr. I. N. Nicollet, During his Exploration of the Region Between the Mississippi and Missouri Rivers, by Profossor John Torrey, M.D. (appendix B.)]

Pound, Roscoe. Notes on Fungi of Economic Interest Observed in Lancaster County During the Summer of 1889. In Bull. of Agr. Exp. St. Nebr., Vol. I., No. 11, 1889.

Rydberg, P. A. Flora of the High Nebraska Plains. Am. Nat. 1891, P. 485 .

On the American Black Cottonwood. In Bull. Torr. Bot. Club XX., No. 2. (1893) [Describes a now cottonwood from Nebraska.

Schofield, J. R. Notes on the Flora on the Artesian Well. In Publ. Nebr. Acad. Sci., II. (1892) 「Gives a list of Algæe found in the artesian well at Lincoln.] 
Smith, J. G. Some Nebraska Grasses. Bot. Gaz., 1889, p. 231. The Grasses of Box Butte and Cheyenne counties. In Rep. Nebr. St. Board of Agr., 1889. (1890) Also in Am. Nat., 1890, p. 181.

Grasses of the Sand Hills of Northern Nebraska. In Rep. Nebr. St. Board of Agr., 1892. (1893) Also in Contrib. Bot. Dept. Univ. Nebr., n. s. V.

Stansbury, Howard. Exploration and Survey of the Valley of Great Salt Lake of Utah, Including a Reconnoisance of a New Route Through the Rocky Mountains. 1853. [Pages 1852 relate to Nebraska and its prairie flora.]

Swezey, G. D. Nebraska Flowering Plants. Doane College Nat. Hist. Studies, 1. 1891. [List and notes].

Additions to the Flora of Nebraska. In Publ. Nebr. Acad. Sci., II. 1892. Also in Bull. Torrey, Bot. Club, 1892, p. 94. Torrey, John. See Fremont, Nicollet.

Warren, G. K. Report on Explorations in Nebraska in 185556-57. In Report of the Secretary of War, 1858. [Botany by F. V. Hayden, at page 726.]

Watson, Sereno. Pentsiemon haydeni. Bot. Gaz, 1891, p. 311. [Notes the rediscovery of this species in Nebraska.]

Williåms, T. A. Notes on Nebraska Lichens. Am. Nat., March, 1889.

Notes on the Canyon Flora of Northwest Nebraska. Am. Nat., 1890, p. 779.

Webber, H. J. A Preliminary Enumeration of the Rusts and Smuts of Nebraska. In Bull. Agr. Exp. Stn. Nebr., Vol. I., No. 11, 1889. [Introduction by Dr. C. E. Bessey. ]

The Fresh Water Algæ of the Plains. Am. Nat., 1889, p. 1011.

The Flora of Central Nebraska. Am. Nat., 1889, p. 633, 1890, p. 76.

The Grasses of Central Nebraska. In Rep. Neb. St. Board Agr., 1889. (1890)

The Grasses of Northwestern Nebraska. In Rep. Neb. St. Board Agr., 1889. (1890)

Catalogue of the Flora of Nebraska. In Rep. Neb. St. Board Agr., 1889. (1890) Also reprinted from the Report by the University, 1890.

Appendix to Catalogue of the Flora of Nebraska. In Trans. St. Louis Acad. Sci. VI., 1, 1892. 


\section{UNIVERSITY OF NEBRASKA.}

\section{Botanical Survey of Nebraska.}

Conducted by the Botanical Seminar.

\section{IV.}

\section{Report on Collections Made in 1894-95.}

Lincoln, Nebraska, U. S. A.

Published by the Seminar.

1896 .

(Distributed January 20, 1896.) 



\section{CONTENTS.}

Note............................... 4

New Species of Fungi..................... 5

Additions to the Reported Flora of the State........... 24

Summary ............................. 48 


\section{NOTE.}

The large number of new species of fungi collected in 1894 delayed the publication so long that we have been able to include in this report a portion of the collections made in 1895. This report is given over entirely to recording additions to our flora, and to descriptions of fungi, the space being fully occupied by them. Work upon the distribution of species of anthophytes in the state is rapidly approaching completion, but is not yet ripe for publication.

The Seminar is indebted to Rev. J. M. Bates, Dr. H. Hapeman, and Messrs. C. L. Shear, M. E. Moore, J. A Warren, and C. C. Engberg for collections. Many others have assisted by sending single specimens. Thanks are due also to Dr. N. L. Britton of Columbia College, New York, for advice and information as to nomenclature.

The list of Anthophyta was prepared by P. A. Rydberg, the algae, except Bacillariaceae by D. A. Saunders, the Bacillariaceae by $\mathrm{C}$. J. Elmore, the fungi by Roscoe Pound and Frederick E. Clements.

From the amount of material already on hand and undetermined, we feel justified in prophesying a total of four thousand species in the next two years.

Charles E. Besset, RosCoe Pound, Frederior E. Clements, Editorial Committee.

December 23, 1895. 


\section{NEW SPECIES OF FUNGI.}

In the following descriptions of new species, Saccardo's Chromotaxia has been faithfully followed. All color-names conform to his charts and to the synonymic lists on p. $6 \mathrm{ff}$.

\section{MUCORACEAE.}

Thamnidium (Helicostylum) cyaneum Pound and Clements.

Terminal sporangium, sporangiophore, and lateral branches as in T. glomeratum; sporangiola borne on the lateral branches, numerous, 30-50, globose or pear-shaped, nodding on deflexed stalks $100 \times 3-5 \mu$, without columella, steelblue, 35-45 $\mu$ in diam., 15-35-spored; spores ellipsoid or ovate, smooth, blue, in age pale blue, 12-15x8-10 $\mu$.

On horse-dung, with Piptocephalis, and Circinella, Lincoln. (4493)

\section{SPHAERIOIDEAE,}

Cytospora celastri Clements.

Perithecia innate, densely aggregated, globulose, ostiole short, erumpent, shorter than the perithecium; sporemasses linear, flexuose-contorted, golden-yellow; conidia hyaline, strongly curved, often semi-circular, obtuse, or acute, $15-20 \times 1 \mu$.

In dead stems of Celastrus scandens, Otowanie Woods. (4742)

\section{MUCEDINACEAE.}

Rhinotrichum doliolum Pound and Clements.

Effused, white, compact, velvety, gray, or drab; sporophore ascending, much branched; filaments hyaline, tortuous, many septate, $5 \mu$ wide, thickly beset with bottle-shaped basidia, $7-8 \times 3-4 \mu$, generally opposite, rarely alternate; 
conidia 2-several on each basidium, borne on short sterigmata, hyaline, ovoid-ellipsoid, 3-5 $\times 2-3 \mu$.

Forming a thick crust on the sporangia and stipes of a slime-mould, bluffs of the Missouri river, Bellevue. (4381)

Mycogone roseola Pound and Clements.

Broadly effused, covering the entire host with a layer 300 $-500 \mu$ thick, at first white, then rosy, or, rarely, pale brick-red; hyphae hyaline, septate, erect, branched, 3-4 $\mu$ wide; conidia borne on short, lateral, often nodding ped. icels, 2-celled, 20×15 $\mu$, superior locule larger, densely and beautifully echinulate, globose, pale brick-red, 12-1 $5 \mu_{\text {, }}$ inferior locule almost hyaline, or very pale rosy, minutely and sparingly punctulate, 8-9 $\mu$.

Parasitic on an Helvella, $H$. albipes (?), Meadville, Keya Paha county. (4743)

DEMATIACEAE.

Helminthosporium phragmidium Pound and Clements.

Broadly effused, velutinous, at first white, then exactly aveIlaneous, $1 \mathrm{~cm}$. wide; sporophore very long, with numerous alternately-disposed, lateral branches, multiseptate, hyaline, $160-300 \times 2 \frac{1}{2}-5 \mu$; conidia solitary, erect, obclavate, smooth, pale gray, constricted between the septa, 3-7-septate, $45-70 \times 10-15 \mu$.

On fragments of wood in the green-house, Lincoln. (4576) Sparodesmium suffultum Pound and Clements.

Cespitula gregarious, superficial, granular, botryoid, black; sterile hyphae creeping, fuliginous, branched, torulose; sporophore erect, unicellular, broad, turgid, hyaline, equalling the conidia in diameter, 25-35x15-20 $\mu$; conidia densely congested, globose, or globose-oblong, rarely oblong, opaque, brown-fuscous, irregularly and densely polygono-areolate, or nearly radiately septate, very rarely muriform, $25-35 \times 25 \mu$. 
On decorticated branches of Populus monilifera, Memphis. $(4578)$

BTILBAOEAE.

Trichurus Clements and Shear nov. gen.

As in Stysanus, but the capitulum densely beset with long, strict bristles.

Trichurus cylindricus Clements and Shear.

Solitary or gregarious; stipe simple, or sometimes two arising from the black base, attenuate above within the capitulum, strict, erect, broad, glabrous, black, opaque, composed of indistinct hyphae $2 \frac{1}{2} \mu$ wide, $\frac{4}{5}-2 \frac{1}{2} \mathrm{~mm}$. long, 35-85 $\mu$ thick; capitula elongate, linear or clavate-cylindrical, equal, or sometimes attenuate towards the apex, gray-fuscous, $\frac{3}{4}-2 \frac{1}{2} \mathrm{~mm}$. long, $18-30 \mu$ thick, densely be. set with acute, rectilinear, fuscous, few-septate, simple, or rarely 2-3-furcate bristles, $60-120 \times 2 \mu$; hyphae brownish-fuscous, short, pseudo-verticillately, or ramosely branched; conidia catenulate, oblong-elliptical, smooth, slightly glaucous, $8-9 \times 3 \mu$.

On decaying seeds of Cucurbita maxima, in the laboratory, Lincoln. (4744)

TUBERCULARIACEAE.

Fusarium hymenula Pound and Clements.

Sporodochia minute, $\frac{1}{1} \sigma-\frac{1}{8} \mathrm{~mm}$., gregarious, orbicular, or oblong, disciform, without white-tomentose, within at first dilutely amber-colored, then black, margin slightly elevated, flexuous, white; sporophore long, 2-4-verticillately branched, branches short, ascending, or erect, appressed, 3 อั $-40 \times 2 \mu$; conidia oblong, wider toward either end, simple, rounded, straight, hyaline, 10-12×3-4 $\mu$.

On dead leaves of Heliathus, Wabash. An Hymenula, with verticillately-branched sporophore.

(4258) 
Volutella gilva albo-pilosa Pound and Clements.

On dead leaves of Ulmus fulva, Weeping Water. (4546) According to Sacc. Fung. Ital., Fig. 728, the specimens in the Survey collection are specifically distinet from $V$. gilva. In Syll. Fung., Saccardo unites with V. gilva $V$. intricata Karst., from which the specimens in hand differ only in the atro-olivaceous, or nearly black sporodochium, covered by the longer, inextricably interwoven, white hairs.

\section{HELVELLACEAE.}

Helvella grisea Clements.

Pileus appressed, persistently bilobed, or sub-reniform, scarcely inflated, reticulated beneath with numerous anastomosing veins, pallid, hymenium pale brownish-gray; stipe concolorous, or scarcely paler, glabrous, at first longitudinally sulcate, then strongly lacunose-sulcate; asci cylindrical, 8 -spored, $200-250 \times 15 \mu$; sporidia broadly elliptical, monostichous, 1-guttate, $18 \times 10 \mu$; paraphyses filiform, septate, hyaline, $4 \mu$ wide.

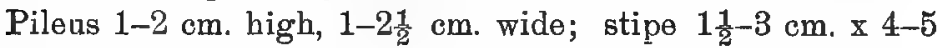
$\mathrm{mm}$.

On the ground, with Conocephalus conicus, Hazel Creek canyons, Brown connty. Related to $H$. palustris Peck, and to $H$. palescens Schaeff,, but distinguished by the color and the persistently saddle-shaped pileus.

Helvella sulcata minor Clements.

Pileus 2-3 mm., rarely $8 \mathrm{~mm}$. wide, $2-5 \mathrm{~mm}$. high; stipe 2-3 mm., rarely $10 \mathrm{~mm}$. high, $1 \frac{1}{2}-3 \frac{1}{2} \mathrm{~mm}$. wide; sporidia $1 \tilde{\mathrm{x}} 10 \mu$.

On shady ground, Otowanie Woods, Lancaster county. PEZIZACEAE.

Peziza brunneo-vinosa Clements.

Ascomata caespitose, sessile, at first cupuliform, at length applanate, often exactly disciform and immarginate, mar- 
gin irregular, hardly lobed, 5-12 $\mathrm{mm}$. in diameter; hy. menium deep brown-wine-color, beneath paler, or orchraceous; asci cylindrical, not turaing blue with iodine, 250 300×12-14 $\mu$; sporidia ellipsoid, smooth, 2-guttate, 16$20 \times 10 \mu$; paraphyses broadly linear, septate, fuliginous above.

On sand in canyons of the Niobrara river, Keya Paha counties.

Peziza paraphysata Clements.

Ascoma orbicular, applanate, margin deflexed, carnose, beneath fusco-ochraceous, hymenium chestnut-colored, 1-2 cm. in diameter; asci cylindrical, 8-spored, elongate, not turning blue with iodine, $325-350 \times 18 \mu$; sporidia broadly ellipsoid, obliquely monostichous, 2-guttate, smooth, 20x $15 \mu$; paraphyses septate, broadly clavate above, densely filled with brown granules, $5 \mu$ wide, clavate apex $50-60 \mu$, long by $10-12 \frac{1}{2} \mu$ wide.

On the ground and on fragments of wood, Meadville, Keya Paha county. Closely related to Discina orbicularis Peck.

Peziza (Plicaria) vinacea Clements.

Ascoma concave, or scutellate, at length applanate, carnose, sessile, margin repand-flexuous, surface of the hymenium uneven, at first orange-vinous, then vinous-brick-colored, beneath even, glabrous, paler, $7-\mathbf{1 5} \mathrm{mm}$. in diam.; asci cylindrical, 8-spored, trunculate, turning blue throughout with iodine, 225-250×12-13 $\mu$; sporidia elliptical, eguttulate, monostichous, hyaline, smooth, 16--20x10-12 $\mu$; paraphyses broadly filiform, scarcely incrassate at the apex, granular, $5 \mu$ wide.

On damp ground in shady woods, Wabash.

Galactinia viridi-tincta Clements.

Ascoma hemispherical, cupulate, sessile, milky, without grayish-tomentose, or minutely verrucose, light-olivaceous, 
hymenium brownish-olivaceous, flesh turning green when wounded, milk grayish-green, $5-10 \mathrm{~mm}$. wide; asci cylindrical, turning blue with iodine, $250-300 \times 12-14 \mu$; sporidia ellipsoid, 1-2-guttate, irregularly disposed, smooth, $15 \times 18 \mu$; paraphyses linear, scarcely, or not at all, incrassate, multiseptae, hyaline, $5-7 \mu$ wide.

On shady ground, Otowanie Woods, Lancaster county.

Barlaea constellatio minuta Clements.

Ascomata generally immarginate, convex, aurantiaceous, rarely reddening with age, $\frac{1}{3}-\frac{1}{2} \mathrm{~mm}$., rarely as much as 1 $\mathrm{mm}$, in diam.

On wet ground, Rock Creek, Keya Paha county.

Humaria clausa Clements.

Ascomata caespitose, carnose, subglobose, or nearly hemispherical, mouth somewhat hysteriform, gaping slightly, pruinulose, drab, $\frac{3}{4}-2 \mathrm{~mm}$., wide; asci clavate, 8-spored, $250 \times 35 \mu$; sporidia iarge, oblong, smooth, hyaline, eguttulate, $32-37 \times 12 \frac{1}{2}-16 \mu$; paraphyses densely packed, filiform, scarcely incrassate above.

On moist ground, Otowanie Woods, Lancaster county.

Humaria phycophila Clements.

Ascomata very minute, sessile, scutellate. at length convex, gregarious, margin entire; hymenium incarnate, or miniate-aurantiaceous, paler without, $300-500 \mu$ in diam.; asci clavate-cylindrical, obliquely operculate, 8-spored, not turning blue with iodine, $60-67 \times 9-15 \mu$; sporidia elliptical, obliquely monostichous, or often distichous, slightly truncate at both ends, generally 1-guttate, smooth, hyaline, $10-11$, rarely $12 \frac{1}{2}$, by $6-7 \frac{1}{2} \mu$; paraphyses filiform, furcate above, stuffed with orange granules, strongly recurved at the apex, 1-2 $\mu$ in diam

Among filaments of Lyngbya on moist ground, Beatrice. A true Humaria, though in some degree related to Ascophanus. 
Humaria subcrenulata Clements.

Ascoma carnose, with a very short, thick, hypogaeous. stipe, at first globose, then hemispherical, or sometimes almost concave, margin slightly incurved, subcrenulate, ochroleucous, or ochraceous, 2-.5 mm. wide; asci narrowly cylindrical, stipitate, $200 \times 10 \mu$; sporidia ellipsoid, hyaline, smooth, eguttulate, $12 \frac{1}{2}-15 \times 8-10 \mu$; paraphyses scarcely equalling the asci, filiform, septate, granular, $3 \mu$ wide.

Among mosses on sandy ground, Rock Creek, Keya Paha county; canyons of Hazel Creek, Brown county.

Humaria tofacea Clements.

Ascoma sessile, carnose, concave, naked, grayish-drab, scarcely margined, or the margin strongly depressed, $2-3 \mathrm{~cm}$. wide; asci cylindrical, not turning blue with iodine, $200 \times 10-12$ $\mu$; sporidia ellipsoid, smooth, eguttulate, monostichous, 15x8-9 $\mu$; paraphyses filiform, $2 \frac{1}{2} \mu$ wide.

On fragments of the wood of Pinus ponderosa, canyons of the Niobrara river, Keya Paha county.

Sarcoscypha roseo-tincta Clements.

Ascomata caespitose, cupuliform, at length often concave, carnose, without beautifully papillate-tomentose, with densely agglutinated cells, 20-느 $\mu$ in diam., white, margin tomentose-ciliate-crenate, sordescent in age; hymenium creamy-ochraceous; stipe broad, rarely 1-3-lacunose. tomentose-papillate, expanded above into the cup, rosy, or rosy-tinged; asci cylindrical, 8-spored, not turning blue with iodine, $125 \times 8-10 \mu$; sporidia ellipsoid, hyaline, monostichous, 2-guttate, $10 \times 5 \mu$; paraphyses linear, stuffed with 1-2-seriate oil-drops above, $2 \frac{1}{2} \mu$ wide.

On horse-dung, Otowanie Woods, Lancaster county.

Sepultaria Cooke Myc. 259, 1879, Lachnea Fr. Syst. Myc. 2:77. 1822; not Lachnaea L. Sp. Pl. Ed. I., 560, 1753 (Lachnea Sp. Pl. Ed. II., 514). Fries cites Lachnum Retz. Flor. Scand. Prod. 329, 1797, as a synonym of Lachnea. Lach. 
num aqaricinum Retz, however, is Dasyscypha virginea (Batsch) Fckl.; hence Lachnum Retz. must stand for the Friesian genus Dasyscypha. In consequence, Sepultaria Cooke is the oldest admissible name, for Lachnea Fr. Kuntze, Rev. Gen. Pl. 868 takes up Scutellinia Cooke, in accordance with his rule of species. majority, a principle not sanctioned by American botanists.

Sepultaria (Eusepultaria) aspera Clements.

Ascoma broadly cupulate, partly subterranean, waxy-carnose, covered with short, thick, flexuous, smoky-brown, 3-6celled hairs, $80-200 \times 12-15 \mu$, chestnut-colored, margin irregularly 3-4-lobed; hymenium bay-chestnut-colored; asci cylindrical, not turning blue with iodine, $200 \times 12 \frac{1}{2}-15$ $\mu$; sporidia fusoid-elliptical, $2-3$ guttate, hyàline, monosti-

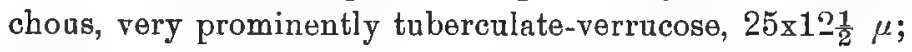
paraphyses entirely fusco-olivaceous, clavulate, 7-8 $\mu$ wide.

Ascoma $3 \mathrm{~cm}$. high, $2 \mathrm{~cm}$. wide.

On shady ground, bluffs of the Missouri river, Bellevue. Related to S. fusicarpa (Ger.) Cooke, but easily distinguished by the lobed margin, sparse hairs, and tuberculate spores.

Sepultaria (Eusepultaria) aurantia Clements.

Ascoma subhypogaeous, hemispherical-cupulate, without fuscous-brown, densely covered with very long, septate, concolorous hairs, 500-600×10 $\mu$; hymenium bright orange, 5-7 $\mathrm{mm}$. wide; asci narrowly cylindrical, longstipitate, not turning blue with iodine, $250 \times 8-10 \mu$; sporidia ellipsoid, smooth, monostichous, 1-guttate, 16-18×8$9 \mu$; paraphyses filiform, filled with orange granules, $2 \frac{1}{2} \mu$ wide.

On the ground among dead leaves, bluffs of the Missouri river, Bellevue. Related to S. lapidaria Cooke; the form and color of the cup are that of S. hemisphaerica (Wigg.) Cooke. 
Sepultaria (Eusepultaria) grisea Clements.

Ascomata subterranean, gregarious, often crowded, hemisspherical-cupulate, without sparsely covered with septate, flexuous hairs, pale brown, or isabel-colored below, hyaline toward the apex, 150-400x6-7 $\mu$, brown-ocbraceous, hymenium gray, 3-4 mm. wide; asci broadly cylindrical, 8-spored, 200x1j-20 $\mu$; sporidia ellipsoid, smooth, 1-2guttate, monostichous, $20-25 \times 12 \frac{1}{2}-15 \mu$; paraphyses gradually but strongly clavate, $7-8 \mu$ wide above.

Among mosses on wet sand, Rock Creek canyon, Keya Paha county.

Sepultrria (Scutellinia) bryophila Clements.

Ascomata gregarious, hemispherical, then deeply scutellate, margin and external surface furnished with septate, brown, obtuse or acuminate hairs, $250-100 \times 8-10 \mu$, hymenium concave, gray, with a pale but distinct rosy tint, $1-2 \mathrm{~mm}$. wide; asci cylindrical, 8-spored, 250-300×12-14 $\mu$; sporidia elliptical, hyaline, smooth, minutely 2 -guttulate, 16-20x10-12 $\mu$; paraphyses guttulate, filiform, 3-4 $\mu$ wide.

On saudy ground among mosses, Rock Creek Canyon, Keya Paha county. Related to Sepultaria cretea (Cooke).

Sepultaria (Scutellinia) pediseta Clements.

Ascomata gregarious, or caespitose, at first hemispherical, scarcely open, then scutellate, without tomentose-tuberculate, the tubercules pale yellow, composed of agglutinated cells $20-25 \mu$ in diam.; bristles sparse, very long, strict, strongly attenuate, pale brown, 9-10-septate, $450-$ $500 \times 18 \mu$ at the base, $5 \mu$ at the apex, arising singly from the tubercules; hymenium bright orange, paler without, 3-5 $\mathrm{mm}$. in diam,; asci cylindrical, not turning blue with iodine, $175 \times 10 \mu$; sporidia ellipsoid, smooth, monostichous, $15 \times 8 \mu$; paraphyses scarcely broader above, $5 \mu$ wide at the apex, slightly green-tinted. 
On horse dung, Otowanie Woods, Lancaster county. Related to $S$. dalmeniensis (Cooke).

Sepultaria (Scutellinia) pseudocrenulata Clements.

Ascomata deeply concave, carnose; hymenium exactly drab, paler without, furnished with short, septate, nearly hyaline hairs, 80-100 $\mu$ long, margin crenulate with fasciculate hairs, $5 \mathrm{~mm}$. wide; asci cylindrical, 8-spored, 200-225x 12-15 $\mu$; sporidia ellipsoid, smooth, hyaline, monostichous, $18 \times 10 \mu$; paraphyses filiform, scarcely incrassate above.

On rich, shady ground among filaments of Lyngbya, Otowanie Woods, Lancaster county. Related to S. laxmanni (Weinm).

Sepultaria (Scutellinia) punicea Clements.

Ascomata subgregarious, superficial, or slightly innate, at first hemispherical, closed, then narrowly open, mouth densely beset with bristles, without furnished with very dense, brown, septate, acute bristles, 300-400x16-20 $\mu$; hymenium bright miniate, $\frac{1}{2}-2 \mathrm{~mm}$. in diam, ; asci cylindrical, 225-230 $\times 10-14 \mu$; sporidia elliptical, smooth, hyaline, monostichous, 10-12x7-9 $\mu$; paraphyses filiform-clavulate, densely filled with orange granules.

On wet, decaying wood, bluffs of the Missouri River, Otoe county.

Sepultaria (Soutellinia) pygmaea Clements.

Ascomata very minute, $\frac{1}{2}-\frac{3}{4} \mathrm{~mm}$., rarely $1 \mathrm{~mm}$. in diam., gregarious, carnose, scutellate; hymenium gray or fuscous, margin and external surface furnished with very strict brown, septate, acute, or often truncate hairs, $175-250 \times 12 \frac{1}{2}$ $\mu$; asci cylindrical, $125-150 \times 9-10 \mu$; sporidia ellipsoid, smooth, $12 \times 7 \mu$; paraphyses exceptionally numerous, filiform.

On rich ground among filaments of Lyngbya, Otowanie woods, Lancaster county. Related to S. bryophila. 
Sepultaria (Scutellinia) rubro-purpurea Clements.

Ascomata at first urceolate, then cupulate, very rarely somewhat scutellate, without brownish-fuliginous, very densely covered with rigid, concolorous, many-septate hairs, rounded at the apex, $150-200 \times 8-10 \mu$, margin scarcely distinctly ciliate, hymenium bright red-purple, $3-5 \mathrm{~mm}$. in diam.; asci cylindrical, turning bright green with iodine, $250 \times 15-18 \mu$; sporidia ellipsoid, verrucose, 2-guttate, monostichous, $23-25 \times 12 \frac{1}{2} \mu$; paraphyses septate, red-purple, 3-4 $\mu$ wide, abruptly incrassate above, $7 \mu$ wide.

On the sandy banks of a brook, Hazel Creek Canyons, Brown county. Related to S. cubensis (Berk.).

Pseudohelotium isabellinum Clements.

Ascoma cupulate, stipitate, margin thick, often flexuous; hymenium concave, isabel-colored, or pale fulvous, paler beneath, puberulent with short hairs, $25-40 \times 8-10 \mu$, $\frac{1}{2}-2$ $\mathrm{mm}$. in diam.; stipe short, $\frac{1}{3}-\frac{1}{2} \mathrm{~mm}$; asci cylindrical, not turning blue with iodine, 75-95x6-7 $\mu$, sporida oblong, monostichous, $7-12 \frac{1}{2} \times 5 \mu$; paraphyses densely filled with large oil-drops.

On wet twigs, Rock Oreek, Keya Paha county. Mollisia lilacina Clements.

Ascomata gregarious, sessile, disciform, lilac or pale livid, testaceous when dry, paler' beneath, margin elevated, white-crenulate, $\frac{1}{3}-1 \mathrm{~mm}$. in diam.; asci small, clavate, stipitate, $30-40 \times 3-4 \mu$; sporidia cylindrical, straight or curved, 1-3-guttato, or spuriously septate, distichous or monostichous, $7-10 \times 1 \frac{1}{2}-2 \mu$; paraphyses cylindrical, granular, $2 \mu$ wide.

On bark of Ulmus americana, Wabash; on decaying twigs, Bellevue, Beatrice, Nebraska City.

Trichopeziza candida Clements.

Ascomata caespitose, sessile, waxy, tenacious, broadly infundibuliform, or scutellate, pure white within and with- 
out, disk becoming cinereous in age, exterior very densely covered with long, hyaline hairs roughened with minute. crowded granules, margin beautifully ciliate, crispatelobate, incurved; asci clavulate, somewhat acute at the apex, $75-100 \times 5-8 \mu$; sporidia lacking.

On bark and twigs of Tilia americana, Wabash. Possibly Trichopeziza tiliae (Peck) Sacc.

Phaeopezia elaeodes Clements.

Ascoma hemispherical-cupulate, rarely scutellate, waxy sessile, beautifully verrucose without, brownish-black; hymenium exactly olivaceous, margin often irregular, flexuous; asci cylindrical, truncate, 8-spored, 200-300x15. $-18 \mu$; sporidia monostichous, spherical, with a large oildrop, $8 \mu$ in diam. densely concentrically verruculose, fuscous, $12-15 \mu$ in diam.; paraphyses linear, many-septate, $5-6 \mu$ wide.

Ascoma 8-20 mm. wide, 5-13 mm. high.

On wet, sandy banks, Hazel Creek Canyons, Brown county. Phaeopezia vinacec Clements

Ascoma at first broadly cupulate or concave, then applanate, sessile, carnose, without pale brownish-verrucose, blackishvinous; hymenium vinous, $5-10 \mathrm{~mm}$.' in diam.; asci cylindrical, turning blue with iodine, obtuse or truncate, 250-300×12-15 $\mu$; sporidia elliptical, pale fuscous, echinate, prominately 1-guttate, monostichous, $15-18 \times 10 \mu$; paraphyses linear, fuscous above, scarcely incrassate, $3 \mu$ wide.

On damp, shady ground, Otowanie Woods, Lancaster county.

\section{BULGARIACEAE.}

Orbilia atropurpurea Clements.

Ascomata superficial, crowded, sessile, gelatinous when wet, corneous when dry, concave, or applanate, dark purple, exciple dense, fuscous, $2-5 \mathrm{~mm}$. in diam.; asci elongate, 
narrowly cylindrical, not turning blue with iodine, 150 160x8-10 $\mu$; sporidia hyaline, granular, occasionally spuriously 1 -septate, broadly fusoid, somewhat obtuse at both ends, crowded, monostichous, $20-25 \times 5-6 \mu$; paraphyses filiform, narrow, $1 \mu$ wide, abové broadened into a minute clava.

On dead wood, Hazel Creek Canyon, Brown county.

\section{AGARICACEAE.}

Mastocephalus carneo-annulatus Clements.

Pileus campanulate, carnose, exstriate, pellicle incarnate, densely fibrillose-silky; free ends of the fibrils agglutinated, pileus hence covered with appressed, granular, atro-incarnate scales; umbo scarcely distinct, slightly depressed, brick-red-incarnate, tomentose, or broken into minute scales; stipe white, fistulose, shining, silky, incrassate at the base, equal above; annulus fixed, superior or inferior, white, erect, appressed to the stipe; limb abruptly spreading, beautifully margined with incarnate, $3 \frac{1}{2} \mathrm{~mm}$. wide; lamellae adnexed to a collar, pure white, slightly crowded; spores hyaline, 1-2-guttate, fusoid-

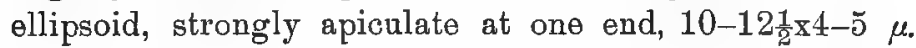
Pileus $3 \mathrm{~cm}$. wide, $2 \mathrm{~cm}$. high; stipe $4 \mathrm{~cm}$. long, $5 \mathrm{~mm}$. wide above, $10 \mathrm{~mm}$. below.

Or shady ground, Otowanie Woods; Wabash, Bellevue. Related to $M$. rhodocephalus (Berk) OK.

Mastocephalus incarnatus Clements.

Subcaespitose; pileus thin, slightly carnose, conical, at length campanulate, rarely convex, slightly silky, pale incarnate, with darker scales, margin striate; umbo distinct, becoming black; stipe stuffed, slender, equal, glabrous, very rarely silky, pallid, or somewhat rosy; annulus median, or superior, fixed, erect, white; lamellae remote, subdistant, scarcely ventricose, white; spores minute, 
ovate-ellipsoid, uniguttate, apiculate at one end, 5-6x3 $\mu$. Pileus 2-4 cm. wide, $1 \frac{1}{2}-3 \mathrm{~cm}$. high; stipe $3-6 \mathrm{~cm}$. long, $2-$ $5 \mathrm{~mm}$. thick.

On the ground, among leaves, Wabash, Otowanie Woods. Mastocephalus repandus Clements.

Pileus convex-repand, carnose, covered with minute, crowded, granular scales, incarnate-ochraceous; umbo distinct; stipe graceful, hollow, equal, minutely floccose-farinaceous, white above, pinkish-ochraceous below; annulus white, superior; lamellae free, white, ventricose; spores ellipsoid or globose, $5-7 \times 5 \mu$.

Pileus 13-18 mm. wide; stipe 2-3 cm. long, 1-1 $\frac{1}{2} \mathrm{~mm}$. wide.

On rich earth, Lincoln. Related to $M$. cristatus (Alb. \& Schw.) OK.

Mastocephalus sulphurinus Clements.

Pileus campanulate, then convex, subcarnose, pellicle sulphur-yellow, silky, torn into crowded, oblong, or elongate scales, margin distinctly striate-plicate, floccose; umbo distinct, elevated, glabrous, or scarcely rimose, incarnate-brickcolored; stipe graceful, fistulose, pruinose, strongly bulbous at the base, yellow-floccose, above incrassate, shining, isabel-colored; annulus superior, fixed, laerate, sulphuryellow; lamellae, touching the collar, linear, crowded, nearly white, or pale straw-colored; spores ovate-ellipsoid, uniguttate, $7-9 \times 4-5 \mu$.

Pileus $1 \frac{1}{2}-3 \mathrm{~cm}$. wide; stipe $4 \mathrm{~cm}$. long, $3-4 \mathrm{~mm}$. wide above, 6-7 mm. below.

On the ground, Lincoln.

Clitocybe megalospora Clements.

Pileus plano-convex, subcarnose, even, glabrous, yelloworchraceous, darker at the center. margin thin; stipe graceful, fistulose, glabrous, becoming white; lamellne white, distant, short-decurrent, pale yellow; spores large, 
hyaline, with a large oil drop $10 \mu$ in diam., ovoid, or slightly limoniform, 17-18×10-12 $\mu$.

Pileus $3 \mathrm{~cm}$. wide; stipe $7 \mathrm{~cm}$. long, '2 mm. thick.

On wet earth, Saltillo.

Collybia discipes Clements.

Pileus convex, at length explanate, subcarnose, even, glab. rous, striate at the margin, grayish-drab; umbo wide, darker, grayish-brown; stipe cartilaginous, stuffed, glabrous, shining, white, arising from a hypogaeous disk; lamellae free, remote, crowded, ventricose, 4-6 $\mu$ wide, pale orchraceous; cystidia exactly cyathiform or sometimes flask-shaped, 2-3 cuspidate at the apex, 45-53 $\mu$ long, $20 \mu$ wide at the base, $10 \mu$ at the apex; spores ellipsoid, or ovate, granular within, 5-6x3-4 $\mu$.

Pileus $5 \mathrm{~cm}$. wide; stipe $3 \frac{1}{2} \mathrm{~cm}$ long, $5 \mathrm{~mm}$. wide.

On damp ground, Beatrice.

Collybia umbrina Clements.

Pileus convex, carnose, umbonate, brown, glabrous, exstriate, very viscid, dotted with thin, black lines; umbo black; stipe very long, attenuate above, carnose, stuffed, glabrous, longitudinally striate towards the apex, radicate, white, becoming fuscous below; lamellae adnate, broad, distant, unequal, white; spores large, irregularly limoniform, with large oil-drop $10 \mu$ in diam., 12-13×17-18 $\mu$.

Pileus $4 \mathrm{~cm}$. wide; stipe $25 \mathrm{~cm}$. long, $5 \mathrm{~mm}$. wide above, 10 mm. below.

On decaying twigs buried in the ground, Bellevue. Perhaps but a variety of $C$. radicata Rehl.

Collybia velutina Clements.

Pileus convex, or plane, carnose-cartilaginous, even, covered with a dense, brown-fulvous tomentum, reddish-chestnutcolored; stipe fistulose, cartilaginous, equal, densely clothed with a silky, fulvous-ochraceous tomentum; lamel- 
lae adnexed, narrow, unequal, crowded, ochraceous; spores ellipsoid, 7-8×5 $\mu$.

Pileus 1-3 cm. wide; stipe $3-5 \mathrm{~cm}$. long, $1 \frac{1}{2}-3 \mathrm{~mm}$. thivk.

On decaying logs, Bellevue.

Lactarius villosus Clements.

Pileus at first convex, margin involute, beset with the fibrils of the veil, then explanate-umbilicate, rarely infundibuliform, spongy-carnose, often irregular, silky-villose, with long, innate, agglutinated fibrils, disk often minutely tomentose-areolate, at first white, then strongly tinged with orange; stipe short, thick, often excentric, attenuated downwards, solid, tomentose, white; lamellae adnexed, decurrent when the pileus is infundibuliform, linear, very crowded, white, then tinged with ochracrous; milk copious, very pungent, white, immutable; spores irregularly ellipsoid, or ovoid, uniguttate, echinulate, $5-6 x t-5 \mu$; cystidia numerous, rugose, lanceolate, $25-30 \times 5 \mu$.

Pileus 6-12 cm. wide; stipe $1 \frac{1}{2}-2 \frac{1}{2} \mathrm{~cm}$. long and thick.

On sandy ground, Hazel Creek Canyons, Brown county. Marasmius albo-marginatus Clements.

Pileus minute, solitary, membranaceous, convex, glabrous, sulcate, purple, paler at the margin; stipe shining, glabrous, equal, lemon-yellow; lamellae few, 7-8, adnate, white; basidia $12-14 \times 6-7 \mu$; spores (?) $5 \times \underline{2}-3 \mu$, ovoid.

Pileus $1 \frac{1}{2} \mathrm{~mm}$. wide; stipe $1 \mathrm{~cm}$. long, $\frac{1}{2} \mathrm{~mm}$. thick.

On the ground in shady woods, Beatrice.

Marasmius fulviceps Clements.

Pileus convex-campanulate, then convex, or almostapplanate, membranaceous, strongly radiate-sulcate, rugose, glabrous, umbonate, fulvo-ferruginous; stipe graceful, with a medulla, flexuose, spirally twisted above, entirely smooth, shining, dark-brown, paler at the apex; lamellae adnexed to a collar around the stipe, with connecting veins, distant, 15-20, edge flexuous, dark-ochroleucous; spores hyaline, fusoid, $18-20 \times 5 \mu$. 
Pileus 5-15 mm. wide; stipe 4-6 cm. long, $\frac{1}{2}-1 \mathrm{~mm}$. thick.

On dead leaves, Bellevue. Related to M. schweinfurthianus P. Henn.

\section{Marasmius hirtipes Clements.}

Pileus plano-convex, membranaceous, scarcely umbilicate, slightly radiate-suleate, glabrous, fulvous; stipe elongated, filiform, hollow, clothed with spreading, white or fulvous hairs, dark-rufous; lamellae somewhat numerous, adnate, linear, white, or dilutely yellow; spores ellipsoid, minutely. 2-guttulate, $7 \times 4 \mu$.

Pileus 3-7 mm.; stipe $3-8 \mathrm{~cm}$. long, $\frac{1}{2}-\frac{3}{4} \mathrm{~mm}$. thick.

Marasmius papillosus Clements.

Pileus conico-papillate, then campanulate, or even explanate, membranaceous, striate, glabrous, cream-colored, or ochraceous; stipe graceful, equal, cartilaginous, pruinose above, densely lanate below, dirty-white, or cream-colored; lamellae few, adnexed, white; spores ellipsoid, $6 \times 4 \mu$.

Pileus 2-6 mm. wide; stipe $1 \frac{1}{2}-3 \mathrm{~cm}$. long, $\frac{1}{2}-1 \mathrm{~mm}$. thick. On decaying logs, Beatrice.

Orcella depressa Clements.

Pileus plano-convex, or depressed in the center, sub-membranaceous, glabrous, even, ochraceous, centre darker; stipe short, solid, glabrous, white, incrassate toward either end; lamellae decurrent, subdistant, light cinnamoncolored; spores irregularly ellipsoid, pale rosy, $8-10 \times 4-5 \mu$.

Pileus $\frac{3}{4}-1 \frac{1}{2} \mathrm{~cm}$. wide; stipe $1 \frac{1}{2}-2 \frac{1}{2} \mathrm{~cm}$. long, $2 \mathrm{~mm}$. thick.

On fallen leaves, Bellevue.

Nolanea atro-cyanea Clements.

Pileus membranaceous, campanulate, glabrous, or minutely verrucose, papillate-umbonate, striate-lacerate at the margin, prussian blue; stipe graceful, equal, cartilaginous, glabrous, bright blue, or slightly tinged with sea-green; lamellae receding slightly, narrow, subdistant, cream- 
colored; spores globose, or ellipsoid, 3-7-angular or apiculate, uniguttate, rosy, $5-7 \times 7-9 \mu$.

Pileus $1-3 \mathrm{~mm}$. wide; stipe $1 \mathrm{~cm}$. long, $\frac{1}{2}-1 \mathrm{~mm}$. thick.

On the ground in woods, Bellevue.

Under the Rochester Rules, Nolanea Fr. seems to be available, notwithstanding the prior Nolance L.

Hebeloma flavum Clements.

Pileus persistently campanulate, fleshy, viscid, covered with nearly concentric, fulvous, scales $2 \mathrm{~mm}$. wide, margin incurved, appendiculate, bright yellow; stipe thick, solid, short, curved, densely beset with concentric, floceose, fulvous scales, except at the base, yellow; lamellae subsinuate, with a decurrent tooth, slightly crowded, drab; spores ovoid, ochroleucous, $7-8 \times 4 \mu$.

Pileus 5-6 cm. wide; stipe $3-5 \mathrm{~cm}$. long, $\frac{2}{3}-2 \mathrm{~cm}$. thick.

Ou ground, Bellevue.

Galera pulchra Clements.

Pileus conical, broad, membranaceous, striate-sulcate to the middle, minutely and densely silky-tomentose; umbo distinct, ochraceous, cream-colored; stipe elongated, cartilaginous, graceful, attenuate, fistulose, characteristically longitudinally lineate striate-pruinose, cream-colored; lamellaeadnexed, narrow, linear, slightly crowded, ochraceous, spores sublimoniform, fulvous, eguttate, 15-16x9-10 $\mu$.

Pileus $2 \frac{1}{2} \mathrm{~cm}$. wide, $2 \mathrm{~cm}$. high; stipe $7-8 \mathrm{~cm}$. long. $2 \mathrm{~mm}$. thick.

On rich, wet ground, Otowanie Woods.

Gomphos caesius Clements.

Pileus at first campanulate-convex, then explanate, carnose, evem, glabrous, not viscid, or obsoletely so, margin involute, dark eye-blue-violet, at length spotted with brown; flesh eye-blue, immutable; stipe fibrous-carnose, solid, with an exactly turbinate bulb, which in age becomes nearly globose, margin of bulb and base of stipe violet, 
stipe violet above, ochrolencous below, with a false annulus composed of the fulvous fibrils of the cortina just beneath the apex; cortina cobwebby, pale eye-blue; lamellae adnate, with a decurrent tooth subdistant, at first white, then cinnamon-colored, never violet; spores brownish-fulvous, verrucose, subelliptical, or globose, 8$10 \times 7-8 \mu$.

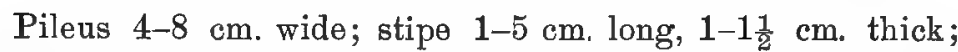
bulb $3-4 \mathrm{~cm}$. high, $4 \mathrm{~cm}$. wide.

On ground in woods, bluffs of the Missouri river, Bellevue. Related to G. glaucopus (Schaff.) OK.

Clarkeinda plana Clements.

Pileus carnose, applanate, exactly plane, even, glabrous, ochraceous, or slightly fulvous; stipe short, stout, solid, attenuate above, fibrillose-aquamulose, becoming fulvous; volva ample, adpressed, membranacoous; lamellae free, ventricose, crowded, black-cinnamon-colored; spores shortellipsoid, or globose, uniguttate, purple-fulvous, 4-6x 5-6 $\mu$.

Pileus $7 \mathrm{~cm}$. wide; stipe $3 \mathrm{~cm}$. high, $2 \mathrm{~cm}$. thick.

On mauured ground, Meadville, Keya Paha county.

Gymnochilus nom. nov.

Psathyra Fr. (1821), not Psathura Commerson, Juss. Gen. (1789).

Gymnochilus roseolus Clements.

Pileus hemispherical or convex, membranaceous, glabrous, or minutely micaceous, irregular regulose, vinous when wet, incarnate when dry; stipe tall, fragile, fistulose, shining, glabrous, apex beset with a few large, farinaceous granules; lamellae slightly remote, purplish-cinnamoncolored; spores ellipsoid, dark-purple, 12-13x7-8 $\mu$.

Pileus 1-21 cm. wide; stipe 4-8 cm. long, $2 \mathrm{~mm}$. thick.

On ground, bluffs of the Missouri river, Bellevue. 
ADDITIONS TO THE REPORTED FLORA OF THE STATE.

\footnotetext{
["Indicates new host only.]

PHYTOMYXINEAE.
}

* Phytomyxa leguminosarum (Frank) Schroet.

On Astragalus carolinianus, Bellevue. (4251)

On Cassia chamaecrista, Bellevue. (4247)

On Meibomia grandiflora, Bellevue. (4248)

NOSTOOACEAE.

Nodularia paludosa Wolle.

In Salt Lake, Lincoln. (4501)

PALMELLACEAE.

Haematococcus lacustris (Girod) Rost.

On horse droppings, Lincoln (4499), in irrigation ditch, Rock Creek, Keya Paha county. (4500)

DESMIDIAOEAE.

Disphinctium notabile (Breb.) Hansg.

Glen Rock. (4502)

BACILLARIACEAE.

Amphiprora conspicua Grev.

Lincoln. (4587)

Amphora ovalis Kuetz.

Plattsmouth. (4588)

Amphora ovalis affinis (Kuetz.) V. H.

Lincoln. (4589)

Amphorc ovalis gracilis (Ehr.) V. H.

Talmage; fossil, Mullen. (4590)

Amphora ovalis pediculus (Kuetz.) V. H.

Peru, Salem, Fairbury, Humboidt, Bellevue, Auburn, Plattsmouth, Sheridan county; Weeping Water. (4591) 
Amphora salina borealis (Kuetz.) Elmore.

Amphora borealis Kuetz. Bacill. 108. (1844)

Amphora salina minor V. H. Syn 57. (1880)

Fairbury. (4592.)

Bacillaria paradoxa (Gmel.) Grun. Crete. (4759)

Campylodiscus campylodiscus (Ehr.) Elnore.

Surivella campylodiscus Ehr. Verb. 424. (1813)

Campylodiscus ehrenbergii Ralfs, Pritchard Inf, 802. (1861)

Talmage, Fairbury, Brock, Lincoln, Auburn, Arago, Brownwille, Weeping Water, Ashland. (4593)

Cocconeis placentula Ehr.

Holt county, Bellevue; fossil, Mullen. (4594)

Cyclotella meneghiniana Kuetz.

Lincoln, Weeping Water, Talmage. (4595)

Cymbella fusidium (Ehr.) Elmore.

Cocconema fusidium Ehr. Inf. 26.

Cymbella affinis Kuetz. Bacill. 80. (1844)

Humboldt. (4596)

Cymbella cuspidata Kuetz.

Bellevue; fossil, Mullev.

(4597)

Cymbella inequalis (Ehr.) Elmore.

Navicula inequatis Ehr. Inf. 184. (1838)

Cymbella ehrenbergii Kuetz. Bacill. 79. (1844)

Holt county, Weeping Water, Talmage; fossil, Mullen. (4598)

Cymbella levis Naeg.

Fossil, Mullen. (4599)

Cymbella cistula (Hempr.) Kirchn.

Fossil, Mullen. (4600)

Cystopleura gibba rentricosa (Kuetz.) Grun.

Rulo, Georgetown, Peru, Arago, Humboldt, Tecumseh, Bellevue, Plattsmouth, Cherry county, Holt county. (4.601) 
Cystopleura gibberula (Ehr.) Kuntze.

Talmage. (4602)

Cystopleura musculus constricta (Breb.) V. H.

Sheridan county. (4603)

Cystopleura turgida vertagus (Kuetz.) Grun.

Fossil, central Nebraska. (4604)

Cystopleura turgida westermannii (Ebr.) Grun.

Fossil, central Nebraska. (4605)

Denticula elegans Kuetz.

Fossil, Mullen. (4606)

Diatoma vulgare Bory.

Fossil, central Nebraska. (4607)

Encyonema caespitosum auerswaldii (Rabenh.) V. H.

Fossil, Mulleu. (4608)

Encyonema prostratum (Berk.) Ralfs.

Fairbury. (4609)

Ernotia formica Ehr.

Fossil, Mullen. (4610)

Fragilaria construens venter Grun

Fossil, central Nebraska, Mullen.

Fragilaria elliptica Schum.

Fossil, central Nebraska, Mullen. (4612),

Eragilaria virescens Ralfs.

Fossil, Mullen. (4613)

Frustulia bohemica (Ehr.) Rabenh.

Sheridan and Cherry counties. (4614)

Gomphonema acuminatum Ehr.

Bellevue. (4615)

Gomphonema gracile Ehr.

Fossil, Mullen. (4616)

Gomphonema micropus Kuetz.

Lincoln. (4617)

Gomphonema montanum Schum.

Auburn, fossil, central Nebraska. (4618) 
Gomphonema montanum commutatum Grun.

Talmage, Weeping Water. (4619)

Gomphonema montanum subclavatum Grun.

Bratton, Talmage; fossil, Mullen. (4620)

Gomponema rostellatum lanceolatum V. $\mathrm{H}$.

Talmage. (4621)

Gomphonema turris Ehr.

Talmage. (4622)

Hantzschia amphioxys (Ehr.) Grun.

Lincoln, Talmage, Weeping Water, Sheridan, Cherry aud Holt counties; fossil Mullen, central Nebraska. (4623), Hantzschin elongata. (Hantzsch) Grun.

Talmage, Holt county; fossil, Mullen.

Homoeocladia filiformis W. Sm.

Fairbury. (46:5)

Melosira distans (Ehr.) Kuetz.

Bellevue; fossil, central Nebraska, Mullen. (4626) Navicula ambigua Ehr.

Talmage, Tecumseh, Humboldt, Brownville, Weeping Water

Sheridan county. (4627)

Navicula atomoides Grun.

Falls City, Talmage, Fairbury, Humboldt, Bellvue, Auburn. (4628)

Navicula atomis (Kuetz.) Grun.

Humboldt, Talmage, Peru. (4629)

Navicula bacilliformis Grun.

Fossil, Mullen. (4630)

Navicula bahusiensis Grun.

Fairbury. (4631)

Nuvicula cocconeiformis Greg.

Humboldt. (4632)

Navicula cryptocephala veneta (Knetz.) Rabenh.

Brock, Talmage, Peru, Pawnee City, Humboldt, Julian, Falls City, Rulo, Dawson, Salem, Aspinwall, Fairbury, Tecumseh, Auburn, Weeping Water, Ashland. (4633) 
Navicula cuspidata Kuetz.

Lineoln, Talmage. (4634)

Nuvicula decurrens Kuotz.

Talmage. (4635)

Navicula dicephala Ehr.

Fairbury. (4636)

Navicula digitato-radiata cyprinus (W. Sm.) V. H.

Talmage. (4637)

Navicula elliptica minutissima Grun.

Talmage. (4638)

Navicula falaisensis Grun.

Fairbury. (4639)

Navicula fasciata Lagerst.

Talmage. (4640)

Navicula inflata Kuetz.

Lincoln, Fairbury. (4642)

Navicula iridis Ehr.

Auburn; fossil, central Nebraska. (4643)

Navioula iridis affinis (Ehr.) V. H.

Talmage, Auburn; fossil, Mullen. (4644)

Navicula iridis amphigomphus (Ehr.) V. H.

Fossil, central Nebraska, Mullen.

Navicula iridis dubia (Ehr.) V. H.

Lincoln, Talmage. (4647)

Navicula levissima Kuetz.

Sheridan county. (4646)

Navicula liburnica Grun.

Lincoln. (4648)

Navicula limosa Kuetz.

Cook, Talmage. (4649)

Navicula macilenta Ehr. Inf. 183. 1838.

Navicula oblonga Kuetz. Bacill. 97. 1844.

Talmage; fossil, central Nebraska, Mullen. (4650)

Navioula mesolepta Ehr.

Lincoln, Auburn. (4651) 
Navicula mesolepta therones (Ehr.) V. H.

Talmage. (4652)

Nrvicula mutica goeppertiana (Bleisch) Cl. \& Grun.

Talmage, Rulo. (46อ̆3)

Navicula parallelistriata Pant.

Fossil, Mullen. (4654)

Navicula parva (Ehr.) Elmore.

Stauroptera parva Ehr. Verb 135. 1843.

Navicula stanroptera Grun. Wien Verhundl 1860:516. 1860.

Fossil, Mullen. (4655)

Navicula parva parva (Grun.) Elmore.

Navicula stauroptera parva Grun.

Talmage, Julian, Arago. (4656)

Navicula peregrina (Ehr.) Kuetz.

Lincoln. (4657)

Navicula placentula (Ehr.) Kuetz.

Fossil, Mullen. (4658)

Navicula placentula tumida (W. Sm.) Elmore.

Navicula tumida W. Sm. Brit. Diat. 1:52. 1853.

Navicula anglica Ralfs, Pritch. Inf. 900. 1861.

Fossil, Mullen. (4659)

Novicula pupula Kuetz.

Nebraska City, Talmage; fossil, central Nebraska, Mullen. (4660)

Navicula pygmaea Kuetz.

Talmage, Salem, Humboldt, Weeping Wuter. (4661)

Navicula radiosa Kuetz.

Bellevue. (4662)

Navicula rhyncocephala amphiceros (Kuetz.) Grun.

Talmage, Humboldt. (4663)

Navicula rostrata Ebr.

Talmage; fossil, central Nebraska. (4664)

Navicula saugerii Des.

Salem. (4665) 
Navicula subcapitata paucistriata V. H.

Bellevue. (4666)

Navicula viridis commutata Grun.

Lincoln. (4667)

Navicula viridis sublinearis Grun.

Auburn. (4668)

Nitzschic acicularis (Kuetz.) W. Sm.

Julian, Rulo, Lincoln, Salem, Fairbury, Humboldt, Talmage, Arago, Weeping Water, Ashland, Cherry county. (4669) Nitzschia amphibia Grun.

Talmage, St. Deroin, Fairbury, Humboldt, Auburn, Weeping Water, Ashland; fossil, Mullen. (4670)

Nitzschia communis obtusa Grun.

Lincoln (4671)

Nitzschia debilis (Arnott \& Ryl.) Grun.

Talmage, Brownville, Weeping Water, Lincoln

Nitzschia fasciculata Grun.

Auburn. (4673)

Nitzschia franenfeldii Grun.

Lincoln, Weeping Water. (4674)

Nitzschia frustulum (Kuetz.) Grun.

Salem, Holt county. (4675)

Nitzschia hungarica Grun.

Lincoln, Salem, Auburn, Talmage, Fairbury. (4676) Nitzschia intermedia Hantzsch.

Dawsons, Salem, Peru. (4677)

Nitzschia lanceolata W. Sm.

Lincoln. (4678)

Nitzschia linearis tenuis (W. Sm.) Grun.

Humboldt, Fairbury, Weeping Water. (4679)

Nitzschia obtusa nana Grun.

Fairbury. (4680)

Nitzschia obtusa scapelliformis Grun.

Talmage. (4681) 
Nitzschia palea debilis (Kuetz.) Grun.

Holt County. (4682)

Nitzschia palea fonticola Grun.

Talmage, Brock, Tecumseh, Pawnee City, Humboldt. Dawson, Aspinwall, St. Dercin, Fairbury, Bellevue, Weeping

Water, Sheridan County, Cherry County. (4683)

Nitzschic palea tenuirostris V. $\mathrm{H}$.

Talmage. (4684)

Nitzschia sigma (Kuetz.) W. Sm.

Sheridan County. Julian, Auburn, Ashland. (4685)

Nitzschia sigma diminuta V. H.

Talmage. (4686)

Nitzschia sigma intercedens Grun.

Talmage. (4687)

Nitzschia sigma lamprocarpa (Ehr.) Elmore.

Navicula lamprocarpa Ehr., Kuetz., Bacill. 22. 1844.

Nitzschid sigma rigida (Kuetz.) Grun., Kasp. Alg. 119. 1878.

Fairbury, Auburn, Lincoln. (4688)

Nitzschic sigma rigidula Grun.

Lincoln. (4689)

Nitzschia sigma subcapitata Rabenh.

Talmage. (4690)

Nitzschia stagnorum Rabenh.

Talmage. (4691)

Nitzschia subtilis (Kuetz. ?) Grun.

Arago, Julian, Talmage, Lincoln. (4692)

Nitzschia subtilis paleacea Grun.

Humboldt, Weeping Water, Talmage.

Nitzschia tryblionella Hantzsch.

Fairbury, Lincoln. (4730)

Nitzschia tryblionella levidensis (W. Sm.) Grun.

Talmage, Nemaha City, Cherry county, Auburn, Weeping

Water, Lincoln. (4700)

Nitzschia tryblionella salinarum Grun. 
Fairbury. (4712)

Nitzschia vitrea recta (Hantzsch.) V. H.

Arago, Peru. (4729)

Odontidium pinnatum (Ehrb.) Kuetz. Bacil. 44. 1844.

Fragilaria pinnata Ehrb. Verbreit. 127. 1843

Dimeregramma mutabile Pritchard Infus. 790. 1845.

Odontidium mutabile W. Sm. Brit. Diat. 2:17. 1853.

Odontidium pinnatum intermedium (Grun.) Elmore.

Fragilaria mutablis intermedia Grun. Verb. Wien. Zool. BotGes. 12, pl. $7, f .9 .1862$.

Bellevue. (4701)

Opephora pacifica (Grun.) Petit.

Fossil, Mullen. (4713)

Pleurosigma thuringicum (Kuetz.) Elmore.

Navicula thuringica Kuetz. Bacil. 102. 1844.

Navicula angulata Queck. Pract. Treat. on the Microsc. 438. 1848.

Pleurosigma angulatum W. Sm. Ann. Nat. Hist. 1853:7. 185 3. Pleurosigma thuringicum elongatum (W. Sm. ) Elmore.

Pleurosigma elongatum W. Sm Brit. Diat. 1:64. 1853.

Pleurosigma angulatum elongatum V. H. Syn. 115. 1880.

Pleurosigma thuringicum elongatum forma fallax Grun.

Lincoln. (4721)

Pleurosigma obscurum W. Sm.

Julian, Ashland, Lincoln. (4702)

Pleurosigma scalproides Rabenh.

Talmage. (4728)

Pleurosigma spencerii nodiferum Grun.

Talmage, Peru. (4705)

Pseudoeunotia lunaris (Ehr.) Grun.

Peru, Bellevae, Auburn, Talmage; fossil, Mullen. (4720) Rhoiconeis trinodis inflata Schultze.

Auburn, Arago, Talmage, Weeping Water; fossil, Mullen. (4711) 
Stauroneis anceps producta Lagerst.

Weeping Water. (4703)

Stauroneis gracilis W. Sm.

Plattsmouth. (4722)

Stauroneis heufleriana Grun.

Holt county. (4710)

Suriraya elegans Ehr.

Holt county. (4709)

Suriraya ovalis Breb.

Talmage, Cherry county. (4727)

Suriraya ovalis minuta (Breb.) V. H.

Talmage, Julian, Salem, Fairbury. (4719)

Suriraya ovalis pinnata (W. Sm.) V. H.

Cook, Auburn, Salem, Fairbury, Arago, Talmage, Weeping

Water. (4714)

Surivaya splendida (Ehr.) Kuetz.

Talmage. (4726)

Synedra affinis Kuetz.

Holt county. (4708)

Synedra affinis delicatula Grun.

Weeping Water. (4723)

Synedra affinis tabulata (Ag.) V. H.

Lincoln. (4715)

Synedra capitata Ehr.

Bellevue; fossil, central Nebraska, Mullen.

Synedra famelicr minuscula Grun.

Fossil, central Nebraska, Mullen. (4725)

Synedra radians Kuetz.

Fossil, Mullen. (4706)

Synedra rumpens Kuetz.

Talmage. (4707)

Synedra tenuissima Kuetz. Bacil. 68. 1844.

Synedra acus Kuetz. 1. c.

Frustulia tenuissima Kuetz. Syn. Diat. 24. 1834. 
Synedra tenuissima angustissima (Grun.) Elmore.

Synedra acus angustissima Grun. in V. H. Syn. 151. 1880.

Weeping Water. (4716)

Synedra tenuissima delicatissima (W. Sm.) Elmore.

Symedra delicatissima W. Sm. Brit. Diat. 1:72. 1853.

Synedra acus delicatissima Grun. in VH. Syn. 151. 1880.

Tecumseh, Cherry county. '(4718)

Synedra ulna amphirhynchus (Ehr.) Grun.

Talmage; fossil, Mullen. (4731)

Synedra ulna danica (Kuetz.) VH.

Fairbury, Holt couuty. (4717)

Synedra ulna oxyrhynchus (Kuetz.) VH.

Fossil, Mullen. (4732)

Synedra ulna vitrea (Bory) VH.

Arago. (4733)

Synedra vaucheriae Kuotz.

Lincoln. (4735)

Tabellaria fenestrata (Lyngb.) Kuetz.

Fossil, Mullen. (4734)

ZYGNEMACEAE.

Spirogyra jugalis (Dillw.) Kuetz.

In ponds, Lincoln. (4504)

MUCORAOEAE.

Circinella umbellata Van. Tieg. \& Ie M. "

On horse dung, Lincoln. (4495)

Piptocephalis freseniana De Bary \& Wor.

On horse dung, with the preceding, Lincoln. (4494)

PERONOSPORACEAE.

*Albugo candida (Pers.) S. F. Gray.

On Lepidium sativum, Lincoln.

ULOTRICHIACEAE.

Conferva tenerrima Kuotz.

In tank in the zoological laboratory, Lincoln.

(4496) 
SPHAERIACEAE.

Eutypella scoparia (Ell.) Sacc.

On dead twigs of Ulmus, Weeping Water. (4434)

Diaporthe tuberculosa (Ell.) Sacc.

On stems of Amelanchier canadensis, Weeping Water. (4435)

Diaporthe claviceps Ell. \& Ever.

On twigs of Ostrya virginiana, Weeping Water.

Valsa toxici (Schw.) Ell. \& Ever.

On dead stems of Rhus radicans, Weeping Water.

Ohleria modesta $\mathrm{Fkl}$.

On decorticated branches of Ulmus, Lincoln.

Melanomma alpinum Speg.

On decorticated wood of Rhamnus lanceolata, Woeping

Water. (4581)

Sporormia minima Auersw.

On horse dung, Lincoln.

$(4487)$

Massaria conspurcata (Wallr.) Sacc.

On dead branches of Prunus americana, Lincoln. (4432) Massaria vomitoria B. \& C.

On fallen twigs of Amelanchier canadensis, Weeping Water. (4485)

HYPOCREACEAE.

Cordyceps militaris (L.) Lk.

In the larva of a butterfly, Bellevue.

HYSTERIACEAE.

Hysterium pulicare lenticulare Fr.

On the bark of Ulmus fulva, Lincoln.

Hysterographium elongatum (Wahlen.) Corda.

On decaying wood of Rhamnus lanceolata, Weөping Water. $(4583)$

Hysterographium kansense Ell. \& Ever.

On bark of Quercus macrocarpa, Weeping Water. 
Hysterographium rousselii (De Not.) Saco.

On decorticated wood, Bellevue.

(4422)

Hysterographium syringae (Schw.) Sacc.

On dead wood, Brownville. (4497)

UREDINEAE.

Uromyces plumbarius Peck.

On Oenothera biennis, Crete. (4358)

Aecidium allenii Clinton.

On Lepargyraea crgentea, Valentine. (4396)

Aecidium anisotomes Reichardt.

On leaves and fruits of Pencedanum foeniculaceum, Weeping Water. (4395)

SPHAERIOIDEAE.

Phyllosticta gentianicola (DC.) Fr.

On Gentiana andrewsii, Ewing.

Macrophoma ricini (Cke.) Berl. \& Vogl.

On Ricinus communis (dead stalks) Lincoln. (4384)

Cytospora ambiens Sacc.

On twigs of Ulnus, Weeping Water.

ytospora ampelopsidis C. Mass.

In stems of Parthenocissus quinquefolius, Lincoln.

(4498)

Cytospora cincta Sacc.

In twigs of Prunus cerasus, Lincoln.

Sphaeropsis albescens Ell. \& Ever.

On dead twigs of Acer negundo, Lincoln.

iplodia maydis (Berk.) Sacc.

On old stalks of Zea nays, Weoping Water. (4483)

Chaetomella atra Fckl.

On dead stems of Allionia nyctaginea, Lincoln.

Septoria chenopodii West.

On Chenopodium album, Valentine. (4449)

If the forms found on Chenopodium album described as Septoria chenopodii, S. atriplicis, and S. weslendorpii are 
to be regarded as distinct, the specimens reported should be referred to $S$. atriplicis; but there seems to be no sufficient reason for distinguishing them. See Syl. Fung. 10 : 380, Farlow \& Seymour, Host Index 2:89. If the several forms are united as one species, the name S. chenopodii has priority.

Septoria helianthi Ell. \& Kell.

On leaves of Helianthus annuus, Lincoln. (4369) Rhabdospora helianthicola (Cke. \& Hark.) Sacc.

On stems of Helianthus annuus, Lincoln.

NECTRIOIDEAE.

Cyphina lanuginosa (Peck) Sacc.

On dead leaves, Beatrice. (4417)

EXCIPULACEAE.

Dinemasporium graminum Lev.

In stems of Zea mays, Beatrice. (4415)

Dinemasporium strigosum leptosporum Sacc.

On stems of Heliathus, Lincoln.

MELANCONIEAE.

Gloeosporium tuberculoides Sacc.

On dying leaves of Acer saccharinum, McCook. Marsonia juglandis (Lib.) Sacc.

On Juglans nigra, Saltillo. (4328)

MUCEDINACEAE.

Monilia fumosa Sacc. ?

On decaying seedlings of Cucurbita maxima in the laboratory, Lincoln. (4586)

Alysidium fasciculatum (Grev.) Pound \& Clements, nom. nov. Acrospermum fasciculatum Grev. Fl. Edin, 469. 1824

Oidium fasciculatum Berk., Smith's Engl. Flor. 5: 349. 1836. 
Oospora fasciculata Sacc. \& Vogl. Sacc. Syl. Fung. 4: 11. 1886.

On lemon peel, Lincoln. (4388)

Alysidium Kunze. Myk. Heft. 1: 11, 1817 must be used instead of Oospora Wallr. Fl. Crypt. Ger. 2 : 182, 1833, the name adopted by Saccardo. Alysidium was founded for $A$. fulvum Kunze.= Oospara fulva Sacc. \& Vogl. 1. c. Moreover, Bonorden, Handb. 35, 1851, took up Alysidium and described several species, one a Torula, but the rest placed in Oospora by Saccardo. Even those who would oppose a year limit of fifty years to the rule of priority, must restore the name Alysidium. The saprophytic species were generally included with the parasitic ones in Oidium until 1886, and neither Oospora nor Alysidium were in general use. But the latter having been adopted in 1851 by Bonorden cannot be called obsolete. Acrospermum Nees 1816 was founded on Oidium monilioides and was applied to this genus by Persoon in 1822 .

Rhopalomyces elegans Corda.

On decaying pericarps and growing seedlings of Beta alba, Lincoln. (4503)

Sterigmatocystis variabilis (Gasp.) Sacc.

On bread, Lincoln. (4506)

Rhinotrichum corticioides Cooke.

On wood, Saltillo. (4309)

Trichothecium sublutescens (Peck) Sacc.

On bark of Populus monilifera, Lincoln.

Jacobaschella alba (Bon.) OK.

On decaying Pleurotus, Lincoln.

DEMATIAOEAE. 
Diplosporium cookei (Sacc.) OK.

On rotten wood, Endicott. (4316)

Heterosporium allii sisyrinchii Speg.

On leaves of Iris versicolor, Peru.

Heterosporium variabile Cooke.

In the sap of living trees of Ulmus fulva, Peru.

Clasterosporium hirudo Sacc.

On decaying wood, Saltillo.

Septonema atrum Sacc.

On dead branches of Ulmus, Weeping Water. (4580) Septonema toruloideum Cke. \& Ell.

On an insect-gall, Franklin. Sporoschisma mirabile B. \& Br.

On rotten wood, Bellevue.

Coniothecium effusum Corda.

On decorticated twigs of Populus monilifera, Memphis. (4577)

Macrosporium caudatum Cke. \& Ell.

On leaves of Ficus elastica, Lincoln.

Macrosporium fasciculatum Cke. \& Ell.

On pods of the cultivated bean, Kennedy.

Macrosporium inquinans Cke. \& Ell.

On sun-flower stalks, Lincoln.

Macrosporium maydis Cke. \& Ell.

On leaves of Zea mays, Lincoln.

Speira toruloides Corda.

On decorticated wood of Rhamnus lanceolata, Weeping

Water. (4582)

Helicosporium vegetum Nees.

On decaying oak twigs, Brownville.

\section{STILBACEAE.}

Siltbum fasciculatum B. \& Br.

On Dianthus sinensis, Lincoln.

$(3321)$ 
Isaria candida Schw.

On decaying wood, Ewing. (45̆17)

Stysanus stemonites (Pers.) Corda.

On horse-dung in culture jar, Lincoln. (4521)

Graphium stilboideum Corda.

On sawdust in culture jar, Lincoln. (4566)

\section{TUBERCULARIACEAE.}

Knyaria fatiscens (Schw.) OK.

On twigs of Prunus americana, Wabash. (4264)

Fusarium nucicola Karst. \& Har.

On nuts of Juglans nigra, and on acorn cups of Quercus macrocarpa, Otowanie Woods. (4456)

Fusarium salmonicolor B. \& C.

On sunflower stalks, Beatrice.

Fusarium sarcochroum (Besm.) Sacc.

On fruits of Acer saccharinum, Lincoln.

Fusarium tenuissimum (Pock.) Sacc.

On sunflower stalks, Beatrice.

HELVELLACEAE.

Helvella crispu (Scop.) Fr.

In shady places, Bellevue. (4421)

Helvella elastica Bull.

In sandy canyons, Long Pine. (4407)

Helvella macropus (Pers.) Karst.

On sandy ground, Hazel Creek Canyons, Brown county. (4531)

Helvella pezizoides Afz.

On sandy ground, Meadville. (4479)

Geoglossum nigritum Pers.

Canyons of the Niobrara River, Brown county.

Geoglossum ophioglossoides (L.) Sacc.

On shady ground, Bellevue. (4420) 


\section{PEZIZACEAE.}

Peziza atro-vinosa Ger. \& Cle.

On rich ground, Meadville, Keya Paha county. (4539) Peziza badia Pers.

On ground, Weeping Water, Bellevue. (4455)

Pezizr sepiatra Cooke. (?)

On wet, decaying wood, Niobrara river. (4555)

Pyronema omphalodes Bull.

On wot, ploughed ground, Lincoln. (4468)

Barlaea constellatio B. \& Br.

On ground among mosses, Rock Creek, Keya Paha county, Bellevue. (4469)

Sarcoscypha floccosa Schw.

On wet, dead twigs, Bellevue, Lincoln.

Sepultaria cretea (Cooke.) Clements.

Lachnea cretea Cooke.

On sandy ground, Hazel Creek Canyons, Brown county. (4548)

Sepultaria livida (Schum.) Clements.

- Lachnea livida Schum.

On sandy ground, Hazel Creek canyons, Brown county. (4458)

Sepultaria setosa (Nees) Clements.

Lachnea setosa Nees.

On wood among mosses, Meadville.

Sepultaria umbrarum (Fr.) Clements.

Lachnea umbrarum Fr.

On wet ground Otowanie woods.

Sepultaria umbrata (Fr.) Clements.

Lachnea umbrata Fr.

On wet wood, canyon of the Niobrara river, Brown county. (4588) 
Helotium citrinum (Hedw.) Fr.

On wet, decaying wood, Mead's ranch, Brown county; Rock creek, Keya Paha county. (4455) (4409)

Helotium conformatum Karst.

On dead wood, Long Pine.

Helotium parile languidum Karst.

On stems of Helianthus, Wabash. (4263)

Helotium rhizogenum Ell. \& Ever.

On corticated branches of Prunus, Bellevue. (4259)

Pseudohelotium hyalinum (Pers.) Fkl.

On decorticated wood, Wabash.

Molissia cinerea luteola Sacc.

On decorticated branches of Prunus, Wabash.

ASCOBOLACEAE.

Ascophanus aurora (Cr.) Boud.

On cow-dung, Wabash. (4478)

Lasiobolus equinus (Mull.) Karst.

On horse-dung, Lincoln. (4401)

DERMATEACEAE.

Cenangium crataegi Schw.

On living branches of Crataegus tomentosa, with the pycnidial stage, Sphaeronema longirostre Clements, Saltillo. (4398)

Cenangium populneum carpini (Rehm) Clements.

Cenangium carpini Rehm.

On corticated wood, Lincoln. (4402)

STICTACEAE.

Propolis faginea (Schrad.) Karst.

On dead stumps of Quercus macrocarpa, Bellevue.

Stictis mollis Pers.

On fallen twigs, Otowanie woods; Mead's Ranch. 


\section{PATELLARIAOHAE.}

Hysteropatella elliptica (Fr.) Rehm.

On the bark of Olmus americanc, Lincoln. (4410)

Hysteropatella prostii (Desm.) Rehm.

On decorticated wood, Beatrice.

\section{AGARICAOEAE.}

Amanita muscaria L.

On wooded bluffs of the Missouri river, Bellevue.

Pseudofarinaceus speciosior Batt.

On the ground, Bellevue.

Mastocephalus cristatus (Alb. \& Schw.) OK

In shaded places, Otowanie woods. (4058)

Tricholoma patulum Fr.

On the ground among dead leaves, Otowanie woods. (4559) Tricholoma virgatum Fr.

In woods, Bellevue. $(4237)$ Collybia dryophila Bull.

On decaying leaves and manure, in densely shaded places, Otowanie woods. (4560)

Collybia radicata Relh.

In shady woods, Wabash. (4561)

Mycena iris Berk.

On the ground among twigs, Otowanie woods. (4562) Russula emetica Fr.

In shady woods, Bellevue, Lincoln, Milford, Beatrice.

Marasmius coniatus B. \& Br.

Among dead leaves, Wabash. (4465)

Marasmius rotula microcephalus Sacc.

On dead trunks and leaves, Wabash.

Volvaria bombycina (Pers.) Fr.

On a living tree, Lincoln.

Eccilia tristis Bres.

Among grass in woods, Wabash. (4558) 
Pholiota gibberosa Fr. ?

In woods, Wabash. (4255) *

Naucoria semiorbicularis Bull.

In grass, University campus, Lincoln. (4999) Galera bryorum Pers.

On mossy banks, Bellevue.

Gomphos collisteus (Fr.) OK.

On the ground, Bellevue. (4563)

Stropharia merdaria Fr.

On horse dung, Meadville, Keya Paha county. (5000)

Stropharia stercoraria Fr.

In manured places in woods, Bellevue. (4235)

Gymnochilus flavo-griseus (Berk.) Clements.

Psathyra flavo-grisea Berk.

Among dead leaves, Bellevue. (4236)

Coprinus filiformis B. \& $\mathrm{Br}$.

On wet straw in culture-jar, Lincoln. (4564)

Coprinus floccosus (DC.) Fr.

On twigs and ground in woods, Bellevue. (4232)

Coprinus ovatus (Schaeff.) Fr.

On wet earth, Saltillo. (4238)

Panaeolus papilionaceus Fr.

In manured places, Lincoln. (4228)

POLYPORACEAE.

Boletus chrysenteron Fr.

On the ground in shady woods, Bellevue.

Boletus edulis Bull.

On the ground in woods, Bellevue.

(5026)

Boletus subtomentosus L.

In leaf-mould, Otowanie woods. (4565) Pileus olivaceous when young, brick-red, and densely silky in age; flesh becoming blue when cut. 
THELEPHORACEAE.

Peniophora flavido-ralba Cooke.

On decaying bark, Lincoln. (4408)

ALISMACEAE.

Šagittaria longiloba Engelm.

Minden. (4505)

LEMNACEAE.

Wolffia brasiliensis Weddell.

Bellevue. (4507)

GRAMINEAE.

Poa reflexa Vasey \& Scribner.

Norfolk. (4520)

Chromaeraphis verticillata (L.) Porter.

Washington county. (4508) Fremont. (4523)

CYPERACEAE.

Scirpus panciflorus Lightf.

Fremont. (4528)

Fuirena squarrosa Michx.

Fremont island, Dodge county. (4509)

Carex granularis Muhl.

Platte river, near Fremont. (451t)

Carex lupulina Muhl.

Fremont. (4510)

ORUCIFERAE.

Cardamine bulbosa (Schreb.) B. S. P.

Fremont. (4อ̃15)

Thlaspr arvense $\mathrm{L}$.

Fremont. (4512)

CARYOPHYLLACEAE.

Alsine media L.

Fremont. (4511) 
Lechea stricta Legett.

CISTACEAE.

Adams county. (4527)

Vitis cinerea Engelm.

VITACEAF.

Peru. (4530)

Ampelopsis cordata Michx.

Peru. (4532)

CHENOPODIACEAE.

Chenopodium urbicum $\mathbf{L}$.

Cherry county. (4513)

AMARANTHACEAE.

Amaranthus torreyi (Gray) Wats.

Thomas county (4524), Hooker county (4535)

Acnida tamariscina dehiscens Uline and Bras.

Lincoln (4518), Wahoo (4534)

Rosa engelmanii Wats.

ROSACEAE.

Sowbelly Canyon, Sioux county. (4526)

PAPILIONACEAE.

Astragalus lotiflorus nebraskensis Bates Am. Nat, $29: 670$, 1895.

Long Pine. (4749) Clay county. (4750)

Astragalus giganteus (Pall.) Sheld.

North Platte. (4751)

Psoralea collina, Rydberg Flor. Nebr. 16 : 54, 189 5.

Fort Robinson (Dr. Bessey, 1887), Scott's Bluff county. (50)

Kuhnisterc candida diffusa, Rydberg Flor. Nebr. 16 : 59, 1895.

Deuel county. (58)

Lathyrus ornatus flavescens, Rydberg Flor. Nebr. 16 : 64, 189 .

Dodge, Kearney, and Cherry counties.

Lathyrus ornatus incanus, Smith \& Rydberg, Flor. Nebr. 16 : $64,1895$.

Sheridan county (49); Fort Robinson (Dr. Bessey, 1887). 
GROSSULARIACEAE.

Ribes aureum chrysococcus, Rydberg Flor. Nebr. 16 : 71, 1895. Scott's Bluff, Banner, and Cherry counties. $\quad(106,1601)$

\section{ONAGRACEAE.}

Oenothera fremontii, Wats.

Bloomington. (4737)

Oenotherc serrulata spinulata, Torr. \& Gr.

Lancaster county. (4519) Fairbury. (4536)

Panax quinquefolia $\mathbf{L}$.

ARALIACEAE.

Bellevue. (4522)

Daucus carota L.

UMBELLIFERAE.

Nebraska City. (4525).

Eryngium yuccaefolium Michx.

Richardson county. (4529)

LOBELIACEAE.

Lobelia cardinalis $\mathrm{L}$.

Franklin. (4739)

CAMPANULACEAE.

Specularia leptocarpa Gr.

Fairbury. (4740)

Anagallis arvensis $\mathrm{L}$.

PRIMULACEAE.

Fairbury. (4738)

ASCLEPIADACEAE.

Ampelanus albidus (Nutt.) Britt.

Auburn. (4533)

Phlox kelseyi Britt.

POLEMONIACEAE.

Sioux county.

$(4554)$ 
BORAGINACEAE.

Cynoglossum officinale $\mathrm{L}$.

Weeping Water. (4537)

Mertensia paniculata (Ait.) Don.

Sheridan county. (4553)

VERBENACEAE.

Verbena stricta $\mathrm{x}$ bracteosa.

Fremont. (4538) Kearnèy. (4552)

Verbena bracteosa $\mathrm{x}$ urticifolia.

Tecumseh. (4540)

COMPOSITAE.

Vernonia marginata (Torr.) Britt.

Fremont. (4551)

Vernonia baldwinii Torr.

Bertrand, Phelps county. (4541)

Helianthus hirsutus trachyphyllus Torr. \& Gr.

Bellevue. (4550)

Hymenopappus flavescens Gray.

Fairbury. (4อ̆42)

Hieracium umbellatum $\mathrm{L}$.

Squaw canyon, Sioux county. (4549)

SUMMARY.

Previously reported . . . . . . . . . . . . . . . 2820

In this report:

New species..................... 5็

Other additions........................ 323

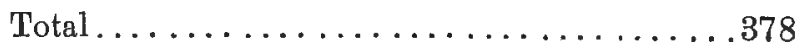

Less new hosts only ................. 2

$376 \quad 376$

Total......................... 3196 
UNIVBRSITY OF NEBRASKA

BOTANICAL SURVEY OF NEBRASKA

Conducted by the Botanteal Seminas

\section{V.}

\section{Report on Recent Collections}

\section{Studies in the Uegetation of the State, I.}

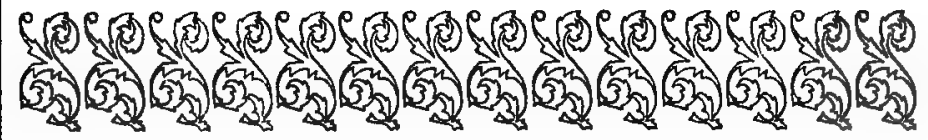

IINCOIN, NEBRASKA

PUbliged by the Seminar 1901

(Distributed March 30, 1901) 



\section{REPORT \\ ON \\ RECENT COLLECTIONS}

STUDIES

IN THE

VEGETATION OF THE STATE, I. 
Jacob North \& Co., General Printers, Lincoln vis 


\section{TABLE OF CONTENTS}

Note $. . . \ldots \ldots \ldots \ldots \ldots \ldots \ldots \ldots \ldots \ldots, 4$

Report on Recent Collections................ 5

New Species of Fungi.................... 5

Additions to the Reported Flora of the State........ 12

The Prairiegrass Formation in Region I. . . . . . . 29

Introduction ........................ 29

Discussion of Ecological Factors............... 32

Ecological Factors........................ 38

Temperature and Atmosphere.............. 38

Water-content ....................... 43

Light Intensity.....................46

Enumeration of Species in the Formation.........51

Structure of the Formation................ 54

Elements of the Prevernal Floral Aspect.......... 57

Elements of the Early Vernal Floral Aspect........660

Elements of the Late Vernal Floral Aspect.........66

Elements of the Early Estival Floral Aspect......... 71

Elements of the Late Estival Floral Aspect........ 81

Elements of the Serotinal and Floral Aspect........ 86

Quadrat Lists......................... 95

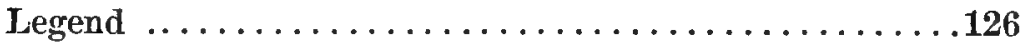

Map of the Formation.................... 126

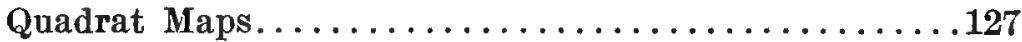

Phenological Record......................137 


\section{NOTE}

The necessary precursor of all phytogeographical investigation is accurate and thorough acquaintance with the flora of the territory in question. Hence the reports of the Survey hitherto have been devoted chiefly to bare lists of additions and the results of collections in the various parts of the State. Much that has been published in this way stands in need of revision, and the catalogue of species, now ten years old, requires complete overhauling, in the light of the subsequent development of the science and of more complete data as to the distributional boundaries of species. If the Seminar can command the means therefor, it is hoped that a descriptive catalogue of the flora of the State may be issued soon as one of the publications of the Survey. But all this work is preliminary to the study of the vegetation, the foundation of which has been laid during the past sixteen years; and study of the regetation as such has been looked forward to as the ultimate purpose of the Survey.

Under the direction of the Seminar, Mr. J. J. Thornber has been engaged for some years past in the investigation of the prairiegrass formation in the best known and most accessible region of the State. The results of his work are given chief place in this report.

In the list of additions to the reported flora, Messrs. Pound and Clements have identified the fungi, Mr. C. J. Elmore the diatoms, and Messrs. J. L. Sheldon, G. G. Hedgcock, and A. A. Hunter have assisted in various groups. The thanks of the Seminar are due to Rev. J. M. Bates and to Messrs. M. E. Moore, of Springview, and J. P. Anderson, of Lamoni, Iowa, for collections. Above all, however, the Seminar is indebted to Mr. G. W. Holdrege, without whose generous assistance adequate investigation in an area as extensive as Nebraska would have been impossible.

\footnotetext{
Charles E. Bessey,

Roscom Pound,

Frederic E. Clements,

Editorial Committee.
}

March 12, 1901. 


\section{NEW SPECIES OF FUNGI}

All color-terms used below conform to the charts and synonymic lists in Saccardo's Chromotaxia.

\section{SPORODESMIACEAE}

Septonema isthmium Pound and Clements.

Caespituli thin, broadly effused, very dark; sporophore obsolete; chains of conidia simple, 90-150 $\mu$ long, very often with a hyaline, simple or 1-2-septate terminal conidium, conidia sooty brown, clavatecylindrical, straight, or rarely slightly curved, 3-7-septate, constricted at the partitions, connected by a short, narrow isthmus $3-4 \mu$ wide, cells with a single large gutta, 35-70x10 $\mu$.

On dead twigs of Smilax hispida, Wabash. (4597)

\section{EXCIPULACEAE}

Scaphidium Clements nov. gen.

Apopycnidium oblong or linear-disciform, at length hysterioid, waxy-membranaceous, dark brown; basidia simple; sporidia uniseptate, hyaline, fusoid. Corresponds to Sporonema in the Hyalodidymae.

Scaphidium boutelouae Clements.

Apopycnidia minute, grouped in lines, upon indistinct brownish spots, innate-erumpent, dark brown, membranaceous, or waxy-membranaceous, linear, hysterioid, glabrous, $100 \mu$ in diameter; basidia simple, very short, hyaline, $10 \times 2 \mu$; sporidia hyaline, solitary, uniseptate, sigmatoid, $25 \times 3-4 \mu$.

On leaves of Bouteloua hirsuta, Simeon. 


\section{HYPODERMIACEAE}

Graphyllium Clements nov. gen.

Hysterothecium innate, then erumpent, linear, simple, membranaceous-plectenchymatous, black; asci ovoid or cylindrical-clavate, 8-spored; spores brown, elliptical to oblong, with transverse and longitudinal septa, but not muriform; paraphyses simple or branched, septate, forming an epithecium.

Graphyllium chloes Clements.

Hysterothecia minute, sparse or gregarious, sometimes lineate, innate, at length erumpent, glabrous, elongate-elliptical or linear, membranaceous, composed of irregularly polygonal cells, $5 \times 6 \mu$, lips thin, gaping at maturity, black, 30-50 $\mu$ wide by 100-300 $\mu$ long; asci 8-spored, of two forms, ovoid-clavate, $60 \times 15 \mu$, or cylindrical-clavate, $90-110 \times 12 \mu$, stipitate, not blue with iodin; paraphyses few, septate, simple or ramose, hyaline, globose above, $2-3 \mu$ wide; spores distichous or irregularly monostichous, brown or yellow-brown, oblong or ovate-oblong, obtusely rounded at the ends, 3-, very rarely 4-, septate, at maturity the two middle loculi divided longitudinally, the others also sometimes, smooth, somewhat compressed, distinctly constricted at the septa, tre second loculus usually inflated, $20-22 \times 8-10 \mu$.

On stems of Aristida purpurea and Bouteloua oligostachya, Long Pine. (12101)

\section{PEZIZACEAE}

Peziza (Plicaria) elaeodes Clements.

Ascoma small, sessile, convexo-explanate, the margin elevated, thick, smooth, olivaceous within and without, $1-1 \frac{1}{2} \mathrm{~cm}$. wide; asci 8 -spored, cylindrical, scarcely stipitate, filled below with a dense, olivaceous protoplasm, blue with iodin, $250 \times 15 \mu$; paraphyses 
broadly linear, slightly clavulate, nearly colorless; spores ellipsoid, asperate, becoming olivaceous, apiculate both ways, $25 \times 10 \mu$.

On twigs of Juniperus scopulorum, Mead's spring, Brown county. (12102)

'Peziza (Plicaria) roseo-lilacina Clements.

Ascoma sessile, waxy-carnose, persistently concavecupuliform, smooth, ochroleucous, margin often irregularly lobed, hymenium rose-lilac, $1-1 \frac{1}{2} \mathrm{~cm}$. wide; asci 8-spored, cylindrical, apex light blue with iodin, $300 \times 15 \mu$; paraphyses linear, scarcely incrassate above, densely filled with lilac granules, apex 6-7 $\mu$; spores monostichous, ovoid-ellipsoid, smooth, 1-2-guttate, $17-18 \times 10 \mu$.

On wet sandy ground among mosses, Barney creek, Keya Paha county. (12103)

'Humaria silvosa Clements.

Ascoma minute, sessile, sparse or gregarious, at first hemispherical, closed, then open, margined, entire, concave, saffron-luteous, $\frac{1}{4-1} \mathrm{~mm}$. wide; asci 4-spored, irregular, stipitate, truncate above, inoperculate, blue with iodin, $150 \times 18 \mu$; paraphyses linear, long-clavate, clava $30 \times 7-8 \mu$, simple, filled with large pale luteous granules, $5 \mu$ wide; spores ellipsoid, obtuse both ways, straight or obliquely monostichous, with 2-3 large guttae, $25 \times 12 \mu$.

On wet mossy banks, Dry Branch, Humboldt. (12104)

Pelodiscus Clements nov. gen.

Ascoma minute, superficial, sessile, carnose, cupulate, at length disciform or explanate, pilose without, but not ciliate at the margin; asci cylindrical, 4-8-spored, paraphysate; spores ellipsoid or oblong, hyaline.

Pelodiscus agrees with Humaria and Scutellinia in having the ascoma superficial, while in Sepultaria and in Sarcosphaera it is at first hypogaeous. It agrees with Sepultaria in being simply tomentose or lanose with- 
out, in which it differs from the smooth Humaria and the ciliate-tomentose Scutellinia.

Pelodiscus miniatus Clements.

Ascoma carnose, single, or in groups of two or three, miniate, margin naked, sparsely clothed without, the base excepted, with very short, continuous, flexuous, pale dark yellow pila, $70-80 \times 10 \mu$, hymenium concave or plane, exactly circular, miniate, 1-2 mm. wide, $\frac{1}{2}-1 \mathrm{~mm}$. high; asci 8-spored, clavate, not blue with iodin, $250 \times 15 \mu$; paraphyses septate, with a large redorange clava, 9-12 $\mu$, filled with a red protoplasm which turns a deep blue with iodin; spores straight or obliquely monostichous, elliptical, four often somewhat abortive and diverse, beautifully papillate, the papillae 2-3 $\mu$ high, hyaline, 26-28x12-14 $\mu$.

On stercorate mud, Beal's slough, Otowanie woods. (12105)

Pelodiscus piliseta Clements.

Ascoma carnose, applanate, rarely concave, margin entire, naked, luteo-fulvous, pila pointed, strict, sparse, brown, 2-3-septate, attenuate at the base, 300-400×25 $\mu$, hymenium bright luteo-fulvous, $1 \mathrm{~cm}$. wide; asci 8-spored, cylindrical, not blue with iodin, $250 \times 25 \mu$; paraphyses septate, clavate, $4-5 \mu$, clava $8 \mu$ wide, nearly colorless; spores large, broadly elliptical, densely filled with polygonal granules, indistinctly crenulate or verrucose, obliquely monostichous, 30x 16-18 $\mu$.

On wet wood, Barney creek, Keya Paha county. (12106)

Sepultaria gigantea Clements

Ascoma cupulate-hemispherical, $3-8 \mathrm{~cm}$. wide and high, hypogaeous, gregarious, opening widely, subcoriaceous, margin contorted, entire, strongly lacunose at base, brown-ochraceous, densely clothed with septate, flexuous, fuligineous pila, 250-700x17 $\mu$, hymenium 
ochroleucous; asci 8-spored, elongate, stipitate, not blue with iodin, $350 \times 15 \mu$; paraphyses linear, apex fuscescent, scarcely incrassate, $7 \mu$ wide; spores straight or obliquely monostichous, fusoid-elliptical, bi- or multi-guttate, strongly tuberculate, 30-35x 13-15 $\mu$.

In rich soil beneath leaves, Otowanie woods. (12107)

\section{ASCOBOLACEAE}

'Ascobolus stercorarius retispora Clements.

Ascoma at first luteo-olivaceous, then brown, plane, limicolous; margin none; spore densely and minutely reticulate, elongate-elliptical $12-13 \times 20-22 \mu$.

Related to Ascobolus stercorarius nudus (Kickx) Boud.

On stercorate mud, Otowanie woods. (12108)

Ascophanus isabellinus Clements.

Ascoma humicolous, carnose, at first nearly doliform, then convex or explanate, naked, white, then isabelcolored, 1-3 mm.; asci broad, 8-spored, stipitate, blue with iodin, $250 \times 30-40 \mu$; paraphyses of two sorts, linear, much exceeding the asci, and clavate, only slightly longer than the asci, $3 \mu$ wide; spores distichous, elliptical, hyaline, smooth, with a thick, lamellose wall $2-3 \frac{1}{2} \mu$ wide, $27-30 \times 12-13 \mu$.

On stercorate mud, Otowanie woods. (12109)

\section{AGARICACEAE}

Volvaria concinna Clements.

Pileus submembranaceous, expanded, not at all or only slightly umbonate, smooth, pale avellaneous, $\frac{1}{2}-1 \frac{1}{2} \mathrm{~cm}$. wide; stipe graceful, concolorous, 1-2 mm. $x_{\frac{1}{2}}-1 \frac{1}{2}$ cm.; volva minute, strictly adpressed, limb obsolescent; lamellae free, rose-colored; spores ovate-ellipsoid, granular or guttate, smooth, rasy, 8-11x5-7 $\mu$; cystidia lacking. 
On moist shaded ground and on flooded banks, Nemaha river, Humboldt, 1897; Marysville, Kansas, 1896. (12110)

Volvaria submyochrous Clements.

Pileus subcarnose, convex, almost plane, scarcely umbonate, silky, shining, umbo densely covered with larger silky fibrils, becoming innate toward the strongly striate margin, pale avellaneo-murinous, 3-4 cm. wide; stipe carnose, equal, solid, white, shining, farinose at the apex, $5 \mathrm{~mm} . \times 3-4 \mathrm{~cm}$.; volva small, hirsute, 2-3-fid, concslorous; lamellae free, remote, subconfertous, ventricose, at first flesh-colored, then isabel-colored; spores ellipsoid, uniguttate, smooth, 6-7x4 $\mu$.

On wet earth in a basement, University Campus, Lincoln. (12111)

Pluteolus glutinosus Clements.

Pileus membranaceous, conico-campanulate, then explanate and repand, glabrous, umbonate, thickly covered with a tenacious mucilage, margin striate to the middle, at length split, gray-stramineous, fuscescent toward the margin, $1 \frac{1}{2}-2 \mathrm{~cm}$. wide; stipe graceful, hollow, shining, equal, densely fibrillose-pulverulent, pale ochroleucous, white-striate toward the apex, $3 \mathrm{~mm}$ x 5-10 cm.; lamellae free, distint, ventricose, brown; spores ovate or ovate-ellipsoid, smooth, amber, 13-16×10-12 $\mu$.

Among stercorate leaves, Otowanie woods.

Naucoria striata Clements and Shear.

(12112)

Pileus carnose, convex, at length explanate or repand, glabrous, glutinous, ochroleucous, paler toward the margin, gregarious, 3-6 cm. ; stipe broad, equal or subincrassate at the base, sometimes compressed, solid or medullate in age, fibrous, beautifully lineate from the apex to the middle, silky-fibrillose or squamose, above shining, pale cremeous, below fuscescent, 13-15 
mm. x 4-7 cm.; lamellae free, somewhat crowded, ventricose, at first pale umber, then ferruginous; spores ovoid, smooth, ferruginous, $7-8 \times 5 \mu$.

On rich ground, Lincoln. (12113)

Cortinarius squarrosus Clements.

Pileus carnose, campanulate, at length convex, dry, scarcely umbonate, markedly squarrose at the center with umber, fibrillose-fasciculate scales, densely covered toward the margin with subsquarrose fibrils, umber or umber-ochroleucous, $2 \frac{1}{2}-3 \mathrm{~cm}$.; stipe hollow, fibrose-carnose, flexuous, subequal, subsquarrose with fulvous-umber fibrillose scales, $5 \mathrm{~mm}$. $\times 3-4 \mathrm{~cm}$. ; lamellae slightly adnexed, ventricose, somewhat crowded, fulvous or umber; fibrils of the veil umber, fugacious; spores irregularly ellipsoid, smooth, eguttate, $12 \times 6 \mu$. Among grasses in the woods, Little Blue river, Endicott. A small form of this species has the pileus and stipe paler and the stipe scarcely squarrose; pileus $12 \mathrm{~mm}$. wide, stipe $2 \mathrm{~mm} . \times 16 \mathrm{~mm}$. (12114). 
ADDITIONS TO THE REPORTED FLORA OF THE STATE

$$
\begin{aligned}
& \text { [* Indicates new host only.] } \\
& \text { CHROOCOCCACEAR }
\end{aligned}
$$

Chroococcus minor (Kuetz.) Naeg.

In aquarium, Lincoln. (12115)

Gomphosphaeria aponina Kuetz.

In aquaria, Lincoln. (12116)

\section{NOSTOCACEAE}

Nostoc linkia (Roth) Born.

In ponds, South Bend. (12117)

Nostoc macrosporum Menegh.

On pots in greenhouse, Lincoln. (12118)

Nostoc sphaericum Vaucher.

On soil in greenhouse, Lincoln. (12119)

Nostoc verrucosum Vaucher.

In culture in greenhouse, Lincoln. (12120)

'Anabaena hallensis (Jancz.) Born. and Flah.

In aquaria, Liccoln. (12121)

Nodularia harveyana Thuret.

In ponds and running water, South Eend.

oscillatoriacean

Lyngbya confervoides C. Ag.

In culture from salt basin, Lincoln. (12123)

Lyngbya major Menegh.

In aquaria, Lincoln. (12124)

Lyngbya semiplena J. Ag.

In pond, South Bend. (12125) 
Lyngbya spirulinoides Gomont.

On moist earth, Lincoln.

Vaginaria chthonoplastes (Hofman-Bang).

On moist saline earth, Lincoln.

Vaginaria oligothrix (Kuetz.) OK.

(12127)

On wet soil, Lincoln.

Vaginaria paludosa (Kuetz.) OK.

On wet soil in greenhouse, Lincoln.

(12129)

Oscillatoria amphibia Ag.

In Salt creek; in cultures, Lincoln.

Oscillatoria chalybea Mertens.

In stagnant water, Waverly.

Oscillatoria chlorina Kuetzing.

In culture in greenhouse, Lincoln.

Oscillatoria curviceps Ag.

On moist soil, greenhouse, Lincoln.

Oscillatoria formosa Bory.

In culture in greenhouse, Lincoln.

Oscillatoria geminata Menegh.

In aquaria, Lincoln.

(12135)

Phormidium ambiguum Gomont.

In aquaria, Lincoln. (12136)

Phormidium corium (Ag.) Gomont.

In ponds, South Bend; Long Pine.

Phormidium fragile (Menegh.) Gomont.

(12137)

In aquaria, Lincoln. (12138).

Phormidium laminosum (Ag.) Gomont.

In running water in greenhouse, Lincoln.

Phormidium subuliforme Gomont.

In aquaria, Lincoln. (12140)

Phormidium tenue (Menegh.) Gomont.

On boards of mill-dam, Milford.

Phormidium uncinatum (Ag.) Gomont.

In aquaria, Lincoln. (12142). 
Schizothrix tinctoria (Ag.) Gomont.

On Cladophora, Fisher's Lake, Glen Rock.

Spirulina major Kuetzing.

In Salt creek, Lincoln. '(12144)

Spirulina subtilissima Kuetzing.

In Salt creek, Lincoln. (12145)

SCYTONEMATACEAE

Scytonema cincinnatum (Kuetz.) Thur.

In ponds, Nebraska City. (12146)

Soytonema ocellatum Lyng.

On pots in greenhouse, Lincoln.

BACTERIACEAE

Spirillum sanguineum (Ehrenb.) Cohn.

In a culture of algae, Lincoln.

Spirochaete plicatilis Ehrenb.

In cultures of algae, Lincoln. (12149)

Bacterium lactis Lister.

In sour milk, Talmage. (12150)

DESMIDIACEAE

Cosmarium margaritiferum Menegh.

In tank, Salem. (12151)

BACILLARIACEAE

Achnanthes affinis Grun.

Fairbury. (12152)

Achnanthes hangarica Grun.

Fossil, Mullen. (12153)

Amphipleura pellucida (Ehr.) Kuetz.

Arago, Weeping Water. (12154)

Cymbella bengalensis De Toni.

Wahoo. (12155) 
Cymbella lanceolata (Ehr.) Kirchn.

Fossil, Mullen. (12156)

Cymbella levis Naeg.

Fossil, Mullen. (12157)

Cymatopleura solea (Breb.) W. Sm.

Rulo, Talmage, Brock, Plattsmouth, Nemaha City,

Weeping Water, Fairbury, Lincoln. (12158)

Cystopleura ocellata (Ehr.) Kuntze.

Fossil, Mullen. (12159)

Eunotia arcus Ehr.

Fossil, Greeley county, Mullen. (12160`

Eunotia diodon Ehr.

Fossil, Mullen. (12161)

Fragilaria construens (Ehr.) Grun.

Fossil, Mullen, Thedford, Wheeler county.

Frustulia vulgaris (Thwaites) De Toni.

Brock, Peru, Julian, Talmage (12163)

Gomphonema eriense Grun.

Long Pine. (12164)

Gomphonema herculaneum Ehr.

Fossil, Mullen. (12165)

Gomphonema insigne Greg.

Crete. (12166)

Comphonema vibrio Ehr.

Fossil, Mullen, Greeley county. (12167)

Navicula appendiculata (Ag.) Kuetz.

Brock. (12168)

Navicula bácillum Ehr.

Auburn. (12169)

Navicula braunii Grun.

Talmage. (12170)

Navicula cincta (Ehr.) Kuetz.

Talmage, Nemaha City, Arbor. (12171)

Navicula crucicula (W. Sm.) Donkin.

Arbor. (12172) 
Navicula elliptica Kuetz.

Holt county, Johnson, Long Pine; fossil, Mullen. (12173)

Navicula gigas (Ełr.) Kuetz.

Fossil, Mullen. (12174)

Navicula hungarica Grun.

Fossil, Mullen. (12175)

Navicula legumen Ehr.

Brock, Auburn; fossil, Mullen.

Navicula menisculus Schum.

Long Pine. (12177)

Navicula minuscula Grun.

Nebraska City, Peru. (12178)

Navicula mormonorum De Toni.

Auburn. (12179)

Navicula nobilis (Ehr.) Kuetz.

Fossil, Mullen. (12180)

Navicula reinhardtii Grun.

Long Pine, Fairbury. (12181)

Navicula rhynchocephala Kuetz.

Fairbury. (12182)

Nitzschia balatonis Grun.

Lincoln. (12183)

Nitzschia dissipata (Kuetz.) Grun.

Long Pine. (12184)

Nitzschia obtusa W. Sm.

Fossil, Mullen. (12185)

Nitzschia sinuata (W. Sm.) Grun.

Fossil, Mullen. (12186)

Nitzschia spectabilis (Ehr.) Ralfs.

Holt county; fossil, Mullen. (12187)

Pleurosigma eciotoense Sulliv.

Crete. (12188)

Rhoicosphenia curvata (Kuetz.) Grûn.

Brock. (12189) 
Rhoiconeis rhomboides Elmore.

Arago, Fairbury, Brock, Long Pine. (12190)

Stauroneis acuta W. Sm.

Brock. (12191)

Stauroneis bicapitata Elmore.

Holt county. (12192)

Synedra bicurvata Biene.

Talmage, Johnson. (12193)

Stauroneis minutissima Lagerst.

Fossil, Mullen. (12194)

Tetracyclus lacustris Ralfs.

Fossil, Greeley county. (12195)

\section{ZYGNEMATACEAE}

Spirogyra nitida Dillw.

In Fisher's Lake, Glen Rock. (12196)

MUCORACEAE

Hydrogera oedipus (Mont.) O. K.

On horse dung, Little Salt creek. (14595)

HYDROGASTRACEAE

Protosiphon botryoides (Kuetz.) Klebs.

On wet earth in greenhouse, and in fields, Lincoln. (12302)

\section{PERONOSPORACEAE}

Peronospora trifoliorum De Bary.

On Astragalus canadensis, Long Pine.

ULOTRICHIACEAE

Conferva tenerrima rhypophila (Kuetz.) Hansg.

In tanks, Peru, Salem, Talmage, Dawson, Brock. (12197)

Hormiscia rivularis (Kuetz.) De Toni.

In creek, Arbor; Lincoln. (12198)

Hormiscia subtilis variabilis Kuetz.

In tanka, Talmage, Auburn, Salem. (12199) 


\section{Microthamnion kuetzingianum Naeg.}

In trough, Peru. (12200)

Stigeoclonium longipilum minus Hansg.

In trough, Brock. (12201)

\section{CHROOLEPIDACEAB}

Trentepohlia umbrina Kuetz.

On bark of Ulmus americana, Georgetown.

\section{SPHAERIOIDACEAT}

Phoma nebulosa (Pers.) Mont.

On Urtica gracilis, Valentine. (12038)

Phyllosticta apii Halsted.

On Apium petroselinum, Kearney. (12204) Phyllosticta iridis E. \& E.

On Rhus toxicodendron, Long Pine. (4658)

Cytospora negundinis E. \& E.

On Acer negundo, Atkinson.

(12087)

Septoria aurea E. \& E.

On Ribes aureum.

$$
\text { (12C5) }
$$

Septoria avenae Frank.

On Avena striata, Long Pine.

Septoria caricis Pass.

On Carex pennsylvanica, Merriman. (12050)

Septoria lycopersici Speg.

On Solanum lycopersicum, Pawnee.

(12839)

Septoria menispermi E. \& H.

On Menispermum canadense, Ewing. (4661)

Septoria pachyspora E. \& H.

On Xanthoxylum americanum, Kennedy.

Septoria petroselini apii B. \& C.

On Apium petroselinum, Kearney. (4724)

Septoria steironcmatis E. \& E.

On Steironema ciliatum, Kennedy. (4672)

Camarosporium compositarum (C. \& Harkn.)

On Artemisia frigida, Atkinson.

(12049) 
LEPTOSTROMATACTAE

- Discosia artocreas (Tode) Fr.

On leaflets of Gleditsia triacanthos, Brownville. (4590)

\section{EXCIPULACEAE}

Dinemasporium decipiens De Not. \& Sacc.

On decorticated twigs of Acer negundo, Lincoln. (4793)

* Dinemasporium graminum Lev.

On Sporobolus vaginiflorus, Lincoln.

(12206)

\section{MELANCONIACEAE}

Cylindrosporium tradescantiae Ell. \& Kell.

On Tradescantia virginica, Inman. (12207)

Cylindrosporium padi Karst.

On Prunus demissa, Valentine. (4654)

Didymosporium corticola Schw.

On Rhus toxicodendron, Long Pine. (4658)

\section{MUCEDINACEAE}

Sterigmatocystis candida Sacc.

In human ear, Lincoln. (12208)

Streptothrix abictina Peck.

On decorticated wood, Lincoln. (12210)

\section{SPORODESMIACEAT}

Alternaria tenuis Nees.

On Calamagrostis confinis, Poa pratensis, Long Pine (947, 12218)

Cercospora cleomes Ell. \& Halst.

On Cleome serrulata, Ewing. (4660)

Cercospora desmodii Ell. \& Kell.

On Meibomia paniculata, Long Pine.

Cercospora eff usa Ell. \& Ev.

(12083)

On Lobelia syphilitica, Long Pine.

(12025) 
Cercospora tuberosa Ell. \& Kell.

On Apios apios, Long Pine.

Frusicladium depressum B. \& Br.

On Sium cicutifolium, Kennedy.

(12211)

Heterosporium gracile (Wallr.) Sacc.

On IVis pumila, Long Pine.

(936)

Coniosporium miserrimum Karst.

Long Pine. (12209)

Stigmina clavulata (C. \& Farkn.) Pound \& Clements.

Valentine. (12212)

STILBACEAE

Coremium bicolor (Web.) Pound \& Clements.

On dung in culture, Lincoln. (4593)

\section{ERYSIBACEAE}

* Sphaerotheca humuli (DC.) Burrill.

On Rosa arkansana, Rosa sp. (cult.), Geum canadense.

Lincoln. (12213, 12213)

On Iris versicolor, Otoe county. (12077)

Sphaerotheca humuli fuliginea (Schlecht.) Salmon.

On Leptilon canadense, Lincoln (12221); Verbcno urticifolia, Otoe county. (12076)

* Podosphaera oxyconthae (DC.) De Bary.

On Prunus besseyi, Long Pine. (4630)

* Microsphaera alni (DC.) Wint.

Juglans nigra, Lincoln.

* Erysibe cichoracearum DC.

(12215)

On Scutellaria laterifora, Atkinson (12031); Aster paniculatus (1047), Helianthus rigidus (12227), Helianthus grosse-serratus (12230), Lappula virginica (12228), Parictaria pennsylvanica (12229), Plantago rugellii (1225), Lincoln.

- Erysilue graminis DC.

On Poa pratensis, Lincoln. 
- Erysibe polygoni DC.

On Polygonum aviculare (12238) and $P$. erectum (12241), Falcata pitcheri (12242), Pisum sativum (12240), Rumex salicifolius (12237), Lotus americanils (12239), Lincoln; Solanum carolinense, Cass county (12078).

\section{SPHAERIACEAE}

Calosphaeria barbirostris (Dufour) Ell. \& Ev.

On dead wood of Quercus, Weeping Water. (4701) Eutypella stellulata (Fr.) Sacc.

On dead twigs of Ulmus, Lincoln. (4709)

Leptosphaeria culmicola (Fr.) Karst.

On Elymus canadensis, Kennedy. (12089)

Leptosphaeria doliolum (Pers.) Ell. \& Ev.

On Helianthus maximiliani. (12088)

Pleospora andropogonis Niessl.

On Muhlenbergia racemosa, Kennedy. (12217)

Pleospora permunda Cooke.

On Asclepias syriaca, Johnstown.

(12090)

\section{HYPOCREACEAE}

Nectria epichloe Speg.

On Chrysopogon avenaceus, Long Pine. Claviceps nigricans Tul.

On Eleocharis glaucescens, Lincoln. (1264)

DOTHIDIACEAE

- Phyllachora graminis (Pers.) Fckl.

On Sporobolus cuspidatus, Long Pine.

(12220)

Dothidea muhlenbergiae Ell.

On Muhlenbergia racemosa, Ewing.

(12047)

\section{Mollisiaceae}

Mollisia sublividula (Nyl.) Karst.

On old wood, Barney creek, Keya Paha county. (12222) 
Lachnum agaricinum Retz.

On wet wood, Dry Branch. (12285)

\section{ASCOBOLACEAE}

Ascobolus stercorarius (Bull.) Rehm.

On horse dung in greenhouse, Lincoln.

(12223)

Ascobolus atrofuscus Phill. \& Plow.

On wet sand- ground in the North Platte river, Gering. (12224)

Ascophanus carneus difformis Karst.

On manure in greenhouse, Lincoln.

\section{(12226)}

\section{PEZIzaCeat}

Plicaria sepiatra (Cooke) Rehm.

On wet, decaying wood and on ground among mosses,

Niobrara river, Keya Paha county. (12231)

Barlaca constellatio humosa (Fuckel) Clements.

Crouania humosa Fuckel.

On ground, Mead's Spring, Brown county.

Humaria salmonicolor B. \& Br.

On wet earth, Lincoln. (4706)

Neottiella ollaris (Fr.) Clements.

Humaria ollaris Fr.

On leafy ground, Otowanie woods. (12244)

Bcutellinia stercorea (Pers.) Cooke.

On horse dung, Lincoln. (4597)

Scutellinia pu'cherrina (Cr.) Cooke.

On muddy creek banks, Otowanie woods, zincoln. (12284)

(12243) 
Uromyces pecliauns Farl.

On subterranean stems of Distichlis maritima, Alliance. (12043)

Uromyces rosicola E. \& E.

On Rosa sp. Crawford. (12266)

* Uromyces trifolii (A. \& S. ) Wint.

On Trifolium repens, Experiment Station. (12246)

Puccinia asparagi D.C.

On Asparagus officinalis, Peru (12251), Lincoln (12029), Purdum (12248), Atkinson (12019)

- Puccinia asteris Duby.

On Aster multiflorus, Merriman (12249), on A. paniculatus, Experiment Station (12247)

Puccinia batesiana Arthur.

On Heliopsis scabra, Long Pine. (12023)

* Puccinia caricis (Schum.) Reb.

On Carex stricta, Long Pine (12250), on Carex laxiflora blanda, Long Pine (12253)

Puccinia eleocharidis Arthur.

On Eleocharis glaucescens, Long Pine. (12254)

* Puccinia emaculata Schw.

On Eragrostis pectinacea, Experiment Station. (12260) Puccinia gentianae Lk.

On Gentiana puberula, Lincoln.

Puccinia heterospora B. \& C.

On Abutilon abutilon, Peru (12259), Nebraska City (12264)

Puccinia kuhniae Sch

On Kuhnia eupatorioides, Lincoln. (1225\&)

- Puccinia nigrescens Pk.

On Salvia pitcheri, Lincoln. (12258)

- Puccinia obtecta Pk.

On Scirpus pungens, Chadron.

(12255)

- Puccinia polygoni Pers.

On Polygonum convolvulus, Peru (12256), Experiment Station (12257) 
- Puccinia pruni Pers.

On Prunus besscyi, Experiment Station (12262), on $\boldsymbol{P}$. serotina, Experiment Station (12263)

- Puccinia solidaginis Pk.

On Solidago nemoralis, Wood Lake.

Melampsorella cerastii (Pers.) Schultz.

On Cerastium vulgatum, Long Pine. (12267).

Peridermium cerebrum $\mathrm{Pk}$.

On Pinus scopulorum, Long Pine. (12268)

Uredo gaurina (Pk.) Sace.

On Gaura biennis, Lincoln (12270), on G. parviflora, Lincoln (12271)

- Aecidium compositarum Mart.

On Aster paniculatus, Peru (12272), A. levis, Peru (12273), A. multiflorus, Peru (12274), Solidago rig. ida, Lincoln (12275), S. serotina, Lincoln (12276), S. missouriensis, Belmont (12277), Erigeron philadelphicus, Peru (12278)

Aecidium compositarum xanthii Burrill.

On Xanthium canadense, Peru. (12279)

Aecidium dracontii Selm.

On Arisaema dracontium, Peru. (12280)

'Aecidium gaurae E. \& E.

On Gaura parviflora, Peru.

- Aecidium polemonii Pk.

On Phlox divaricata, Cedar creek (12282)

- Aecidium punctatum Pers.

On Anemone caroliniana, Lincoln.

(12283)

\section{USTILAGINACEAE}

Burrillia pustulata Setch.

On Sagittaria latifolia. (12269)

\section{LABOULBENIACEAE}

Laboulbenia brachini Thaxter.

On Brachinus kansanus, South Bend. 
Laboulbcnia elongata Thaxter.

On Platynus sp. (4722)

Laboulbenia galeritae Thaxter.

On Galerita jamus (4719) and $G$. nigropes. (4718)

Laboulbenia variabilis Thaxter.

On Chlaenius pennsylvanicus (4715), prasinus (4716), sericeus (4717), and solitarius (4714)

CLAVARIACEAD

Clavaria subtilis Pers.

On acorn cups, Naponee. (4708)

POLYPORACEAE

Trametes sepium Berk.

On decaying oak, Lincoln. (4710)

AGARICACEAE

Tricholoma melaleucum Fr.

On ground in woods, Lincoln. (12286)

Tricholoma personatum Fr.

On ground in Otowanie woods. (12287)

Pleurotus petaloides Bull.

Lincoln. (12288)

Volvaria parvula Weinm.

On wet ground, Lincoln. (12289)

Annularia levis Krombh.

On ground among grasses, Lincoln. (12290)

Agaricus silvaticus Schaeff.

On ground in Otowanie woods. (12291)

Hypholoma candolleanum Fr.

On wet earth, Lincoln; on ground in Otowanie woods. (12292)

Panaeolus phalaenarum Fr.

On horse dung, Lincoln. (12293)

Panaeolus solidipes Peck.

On dung, Otowanie woods. (12294) 


\section{NIDULARIACRAF}

Cyathus rufipes E. \& E.

On dung, Kennedy. (12295)

\section{RICCIACEAE}

Riccia crystallina $\mathbf{L}$.

On ground, Wymore. (12296)

Sphaerocarpus terrestris Smith.

On ground, Wymore.

JUNGERMANNIACGAN

Aneura pinguis (L.) Dum.

On ground, Peru. (12298)

BRYAC̈EAF

Barbula subulata Beauv.

Long Pine. (6697)

Encalyptra ciliata Hedw.

Long Pine. (6684)

Leskea obscura Hedw.

Franklin county. (6687)

Polytrichum commune $\mathbf{L}$.

Franklin county. (6683)

Webera cruda Schimp.

Long Pine. (6688)

\section{LILIACEAE}

Lilium umbellatum Pursh.

Watt's Lake, Cherry county. (12015)i

\section{GRAMINACEATO}

Bromus hordeaceus L.

Long Pine. (12057)

Bromus tectorum L.

Minden. (12299) 


\title{
ADDITIONS TO REPORTED FLORA
}

\section{CYPERACFAD}

C'arex fusca All.

lírkwood, Rnck county. (12055).

Be:rpus campestris Britton.

Long Pine.

\section{CRUCIFERACEAD}

Sisymbrium altissimum, $\mathbf{I}$.

Ewing. (12012)

PIOLACEAT

Viola tenella Muhl.

Stella. (12300)

\author{
ERICACEAT
}

Pirola elliptica Nutt.

Long Pine. (12001)

CAPRIFOLAACEAE

Viburnum prunifolium $\mathrm{L}$.

Johnson. (12301) 



\title{
THE PIRAIRIE-GRASS FOIMATION IN REGION I
}

\author{
BY JoHN J. Thor
}

\section{INTRODUCTION}

During the last three years the writer has carried on several lines of phytogeographical investigation about Nebraska City, Neb., with a view of bringing results together ultimately and presenting them to the Faculty of the University of Nebraska as a thesis for the degree of master of arts. On account of school duties, the work has not been pursued as vigorously at all times, particularly during late spring and autumn, as was desirable. When circumstances were favorable, however, all possible iaste, consistent with a proper degree of accuracy, was made.

The pieces of apparatus needed were designed largely as necessity dictated, and, as is generally the case under similar circumstances, a desirable degree of accuracy was not always obtained by their use. It is gratifying to know that now we may expect greater precision in determining physical factors, from the excellent and inexpensive apparatus designed by Dr. Clements and Mr. Hedgeock of the University.

To Dr. Clements in particular the writer is deeply indebted for the invaluable assistance rendered from time to time, for the many personal conferences, and for the timely suggestions ever freely given. More than this, Dr. Clements has furnished many invaluable outlines for extended rescarch, and under his supervision alone what is now presented was made possible. The "Phytogeography of Nebraska," by Drs. Pound and Clements, was also a valuable incentive to the work. To Mr. E. R. Yundt, of the Nebraska City high school, the writer is indebted for the accurate topographical survey 
of the prairie on which the formational work was conducted. Mr. Frank Zimmerer has rendered invaluable assistance in photography and map-finishing in water colors, and also along other lines.

Throughout this treatise, the metric system of measures, the Fahrenheit thermometer scale, and the nomenclature based upon Britton and Brown's "Illustrated Flora" are used. The author-citation is not considered imperative here, and accordingly is omitted.

Before any work of importance can be accomplished in phytogeography, the physiography of the region as well as the flora must be adequately in hand. This is especially emphasized in the study of the formation, where it will be discussed to some extent. The work of becoming acquainted with the flora and the physiography of the country about Nebraska City was begun in the autumn of 1897 and continued throughout the following year. During this time, also, a complete collection of the plants was made and deposited with the botanical department of the University of Nebraska. The phenological notes taken during this year were not considered extensive enough to be of importance; this leaves the phenological records for 1899 and 1900, which were made as accurate and complete as conditions would permit.

The more important lines of investigation, with brief explanatory notes, are here given:

I. Physical factors.

1. Physical data consisting of,

a. Soil temperature at a depth of ten centimeters.

b. Psychrometer readings (wet and dry bulb thermometers) at surface of ground and one meter above.

c. Meteorological conditions as to amount and direction of wind, amount and seasonal distribution of precipitation and sky conditions, whether clear, overcast, or cloudy.

d. Determination of physical water-content. 
e. Determination of light-intensity.

II. Vegetation-forms of the flora, including accessory biological characters.

1. Classification of the flora according to vegetationforms.

2. The study of rosettes and rosette-forming plants.

3. The study of buds with especial reference to protection.

4. Observations concerning seed-production and dissemination.

5. The study of the pollination of plants.

6. The preparation of a complete phenological record for each species to include the following:

a. Earliest appearance of leaves.

b. Earliest appearance of flowers.

c. Maximum flowering period.

d. Length of flowering period.

e. Earliest maturation.

$f$. Period of maturation.

g. Dying of parts of herbs, and leaf-fall of trees.

7. Observations upon various other phenomena, as foliage movements, etc., falling under accessory biological characters.

III. Ecological relations.

1. The classification of the flora into habitat-groups.

IV. The study of the formation, summing up all the previous lines of investigation. 


\section{DISCUSSION OF ECOLOGICAL FACTORS}

With the exception of light and physical water-content factors, observations were taken daily, except Sunday, throughout the period of active vegetative growth, from March 20 to December 7,1900 , in the four locations, prairie or I, thicket or II, open woodlands or III, and deep woodlands or IV. Observations were taken between 5:00 and 6:00 P.M. from March 20 to May 5, beginning with location I and ending with IV; between $6: 00$ and $7: 00$ P.M. from May 5 to September 7 ; between 5:00 and 6:00 P.M. from September 8 to October 26 . After the latter date conditions were noted daily from 4:00 to 5:00 P.M. During a considerable portion of the time the psychrometer readings were taken at the surface and one meter above the surface of the ground. Beginning with July 1 the psychrometer data in the first column represent conditions at the earth's surface, and those in the second column conditions at the height of one meter. In all cases soil temperatures were taken ten centimeters below the surface.

The direction of the wind, indicated in the factor record by the letters N., S., E., W., N. E., N. W., S. E., S. W., etc., and amount of wind are at best approximations, as no anemometer was used. The sky condition for each day is indicated by the characters SS 1, signifying few or no clouds; SS $\frac{1}{2}$, half overcast; $\mathrm{Cl} \frac{3}{4}$, mostly overcast; and $\mathrm{Cl} 1$, entirely overcast.

Relative precipitation factors were obtained by measuring the rainfall in a vessel partially sunk in the ground, the amount being indicated in each case in centimeters. The physical water-content was determined from samples of soil, taken to a depth of six to ten cm., collected semi-weekly and placed in air-tigit tin boxes of known weight. These were 
carefully weighed, the contents dried in an oven, and again weighed. The loss in weight represents the physical water. content.

To obtain light factors in the four locations, strips of "Solio" paper were ruled lightly with a violet lead and exposed to the sun's rays until the lines entirely disappeared, the time required in each instance being noted. Observations were made on clear days between 12:00 and 2:30 P.M. In determining light factors, there are many difficulties to be overcome before even fairly accurate results may be expected. The angle of inclination of the sensitized paper to the sun's rays is not always the same, the sky is not uniformly clear even though cloudless, and, disregarding defiection, reflection, and absorption, the amount of light received on a given surface varies as the sine of the angle of elevation. Accordingly, paper exposed in woodland or thicket for a given period will receive much more than one-half of the total amount of light during the first half of the exposure. As stated heretofore, these difficulties are already being anticipated.

A careful examination of the ecological factors found in another part will reveal many interesting relations, the more important of which are here noted. Atmospheric and soil temperatures, humidity, physical water-content, light ratios, and, in fact, most other ecological factors are minimum in the four locations during the spring and autumn months and become maximum only during the summer months. The air temperature of the prairie formation is from $.5^{\circ}-\mathbf{1 0}^{\circ}$ higher than corresponding temperatures in deep woodlands, from $.2^{\circ}-3.5^{\circ}$ higher than temperatures in open woodlands, and from $.5^{\circ}-10^{\circ}$ higher than corresponding temperatures in dense thickets. If for the time we consider prairie conditions normal, we shall conclude that these differences are due to the controlling woody plants in locations II, III, and IV, since where the trees are less controlling, as in III, temperature differences are accordingly reduced. T'em- 
perature differences between the prairie formation on the one hand and thicket and deep woodland formations on the other range from $.2^{\circ}-5^{\circ}$ during the early spring months, and from $2^{\circ}-12^{\circ} 15^{\circ}$ during the summer months.

Soil temperatures are invariably higher in I than in II, III, or IV. During the spring and autumn these differences range from $2^{\circ}-5^{\circ}$, while in midsummer it is not uncommon for the soil temperature of the prairie to range $12^{\circ}-20^{\circ}$ higher than corresponding temperatures in thickets and woodlands. Soil temperatures of location I show greatest variations, $10^{\circ}-20^{\circ}$ in twenty-four hours being not infrequent. Similar temperatures in woodlands and thickets are relatively stable, the changes being gradual and of a less degree. It is also to be noted that soil temperatures taken at a number of stations in the same formation show little variation if conditions are similar. Immediately after a heavy precipitation, temperatures of the soil in all formations are observed to have approached almcist a common degree.

Psychrometer data indicate the least amount of atmospheric humidity in the prairie formation and the greatest amount in thickets and deep woodlands; in this relation, as would be expected, open woodlands are intermediate. As a rule, the wet-bulb thermometer registered $.2^{\circ}-3^{\circ}$ lower at a height of one meter than at the earth's surface, thus indicating a greater evaporation of the water particles from the bulb and hence less humidity in the atmosphere at this height. The dry-bulb thermometer recorded in most cases a temperature of $.2^{\circ}-2.5^{\circ}$ higher one meter above than at the earth's surface. These conditions are found to be true, in general, for the various formations, and they explain to a considerable extent the habits of certain plants of our flora, which tend to develop mesophytic leaves near the ground and xerophytic leaves above. It goes without saying that, when the atmosphere is laden with moisture, little or no difference is observed between the wet- and dry-bulb ther- 
mometers, and that conditions of humidity at the earth's surface are essentially those one meter above.

The physical water-content of the soil is least in location I, greatest in II and IV, and intermediate in III. These conditions in a general way hold good throughout the active growing period. Immediately after a heavy precipitation, the water-content of the soil, theoretically at least, is the same for all locations, but the direct exposure of the sun's rays, combined with the greater atmospheric movements in I, produce a much more rapid evaporation than is possible in wooded formations. Accordingly, variations of the physical water-content of the prairie formation are more abrupt than in any other, assuming of course that the soil composition is the same throughout. During certain portions of May and June, 1900, the physical water-content of location I ranged from 3-9 per cent; at other times in June, July, and August, this soil contained 25 per cent and even 35 per cent of water for a short time. Locations II and IV maintained 18-26 per cent of water in the soil for the greater portion of the year. Immediately after precipitation, 30-38 per cent of soil water is not uncommon in woodlands; this is reduced to 9-14 per cent during the drier parts of the year.

Since the amount of light on a given surface varies as the sine of the angle of elevation, light conditions in the prairie formation are minimum in early spring and autumn, and maximum during midsummer. For each day, there is also a maximum of light (12:00 M.) and two minima (morning and evening). As a result of the foliage, the light relations in locations II, III, and IV are just the reverse of those in location I, viz., maximum light obtaining in the spring and autumn, and minimum light during midsummer. From the above, it follows that the amounts of light in the four locations are nearest equal in early spring, before the foliation of the woody plants, and in autumn after the leaves have fallen. This can readily be seen by a comparison of light ratios given below, which are taken from the ecological fac- 
tors. The relative amount of light in each of the above locations on March 31 may be expressed by the following ratios:

(Light amount) I : (Light amount) II :: $1: 1.583$

(Light amount) I : (Light amount) III :: $1: 1.250$

(Light amount) I : (Light amount) IV :: $1: 1.500$

On June 6 the light relations between the four locations were as indicated:

(Light amount) $1:$ (Light amount) II :: $1: 220$

(Light amount) I : (light amount) III :: $1: 5$

(Light amount) I : (Light amount) IV :: $1: 32$

The following light ratios obtained September 8 and November 3 , respectively:

(Light amount) I : (Light amount) II :: $1: 100$

(Light amount) I : (Light amount) III :: $1: 4$

(light amount) $\mathrm{I}:$ (Light amount) IV $:: 1: 12$

(Light amount) I : (Light amount) II :: $1: 2$

(Light amount) I : (Light amount) III :: $1: 1.3$

(Light amount) $I:$ (Light amount) IV :: $1: 2.6$

In every location results clearly indicate more light at the height of one meter than at the earth's surface. It is more than likely that considerable error will be found in thicket and deep woodland factors owing to the length of time required to sufficiently blacken the paper, but, after allowing for all possible error, the facts remain materially unchanged.

The thicket formation from which the light factors were obtained is a veritable jungle of Xanthoxylum, Corylus, Cornus, and Prunus, sparingly interspersed with Ulmus, Robinia, Gleditsia, and Crataegus. Rhus glabra and Ribes gracile with Menispermum as a climber are common along its borders, and in many places it is overgrown with Vitis, Parthenocissus, and Smilax. It is worth while to note the species of herbaceous plants growing in this thicket, also their flowering period. Viola obliqua, V. scabriuscula, Erythronium albidum, Bicuculla cucullaria, Carex grisea, $C$. 
laxiflora, Phlox divaricata, Hydrophyllum virginicum, Washingtonia longistylis, Ranunculus abortivus, and a few other species of lesser importance make up the entire list of herbaceous bloomers, and these, with the exception of Geum canadense, have completed anthesis before June 1. Plants growing in dense woodlands are compelled, as is evidenced by the specialization and adaptation of parts, to blossom before the dense foliage of the upper woody layers appears, in order to secure a good exposure to light and heat. Upon examining these plants, we find seven, Viola obliqua, V. scabriuscula, Carex grisea, C. laxiflora, Phlox divaricata, Ranunculus abortivus, and Geum canadense, are rosette formers, two, Erythronium and Bicuculla, produce bulbs, and one, Washingtonia, develops thickened fleshy roots. These adaptations enable plants to gain an early start in the spring when maximum light obtains in woodlands. Similar conditions are found in location IV, but not to the same extent, since here the struggle for light is not so desperate, and a larger number of species are enabled to secure favorable conditions. 


\section{ECOLOGICAL FACTORS}

\section{TEMPERATURE AND ATMOSPHERE}

[Owing to the length of the complete record, it is given only for a few days in each month.]

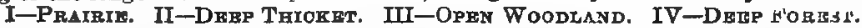

\begin{tabular}{|c|c|c|c|c|c|c|c|c|c|c|c|c|c|}
\hline \multirow{2}{*}{\multicolumn{2}{|c|}{ DATE. }} & \multirow{2}{*}{$\begin{array}{c}\text { HOOR } \\
\text { P.M. }\end{array}$} & \multirow{2}{*}{ 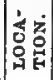 } & \multicolumn{2}{|c|}{ AIR TEMP. } & \multicolumn{2}{|c|}{ WET BOLB. } & \multicolumn{2}{|c|}{ BEL. HOM. } & \multirow{2}{*}{$\begin{array}{l}\text { SOIL } \\
\text { TEMP. }\end{array}$} & \multicolumn{2}{|c|}{ MISTEOROL. } & \multirow{2}{*}{ PRBCIP. } \\
\hline & & & & SURF. & $1 \mathrm{k}$. & SURF. & 1 м. & SURF. & $1 \mathrm{м.}$ & & BKY. & WIND. & \\
\hline Mar. 2 & & $-6: 00$ & I & $33^{\circ}$ & & $30^{\circ}$ & & 70 & $\cdots$ & $32^{\circ}$ & SS 1.. & N. W. & \\
\hline & 20 & “ & II & $32.1^{\circ} \mid$ & & $30^{\circ}$ & & 69 & & $32^{\circ}$ & " & $\because$ & \\
\hline “ & & “ & IIII & $31^{\circ}$ & & $29^{\circ}$ & & 79 & .... & $32^{\circ}$ & “6 & “ & \\
\hline “2 & & “ & IV & $31^{\circ}$ & & $28^{\circ}$ & & 68 & & $32^{\circ}$ & " & 16 & \\
\hline “ & & “ & I & $55^{\circ}$ & & $43^{\circ}$ & & 34 & $\ldots$ & $33^{\circ}$ & $"$ & S. W. & ..... \\
\hline " & & " & II & $\left|52.2^{\circ}\right|$ & & $42^{\circ}$ & & 40 & $\ldots$. & $33^{\circ}$ & “ & “ & \\
\hline "1 & & “ & III & $51^{\circ}$ & & $41.8^{\circ}$ & & 45 & $\ldots$. & $33^{\circ}$ & " & " & $\ldots$ \\
\hline “ & & “" & IV & $50^{\circ}$ & & $40^{\circ}$ & & 38 & $\ldots$ & $33^{\circ}$ & " & " & \\
\hline " & & “ & I & $67^{\circ}$ & & $51^{\circ}$ & & 30 & $\ldots$ & $38^{\circ}$ & “ & " & \\
\hline “ & 22 & “ & $\overline{\mathbf{I I}}$ & $66^{\circ}$ & & $51.5^{\circ}$ & & 34 & $\ldots$ & $36^{\circ}$ & “ & “ & $\ldots \ldots$ \\
\hline 1 & & " & ÜI & $65^{\circ}$ & & $50^{\circ}$ & & 31 & (... & $36^{\circ}$ & $" 1$ & " & \\
\hline “" & 22 & “ & IV & $65.5^{\circ}$ & & $49.1^{\circ}$ & & 26 & .... & $35^{\circ}$ & “" & “ & $\cdots \cdots$ \\
\hline “" & & “ & I & $50^{\circ}$ & & $46^{\circ}$ & & 74 & $\ldots$ & $42^{\circ}$ & Cl $1 \ldots$ & & \\
\hline “ & 23 & “ & II & $50^{\circ}$ & & $47^{\circ}$ & $\ldots$ & 80 & $\ldots$ & $34^{\circ}$ & " & & \\
\hline & 23 & 14 & IIII & $50^{\circ}$ & & $48^{\circ}$ & & 87 & $\ldots$ & $40^{\circ}$ & “ & & . \\
\hline as & & " & $\mathrm{IV}$ & $49.5^{\circ}$ & & $46^{\circ}$ & & 76 & $\ldots$ & $40^{\circ}$ & " & & \\
\hline$\because$ & 24 & 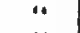 & $I$ & $40.7^{\circ}$ & & $38.5^{\circ} \mid$ & $\ldots$ & 88 & $\ldots$. & $38^{\circ}$ & " & E. & $1 / 8 \mathrm{~cm}$. \\
\hline & 24) & “ & III & $41^{\circ}$ & & $39^{\circ}$ & & 84 & $\ldots$ & $36^{\circ}$ & “ & "s & 16 \\
\hline 11 & 24 & 16 & III & $41^{\circ}$ & & $39^{\circ}$ & & 84 & & $38^{\circ}$ & " & $" 1$ & “" \\
\hline " & 24 & $\because$ & IV & $42^{\circ}$ & & $\left|39.4^{\circ}\right|$ & & 81 & $\ldots$ & $36^{\circ}$ & " & $"$ & $" 1$ \\
\hline Apr. & 2 & $" 1$ & I & $60.5^{\circ}$ & & $48^{\circ}$ & & 37 & $\ldots$ & $59^{\circ}$ & SS 1.. & S.W. & \\
\hline$" 7$ & & $\because$ & II & $59.9^{\circ}$ & & $45^{\circ}$ & & 26 & $\cdots$ & $44^{\circ}$ & $\because 0$ & 11 & \\
\hline “ & & “ & IIII & $59.6^{\circ}$ & & $45.5^{\circ} \mid$ & ... & 29 & $\ldots$ & $54^{\circ}$ & " & " & \\
\hline " & & "4 & IV & $57^{\circ}$ & & $45^{\circ}$ & & 36 & .... & $52^{\circ}$ & " & "، & \\
\hline & & "“ & $I$ & $58^{\circ}$ & & $44^{\circ}$ & & 28 & $\ldots$ & $53^{\circ}$ & SS $3 / 4$. & N.E. & \\
\hline “. & & “ & II & $58.1^{\circ}$ & & $45^{\circ}$ & & 33 & $\ldots$ & $46^{\circ}$ & 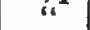 & " & $\because$ \\
\hline " & & $"$ & III & $58^{\circ}$ & & $49.9^{\circ}$ & $\ldots \ldots$ & 56 & $\ldots$ & $51^{\circ}$ & “ & “" & \\
\hline “ & 3 & “ & IV & $58^{\circ}$ & & $44.5^{\circ}$ & & 30 & $\ldots$ & $50^{\circ}$ & " & ، & . \\
\hline & 4 & “ & I & $56^{\circ}$ & & $42^{\circ}$ & & 25 & $\ldots$ & $55^{\circ}$ & SS 1.. & S.E. & \\
\hline “ & 4 & “ & II & $55^{\circ}$ & & $42.3^{\circ} \mid$ & & 31 & $\ldots$ & $46^{\circ}$ & “ & "1 & 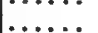 \\
\hline “ & 4 & “ & IIII & $56^{\circ}$ & & $42^{\circ}$ & & 25 & $\ldots$ & $51.7^{\circ}$ & “ & " & \\
\hline$\ddot{\prime}$ & 4) & “" & IV & $54^{\circ}$ & & $43^{\circ}$ & & 32 & & $48^{\circ}$ & " & “ & \\
\hline " & 5 & “" & I & $66.2^{\circ}$ & & $47.5^{\circ} \mid$ & & 22 & & $60^{\circ}$ & “" & “ & $\cdots$ \\
\hline " & 5 & “ & II & $65^{\circ}$ & & $47.7^{\circ}$ & .. & 24 & .. & $52^{\circ}$ & “" & “ & $\cdots$ \\
\hline “ & 5 & “ & IIII & $65.7^{\circ}$ & & $47.8^{\circ}$ & ... & 22 & & $55^{\circ}$ & " & "، & \\
\hline “" & 5 & “ & IV & $64^{\circ}$ & & $47.6^{\circ} \mid$ & & 25 & & $54^{\circ}$ & a & "6 & \\
\hline " & & “ & $I$ & $70^{\circ}$ & & $50.2^{\circ}$ & & 19 & & $63.7^{\circ}$ & SS $3 / 4$. & & ….... \\
\hline " & 6 & “ & II & $67.8^{\circ}$ & 0. & $9^{\circ}$ & & 31 & .. & $52.5^{\circ}$ & $\therefore$ & & . \\
\hline “ & 6 & “ & IIII & $68^{\circ}$ & & $51^{\circ}$ & & 27 & $\ldots$ & $58^{\circ}$ & “" & & \\
\hline "̈ & 6 & " & IV & $66^{\circ}$ & & $51^{\circ}$ & & 32 & & $52^{\circ}$ & “ & & ...... \\
\hline “ & 7 & " & I & $67.5^{\circ}$ & & $58^{\circ}$ & & 55 & .. & $64^{\circ}$ & " & $\cdots$ & . \\
\hline · & 7 & 4 & II & $67.5^{\circ}$ & & $58^{\circ}$ & & & & $56^{\circ}$ & " & & $\cdots$ \\
\hline 4 & 7 & "“ & III & $66.1^{\circ}$ & & $58^{\circ}$ & & 6 & & $61^{\circ}$ & s & & 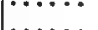 \\
\hline " & 7 & " & IV & $649^{\circ}$ & & {$\left[57.2^{\circ} \mid\right.$} & & 61 & & $54^{\circ}$ & "r & $\cdots \cdots$ & $\cdots \cdots$ \\
\hline May & 2 & “ & I & $59^{\circ}$ & & {$\left[04.4^{\circ} \mid\right.$} & & 75 & $\ldots$ & $64^{\circ}$ & " & $\mathrm{N} . \overline{\mathrm{w}}$ & $\cdots$ \\
\hline & & $"$ & II & $58^{\circ}$ & & $51.5^{\circ}$ & & 64 & ... & $63.5^{\circ}$ & " & if & \\
\hline$\because$ & & “" & IIII & $56^{\circ}$ & & & & & & $64^{\circ}$ & " & 34 & \\
\hline & $2 i$ & “ & $|\mathrm{IV}|$ & $55^{\circ}$ & & & & 59 & & $62^{\circ}$ & " & 17 & $\ldots$ \\
\hline
\end{tabular}




\section{ECOLOGICAL FACTORS-Continued}

\section{TEMPERATURE AND ATMOSPHERE}

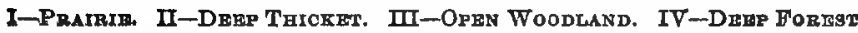

\begin{tabular}{|c|c|c|c|c|c|c|c|c|c|c|c|c|c|}
\hline \multirow{2}{*}{\multicolumn{2}{|c|}{ DATE. }} & \multirow{2}{*}{$\begin{array}{l}\text { HODR } \\
\text { P.M. }\end{array}$} & \multirow{2}{*}{ 㻤 } & \multicolumn{2}{|c|}{ AIR TEMP. } & \multicolumn{2}{|c|}{ WET BOLB. } & \multicolumn{2}{|c|}{ 툐IEL. HOX. } & \multirow{2}{*}{$\begin{array}{l}\text { SOIL } \\
\text { TEMP. }\end{array}$} & \multicolumn{2}{|c|}{ METEOROL. } & \multirow{2}{*}{ PRECIP. } \\
\hline & & & & sURF. & $1 \mathrm{M}$. & SORP. & $1 \mathbf{x}$. & $60 \mathrm{RF}$. & $1 \mathrm{M}$. & & SKY. & WIND. & \\
\hline May & & $5-6: 00$ & I & $66^{\circ}$ & & $53^{\circ}$ & & 40 & $\cdots$ & $67.3^{\circ}$ & SS $3 / 4$. & N. W. & $\cdots$ \\
\hline 4 & 3 & $\because$ & II & $65^{\circ}$ & & $52.5^{\circ}$ & & 42 & $\ldots$ & $63^{\circ}$ & 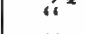 & 16 & \\
\hline ". & & $\because$ & III & $66^{\circ}$ & & $52^{\circ}$ & & 36 & $\ldots$ & $65^{\circ}$ & “" & “" & $\ldots \ldots$ \\
\hline$"$ & & 11 & IV & $64^{\circ}$ & & $52^{\circ}$ & & 43 & $\ldots$ & $60^{\circ}$ & " & $"$ & $\ldots .$. \\
\hline$"$ & 4 & “ & I & $64^{\circ}$ & & $55^{\circ}$ & $\ldots \ldots$ & 56 & $\ldots$ & $69^{\circ}$ & SS 1.. & N.E. & $\cdots$ \\
\hline “ & & “ & II & $63^{\circ}$ & & $55^{\circ}$ & $\ldots$ & 60 & .... & $64^{\circ}$ & 11 & is & $\cdots$ \\
\hline “ & & “ & III & $63^{\circ}$ & & $54^{\circ}$ & $\ldots \ldots$ & 55 & $\ldots$ & $65^{\circ}$ & $" 1$ & “" & $\cdots$ \\
\hline "4 & & “ & $\overline{I V}$ & $62.2^{\circ}$ & & $54^{\circ}$ & & 59 & $\ldots$ & $62^{\circ}$ & " & " & \\
\hline “ & 5 & $6-6: 30$ & I & $70^{\circ}$ & & $62^{\circ}$ & $\ldots$ & 64 & $\ldots$ & $70^{\circ}$ & SS $1 / 2$. & E. & $1.2 \mathrm{~cm}$ \\
\hline •" & 5 & “ & II & $69^{\circ}$ & & $61.3^{\circ}$ & $\cdots$ & 65 & $\ldots$ & $63^{\circ}$ & “" & " & “ \\
\hline " & 5 & ““ & III & $69^{\circ}$ & & $61^{\circ}$ & $\ldots$. & 63 & .... & $64.5^{\circ}$ & “ & “ & " \\
\hline “" & 5 & “ & IV & $69^{\circ}$ & & $61^{\circ}$ & & 63 & & $64^{\circ}$ & " & “4 & “ \\
\hline " & 7) & " & I & $64^{\circ}$ & & $58^{\circ}$ & ... & 70 & $\ldots$ & $68^{\circ}$ & “ & " & $1 / 2 \mathrm{~cm}$. \\
\hline ". & 7 & “ & II & $64^{\circ}$ & & $59.8^{\circ}$ & & 79 & $\ldots$ & $65.7^{\circ}$ & " & “" & " a \\
\hline “ & 7 & " & IIII & $64^{\circ}$ & & $60^{\circ}$ & $\ldots$ & 79 & ... & $66^{\circ}$ & $\ddot{1}$ & “ & “ \\
\hline “ & 7 & “ & IV & $66^{\circ}$ & & $60.8^{\circ}$ & & 73 & & $68^{\circ}$ & 11 & " & "“ \\
\hline June & 1 & $6-6: 40$ & I & $71^{\circ}$ & & $65^{\circ}$ & $\ldots$. & 72 & ... & $79^{\circ}$ & SS 1... & N. & ..... \\
\hline “4 & 1 & “ & II & $70^{\circ}$ & & $64^{\circ}$ & $\cdots$ & 72 & $\ldots$ & $68^{\circ}$ & “ & “ & ..... \\
\hline “" & 1 & “ & III) & $70.5^{\circ}$ & & $64^{\circ}$ & . & 70 & .... & $73^{\circ}$ & “ & “ & $\ldots .$. \\
\hline " & 1 & "4 & IV & $71^{\circ}$ & & $64^{\circ}$ & & 68 & & $72^{\circ}$ & $"$ & " & 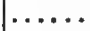 \\
\hline ". & 2 & "4 & I & $70.1^{\circ}$ & & $62^{\circ}$ & $\ldots \ldots$ & 64 & $\ldots$ & $82^{\circ}$ & " & “ & \\
\hline " & 2 & "“ & II & $66^{\circ}$ & & $60^{\circ}$ & $\cdots$ & 71 & $\ldots$ & $66^{\circ}$ & “" & “ & $\ldots \ldots$ \\
\hline .• & 2 & “6 & III & $67^{\circ}$ & & $62.3^{\circ}$ & & 78 & $\ldots$ & $69^{\circ}$ & “" & " & $\ldots$. \\
\hline “ & 2 & " & IV & $67^{\circ}$ & & $61^{\circ}$ & & 71 & & $67^{\circ}$ & “" & “ & $\ldots$. \\
\hline “ & 4 & " & $I$ & $76^{\circ}$ & & $68^{\circ}$ & . & 66 & $\ldots$ & $85^{\circ}$ & " & S. & $\cdots$ \\
\hline "“ & 4 & " & III & $73^{\circ}$ & & $67^{\circ}$ & $\cdots$ & 73 & .... & $70^{\circ}$ & “” & “" & $\ldots \ldots$ \\
\hline “ & 4 & "4 & III & $76^{\circ}$ & & $66^{\circ}$ & , & 59 & .... & $72^{\circ}$ & “" & " & $\ldots \ldots$ \\
\hline ." & 4 & “" & IV & $76^{\circ}$ & & $67^{\circ}$ & & 63 & $\ldots$ & $70^{\circ}$ & " & " & $\ldots$. \\
\hline " & 5 & "6 & I & $78.2^{\circ}$ & - & $70^{\circ}$ & .... & 67 & .... & $86^{\circ}$ & " & S.E. & - \\
\hline “" & 5 & " & II & $77^{\circ}$ & & $70^{\circ}$ & $\cdots$ & 71 & $\ldots$ & $71^{\circ}$ & "“ & "“ & $\ldots$ \\
\hline ." & 5 & "1 & III & $77^{\circ}$ & & $70^{\circ}$ & & 71 & $\ldots$ & $72^{\circ}$ & "“ & " & $\ldots \ldots$ \\
\hline “ & 5 & " & IV & $78^{\circ}$ & & $70^{\circ}$ & & 67 & $\ldots$ & $72^{\circ}$ & " & " & 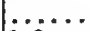 \\
\hline “" & 6 & " & $I$ & $90^{\circ}$ & & $76^{\circ}$ & 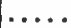 & 53 & & $90^{\circ}$ & "“ & & $1.6 \mathrm{~cm}$ \\
\hline “. & 6 & $"$ & $I I$ & $82^{\circ}$ & & $72.6^{\circ}$ & & 65 & $\ldots$ & $77^{\circ}$ & " & $\ldots \ldots$ & " \\
\hline " & 6 & ". & IIII & $83^{\circ}$ & & $73.5^{\circ}$ & $\cdots$ & 66 . & & $79^{\circ}$ & “" & & " \\
\hline .. & 6 & “6 & $I V$ & $83.5^{\circ}$ & & $76^{\circ}$ & $\cdots$ & 71. & $\ldots$ & $78^{\circ}$ & " & & " \\
\hline ." & 7 & “ & I & $81^{\circ}$ & & $73.8^{\circ}$ & & $7 \overline{2}$ & .... & $84^{\circ}$ & SS $4 / 8$. & N.W. & $.5 \mathrm{~cm}$. \\
\hline$\because$ & 7 & "r & II & $71.6^{\circ}$ & & $70^{\circ}$ & & 94 & .... & $72^{\circ}$ & & " & \\
\hline "u & 7 & “" & IIII & $77.8^{\circ}$ & & $69.8^{\circ}$ & & 67 & $\ldots$ & $80^{\circ}$ & " & " & “ \\
\hline$\because$ & 7. & " & IV & $77^{\circ}$ & & $70^{\circ}$ & & 71 & & $73^{\circ}$ & “ & “ & \\
\hline fuly & 2 & $6-7: 00$ & I & $88^{\circ}$ & $87.5^{\circ}$ & $78^{\circ}$ & $76.1^{\circ}$ & 64 & 60 & $90^{\circ}$ & SS $8 / 4$. & S. & $33 \mathrm{~cm}$. \\
\hline & 2 & “' & II & $83^{\circ}$ & $82.2^{\circ}$ & $76.4^{\circ}$ & $76^{\circ}$ & 73 & 76 & $76^{\circ}$ & & & “ \\
\hline “" & 2 & “ & III & $84^{\circ}$ & $85^{\circ}$ & $77.2^{\circ}$ & $76.1^{\circ}$ & 73 & 66 & $83^{\circ}$ & " & " & $"$ \\
\hline " & 2 & " & IV & $84^{\circ}$ & $84^{\circ}$ & $76^{\circ}$ & $76^{\circ}$ & 69 & 69 & $80^{\circ}$ & " & "4 & " \\
\hline " & 3 & “ & I & $92^{\circ}$ & $92^{\circ}$ & $76.1^{\circ}$ & $76^{\circ}$ & 48 & 48 & $90^{\circ}$ & SS 1. & ..... & $\because$ \\
\hline •. & & " & II & $87.5^{\circ}$ & $88^{\circ}$ & $77.5^{\circ}$ & $76^{\circ}$ & 64 & 58 & $80^{\circ}$ & $"$ & $\ldots \ldots$ & $\cdots$ \\
\hline$"$ & & $" \because$ & IIII & $90^{\circ}$ & $89.5^{\circ}$ & $78.1^{\circ}$ & $77^{\circ}$ & 59 & 58 & $88^{\circ}$ & " & $\ldots \ldots$ & $\ldots \ldots$ \\
\hline 0 & & $"$ & IIVI & $88.2^{\circ}$ & $89^{\circ}$ & $178^{\circ}$ & $77^{\circ}$ & 64 & 58 & $81.5^{\circ}$ & “" & 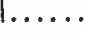 & $\ldots \ldots$ \\
\hline
\end{tabular}




\section{ECOLOGICAL FACTORS-Continued}

\section{TEMPERATURE AND ATMOSPHERE}

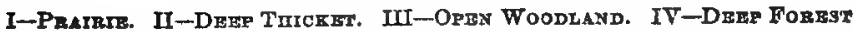

\begin{tabular}{|c|c|c|c|c|c|c|c|c|c|c|c|c|c|}
\hline \multirow{2}{*}{\multicolumn{2}{|c|}{ DATE. }} & \multirow{2}{*}{$\begin{array}{l}\text { HOOR } \\
\text { P.M. }\end{array}$} & \multirow{2}{*}{ 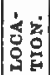 } & \multicolumn{2}{|c|}{ AIR THAP. } & \multicolumn{2}{|c|}{ WET BULB. } & \multicolumn{2}{|c|}{ RELL. HUM. } & \multirow{2}{*}{$\begin{array}{l}\text { SOIL } \\
\text { TEAKP. }\end{array}$} & \multicolumn{2}{|c|}{ METEOROL. } & \multirow{2}{*}{ PEBOIF } \\
\hline & & & & SERF. & 1 м. & SURP. & 1 м. & SERF. & $1 \mathrm{M}$. & & SKY. & WIND. & \\
\hline aly & 5 & $6-7: 00$ & $I$ & $88.1^{\circ}$ & $38^{\circ}$ & $71^{\circ}$ & $69^{\circ}$ & 43 & 38 & $90^{\circ}$ & SS $1 / 4$ & S. & \\
\hline & 5 & & II & $80^{\circ}$ & $80.5^{\circ}$ & $70.5^{\circ}$ & $70^{\circ}$ & 62 & 60 & $76^{\circ}$ & & " & \\
\hline " & 5 & “ & IIII & $37^{\circ}$ & $88^{\circ}$ & $69^{\circ}$ & $68^{\circ}$ & 40 & 35 & $86^{\circ}$ & “ & " & \\
\hline “ & 5 & “ & IV & $85.0^{\circ}$ & $86^{\circ}$ & $170^{\circ}$ & $69.8^{\circ}$ & 45 & 45 & $78^{\circ}$ & “ & “ & \\
\hline a & 6 & "4 & I & $91.7^{\circ}$ & $91.7^{\circ}$ & $76^{\circ}$ & $75^{\circ}$ & 48 & 45 & 0 & SS 1. & “ & \\
\hline "6 & 6 & “ & II & $89^{\circ}$ & $89^{\circ}$ & $76^{\circ}$ & $76^{\circ}$ & 55 & 55 & $79.8^{\circ}$ & " & " & \\
\hline “ & 6 & “ & III & $90^{\circ}$ & $91^{\circ}$ & $75^{\circ}$ & $74.3^{\circ}$ & 50 & 45 & $90^{\circ}$ & “" & " & \\
\hline “؛ & $\theta$ & . & ITV & $90^{\circ}$ & $91^{\circ}$ & $75^{\circ}$ & $75^{\circ}$ & 50 & 47 & $2.5^{\circ}$ & "“ & " & \\
\hline at & & “ & I & $82^{\circ}$ & $82^{\circ}$ & $63.9^{\circ}$ & $64^{\circ}$ & 36 & 36 & & " & N.E. & \\
\hline at & 2 & “ & II & $78.2^{\circ}$ & $79^{\circ}$ & $65.9^{\circ}$ & $64^{\circ}$ & 53 & 43 & $76^{\circ}$ & "4 & "6 & \\
\hline ، & 7 & " & III & $79.9^{\circ}$ & $80^{\circ}$ & $63^{\circ}$ & $62^{\circ}$ & 38 & 35 & $89^{\circ}$ & “ & " & \\
\hline " & 7 & “ & IV & $78.8^{\circ}$ & $79^{\circ}$ & $64.2^{\circ}$ & $63^{\circ}$ & 43 & 40 & $79^{\circ}$ & " & "6 & \\
\hline ag. & 1 & $6-7: 30 \mid$ & $\mathrm{I}$ & $85^{\circ}$ & $85.5^{\circ}$ & $73^{\circ}$ & $71.9^{\circ}$ & & 52 & 90 & "6 & S. & \\
\hline$\because$ & 1 & a & II & $82^{\circ}$ & $81.7^{\circ}$ & $72.8^{\circ}$ & $72.3^{\circ}$ & 65 & 64 & 7 & "، & $\therefore$ & \\
\hline “ & 1 & " & III & $83^{\circ}$ & $83.9^{\circ}$ & $73^{\circ}$ & $71.8^{\circ}$ & 62 & 55 & $80.1^{\circ}$ & " & “ & \\
\hline “" & 1 & ." & IV & $82^{\circ}$ & $82^{\circ}$ & $73^{\circ}$ & $72^{\circ}$ & 65 & 62 & 77 & “ & “ & \\
\hline ، & 2 & $6-7: 00$ & I & $88^{\circ}$ & $88.1^{\circ}$ & $71^{\circ}$ & $68.1^{\circ}$ & & & $g$ & "6 & " & \\
\hline “" & 2 & “ & $\overline{I I}$ & $82.6^{\circ}$ & $83^{\circ}$ & $72.2^{\circ}$ & $71^{\circ}$ & 62 & 55 & 7 & “ & “ & \\
\hline "4 & 2 & “ & III & $85^{\circ}$ & $85.5^{\circ}$ & $70.1^{\circ}$ & $69^{\circ}$ & 47 & 43 & $83^{\circ}$ & " & 16 & \\
\hline " & & " & IV & $83^{\circ}$ & $82.8^{\circ}$ & $70^{\circ}$ & $70^{\circ}$ & 52 & 52 & $0^{\circ}$ & " & " & \\
\hline " & 3 & .1 & $\mathrm{I}$ & $88^{\circ}$ & $87.8^{\circ}$ & $72^{\circ}$ & $71.9^{\circ}$ & & 46 & 9. & ") & S.E. & \\
\hline " & 3 & " & II & $80.2^{\circ}$ & $83.7^{\circ}$ & $70.2^{\circ}$ & $71.9^{\circ}$ & 61 & $\overline{5}$ & 76 & “' & $" 7$ & \\
\hline "I & & . & III & $85^{\circ}$ & $85.6^{\circ}$ & $73^{\circ}$ & $71.3^{\circ}$ & 56 & 5 & 8 & " & " & \\
\hline : & & "1 & $\mathbf{I V}$ & $84^{\circ}$ & $85^{\circ}$ & $72^{\circ}$ & $70.8^{\circ}$ & 56 & 50 & 79 & "1 & "6 & \\
\hline " & 4 & " & I & $87^{\circ}$ & $87^{\circ}$ & $75.5^{\circ}$ & $74^{\circ}$ & & & & ' & " & \\
\hline 4 & 4 & " & II & $83.4^{\circ}$ & $84^{\circ}$ & $74.3^{\circ}$ & $74^{\circ}$ & 66 & 63 & & & ، & \\
\hline "1 & & " & III & $84.6^{\circ}$ & $85.2^{\circ}$ & $74.2^{\circ}$ & $73.9^{\circ}$ & & & & at & " & \\
\hline •. & 4 & " & IV & $84.1^{\circ}$ & $84^{\circ}$ & $74^{\circ}$ & $73^{\circ}$ & & 5 & & ' & " & \\
\hline “ & 6 & " & $i$ & $87^{\circ}$ & $87.3^{\circ}$ & $73^{\circ}$ & $72.1^{\circ}$ & & 48 & & “ & " & \\
\hline "* & & • & II & $83.4^{\circ}$ & $84^{\circ}$ & $73.7^{\circ}$ & $73^{\circ}$ & & 5 & & & & \\
\hline " & & It & III & $85.8^{\circ}$ & $85.5^{\circ}$ & $73.2^{\circ}$ & 7 & & & & " & " & \\
\hline “' & 6 & " & IV & $84.2^{\circ}$ & $85^{\circ}$ & $72^{\circ}$ & $72^{\circ}$ & & & & c & & \\
\hline " & 7 & " & I & $188^{\circ}$ & $86^{\circ}$ & $72.4^{\circ}$ & $72.1^{\circ}$ & & & & ' & & \\
\hline “ & & “ & II & $83^{\circ}$ & $82^{\circ}$ & $73.2^{\circ}$ & $72.1^{\circ}$ & & & 7 & ، & & \\
\hline “" & 7 & “ & III & $85^{\circ}$ & $85^{\circ}$ & 73 & $72^{\circ}$ & & & & . & & \\
\hline$"$ & 7 & “" & IV & $85.4^{\circ}$ & $85.4^{\circ}$ & $73^{\circ}$ & $72.1^{\circ}$ & 5 & & & ، & " & \\
\hline ept.1 & 11 & $5-6: 00$ & I & $71^{\circ}$ & $71.2^{\circ}$ & $64.1^{\circ}$ & $64.1^{\circ}$ & 68 & 68 & 78 & SS $3 / 4$. & N. & $3.5 \mathrm{c}$ \\
\hline is & 11 & “ & II & $70^{\circ}$ & & $66.4^{\circ}$ & & & & & & & " \\
\hline "' & & “" & III & $70.4^{\circ}$ & $70.4^{\circ}$ & $65^{\circ}$ & $65^{\circ}$ & & & & "1 & " & . \\
\hline " & 11 & “ & IV & $70^{\circ}$ & $70^{\circ}$ & $66^{\circ}$ & $65^{\circ}$ & & 7 & & " & & \\
\hline " & 1. & $5-6: 30$ & I & $71^{\circ}$ & $71.7^{\circ}$ & $63.9^{\circ}$ & $62.5^{\circ}$ & 6 & 61 & & SS 1. & S. & \\
\hline “ & 1 & $"$ & II & $67^{\circ}$ & $66.5^{\circ}$ & $64^{\circ}$ & & & 8 & & 16 & " & \\
\hline ". & 12 & " & III & $68^{\circ}$ & 68 & $64^{\circ}$ & 6 & & & & 。 & 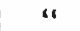 & \\
\hline " & 12 & " & IV & $67.5^{\circ}$ & $67.5^{\circ}$ & $64^{\circ}$ & $63.8^{\circ}$ & 83 & 80 & $.5^{\circ}$ & “ & ، & \\
\hline “. & & $-6: 00$ & $I$ & $84^{\circ}$ & $84.5^{\circ}$ & $72.4^{\circ} \mid$ & $72.2^{\circ}$ & 5 & 5 & & "6 & “4 & \\
\hline "I & 13 & 11 & $\tilde{u}$ & $80^{\circ}$ & $81^{\circ}$ & $70.9^{\circ}$ & $70.4^{\circ}$ & & 6 & & " & $" 1$ & \\
\hline "6 & & “ & III & $81.5^{\circ}$ & $82^{\circ}$ & $71.5^{\circ}$ & $71^{\circ}$ & & & & 10 & 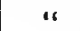 & \\
\hline s" & & “ & IIV & $80^{\circ}$ & $180.8^{\circ}$ & $\mid 70.8^{\circ}$ & $70.3^{\circ}$ & & 6 & & "1 & " & \\
\hline
\end{tabular}


ECOLOGICAL FACTORS-Continued

TEMPERATURE AND ATMOSPIERE

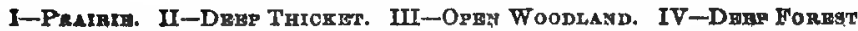

\begin{tabular}{|c|c|c|c|c|c|c|c|c|c|c|c|c|c|}
\hline \multirow{2}{*}{\multicolumn{2}{|c|}{ DATE. }} & \multirow{2}{*}{$\begin{array}{l}\text { HOOR } \\
\text { P.MS. }\end{array}$} & \multirow{2}{*}{$\begin{array}{l}4 \\
0 \\
0 \\
0\end{array}$} & \multicolumn{2}{|c|}{ AIR TEMP. } & \multicolumn{2}{|c|}{ WET ДULE. } & \multicolumn{2}{|c|}{ REL. HUM. } & \multirow{2}{*}{$\begin{array}{l}\text { SoII } \\
\text { TIMP. }\end{array}$} & \multicolumn{2}{|c|}{ METEOROI. } & \multirow{2}{*}{ PREGIP } \\
\hline & & & & 80RP. & $1 x$ & SURF. & 1 ม. & SORP. & [ ] $\mathbf{3}$ & & BKY. & WIND. & \\
\hline \multicolumn{2}{|c|}{ Sept. 14} & $6: 00$ & I & $78^{\circ}$ & $78.2^{\circ}$ & $66.3^{\circ}$ & $66^{\circ}$ & 53 & 53 & $76^{\circ}$ & SS $1 / 3$. & S.E. & $20 \mathrm{~cm}$ \\
\hline & 14 & 16 & II & $74^{\circ}$ & $76^{\circ}$ & $68^{\circ}$ & $66^{\circ}$ & 74 & 59 & $73^{\circ}$ & & $"$ & " \\
\hline “ & & “ & III & $76^{\circ}$ & $77^{\circ}$ & $67^{\circ}$ & $66^{\circ}$ & 63 & 56 & $75^{\circ}$ & “ & " & $" 6$ \\
\hline " & & “ & IV & $74.9^{\circ}$ & $76^{\circ}$ & $67^{\circ}$ & $66^{\circ}$ & 66 & 59 & $72^{\circ}$ & 11 & “" & \\
\hline " & & " & I & $68^{\circ}$ & $68^{\circ}$ & $58^{\circ}$ & $57^{\circ}$ & 54 & 50 & $74^{\circ}$ & SS 1.. & N. & $\ldots$ \\
\hline “، & & “، & II & $66^{\circ}$ & $66^{\circ}$ & $59^{\circ}$ & $58^{\circ}$ & 66 & 61 & $70^{\circ}$ & " & 16 & $\cdots$ \\
\hline “* & & “ & III & $68^{\circ}$ & $66.2^{\circ}$ & $58^{\circ}$ & $57^{\circ}$ & 61 & 57 & $72^{\circ}$ & “ & “ & $\ldots$ \\
\hline " 1 & & “ & $\mathrm{IV}$ & $65.8^{\circ}$ & $66.6^{\circ}$ & $58^{\circ}$ & $56.9^{\circ}$ & 61 & 53 & $69^{\circ}$ & “ & “ & \\
\hline Oct. & 1 & “ & I & $67.7^{\circ}$ & $67.7^{\circ}$ & $65^{\circ}$ & $64.4^{\circ}$ & 88 & 85 & $67^{\circ}$ & SS $1 / 4$ & $\mathbf{E .}$ & $T$. \\
\hline " & 1) & “ & II & $67^{\circ}$ & $67^{\circ}$ & $65^{\circ}$ & $65^{\circ}$ & 90 & 90 & $66^{\circ}$ & & "s & “ \\
\hline “ & 1 & “4 & III & $67^{\circ}$ & $67^{\circ}$ & $65^{\circ}$ & $64.7^{\circ}$ & 90 & 89 & $68^{\circ}$ & “ & " & 46 \\
\hline "6 & 1 & "1 & IV & $66^{\circ}$ & $66.1^{\circ}$ & $64.4^{\circ}$ & $64^{\circ}$ & 92 & 90 & $66^{\circ}$ & “" & " & "1 \\
\hline "s & 2 & “" & $\mathbf{I}$ & $80.2^{\circ}$ & $81.9^{\circ}$ & $73^{\circ}$ & $73^{\circ}$ & 72 & 65 & $76^{\circ}$ & SS 1 & S. & \\
\hline “ & 2 & “4 & II & $77^{\circ}$ & $78^{\circ}$ & $72.8^{\circ}$ & $72.7^{\circ}$ & 83 & 79 & $73^{\circ}$ & "، & $\because$ & \\
\hline "، & 2 & “ & III & $79^{\circ}$ & $79.4^{\circ}$ & $73^{\circ}$ & $72.8^{\circ}$ & 75 & 73 & $75^{\circ}$ & “ & “ & $\cdots$ \\
\hline “" & 2 & ." & IV & $76^{\circ}$ & $76^{\circ}$ & $72.8^{\circ}$ & $72.7^{\circ}$ & 87 & 86 & $72^{\circ}$ & " & 16 & \\
\hline "s & 4 & “ & 21 & $81.9^{\circ}$ & $83^{\circ}$ & $73^{\circ}$ & $72^{\circ}$ & 65 & 59 & $76^{\circ}$ & " & " & \\
\hline "6 & 4 & “ & III & $79^{\circ}$ & $79^{\circ}$ & $72.1^{\circ}$ & $72^{\circ}$ & 71 & 71 & $75^{\circ}$ & " & "، & \\
\hline “، & 4 & “ & III & $79^{\circ}$ & $180^{\circ}$ & $73.2^{\circ}$ & $73.6^{\circ}$ & 75 & 73 & $77^{\circ}$ & " & “" & ..... \\
\hline “، & 4 & “، & $\overline{I V}$ & $78^{\circ}$ & $78^{\circ}$ & $73.4^{\circ}$ & $73.3^{\circ}$ & 81 & 80 & $75.5^{\circ}$ & " & " & \\
\hline “" & 5 & ." & $i$ & $78.1^{\circ}$ & $78.5^{\circ}$ & $70.3^{\circ}$ & $70^{\circ}$ & 67 & 65 & $78^{\circ}$ & a & “. & 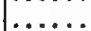 \\
\hline “، & 5 & “" & $\overline{\mathrm{II}}$ & $78^{\circ}$ & $78^{\circ}$ & $71^{\circ}$ & $70.5^{\circ}$ & 71 & 69 & $76^{\circ}$ & " & “" & . \\
\hline “ & 5 & "• & IIII & $78.1^{\circ}$ & $78.5^{\circ}$ & $70.5^{\circ}$ & $70^{\circ}$ & 69 & 65 & $77^{\circ}$ & “ & " & ..... \\
\hline “6 & 5 & ." & IV & $76^{\circ}$ & $78^{\circ}$ & $71^{\circ}$ & $70.5^{\circ}$ & 71 & 69 & $75^{\circ}$ & " & " & \\
\hline or & 6 & “" & I & $61^{\circ}$ & $61^{\circ}$ & $53^{\circ}$ & $52.7^{\circ}$ & 58 & 57 & $73^{\circ}$ & SS 36 & $\mathbf{N} \mathbf{W}$. & .... \\
\hline ". & 6 & 46 & II & $61^{\circ}$ & $61^{\circ}$ & $54^{\circ}$ & $53.8^{\circ}$ & 63 & 63 & $70.5^{\circ}$ & & “ & $\cdots$ \\
\hline 18 & 6ᄋ & " & III & $61^{\circ}$ & $61^{\circ}$ & $53^{\circ}$ & $52.9^{\circ}$ & 58 & 58 & 71 & " & " & ..... \\
\hline ، & 6 & " & IV & $61^{\circ}$ & $61^{\circ}$ & $54^{\circ}$ & $53.9^{\circ}$ & 63 & 63 & $69^{\circ}$ & " & “ & \\
\hline Nor. & 1 & $5: 30$ & $I$ & $50.5^{\circ}$ & & $45^{\circ}$ & $\ldots$. & 64 & $\ldots$ & $56^{\circ}$ & SS 1 & $\ldots$ & $\ldots$ \\
\hline “ & 1 & & II & $47^{\circ}$ & & $43^{\circ}$ & $\ldots \ldots$ & 72 & $\ldots$ & $53^{\circ}$ & " & ...... & $\cdots$ \\
\hline “ & 1 & “ & III & $48^{\circ}$ & & $43^{\circ}$ & $\ldots$ & 66 & $\ldots$ & $54^{\circ}$ & $"$ & $\ldots \ldots$ & $\ldots \ldots$ \\
\hline " & 1 & a & $\mid \boldsymbol{I V}$ & $46.5^{\circ}$ & & $43^{\circ}$ & & 75 & $\ldots$ & & “ & & \\
\hline . & 2 & " & I & $53^{\circ}$ & & $47.8^{\circ}$ &. & 66 & .... & $56^{\circ}$ & " & S. & \\
\hline "، & 2 & ،" & II & $50^{\circ}$ & & $45^{\circ}$ & . . & 67 & $\ldots$ & $55^{\circ}$ & " & $" 1$ & $\cdots$ \\
\hline “ & 2 & "، & III & $50.5^{\circ}$ & & $46.5^{\circ}$ & $\ldots$ & 74 & $\ldots$ & & " & “" & \\
\hline c6 & 2 & " & IV & $50.5^{\circ}$ & & $46^{\circ}$ & & 70 & & & " & " & \\
\hline a & 3 & " & $I$ & $63^{\circ}$ & & $54^{\circ}$ & & 56 & & & 4 & "1 & \\
\hline “، & 3 & “ & II & $59^{\circ}$ & & $53^{\circ}$ & $\cdots$ & 67 & & $5 t$ & " & " & $\cdots$ \\
\hline & 3 & "6 & III & $62^{\circ}$ & & $54^{\circ}$ & & 59 & $\ldots$ & $54.5^{\circ}$ & " & " & \\
\hline “. & 3 & "1 & IV & $61^{\circ}$ & & $53.9^{\circ}$ & & 63 & & $57.5^{\circ}$ & " & “ & \\
\hline Dec. & 4 & $4-5: 00$ & I & $46^{\circ}$ & & $38^{\circ}$ & & 46 & .... & $40^{\circ}$ & SS 16 & N.W. & .. \\
\hline & 4) & " & II & $44^{\circ}$ & & $38^{\circ}$ & & 57 & & & 6 & " & \\
\hline “6 & 4 & " & III & $45^{\circ}$ & & $38^{\circ}$ & & 51 & & & 6 & " & \\
\hline " & 4 & " & IV & $44^{\circ}$ & & $38^{\circ}$ & & 57 & & & " & " & \\
\hline .6 & 5 & 46 & $\mathbf{I}$ & $43^{\circ}$ & & $38.5^{\circ}$ & & 66 & .... & $39.5^{\circ}$ & " & S.E. & \\
\hline “. & 5 & "6 & III & $41^{\circ}$ & & $37^{\circ}$ & & 69 & & $39.5^{\circ}$ & "“ & " & \\
\hline 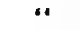 & 6 & “ & III & $42^{\circ}$ & & $39^{\circ}$ & & 77 & .... & $38.5^{\circ}$ & "s & " & \\
\hline$" \leadsto$ & 5] & “" & $|I V|$ & $\left|41.8^{\circ}\right|$ & & $\mid 38^{\circ}$ & & 69 & & $39^{\circ}$ & $"$ & " & $1 \ldots$ \\
\hline
\end{tabular}




\section{ECOLOGICAL FACTORS-Conciuded}

\section{TEMPERATURE AND ATMOSPHERB}

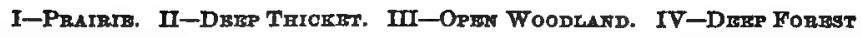

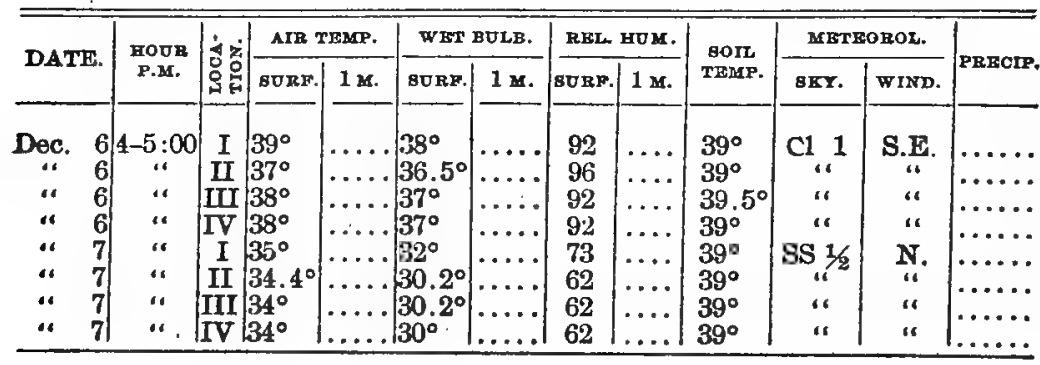


PHYSICAL WATER-CONTENT

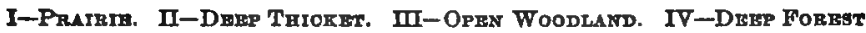

\begin{tabular}{|c|c|c|c|c|c|c|}
\hline & DATE & $\begin{array}{l}\text { LOOA- } \\
\text { TYON }\end{array}$ & $\begin{array}{l}\text { TOTAL } \\
\text { WIGET }\end{array}$ & $\begin{array}{c}\text { WEIGHT' AFTERR } \\
\text { DRYING }\end{array}$ & $\begin{array}{l}\text { WATER- } \\
\text { CONTEAT }\end{array}$ & $\underset{\text { PENT }}{\text { PER }}$ \\
\hline March & 27. & $\mathrm{I}$ & $18.715 \mathrm{~g}$ & $14.220 \mathrm{~g}$ & $4.495 \mathrm{~g}$ & 24 \\
\hline .6 & $27, \ldots \ldots \ldots \ldots$ & II & $23.570^{\circ}$ & $16.975^{\circ}$ & 6.595 & 28 \\
\hline “ & $27 \ldots \ldots \ldots \ldots \ldots$ & III & 20.330 & 13.490 & 6.840 & 34 \\
\hline “4 & $27 \ldots \ldots \ldots \ldots \ldots$ & IV & 21.125 & 14.150 & 6.975 & $\mathbf{3 3}$ \\
\hline “ & $30 \ldots \ldots \ldots \ldots \ldots$ & $I$ & 17.590 & 14.800 & 3.790 & 22 \\
\hline " & $30 \ldots \ldots \ldots \ldots \ldots$ & II & 17.645 & 12.250 & 5.395 & 31 \\
\hline “ & ......... & III & 16.945 & 12.585 & 4.360 & 26 \\
\hline “ & $30 \ldots \ldots \ldots \ldots \ldots$ & IV & 20.535 & 14.915 & 5.620 & 27 \\
\hline April & $16 \ldots$ & I & 22.790 & 17.870 & 4.920 & 22 \\
\hline 10 & $16 \ldots$ & II & 17.395 & 11.000 & 6.395 & 37 \\
\hline " & $16 \ldots \ldots \ldots \ldots \ldots$ & III & 22.360 & 16.570 & 5. 790 & 26 \\
\hline " & $16 \ldots \ldots \ldots \ldots \ldots$ & IV & 21.205 & 15.855 & 5.350 & 25 \\
\hline “4 & $20 \ldots \ldots \ldots \ldots$ & I & 13.975 & 12.015 & 1.960 & 14 \\
\hline “ & $20 \ldots$ & II & 19.960 & 14.600 & 5.30 & 27 \\
\hline " & $20 \ldots$ & III & 15.930 & 12.220 & 3.715 & 23 \\
\hline " & $20 \ldots \ldots \ldots \ldots \ldots$ & IV & 19.455 & 13.485 & 5.970 & 31 \\
\hline May & $16 \ldots \ldots \ldots \ldots$ & $I$ & 23.715 & 18.795 & 4.920 & 21 \\
\hline .6 & $16 \ldots \ldots \ldots \ldots \ldots$ & $\tilde{\Pi}$ & 18.605 & 12.375 & 6.230 & 33 \\
\hline "A & $16 \ldots \ldots \ldots \ldots \ldots$ & III & 24.835 & 17.525 & 7.310 & 29 \\
\hline “ & $16 \ldots \ldots \ldots \ldots$ & IV & 19.850 & 13.030 & 6.820 & 34 \\
\hline .• & $19, \ldots \ldots \ldots \ldots$ & $I$ & 17.470 & 15.840 & 1.630 & 9 \\
\hline$"$ & $19 \ldots \ldots \ldots \ldots \ldots$ & $\overline{\text { II }}$ & 19.045 & 14.935 & 4.110 & 22 \\
\hline " & $19 \ldots \ldots \ldots \ldots$ & III & 23.170 & 17.625 & 5. 545 & 24 \\
\hline$\because$ & $19 \ldots \ldots \ldots \ldots \ldots$ & IV & 18.220 & 13.945 & 4.275 & 23 \\
\hline Jnine & $19 \ldots \ldots \ldots \ldots$ & $I$ & $20.7: 0$ & 17.890 & 2.830 & 14 \\
\hline " & $19 \ldots \ldots \ldots \ldots$ & II & 24.115 & 16.085 & 8.030 & 33 \\
\hline "1 & $19 \ldots \ldots \ldots \ldots \ldots$ & III & 21.635 & 16.435 & 5.200 & 24 \\
\hline “ & $19 \ldots \ldots \ldots \ldots \ldots$ & IV & 22.285 & 15.135 & 7.150 & 32 \\
\hline “ & $22 \ldots \ldots \ldots$ & 1 & 18.040 & 16.550 & 1.490 & 8 \\
\hline .4 & 22. & $\overline{\text { II }}$ & 19.585 & 15.585 & 4.000 & 21 \\
\hline "4 & $22 \ldots$ & III & 17.010 & 13.600 & 3.410 & 20 \\
\hline “، & $22 \ldots$ & IV & 19.145 & 14.065 & 5.080 & 27 \\
\hline “" & $26 \ldots \ldots \ldots$ & $I$ & 16.845 & 16.745 & 0.100 & 1 \\
\hline "6 & $26 \ldots \ldots \ldots \ldots \ldots$ & $\overline{\text { II }}$ & 18.365 & 15.665 & 2.700 & 15 \\
\hline “ & $26 \ldots \ldots \ldots \ldots \ldots$ & III & 17.865 & 15.183 & 2.682 & 15 \\
\hline “ & $26 \ldots \ldots \ldots \ldots$ & IV & 17.970 & 15.580 & 2.390 & 13 \\
\hline July & $14 \ldots \ldots \ldots \ldots \ldots$ & $\mathrm{I}$ & 15.365 & 14.525 & 0.840 & 5 \\
\hline & $14 \ldots \ldots \ldots \ldots \ldots$ & II & 14.665 & 11.515 & $\mathbf{3 . 1 5 0}$ & 22 \\
\hline “ & $14 \ldots \ldots \ldots \ldots \ldots$ & III & 14.365 & 13.020 & 1.345 & 9 \\
\hline .. & $14 \ldots \ldots \ldots \ldots \ldots$ & IV & 16.965 & 14.385 & 2.580 & 15 \\
\hline . & $16 \ldots \ldots \ldots \ldots \ldots$ & I & 23.740 & 17.390 & 6.350 & 27 \\
\hline “4 & $16 \ldots \ldots \ldots \ldots \ldots$ & $\overline{\mathbf{H}}$ & 21.445 & 13.835 & 7.615 & 36 \\
\hline " & $16 \ldots \ldots \ldots \ldots$ & III & 24.085 & 16.335 & 7.750 & 32 \\
\hline ". & $16 \ldots \ldots \ldots$ & IV & $24: 420$ & 16.275 & 8.150 & 33 \\
\hline “4 & $21 \ldots \ldots \ldots \ldots \ldots$ & $I$ & 18.720 & 15.270 & $\mathbf{3 . 4 5 0}$ & 18 \\
\hline “ & $21 \ldots \ldots \ldots \ldots$ & II & 19.475 & 12.645 & 6.830 & 35 \\
\hline 4" & 21. & III & 21.115 & 15.265 & 5.850 & 28 \\
\hline .1 & $21 \ldots \ldots \ldots \ldots$ & $\overline{\mathrm{IV}}$ & 21.245 & 15.855 & 5.390 & 25 \\
\hline
\end{tabular}




\section{PHYSICAL WATER-CONTENT-Continued}

I-Praikin. II-Dhep Thicket. III-Opan Wooduand. IV-Danp Fonest

\begin{tabular}{|c|c|c|c|c|c|c|}
\hline & DATE & $\begin{array}{l}\text { LOCA: } \\
\text { TION }\end{array}$ & $\begin{array}{l}\text { TOTAL } \\
\text { WEIGHT }\end{array}$ & $\begin{array}{c}\text { WEIGHT AFTER } \\
\text { DEYING }\end{array}$ & $\begin{array}{l}\text { TATER- } \\
\text { CONTERT }\end{array}$ & $\begin{array}{l}\text { PEE } \\
\text { CENT }\end{array}$ \\
\hline August & $14 \ldots \ldots \ldots \ldots$ & I & $19.615 \mathrm{~g}$ & $14.935 \mathrm{~g}$ & $4.680 \mathrm{~g}$ & 24 \\
\hline "1 & $14 \ldots \ldots \ldots \ldots$ & II & $91.415^{\circ}$ & $16.545^{\circ}$ & $4.870^{\circ}$ & 23 \\
\hline 4 & $14 \ldots \ldots \ldots \ldots$ & III & 24.185 & 18.685 & 5.500 & 23 \\
\hline$\because 1$ & $14 \ldots \ldots \ldots$ & IV & 21.635 & 15.445 & 6.190 & 29 \\
\hline "، & $17 . . . \ldots \ldots$ & I & 19.020 & 13.530 & 5.490 & 21 \\
\hline 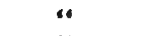 & $17 \ldots \ldots \ldots \ldots$ & II & 22.925 & 15.185 & 7.740 & 34 \\
\hline “ & $17 \ldots \ldots \ldots \ldots$ & III & 21.360 & 15.380 & 5.980 & 28 \\
\hline “ & $17 \ldots \ldots \ldots \ldots$ & IV & 20.305 & 13.435 & 6.870 & 34 \\
\hline$\because$ & $22 \ldots \ldots \ldots \ldots$ & $I$ & 22.085 & 18.015 & 4.070 & 19 \\
\hline$\because 1$ & $22 \ldots \ldots \ldots \ldots$ & II & 21.770 & 15.070 & 6.700 & 31 \\
\hline “ & $22 \ldots \ldots \ldots \ldots$ & III & 21.725 & 15.150 & 6.570 & 30 \\
\hline “ & $22 \ldots \ldots \ldots \ldots$ & IV & 24.130 & 17.220 & 6.910 & 29 \\
\hline September & $22 \ldots \ldots \ldots \ldots$ & I & 18.600 & 15.300 & $\mathbf{3 . 3 0 0}$ & 18 \\
\hline .4 & $22 \ldots \ldots \ldots \ldots$ & $\overrightarrow{\text { II }}$ & 25.045 & 17.765 & 7.280 & 29 \\
\hline 11 & $22 \ldots \ldots \ldots \ldots$ & III & 19.325 & 13.835 & 5.490 & 28 \\
\hline$\because$ & $22 \ldots \ldots \ldots \ldots$ & $\mathrm{IV}$ & 19.715 & 13.085 & 6.680 & 34 \\
\hline$“$ & $25 \ldots \ldots \ldots$ & I & 19.700 & 14.150 & 5.550 & 28 \\
\hline “ & $25 \ldots \ldots$ & II & 22.315 & 16.070 & 6.245 & 28 \\
\hline 11 & $25 \ldots \ldots \ldots \ldots$ & III & 19.755 & 15.665 & 4.090 & 21 \\
\hline 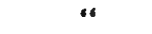 & $25 \ldots \ldots \ldots \ldots$ & IV & 24.225 & 17.815 & 6.410 & 26 \\
\hline ar & $29 \ldots \ldots \ldots \ldots$ & I & 21.070 & 16.010 & 5.000 & 24 \\
\hline “ & $29 \ldots \ldots \ldots$ & $\overline{\mathrm{II}}$ & 23.295 & 15.895 & 7.400 & 32 \\
\hline “4 & $29 \ldots \ldots \ldots \ldots$ & III & 23.710 & 17.970 & 5.740 & 24 \\
\hline “ & $29 \ldots \ldots \ldots \ldots$ & IV & 22.375 & 15.365 & 7.010 & 31 \\
\hline October & $13 \ldots \ldots \ldots \ldots$ & I & 18.790 & 15.420 & 3.370 & 18 \\
\hline if & $13 \ldots \ldots \ldots$ & II & 20.095 & 14.215 & 5.880 & 29 \\
\hline “ & $13 \ldots \ldots \ldots \ldots$ & III & 18.400 & 15.400 & 3.000 & 16 \\
\hline “ & $13 \ldots \ldots \ldots \ldots$ & IV & 18.475 & 14.985 & 3.490 & 10 \\
\hline “ & $16 \ldots \ldots \ldots \ldots$ & I & 17.545 & 14.515 & 3.030 & 18 \\
\hline$"$ & $16 \ldots \ldots \ldots \ldots$ & II & 21.915 & 16.665 & 5.250 & 24 \\
\hline “ & $16 \ldots \ldots \ldots$ & III & 19.155 & 15.965 & 3.190 & 17 \\
\hline$"$ & $16 \ldots \ldots \ldots$ & IV & 17.160 & 14.030 & 3.130 & 18 \\
\hline a & $19 \ldots \ldots \ldots$ & I & 17.760 & 14.030 & 3.730 & 21 \\
\hline$\because$ & $19 . \ldots \ldots \ldots$ & II & 20.865 & 16.535 & 4.3930 & 21 \\
\hline 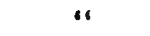 & $19 \ldots \ldots \ldots$ & III & 17.830 & 14.900 & 2.930 & 16 \\
\hline 4 & $19 \ldots \ldots \ldots$ & IV & 20.485 & 15.165 & 5.320 & 26 \\
\hline November & $20 \ldots \ldots \ldots$ & I & 24.265 & 18.285 & 5.980 & 25 \\
\hline 14 & $20 \ldots \ldots \ldots$ & II & 23.910 & 16.860 & 7.050 & 29 \\
\hline 4 & $20 \ldots \ldots \ldots \ldots$ & III & 21.415 & 14.465 & $6.8 \div 0$ & 32 \\
\hline “ & $20 \ldots \ldots \ldots \ldots$ & IV & 28.060 & 20.820 & 7.240 & 26 \\
\hline$"$ & $24 \ldots \ldots \ldots \ldots$ & I & 23.405 & 17.815 & 5.590 & 23 \\
\hline$“$ & $24 \ldots \ldots \ldots \ldots$ & II & 26.115 & 18.925 & 7.190 & 28 \\
\hline$\because$ & $24 \ldots \ldots \ldots \ldots$ & III & 22.585 & 16.665 & 5.920 & 26 \\
\hline " & $24 \ldots \ldots \ldots \ldots$ & IV & 21.930 & 15.600 & 6.3350 & 29 \\
\hline “ & $27 \ldots \ldots \ldots \ldots$ & I & 18.680 & 15.380 & 3.300 & 18 \\
\hline$\because$ & $27 \ldots \ldots \ldots \ldots$ & II & 21.795 & 16.645 & 5.150 & 24 \\
\hline 4 & $27 \ldots \ldots \ldots \ldots$ & IIII & 25.360 & 20.230 & 5.130 & 20 \\
\hline “ & $27 \ldots \ldots \ldots$ & IV & 22.780 & 16.630 & 6.150 & 27 \\
\hline
\end{tabular}




\section{DISCUSSION OF ECOLOGICAL FACTORS}

\section{PHYSICAL WATER-CONTENT-Concluded}

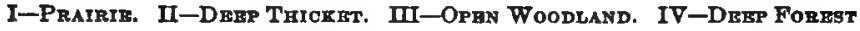

\begin{tabular}{|c|c|c|c|c|c|c|}
\hline & DATE & $\begin{array}{l}\text { LOCA- } \\
\text { TION }\end{array}$ & $\begin{array}{c}\text { TOTAL } \\
\text { WHIGHY }\end{array}$ & $\underset{\text { DRYIGET AFTRER }}{\text { DRYING }}$ & $\begin{array}{l}\text { WATER- } \\
\text { CONTENT }\end{array}$ & $\begin{array}{l}\text { PER } \\
\text { CBNT }\end{array}$ \\
\hline December & & I & $19.765 \mathrm{~g}$ & $15.475 \mathrm{~g}$ & $4.290 \mathrm{~g}$ & 22 \\
\hline . & $1 \ldots$ & II & $25.945^{8}$ & $20.115^{8}$ & $5.740^{\circ}$ & $\overline{22}$ \\
\hline$\leadsto$ & $1 \ldots$ & III & 26.135 & 20.065 & 6.070 & 23 \\
\hline . & 1. & IV & 23.305 & 16.805 & 6.500 & 28 \\
\hline " & $5 .$. & I & 21.420 & 16.820 & 4.600 & 21 \\
\hline “ & $5 .$. & II & 23.315 & 17.535 & 5.780 & 25 \\
\hline .4 & $5 .$. & III & 22.585 & 17.315 & 5.270 & 23 \\
\hline " & $5 \ldots$ & IV & 22.945 & 17.485 & 5.460 & 24 \\
\hline
\end{tabular}




\section{LIGHT INTENSITY}

I-Prarryg. II-D hesp Thickex. III-Ophy Woodtakd. IV-Dhep Formst

\begin{tabular}{|c|c|c|c|c|c|c|}
\hline \multicolumn{2}{|c|}{ DATE } & \multirow{3}{*}{$\begin{array}{c}\begin{array}{c}\text { HoURS } \\
\text { P.M. }\end{array} \\
1: 30 \\
1: 40\end{array}$} & \multirow{2}{*}{$\frac{\begin{array}{c}\text { LOOA- } \\
\text { TION }\end{array}}{I}$} & \multirow{3}{*}{$\frac{\text { BEY OONDITIOK }}{\text { Clear }}$} & \multirow{2}{*}{$\begin{array}{c}\text { LENATH OF } \\
\text { EXPOSORA } \\
\text { IN SHCONDS } \\
60\end{array}$} & \multirow{2}{*}{$\frac{\text { Fatio* }}{100}$} \\
\hline March & 31. & & & & & \\
\hline ". & $31 \ldots$ & & II & & 95 & 62 \\
\hline “" & $31 \ldots .$. & $1: 50$ & III & " & 75 & 80 \\
\hline 4 & 31. & $2: 00$ & IV & " & 90 & 66 \\
\hline April & $21 \ldots \ldots$ & $2: 00$ & I & Rather hazy & 80 & 75 \\
\hline "4 & $21 \ldots$ & $2: 10$ & II & "4 & 150 & 40 \\
\hline .4 & 21.. & $2: 20$ & III & " & 90 & 66 \\
\hline '“ & 21 . & $2: 30$ & $\overline{I V}$ & as & 120 & 50 \\
\hline May & 12. & $1: 00$ & I & Clear & 50 & 111 \\
\hline "“ & $12 \ldots \ldots$ & $1: 10$ & II & " & 480 & 12 \\
\hline$" 1$ & $12 \ldots \ldots$ & $1: 20$ & III & 16 & 160 & 37 \\
\hline “ & 12. & $1: 30$ & IV & a & 420 & 14 \\
\hline June & $6 \ldots \ldots$ & $1: 30$ & $\mathbf{I}$ & “ & 48 & 125 \\
\hline “ & $6 \ldots \ldots$ & $1: 40$ & II & “" & $3900 \dagger$ & 1.5 \\
\hline “ & $6 .$. & $1: 50$ & III & '6 & 180 & 33 \\
\hline$"$ & $6 \ldots$ & $2: 00$ & IV & “ & 1380 & 4 \\
\hline 11 & $20 \ldots \ldots$ & $2: 00$ & I & " & 45 & 133 \\
\hline “ & 20 . & $2: 10$ & II & “" & 10800 & 0. \\
\hline$"$ & $20 .$. & $2: 20$ & III & “ & 240 & 25 \\
\hline$"$ & 20. & $2: 30$ & $\overline{I V}$ & $\because$ & 1500 & 4 \\
\hline July & 9. & $2: 00$ & I & “ & 47 & 128 \\
\hline 16 & $9 .$. & $2: 10$ & II & "s & 11700 & 0. \\
\hline$“$ & $9 . \ldots$. & $2: 20$ & III & " & 210 & 28 \\
\hline " & $9 \ldots \ldots$ & $2: 30$ & IV & 4 & 1020 & 6 \\
\hline $1 "$ & $27 \ldots \ldots$ & $1: 00$ & I & $"$ & $50-45$ & 111 \\
\hline “ & 27. & $1: 10$ & II & " & $11700-11400$ & 0. \\
\hline “ & $27 \ldots \ldots$ & $1: 20$ & III & $" 6$ & $270-240$ & 23 \\
\hline " & $27 \ldots \ldots$ & $1: 30$ & IV & " & $1200-1020$ & 0.5 \\
\hline August & $20 \ldots \ldots$ & $1: 00$ & I & “ & $53-49$ & 111 \\
\hline a & $20 \ldots \ldots$ & $1: 10$ & II & $\because$ & $10800-9300$ & 0.5 \\
\hline$" 1$ & $20 \ldots \ldots$ & $1: 20$ & III & “ & $360-315$ & 16 \\
\hline " & $20 \ldots \ldots$ & $1: 30$ & IV & " & $1350-1110$ & 4 \\
\hline Septembe & r $8 \ldots \ldots$ & $1: 00$ & I & Slightly Cloudy & $60-53$ & 100 \\
\hline " " & $8 \ldots \ldots$ & $1: 10$ & II & "1 & $6600-6000$ & 0.8 \\
\hline “ & $8 \ldots \ldots$ & $1: 20$ & III & “ & $240-210$ & 25 \\
\hline “ & $8 \ldots \ldots$ & $1: 30$ & IV & “ & $720-720$ & 8 \\
\hline October & $13 \ldots \ldots$ & 1:00 & I & Clear & $46-44$ & 133 \\
\hline " & $13 \ldots \ldots$ & $1: 10$ & II & 16 & $600-480$ & 10 \\
\hline " & $13 \ldots \ldots$ & $1: 20$ & III & i & $110-90$ & 55 \\
\hline 11 & $13 \ldots \ldots$ & $1: 30$ & IV & $“$ & $347-300$ & 16 \\
\hline Novembe & r $3 \ldots \ldots$ & 1:00 & $I$ & 16 & $48-48$ & 125 \\
\hline " & 3..... & $1: 10$ & II & " & $140-80$ & 50 \\
\hline “" & $3 \ldots \ldots$ & $1: 20$ & III & $\because$ & $90-81$ & 66 \\
\hline " & $3 \ldots \ldots$ & $1: 30$ & IV & " & $180-180$ & 33 \\
\hline
\end{tabular}

* For comparison the light on the prairie for March 31 is taken as 100 .

$t$ At the pud of 65 minutes the lines were still visible. 
The influence of the more important ecological factors upon the prairie formation is briefly discussed in the following pages. The prairie upon which the formational work was conducted lies about three miles west by south of Nebraska City, along the Burlington railroad. It measures 800 meters on a side and has an elevation of 305 meters above sea-level. The highest point is located south of the center on the most important ridge called, for convenience, Great ridge. This elevation, which is an extension of higher land lying to the south, passes north and northeast through the formation, dividing it into two unequal portions, and terminates abruptly in the northeast corner of the prairie. In its course it gradually decreases in altitude and gives off to the northwest and east secondary ridges of note. The lowest point, exclusive of the creek-bed, is northwest from the center in the meadow formation. The difference of elevation between the highest and lowest points is, by actual measurements, 24 meters.

On the south of this prairie lies the regular section road, beyond which are cultivated fields; to the east and west also are culture formations, and a pasture and railroad bounds the north. A hedge of Toxylon pomiferum (osage orange) forms a dividing line on the east between prairie and field for 400 meters, and for the same distance on the north between prairie and pasture. This hedge serves as a basis for woodland species to gain access to the prairie.

The surface as a whole gradually inclines to the north and is rolling, a character due entirely to the erosive action of water. The slopes of the south are more gradual than those of the north; likewise those of the east are less abrupt than those of the west. South Table creek flows through the northern half of the prairie, cutting off perhaps ten or twelve hectares from the main body. It enters midway on the west, meanders east by northeast, and leaves at the northeast corner. The banks of this creek in many places are steep, ranging from six to twelve meters high, and generally 
covered with a thicket-like growth of Rhus, Corylus, Cornus, and, lower down, Salix, scattered here and there with a few large specimens of Populus. Several ravines of lesser importance flow into this creek from either side. Through the eastern and the western borders of this prairie, wide draws pass from the south, tend northward, and empty into Table creek. " The draw on the west broadens out, as it approaches the creek, and loses itself, thus giving rise to a typical meadow formation. On the east, the swale-like draw lies at the foot of Great ridge and makes possible the one wet meadow formation, which is in extent $400 \times 100$ meters. Immediately after excessive precipitation, large volumes of water from the surrounding region pass through these draws.

The above description, with a careful study of the contour map, conveys a fairly accurate idea of the prairie formation under discussion. The extreme diversity of elevation, the various waterways, and the direction and degree of the numerous slopes and exposures exert a pronounced influence upon the other controlling physical factors, viz., light, soil and air temperatures, and water-content. Since physiographical factors, indirectly, produce favorable conditions for one species and unfavorable conditions for another by influencing the other physical factors, they are largely controlling in the migration and distribution of species in a prairie formation, as will be pointed out from time to time. It can readily be seen that the ecological factors of light, soil and air temperatures, and water-content exert a far greater influence upon this formation than all other physical and biological factors combined. The crests and southern exposures of ridges maintain maximum soil and atmospheric temperatures and light, while other slopes, northern and eastern or western, maintain respectively minimum and medium temperature and light conditions. Northern slopes never receive maximum heat and light from the sun; they are

\footnotetext{
* Table creek for the most part is enclosed for pasture, as the map indicates, and hence will not appear in the discussion hereafter.
} 
the last to recover from a state of winter; their conditions favoring growth are retarded more by north winds and accelerated less by south winds than those of any other exposure. It is not surprising, then, that we find their prevernal and vernal bloomers 7-17 days behind those of southern exposures. The soil of crests and steep, exposed slopes of ridges contains the least amount of water-content on account of excessive drainage and evaporation; that of draws and meadow formations the largest amount. Other things being equal, northern exposures lead all others in water-content. The more extensive vegetative covering of the meadow formations as compared with that of the ridges will undoubtedly exert some infiuence upon soil temperatures and water-content.

The soil about Nebraska City is largely of glacial origin overlaid with a rich deposit of loess. It is very porous, darkgray in color, and of a light, pulverulent, uniform texture, particularly rich in organic matter and potash. The general physical properties of the soil correspond with the characteristics of sandy loam. Porous soil, especially when sandy, has a great capacity for water and air. The water falling upon the surface will largely be absorbed, the water-content of the soil will be accordingly increased, thus reacting upon the fioral covering, and erosion will be reduced to a minimum. It is estimated that at least 90 per cent of all water falling upon this soil is absorbed.

Through the courtesy of J. Sterling Morton, under whose direction the work was carried on, we are permitted to publish the following chemical analysis of soil collected about Nebraska City, made in 1873 by Charles A. Goessmann, Professor of Chemistry, Massachusetts Agricultural College. "The soil lost, when kept over dry calcium chloride, 1.6 per cent of water; it absorbed, in an air saturated with moisture at $56^{\circ} \mathrm{F}$., 5.04 per cent of moisture; when dried at $212^{\circ} \mathrm{F}$., it lost 4.05 per cent of moisture; upon being calcined, it losi 
13.08 per cent of its weight, consisting mainly of water and organic matter.

"One thousand parts of the soil treated with 2,000 parts water produced a solution which left, at $212^{\circ}$ to $220^{\circ} \mathrm{F}$., a residue equal to 1.184 parts, which consisted of .414 parts of ash constituents and .770 parts of organic and volatile matter.

"One thousand parts of the soil, being treated with hydrochloric acid of 1.12 specific gravity, produced a solution which contained as follows:

Potassa ..................2.3259 parts

Soda ..................... 0.2647 parts

Lime ...............6.6.4283 parts

Magnesia ................ 2.7237 parts

Sesquioxide of iron, with traces of

Manganese oxide..........44.8332 parts

Alnmina ................. 1.4375 parts

Phosphoric acid............. 0.6660 parts

Sulphuric acid............. 0.5792 parts

"Quantitative analytical determinations of nitric acid, ammonia, and organic matter (humus) have not been carried out." 


\section{ENUMERATION OF SPECIES IN THE FORMATION}

\section{ELEMENTS PROPER TO THE PRAIRIE FORMATION}

Acerates viridiflora ivesii.

$\Lambda$. viridifiora linearis.

Agrostis hiemalis.

Allionia nyctaginea ovata.

Amorpha canescens.

Andropogon furcatus.

A. scoparius.

Androsace occidentalis.

Anemone caroliniana.

A. cylindrica.

Antennaria campestris. Artemisia gnaphalodes. Asclepias obtusifolia.

A. syriaca.

A. tuberosa.

A. verticillata.

Aster multiflorus.

A. oblongifolius.

A. sericeus.

Astragalus crassicarpus.

Baptisia bracteata.

Bouteloua curtipendula.

Brauneria pallida.

Carex meadii.

C. pennsylvanica.

Ceanothus ovatus.

Comandra umbellata.

Coreopsis palmata.

Delphinium carolinianum.
Eatonia obtusata.

Eragrostis pectinacea.

Erigeron ramosus.

Euphorbia corollata.

Gentiana puberula.

Helianthus maximiliani.

H. scaberrimus.

Hieracium longipilum.

Koeleria cristata.

Kuhnistera candida.

K. purpurea.

Kuhnia eupatoroides.

Lespedeza capitata.

Laciniaria scariosa.

L. squarrosa.

Linum sulcatum.

Lithospermum canescens.

L. angustifolium.

Meibomia illinoensis.

Mesadenia tuberosa.

Nabalus asper.

Onosmodium mollc.

Oxalis violacea.

Panicum depauperatum.

P. pubescens.

P. scribnerianum.

Phlox pilosa.

Physalis heterophylla.

P. virginiana var. 
Polygala verticillata.

Psoralea argophylla.

P. esculenta.

P. tenuiflora.

Rosa arkansana.

Ruellia ciliosa.

Salix humilis.

Senecio plattensis.

S. integerrimus.

Silphium laciniatum.

Sisyrinchium angustifolium.
Solidago memoralis.

S. rigida.

S. rigidiuscula.

S. rupestris.

Sporobolus asper.

S. heterolepis.

Stipa spartea.

Verbena stricta.

Vernonia gigantea.

V. fasciculata.

Viola pedatifida.

ELEMENTS PECULIAR TO RAVINE, MEADOW, AND SWAMP

Anemone canadensis.

Apocynum cannabinum.

Asclepias incarnata.

A. sullivantii.

Aster paniculatus.

Baptisia leucantha.

Calamagrotis canadensis.

Carex cephaloidea.

C. festucacea.

C. stricta.

C. vulpinoidea.

Chrysopogon avenaceus.

Cicuta maculata.

Cuscuta paradoxa.

Eleocharis palustris.

Elymus canadensis.

Equisetum arvense.

E. laevigatum.

Euthamia graminifolia.

Falcata pitcheri.

Galium tinctorum.

Gyrostachys cernua.
Habenaria bracteata.

H. leucophaea.

Helenium autumnale. Helianthus giganteus.

H. grosse-serratus.

Juncus tenuis.

Laciniaria pycnostachya.

Lilium canadense.

Lobelia spicata hirtella.

Lycopus americanus.

Lythrum alatum.

Mentha canadensis.

Muhlenbergia racemosa.

Panicum virgatum.

Phalaris arundinace.

Physalis longifolia.

Polygonum emersum.

Oxalis stricta.

Rumex altissimus.

Salix fluviatilis.

Scirpus atrovirens.

Scutellaria laterifiora. 
S. parvula.

Silphium integrifolium.

Solidago canadensis.

Spartina cynosuroides.

Steironema ciliatum.

Teucrium occidentale.

Thalictrum purpurascens.
Tradescantia virginiana.

Tripsacum dactyloides.

Verbena hastata.

Vicia americana.

Viola obliqua.

Zizia aurea.

ELEMENTS DERIVED FROM THICKETS AND WOODLANDS

Acalypha virginica.

Aster azureus.

A. levis.

Astragalus carolinianus.

Carduus altissimus.

Cornus asperifolius.

Corylus americana.

Fraxinus lanceolata.

Felianthus tuberosus.

Heliopsis scabra.

Lactuca canadensis.
Meibomia canadensis.

Monarda fistulosa.

Quercus macrocarpa.

Ratibida pinnata.

Rhus glabra.

12. radicans.

Sambucus canadensis,

Silene stellata.

Silphium perfoliatum.

Teucrium canadense.

ADVENTIIIOUS ELEMENTS AND OTHER RUDERAL SPECIES

Acnida tamariscina.

Agrostis alba.

Amaranthus graecizans.

A. torreyi.

Ambrosia trifida.

Cassia chamaecrista.

Chaetochloa viridis.

C. glauca.

Chenopodium album.

Convolvulus sepium.

Euphorbia glyptosperma.

E. maculata.

E. nutans.

Helianthus annuus.

Iva xanthifolia.
Lactuca canadensis.

L. ludoviciana.

L. pulchella.

Onagra biennis.

Panicum capillare.

Phleum pratense.

Plantago major.

P. rugellii.

Poa pratensis.

Polygonum pennsylvanicum.

Potentilla monspeliensis.

Rumex crispus.

Solanum carolinense.

Trifolium pratense.

T. repens. 


\section{STRUCTURE OF THE FORMATION}

Within the limits of this prairie one hundred and eightyeight species are noted, of which eighty are elements proper to the prairie formation, fifty-seven are peculiar to ravine, meadow, and swamp, twenty-one are elements derived from woodlands and thickets, and thirty are adventitious or ruderal species. When the diversity of the formation is considered, the facies are relatively few in number. Andropogon scoparius is the important facies on the slopes and crests of ridges and is always accompanied by $A$. furcatus, which ranks next in importance. Throughout its entire range $A n$ dropogon scoparius resorts to the "bunch" habit but once or twice, viz., on steep, exposed, gravelly slopes to the west and southwest of Great ridge. Everywhere else it plays the rôle of a "sod-former," giving rise to a closed formation. Toward the foot of ridges and in low prairie, Andropogon scoparius and $A$. furcatus become of equal importance. Bouteloua curtipendula and Kocleria cristata are also facies. Frequently Bouteloua does not blossom, in which case it is likely to be confused with Andropogon. Stipa spartea becomes a facies in but one location in low prairies, where for a few hectares it is almost exclusive. In the meadow formation, Andropogon furcatus attains its maximum in size and numbers. In somestationsitbecomes thesole facies; again it shares equally with two tall-growing meadow grasses, Chrysopogon avenaceus and Panicum virgatum. Panicum is controlling in the wetter parts of the formation, in the beds of draws, etc. Spartina cynosuroides occurs in meadow formations sparingly scattered throughout, never in numbers sufficient to rank as an important facies. In the wet meadow formation Elcocharis palustris covers the ground with a dense carpet-like layer during May and June. This is soon hidden from view by the 
taller-growing Carex stricta and $C$. cephaloidca, and later by Spartina cynosuroides which frequently attains a height of three meters.

The distribution, alternation, and zonation of some of the more important species are worthy of note. Comandra umbellata commonly occupies crests and exposed slopes of ridges, where it often numbers 2,000 to the quadrat. Comandra is not continuous, however; it disappears entirely for a time, reappearing in greater numbers than before. Coreopsis palmata and Aster multiflorus inhabit crests of ridges and high prairies. These two species occur in copious, definite patches, which frequently alternate. The individuals of a patch are most numerous toward the center, frequently 2,500 to the five-meter quadrat, and gradually diminish in size and number as the margins are approached. The above species are rarely found in controlling numbers in the same quadrat; the one or the other ranks as copious 1 , the remaining ones being subordinate. Aster multifiorus also frequents meadows, where it forms rather definite patches of robust individuals. Alternation of species is beautifully shown in the case of Kuhnistera purpurea and $K$. candida. The explanation is evident. The former species is much more of a xerophyte and almost invariably grows on crests of ridges and abrupt or exposed slopes. About midway up the ridge, these two species are of equal importance, while a little lower down the white-flowered form will have almost completely displaced K. purpurea. Kuhnistera candida is a taller-growing plant and possesses a greater leaf surface than $K$. purpurea. Accordingly, it is not as well adapted to xerophytic conditions. The distribution of the blazing-stars is also largely dictated by the water-content, and if these plants were as abundant as Kuhnistera, conditions at least as striking would obtain. Laciniaria squarrosa is found on crests of high ridges, a typi-

* Alternation is here ueed in the sense in which it has been employed by Dr. Cloments, 1.e.. to apply to succession in snace of species and formations, though alternation of apecie日 is alone concerned in this place. The term "succession" is applied by the same authority to the phenomenon of euccession in time. 
cai xerophyte reluced in size of leaf surface. L. scariosa frequents low prairies and extends about half way up the ridges, while $L$. pycnostachya is a tall species, invariably inhabiting meadow formations. Baptisia bracteata and $B$. leucantha arrange themselves in concentric zones, ten to thirty meters wide, about draws and meadows and about ridges. The taller, glabrous form, $B$. leucantha, always occupies the moister zone, and it is seldom that one finds individuals of the one species within the zone of the other. $B$. bracteata is frequently found half way up the slopes. Erigeron ramosus finds the most favorable conditions on the tops of ridges and southern exposures. Euphorbia corollata is a gregario-copious plant, found mostly on ridges and slopes. It gradually diminishes in numbers as the foot of the ridge is approached, and hardly occurs at all in meadow formations.

Certain thicket and open woodland species have established themselves in the prairie and meadow formation. Among these are Aster azureus and A. levis, two gregario-copious, rosette-forming species, which thrive abundantly in low prairies. Corylus americana has gained a foothold at several locations in the formation, particularly near the creek and hedges. This species takes up its characteristic thicket growth, which makes possible the migration of such woodland forms as Ratibida pinnata, Silene stellata, Acalypha virginica, Helianthus tuberosus, Monarda fistulosa, and Heliopsis scabra. The invasion of Corylus in a prairie formation clearly demonstrates its superiority of occupation over the prairie species. With Corylus also are found a few specimens of Sambucus canadensis, Cornus asperifolia, Rhus glabra, Quercus macrocarpa, and Fraxinus lanceolata.

of the ruderal species the aianthous Cassia chamaecrista is the most conspicuous during its flowering period, as well as the most important in numbers. To the south along the section road, Cassia has pushed into the prairie formation twenty-five to thirty-five meters, and in many places the principal and secondary species have been crowded out; even the facies are subordinated, and to find them one has to look be- 
low the dense mass of yellow and green. In the vicinity of olu stock yards, Cassia immediately takes full possession of the denuded areas. Helianthus annuus and A mbrosia trifida attain considerable prominence along the wet meadow formation and in roadside ditches. Parts of the wet meadow formation from which the grass is cut several times during the year afford suitable location for such plants as Plantago major, $P$. rugellii, Trifolium pratense, T. repens, and Poa pratensis. Helianthus grosse-serratus and $H$. giganteus, though not ruderal species, have found bome-like conditions on the banks of a ditch extending the entire length of the wet meadow formation.

SPECIES OF THE PREVERNAL FLORAL ASPECT

Principal Species

Carex pennsylvanica.

Antennaria campestris.

Secondary Species

Androsace occidentalis. Anemone caroliniana.

Equisetum arvense.
Salix humilis.

S. fluviatilis.

Corylus americana.

\section{THE PRIVERNAI FLORAL ASPECT}

The earliest prevernal bloomers of the prairie formation appear on the southern slopes of the higher ridges during the first or second week in April. Of the four species proper to the formation, one, Carex pennsylvanica, not infrequently ranks as a facies, and the others, Anemone caroliniana, Antennaria campestris, and Androsace occidentalis, occur as copious or gregarious species. Carex pennsylvanica is a xerophyte, forming patches or mats several meters in extent on slopes or crests of ridges; toward the midule of April these patches are rendered noticeable by the yellow and purple of the numerous small spikes. Carex pennsylvanica also occur in low meadows, where it grows with reduced numbers, and blossoms about a week later, for which reason it never as- 
sumes the same importance as in the higher situations. This early spring sedge is perennial by virtue of its rootstalks and stolons. The flowers of $C$. pennsylvanica are proterogynous, macrobiostigmatous, and allogamous. The stigmas are borne below the longistaminate anthers, and if xenogamy fails geitonogamy may thus obtain.

The blue and white dalisy-like flower of Anemone caroliniana appear with or soon after the first yellow spikes of Carex pennsylvanica, and when abundant they present a striking contrast with the faintly green sward. A. caroliniana is also a xerophyte and a frequenter of southern slopes and crests; its bulbous root explains its prevernal appearance. The pollen is protected by the corolla-like sepals, which open at $8: 30$ A.M. and close at $4: 30$ P.M., remairing closed on cloudy days. Fertilization is allo-autogamous. If allogamy is not secured by visiting insects, which are by no means abundant at this time, autogamy is sure to result with the closing of the flower parts, as the anther's are brought in direct contact with the stigmas. Last year, flowers excluded from all insects were fertilized, and matured seeds.

Androsace accidentalis is in all respects one of the smallest and most inconspicuous of our flowering plants. Androsace appears on high ridges about one week before $A$ nemone, and were it not for the massing of its individuals, the plant might easily pass unnoticed. Although Androsace is hapaxanthous, it is wonderfully stable in the formation, since it occupies the same ridge year after year. So far as observed, the plant has no direct means of dissemination, hence its infrequent distribution but copious numbers. The plants begin growth in the autumn, forming a very small but closed rosette, which passes the winter in excellent condition, and, during the first warm days of spring, sends up a slender stalk bearing a cluster of flowers. The inconspicuous, gamopetalous corollas are constricted immediately above the point of attachment of stamens to the corolla, which serves to keep the pollen dry. In case allogamy fails, it would seem that 
mychogamy might insure autogamy. Nevertheless it is to be observed that only a small percentage of ovaries is fertilized.

Antennaria campestris, the one mat-forming species of our prairies, is a frequenter of high and low prairies and meadows, though, from a standpoint of numbers, finding its most favorable conditions in the low prairies. The prairie everlast. ing appears with the other prevernal bloomers, being from ten days to two weeks earlier on southern than on northern exposures. The plant is stoloniferous, which character renders it highly gregarious; this latter habit, together with its white-woolly apearance, makes it one of the most conspicuous elements of the prevernal aspect. Anthesis in this species, as in other prevernal bloomers, is closely followed by maturation. The elongation of the scape is simultaneous with the spreading of the pappus, which causes the achene to become free from the receptacle. The general distribution of the species over the prairie is evidence of the perfect dissemination secured through the agency of the wind. The above species, though beginning to blossom during the prevernal period, mostly continue during the early vernal, and frequently appear more prominent during the vernal than the prevernal aspect. This is especially the case with Antennaria, since the mats are more prominent during the period of maturation than during anthesis.

The other prevernal bloomers need only bare mention. A few bluish-gray cones of Equisetum arvense are found dotted here and there in the meadow formations during the latter half of the prevernal period. Salix humilis, a xerophilous woody shrub, one to two meters in height, occurs at one station in the prairie. Its short catkins of dioecious flowers are observed on the first or second day of April; these flowers are frequented by bees and flies which undoubtedly assist in pollen transfer. The swamp willow, Salix fuviatilis, is found in the meadow formation near the creek, especially in those parts flooded during the spring time. It is to be looked upon as an intruder. The swamp willow is about two 
weeks later than the prairie willow, S. humilis. The distribution of Corylus americana, the hazel nut, has already been given. The flowers of this species are anemophilous and accordingly allogamous; the copious, powdery pollen is produced in pendulous aments, protection being afforded by the expanded bracts. It is well known that blue jays, crows, and squirrels look after the dissemination of the hazel nut. $\Lambda$ blue jay will easily crowd four to eight of these nuts in his throat and thus carry them from one place to another.

ELEMENTS OF THE EARLY VERNAL FLORAL ASPECT

Facies (Wet Meadow Formation)

Carex stricta.

Eleocharis palustris.

Carex cephaloidea.

\section{Principal Specics}

Comandra umbellata.

Carex vulpinoidea (wet meaBaptisia bracteata. dow).

\section{Secondary Species}

Astragalus crassicarpus.

Carex festucacea.

Carex meadii.

Fragaria virginiana.

Lithospermum angustifolium.

Lithospermum canescens.
Scutellaria parvula.

Senecio integerrimus.

S. plattensis.

Sisyrinchium angustifolium.

Vicia americana.

Viola obliqua.

Viola pedatifida.

\section{DISCUSSION OF THE EARLY VERNAL TLORAL ASPECT}

The facies of the prairie formation do not contribute to the general aspect and need not be considered now. The sedge meadow facies are Carex stricta, C. vulpinoidea, and Eleocharis palustris. Carex stricta alternates with Spartina cynosuroides in the sedge meadow formation, occupying the wetter portions, where, with.C.vulpinoidea, it forms a close, 
permanent sod, which resists largely the encroachment of other species. The lower purple spikes contrast with the upper yellow staminate ones and form a conspicuous element of the aspect. The copious pollen of the much exserted stamens is either transferred by means of the wind (xenogamy), or showered down upon the receptive stimmas, which are proterogynous and macrobiostigmatous, in such masses as to color them yellow. In the latter case geitonogamy results. Elcocharis palustris is a hydrophytic rootstalk plant, by means of which it occupies a considerable area of the wet meadow formation, over which it forms a dense carpet-like covering. This plant is inconspicuous in every respect and soon hidden from view by the taller sedges. Carex vulpinoidea does not form definite patches, but is dispersed through the wet meadow formation. It is at least two weeks later than C. stricta; its dull-colored spikes do not contribute to the general aspect, even though the plant is later and more robust. Carex cephaloidea occupies the margins of the wet meadow formation at a few stations, but at no place is it prominent.

Comandra umbellata is the one copious species in the early vernal aspect of the prairie formation. Its general distribution has already been given. Comandra is a small, rather inconspicuous, pale green plant with umbels of greenish-white, odorless flowers, and were it not for the aggregation of its individuals and its blossoming at a time when the floral covering is almost destitute of color, it would produce little effect in the fiforal aspect. As it is, the bastard toad-flax is lost sight of before it has completed anthesis. Comandra is perennial by means of rootstalks, a fact which explains its habit of growing in patches. It is a typical xerophyte. In fourteen quadrats, including various exposures of Great ridge, its numbers range between 400 and 1,900 individuals, the average being 1,000 per quadrat. It is the most abundant of the principal species within the limits of its distribution. The anthers, at least, of the newly opened flowers are frequently in contact with the stigmas by inflection of the filaments, the 
pistils elongating as they become older. Very few ovaries become fertilized.

The large bright blue blossoms of Viola pedatifida appear about a week later than those of $V$. obliqua, and, when mingled with the yellow of Lithospermum and of Carex meadii, give a typica l vernal effect. Viola pedatifida is one of the most evenly distributed of our prairie species. It is found on all of the slopes, crests of ridges, and to some extent in the meadow formations. The greatest number found in any quadrat is forty-two, and the average for seventeen quadrats, for all except northern exposures, is twenty individuals. On northern slopes two to twelve plants were growing in each quadrat examined. Viola pedatifida, V. obliqua, and others of the genus have developed short, thick, fleshy rootstalks, by virtue of which they are enabled to secure an early start in the spring. I have not observed that rosettes of Viola pass the winter months. Viola perlatifida is chasmo-cleistogamous, but so far as observed few chasmogamous flowers are fertile. The cleistogamous flowers are usually developed later and are quite productive. The chasmogamous flowers are herkogamous, and inclined so as to receive no water upon the pollen. The bright colored petals are folded and bearded, apparently to protect the pollen. Dissemination is brought about in the various violets by the closing together of the walls of the valves of the ovary in drying.

Oxalis violacca frequents low prairies and meadows, where it forms circular, carpet-like patches of green and rose 2-1 meters in diameter. Oxalis has also established itself along southern exposures of hedges, growing profusely in dense carpets 15-25 meters in extent. The wood sorrel is perennial by means of a scaly bulb, which explains its early appearance. It is one of our prettiest vernal bloomers, appearing at a time when the floral covering is sparse. Its flowers are hemeranthous and usually ephemeral, opening at $7: 30$ A.M., and closing at $5: 30$ P.M., remaining closed on clondy days. The fower pedicels are reflexed before and after anthesis. The filaments 
and styles are reciprocally of two lengths (heterodistyly), xenogamy alone being possible.

Carex meadii frequents low prairies and meadow's. Its individuals are somewhat aggregated on account of rootstalk propagation. Its bluish green leaves and large, yellow staminate spikes appear where there is little else to relieve the monotone left by winter. Carex meadii does not occur widely distributed, nor does it exceed copious 4 in abundance. The copious pollen of the exserted stamens is borne above the pistillate spikes, and matures before the stigmas are receptive (proterandry). Allogamy, consisting of xenogamy or geitonogamy, results. Sisyrinchium angustifolium, a grass-like plant in appearance, occurs typically in low prairies, but is also found in meadows. Its "bunch" habit, causing the small blue and white flowers to be aggregated, renders it conspicuous, especially since it appears before the facies play any part in the aspect. The flowers of Sisyrinchium are herkogamous, the stigmas projecting beyond and alternating with the extrorse anthers, and hemeranthous, opening at 8 A.M. and closing at $5: 30$ P.M. They remain closed on cloudy days. It is to be noted that almost every ovary is fertile. Senecio integerrimus is conspicuous on account of being represented in the formation by one individual.

Senecio plattensis is met rarely in the low prairies. Its numerous bright yellow heads, especially when the individuals are semi-aggregated, as is usually the case, are conspicuous objects of the early vernal period. Wind dissemination obtains by means of the copious pappus. Both Senccio plattensis and S. integerrimus produce open rosettes through the agency of which these plants secure greater recognition on account of blossoming earlier.

Fragaria virginiana haunts low prairies and meadows, where, on account of numerous runners, it forms rather definite patches, of which two or three are observed in the formation. Fragaria forms open rosettes, the inner silky leaves of which are covered over with the broad stipules of the outer 
leaves, thus passing the winter unharmed. The periect rosette conditions explains the appearance of the clusters of white flowers during the last week of April. Dissemination is secured through animal agency, by virtue of the color and taste of the fruit. Lithospermum canescens is sparsely distributed over the entire prairie, most abundantly, however, in low prairies, where it averages one or two individuals per quadrat. The throat of its hypocrateriform corolla is partially closed by projecting folds or sinuses. The nectar-bearing flowers are heterodistylous, the stamens and stigmas being scparated by nearly the length of the corolla tube, xenogamy always resulting. A visiting insect carries pollen from the high stamens of one plant to the high stigma of another, and from the low stamens to the low stigmas. Dioecism practically obtains, with the advantage that no plant is unfruitful. Lithospermum angustifolium has about the same distribution as the above species, but it occurs only now and then, and is a rare plant in the formation. In $L$. angustifolium the tube of the nectariferous corolla is much elongated, at least in earlier flowers, and its throat is entirely closed with the prominent appendages. The pollen is thus well protected from insect enemies and excessive moisture. Allogamy results, but the means are not clear. The later cleistogamous flowers of this species are much more fertile than the earlier chasmo. gamous ones. These two species, even with sparse numbers, brighten the prairie with their shades of yellow and give a decided tone to the aspect. Of the two plants, $L$. canescens is the more conspicuous on account of its bright colors and its almost leafless, compactly flowered racemes. The flowers of L. canescens are also very fragrant. Both of the puccoons are perennial by deep, thick roots, from which the stems sprout up very early in the spring. Plants of $L$. angustifolium frequently pass the winter in the rosette condition.

The most striking plant of the vernal aspect is Baptisia bracteata, which begins blossoming during the first week in May and continues for about twenty days. Its massiveracemes 
of showy, cream-colored flowers, too heavy to be sustained by the plant, are strewn over eastern, western, and southern slopes, sometimes extending almost to the crests of ridges. It is normally a low prairie species, as is shown by its distribution. As to duration, it is perennial, and its average is about one individual for three or four quadrats. The strong papilionaceous corolla admirably protects the pollen from rain and admits of fertilization by means of bumble bees only. As the bee's head is thrust far into the flower to reach the nectar, the keel and wings of the corolla are forced down, thus uncovering the incurved stamens and styles, and bringing the anthers and hairy stigma into direct contact with the abdomen of the bee. Since the bee has no means of determining whether nectar is present, except by trying, the flowers are visited many times during the day and thus allogamy is secured. Upon examination, I found the abdomens of several bees colored yellow with the abundant pollen masses. Astrag. alus crassicarpus is found at only one station in the prairie, viz., on the western slopes of Great ridge near the south side. It is a low prairie species tending toward xerophilous conditions. The purplish blue racemes of the ground plum appear at a time when there is little else in the floral covering, hence the important part they play. The low, reclining stems spread from the perennial, woody root in all directions, a single individual frequently occupying considerable space. Vicia americana is a rootstalk plant and finds its favorite luaunts in meadows near streams and woodlands. This plant grows at a few locations in the meadow formation near the creek, where it occupies rather definite patches. Its climbing habit renders all the more conspicuous the numerous showy racemes of purplish and light blue flowers, so that a patch may be recognized at a distance of twenty to forty meters. During the month of May, Vicia dominates the floral aspect within its limited range. Patches of Vicia are the favorite resorts of bumble bees, fertilization being accomplished as 
for Baptisia. Carex festucacea frequents meadows, where it occurs as a secondary species. It adds little to the floral aspect. Allogamy is secured in pollination by means of proterogynons stigmas. Scutellaria parvula, a small herb, perennial by means of tuberous rootstalks, occurs rarely in the meadows. Scutellaria is scarcely noticeable, owing to the denser meadow facies which soon overtop it. Viola obliqua is a hydrophyte, occurring as a secondary species in the sedge meadow, and meadow formations. Its flowers are chasmocleistogamous, the cleistogamous ones being abundantly fertile.

ELEMENTS OF THE LATE VERNAL FLORAL ASPECT

$$
\text { Facies }
$$

Koeleria cristata.

Panicum scribnerianum.

Stipa spartea.

$$
\text { Principal Species }
$$

Ceanothus ovatus.

Secondary Species

Agrostis hiemalis.

Allionia nyctaginea ovata.

Anemone canadensis

Asclepias obtusifolia.

Calamagrostis canadensis.

Delphinium carolinianum.

Eatonia obtusata.

Galium tinctorum.

Juncus tenuis.

Onosmodium molle.

Oxalis stricta.
Panicum depauperatum

P. pubescens.

Phlox pilosa.

Physalis virginiana var.

Psoralea esculenta.

Rosa arkansana.

Rumex altissimus.

R. crispus.

Tradescantia virginiana.

Trifolium pratense.

T. repens.

Tripsacum dactyloides.

Zizia aurea. 


\section{TUE LATE VERNAL ASPECT}

Many of the plants of the early vernal period continue throughout the late vernal aspect, so that this division is one for convenience rather than otherwise. The facies of the prairie formation are represented by three sod formers, Panicum scribnerianum, Stipa spartea, and Koeleria cristata, all of which occur throughout the prairie, but only in localities become of sufficient importance to rank as facies. Koeleria is most abundant on the higher slopes and crests of ridges, Stipa and Panicum in the lower prairies and mead. ows. Panicum averages twenty to twenty-five bunches per quadrat, Stipa from five to fifteen, and Koeleria, when present, twenty to twenty-five. Panicum is the first of the vernal facies to blossom, closely followed by Koeleria and Stipa. The flowers of all are anemophilous and belong to the Longistamineae; as would be expected, the stigmas are plumose and exserted. Ceanothus ovatus is a xerophytic shrub occupying the exposed tops of the various ridges. Where most abundant, it controls to a considerable extent the numbers of the secondary species. Ceanothus occurs in every count made upon the crest of Great ridge, ranging from three to twentynine individuals to the quadrat. When we remember that a singie shrub of redroot contains from five to sixty woody stems, some of which are branched, we get some idea of the dominating influence it exerts. During May and the early part of June, the crest of Great ridge is white with the innumerable panicled flowers of this plant. Two xerophytes, Panicum pubescens and $P$. depauperatum, occur as small, tufted perennials on Great ridge and some exposed slopes. $P$. pubescens is the less abundant and is easily recognized by its villous appearance and its smaller spikelets. Panicum depauperatum is frequent, averaging about six bunches per quadrat throughout its range. Neither of these grasses contributes anything of note to the vegetative covering. Eatonia 
obtusata occurs only sparsely and infrequently on the ridge, and also on the low prairies.

Rosa arkansana is a low bush two to five decimeters in height, frequenting high prairies and slopes. At times it tends to become gregario-copious; at other times its individuals are scattered rather evenly over the formation. The rose averages twelve to fifteen individuals to the quadrat, which number may be doubled under more xerophytic conditions, or reduced to one or two plants in meadows. The prairie rose begins anthesis during the late vernal period and continues throughout the estival aspect. Its large pink or white fragrant blossoms are among the more conspicuous objects of the early summer aspect, especially when slightly raised above the surrounding prairie, as is the case when the plant grows on slopes. The flowers open at 5:30 A.M., lasting for one or two days. One of the most deceptive of the prairie species, so far as numbers are concerned, is Physalis virginiana. On a walk over the prairie, even with careful examination, one seldom sees more than half a dozen individuals; however, the plant is found in almost every quadrat throughout meadow and prairie formation, sparsely, or more rarely as subcopious 2. It is needless to say the groundcherry adds nothing to the tone of the aspect. The plants are small, one or two decimeters high, and perennial by fleshy rootstalks. Allionia nyctaginea ovata was discovered at only one station, viz., on Great ridge. In duration, it is a perennial. The flowers are nyctanthous, opening at $4: 30$ P.M., and closing at 9:00 A.M., and ephemeral, except on cloudy days. If allogamy is not secured through the agency of "night flyers," the much exserted filaments and style become reflexed, frequently describing one or two complete circles and bringing anthers and stigmas together. Autogamy may thus obtain. Asclepias obtusifolia is a solitary plant occurring only rarely on the higher prairies throughout its distribution. Its many-flowered, mostly solitary umbel of greenish-purple blossoms is raised above the surrounding vegetation by means of a long 
peduncle. The plant is a perennial. Only a few specimens of Onosmodium molle were found within the prairie. Unless several stems grow from the perennial root, as is usually the case, these shaggy plants with greenish white clustered flowers pass unnoticed.

Psoralea esculenta is noted as a sparse plant on the slopes of Great ridge and to a limited extent on low prairies. The plant body, which is clothed with white villous hairs, hardly contrasts with its densely flowered spikes of grayish-blue flowers. This peculiarity, with the solitary habit of the plant and the overtopping facies, renders it quite obscure. $P$. esculenta is perennial by means of large tubers. During July the stem becomes freed from the caudex and rolls about over the prairies, thus disseminating the seeds. Delphinium carolinianum is recorded at one location in rather low prairies. The appearance of the slender, showy racemes of white flowers indicates the close of the vernal and the beginning of the estival period. The individuals are not numerous enough to contribute to the general aspect. From various observations made, the bumble bee appears to attend to pollination, which is in general similar to that of Baptisia bracteata. When ripe the follicles open at the top, thus allowing the seeds to scatter gradually. Tuberous clustered roots render the larkspur perennial. Agrostis hiemalis occurs only now and then in meadows, especially where the formation is somewhat open. Unless abundant, the fine, hair-like bunches are hardly noticeable. The plant is perennial by means of rosettelike tufts of crowded linear leaves which pass the winter nicely. During early summer the lax panicles containing the ripened seeds are rolled about by the wind.

Galium tinctorum is a weak stemmed, much branching, rootstalk plant frequenting wet meadows and ravines, where it forms a laver beneath the tall facies. At times Galium becomes controlling in small areas, in which case it develops an almost impenetrable carpet. This bedstraw is of little prominence in the formation from any standpoint. Anemone 
canadensis is a ferennial mesophyte, occurring at a few stations in draws and ravines, and having the same distribution as Gaiium tinctorum. Toward the middle of May the large white blussoms of Anemone begin to appear and continue for about a month. Phlox pilosa is a perennial mesophyte found sparsely in meadows. Its cymes of bright pink or purple fragrant blossoms, with now and then a white flowered individual, lend a decided tone to the floral aspect. The hypocrateriform corolla is nectar-bearing and the stamens are unequally inserted upon its tubes above the style. If allogamy is not secured through insect visitors, autogamy may result from pollen falling upon the receptive stigmas. On account of its grass-like leaves, $P h l o x$ practically disappears with the close of anthesis. Tradescantia virginiana is noted only sparsely at a few stations in the meadow formation. These plants develop from perennial roots, and during the vernal period produce bunches of showy blue, pink, or purplish fiowers. On account of its semblance to the facies, the plant disappears with the close of anthesis. Another pleiocyclic species occurring with sparse or subcopions abundance throughout meadow formations is Zizia aurca. This plant begins blossoming about the middle of May and continues for a month, varying the aspect with yellow here and there.

Tripsacum dactyloides is found at one of two stations along the margins of the scdge meadow, becoming occasionally quite controlling. Its coarse leaves and stems, together with its monoecious flowers, easily distinguish it from any other grass in the prairie. The flowers are proterandrous and xenogamous, the long, roughened stigmas appearing after the stamens. In maturation the upper spikes ripen first and with that portion of the rhachis drop off, and thus are carried away with the first flood. Tripsacum is perennial with very thick, woody rootstalks.

Calamagrostis canadonsis is a hydrophilous, rootstalk pleiocyclic growing in small clumps in the wet meadow. During the flowering period these clumps are especially con- 
spicuous on account of the plumose stigmas and the much exserted anthers.

Rumex crispus and $R$. altissimus occur sparsely, the one within the wet meadow, the other about its margins. Both species owe their very early start in the spring to their thickened perennial roots, as the loose rosettes formed by these plants do not withstand the winter. The numerous, green, inconspicuous, pendulous flowers of these docks are polygamo-monoecious and anemophilous; geitonogamy and xenogamy accordingly result. The least movement of the plant envelops the flowers with a cloud of pollen. The three valves or wings, which are commonly grain-bearing, enable the achene to float, which explains the distribution of these plants along water ways. If these valves are removed, the achenes sink. The remaining species of the vernal aspect need only brief mention. Juncus tenuis frequents a few places in the wet meadow, as a rather inconspicuous, grass-like perennial. Oxalis stricta forms an interrupted layer below the tall facies, in parts of the wet meadow and meadow formations. During the fall Oxalis forms small, compact mats, which, in protected situations, pass the winter in good condition. The yellow flowered oxalis is one of the first plants to begin growth in the spring. Trifolium pratense and T. repens are growing in the drier portions of the wet meadow. T. pratense forms open rosettes which, with protection, frequently withstand the hardships of winter.

ELEMENTS OF THE EARLY ESTIVAL FLORAL ASPECT

\section{Facies}

Same as for vernal aspect.

\section{Principal Specues}

Amorpha canescens.

Ceanothus ovatus.*

Coreopsis palmata.

Erigeron ramosus.
Kuhnistera candida.

K. purpurea.

Meibomia illinornsis.

* Extending into this aspect from the vernal. 


\section{Secondary Species}

Acerates viridiflora ivesii.

A. viridiflora linearis.

Agrostis alba.

Anemone cylindrica.

Amaranthus graecizans.:

Apocynum cannabinum.

Asclepias sullivantii.

A. syriaca.

A. tuberosa.

A. verticillata.

Astragalus carolinianus.

Baptisia leucantha.

Brauneria pallida.

Chaetochloa viridis.

\section{C. glauca.}

Chenopodium album.

Convolvulus sepium.

Equisetum laevigatum.

Euphorbia glyptosperma.

E. nutans.

E. maculata.

Habenaria leucophaea.

Lactuca pulchella.
Lilium canadense.

Lythrum alatum.

Mesadenia tuberosa.

Oxalis stricta.*

Phalaris arundinacea.

Phleum pratense.

Physalis heterophylla.

Physalis longifolia.

Plantago major.

P. rugellii.

Polygala verticillata.

Potentilla monspeliensis.

Psoralea argophylla.

$P$. tenuiflora.

Rosa arkansana.*

Ruellia ciliosa.

Scirpus atrovirens.

Solanum carolinense.

Steironema ciliatum.

Teucrium occidentale.

T. canadense.

Thalictrum purpurascens.

Verbena stricta.

The estival period begins about the tenth of June, and is characterized by the rapid diminution of the vernal bloomers rather than by the addition of the important estival flowers. The physical factors are changing rapidly and in most cases approaching their maximum. The floral covering accordingly undergoes a complete change. The white flowers of Ceanothus ovatus continue on the ridges in maximum numbers, but the white-rayed flowers of the more frequent and equally abundant Erigeron ramosus render them of secondary prominence. Bright yellow patches of Coreopsis palmata

* Extending into this aspect from the verndh. 
are found on every, ridge and slope. Before these flowers have reached their maximum numbers, three other showy bloomers, Amorpha canescens, Kuhnistera purpurea, and $K$. candida are added, and the estival aspect attains its maximum in color and complexity.

No new facies appear during the early estival aspect. Erigeron ramosus occurs principally on ridges and slopes where it averages 15-18 individuals to the quadrat, also to some extent in low prairies and meadows. In the latter situation it occurs sparsely, and its flowers are not conspicuous owing to the denser facies. The flea-bane is dicyclic and forms moderately close rosettes, which secure for it an early start in the spring and a nearly uniform distribution. Wind dissemination is brought about by means of the pappus. Kuhnistera candida, $K$. purpurea, and Amorpha canescens are both frequent and copious, occurring in varying numbers in every quadrat. The abundance of Kuhnistera candida, $K$. purpurea, Amorpha canescens, and Coreopsis palmata in quadrats I-XXII is as follows:

\begin{tabular}{|c|c|c|c|c|c|c|}
\hline QTADRAT. & LOCATION. & EXPOSURE. & K. CANDIDA. & E. PURPUREA. & $\begin{array}{l}\text { A. CANE- } \\
\text { SOENSE. }\end{array}$ & O. PALMATA. \\
\hline$I$ & Low ..... & West .... & 643 & 28 & 40 & \\
\hline II & Crest .... & Ridge.... & 7 & 51 & 128 & 1416 \\
\hline III & " $\quad .$. & " $\quad .$. & 12 & 150 & 138 & 593 \\
\hline IV & Low ..... & North.... & 115 & $\cdots$ & 2 & ........... \\
\hline $\mathrm{V}$ & $" \quad \ldots$. & West .... & 81 & 3 & 2 & ........... \\
\hline VI & $" \ldots \ldots$ & East ..... & 190 & 49 & 3 & \\
\hline VII & Ridge.... & North.... & 10 & 1032 & 23 & 1100 \\
\hline VIII & Crest .... & Ridge.... & 10 & 176 & 10 & 375 \\
\hline $\mathbf{I X}$ & Ridge 1/2. & S. E..... & 225 & 476 & 42 & 5 \\
\hline $\mathbf{X}$ & " & $" \quad \ldots$. & 482 & 360 & 8 & .. \\
\hline XI & Crest .... & Soath.... & 4 & 163 & 134 & 129 \\
\hline XII & “ $\quad \ldots$ & Ridge.... & 5 & 425 & 326 & 900 \\
\hline XIII & Ridge $1 / 2$. & S. E..... & 169 & 10 & 425 & 125 \\
\hline XIV & $\because 20$ & North.... & $\because \cdots$ & 575 & 90 & $\ldots$ \\
\hline $\mathrm{XV}$ & " & $" \quad \ldots$ & 115 & 75 & 92 & 86 \\
\hline XVI & " & West .... & 32 & 116 & 325 & 206 \\
\hline XVII & Base ..... & $" \ldots$ & 20 & 19 & 142 & in.... \\
\hline XVIII & Crest .... & N. W.... & 4 & 34 & 500 & 190 \\
\hline $\mathrm{XIX}$ & Base..... & $\because \quad \ldots \ldots$ & 356 & 8 & 88 & 137 \\
\hline$\overline{X X}$ & Crest .... & North.... & 9 & 18 & 3 & 1100 \\
\hline XXI & $\because \quad \ldots$ & N. W.... & 7 & 119 & 26 & 178 \\
\hline$\overline{X X I I}$ & Base ..... . & North.... & 45 & 16 & 180 & 7 \\
\hline
\end{tabular}


Kuhnistera candida ranges from 150-600 individuals to the five-meter square in the low prairies, averaging 250-275, and from 4-45 individuals on the ridges and exposed slopes. Individuals of $K$. purpurea number 150 to 1,000 to the quadrat in the higher prairies about Great ridge and 5-50 in the low prairies. The average of the plant under normal conditions is about 350 to the quadrat. The prairie clovers are perennial, so that their distribution in the formation is rather permanent and dictated perhaps wholly by the water-content, since conditions frequently obtain where the two species occur in equal numbers. The showy white or purple flowers of these species are arranged in compact cylindrical spikes; anthesis begins from below, extending upward over a zone of about one centimeter daily. 'The Kuhnisteras have proterandrous, pollen flowers, the stamens of which become exserted during the morning hours (5:00-7:00 A.M.) and disappear very soon, while the styles elongate in the afternoon or on the following morning. Allogamy, xenogamous or geitonogamous, obtains through insect agency. The orange anthers of $K$. purpurea produce copious masses of globose, roughened pollen cells covered with a yellowish viscin. On high prairjes Amorpha canescens ranges from 125-500 individuals per quadrat, averaging perhaps 300 , while on low prairies from 12-50 plants are counted in the five-meter plot. Its abundance is sparse to subcopious in meadows. The leadplant or shoestring is a low undershrub controlling by virtue of its deep-seated, woody roots, which eminently fit it for xerophytic conditions. The leaves and stem of this plant are leadcolored, and when the terminal, clustered blue racemes appear it becomes one of the most conspicuous plants in the aspect. The flowers are proterogynous and accordingly allogamous. The yellow anthers with red filaments are exserted during the early morning hours and by noon have mostly disappenred.

Corcopsis palmata occurs in definite patches on Great ridge, ranging from $200-1,400$ individuals per quadrat, the 
number depending largely upon the location of the quadrat within the Coreopsis patch. During June these masses of yellow-rayed bloomers contrast sharply with the other mostly white and blue flowers. The plants are perennial; root propagation obtains, hence the patches. Brauneria pallida is a xerophyte with thick, black roots and rigid stems and leaves. It invariably occurs on high prairies and ridges, where it averages $25-30$ individuals to the quadrat. Brauneria begins blossoming during the first week of June and continues for over a month. The spreading rose rays surround a cone of brown and altogether produce a pretty effect. These flowers are easily recognized at a distance of fifty meters. Acerates viridiflora linearis and $A$. viridiflora ivesii are two typical xerophytes of Great ridge. Both are perennial, solitary plants, recognized only with difficulty in the floral covering on account of the uniform color of flowers and leaves. Their follicles containing comose seeds mature September 1 . Anemone cylindrica is neither frequent nor abundant, occurring only at a few stations on Great ridge. It is a perennial xerophyte. The few greenish-white, unattractive flowers appear during the first or second week of June, lasting for a few days only. The ragged heads are common on the prairies during July and August, the woolly achenes of which are carried by gophers to their burrows.

The tuberous-rooted Indian plantain, Mesadenia tuberosa, is a rare plant in the low prairies. The stems are at least one meter high and terminate with a showy, white corymb easily distinguishable for 200 meters. Verbena stricta occurs occasionally in low prairies and meadows. Its hairy, rigid stems, bearing terminal racemes of blue flowers, grow from perennial roots. The curved salver-form corolla includes the didynamous stamens which exceed the style (herkogamy). When the insect, usually one of the Hymenoptera, is securing the nectar, he is quite sure to leave pollen of some other vervain flowers upon the stigma, allogamy resulting. It is to be noted that $V$. stricta is rather abundant in a pasture of two 
years' duration, joining the above meadow formation. The same is likewise true for $V$. hastata and Vernonia gigantea, all three of which possess a bitter juice, rendering them distasteful to stock. Polygala verticillata is a frequent, but sparse, inconspicuous annual of low prairies and meadows. It adds nothing to the floral covering nor to the aspect. Ruellia ciliosa is a low, hairy perennial occurring sparsely at a few stations in low prairies, where it aggregates in loose patches. The beautiful lilac-colored flowers are ephemeral, opening during the night and falling away by $12: 00 \mathrm{M}$. They scarcely contribute to the floral aspect on account of the taller facies. Flowers of Ruellia ciliosa are funnel-form, with a long tube, at the base of which is secreted the nectar. The period of anthesis lasts for sixty days. Psoralea argophylla is a low, branching perennial of moist prairies, where it assumes a gregarious habit. Its characteristic white stems and leaves render patches of this plant conspicuous objects of the aspect. On high prairies it is usually loosely interspersed throughout and hence never noticeable. The small, blue flowers do not add to the plant. After maturation, the plant becomes freed from the caudex and rolls about on the prairies as a tumble-weed. Psoralea tenuiflora is found at a few stations in low prairies and meadows. It is a rather tall, diffusely branched perennial, conspicuous on account of its many small spikes of blue flowers, which continue for a month or more.

Asclepias verticillata occurs as a subcopious plant throughout low prairies and meadows. Its individuals are hardly distinguishable from the facies until the small umbels of white flowers appear. The small milkweed is perennial. Maturation begins during the latter part of August, at which time the follicles burst, setting free the comose seeds. Asclepias tuberosa is a rare plant in the low prairies. Its mostly clustered stems proceed from thickened perennial roots and terminate with conspicuous, orange-red umbels which are among the brightest object, of the aspect. It is to be noted 
that very few of the ovaries become fertile. Maturation begins September 1, dissemination occurring as for $A$. vcrticillata. Asclepias syriaca and $A$. sullivantii are frequenters of low prairies, meadows, and draws, where they occur only rarely; the latter species prefers the wetter situations. $A$. syriaca also grows in cultivated fields, wastes, and along borders of thickets. The tall, leafy stems, bearing numerous umbels of purplish flowers, are striking objects in the formation. These flowers continue for twenty-five to thirty days, during which time they are visited frequently by the redbrown milkweed butterfly (Danais archippus). Dissemination is the same as for other milkweeds.

The distribution of Baptisia leucantha has already been indicated. The plant is found but sparsely in low prairies and meadows; nevertheless it is a conspicuous element of the floral aspect. The erect stems develop from a perennial root and are crowned with a long, loosely flowered raceme of cream-colored flowers, which continue during June, After anthesis these plants give the appearance of miniature trees scattered throughout the formation. Fertilization obtains through the agency of bumble bees and is in all respects as for $B$. bracteata. The inflated pods mature during the latter part of July, but give no clue to methods of dissemination. Meibomia illinoensis, a tall, rigid, branching perennial, one to two meters high, abounds at a few stations in the low prairies, meadows, and draws. It ordinarily averages $25-60$ individuals to the five-meter plot; however, in one quadrat 672 plants were enumerated. In this particular case, Meibomia has become a controlling element in the formation, either crowding out or subordinating the secondary species. The long raceme of light blue flowers is especially noticeable during the morning hours. The easily separable loments are bristly uncinate-pubescent, and adhere to any surface with which they come in contact. Lythrum alatum inhabits ravines, draws, and wet meadows. It usually grows in definite crowded patches, in which few secondary species are found, 
and hence the greater prominence it derives. Its period of anthesis lasts about sixty days, during which time its numer-. ous purple flowers give a characteristic coloring to the patches. The flowers are heterodistylous and accordingly xenogamous; the insect, in securing nectar from the base of the calyx tube, carries the pollen from the long stamens to the long pistil, etc. Steironema ciliatum occurs in draws with Lythrum alatum and at one or two stations in the meadows. It is a rootstalk plant, by means of which it forms ráther definite patches. Steironema is not abundant enough to be of importance. Teucrium occidentale and $T$. canadense are inhabitants of low meadows and draws, in which situations their individuals become aggregated. $T$. occidentale is also sparsely scattered through the sedge meadow formation. The two species frequently grow in the same patch, the type specimens of each, however, being recognized at a glance. Neither species is abundant enough to add materially to the formation or the aspect.

Habenaria lcucophaea, Thalictrum purpurascens, and Lilium canadense are frequenters of low meadows and ravines where they occur sparsely. The showy spikes of white fragrant flowers of Habenaria are conspicuous objects in the vegetation, though never abundant enough to give color to the aspect. Xenogamy or geitonogamy results from insect pollination. The nectar is secreted in large quantities in the very elongated spur of the lip. The insect, in order to reach the nectar, (of necessity an insect with a long proboscis) brings his head or proboscis in contact with a viscid disc, to which are attached the two pairs of pollinia. These pollinia adhering to the head parts are thus torn away from the flower and stand a good chance of coming in contact with the broad stigmatic surfaces of the succeeding flowers, to which they readily adhere if the stigmas are receptive, i. e., covered with very adhesive viscin. The above is practically what obtained when these flowers were exposed to "night flyers." On account of the resemblance to the facies, the 
leafy plants are scarcely observed after flowering. The white fringed orchis is perennial by means of thick, fibrous roots. Habenaria bracteata is a rare, inconspicuous plant found only once in the meadows. Thalictrum purpurascens is a leafy, tall-growing perennial, the numerous male plants of which are especially prominent in the aspect during anthesis. At this time the staminate racemes are one mass of yellow, linear anthers, which give off a cloud of "pollen dust" at the least movement. The flowers are anemophilous and dioecious, xenogamy alone being possible. By far the most showy flower in the entire prairie is Lilium canadense. Its erect, leafy stems are about one meter high and terminate with a single large, nodding blossom, bright orange in color and beautifully spotted with brown. The lily is perennial by means of scaly bulbs developed from a short, stout rootstalk. Equisetum laevigatum is one of the two fernworts of meadows, $E$. arvense being the other. $H$. laevigatum occurs at two stations in the low meadows, and ranges from 44-260 individuals to the quadrat. Its dark green, jointed stems are hardly to be distinguished from the coarser facies. Equisetum is a perennial with deep-seated rootstalks, from which frequently many stems develop.

Scirpus atrovirens and Phalaris arundinacea are hydrophilous species of the wet meadow. Scirpus develops short rootstalks, by virtue of which it forms dense clumps a few meters in extent. The dark green foliage and the brown umbels render the clumps of this species prominent in meadows. The flowers are proterogynous, allogamy being the preferred method of pollination. Phalaris is a tall-growing perennial grass, forming rather indefinite bunches of several dozen individuals. Anthesis takes place during June and is immediately followed by maturation. The seeds are soon thrashed out of the dry panicles with the wind. Scirpus atrovirens and Phalaris arundinacea are almost invariably associated with Cicuta maculata, Asclepias incarnata, and Lythrum alatum. Apocynum cannabinum occurs sparsely 
in meadows and swamps. Its clusters of white flowers apyear about June 1 and continue for forty days. The Indian hemp is a perennial with deep, thick roots, from which propagation results; hence its tendency to form clumps. Dry, grassy banks of streams are the favored haunts of A. cannabinum. Dissemination is as for the milkweeds. Physalis longifolia is a tall, bushy perennial inhabiting mostly the Carex facies of the wet meadow. It averages four to ten individuals per quadrat.

The remaining plants of the early estival floral aspect are of importance principally because of their presence. They neither add to the aspect nor the floral covering. With perhaps one exception, Physalis heterophylla, which occurs rarely throughout the prairie, all are ruderal or adventitious species. Agrostis alba, Phleum pratense, and Poa pratensis form a zone a few meters in width on the west of the wet meadow. Plantago major and $P$. rugellii occur in the drier parts of the wet meadow. They are perennial plants with short, thick rootstalks, forming open rosettes which "freeze out" during the winter. The flowers of these plantains are aianthous, anemophilous, proterogynous, and xenogamous. The long, exserted stamens appear several days after the stigmas. Potentilla monspeliensis is a dicyclic herb growing below the tall, wet meadow facies. It forms open rosettes, the inner leaves of which are protected with the broad stipules and bases of the outer ones.

During excessive precipitation, soil from neighboring culture formations is transported to parts of the sedge meadow. These deposits are soon covered over with a dense growth consisting of such ruderals as Solanum carolinense, Lactuca pulchella, Chactochloa viridis, C. glauca, Chcnopodium album, Amaranthus graecizans, and Convolvulus sepium. The common tumbling weed, Amaranthus graecizans, and also the following spurges, Euphorbia glyptosperma, E. nutans, and $E$. maculata, grow abundantly on the mounds in the 
prairie made by the pouched gopher. After some time the encroaching facies again claim the ground.

ELEMENTS OF THE LATE ESTIVAL FLORAL ASPECT

Facies

Bouteloua curtipendula.

\section{Principal Species}

Euphorbia corollata. Kuhnistera candida.
Kuhnistera purpurea. Meibomia illinoensis.

Secondary Species

Amaranthus torreyi. Asclepias incarnata. A. verticillata.

Astragalus carolinianus.

Cassia chamaecrista.

Cicuta maculata.

Convolvulus sepium. Elymus canadensis.

Heliopsis scabra.

Hieracium longipilum.

Laciniaria squarrosa.

Lactuca canadensis.

L. Iudoviciana.

Lespedeza capitata.

Linum sulcatum.

Lycopus americanus.

Lythrum alatum.
Mentha canadensis.

Meibomia canadensis.

Polygonum pennsylvanicum.

Panicum capillare.

Ratibida pinnata.

Silphium laciniatum.

S. integrifolium.

S. perfoliatum.

Solidago rupestris.

Scutellaria lateriflora.

Teucrium canadense.

T. occidentale.

Verbena hastata.

V. stricta.

Vernonia fasciculata.

V. gigantea.

The late estival floral aspect is set off here în order to note more accurately the gradual changes in the floral covering. It is not considered an aspect complete in itself but rather a division of one. Like the late vernal aspect, it is a period of transition. Bouteloua curtipendula is the only new facies, and its distribution has already been noted. Kuhnistera purpurea, $K$. candida, and Meibomia illinoensis, 
respectively, continue in diminishing numbers in the higher prairies, the low prairies, and the meadows, and pass out entirely with the advent of the serotinal period. The grayishwhite plants of Amorpha canescens are still conspicuous objects on ridges, though long past anthesis. They give the floral covering a "ragged" appearance. Ceanothus ovatus is represented now by a mass of low, leafy bushes, abundant on ridges and scattered here and there on the higher slopes. The white flowers of Erigeron ramosus and the bright yellow of Corcopsis palmata have disappeared entirely from the formation, and their places have been taken by the taller and more prominent Euphorbia corollata, the flat-topped flower clusters of which are conspicuous on every ridge and slope. Others that lend coloring to the aspect are Laciniaria squarrosa on ridges, Solidago rupestris in high and low prairies, Silphium laciniatum in low prairies and meadows, $\mathbb{S}$. integrifolium and Vernonia gigantea in low meadows, and Cassia chamaccrista in locations heretofore given.

Euphorbia corollata occurs, with two exceptions, in every quadrat from II-XXII. It averages 50 individuals to the five-meter square, though in several instances more than twice that number have been counted. E. corollata is prominent in this formation, not alone on account of its size, overtopping as it does all other species within its patch, but also because of its advantageous distribution and gregario-copious disposition. The strict stems are about one meter high, clothed with bright green leaves and crowned with large, white, flat-topped umbels. This milk spurge is an aianthous bloomer; its flower's appear about the middle of June, increasing in numbers until August 1, after which time they gradually diminish. The stems and leaves are not eaten by stock on account of the milky juice they contain, a property which gives the plant a decided advantage in pastures. The plant is perennial by means of long rootstalks.

Laciniaria squarrosa is a xerophyte of the crest of Great ridge, occurring rather infrequently. It ranges from 6-55 
individuals to the quadrat with an average of 18 (subcop. 1). The short spikes of bright purple flowers render the plant prominent in the late floral aspect. Dissemination is by moans of the plumose pappus. Solidago rupestris frequents low prairies and especially slopes; it is found to some extent in xerophilous situations. The individuals of this species, in the various quadrats examined, range from 2-275, with an average of 45 . The plants are copio-gregarious in the lower prairies, frequently forming patches 1-3 meters in extent, while in the more xerophilous situations they are often loosely dispersed throughout. $\$$. rupestris forms rather open rosettes, which tend toward the closed type; it is also a rootstalk plant, which partially explains its gregarious luabit. $S$. rupestris is among the earliest of the goldenrods to blossom and the only estival one in the formation. Its bright yellow flower clusters, especially when aggregated, are among the more conspicuous objects of the aspect. The achenes are modified for wind dissemination. Linum sulcatum is a small, slender annual occurring frequently but sparsely throughout the prairie, its average for sixteen quadrats for all conditions being five individuals. It is interesting to note that in quadrats XXI and XXII (see map) both with northern exposures, Linum averages 40 individuals, (subcop. 1). The bright yellow ephemeral corollas open at 4:00 A.M., and fall off between 10:00 and 11:00 A.M. Dissemination results from the capsules splitting open from above when dry, thus scattering the seeds.

Hieracium longipilum is a sparse or subcopious perennial of low prairies. The plant is not abundant enough to be of importance to the covering. Hieracium forms rather close rosettes, the leaves of which are densely covered with brownish, bristly hairs 1-2 cm. long. Silphium laciniatum is a tall, coarse perennial growing sparsely in low prairies and to some extent on the crest of Great ridge. It is a prominent feature of the formation on account of its extreme size (2 meters) and the large, yellow flowers. Its laciniate leaves 
are arranged with reference to morning and evening light. Lespedexa capitata is a strict, silky-canescent perennial occurring infrequently in low prairies and meadows. In quadrats I, II, and IV of low prairies and meadows it averages 55 individuals, while in quadrats XXII, XXIV, XXV, and XXVII of low meadows it averages but three. When abundant it becomes rather a prominent factor in the floral covering on account of its size, color, and aggregation of plants. The capitate clusters of small flowers do not add to the prom. inence of the plant in the aspect. L. capitata is also an inhabitant of high, dry prairies and bluffs along the Missouri river.

Silphium integrifolium and Vernonia gigantea are two tall, leafy perennials of low meadows and edges of sloughs. In such situations the iron-weed ranges from 15-65 individuals per quadrat and the rosin-weed from 15-100. Silphium frequently grows in dense impenetrable clumps which emphasize all the more its prominence. These two species form a zone about 30 meters wide in the wet meadow, and similar zones in other parts of the formation. In these zones Silphium invariably occupies the inside, next to, but never invading, the Spartina facies, while Vernonia begins about the middle and extends in diminishing numbers to the outside, where it ends in scattered bunches. During July and August these zones are prominent features in the formation, from the masses of yellow flowers of Silphium within, and the purple flowers of Vernonia without, the two colors blending at the center. $V$.gigantea occurs abundantly in low pastures, frequently in company with $V$. fasciculata.

Two hydrophytes, Cicuta maculata and Asclepias incarnata, occur sparsely in the sedge meadow. The large, leafy, robust individuals of $C$. maculata tend to grow in groups; its numerous white-flowered umbels continue during July and are in striking contrast with the rose-purple of the swamp milkweed. Both plants are perennials, the water hemlock developing fleshy tuber-like roots. Another inhal)- 
itant of the sedge meadow, though not a hydrophyte, is Vernonia fasciculata, a plant growing mostly throughout the Carex facies. A few plants of Lycopus americanus, Mentha canadensis, and Scutellaria lateriflora are also scattered through this formation. The taller, overtopping facies render them hardly discernible. All are perennial amphibious plants, by means of suckers or stolons.

Ratibida pinnata, Lactuca canadensis, Meibomia canadensis, Heliopsis scabra, Silphium perfoliatum, and As. tragalus carolinianus are typically frequenters of thickets, open woodlands, and grassy banks of streams. With the exception of Lactuca canadensis, which is a dicyclic, all are pleiocyclic herbs. Ratibida pinnata occurs at but a few stations in the prairies near the creek. Under such conditions it hardly ever blossoms and is of no consequence in the formation. A few individuals of Lactuca canadensis are found in the sedge meadow and in low prairies. The wide dissemination of the achenes of the wild lettuce explains its presence. $L$. canadensis and $L$. ludoviciana develop rosettes, the leaves of which are mostly killed during the winter months. Meibomia canadensis is found near the creek or hedges, where it forms rather dense clumps of individuals. During anthesis these clumps are especially prominent on account of the crowded, terminal racemes of purple flowers. Heliopsis scabra occurs at a few stations in the meadows. It has the appearance of a small sunflower. The square stems and connate leaves of Silphium perfoliatum render individuals of this species noticeable in any formation. A few straggling plants of the square-stemmed rosin-weed are found in the meadows. Astragalus carolinianus also grows in meadows. Its large racemes of greenish-white flowers are rendered less noticeable on account of the many other brighter colored forms. Astragalus is one of the first plants to begin growth in the spring, in spite of its late flowering period.

The remaining six species, Amaranthus torreyi, Convolomlulus sepium, Cassia chamaecrista, Lactuca ludoviciana, 
Panicum capillare, and Polygonum pennsylvanicum are adventitious or ruderal elements. With one exception, Convolvulus sepium, which is perennial by rootstalks, all are hapaxanthous herbs, Lactuca ludoviciana being dicyclic. Convolvulus sepium is an inhabitant of the meadows, where it averages about $\mathbf{3 0}$ to the quadrat. The large, white flowers of the bindweed are conspicuous objects among the facies, the vines being mostly hidden from view. Amaranthus torreyi and Polygonum pennsylvanicum occur sparsely in the wet meadow. Panicum capillare grows on the mounds of the pouched gopher. With the close of maturation, the globose panicle becomes free and rolls about over the prairies. Cassia chamaecrista is undoubtedly an intruder from the section road. It is also found in quantity along the eastern margins of the wet meadow. During maturation the pods split open, thus exposing the seeds for dissemination.

FLEMENTS OF THE SEROTINAL FLORAL ASPECT

\section{Facies}

Andropogon scoparius.

A. furcatus.

Bouteloua curtipendula.

Chrysopogon avenaceus.
Elymus canadensis.

Panicum virgatum.

Spartina cynosuroides. :

\section{Principal Species}

Aster multiflorus.

Helianthus scaberrimus.

Secondary Species

Aster levis.

A. azureus.

A. paniculatus.

A. oblongifolius.

A. sericeus.

Artemisia gnaphalodes.

Ambrosia trifida.

Acalypha virginica.
Carduus altissimus.

Cuscuta paradoxa.

Euthamia graminifolia.

Lespedeza capitata.

Laciniaria pycnostachya

L. scariosa.

Lygodesmia juncea.

Muhlenbergia racemosa. 
Monarda fistulosa.

Nabalus asper.

Polygonum emersum.

Eragrostis pectinacea.

Falcata pitcheri.

Gentiana puberula.

Gyrostachys cernua.

Helianthus maximiliani.

H. annuus.

H. grosse-serratus.

H. giganteus.
H. tuberosus.

Helenium autumnale.

Kuhnia eupatorioides.

Solidago rigida.

S. rigidiuscula.

S. nemoralis.

S. canadensis.

Sporobolus asper.

S. heterolepis.

Vernonia fasciculata.

V. gigantea.

The serotinal period begins during the last days of July and continues with a gradual diminution of bloomers until the close of the flowering season. Its arrival is made known by the blossoming of such species as Solidago rigida and $S$. nemoralis in the high prairies and $S$. canadensis and Laciniaria pycnostachya in the meadows. New species continue to be added until the 15th of September, Aster azureus, A. levis, and Solidago rigidiuscula being among the last. After September 10, the decrease of bloomers in the aspect is very noticeable, the field being left entirely to the asters, goldenrods, blazing stars, sunflowers, and ironweeds. These autumnal bloomers secure the same recognition in the floral covering as the vernal bloomers, by analogous means, i. e., appearing late in the year rather than early, when there is little else of prominence in the floral covering. During the early serotinal period the vegetative layer is extremely complicated, since many of the estival flowers persist for a time, besides sporadic aianthous bloomers. Of the forty-seven bloomers of the period, twenty-eight are species of the Compositae. Accordingly, the aspect from beginning to end is preeminently a composite one, the yellow of the goldenrods and sunflowers being varied with the blue and white of the aster's and the purple of the blazing star's and ironweeds. It is interesting to note that one-half of the serotinal species 
secure wind dissemination directly by means of rolling panicles, pappus modifications, etc.

The distribution of the facies having been taken up in the structure of the formation needs no further comment here. It should be noted, however, that in the higher prairies the Andropogons form a low, scanty herbaceous covering with only here and there a culm. In the meadows, on the contrary, the forked heads of Andropogon furcatus, the close panicles of Chrysopogon avenaceus, and the bearded spikes of Elymus canadensis* control the aspect. The large, purplish panicles of Panicum virgatum are observed toward the center of draws and in moist parts of meadows. Since these are all tall grasses and generally closely aggregated, there is little to be seen of the few, mostly over-topped, secondary species; with one exception, Elymus canadensis, these grasses are rootstalk formers and all are pleiocyclic. The Andropogons and Chysopogon enjoy, at least to some extent, dissemination by means of the wind. Spartina cynosuroides is controlling only in parts of the wet meadow where its tall, coarse culms

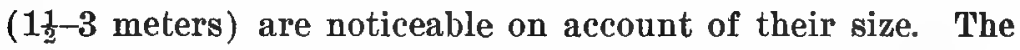
slough grass is perennial with thick, scaly rootstalks.

Aster multiflorus is present, with two exceptions, in every quadrat from I-XXVII; $275-300$ is perhaps a fair average, but not infrequently as many as 2,000 individuals may be enumerated within the five-meter square. The dense, white flowered aster is normally a xerophyte, but it also occurs in low prairies and meadows and with nearly the same abundance as in the higher situations; in the latter, however, the patches are usually of greater extent and the individuals more hirsute and reduced in size and leaf surface. As would be expected, the patches are hardly at all noticeable until anthesis, when they give to the floral covering a characteristic white color for almost two months. Aster multiflorus is perennial by rootstalks and also derelops small, rather closed rosettes.

* Elymas canadensis is an estival bloomer reforred to this place for convenfence. 
Helianthus scaberrimus is a gregario-copious or subcopious xerophyte of Great ridge. It grows mostly in patches of considerable extent, though frequently occurring sparsely for some distance, especially in low prairies. The rigid, scabrous stems, often much reduced on the ridge, attain a height of $\frac{1}{2}-$ $1 \frac{1}{2}$ meters and terminate with a few long-pedunculate, conspicuous, yellow and purple heads, about the middle of July, and continue until October 1 , during a portion of which time patches of these plants contrast sharply with the surrounding floral covering. Helianthus maximiliani grows at a few places along the slopes of the ridge; the individuals are usually low, gregarious, and grayish-canescent. The dwarf, few-flowered plants of this species growing in xerophytic conditions are in striking contrast with the tall, robust, many-flowered individuals of more favorable circumstances. H. maximiliani and $H$. scaberrimus are perennial by thickened rootstalks.

Of the three xerophytic goldenrods, Solidago rigida, $S$. rigidiuscula, and S. nemoralis, all frequenters of the ridge, the first is the most frequent and also the most abundant, occurring in eighteen out of twenty-six quadrats with an average of 12-15; the second is found in nine quadrats out of twenty-six with an average of five individuals; the third, S. nemoralis, is found at only a few stations on the ridge and northern slopes near the creek. Solidago rigida is distributed rather evenly throughout its range and during its flowering period. The rigid, coarse stems, crowned with a dense, yellow corymb, are conspicuous objects of ridges and slopes. The bitter juice of the stem prevents the plant from being eaten, and hence we find this species one of the commonest of the "pasture weeds." A few leaves at the base of the stem usually remain green through the winter, which means, perhaps, the beginning of a rosette. The most showy goldenrod in the prairies is S. rigidiuscula; its terminal oblong heads, usually 5-20 from one root, appear with the last flowers of the serotinal period and continue for a month or more, during which time they are the most showy of the gold- 
enrods of high prairies and bluffs. Solidago nemoralis, the least of our goldenrods, is one of the most beautiful; the ashen-gray plants are gregario-copious, a few to several hundred ,ccurring in a patch. The dull purplish stems terminate with large, one-sided, sparsely branched panicles, which render groups of this plant very noticeable. It goes without saying that the goldenrods are perennial. The latter plant, S. nemoralis, also develops closed rosettes from near the base of the old stem, which present the appearance of a rudimentary mat. Aster oblongifolius and $A$. sericeus are xerophytes of Great ridge; the latter species, $A$. sericens, is conspicuous in the formation throughout its period of growth on account of its silvery white, silky pubescence, and, though the two species enjoy the same distribution, frequence, and abundance, A. oblongifolius is rarely seen except during anthesis. The flowers of both species are large (2-3 cm. broad) with numerous violet-purple or light blue rays surrounding a disc of yellow, and are in striking contrast with the sear brown or greenish brown prairie facies. The individuals of both species tend to become loosely aggregated; both are perennial and frequently form small closed rosettes.

Other xerophytes or species with xerophilous tendencies are Laciniaria scariosa, Kuhnia eupatorioides, Lygodesmia juncea, Sporobolus asper, $\mathbf{S}$. heterolepis, and Eragrost is pectinacea. Laciniaria scariosa is a xerophilous species of Great ridge, with a very strong tendency to grow in low prairies, where it becomes more robust and abundant. In the thirteen quadrats, representing varied conditions, in which the plant occurs, it maintains an average of seven individuals; however, in certain locations of the low prairies fifty plants have been counted within the five-meter square. The hemispheric heads of rose-purple flowers are produced on long, strict racemes, and so far as coloring the aspect is concerned this plant ranks equally with the asters and goldenrods. The blazing stars are perennial by means of tubers. Kuhnia 
cupatorioides inhabits high prairies and to some extent meadows; it is, however, of a xerophytic tendency. Iruhnia occurs in seventeen quadrats out of twenty-seven for all locations, with an average of five individuals. When seen from a distance, especially upon ridges, the plant has a characteristic bush-like appearance, owing to the numerous leafy stems proceeding from the same perennial root. The greenish, terminal flower clusters are of secondary note in the aspect; nevertheless the abundant whitish pappus renders the plant prominent during maturation. Lygodesmia juncea is an inconspicuous xerophilous perennial of Great ridge, occurring only rarely. It belongs properly to the summer aspect. On account of its wiry stems and linear leaves the plant is rarely seen among the facies. Sporobolus asper and $\mathbb{S}$. heterolepis are two pleiocyclic grasses sparsely distributed over Great ridge, neither one being abundant enough, however, to add to the floral covering. It is interesting to note that a xerophyte like sporobolus asper should hare obtained a foothold along the southern border of the wet meadow and become almost controlling for fifty meters or more in extent. Eragrostis pectinacea also is found on Great ridge, but more especially on the lower slopes; in addition to this it is a frequenter of roadsides. It grows in definite bunches which are not recognized until the large purplish panicles appear. The plant is never abundant enough to add color to the aspect. During maturation the panicles become free and roll about as for Panicum capillare, thus dispersing their seeds.

Gentiana puberula is a rare and infrequent inhabitant of low prairies. Its rather short, lcafy stems, mostly clustered from perennial roots, produce, during September and October, the few large, bright blue flowers. Gentiana puberula, Aster sericeus, A. oblongifolius, and Solidago rigidiuscula are among the last bloomers of the year. From various observations made, the flowers of Gentiana are undoubtedly proterandrous, insect pollination obtaining. Artemisia gnaphalodes is normally a xerophyte; its stem and leaves are covered 
over with a dense, woolly coat, thus greatly reducing evaporation. However, in this formation the species is growing in the meadows near the sloughs. The white tomentose character renders the patches prominent in the floral covering. The mugwort is pleiocyclic, root propagation obtaining. Tabalus asper is an infrequent plant of low prairies and meadows. The erect stem, bearing the numerous heads of inconspicuous flowers, proceeds from a tuber. Seedlings of this plant pass the winter in a rosette condition.

Gyrostachys cernua is a frequenter of meadows and draws where it occurs sparsely. It has practically the same distribution and abundance as Lilium canadense, Habenaria leucophaea, and Lobelia spicata hirtella. The small spikes of white flowers are seen only with difficulty, since they appear at a time when the facies equal or overtop them. It is curious to note that some individuals of this species have been found on the crest of Great ridge, others in the wet meadows. This orchis, as for Habenaria leucophaea, is perennial with fleshy-fibrous roots. Euthamia graminifolia is found in draws near the south end of the prairie. The grassy-leaved stems are not easily recognizable until the flat-topped, yellow flower cluster appears. The plants are perennial by long rootstalks. Laciniaria pycnostachya occurs in meadows as a sparse to subcopious plant. Its tall, erect stems, usually in clusters of two to five, terminate with a showy, purple spike (20-40 cm. long) which easily makes the plant the most striking of its time. Where a number of the plants are found together the facies is tinged purplish. The stems are clothed with grass-like leaves, the surfaces of which are arranged for the milder light of morning and evening. The plants are perennial as in L. scariosa. Solidago canadensis is an inhabitant of edges of woodlands, meadows, and draws -the latter especially where erosion is taking place. As rapidly as the facies are dislodged, $S$. cunadensis, Helianthus grosse-serratus, and $H$. giganteus push in and occupy the space. When growing in draws, $S$. canadensis forms rather 
small but definite patches. The yellow flowers of these three species in draws, and of the goldenrod in meadows, color the autumnal meadow aspect. $\boldsymbol{S}$. canadensis is perennial with rootstalks, and is also a rosette former. Helianthus grosseserratus and $H$. giganteus are frequenters of banks of streams, draws, ditches, etc. It has already been stated that these two flowers occupy almost completely the banks of a ditch extending the length of the sedge meadew; in this location, however, $H$. annuus grows with them, all three giving a bright yellow to the eastern margin of the slough. $H$. grosseserratus and $H$. giganteus are perennial by fleshy roots and rootstalks.

Helenium autumnale and Polygonum emersum are two hydrophilous inhabitants of low wet meadows. Helenium is pleiocyclic and occurs sparsely at a few locations in the sedge meadow. In the meadows $P$. emersum is diffused through the facies, as a sparse or subcopious plant, never attaining much size, nor blossoming, while in the sedge meadow it forms a dense clump or patch from which all other species are excluded, and which during anthesis becomes scarlet from the many flower spikes. Polygonum persicaria is perennial with horizontal, creeping rootstalks. Aster paniculatus is a low meadow inhabitant frequently growing in hydrophilous situations. It is found in four quadrats out of six in the sedge meadow, with an average of ten (subcopious 2). When growing in meadows proper or on the banks of streams it often forms patches or clumps which become conspicuous objects during September and October from the many panicles of white-rayed flowers. In the wet meadow the flowers are hardly noticeable on account of the taller facies. The tall white aster is perennial by means of rootstalks. The remaining species, Aster levis, $A$. azureus, Ambrosia trifida, Carduus altissimus, Helianthus annuus, $H$. tuberosus, Muhlenbergia racemosa, Acalypha virginica, Cuscuta paradoxa, and Falcata pitcheri are elements of woodlands and thickets, or adventitious and ruderal 
species occurring only occasionally in the formation. Aster levis and A. azureus are woodland elements growing in prairies and meadows adjacent to woody formations. These two woodland asters occur sparsely to subcopiously in thirteen out of twenty-seven quadrats representing important physiographical variations. In other meadows, I have counted 400 individuals of each of these species in one quadrat. The numerous light blue flower heads appear about the middle of September and stand in marked contrast with the withering facies. Both species are pleiocyclic, and rosette formers, with generally several stems proceeding from the same perennial root. Ambrosia trifica is a robust annual forming herbaceous thickets in draws, ditches, and along roadsides. The great ragweed is one of the worst roadside ruderals, since it adapts itself to almost all conditions, though preferring rather moist land. Its diclinic, anemophilous flowers produce copious, powdery pollen masses which are protected by the inverted saucer-shaped involucres. Carduus altissimus is a tall, branched thicket and woodland species occurring rarely in the wet meadow; it is a pleiocyclic herb and forms closed rosettes. Helianthus annuus is prominent on account of its extreme size by virtue of which it often becomes controlling in the floral covering. Its distribution has been indicated heretofore. The Jerusalem artichoke, Hclianthus tuberosus, and the horsemint, Monarda fistulosa, grow in thickets of Corylus within the prairie, and also along the creek. Both are pieiocyclic woodland species, perennial by rootstalks, the former also with tubers and fleshy rootstalks. Muhlenbergia racenosa, a frequenter of banks of streams and edges of woodlands, grows rather commonly in the low meadows. It is also perennial by means of rootstalks. Acalypha virginica is a small annual of deep woodlands found at a few stations in the low meadows where it forms patches of rather robust individuals; it is not abundant enough, however, to be of importance in the formation. Cuscuta paradoxa forms an interrupted layer in the sunflower patches on the east of the 
wet meadow. The masses of yellowish stems are often abundant enough to be noticeable, and frequently to become controlling for a short distance, in which case they cover over or drag down many of the plants within their reach. The whitish flowers are arranged in glomerate, ropy masses about the stems to which the parasite has grafted itself. The occurrence of Cuscuta, like that of other parasites, is dependent upon the distribution of its host. F'alcata pitcheri is a pleiocyclic woodland twiner growing in the eroded beds of a few draws near the creek. On account of the location, the short racemes of blue flowers do not influence the aspect.

\section{QuAdrat I*-June 29, 1900}

PRAIRIE FORMATION: EASTLRN EXPOSURE: ESTIVAL FLORAT, A.SPECT

Kuhnistera candida, 2, 1, 1, 1, 1, 1, 1, 2, 4, 1, 1, 1, 1, 1, 1, $2,1,4,1,1,3,2,2,1,3,3,1,1,1,1,1,1,1,2,1,1,1$, $1,1,1,2,1,1,1,1,1,2,1,2,1,1,1,1,3,1,2,1,1,1$, $1,1,1,1,1,2,1,1,1,1,1,1,2,1,3,1,1,1,1,1,1,1$, $2,1,1,1,1,1,1,1,1,1,1,4,1,1,1,3,1,2,1,3,1,1$, $2,1,1,1,1,1,2,1,1,1,1,1,1,1,1,2,1,2,1,1,1,1$, $2,1,1,3,1,1,1,1,2,1,1,1,1,1,1,1,2,1,1,1,2,1$, $1,1,1,1,1,2,1,1,3,1,1,1,1,1,2,1,1,1,1,1,1,1$, $1,1,1,1,3,2,1,2,5,2,2,1,1,1,1,1,1,1,1,1,2,1$, $1,1,1,1,1,2,2,1,2,2,2,1,1,1,1,1,1,2,1,1,1,2$, $1,2,3,2,3,1,1,2,2,3,1,1,1,4,2,1,1,2,1,1,1,1$, $1,1,2,1,1,1,4,1,1,2,1,2,1,1,2,1,1,1,2,1,1,1$, $2,1,1,7,1,3,3,1,1,1,1,4,3,1,2,1,1,1,1,1,1,1$, $4,2,1,2,2,2,2,1,2,1,2,1,3,1,1,2,2,1,2,1,3,1$, $1,1,1,2,1,2,3,3,2,1,1,2,3,1,2,2,1,1,1,1,2,1$, $1,1,1,1,1,1,1,1,1,2,2,2,1,1,1,1,1,3,1,1,1,4$, $1,2,1,1,1,2,2,1,3,1,1,1,1,1,3,2,1,1,2,3,1,1$, $1,2,2,1,1,1,2,1,1,1,1,1,2,1,1,2,1,1,1,1,1,1$, $1,1,1,1,1,1,1,1,1,1,2,2,1,2,1,1,1,1,1,3,1,1$,

*In Quadrat I the number of stems from ong root is indicated by eqch number. To save epace this enumeration has been omitted in the remaining quadrat lists. 
$1,1,1,1,4,1,2,1,2,2,3,2,1,1,2,1,1,1,1,1,1,1$, $1,1,1,1,1,1,2,1,2,2,1,2,1,1,1,2,1,2,1,1,1,1$, $1,1,1,2,3,1,3,1,1,1,1,1,1,1,1,2,1,2,1,2,2,3$, $1,4,1,1,2,2,1,1,1,2,2,2,1,2,1,1,2,1,3,1,1,1$, $4,1,2,2,1,1,2,1,1,2,1,3,1,1,1,1,1,1,1,2,1,1$, $1,1,1,1,1,1,1,1,2,2,3,2,1,2,1,2,1,1,1,2,1,1$, $1,1,1,2,2,2,1,1,2,1,1,2,1,1,2,1,2,2,2,1,2,1$, $1,1,1,1,1,2,1,1,2,1,1,1,1,2,1,2,1,1,1,1,1,1$, $2,4,1,2,1,1,1,2,2,1,1,4,1,1,1,1,4,2,1,2,2,3$, $1,1,2,1,2,1,1,2,2,2,2,1,1,1,1,3,2,3,1,1,2,2$, $1,1,2,1,2,1,1,2,2,1,1,1,1,1,2,2,1,1,1,2$,

(cop. 1) 643

Antennaria campestris (five mats) .......... (cop. 1) 324 Aster multiflorus...................... (cop. 1) 249 Lespedeza capitata, $2,1,1,2,1,1,2,1,1,1,1,1,1,1,1$, $1,2,1,1,1,1,1,1,1,1,1,1,1,1,1,1,1,1,1,1,1,1$, $1,1,1,1,2,1,1,1,1,2,1,1,1,1,1,1,1,1,1,1,1,1$, $1,1,1,2,1,1,1,1,1,1,1,1,1,1,1,1,1,1,1,1,1,1$, $1,1,1,1 \ldots \ldots \ldots \ldots \ldots \ldots \ldots \ldots \ldots \ldots$ (cop. 4) 90 Cassia chamaecrista ................... (cop. 4) 57 Amorpha canescens, 3, 4, 1, 2, 1, 3, 2, 2, 7, 1, 1, 1, 1, 1, 3, $3,1,3,4,2,2,1,2,3,1,1,1,6,2,2,1,1,3,1,3,5,3$, $2,2,3 \ldots \ldots \ldots \ldots \ldots \ldots \ldots$ (subcop. 1)

Kuhnistera purpurea, 4, 1, 1, 1, 4, 1, 2, 4, 2, 1, 5, 1, 4, 1, $2,1,1,1,5,1,1,2,1,1,1,1,1,1 \ldots \ldots$ (subcop. 1 ) Koeleria cristata (bunches) ............ (subcop. 1) 20 Physalis virginiana var............. (subcop. 2) 15

Vernonia gigantea................. (subcop. 2) 13

Sisyrinchium angustifolium (bunches).... (subcop. 2) 10 Polygala verticillata................(subcop. 2) 7 Panicum scribnerianum (bunches) ........(subcop. 2) 6 Kuhnia eupatorioides, $8,6,1,1,1 \ldots \ldots \ldots$ (sparse) 5 Meibomia illinoensis, $1,1,1,1,2 \ldots \ldots \ldots$ (sparse) 5 Asclepias verticillata.................. (sparse) 4

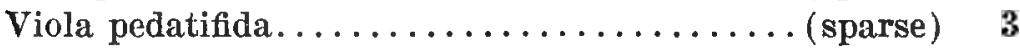
Anemone cylindrica, $1,1 \ldots \ldots \ldots \ldots \ldots$ (sparse) 2 
Aster levis, 2, 3................... (sparse) $\quad 2$

Baptisia bracteata..................... (sparse) 2

Lithospermum canescens................ (sparse) 2

Lactuca ludoviciana..................... (sparse) 1

Psoralea argophylla.................... (sparse) 1

Facies:-

Andropogon scoparius.

A. furcatus.

\section{QUADRAT IJ}

PRAIRIE FORMATION : CREST OF RIDGE: ESTIVAL ASPECT

Aster multiflorus. ................... (cop. 1) 1995

Coreopsis palmata...................... (cop. 1) 1416

Comandra umbellata.....................(cop. 1) 402

Amorpha canescens................... (cop. 3) 128

Kuhnistera purpurea .................... (cop. 4) $\mathbf{5 1}$

Antennaria campestris (two mats) ....... (subgreg. 1) 37

Ceanothus ovatus.................... (subcop. 1) 29

Solidago rigida.................... (subcop. 1) 28

Brauneria pallida................... (subcop. 1) 21

Laciniaria scariosa ................... (subcop. 1) 21

Panicum scribnerianum (bunches) ........ (subcop. 1) 20

Viola pedatifida...................... (subcop. 1) 18

Koeleria cristata (bunches)............. (subcop. 2) 8

Aster oblongifolius................. (subcop. 2) 7

Kuhnistera candida..................(subcop. 2) 7

Rosa arkansana.................... (subcop. 2) 7

Euphorbia corollata................. (greg-cop. 2) 6

Physalis virginiana var.................(subcop. 2) 6

Carex pennsylvanica (bunches)............ (sparse) $\mathbf{5}$

Stipa spartea (bunches)................ (sparse) 4

Panicum depauperatum (bunches)........... (sparse) 3

Aster sericeus.......................... (sparse) $\quad 2$

Baptisia bracteata .................... (sparse) $\quad$ 2

Erigeron ramosus..................... (sparse) 2

Ruellia ciliosa...................... (sparse) 2 
Kuhnia eupatorioides ............... (sparse) 1

Lactuca ludoviciana................... (sparse) 1

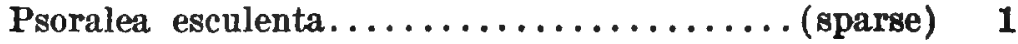

Facies :-

Andropogon scoparius.

A. furcatus.

Bouteloua curtipendula.

\section{QUADRAT III}

PRAIRIE FORMATION : ESTIVAT ASPECT: CREST OF RIDGE

Aster multiflorus. ................... (cop. 1) 1925

Comandra umbellata................... (cop. 1) 1460

Coreopsis palmata......................... 1) 593

Kuhnistera purpurea....................... 2) 150

Amorpha canescens.................... (cop. 3) 138

Solidago rupestris................ (greg-cop. 4) 54

Euphorbia corollata ................(greg-cop. 4) 51

Brauneria pallida................. (subcop. 1) 48

Rosa arkansana...................(subcop. 1) 23

Viola pedatifida.................. (subcop. 1) 17

Erigeron ramosus...................(subcop. 1) 16

Koeleria cristata (bunches) ............ (subcop. 2) 12

Kuhnistera candida.................. (subcop. 2) 12

Panicum scribnerianum (bunches)......... (subcop. 2) 10

Helianthus scaberrimus .............. (subcop. 2) 8

Cassia chamaecrista.................(subcop. 2) 7

Stipa spartea (bunches) ................ (sparse) 5

Bouteloua curtipendula (bunches) .......... (sparse) 4

Sisyrinchium angustifolium.............. (sparse) 3

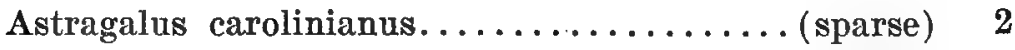

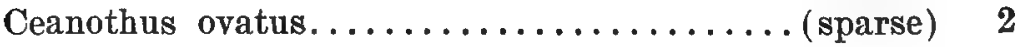

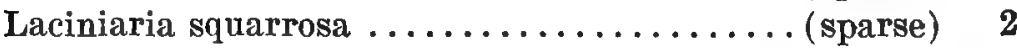

Panicum depauperatum (bunches)..........(sparse) 2

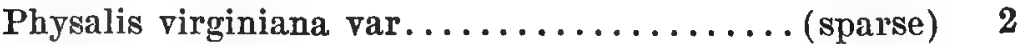

Solidago rigida................... (sparse) 2 
Laciniaria scariosa

Facies :-

Andropogon scoparius.

A. furcatus.

\section{QUAdrat IV.}

PRAIRIE FORMATION : NORTHERN EXPOSURE NEAR BASE OF RIDGE : LATE ESTIVAL FLORAL ASPECT

Aster multiflorus................... (cop. 1) 1325

Antennaria campestris (four mats) ........ (greg. 1) 262

Kuhnistera candida ........................... 3) 115

Meibomia illinoensis....................(cop. 4) 87

Vernonia gigantea.................... (greg-cop. 4) 61

Equisetum laevigatum................ (subcop. 1) 44

Viola pedatifida.......................(subcop. 1) 42

Helianthus scaberrimus................. (subcop. 1) 36

Aster levis....................... (subcop. 1) 25

Panicum scribnerianum................. (subcop. 1) 25

Poa pratensis...................... (subcop. 1) 24

Erigeron ramosus.................... (subcop. 1) 21

Kuhnia eupatorioides.................. (subcop. 1) 19

Lespedeza capitata................... (subcop. 1) 18

Scutellaria parvula................... (subcop. 2) 13

Panicum virgatum................... (subcop. 2) 12

Physalis virginiana var............... (subcop. 2) 12

Solidago rigida.....................(subcop. 2) 12

Solidago rupestris.................. (greg-cop. 2) 11

Muhlenbergia racemosa................ (subcop. 2) 9

Aster azureus..................... (subcop. 2) 8

Anemone cylindrica...................(sparse) 5

Asclepias verticillata.................. (sparse) $\mathbf{5}$

Bouteloua curtipendula (bunches) ........... (sparse) 3

Euphorbia glyptosperma.................(sparse) 3

Amorpha canescens.................... (sparse) 2

Euphorbia nutans.................... (sparse) 2

Silphium integrifolium................ (sparse) $\quad 2$ 


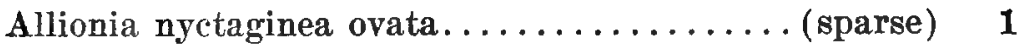

Cornus asperifolia.................... (sparse) 1

Fragaria virginiana ................. (sparse) 1

Polygala verticillata $\ldots \ldots \ldots \ldots \ldots \ldots \ldots$ (sparse) 1

Vicia americana .................. (sparse) 1

Facies :-

Andropogon furcatus.

A. scoparius.

\section{QUADRAT V}

PRAIRIE FORMATION: WESTERN EXPOSURE : LATE ESTIVAL FLORAI، ASPECT

Aster multiflorus.................... (cop. 1) 2275

Meibomia illinoensis................... (cop. 1) 672

Antennaria campestris (eight mats) ....... (greg. 1) 350

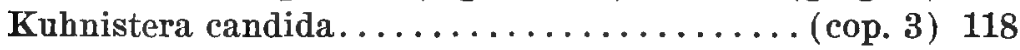

Equisetum laevigatum................ (cop. 4) 70

Lespedeza capitata................ (cop. 4) 57

Silphium integrifolium................(cop. 4) 50

Poa pratensis.................... (subcop. 1) 24

Panicum scribnerianum (bunches) ........ (subcop. 1) 22

Vernonia gigantea.............. (sub-greg-cop. 1) 18

Asclepias verticillata................(subcop. 1) 16

Helianthus scaberrimus............... (subcop. 2) 15

Viola pedatifida................... (subcop. 2) 15

Bouteloua curtipendula (bunches)........ (subcop. 2) 12

Solidago rigida.................... (subcop. 2) 9

Kuhnia eupatorioides................ (subcop. 2) 8

Aster levis . . . . . . . . . . . . . . .... (subcop. 2) 7

Polygala verticillata.................(subcop. 2) 7

Solidago rupestris.................(greg-cop. 2) 6

Aster azureus ...................... (sparse) 5

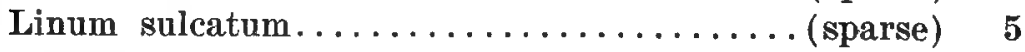

Scutellaria parvula................... (sparse) 4

Heliopsis scabra....................... (sparse) 3

Kuhnistera purpurea................ (sparse) 3 


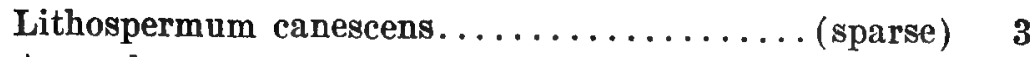

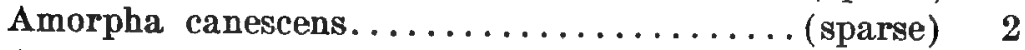

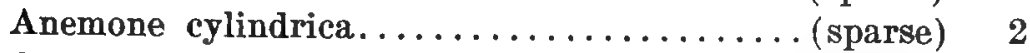

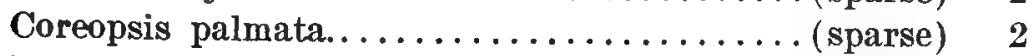

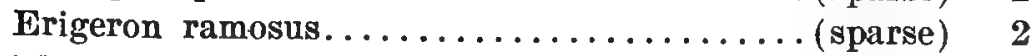

Physalis virginiana var................ (sparse) 2

Acalypha virginica.................. (sparse) 1

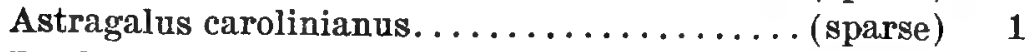

Euphorbia glyptosperma................ (sparse) 1

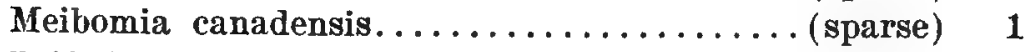

Trifolium pratense................... (sparse) 1

Facies:-

Andropogon furcatus.

A. scoparius.

Panicum virgatum.

\section{QUAdrat VI}

PRAIRIE FORMATION : EASTERN EXPOSURE : LATE ESTIVAL FLORAL ASPECT

Antennaria campestris (nine mats) . . . . . . (greg. 1) 322

Solidago rupestris................. (greg-cop. 1) 273

Kuhnistera candida................... (cop. 2) 190

Aster multiflorus..................... (cop. 3) 105

Kuhnistera purpurea................(subcop. 1) 49

Panicum scribnerianum (bunches) ........ (subcop. 1) 25

Viola pedatifida...................(subcop. 1) 21

Rosa arkansana..................(subcop. 1) 17

Euphorbia corollata...............(subcop. 2) 14

Physalis virginiana var..............(subcop. 2) 12

Solidago rigida.................... (subcop. 2) 10

Panicum depauperatum..............(subcop. 2) 9

Brauneria pallida..................(subcop. 2) 8

Bouteloua curtipendula (bunches) ........ (subcop. 2) 7

Erigeron ramosus................. (subcop. 2) 6

Kuhnia eupatorioides ............... (subcop. 2) 6

Linum sulcatum................(subcop. 2) 6 
Stipa spartea (bunches) ..............(subcop. 2) 6

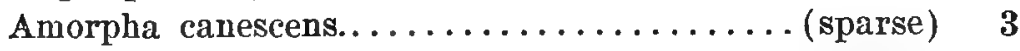

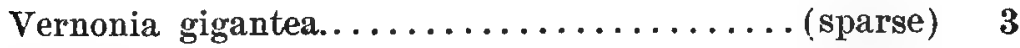

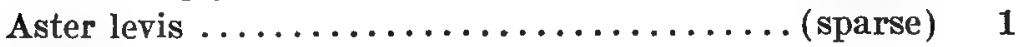

Baptisia bracteata .................... (sparse)

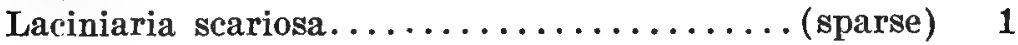

Lithospermum canescens ............... (sparse) 1

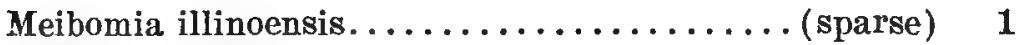

Polygala verticillata................... (sparse) 1

Ruellia ciliosa...................... (sparse) 1

Facies:-

Andropogon furcatus.

A. scoparius.

\section{QUADRAT VII}

PRAIRIE FORMATION: NORTHERN EXPOSURE: LATE ESTIVAL FLORAL ASPECT

Comandra umbellata................... (cop. 1) 1650

Antennaria campestris (fourteen mats) ...... (greg. 1) 1250

Coreopsis palmata.................... (cop. 1) 1100

Kuhnistera purpurea................... (cop. 1) 1032

Helianthus scaberrimus ................ (cop.4) 83

Solidago nemoralis................. (greg. 4) 71

Brauneria pallida........................... 4) 63

Laciniaria squarrosa.................(cop.4) $\mathbf{5 5}$

Aster levis ........................ (cop.4) 55

Euphorbia corollata................(subcop. 1) 39

Panicum virgatum................... (subcop. 1) 29

Bouteloua curtipendula (bunches) ........ (subcop. 1) 28

Amorpha canescens.................. (subcop. 1) 23

Laciniaria scariosa .................. (subcop. 1) 23

Viola pedatifida.................. (subcop. 1) 21

Aster sericeus...................... (subcop. 1) 19

Aster azureus.................... (subcop. 1) 18

Koeleria cristata (bunches) ............ (subcop. 2) 12

Rosa arkansana................... (subcop. 2) 11 
Kuhnistera candida.................. (subcop. 2) 10

Stipa spartea (bunches)................ (sparse) 5

Panicum depauperatum (bunches).......... (sparse) 3

Linum sulcatum...................... (sparse) $\quad 2$

Psoralea esculenta..................... (sparse) 2

Sisyrinchium angustifolium............... (sparse) $\quad \mathbf{2}$

Acerates viridifiora ivesii................ (sparse) 1

Ceanothus ovatus...................... (sparse) 1

Facies:-

Andropogon furcatus.

A. scoparius.

\section{QUADRAT VIII}

PRAIRIE FORMATION: SOUTHEAST EXPOSURE: ON GREAT RIDGE: LATE ESTIVAL ASPECT

Comandra umbellata.................... (cop. 1) 850

Coreopsis palmata...................... (cop. 1) 375

Erigeron ramosus.....................(cop. 1) 231

Kuhnistera purpurea................... (cop.2) 176

Aster multiflorus ..................... (cop. 4) $\quad \mathbf{7 4}$

Solidago rigida..................... (subcop. 1) $\quad 46$

Brauneria pallida................... (subcop. 1) 34

Laciniaria squarrosa ................. (subcop. 1) 28

Viola pedatifida...................... (subcop. 1) 18

Euphorbia corollata................. (greg-cop. 2) $\quad 15$

Aster levis........................ (subcop. 2) 14

Antennaria campestris................ (subgreg. 2) 11

Aster sericeus..................... (subcop. 2) 11

Rosa arkansana..................... (subcop. 2) 11

Amorpha canescens.................. (subcop. 2) 10

Kuhnistera candida.................. (subcop. 2) 10

Stipa spartea (bunches) .............. (subcop. 2) 9

Laciniaria scariosa ................... (subcop. 2$) \quad 7$

Solidago nemoralis ................. (subgreg. 2) 7

Koeleria cristata (bunches) ............. (subcop. 2) 6

Physalis virginiana var................ (sparse) 5 
Eelianthus scaberrimus .............. (sparse) 4

Panicum depauperatum (bunches) .......... (sparse) 4

Bouteloua curtipendula (bunches) ........... (sparse) 3

Solidago rupestris.................. (vix-greg.) 3

Panicum scribnerianum (bunches)......... (sparse) 2

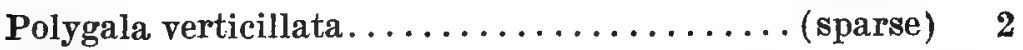

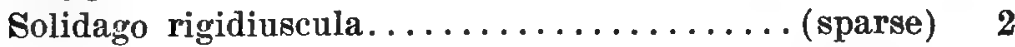

Stipa spartea (bunches) ............... (sparse) 2

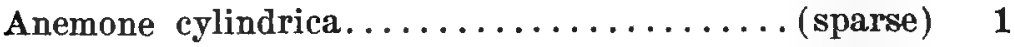

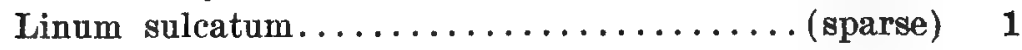

Lithospermum canescens................. (sparse) 1

Facies:-

Andropogon scoparius.

A. furcatus.

Carex pennsylvanica.

QUADRAT İX

PRAIRIE FORMATION : HALF WAY BETWEEN FOOT AND CREST OF RIDGE : SOUTHEASTERN EXPOSURE : ESTIVAL FLORAL ASPECT

Kuhnistera purpurea................. (cop. 1) 476

Comandra umbellata.................... (cop. 1) 408

Helianthus scaberrimus ................. (cop. 1) 365

Kuhnistera candida................... (cop. 1) 225

Solidago rupestris................. (greg-cop. 1) 204

Aster multiflorus.................... (cop. 2) 175

Antennaria campestris (three mats) ......... (greg. 4) 62

Amorpha canescens. ................. (subcop. 1) 42

Viola pedatifida.................... (subcop. 1) 30

Brauneria pallida................. (subcop. 1) 28

Physalis virginiana var..............(subcop. 1) 17

Rosa arkansana.................. (subcop. 1) 16

Erigeron ramosus ................. (subcop. 2) 14

Stipa spartea (bunches) . . . . . . . . . . (subcop. 2) 12

Aster levis........................(subcop. 2) 10

Euphorbia corollata.............. (subgreg-cop. 2) 8

Panicum depauperatum (bunches).......(subcop. 2) \& 
Solidago rigida................... (subcop. 2) 7

Solidago rigidiuscula................(subcop. 2) 7

Asclepias obtusifolia................(subcop. 2) 6

Stipa spartea (bunches) ............. (subcop. 2) 6

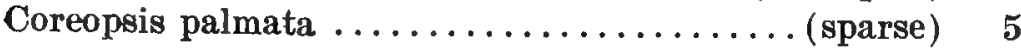

Kuhnia eupatorioides ............... (sparse) 4

Laciniaria squarrosa.................. (sparse) 4

Panicum scribnerianum (bunches).......... (sparse) 4

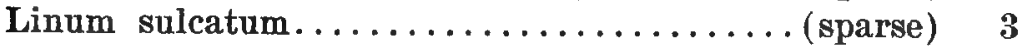

Aster oblongifolius.................... (sparse) 2

Baptisia bracteata ................... (sparse) 2

Aster azureus..................... (sparse) 1

Facies:-

Bouteloua curtipendula.

Andropogon scoparius.

A. furcatus.

\section{QUadrat X}

PRAIRIE FORMATION: AT BASE OF GREAT RIDGE: EASTERN EXPOSURE : ESTIYAL ASPECT

Kuhnistera candida................. (cop. 1) 482

Kuhnistera purpurea................. (cop. 1) 360

Antennaria campestris (seven mats) ........ (greg. 1) 284

Erigeron ramosus................... (cop. 3) 117

Comandra umbellata.................. (cop. 4) 101

Solidago rupestris................. (greg-cop. 3) 101

Aster multiflorus...................... (cop.4) 80

Euphorbia corollata.............. (subgreg-cop. 1) 48

Rosa arkansana..................(subcop. 1) 30

Brauneria pallida.................. (subcop. 1) 25

Panicum scribnerianum (bunches)........ (subcop. 1) 23

Viola pedatifida .................. (subcop. 2) 14

Amorpha canescens.................. (subcop. 2) 8

Bouteloua curtipendula (bunches) ........ (subcop. 2) 8

Stipa spartea (bunches) .............. (subcop. 2) 8

Aster oblongifolius ................ (subcop. 2) 6 
Physalis virginiana var .............. (sparse) $\mathbf{5}$

Solidago rigida..................... (sparse) 5

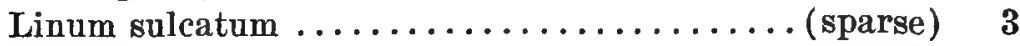

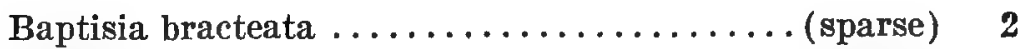

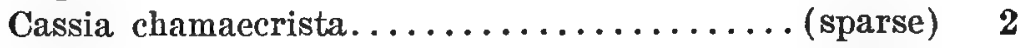

Kuhnia eupatorioides ................ (sparse) 2

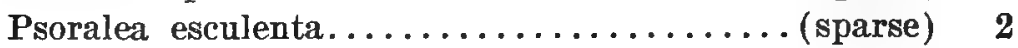

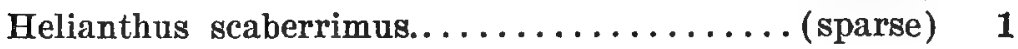

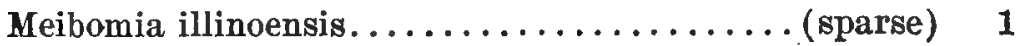
Facies:-

Andropogon scoparius.

A. furcatus.

\section{Quadrat XI}

PRAIRIE FORMATION : NEAR CREST OF GREAT RIDGE : EASTERN EXPOSURE: LATE ESTIVAL FLORAL ASPECT

Comandra umbellata................... (cop. 1) 1750 Aster multiflorus......................(cop. 1) 625

Helianthus scaberrimus ................ (cop. 1) 550 Kuhnistera purpurea................... (cop. 2) 163 Amorpha canescens................... (cop. 3) 134 Coreopsis palmata..................... (cop. 3) 129 Antennaria campestris (one mat) .......... (greg. 4) 89 Panicum scribnerianum (bunches) . . . . . . (subcop. 1) 29 Viola pedatifida...................(subcop. 1) 28 Euphorbia corollata .............. (subgreg-cop. 1) 25 Panicum depauperatum (bunches)......... (subcop. 1) 23 Solidago rupestris...............(subgreg-cop. 1) 20 Rosa arkansana ...................... (subcop. 1) 17 Bouteloua curtipendula (bunches)........ (subcop. 2) 12 Brauneria pallida.................. (subcop. 2) 12 Erigeron ramosus. ................ (subcop. 2) 6 Solidago rigida................... (subcop. 2) 6 Stipa spartea (bunches) .............. (subcop. 2) 6

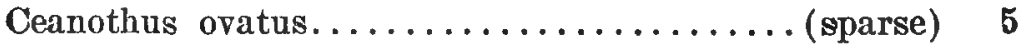
Laciniaria scariosa ................. (sparse) 5 
Kuhnistera candida..................... (sparse) 4

Chenopodium album .................. (sparse) 1

Physalis virginiana var............... (sparse) 1

Psoralea esculenta...................... (sparse) 1

Facies:-

Andropogon scoparius.

A. furcatus.

Carex pennsylvanica.

\section{Quadrat XII}

PRAIRIE FORMATION: CREST OF RIDGE: LATE ESTIVAL FLORAL ASPECT

Antennaria campestris................ (greg. 1) 1950

Comandra umbellata.................... (cop. 1) 1900

Coreopsis palmata....................(cop. 1) 900

Kuhnistera purpurea................... (cop. 1) 425

Amorpha canescens.................... (cop. 1) 326

Euphorbia corollata............... (greg-cop. 4) 90

Aster multiflorus ..................... (cop. 4) 75

Brauneria pallida................. (subcop. 1) 45

Viola pedatifida.................... (subcop. 1) 34

Panicum scribnerianum (bunches) ........ (subcop. 1) 29

Bouteloua curtipendula (bunches) ........ (subcop. 1) 25

Stipa spartea (bunches) ............. (subcop. 1) 17

Solidago rigida................... (subcop. 2) 15

Aster levis....................... (subcop. 2) 12

Erigeron ramosus.................. (subcop. 2) 11

Panicum depauperatum (bunches) ........ (subcop. 2) 9

Laciniaria scariosa................ (subcop. 2) 6

Laciniaria squarrosa................. (subcop. 2) 6

Kuhnistera candida................... (sparse) 5

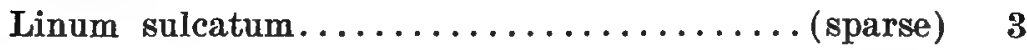

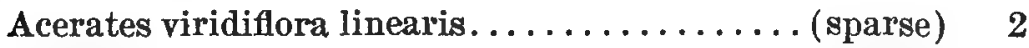

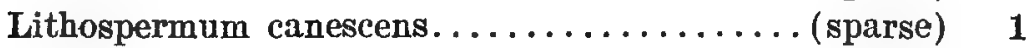

Physalis virginiana $\operatorname{var} . \ldots \ldots \ldots \ldots \ldots$ (sparse) 1 


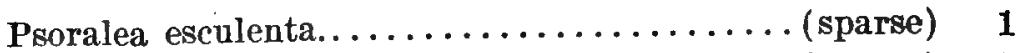
Solidago rigidiuscula ................ (sparse). 1 Facies :-

Andropogon scoparius.

A. furcatus.

\section{Quadrat XIII}

PRAIRIE FORMATION : TOWARDS BASE OF RIDGE: SOUTHEASTERN EXPOSURE : LAATE ESTIVAL FLORAL ASPECT

Amorpha canescens................... (cop. 1) 425 Aster multiflorus.................... (cop. 2) 178

Kuhnistera candida................... (cop. 2) 169 Solidago rupestris................... (greg-cop.) 75 Meibomia illinoensis .................(subcop. 1) 25

Panicum scribnerianum (bunches) ......... (subcop. 1) 25

Euphorbia corollata .............. (subgreg-cop. 1) 22

Viola pedatifida..................(subcop. 1) 21

Stipa spartea (bunches) .............. (subcop. 2) 14

$\Lambda$ sclepias verticillata ................. (subcop. 2) 12

Kuhnistera purpurea................ (subcop. 2) 10

Erigeron ramosus.................. (subcop. 2) 6

Linum sulcatum ............... (subcop. 2) 6

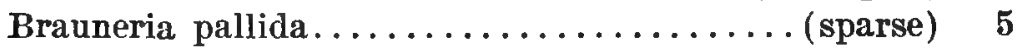

Acerates viridiflora ivesii................ (sparse) 4

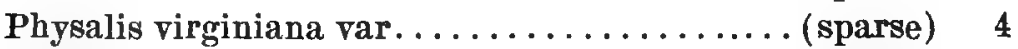

Solidago rigidiuscula.................. (sparse) 4

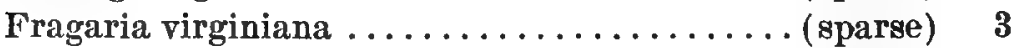

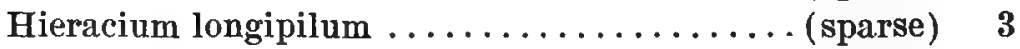

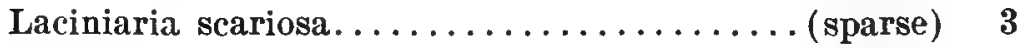

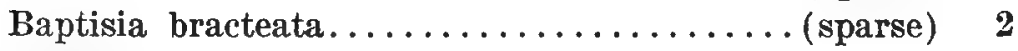

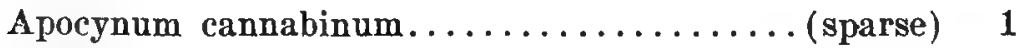

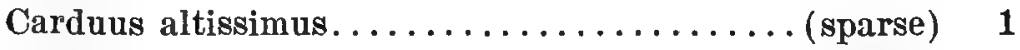

Kuhnia eupatorioides ................. (sparse) 1

Silphium laciniatum................ (sparse) 1 
Facies :-

Andropogon scoparius.

A. furcatus.

Bouteloua curtipendula.

\section{QUADRAT XIV}

PRAIRIV FORMATION : NORTHEAST EXPOSURE: HALF TVAY UP RIDGE : LATE ESTIVAL FLORAL ASPECT

Comandra umbellata. ................. (cop. 1) 1275

Kuhnistera purpurea.................(cop. 1) 575

Aster multiflorus.......................... 1) 275

Coreopsis palmata..................... (cop. 3) 125

Amorpha canescens...................(cop. 4) 90

Euphorbia corollata...............(greg-cop. 4) 86

Solidago rupestris................(greg-cop. 4) 51

Amorpha canescens .................(subcop. 1) 44

Brauneria pallida.................... (subcop. 1) 29

Aster levis . . . . . . . . . . . . . . . (subcop. 1) 20

Equisetum laevigatum................ (subcop. 1) 19

Rosa arkansana....................(subcop. 1) 16

Cassia chamaecrista.................(subcop. 2) 15

Viola pedatifida................... (subcop. 2) 12

Antennaria campestris (bunches) ........ (subgreg. 2) 10

Bouteloua curtipendula (bunches) ......... (subcop. 2) 10

Erigeron ramosus................... (subcop. 2) 10

Linum sulcatum................. (subcop. 2) 9

Kuhnia eupatorioides .............. (sparse) 5

Laciniaria scariosa............................... 5

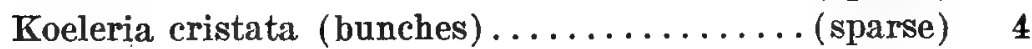

Nabalus asper....................... (sparse) 4

Sisyrinchium angustifolium (bunches)....... (sparse) 3

Solidago rigida................... (sparse) $\mathbf{3}$

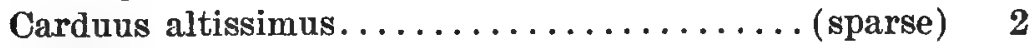

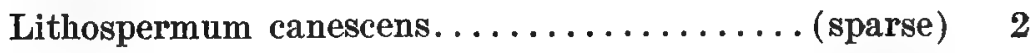

Solidago rigidiuscula................ (sparse) 2

Vernonia gigantea................... (sparse) 2

Ambrosia trifida................................ 1 
Lactuca ludoviciana (sparse)

Polygala verticillata (sparse)

Facies:-

Andropogon scoparius.

A. furcatus.

Panicum scribnerianum.

\section{QUAdRAT XV}

PRAIRIE FORMATION: NORTHEAST EXPOSURE: HALF WAY UP RIDGE : LATE ESTIVAL ASPECT

Antennaria campestris (estimated) ......... (greg. 1) 2050 Comandra umbellata.....................(cop. 1) 375 Cassia chamaecrista..................... (cop. 3) 140 Kuhnistera candida ....................(cop. 3) 115 Aster multiflorus ....................... (cop. 3) 105 Amorpha canescens .................... (cop.4) 92 Coreopsis palmata.....................(cop.4) 86 Kuhnistera purpurea .................... (cop.4) 75 Euphorbia corollata .............. (subgreg-cop. 1) 39 Brauneria pallida .................... (subcop. 1) 27 Rosa arkansana ..................... (subcop. 1) 26 Solidago rupestris ............... (subgreg-cop. 1) 24 Helianthus scaberrimus ................ (subcop. 1) 21 Solidago rigida.................... (subcop. 1) 17

Viola pedatifida ..................... (subcop. 1) 17 Erigeron ramosus .................... (sparse) 5 Gentiana puberula ................... (sparse) 4 Acerates viridiflora ivesii................. (sparse) 3 Panicum depauperatum (bunches) .......... (sparse) 3 Polygala verticillata ................... (sparse) 3 Lactuca ludoviciana.................... (sparse) 2 Physalis virginiana var................. (sparse) 2

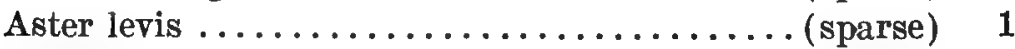
Baptisia bracteata ..................... (sparse) 1 Kuhnia eupatorioides .................. (sparse) 1 
Laciniaria scariosa ...................... (sparse) 1

Linum sulcatum ..................... (sparse) 1

Facies:-

Andropogon scoparius.

A. furcatus.

Panicum scribnerianum.

\section{Quadrat XVI}

PRAIRIE FORMATION: WESTERN EXPOSURE : HALF WAY UP RIDGE: LATE ESTIVAL ASPECT

Comandra umbellata ...................... 1) 550

Amorpha canescens ..................... (cop. 1) 325

Aster multiflorus ........................... 1) 275

Coreopsis palmata ......................... (cop. 1) 206

Kuhnistera purpurea ................. (cop. 3) 116

Euphorbia corollata .................(cop.4) 87

Antennaria campestris (1 mat) ............(greg.4) 51

Brauneria pallida ..................(subcop. 1) 33

Kuhnistera candida ................(subcop. 1) 32

Solidago rigidiuscula ............... (subcop. 1) 17

Koeleria cristata (bunches) ........... (subcop. 2) 15

Cassia chamaecrista ................. (subcop. 2) 10

Panicum scribnerianum (bunches) ....... (subcop. 2) 10

Linum sulcatum . . . . . . . . . . . . (subcop. 2) 9

Laciniaria scariosa ................. (subcop. 2) 7

Panicum depauperatum (bunches)......... (sparse) 5

Bouteloua curtipendula (bunches) .......... (sparse) 4

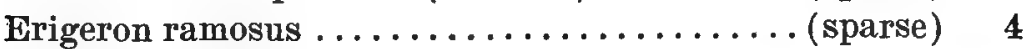

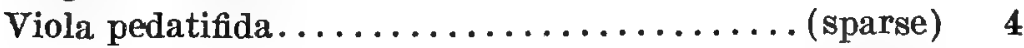

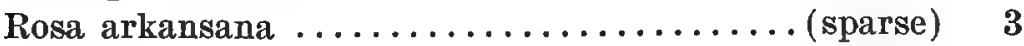

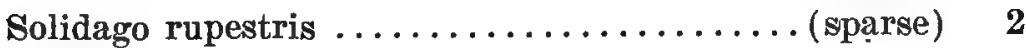

Acerates viridiflora ivesii................ (sparse) 1

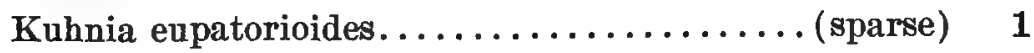

Physalis virginiana var ................ (sparse) 1 
Facies:-

Andropogon scoparius.

A. furcatus.

\section{QUADRAT XVII}

PRAIRIE FORMATION : EXPOSURE WESTERN, ABRUPT SLOPE : LATE ESTIVAL FLORAL ASPECT

Antennaria campestris (14 mats) .......... (greg. 1) 1750

Comandra umbellata .................. (cop. 1) 1450

Àster multiflorus ..................... (cop. 1) 575

Amorpha canescens ................... (cop. 3) 142

Kuhnistera candida................... (subcop. 1) 20

Kuhnistera purpurea ................. (subcop. 1) 19

Bouteloua curtipendula (bunches) ........ (subcop. 2) 12

Stipa spartea (bunches) . . . . . . . . . . (subcop. 2) 12

Erigeron ramosus ................. (subcop. 2) 10

Panicum scribnerianum (bunches) ........(subcop. 2) 9

Rosa arkansana .................... (subcop. 2) 6

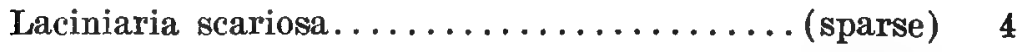

Panicum depauperatum (bunches)......... (sparse) 4

Koeleria cristata (bunches) ............... (sparse) 3

Ambrosia trifida ..................... (sparse) 2

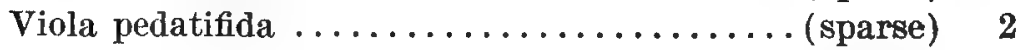

Acerates viridiflora ivesii................ (sparse) 1

Agrostis hiemalis ................... (sparse) 1

Astragalus crassicarpus ................. (sparse) 1

Euphorbia corollata .................. (sparse) 1

Gentiana puberula .................... (sparse) 1

Linum sulcatum .................... (sparse) 1

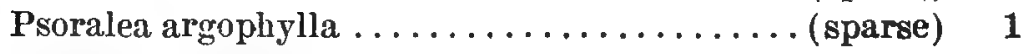

Solidago rigidiuscula $\ldots \ldots \ldots \ldots \ldots \ldots \ldots \ldots$ (sparse) 1

Vernonia gigantea ................... (sparse) 1

Facies:-

Andropogon scoparius.

A. furcatus. 


\section{QUAdRAT XVIII}

PRAIRIE FORMATION : NORTHWEST EXPOSURE : MIDWAY BETWEEN CREST AND FOOT OF RIDGE : LATE ESTIVAL ASPECT

Comandra umbellata................... (cop. 1) 1900 Antennaria campestris................... (cop. 1) 929 Amorpha canescens.................... (cop. 1) 500 Coreopsis palmata.................... (cop. 2) 190 Euphorbia corollata .................. (greg-cop. 3) 110 Brauneria pallida................... (subcop. 1) $\quad 49$ Kuhnistera purpurea................. (subcop. 1) $\mathbf{3 4}$ Aster levis....................... (subcop. 1) 28 Aster multiflorus................... (subcop. 1) 28 Panicum scribnerianum (bunches)......... (subcop. 1) 25 Viola pedatifida...................... (subcop. 1) 25 Laciniaria squarrosa................... (subcop. 2) 12 Laciniaria scariosa................... (subcop. 2) 11 Rosa arkansana..................... (subcop. 2) 11 Solidago nemoralis................ (sub-greg. 2) 8 Erigeron ramosus.................. (subcop. 2$) \quad 6$ Helianthus scaberrimus ................. (sparse) 5 Panicum depauperatum (bunches) ........... (sparse) 5 Kuhnistera candida................... (sparse) 4 Linum sulcatum..................... (sparse) 4 Bouteloua curtipendula (bunches).......... (sparse) $\quad 2$ Kuhnia eupatorioides .................. (sparse) 1 Panicum pubescens................... (sparse) 1 Vernonia gigantea.................... (sparse) $\quad 1$ Facies :Andropogon scoparius. A. furcatus. 


\section{Quadrat XIX}

PRAIRIE FORMATION : TOWARD FOOT OF RIDGE : NORTHWIST EX. POSURE : LATE ESTIVAL FLORAL ASPECT

Antennaria campestris (twelve mats) ........ (greg. 1) 855

Comandra umbellata..................... (cop. 1) 450

Kuhnistera candida................... (cop. 1) 356

Coreopsis palmata................... (cop. 3) 137

Euphorbia corollata ................ (greg-cop. 3) 135

Carex pennsylvanica (bunches) .............. (cop. 4) 100

Amorpha canescens....................(cop. 4) 88

Brauneria pallida................... (subcop. 1) 20

Bouteloua curtipendula (bunches) ........ (subcop. 2) 12

Erigeron ramosus .................. (subcop. 2) 11

Panicum scribnerianum (bunches) ........(subcop. 2) 10

Rosa arkansana.................. (subcop. 2) 9

Kuhnistera purpurea................(subcop. 2) 8

Solidago rigidiuscula..............(subcop. 2) 7

Viola pedatifida.................. (subcop. 2) 7

Aster azureus................... (subcop. 2) 6

Laciniaria scariosa ................ (subcop. 2) 6

Panicum depauperatum (bunches) .......... (sparse) 5

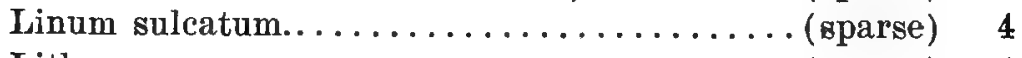

Lithospermum canescens............... (sparse) 4

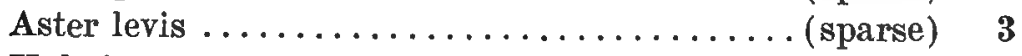

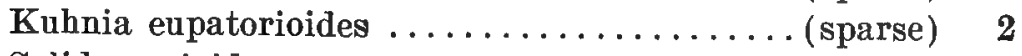

Solidago rigida................... (sparse) 2

Acerates viridiflora ivesii.................. (sparse) 1

Sisyrinchium angustifolium (bunches)........ (sparse) 1

Solidago rupestris.................... (sparse) 1

Facies :-

Andropogon scoparius.

A. furcatus.

Koeleria cristata. 


\section{QUadrat XX}

PRAIRIE FORMATION : NORTHERN EXPOSURE : ON CREST OF RIDGE : LATE ESTIVAL FLORAL ASPECT

Coreopsis palmata................... (cop. 1) 1100

Comandra pallida...................... (cop. 1) 1050

Antennaria campestris (four mats) ......... (greg. 1) 237

Brauneria pallida............................ 4) 60

Aster multiflorus....................(cop. 4) 56

Euphorbia corollata...............(greg-cop. 4) 50

Laciniaria scariosa ................. (subcop. 1) 33

Erigeron ramosus................. (subcop. 1) 24

Kuhnistera purpurea..............(subcop. 1) 18

Silphium laciniatum................(subcop. 2) 13

Solidago rigida.................. (subcop. 2) 13

Kuhnistera candida................ (subcop. 2) 9

Bouteloua curtipendula (bunches) ........(subcop. 2) 8

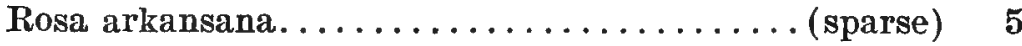

Stipa spartea (bunches) .............. (sparse) 5

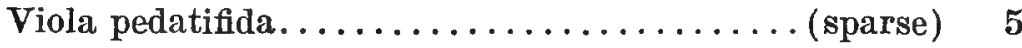

Panicum depauperatum (bunches) .......... (sparse) 4

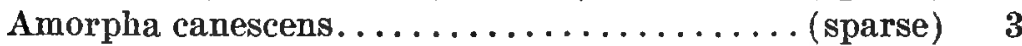

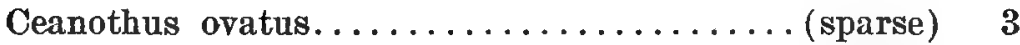

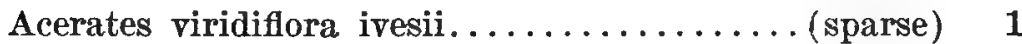

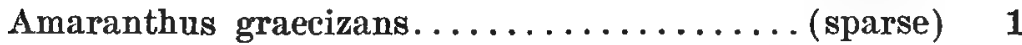

Eatonia obtusata (bunches) .............. (sparse) 1

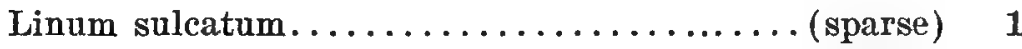

Lithospermum canescens................ (sparse) 1

Facies :-

Andropogon scoparius.

A. furcatus.

Koeleria cristata. 


\section{QUADRAT XXI}

PRAIRIE FORMATION : NORTHWEST EXPOSURE: MIDWAY BETWEEN FOOT AND CREST OF RIDGE : LATE ESTIVAL FLORAL ASPECT

Antenuaria campestris (seven mats) ....... (greg. 2) 185

Coreopsis palmata....................(cop. 2) 178

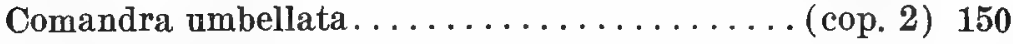

Kuhnistera purpurea..................(cop. 3) 119

Brauneria pallida....................... 3) 117

Euphorbia corollata............... (greg-cop. 3) 107

Linum sulcatum..................... (cop.4) 63

Erigeron ramosus.................. (subcop. 1) 32

Amorpha canescens................. (subcop. 1) 26

Koeleria cristata (bunches) ............ (subcop. 1) 16

Solidago rigida................... (subcop. 2) 13

Laciniaria squarrosa . . . . . . . . . . . . . (subcop. 2) 12

Aster multiflorus. ................. (subcop. 2) 10

Stipa spartea (bunches) .............. (subcop. 2) 8

Kuhnistera candida................. (subcop. 2) 7

Panicum depauperatum (bunches).......... (sparse) 5

Viola pedatifida............................

Sisyrinchium angustifolium (bunches)...... (sparse) 4

Acerates viridiflora linearis .............. (sparse) 3

Solidago nemoralis................. (vix-greg.) 3

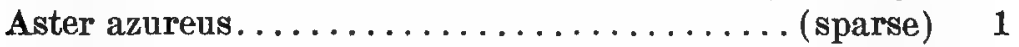

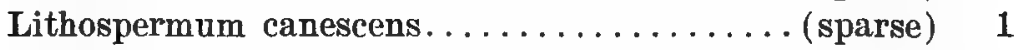

Physalis virginiana var................ (sparse) 1

Polygala verticillata................. (sparse) 1

Facies :-

Andropogon scoparius.

A. furcatus.

Bouteloua curtipendula. 


\section{QUADRat XXII}

PRAIRIE FORMATION : NORTHWEST EXPOSURE : AT FOOT OF RIDGE : LATE ESTIVAL FLORAL ASPECT

Antennaria campestris (twenty mats) ....... (greg. 1) 1300 Comandra umbellata.................... (cop. 1) 850 Aster multiflorus...................... (cop. 1) 625 Amorpha canescens................... (cop. 2) 180 Kuhnistera candida.................(subcop. 1) 45

Linum sulcatum.................. (subcop. 1) 37

Erigeron ramosus.................. (subcop. 1) 23

Rosa arkansana....................(subcop. 1) 18

Kuhnistera purpurea................(subcop. 1) 16

Solidago rupestris................. (greg-cop. 2) 13

Viola pedatifida....................(subcop. 2) 12

Polygala verticillata.................(subcop. 2) 10

Agrostis hiemalis................... (subcop. 2) 7

Coreopsis palmata................. (subcop. 2) 7

Panicum scribnerianum (bunches) .........(subcop. 2) 7

Psoralea argophylla................(subcop. 2) 7

Euphorbia corollata............... (greg-cop. 2) 6

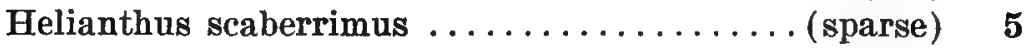

Brauneria pallida.................... (sparse) 4

Hieracium longipilum................................ 4

Panicum pubescens (bunches) ............ (sparse) 4

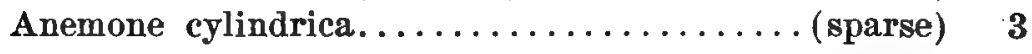

Kuhnia eupatorioides ................ (sparse) $\mathbf{3}$

Vernonia fasciculata................................... 3

Eatonia obtusata (bunches) .............. (sparse) 2

Lespedeza capitata..................... (sparse) 2

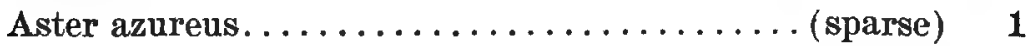

Koeleria cristata (bunch) ............... (sparse) 1

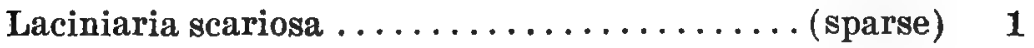

Solidago rigida $\ldots \ldots \ldots \ldots \ldots \ldots \ldots \ldots \ldots$ (sparse) 1 
Facies :-

Andropogon scoparius.

A. furcatus.

\section{QUADRAT XXIII}

MEADOW FORMATION: NORTHERN EXPOSURE: LATE ESTIVAL FLORAL ASPECT

Chaetochloa glauca.................. (cop. 3) 125

Teucrium occidentale............. (subcop. 1) 37

Convolvulus sepium ................ (subcop. 1) 36

Vernonia gigantea................. (subcop. 1) 33

Amorpha canescens................. (subcop. 1) 21

Acalypha virginica..................(subcop. 2) 13

Panicum scribnerianum (bunches) ........ (subcop. 2) 13

Euphorbia glyptosperma ..............(subcop. 2) 11

Thalictrum purpurascens............. (subcop. 2) 8

Asclepias verticillata. .............. (subcop. 2) 6

Kuhnia eupatorioides .............. (subcop. 2) 6

Oxalis stricta ................... (subcop. 2) 6

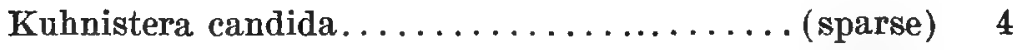

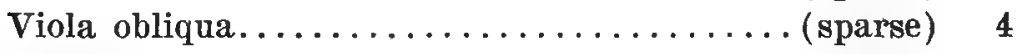

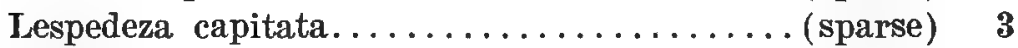

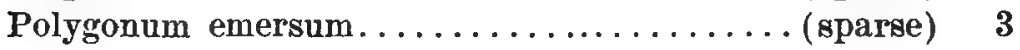

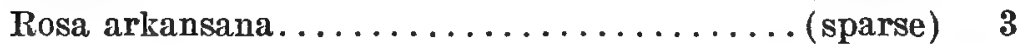

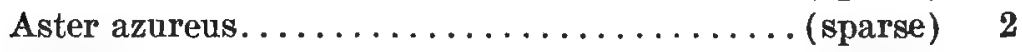

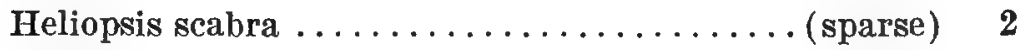

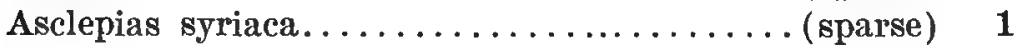

Aster multiflorus.................... (sparse) 1

Euphorbia corollata............... (vix-greg.) 1

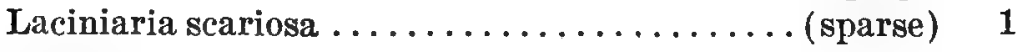

Physalis virginiana var............... (sparse) 1

Solanum carolinense................... (sparse) 1

Solidago rigida................... (sparse) 1

Facies :-

Andropogon furcatus.

A. scoparius. 
Panicum virgatum.

Chrysopogon avenaceus.

Elymus canadensis.

\section{QUADRAT XXIV}

MEADOW FORMATION: OPEN EXPOSURE: LATE ESTIVAL ASPECT Asclepias verticillata.................... (cop. 2) 144 Antennaria campestris (four mats) ......... (greg. 4) 75 Polygonum emersum.................(cop. 4) 54 Kuhnistera candida................(subcop. 1) 48 Convolvulus sepium ................. (subcop. 1) 31 Kulnnia eupatorioides ................(subcop. 2) 13 Anemone canadensis.............. (subgreg-cop. 2) 12 Panicum scribnerianum (bunches) ........ (subcop. 2) 10 Vernonia gigantea............... (subgreg-cop. 2) 10 Aster paniculatus................(subcop. 2) 9 Kuhnistera purpurea.............(subcop. 2) 8 Aster multiflorus. . . . . . . . . . . . . (subcop. 2) 7 Amorpha canescens..................(subcop. 2) 6 Oxalis stricta...................... (subcop. 2) 6 Polygala verticillata ................(subcop. 2) 6

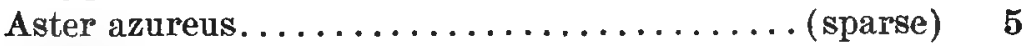

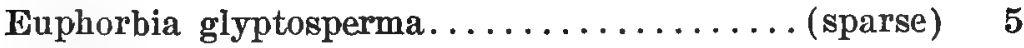
Lactuca pulchella ................... (sparse) 4 Physalis virginiana var................ (sparse) 4 Solidago rigida.................... (sparse) 4

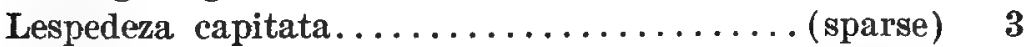

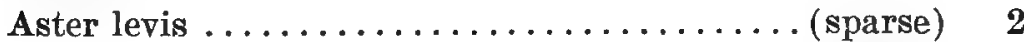

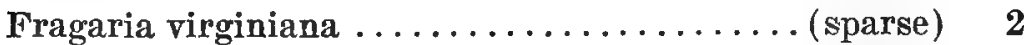

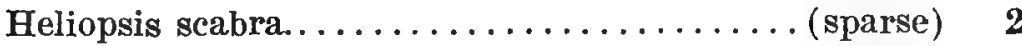

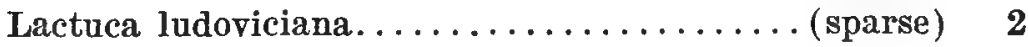

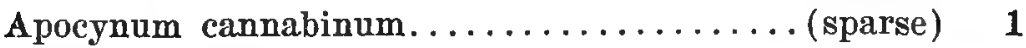

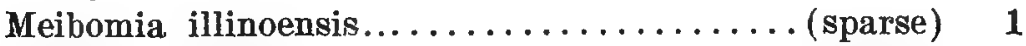
Monarda fistulosa.................... (sparse) 1 Silphium perfoliatum.................. (sparse) 1 
Viola pedatifida. (sparse)

Facies:-

Andropogon furcatus.

Andropogon scoparius.

Panicum virgatum.

Chrysopogon avenaceus.

Elymus canadensis.

\section{QUADRAT XXV}

MEADOW FORMATION: OPEN EXPOSURE: LATE ESTIVAL ASPECT Asclepias verticillata................ (cop. 4) 79 Anemone canadensis................. (greg-cop. 4) 64 Antennaria campestris (three mats) ........ (greg. 4) 54 Aster multiflorus.................... (subcop. 1) 29 Panicum scribnerianum (bunches)........ (subcop. 1) 29 Salix fluviatilis.................... (subcop. 1) 29 Kuhnistera candida................(subcop. 1) 27 Meibomia illinoensis................(subcop. 1) 27

Oxalis stricta.................... (subcop. 1) 20

Euphorbia glyptosperma .............. (subcop. 1) 19

Polygonum . emersum................. (subcop. 1) 17

Aster azureus .................. (subcop. 2) 14

Fragaria virginiana.................(subcop. 2) 14

Helianthus scaberrimus ............. (subcop. 2) 14

Silphium integrifolium.............. (subcop. 2) 14

Amorpha canescens................. (subcop. 2) 11

Vernonia gigantea.................. (greg-cop. 2) 11

Aster levis . . . . . . . . . . . . . . . . . (subcop. 2) 10

Laciniaria pycnostachya...............(subcop. 2) 10

Apocynum cannabinum..............(subcop. 2) 9

Kuhnia eupatorioides ................ (subcop. 2) 9

Aster paniculatus. ................. (subcop. 2$) \quad$ T

Polygala verticillata................ (subcop. 2) 7

Lespedeza capitata................... (subcop. 2) 6

Baptisia leucantha.................. (sparse) 4 
Physalis virginiana var................ (sparse) 4

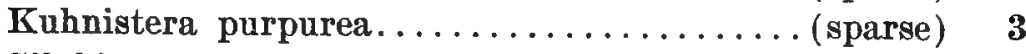

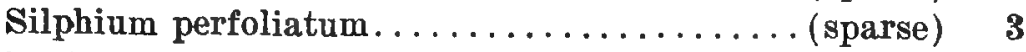

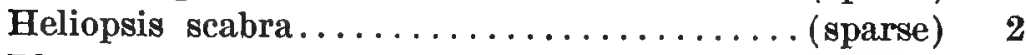

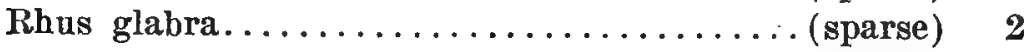

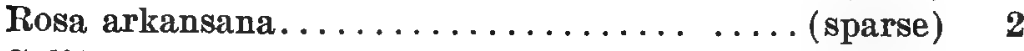

Solidago rigida.................. (sparse) 1

Facies:-

Andropogon furcatus.

A. scoparius.

Panicum virgatum.

Elymus canadensis.

\section{Quadrat XXVI}

MEADOW FORMATION : OPEN EXPOSURE: LATE ESTIVAL FLORAL ASPECT

Antennaria campestris (fourteen mats) . . . . (greg. 1) 2500 Comandra umbellata................... (cop. 1) 320 Equisetum laevigatum . ........................ 1) 260 Kuhnistera candida................... (subcop. 1) 45 Panicum scribnerianum (bunches).........(subcop. 1) 35 Aster multiflorus................... (subcop. 1) 20 Kuhnistera purpurea................. (subcop. 1) 20 Helianthus scaberrimus . . . . . . . . . . . (subcop. 1) 19 Bouteloua curtipendula (bunches) ........ (subcop. 2) 14 Euphorbia corollata .............. (subgreg-cop. 2) 14 Amorpha canescens................. (subcop. 2) 8

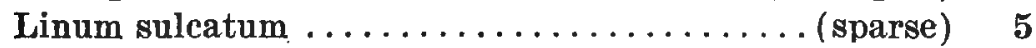

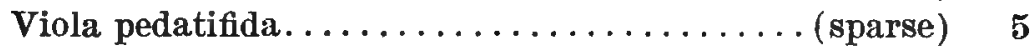

Coreopsis palmata.................... (sparse) 4

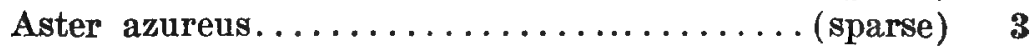

Laciniaria pycnostachya ................ (sparse) $\mathbf{3}$

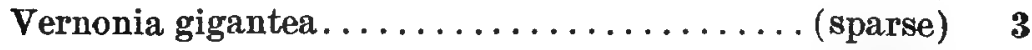

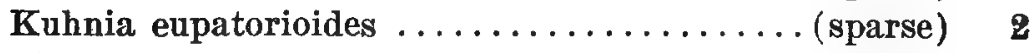
Anemone cylindrica................. (sparse) । 
Meibomia illinoensis .................. (sparse) 1

Rosa arkansana...................... (sparse) 1

Silphium laciniatum.................. (sparse) $\quad 1$

Solidago rigidiuscula.................. (sparse) 1

Facies:-

Andropogon scoparius.

Andropogon furcatus.

Chrysopogon arenaceus.

\section{QUADRAT XXVII}

MEADOW FORMATION: OPEN EXPOSURE: LATE ESTIVAL ASPECT

Antennaria campestris (twelve mats) ........ (greg. 1) 475

Aster multiflorus....................... (cop. 1) $\mathbf{4 3 5}$

Helianthus scaberrimus ................. (cop.4) 70

Euphorbia corollata ............... (subgreg-cop. 1) 49

Kuhnistera candida.................... (subcop. 1) 42

Asclepias verticillata ................ (subcop. 1) 28

Viola pedatifida .................... (subcop. 2) 12

Panicum scribnerianum (bunches) ........ (subcop. 2) 11

Erigeron ramosus .................. (subcop. 2$) \quad 8$

Physalis virginiana var............... (subcop. 2) 8

Amorpha canescens................... (subcop. 2) $\quad 6$

Convolvulus sepium................. (subcop. 2) $\quad 6$

Kuhnia eupatorioides ............... (subcop. 2$) \quad 6$

Polygala verticillata.................... (sparse) 5

Solidago rigida...................... (sparse) 5

Anemone cylindrica................... (sparse) 4

Laciniaria pycnostachya ................. (sparse) 4

Aster levis ......................... (sparse) $\quad 3$

Aster azureus........................ (sparse) $\quad 2$

Kuhnistera purpurea................... (sparse) 2

Lespedeza capitata................... (sparse) 2

Lactuca ludoviciana.................... (sparse) 1

Meibomia illinoensis................... (sparse) 1

Silphium perfoliatum.................. (sparse) 1 
Vernonia gigantea.................. (sparse) 1

Zizia aurea........................ (sparse) 1

Facies:-

Andropogon scoparius.

A. furcatus.

Panicum virgatum.

\section{Quadrat XXVIII}

WE' MEADOW FORMATION : LATE ESTIVAL FLORAL ASPECT

Rumex crispus ..................... (sparse) 5

Phalaris arundinacea (bunches) ............ (sparse) 4

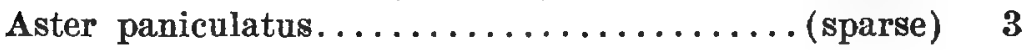

Cicuta maculata.................. (sparse) 2

Facies :-

Poa pratensis.

Calamagrostis canadensis.

Carex vulpinoidea.

Eleocharis palustris.

Carex cephaloidea.

\section{Quadrat XXIX}

WET MEADOW FORMATION : LATE ESTIVAL FLORAL ASPECT Physalis longifolia.................(subcop. 1) 34 Lactuca canadensis................. (subcop. 2) 10 Aster paniculatus................. (subcop. 2) 6 Plantago rugellii..................(subcop. 2) 6 Phleum pratense ................... (sparse) 5 Heliopsis scabra...................... (sparse) 4 Asclepias syriaca..................... (sparse) 1 Carduus altissimus.................. (sparse) 1

Facies:-

Carex stricta.

C. vulpinoidea.

C. cephaloidea.

Poa pratensis. Eleocharis palustris. 


\section{QUadrat XXX}

WET MEADOW FORMATION : LATE ESTIVAL ASPECT

Oxalis stricta............................ 3) 116

Asclepias verticillata...................... 4) 57

Lactuca ludoviciana................. (subcop. 1) 27

Teucrium occidentale................(subcop. 1) 19

Aster paniculatus................. (subcop. 1) 16

Cicuta maculata ................... (subcop. 2) 15

Heliopsis scabra................... (subcop. 2) 14

Onagra biennis..................(subcop. 2) 11

Potentilla monspeliensis.............. (subcop. 2) 8

Aster multiflorus.................. (sparse) 5

Vernonia fasciculata................... (sparse) 2

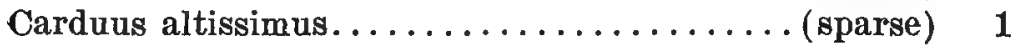

Iva xanthifolia $\ldots \ldots \ldots \ldots \ldots \ldots$ (sparse) ${ }^{\prime} 1$

Plantago rugellii.................. (sparse) 1

Facies :-

Spartina cynosuroides.

Poa pratensis.

Eleocharis palustris.

\section{Quadrat XXXI}

WET MEADOW FORMATION: LATG ESTIYAL FLORAL ASPECT Vernonia fasciculata................. (subcop. 2) 6 Apocynum cannabinum................. (sparse) 1 Facies :-

Carex vulpinoidea.

Carex cephaloidea.

Carex stricta.

Spartina cynosuroides.

Eleocharis palustris. 


\section{QUAdRat XXXII}

WET MEADOW FORMATION : LATE ESTIVAL FLORAL ASPECT -

Phalaris arundinacea ...............(subcop. 2) 14

Aster paniculatus.................. (subcop. 2) 12

Lythrum alatum.................. (subcop. 2) 12

Cicuta maculata.................. (subcop. 2) 10

Vernonia fasciculata............... (subcop. 2) 9

I ycopus americanus................(subcop. 2) 8

Polygonum pennsylvanicum............(subcop. 2) 7

Potentilla monspeliensis................ (sparse) $\mathbf{5}$

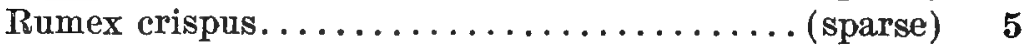

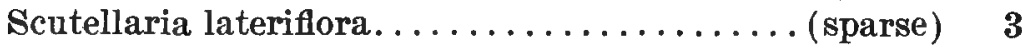

Iva xanthifolia.................... (sparse) 1

Facies :-

Spartina cynosuroides.

Carex cephaloidea.

Carex vulpinoidea.

Carex stricta.

\section{QUamRat XXXIII}

WET MEADOW FORMATION: LATE ESTIVAL FLORAL ASPECT

Vernonia fasciculata................... (sparse) 4

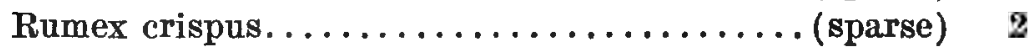

Facies :-

Eleocharis palustris.

Poa pratensis.

Carex vulpinoidea.

Carex cephaloidea.

Carex stricta. 


\section{LEGEND}

: = Antennaria campestris.

$\therefore \quad=$ Aster multiflorus.

a $=$ A. levis.

$a^{\prime}=$ A. azureus.

$\otimes=\mathbf{A}$. paniculatus.

$X$-Amorpha canescens.

$\therefore \quad=$ Asclepias verticillats.

* =Anemone canadensis.

$\mathscr{A}^{*}=$ Apocynam cannabinam.

$\bar{B}=$ Bouteloua curtipendula.

YK =Baptisia bracteata

\# =B. leucantha.

$b=$ Branneria pallida.

$\beta=$ Cicuta maculata.

c. =Coreopsis palmata.

i =Ceanothus ovatus.

al =Equisetom laevigatam.

$\therefore *_{*}=$ Erigeron ramosus.

$\dot{f}=$ Gentiana puberala.

$\mathcal{H}=$ Helianthus scaberrimus.

- =Heliopsis scabra.

$+\quad=$ Kuhnistera candida.

$0=\mathrm{K}$. purpurea.

$\square=$ Kuhnia eupatorioides.

$K$ =Koeleria cristata.

$\mathcal{L}=$ Lespedeza capitats.

- =Laciniaria squarrosa.

F =L. scariosa.

If =L. pycnostachya.

$\mathcal{L}^{\prime}=$ Lactuca ludovicians.

$m_{2}=$ Meibomia illinoenais.

0 = Onagra biennis.

$\Delta=$ Polygonum emersum.

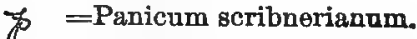

$r=$ Rosa arkansana.

$\&=$ Silphium laciniatum.

s =S. integrifolium.

=Solidago rigidinscala.

if =S. rigida.

A $=\mathrm{S}$. rupestris.

$y=$ S. nemoralis.

$\uparrow$ =Stipa spartea.

- =Salix fluviatilis.

$t$ =Teucrium occidentale.

$V=$ Vernonia gigantea.

$v=V$ iola pedatifida. 


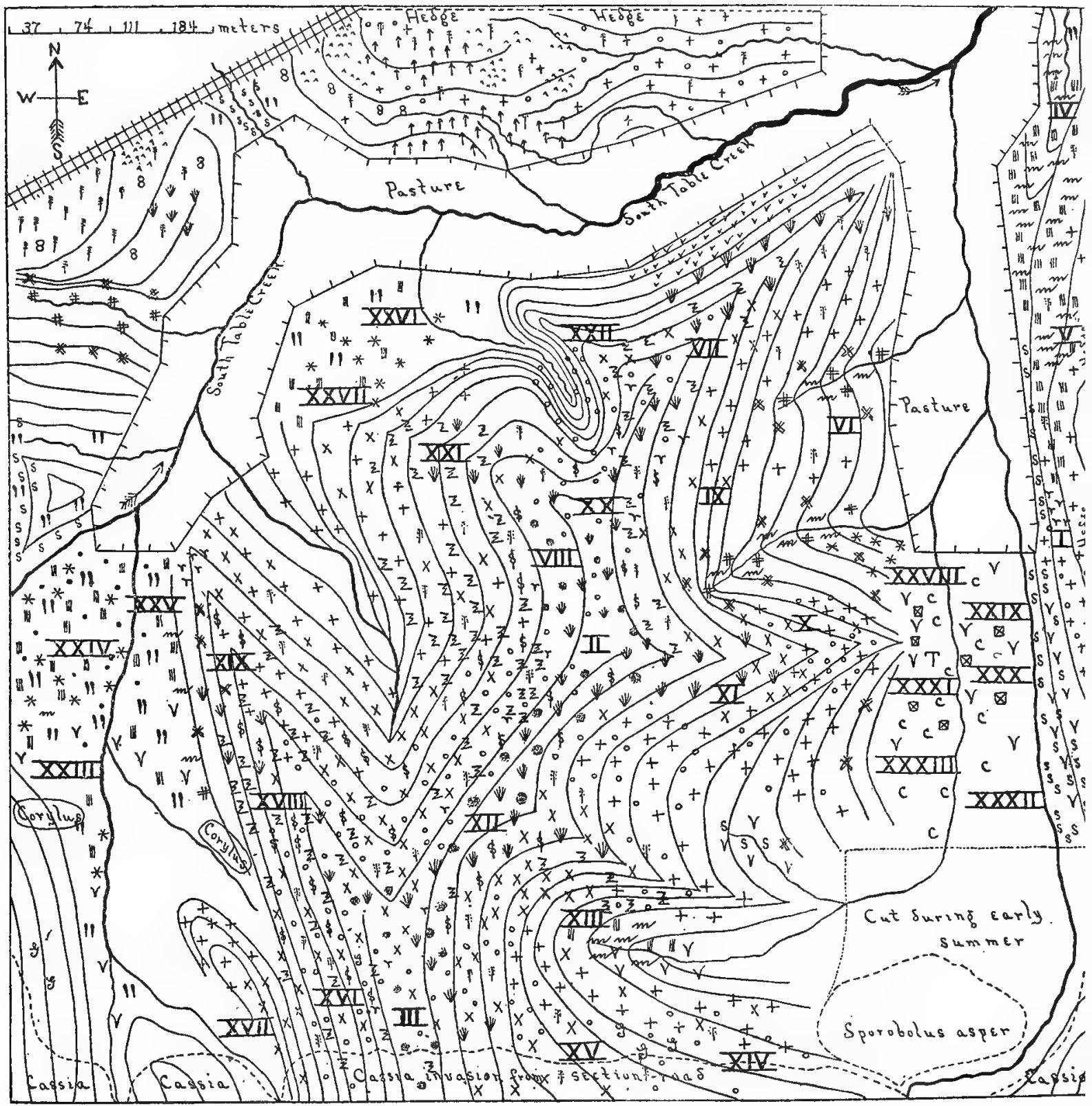





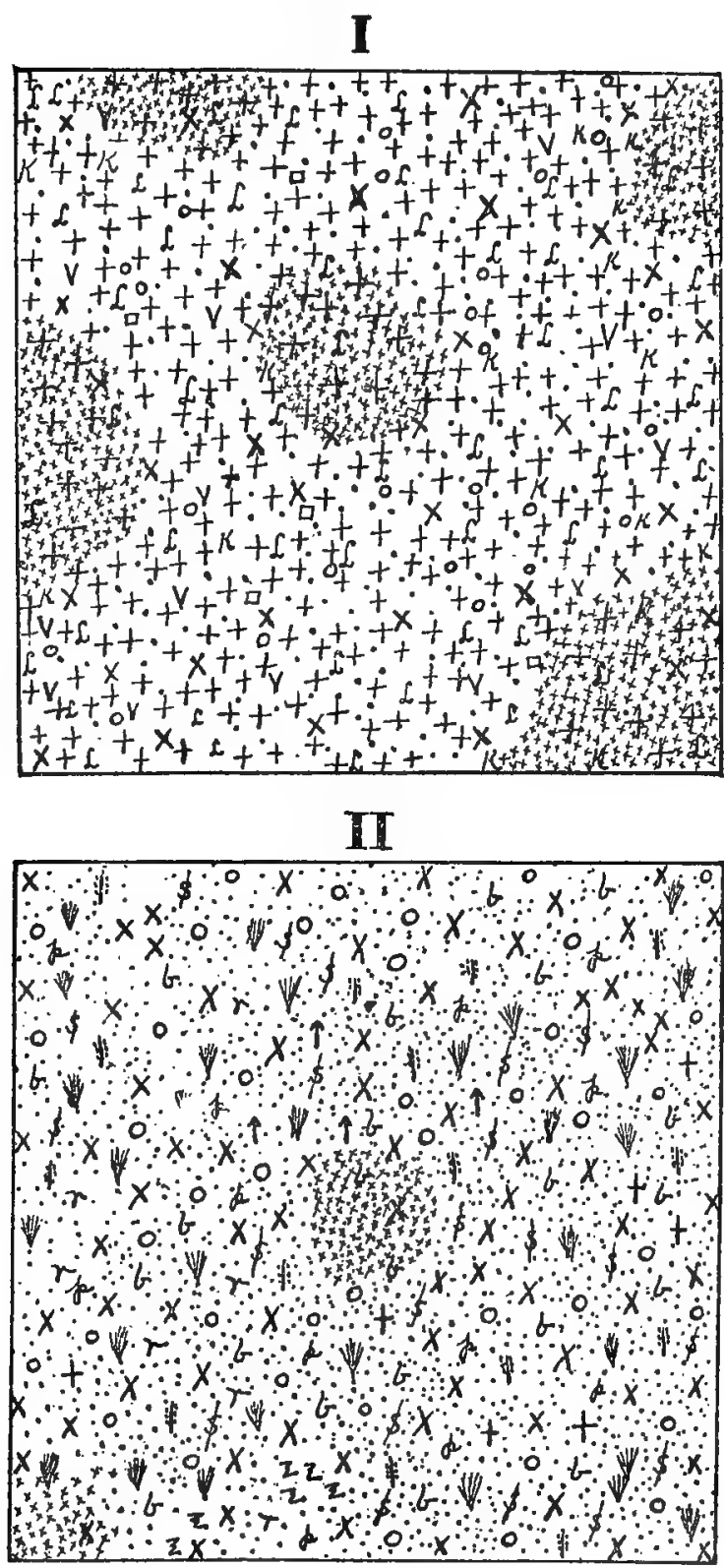




\section{III}

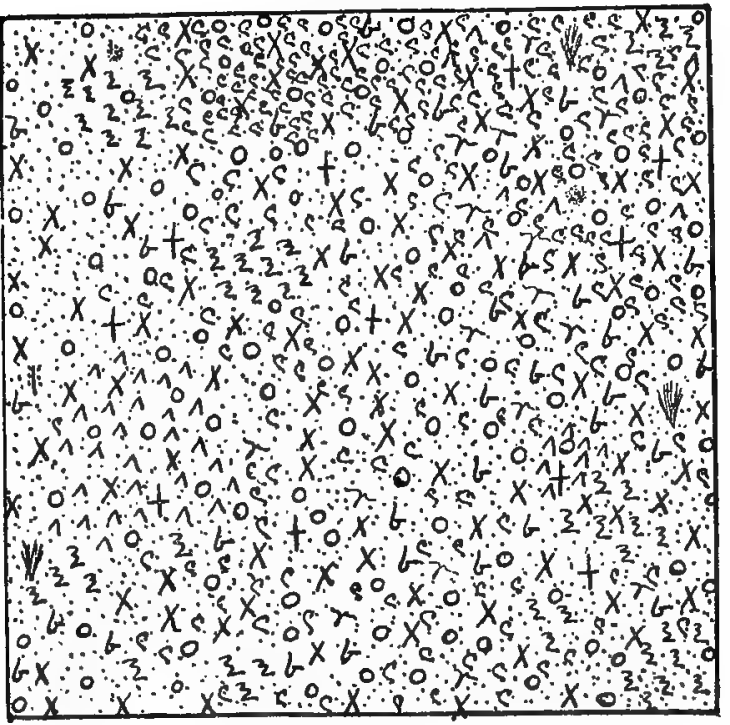

\section{IV}

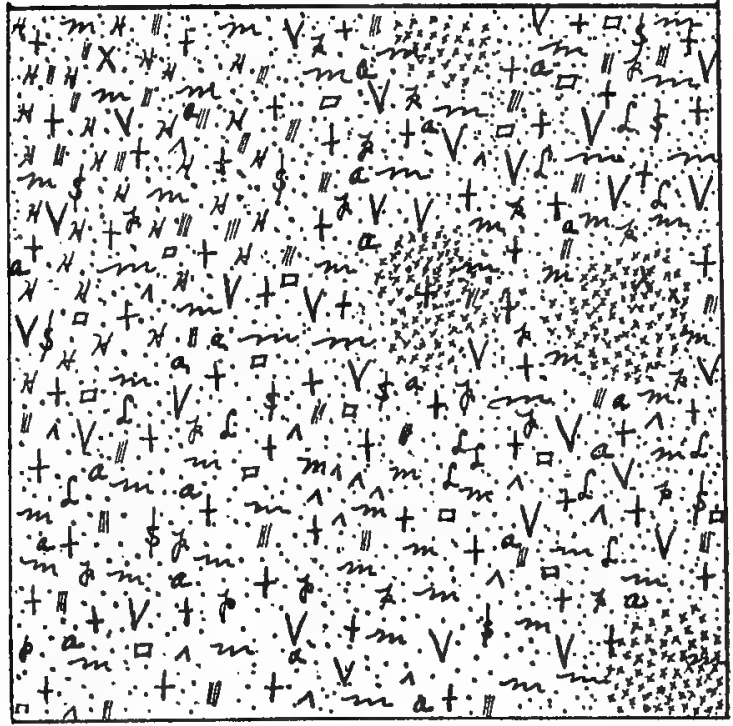




\section{VI}

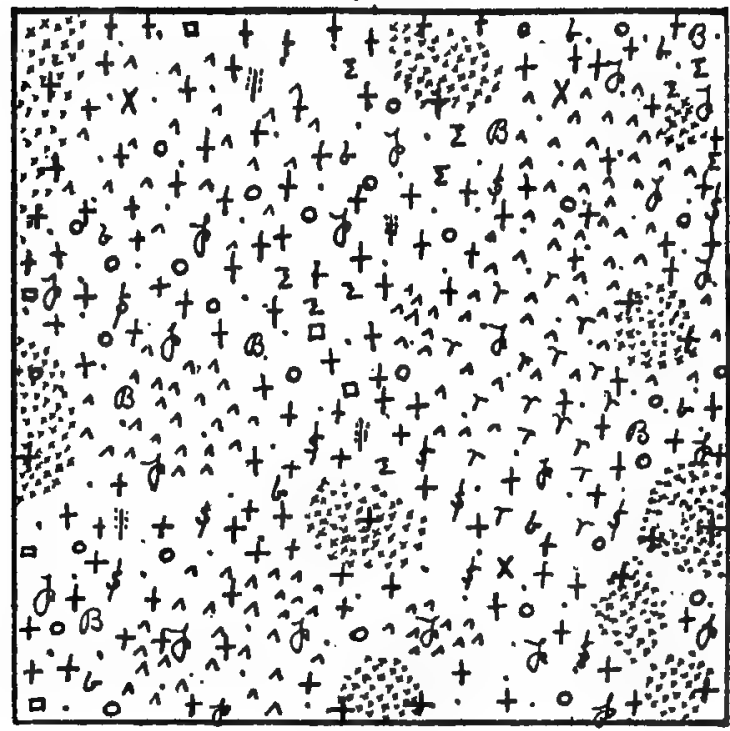

VII

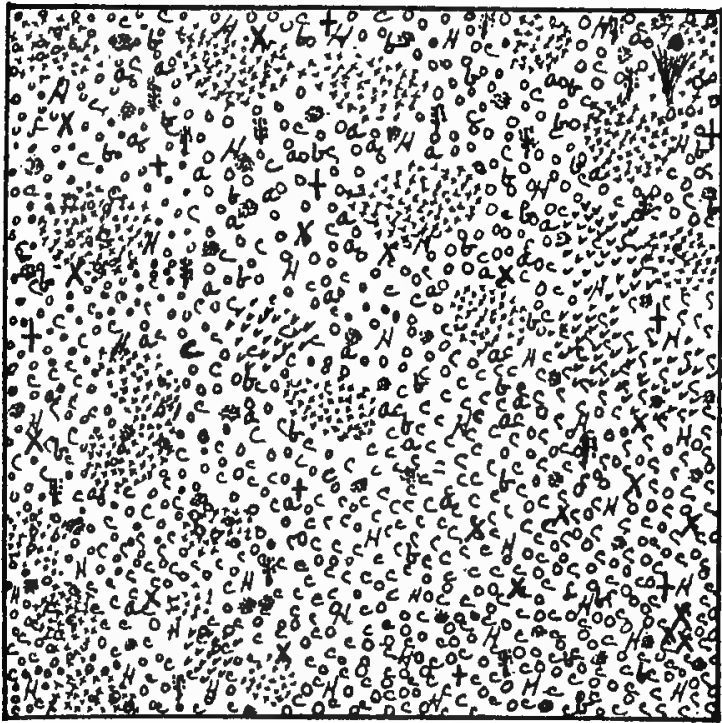




\section{V}

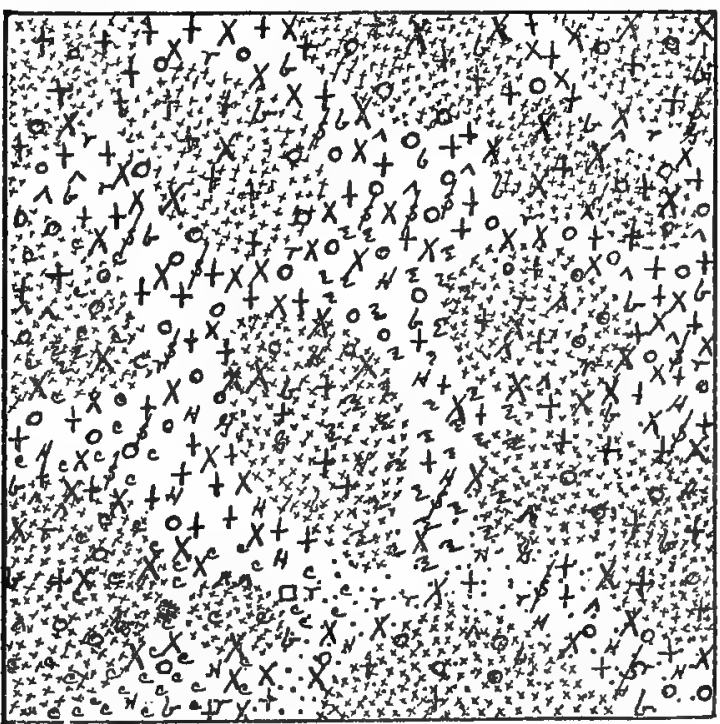




\section{XVI}

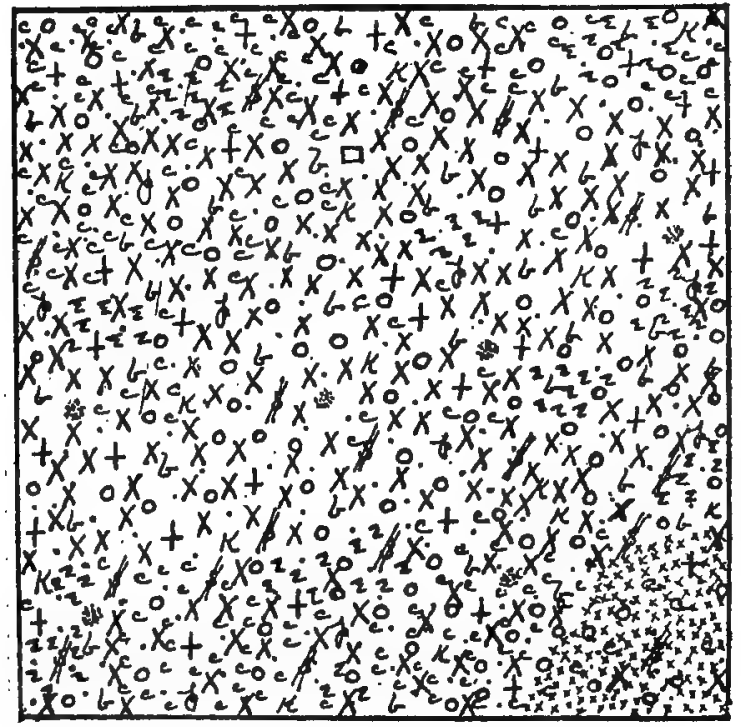

XVII

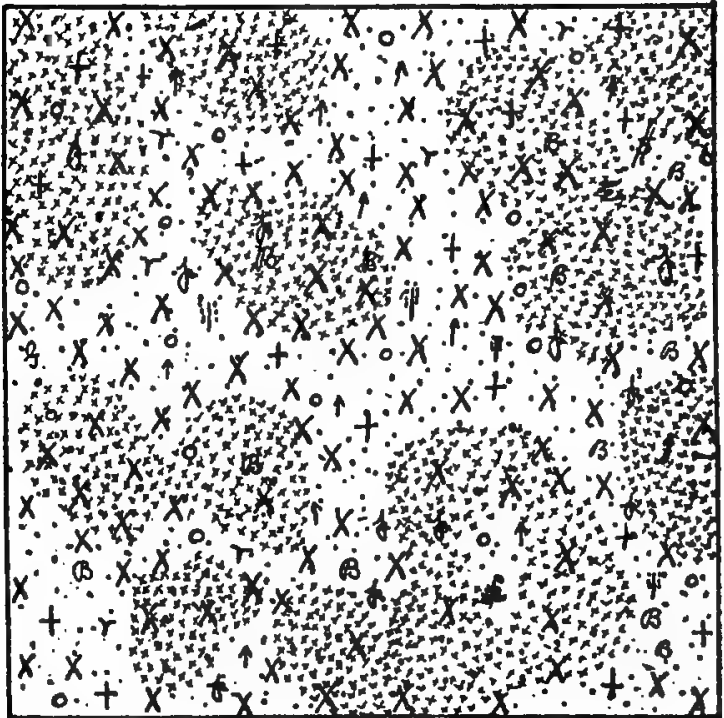




\section{XVIII}

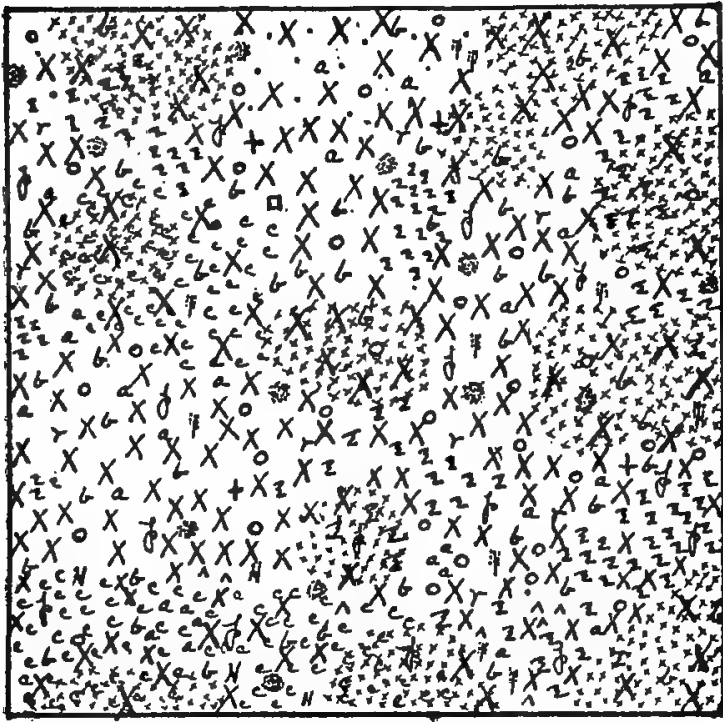

XX

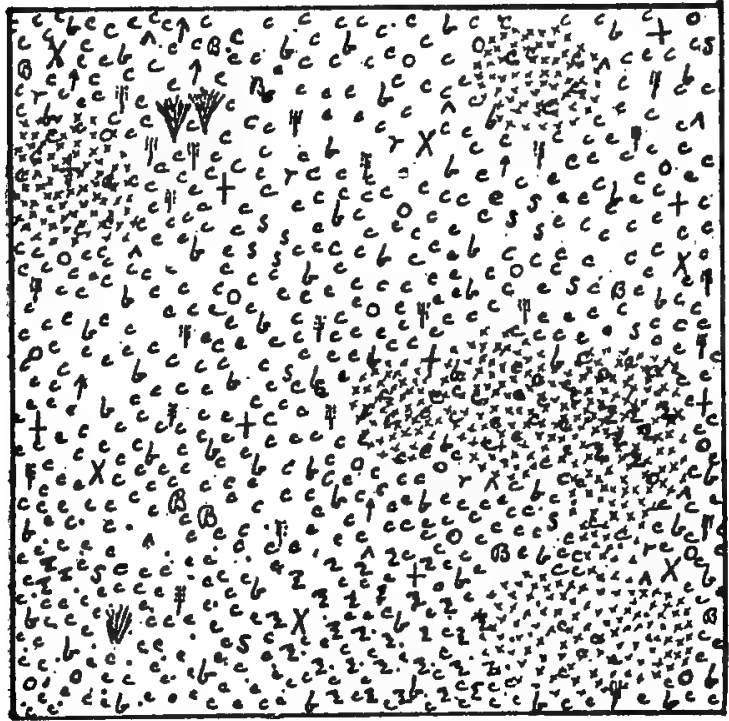




\section{XXVI}

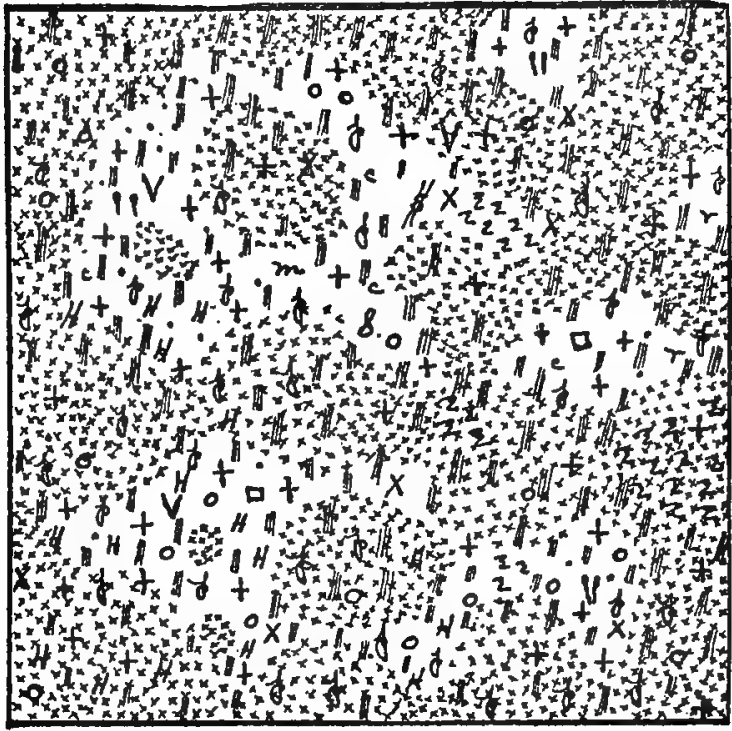

\section{XXVII}

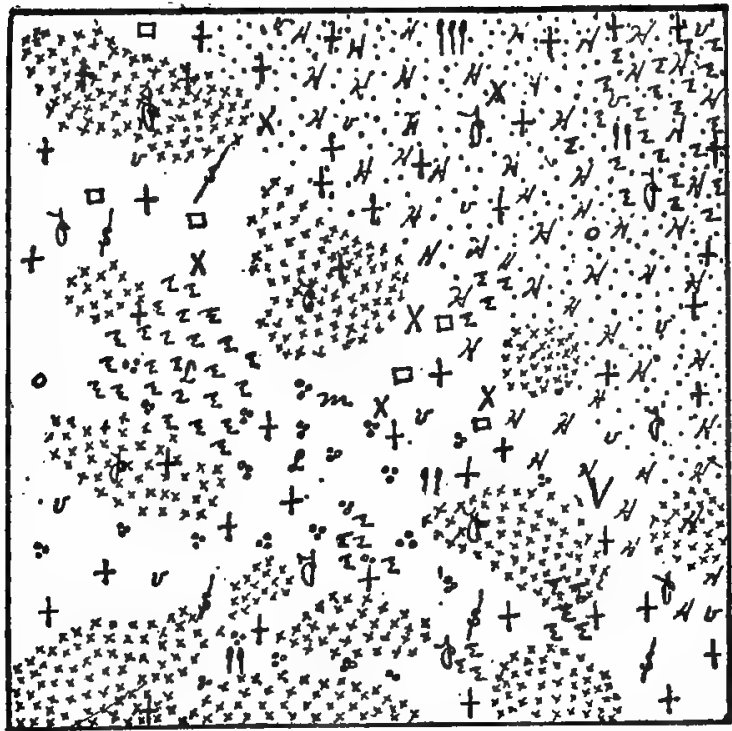


XXX.

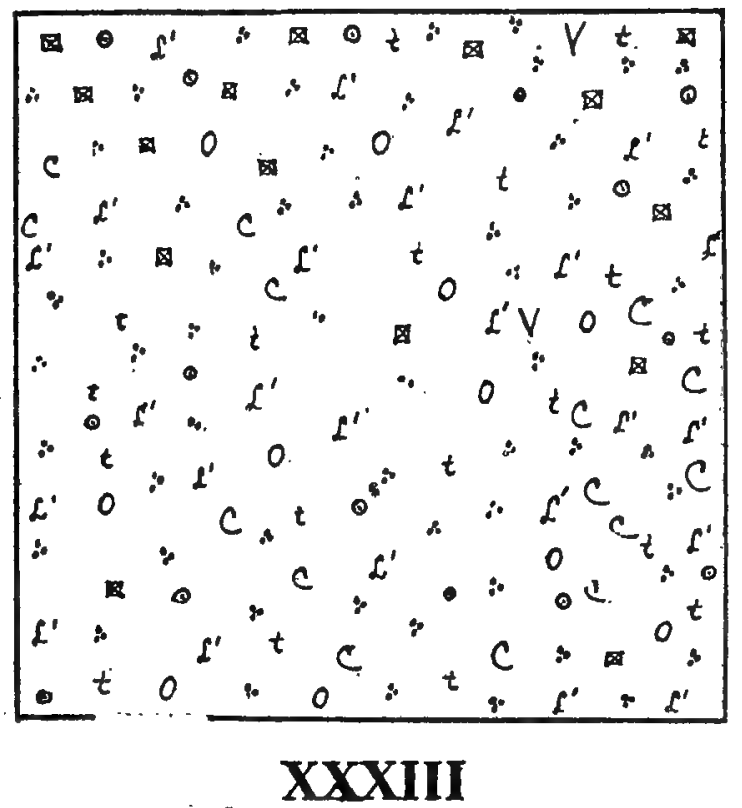

$V^{\prime}$ 


\section{PHENOLOGICAC RECORD OF SPECIES GROWING IN} PRAIRIE FORMATION

\begin{tabular}{|c|c|c|c|c|c|c|c|c|}
\hline & 总 & 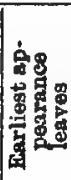 & 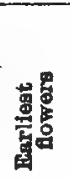 & 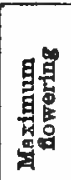 & 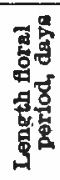 & 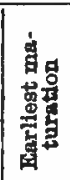 & 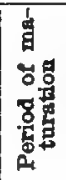 & 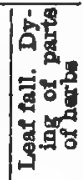 \\
\hline Acalypha virgi & 1899 & & $6-26$ & $7-20$ & 60 & $9-11$ & 30 & \\
\hline Acalypha virginica .. & 1900 & $4-28$ & $6-28$ & 8-1 & 70 & $8-27$ & 40 & 10.12 \\
\hline Acerates viridiflora ivesii. . & 1900 & & 6-13 & $6-23$ & 16 & $2 \overline{5}$ & $\ldots$ & \\
\hline Acerates viridiflora linearis... & 1900 & & $6-13$ & $6-29$ & 16 & & & 8 \\
\hline 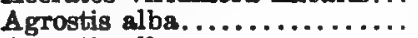 & 1899 & & $6-15$ & $6-21$ & 48 & $7-25$ & & \\
\hline Agrostis alba. & 1900 & & $5-21$ & $6-1$ & 25 & & & \\
\hline Agrostis hiemalis.. & 1899 & & $6-4$ & $6-10$ & 20 & 6.26 & 15 & \\
\hline Agrostis hiemalis. ..... & 1900 & -26 & 5-19 & $5-24$ & 21 & $6-6$ & 20 & $=2$ \\
\hline inea ovats. .... & 1900 & & & & & $6-27$ & & \\
\hline Amaranthus graecizans....... & 1899 & & $6-10$ & $7-17$ & 60 & $7-17$ & 60 & \\
\hline Amaranthus graecizans. . . . . . & 1900 & 423 & $5-17$ & $6-6$ & 120 & $6-9$ & 125 & \\
\hline Amaranthus torreyi .... & 1899 & & $6-28$ & & & & & $9-1$ \\
\hline Amaranthus torreyi .... & $\begin{array}{l}1900 \\
1899\end{array}$ & & 6-21 & $\begin{array}{l}8-19 \\
8-22\end{array}$ & $\begin{array}{l}78 \\
35\end{array}$ & $\begin{array}{l}8-5 \\
9-9\end{array}$ & $\begin{array}{l}60 \\
22\end{array}$ & \\
\hline $\begin{array}{l}\text { Ambrosia tritida.............. } \\
\text { Ambrosis trifida........... }\end{array}$ & 1900 & $\ddot{4 i}$ & $8-4$ & $8-24$ & 35 & $9-1$ & 28 & $9-25$ \\
\hline Amorpha canescens .......... & 1899 & & 6-19 & $7-5$ & 21 & $8-5$ & 30 & $9-29$ \\
\hline Am & 1000 & $4-30$ & $6-18$ & $6-28$ & 21 & $7-25$ & 25 & $9-20$ \\
\hline Andropogon furcatus... & 1899 & & $7-25$ & $8-\overline{5}$ & 30 & $8-16$ & 26 & \\
\hline Andropogon furc & 1900 & 4-21 & 7.23 & 8-20 & 40 & $8-25$ & 35 & \\
\hline Andropogon sce & 1900 & $\$ 21$ & $7-20$ & $8-20$ & 45 & $8-20$ & 30 & \\
\hline isis... & 1899 & & $5-22$ & $5-30$ & 38 & $7-15$ & 25 & \\
\hline Anemone canadensis... & 1900 & $4-10$ & $5-11$ & $5-25$ & 30 & $6-21$ & 25 & 30 \\
\hline Anemone carol & 1899 & & $4-24$ & $4-27$ & 15 & & & \\
\hline Anemone c: & 1900 & & 4-14 & $4-25$ & 30 & $5-5$ & & $6-6$ \\
\hline e cylin & 1899 & & $6-12$ & $6-24$ & 18 & 8-14 & 20 & \\
\hline ne cylindrica .... & 1900 & & $6-11$ & $6-16$ & 11 & $7-10$ & 15 & \\
\hline ennaria cam & 1899 & & 420 & & 25 & $5-17$ & 28 & \\
\hline atris & 1900 & & $4-17$ & $4-28$ & 18 & $5-5$ & 10 & \\
\hline Apocynum cannabinum & 1899 & & $5-30$ & & 60 & & & \\
\hline num & 1900 & & 5-30 & $6-21$ & 40 & $8-20$ & 45 & $8-10$ \\
\hline misia gnsphalodeg. . & 1900 & $4-12$ & 8-14 & $9-10$ & 40 & 9-20 & 30 & $10-20$ \\
\hline & & & -10 & $7-28$ & 45 & $9-4$ & 25 & \\
\hline As & 1900 & & 7. & $7-1$ & 45 & $9-1$ & 28 & $2-30$ \\
\hline Asclepias obtusifolia. . & 1900 & & $5-30$ & & 12 & 8. & 25 & $7-19$ \\
\hline epias sullivantii .. & & & $6-13$ & $6-23$ & 22 & & 25 & $9-15$ \\
\hline Alepias syri & & & $6-14$ & $6-26$ & 44 & $8-25$ & 25 & \\
\hline Asclepias syriaca... & 1900 & $4-27$ & & $6-23$ & 30 & $9-5$ & 25 & $9-25$ \\
\hline Asclepias tubeross... & & & & $6-26$ & 35 & & & \\
\hline Asclepias taberosa.... & & $4-28$ & & $6-25$ & 34 & $8-29$ & 30 & 9-15 \\
\hline Asclopias verticillata . & & & $6-23$ & $7-5$ & 60 & 8-12 & 45 & \\
\hline Asclepias verticillata. & & & $6-16$ & $6-27$ & 50 & $8-27$ & 35 & 10.1 \\
\hline Agter axureas ........ & & & $9-18$ & $9-25$ & 30 & $10-3$ & & \\
\hline Aster azurens ....... & & $3-27$ & $9-17$ & $10-2$ & 40 & $10-10$ & 25 & $11-1$ \\
\hline ster levis .... & & & $9-16$ & 9-27 & 28 & $9-29$ & 20 & \\
\hline ex levis ........ & & & $9-17$ & $10-2$ & 40 & $10-10$ & 25 & $11-5$ \\
\hline Aster multiflorus. & 188 & & $8-14$ & 2-25 & 60 & Q-2.2 & 40 & \\
\hline Aster multiflorus. & & & & $9-29$ & 70 & 220 & 45 & \\
\hline
\end{tabular}


PHENOLOGICAL RECORD OF SPECIES GROWING IN PRAIRIE FORMATION-Continued

\begin{tabular}{|c|c|c|c|c|c|c|c|c|}
\hline & 宥 & 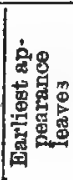 & 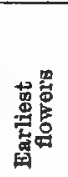 & 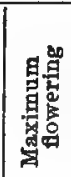 & 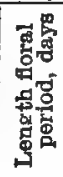 & 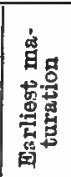 & 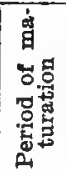 & 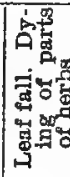 \\
\hline Aster obl & 1899 & & $9-20$ & & & & & \\
\hline Aster o & 1900 & & $9-10$ & $9-25$ & 35 & & & \\
\hline Aster paniculatus. ........... & 1899 & & 9.7 & $9-23$ & 45 & $10-10$ & 29 & \\
\hline Aster paniculatus... & 1900 & $3-3$ & $9-10$ & $10-2$ & 40 & $10-15$ & 40 & \\
\hline Aster sericeus.... & 1899 & & 9-7 & $9-20$ & 29. & $9-25$ & in & \\
\hline $\begin{array}{l}\text { Aster s } \\
\text { Astraga }\end{array}$ & 1900 & $4-8$ & $\begin{array}{l}8-27 \\
7-10\end{array}$ & $\begin{array}{l}9-15 \\
7-18\end{array}$ & 40 & $\begin{array}{l}9-20 \\
8-5\end{array}$ & 40 & \\
\hline $\begin{array}{l}\text { Astragalus carolinian } \\
\text { Astragalus carolinian }\end{array}$ & 1900 & $\ddot{3} \ddot{2} \ddot{24}$ & $7-6$ & $7-20$ & 40 & $9-1$ & $\begin{array}{l}10 \\
25\end{array}$ & \\
\hline Astragalus crassicarp & 1900 & & & & & $6-30$ & 30 & \\
\hline$\ldots \ldots$ & 1899 & & $5-14$ & $5-22$ & 18 & $9-4$ & & \\
\hline & 1900 & $4-16$ & $5-4$ & $5-12$ & 16 & $8-20$ & 20 & \\
\hline 1 & 1899 & & $6-17$ & $6-26$ & 16 & $9-4$ & & \\
\hline a leu & 1900 & 4-15 & $6-23$ & $6-11$ & 27 & $7-25$ & 15 & \\
\hline$\ldots$ & 1899 & & $7-5$ & $7-13$ & 24 & & & \\
\hline ... & 1900 & $4-5$ & $6-27$ & $8-1$ & 40 & $8-25$ & 35 & \\
\hline & 1899 & & $6-18$ & $6-26$ & 40 & $\begin{array}{l}8-5 \\
7-10\end{array}$ & $\begin{array}{l}24 \\
.5\end{array}$ & \\
\hline $\mathrm{Bra}$ & 1900 & & $6-8$ & $\begin{array}{l}6-22 \\
6.19\end{array}$ & 35 & $7-10$ & $\therefore 5$ & \\
\hline $\begin{array}{l}\text { Cala } \\
\text { Cala }\end{array}$ & $\begin{array}{l}1899 \\
1900\end{array}$ & & $\ddot{6-4}$ & $\begin{array}{l}0-1 \\
6-11\end{array}$ & 15 & $6-21$ & 10 & \\
\hline & 1899 & & $8-12$ & $9-4$ & 46 & $9-4$ & 29 & \\
\hline Carduo & 1900 & 3-27 & $8-10$ & $8-27$ & 40 & $8-30$ & 33 & \\
\hline Car & 1900 & 4-1 & $5-4$ & $5-10$ & 14 & $5-31$ & 25 & \\
\hline Carex & 99 & & $5-11$ & & 30 & $6-10$ & & \\
\hline $6 f$ & 1900 & 10 & $5-4$ & $5-22$ & 20 & $5-30$ & 30 & \\
\hline $\mathrm{me}$ & 1899 & & $4-29$ & $5-6$ & 14 & $5-30$ & 21 & \\
\hline Cax & 1900 & & $4-26$ & $5-$ & 20 & $5-20$ & 12 & \\
\hline & & & & $4-2$ & 12 & $5-20$ & 20 & \\
\hline $\mathrm{Cas}$ & 1900 & & $4-14$ & $4-21$ & 11 & $5-16$ & 25 & \\
\hline 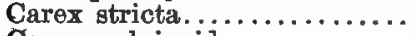 & 1900 & 4 & $4-23$ & 4-28 & 24 & 5-0 & 10 & \\
\hline 8 & & & $5-$ & & 20 & $6-19$ & 25 & \\
\hline & & $4-10$ & & & 20 & & 30 & \\
\hline (c) & & & 6-28 & $7-24$ & 58 & 8-16 & 40 & \\
\hline ista & 1900 & & $6-27$ & $7-25$ & 65 & $8-14$ & 40 & \\
\hline & 1900 & $4-21$ & $5-10$ & $5-16$ & 25 & $6-15$ & 22 & \\
\hline & & & & & 60 & $7-17$ & 60 & \\
\hline & 1900 & 414 & $6-5$ & $6-2$ & 80 & $6-28$ & 70 & \\
\hline & & & $6-$ & $6-20$ & 70 & $7-17$ & 50 & \\
\hline & 1900 & & $6-$ & 6-21 & 120 & $6-25$ & 95 & \\
\hline & & & $6-15$ & $6-30$ & 90 & $7-5$ & 60 & \\
\hline & & & $5-$ & $6-19$ & 125 & $6-20$ & 120 & \\
\hline & & & $8-21$ & 9 & 30 & $8-4$ & 25 & \\
\hline & 1900 & & 8 & & 35 & $8-4$ & 21 & 1. \\
\hline & & & $7=10^{\circ}$ & $7-28$ & 35 & $9-6$ & 25 & \\
\hline & & 4-12 & $6-29$ & $7-19$ & 35 & $8-25$ & 35 & \\
\hline & & & 6.7 & & 26 & $6-27$ & & \\
\hline & & & $4-29$ & 5-12 & 31 & $6-6$ & & \\
\hline & & & & & 70 & $\ddot{7} \ddot{7}$ & & \\
\hline onvolvulus sepium ... & & & & $6-23$ & & $7-25$ & & \\
\hline
\end{tabular}




\section{PHENOLOGICAL RECORD OF SPECIES GROWING IN} PRAIRIE FORMATION-Continued

\begin{tabular}{|c|c|c|c|c|c|c|c|c|}
\hline & d્d & 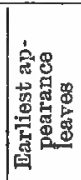 & 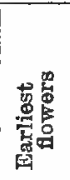 & 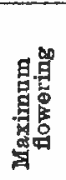 & 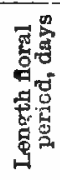 & 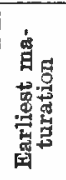 & 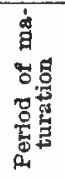 & 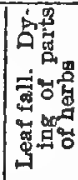 \\
\hline oreopsis palmata. & 1899 & & $6-15$ & $6-22$ & 28 & $8-15$ & 25 & \\
\hline Coreopsis palm & 1900 & 4-14 & $6-7$ & 6-25 & 30 & $7-10$ & 25 & \\
\hline Cornus asperifolia. & 1899 & $5-2$ & $6-7$ & $6-19$ & 22 & $8-8$ & 20 & \\
\hline Cornus asperifolia.. & 1900 & $4-18$ & $6-2$ & $6-10$ & 17 & $7-15$ & 20 & \\
\hline Corylus americe & 1899 & $5-1$ & $4-11$ & $4-15$ & 10 & $9-7$ & 15 & \\
\hline Corylus americ: & 1900 & $4-20$ & $3-24$ & $4-2$ & 18 & $\theta-1$ & 55 & \\
\hline ta parados & 1899 & & $7-30$ & $8-16$ & 36 & 915 & & \\
\hline & 1900 & & $7-14$ & $8-14$ & 50 & $9-1$ & 35 & \\
\hline 1.... & 1899 & & $6-8$ & $f-17$ & 16 & & & \\
\hline colinian & 1900 & $4-21$ & $5-30$ & $6-6$ & 25 & $6-20$ & 20 & \\
\hline$a \ldots$. & 1899 & & & & & $6-9$ & 25 & \\
\hline . & 1900 & 4-14 & $5-28$ & $6-5$ & 15 & 6-14 & 15 & \\
\hline ria & 1899 & & $5-20$ & $5-29$ & & & & \\
\hline is.... & 1900 & 4-5 & $5-\overline{7}$ & $5-20$ & 24 & $5-30$ & 15 & \\
\hline & 1899 & & $7-10$ & $7-20$ & 25 & $8-14$ & 25 & \\
\hline 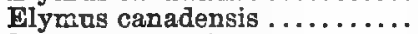 & 1900 & 3-26 & $6-23$ & $7-14$ & 30 & 7.25 & 35 & \\
\hline 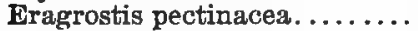 & 1899 & & $7-17$ & $7-20$ & 20 & $8-20$ & 20 & \\
\hline & 1900 & $4-20$ & $7-7$ & $7-18$ & 28 & $8-14$ & 30 & \\
\hline & 1899 & & $5-28$ & $6-15$ & 60 & $6-21$ & 51 & \\
\hline & 1900 & $3-27$ & $5-23$ & $6-9$ & 35 & 6.8 & 35 & \\
\hline & 1899 & & $6-19$ & 7-17 & 80 & 820 & 35 & \\
\hline a & 1900 & & $6-16$ & & 75 & $8-27$ & 45 & \\
\hline erma. & 1900 & & $6-2$ & $7-23$ & 125 & $7-10$ & 10 & \\
\hline & 1899 & & $6-12$ & $6-30$ & 113 & $7-25$ & 65 & \\
\hline & 1900 & $5-5$ & $6-16$ & $7-30$ & 100 & $7-16$ & 65 & \\
\hline & 1899 & & $8-14$ & 8- & 40 & $9-1 L$ & & \\
\hline & 1900 & $4-26$ & $7-28$ & & 40 & $9-5$ & 30 & \\
\hline & 1899 & & $5-3$ & & 28 & $5-30$ & 20 & \\
\hline & 1900 & $3-13$ & 4-27 & & 83 & 5-24 & 18 & \\
\hline & 399 & $4-29$ & $4-25$ & 4-27 & 10 & 9 & 25 & \\
\hline & 1900 & $4-24$ & $4-22$ & $4-28$ & 11 & $8-28$ & 15 & \\
\hline & 1900 & & $5-22$ & & 22 & $6-20$ & 30 & \\
\hline & 1899 & & $6-17$ & 7.20 & 80 & $7-24$ & 80 & \\
\hline & 18 & $3-13$ & $5-29$ & $7-$ & 120 & $6-19$ & 120 & \\
\hline & & & $8-30$ & $9-10$ & 40 & & & \\
\hline & 1900 & $4-18$ & $8-25$ & $9-20$ & 50 & $9-30$ & 30 & \\
\hline & 1900 & & $5-10$ & & & & & \\
\hline & 1900 & & $6-11$ & $6-18$ & 16 & & & \\
\hline & 190 & & $8-1$ & 8.23 & 35 & $9-1$ & 28 & \\
\hline & 1899 & & $7-5$ & 8-16 & 70 & $9-5$ & 30 & \\
\hline us ann & 1900 & $4-5$ & $6-21$ & 8.25 & 100 & $8-28$ & & \\
\hline & & & $8-10$ & & 29 & $8-10$ & & \\
\hline & 1900 & $4-11$ & $7-26$ & 8.25 & 35 & $8-30$ & 40 & $10-$ \\
\hline $\mathrm{s} \mathrm{m}$ & 189 & & $8-26$ & $8-4$ & 29 & 9.10 & & \\
\hline & & & $8-11$ & & 40 & 9-12 & 35 & \\
\hline & & & $7-24$ & $8-16$ & 60 & $9-6$ & 22 & \\
\hline aus & 1800 & |4-14 & $7-20$ & $8-27$ & 50 & $9-1$ & 35 & \\
\hline
\end{tabular}


PHENOLOGICAL RECORD OF SPECIES GROWING IN

PRAIRIE FORMATION-Continued

\begin{tabular}{|c|c|c|c|c|c|c|c|c|}
\hline & $\stackrel{\tilde{E}}{\tilde{\nu}}$ & 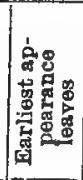 & 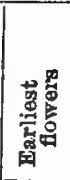 & 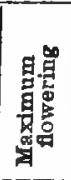 & 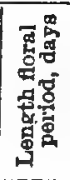 & 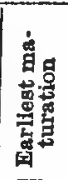 & 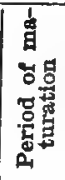 & 密 \\
\hline 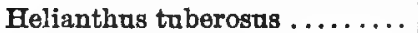 & 1899 & & $8-8$ & $8-21$ & 48 & $9-1$ & & \\
\hline & 1900 & s & & & 45 & & 35 & \\
\hline & 1899 & & $7-13$ & & 75 & $8-1$ & & \\
\hline Hel & 1900 & $4-21$ & $6-29$ & 7-1 & 70 & $7-29$ & 60 & \\
\hline Hie & 1899 & & $7-27$ & $8-$ & 20 & 8-14 & 20 & \\
\hline ... & 1900 & $4-14$ & $7-10$ & $7-25$ & 40 & $7-$ & 25 & \\
\hline & 1899 & & $8-16$ & $9-1$ & 30 & $9-4$ & 17 & \\
\hline Iva & 1900 & & $8-15$ & $9-5$ & 35 & & 25 & \\
\hline$\ldots$ & $\begin{array}{l}1899 \\
1900\end{array}$ & $4-16$ & & $3-25$ & 128 & & & \\
\hline${ }^{\ldots} \ldots \ldots$ & $\begin{array}{l}1900 \\
1899\end{array}$ & & $5-20$ & $\begin{array}{l}5-20 \\
5-20\end{array}$ & $\begin{array}{r}128 \\
25\end{array}$ & & 120 & \\
\hline$\ldots \ldots \ldots$ & $\begin{array}{l}1895 \\
1900\end{array}$ & $4-10$ & $\ddot{5}-22$ & 5.27 & $\begin{array}{l}20 \\
10\end{array}$ & $\begin{array}{l}2-10 \\
6-8\end{array}$ & & \\
\hline les.. & 1899 & & $8-16$ & $9-4$ & 38 & $9-4$ & $\ddot{26}$ & \\
\hline $\mathrm{Ku}$ & 1900 & & 7-14 & $8-21$ & 79 & $8-20$ & 40 & \\
\hline & 1899 & & 6.26 & $7-5$ & 28 & 8-14 & 25 & \\
\hline . & 1900 & $4-23$ & $6-18$ & $6-2$ & 23 & $7-15$ & 30 & \\
\hline$\ldots$ & 1899 & & $6-26$ & $7-5$ & 28 & 8-14 & 25 & \\
\hline $\mathrm{K} \mathbf{\mathrm { a }}$ & 1900 & $4-23$ & $6-22$ & $6-30$ & 35 & $7-20$ & 33 & \\
\hline Lac & 1899 & & & & & & & \\
\hline laria & 1900 & $5-12$ & $7-20$ & $8-10$ & 30 & $8-28$ & 30 & \\
\hline a 8 & 1899 & & $8-14$ & $9-4$ & 35 & $9-15$ & 20 & \\
\hline * & 1900 & 4-24 & 8-11 & 9 & 38 & 9-15 & 25 & \\
\hline . & 1899 & & 8 & 8 & 25 & $9-10$ & & \\
\hline . & 1900 & & $7-10$ & $7-25$ & 38 & $8-20$ & 30 & \\
\hline & 899 & & $7-22$ & 8 & 45 & 8-16 & 40 & \\
\hline & $\begin{array}{l}1900 \\
1899\end{array}$ & & $\begin{array}{l}6-29 \\
7-9\end{array}$ & & $\begin{array}{l}60 \\
50\end{array}$ & $7-20$ & 60 & \\
\hline & 1900 & $3-26$ & $6-16$ & $7-10$ & 50 & $6-25$ & 60 & \\
\hline & 1899 & & $6-19$ & $7-5$ & 45 & $7-5$ & 35 & \\
\hline & 1900 & $4-10$ & $6-13$ & 6-27 & 45 & 6-27 & 35 & \\
\hline & 1899 & .. & $8-10$ & 8-1 & 25 & $9-11$ & 20 & \\
\hline & 1900 & & $7-14$ & 8-1 & 40 & $8-27$ & 30 & \\
\hline & 1899 & & & & 12 & & & \\
\hline & 1900 & $4-14$ & $6-22$ & 6 & 12 & & & \\
\hline & 399 & & & & 45 & $7-19$ & 38 & \\
\hline$M$ & 1900 & $5-5$ & $6-25$ & & 60 & $7-14$ & 40 & \\
\hline & 899 & & & & 18 & $6-10$ & 5 & \\
\hline & $\begin{array}{l}1900 \\
1899\end{array}$ & & & & 18 & $6-1$ & 30 & \\
\hline & 1900 & $4-14$ & -27 & & $\begin{array}{l}28 \\
23\end{array}$ & $\because 15$ & $\ddot{20}$ & $\ddot{8}-1$ \\
\hline & 1900 & & 6 & 7. & 20 & & & \\
\hline & & & $7-12$ & $7-$ & 58 & 8.25 & 30 & \\
\hline & & 4-12 & $6-28$ & & 55 & $8-20$ & 38 & $10-2$ \\
\hline & & & $6-1$ & & 60 & $8-5$ & 40 & \\
\hline & & & & & 55 & & 40 & \\
\hline & & & & & & $8-16$ & 28 & \\
\hline (2) - & & & & & & & & \\
\hline
\end{tabular}


PEENOLOGICAL RECORD OF SPECIES GROWING IN

PRAIRIE FORMATION-Continued

\begin{tabular}{|c|c|c|c|c|c|c|c|c|}
\hline & 恿 & 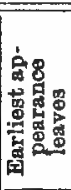 & 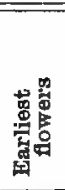 & 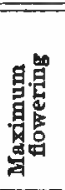 & 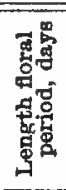 & 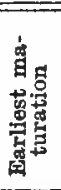 & 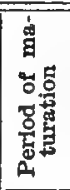 & 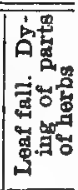 \\
\hline 318 & 1899 & & $7-13$ & $7-25$ & 30 & 8-14 & 25 & \\
\hline$\cdots$ & 1900 & $4-27$ & $6-22$ & & 45 & & 40 & \\
\hline anadensis...... & 1899 & & $7-10$ & $7-28$ & 70 & 8.25 & 45 & \\
\hline Mentha canadensis... & 1900 & $4-12$ & $7-6$ & $8-10$ & 75 & $8-10$ & 55 & \\
\hline Mesadenia tuberosa... & 1899 & & $6-23$ & $7-5$ & 37 & & & \\
\hline esadenia tuberosa.......... & 1900 & $4-7$ & $6-18$ & $6-29$ & 25 & $7-20$ & 20 & \\
\hline arda fistulosa. . . . . . . . . . & 1899 & & $7-15$ & 8-1 & 45 & $8-20$ & 30 & \\
\hline [uhlenbergia racemosa. & 1899 & & $7-7$ & $8-10$ & 68 & $9-1$ & 30 & \\
\hline ahlenbergia racemosa. . & 1900 & $4-18$ & $7-20$ & $8-25$ & 70 & $8-$ & 40 & \\
\hline 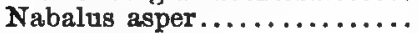 & 1899 & & 8-14 & 8-22 & 28 & $9-1$ & 25 & \\
\hline abalus a & 1900 & $4-14$ & $8-9$ & $8-22$ & 30 & $8-27$ & 25 & \\
\hline a biennis....... & 1899 & & $7-12$ & $8-5$ & 45 & & 30 & \\
\hline a biennis......... & 1900 & 3-24 & $6-27$ & $7-22$ & 90 & $8-5$ & 60 & \\
\hline alis stricta........ & 1899 & & $5-8$ & $5-22$ & 65 & & 60 & \\
\hline ric & 1900 & 4-2 & $5-11$ & $5-22$ & 80 & $6-15$ & 70 & \\
\hline ea. & 1899 & & $5-11$ & $5-27$ & 28 & & & \\
\hline 3 violace & 1900 & 4-18 & $4-27$ & $5-5$ & 35 & $5-25$ & 25 & \\
\hline illare... & 1899 & & $7-25$ & $8-15$ & 40 & $8-25$ & 30 & \\
\hline $\mathrm{m}$ ca & 1900 & $4-10$ & $6-20$ & 7.15 & 55 & $7-20$ & 45 & \\
\hline m dep & 1899 & & & 5-20 & & & & \\
\hline uperatum....... & 1900 & $4-12$ & $5-19$ & $5-25$ & 12 & $6-10$ & 20 & \\
\hline & 1899 & & $6-5$ & 6.12 & 20 & $6-20$ & & \\
\hline bescens........... & 1900 & 4-14 & $5-22$ & $5-28$ & 18 & $6-15$ & 25 & \\
\hline ribnerianum ...... & 1899 & & $5-22$ & 5-30 & & & & \\
\hline bnerianum .. & 1900 & $4-7$ & 5-16 & $5-26$ & 20 & $6-25$ & 25 & \\
\hline im. . & 1899 & & $7-26$ & $8-8$ & 25 & $8-2$ & 32 & \\
\hline & 1900 & $4-29$ & $7-24$ & 8. & 30 & -20 & 25 & \\
\hline dinacea..... & 300 & & $5-30$ & $6-6$ & 10 & $6-10$ & 8 & \\
\hline & 1899 & & $6-14$ & $6-19$ & 28 & $7-17$ & 18 & \\
\hline atense.... & 1900 & $3-24$ & $6-8$ & $6-20$ & 26 & & 30 & \\
\hline & 1899 & & $5-26$ & 5-31 & 31 & $6-25$ & 25 & \\
\hline$\therefore$ & 300 & 4-14 & $5-17$ & $5-25$ & 35 & $6-25$ & 35 & \\
\hline . & 399 & & $6-10$ & & & & & \\
\hline ylla. & 1900 & $4-28$ & $6-2$ & $6-8$ & 45 & & 30 & \\
\hline & 399 & & $6-26$ & 7.10 & 45 & & 23 & \\
\hline Phy & 1900 & $4-28$ & $6-5$ & $6-22$ & 85 & $8-10$ & 60 & \\
\hline giana va & & & & $6-15$ & 25 & $7-19$ & & \\
\hline hiana var. & 1900 & $4-2$ & $5-23$ & $6-2$ & 25 & $7-19$ & & \\
\hline & 1899 & & & $6-26$ & & & & \\
\hline ent $>>>$ & 1900 & $3-23$ & & $6-27$ & 70 & $7-16$ & 45 & \\
\hline & & & $6-5$ & 6-30 & & & & \\
\hline ellii .... & 1900 & $2-23$ & $6-5$ & $7-5$ & 60 & $7-1$ & 45 & \\
\hline & 1899 & & $5-16$ & 5-24 & 34 & $6-5$ & 21 & \\
\hline angis & 1900 & $3-12$ & & $5-25$ & 30 & $6-1$ & 20 & \\
\hline & & & $6-21$ & & 60 & & & \\
\hline llata. & & & $6-11$ & $6-29$ & 100 & $6-29$ & 90 & \\
\hline & & & & $7-28$ & & & & \\
\hline$m g^{\prime}$ & & & & $8-8$ & & 81 & & \\
\hline
\end{tabular}


PHENOLOGICAL RECORD OF SPECIES GROWING IN PRAIRIE FORMATION-Continued

\begin{tabular}{|c|c|c|c|c|c|c|c|c|}
\hline & 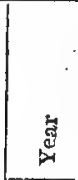 & 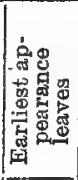 & 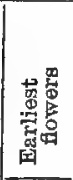 & 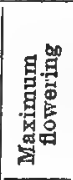 & 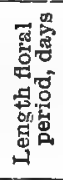 & 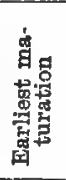 & 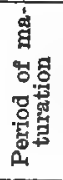 & 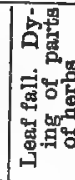 \\
\hline $\mathrm{am}$. & 1889 & & $6-10$ & $6-26$ & 20 & $7-10$ & 20 & \\
\hline cum. & 1900 & & & & 95 & & 90 & \\
\hline sis ...... & 1899 & & & $6-1$ & 45 & & 30 & \\
\hline beliensis .... . . & 1900 & $3-24$ & $6-1$ & $6-22$ & 70 & $7-10$ & 60 & \\
\hline Psoralea argu & 1899 & & $6-19$ & $6-26$ & 25 & & & \\
\hline ylla........... & 1900 & $5-8$ & $6-2$ & $6-18$ & 37 & $8-2$ & 30 & \\
\hline ta............. & 1900 & $5-1$ & $5-17$ & $5-22$ & 15 & 6.10 & 10 & \\
\hline Ps & 1900 & & $5-30$ & $6-6$ & 25 & & & \\
\hline & 1839 & $4 \cdot 23$ & $4-28$ & $5-8$ & 14 & $9-5$ & 20 & \\
\hline tes & 1900 & $4-26$ & $5-2$ & $5-8$ & 13 & $8-27$ & 25 & \\
\hline Kati & 1899 & & $7-13$ & $7-28$ & 45 & 8-2.) & 20 & \\
\hline & 1900 & 4-] & $6-23$ & $7-20$ & 45 & $8-16$ & 30 & \\
\hline & 1899 & $5-10$ & $6-15$ & $6-22$ & 20 & $8-25$ & 30 & \\
\hline Rotus & 1900 & $4-3$ & $6-2$ & $6-19$ & 24 & $8-1$ & 25 & \\
\hline & 1899 & & $6-1$ & $6-10$ & & $9-25$ & & \\
\hline & 1900 & $4-28$ & $5-31$ & $6-1$ & 12 & $8-20$ & 25 & $9-2$ \\
\hline Ro & 1899 & & $6-10$ & $6-19$ & 40 & $8-10$ & 30 & $10-1$ \\
\hline $\mathbf{R}$ & 1940 & 4-12 & $5-20$ & $5-30$ & 60 & $8-15$ & 35 & \\
\hline & 1900 & & 6-26 & $7-13$ & 60 & $8-15$ & 45 & $9-3$ \\
\hline & 1899 & & $5-11$ & $5-30$ & 50 & $6+8$ & 34 & \\
\hline & 1900 & $ن-26$ & $5-8$ & $5-25$ & 30 & $6-7$ & 26 & 7.] \\
\hline & $18 \unlhd 9$ & & $5-29$ & $6-10$ & 44 & $6-17$ & 30 & \\
\hline & $190 \mathrm{~s}$ & $3-24$ & $0-24$ & $5-30$ & 36 & $6-12$ & 40 & \\
\hline & 1899 & $5-1$ & $6-15$ & $6-24$ & 30 & $7-28$ & 30 & $10-1$ \\
\hline Se & 1900 & $4-10$ & $6-9$ & $6-22$ & 48 & $8-1$ & 30 & $10-1$ \\
\hline S: & 399 & $5-3$ & $5-3$ & $5-7$ & & & & \\
\hline$\cdots$ & 1900 & $4-25$ & $5-1$ & $5-7$ & 32 & $5-19$ & 40 & \\
\hline & $18 \backsim 9$ & & & & & & 20 & \\
\hline & 2.0 & $4-27$ & $4-2$ & $4-$ & 25 & $4-29$ & 15 & $10-1$ \\
\hline & 1899 & & $6-9$ & $6-19$ & 20 & $7-10$ & 20 & \\
\hline & 19011 & $4-7$ & $5-30$ & $6-11$ & 20 & $6-16$ & 18 & \\
\hline & 1899 & & $7-24$ & & 45 & $9-$ & 30 & \\
\hline & 100 & $4-15$ & $7-15$ & 8.15 & 50 & $9-1$ & 35 & $10-1$ \\
\hline & 1900 & & $5-10$ & $5-2$ & 30 & $6-1$ & 20 & $6-2$ \\
\hline & & & $5-17$ & $5-22$ & 45 & $6-10$ & & \\
\hline & & $3-27$ & $5-5$ & $3-10$ & 31 & $5-\_3$ & 25 & $6-1$ \\
\hline & & & & & & $5-20$ & 20 & \\
\hline & & & $7-2$ & $7-10$ & 26 & $8-1$ & 20 & \\
\hline & 1900 & $4-11$ & $6-25$ & $7-4$ & 15 & $8-5$ & 20 & \\
\hline & & & $7-7$ & $7-26$ & 45 & $9-7$ & 23 & \\
\hline & & 4-7 & $6-28$ & 7-25 & 60 & $8-18$ & 35 & $9-3$ \\
\hline & & & $7-17$ & $7-26$ & 34 & & & \\
\hline & 1900 & $4-14$ & $6-27$ & $7-19$ & 55 & $8-1$ & 30 & $9-2$ \\
\hline & & & $7-17$ & $7-29$ & 32 & $8-16^{\circ}$ & 30 & \\
\hline & & $4-14$ & $7-7$ & $7-25$ & 38 & 8.29 & 30 & $10-2$ \\
\hline & & & & $5-13$ & 25 & $6-10$ & 20 & \\
\hline Sisyrinchium angustifolium. & & & $4-29$ & & 25 & $5-20$ & 15 & \\
\hline
\end{tabular}




\section{PHENOLOGICAL RECORD OF SPECIES GROWING $N$}

PRAIRIE FORMATION-Concluded

\begin{tabular}{|c|c|c|c|c|c|c|c|c|}
\hline & 橤 & 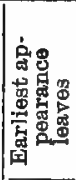 & 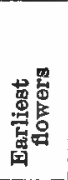 & 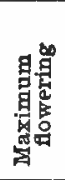 & 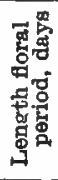 & 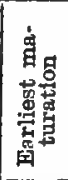 & 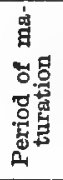 & 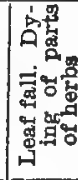 \\
\hline olanum carolinense. & 1899 & & $6-14$ & $6-26$ & 44 & $8-25$ & 25 & \\
\hline Solanum carol & 1900 & $5-3$ & $6-1$ & $6-26$ & 45 & $8-18$ & 30 & 10 \\
\hline Solidago canadensis. . . . . . . & 1899 & & 8-16 & $9-1$ & 46 & $9-20$ & 30 & \\
\hline Solidago canadensis. ....... & 1900 & $4-7$ & $8-4$ & $8-25$ & 45 & $9-10$ & 35 & \\
\hline Solidago nemoralis........... & 1900 & & $8-3$ & $8-23$ & 40 & $9 \cdot 10$ & 30 & $10-$ \\
\hline Solidago rigida . . . . . . . . . . . & 1899 & & $8-5$ & $8-16^{\circ}$ & 55 & $9-15$ & & \\
\hline Solidago rigida ............. & 1900 & 4-4 & $8-2$ & $8-30$ & 56 & $9-10$ & 30 & $10-$ \\
\hline Solidago rigidiuscula......... & 1900 & $4-14$ & $9-15$ & $9-25$ & 30 & $10-1$ & 25 & $10-2$ \\
\hline idago rnpes & 1899 & & $7-20$ & $7-29$ & 30 & $9-7$ & 28 & \\
\hline Solidago rapestrig............ & 1900 & $3-27$ & $7-10$ & $7-23$ & 40 & $8-20$ & 25 & \\
\hline Spartina cynosuroides. ........ & 1899 & & $\begin{array}{l}7-25 \\
7-5\end{array}$ & $\begin{array}{l}8-6 \\
8-10\end{array}$ & 45 & & & i \\
\hline $\begin{array}{l}\text { Spart } \\
\text { Sporc }\end{array}$ & $\begin{array}{l}1900 \\
1899\end{array}$ & $4-21$ & $\begin{array}{l}7-5 \\
8-9\end{array}$ & $\begin{array}{l}8-10 \\
8-20\end{array}$ & 40 & $\begin{array}{l}8-20 \\
9-1\end{array}$ & $\begin{array}{l}25 \\
25\end{array}$ & \\
\hline Sporobolus asper........... & 1900 & $4-28$ & $8-20$ & $9-1$ & 25 & $9-5$ & 25 & $10-10$ \\
\hline Sporobolus heterolepis .... & 1900 & & & $8-23$ & & $8-30$ & 20 & \\
\hline Steironema cili & 1899 & & $6-19$ & 6.26 & 38 & $5-15$ & & \\
\hline a ciliatum ......... & 1900 & 4-14 & $6-15$ & $1-25$ & 30 & $7-25$ & 30 & $9-1$ \\
\hline ppartea ............ & $18\lrcorner 9$ & & $5-25$ & $5-30$ & 10 & $6-111$ & 10 & \\
\hline Stipa spartea.. & 1900 & $4-5$ & $5-24$ & $5-28$ & 10 & $6-11$ & 10 & $6-19$ \\
\hline ənse... & 1899 & & $6-24$ & $7-7$ & 36 & $7-29$ & 30 & \\
\hline Ten & 190 & $4-15$ & $6-17$ & $6-27$ & 60 & $7-25$ & 40 & $9-10$ \\
\hline Teucrium occidentale........ & 1900 & $4-15$ & $6-18$ & $7-5$ & 60 & $7-25$ & 40 & $9-10$ \\
\hline Thalictrum purpurascens.... & 1899 & & $6-6$ & $6-19$ & 24 & $7-30$ & 15 & \\
\hline trum purpurascens. . & 1900 & 4-10 & $6-5$ & $6-11$ & 22 & $7-25$ & 25 & 0 \\
\hline Tra & 1899 & & $5-23$ & $5-30$ & 14 & $6-19$ & 12 & \\
\hline Tradescantia virginiana... & 1900 & 1-16 & 5-11 & $5-18$ & 17 & $6-5$ & 12 & $6-19$ \\
\hline Trifolium pratense. . .... & 1. 99 & & $5-24$ & $6-15$ & 95 & $6-20$ & 90 & \\
\hline olium pratense...... & 1900 & 3-12 & $5-15$ & $6-1$ & 120 & $6-55$ & 90 & $10-30$ \\
\hline Tri & 1899 & & $5-13$ & 5-31 & 60 & $6-10$ & 50 & \\
\hline Trifolin & 1900 & 3-12 & $5-10$ & 6-1 & 70 & $6-25$ & 58 & $10-30$ \\
\hline Vernonia fasciculata......... & 1899 & & & $8-10$ & 35 & $8-22$ & 30 & \\
\hline Vernonia fasciculata.... & 1900 & 427 & $7-10$ & $8-$ & 35 & $9-1$ & 30 & $10-15$ \\
\hline Ve. & 1899 & & $7-10$ & $7-28$ & 45 & $8-16$ & 30 & \\
\hline antea......... & 1900 & $4-25$ & $7-4$ & $8-1$ & 45 & $8-15$ & 35 & $10-1$ \\
\hline Verbena hastata.......... & 1899 & & $7-12$ & $7-25$ & 50 & 8-10 & & \\
\hline pena hastata... & 1900 & $4-12$ & $6-29$ & $7-9$ & 50 & $8-25$ & 40 & $10-1$ \\
\hline Verbe & 1899 & & $6-14$ & $7-10$ & 65 & $7-31$ & 30 & \\
\hline ena stricta. . . . . . . . & 1900 & $4-6$ & 6-11 & $7-4$ & 60 & 8-1 & 40 & 9-1 \\
\hline mericana & 1849 & & $5-10$ & 5-22 & 25 & $6-10$ & 25 & \\
\hline Vicia americana.... & 1900 & 4-7 & $5-4$ & $5-?$ & 30 & $6-10$ & 22 & \\
\hline Viola obliqua. . . . . . & 1899 & & $4-23$ & $5-1$ & 16 & $5-30$ & 40 & \\
\hline & 1900 & 3-24 & $4-18$ & $4--1$ & 28 & $5-19$ & 30 & $6-25$ \\
\hline & $1 \times 99$ & & $5-1$ & 5-8 & 14 & $5-30$ & 30 & \\
\hline pedatifida.... & 1900 & $4-14$ & $4-27$ & $5-5$ & 18 & $5-25$ & 35 & $6-2$ \\
\hline Zizia aurea.............. & 1900 & $4-14$ & $5-14$ & $5-24$ & 28 & $7-5$ & 30 & \\
\hline
\end{tabular}







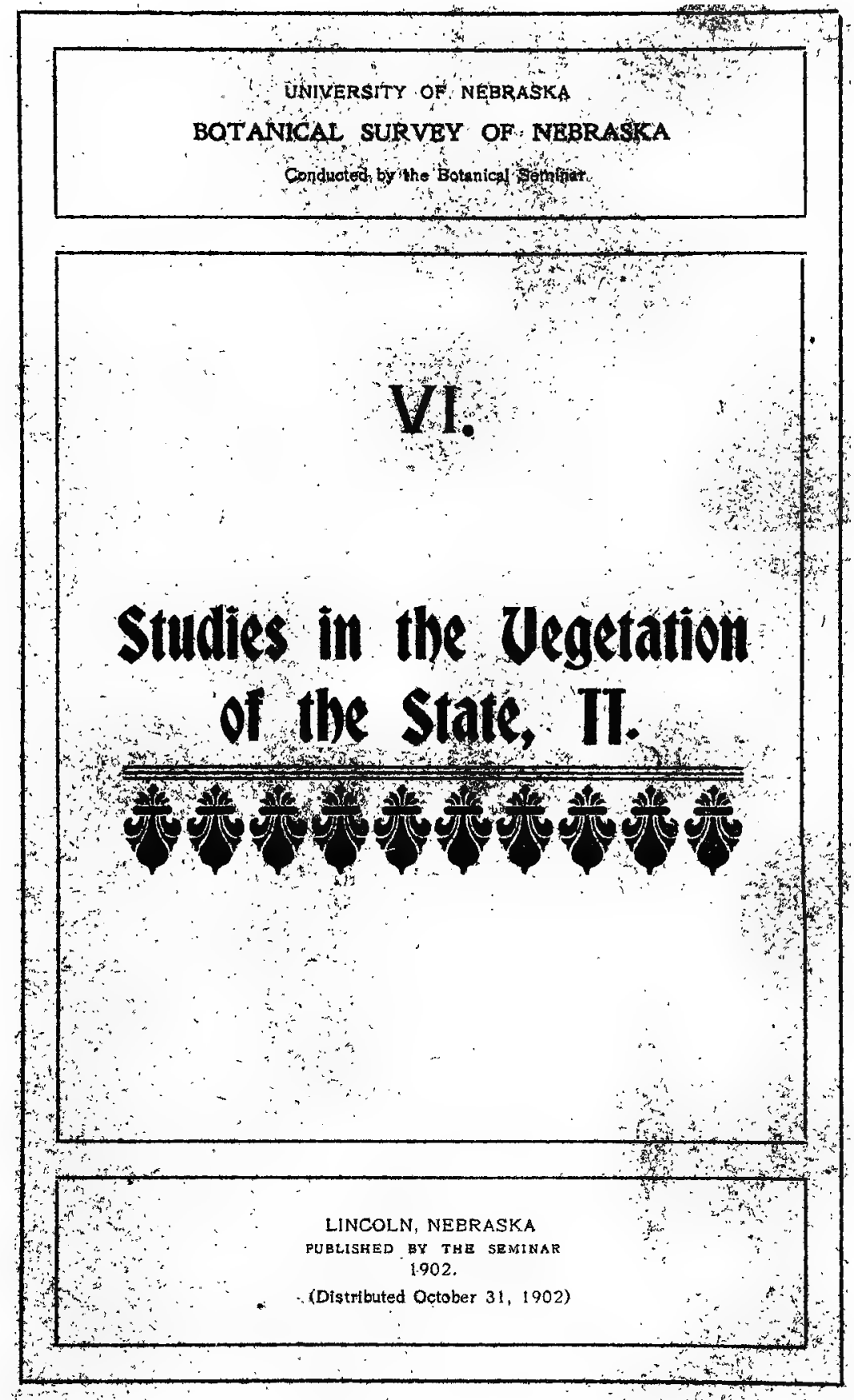







\section{STUDIES}

IN THE

VEGETATION OF THE STATE, II. 
Woodrugh-Collins Printing Co.,

LiNCOLN, NeBRASKa. 


\title{
THE RELATION OF THE WATER CONTENT OF THE SOIL TO CERTAIN PLANTS, PRINCIPALLY MESOPHYTES
}

\author{
By George G. Hedgcock
}

\section{INTRODUCTION*}

A plant is either modified, or destroyed by its environment whenever this is changed even in the slightest degree, and, since the physical conditions vary constantly, a plant must be an adaptive organism in order to meet all these requirements. Being originally an aquatic structure, in order to become an intense xerophyte, it must have undergone a long series of small changes in function and structure as it passed over from the one type to the other. The mesophyte possesses a structure which prevents it from thriving in extreme conditions of dryness or moisture. In periods of drought it may adapt itself in a certain degree to dry conditions, but lacking either a sufficient store of moisture in its tissues or the means of conserving what it has, it perishes long before the true xerophyte.

The mesophyte, since it grows in a substratum of soil, is necessarily affected by the water content of the soil, and by the varying conditions of soil moisture. In times of drought it is quite noticeable that plants of one species die sooner than others of a different species, and certain individuals perish sooner than others of the sarre species, although growing in the same soil plot. The observation of these differences in the drought resistance of plants led to the series of experiments the results of which are given in this paper. Although the study has been made from an ecological

*Accepted by the Faculty of the University of Nebraska as a thesis for the degree of Master of Arts, June, 1901. 
standpoint, it is difficult to decide whether the results are of most importance to plant physiology or to plant pathology, since both are concerned either directly or indirectly in the problems involved. The whole question of resistance to drought is intimately connected with the ability of protoplasm to withstand the effects of unfavorable stimuli. Furthermore the problem resolves itself into inquiries as to the proper amount of light, heat, water, etc., necessary for the natural development of the plant. When a plant does not receive the stimuli necessary for its growth and maturity, it soon passes over into a condition which may or may not be pathological, but which directly concerns the plant pathologist, as well as the plant physiologist. The former is concerned with conditions which lower the vitality of the plant, the latter, with those which are necessary to growth and maturity. It is hardly possible to draw the line between health and disease, or to establish the proper amount of light, heat, moisture, etc., requisite for a healthy plant, since the quantity of each may vary not only with the inherent nature of the species, but also with its adaptability.

When a mesophyte dies from drought, there is always some water remaining in the soil which is not available for the use of the plant. This has been called "non-available" water, in distinction to the physiological water of the soil which is available for the needs of the plant. There are so many factors and combinations of factors, both physical and biological, that may vary the limit of the amount of non-available soil water that only a few of tirese can be considered in one series of experiments. The work that has been done is included in the following outline. No attempt will be made to enter into a complete discussion of any one topic, for that would require the results of many years of investigation. The following outline contains the subjects investigated:

I. Water in Different Soils.

1. Physical Water.

2. Physiological Water.

3. Non-available Water. 
II. The Relative Amount of the Water in the Plant and in the Soils.

1. The amount of water in healthy plants, and in the soil in which they grow.

2. The amount of water in plants and in the soil when they are wilting under measured physical conditions.

3. The amount of water in plants at or near the death point when dying from drought, and the corresponding amount of non-available water in the soil.

III. The Limit of Physiological Water, (non-available water). How affected?

1. By increased heat and light.

2. By increased humidity.

3. By decreased humidity.

4. By decreased light.

5. By lowered temperature.

6. By high temperature.

7. By different soils.

8. How varied in different species and individuals?

IV. Drought Effects. The Manner in Which Mesophytic Herbs Die from Drought.

I. WATER IN DIFFERENT SOILS.

A. METHODS OF EXPERIMENTATION, ETC.

1. Soils. The soils used in the investigation were brick clay, quartz sand not quite pure, loam, loess or subsoil, saline soil from the saline lands near Lincoln, and humus consisting largely of leaf mold. The mechanical analyses of these six soils have been appended to this paper in the form received from the assistant agriculturist of the Nebraska Experiment Station, to whom they were sent for analysis. The experiments in attempting to grow plants in the native saline soil proved the futility of such efforts. Only saltbasin plants thrived in it, then only when slightly diluted with loam. The results noted in the summary appended are from a soil consisting of one part saline mixed with three parts of loam. All soils were sifted with a screen possess- 
ing a quarter inch mesh. In experiments in the plant-house, the suils were usually looser than found in nature. It was impossible during the length of time used for experimentation to measure the degree of hardness, yet this must determine to a greater or less degree the extent and course of the rootlets of the plant. Such being the case, the hard soil may limit the extent of a plant's roots and become an direct factor in times of drought because it may thus hasten the death of the plant by limiting its ability to secure water. In order to roughly estimate the condition of the soil, it has been indicated in the summary of results as loose, medium, and hard in density. In case of plants grown for experimentation, the soil being loose at first, most of them had an opportunity to become well rooted before it became hard, and as a result the hardness of the soil is not considered as a factor affecting the death of the plants to an appreciable degree.

2. Water in Soils. Since the amuunt of water a soil may hold is measured by the space between the particles of soil, the water capacity was determined for each of the six soils used by a simple experiment which is described under Physical Water. The amount of physical water present in the soil was determined by taking samples from the pots with a tubular soil sampler. These were weighed at once to prevent error from evaporation, and subjected to a heat of $212^{\circ} \mathrm{F}$. for about 24 hours; then the per cent was determined from the loss in weight. The evaporation of slight amounts of volatile organic water was not taken into consideration in the results. The amount of 'water in the soil not available for the plant was found in the same way as the physical water, except that it was determined when the plant was near the death point, as evinced by the condition of the organs of the plant. The exact time when a plant can no longer get water from the soil is difficult to decide, but the rule followed in most cases was to take the soil sample at the time the youngest leaves of the plant wilted strongly, though in many cases the plant was actually dead at the time of the estimate. This will account for most of the irregularities 
shown in the results. The physiological water was finally determined by deducting the per cent of non-available water from the per cent of physical water in the soil at the time the process of drying out began. The plants were grown for experimentation in three and four inch porous pots. In order to control the evaporation, the three inch pots were set in soil and kept there during the whole time of experimentation. In order to prevent too rapid loss of water, the four inch pots were placed inside painted pots, during the period of drought to which they were subjected in determining the non-available water. All soil samples were taken from the soil in the lower part of the pot, in the vicinity of the roots, the upper part of the soil being discarded, as it contained very few roots. Owing to the great number of pots used in experiments it was impossible to keep a daily record of the physical water in the soil of each. In the comparative plots, soil samples were taken from one pot in each condition, and the water in the others estimated from this record. The non-available water was always determined by taking a soil sample from each pot used in the experiment.

3. Water in Plants. In determing the amount of water in plants, the plant was carefully removed from the soil, the small particles of soil adhering to the roots were shaken off where possible, and the plant quickly weighed. It was then subjected to a heat of $212^{\circ} \mathrm{F}$. in a drying oven from 8 to 24 hours, depending upon the size of the plant. The per cent of moisture was then determined from the loss in weight. All weights both of plants and soil samples were made with a laboratory balance sensitive to one milligram.

4. Physical Conditions. In order to grow plants under different physical conditions, four plant cases with glass panes overhead and in the sides were constructed and placed in the south windows of the laboratory. One of these had a connection with the outside air and by this means was kept at a lower temperature must of the time, the exception being the last week of the experiment. This case is desig. nated as Case I. The light, though less intense than that in 
the plant house, was nearly equal in intensity to that of Case III and Case IV. The soil moisture was kept at a good growing per cent, usually at from 12 to 25 per cent. physical water. Case II had the direct sunlight shut out. This reduced the light to less than one-hundredth of that of full noon-day sunlight. Other factors were kept near the normal. Case III was intended to be a xerophytic chamber but usually it was nearer normal than otherwise. Case IV was regulated so that the air was almost constantly humid. The soil likewise was kept wet, generally with from 20 to 30 per cent of soil water. In another window, in the air of the laboratory, was a plot designated as Lab. in the records. The air of this plot during the time of experimentatiun was almost constantly deficient in moisture. The averages of temperatures in Case II, Case III, Case IV, and Lab. were about equal.

In the plant-house, plants were grown under five measured sets of physical factors. Those grown under the full light are designated in the species lists as P. H. These were kept in good yrowing condition with a soil moisture of from 12 to 25 per cent of physical water. P. I, P. II, P. III and P. IV were four plots under essentially the same air conditions as the P. H. plot, except that the first two were shaded. P. I and P. III were kept at a high per cent of physical soil water, 20 to 35 per cent, but $P$. II and $P$. IV were kept at a low per cent.

5. Temperature and Humidity. Air temperatures were taken with a psychrometer in the plant-house and with stationary wet and dry bulb thermometers in the plant cases. The relative humidity was calculated from these readings. The maximum and minimum thermometer wis used for recording the extremes of temperature in the plant-house. Soil thermometers were kept constantly in one pot in each plot. Readings of all factors were taken three times daily, morning, noon, and evening, at or near $8 \mathrm{~A}$. M., $12 \mathrm{M}$, and $4 \mathrm{P}$. M. For convenience in comparing the records with those of the local U. S. Weather Bureau all temperatures are given in Fahrenheit. 
6. Light. The intensity of the light was estimated three times daily in each plot until a ratio was established for each plot. This ratio varies somewhat, being relatively greater indoors on cloudy than on sunny days. The method used in estimating light is the photographic method. Strips of solio paper pasted together in a ribbon are placed on rollers in an instrument invented by the author. The ribbon may be exposed any number of seconds by means of a shutter in the bux which contains it, and then rolled back in the dark until needed for making the estimation. Solio paper if exposed in full sunlight at noon during a series of increasing periods of time as $2,3,4,5$ seconds, will print a series of colors changing from a light pink for the shortest exposures to a rich red brown for an exposure of 15 seconds. Such a series of culors printed at meridian noon is taken for a daily or local standard, and the colors obtained by time exposures in each locality were matched with those of the standard. From this the per cent of light at the time of the exposure was estimated. The daily standard of sunlight varies during the year with the sine of the angle of the sun's elevation. Some day of the year must be taken as a fixed standard. June 22 being the time of maximum light is taken in this treatise as the fixed standard, and the light at meridian noon for that day is called 100. In figuring light intensities by this method, it was necessary to estimate each by the light standard of June 22, in order to compare the light of different days in different plots with each other. This was done as follows: Suppose observations of light are taken February 20, morning, noon, and evening. The noon exposure is one of full sunlight, and its per cent of the fixed standard, June 22, is calculated as follows: From the astronomical tables of elevations for Lincoln prepared by Professor Swezey, the angles of elevations are as follows:

\section{June 22, angle $72.6^{\circ}$}

Feb. 20, angle $38.4^{\circ}$

Turning to trigonometric tables for natural sines the sines of these angles are:

$\begin{array}{rr}\text { For } 72.6^{\circ} & .9542 \\ 38.4^{\circ} & .6211\end{array}$


The ratio of the sines obtained by dividing the latter by the former is 65, that is, the light intensity on February 20 is 65 per cent of that of June 22. Again if the exposure taken for 15 seconds in the morning of February 20 equals in color that of 7 seconds at noon the same day, its intensity is $\frac{7}{15}$ of 65 per cent or 30.3 per cent. This method of measuring light is not without error, but until we know more of the nature and effect of the sun's rays, it is an approximate method of measuring those rays which are most stimulating to the chlorophyll of the plant.

The present investigation was suggested by Dr Clements, under whose direction it has been carried out. 'I'he writer is greatly indebted to Dr. Bessey and Dr. Clements for help given in the course of experimentation.

\section{B. RESULTS OF INVESTIGATION}

The results of the series of experiments carried on in the laboratory and the plant house from December, 1900, to June, 1901, are here considered in nearly the same order as that of the outline given in the introduction. With these data are incorporated the observations made in the fields near Lincoln during a short period of excessive dryness in the month of August, 1900.

\section{Water Capacity of Soils.}

The water capacity of different soils varies directly with their porosity, that is, with the air spaces they contain. This is dependent upon the fineness of the soil particles. However, this is a subject that cannot be fully discussed in a treatise of this nature. In order to know the comparative amount of water, each of the six soils used in experimentation was subjected to the following experiment. Pulverized soil of each kind, namely, sand, saline, loam, clay, loess and humus, was placed in a drying oven at a temperature of $250^{\circ} \mathrm{F}$. for 48 hours. Measured quantities of each were weighed and placed in flat crystallizing dishes of approxi- 
mately the same size. Distilled water was then slowly added to each to the point of apparent saluration. The humus probably was not thoroughly saturated owing to the resistance of the numerous capillary air spaces contained in the organic matter. The dishes were placed in similar physical conditions and the water allowed to evaporate from the soil, in order to determine the daily rate of evaporation. After continuing the process for several days the soils were again dried in an oven under the same conditions as before except that they were not pulverized. The experiment of evaporation was now repeated, using the hard soils, with the exception that this time they were allowed to become air dry. The first experiment was performed in the laboratory under dry conditions, the second under more moist conditions in the plant house. The following tables give the data obtained:

Water Capacity of Soils (in per cent of weight)

\begin{tabular}{|c|c|c|}
\hline SOIL & LOOSE & HA RD \\
\hline 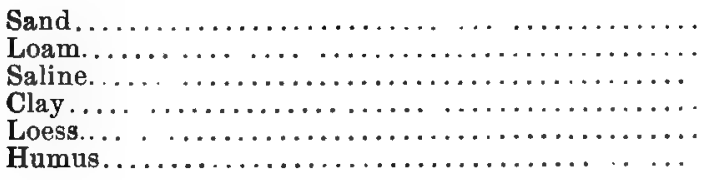 & $\begin{array}{l}125 \\
399 \\
40.8 \\
325 \\
37.1 \\
33.6 ?\end{array}$ & $\begin{array}{l}12.0 \\
33.6 \\
38.6 \\
27.6 \\
303 \\
28.7 ?\end{array}$ \\
\hline
\end{tabular}

Shrinkage of Loose Soils on Becoming Hard as Indicated by the Difference in Bulk.

\begin{tabular}{|c|c|c|c|}
\hline SOIL & LOOSE & HARD & SHRINKAGE \\
\hline 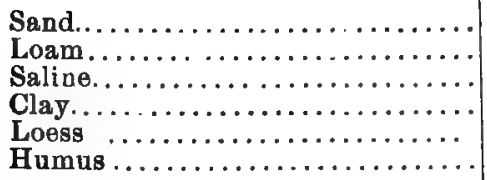 & $\begin{array}{l}510 \text { c.c } \\
510 \text { c c. } \\
600 \text { c.c. } \\
600 \text { c c. } \\
600 \text { c.c. } \\
600 \text { c.c. }\end{array}$ & $\begin{array}{l}500 \text { c.c. } \\
460 \text { c.c. } \\
555 \text { c.c. } \\
540 \text { c.c. } \\
552 \text { c.c. } \\
585 \text { c.c. }\end{array}$ & $\begin{array}{r}20 \% \\
9.8 \% \\
75 \% \\
10.0 \% \\
8.0 \% \\
4.2 \%\end{array}$ \\
\hline
\end{tabular}


Per Cent. of Space Occupied by Water in Soils.

\begin{tabular}{|c|c|c|}
\hline sorl & LOOSE & HARD \\
\hline 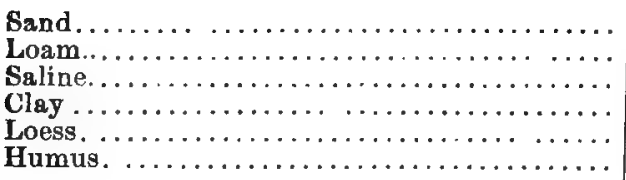 & $\begin{array}{l}27.1 \% \\
603 \% \\
59.2 \% \\
48.6 \% \\
669 \% \\
44.9 \% \text { ? }\end{array}$ & $\begin{array}{l}25.8 \% \\
553 \% \\
582 \% \\
462 \% \\
50.5 \% \\
39.0 \% ?\end{array}$ \\
\hline
\end{tabular}

It will be seen from the above tables that the water capacity of both loose and hard soil in order of the amount contained is as follows: saline, loam, loess, humus, clay, sand, when estimated by the comparative weights. If the water capacity is estimated by the per cent of space occupied, the series is as follows in loose soil: loam, saline, loess, clay, humus, sand. The order of shrinkage in becoming hard is slightly different being as follows: clay, loam, loess, saline, humus, sand.

The loss of water from the loose soil by evaporation, given in grams for each period of 24 hours, and for the final 6 days is given below.

Evaporation of Water from Loose Soils.

\begin{tabular}{|c|c|c|c|c|c|}
\hline SOIL & NET WT & 24 HRS. & 24 HRS. & 24 HRS. & 6 DAYS \\
\hline 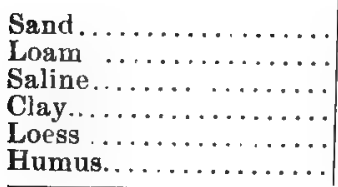 & $\begin{array}{l}941.7 \\
454.6 \\
497.4 \\
6040 \\
577.5 \\
529.6\end{array}$ & $\begin{array}{l}61.8 \\
46.7 \\
77.0 \\
49.5 \\
55.0 \\
55.1\end{array}$ & $\begin{array}{l}46.4 \\
37.3 \\
46.0 \\
45.5 \\
33.5 \\
33.0\end{array}$ & $\begin{array}{l}18.8 \\
33.4 \\
410 \\
\mathbf{3 7 . 0} \\
31.5 \\
30.0\end{array}$ & $\begin{array}{r}7.0 \\
154.8 \\
144.5 \\
141.0 \\
1690 \\
156.0\end{array}$ \\
\hline
\end{tabular}

The evaporation from hard soil for each 24 hours is given in the following table. There is also given in square centimeters the amount of surface exposed to the air by each dish of soil. The number at the head of each column is the date of each daily evaporation. 
Evaporation of Water from Hard Soils.

\begin{tabular}{|c|c|c|c|c|c|c|}
\hline SOIL & SURFACE & $1 \frac{5}{9}$ & 28 & $\frac{5}{21}$ & $\frac{5}{22}$ & $\frac{8}{83}$ \\
\hline Sand....... & $176.7 \mathrm{~cm}$. & 73 & 49 & 21 & 7 & 0 \\
\hline I nom ...... & 167.4 & 78 & 78 & 43 & 23 & 2 \\
\hline raline..... & 179.1 “ & 88 & 87 & 58 & 24 & 11 \\
\hline Clay ...... & 167.7 & 75 & 76 & 47 & 18 & 5 \\
\hline Loess ...... & 167.4 & 77 & 82 & 47 & 18 & 8 \\
\hline Humus. ... & 167.4 & 80 & 72 & 49 & 30 & 15 \\
\hline
\end{tabular}

\begin{tabular}{|c|c|c|c|c|c|c|}
\hline & $\frac{5}{24}$ & $2 \frac{1}{3}$ & $2^{5} 6$ & $\mathbf{8}^{\overline{5}} 7$ & $2^{5} 8$ & $2^{5}$ \\
\hline 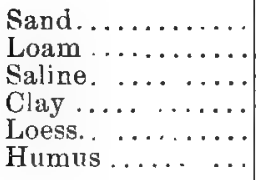 & $\begin{array}{l}0 \\
5 \\
3 \\
3 \\
1 \\
5\end{array}$ & $\begin{array}{r}0 \\
13 \\
21 \\
14 \\
19 \\
28\end{array}$ & $\begin{array}{r}0 \\
8 \\
16 \\
6 \\
13 \\
19\end{array}$ & $\begin{array}{r}0 \\
5 \\
8 \\
6 \\
16 \\
21\end{array}$ & $\begin{array}{l}0 \\
0 \\
3 \\
2 \\
0 \\
5\end{array}$ & $\begin{array}{l}0 \\
0 \\
0 \\
0 \\
0 \\
2\end{array}$ \\
\hline
\end{tabular}

May 23 and 24 were cloudy days, the latter being very humid, as a result of which the weight of the clay and loess increased. When each soil became air dry it was heated in an oven for 24 hours at $250^{\circ} \mathrm{C}$. The sand when air dry contained no measurable amount of water. The other soils contained water as follows: loam 4 grams, loess 7 grams, saline 3 grams, clay 6 grams, humus 5 grams. The sand lost its water most rapidly; the humus retained moisture most tenaciously.

From the foregoing data it is easily inferred that sandy soils lose water by evaporation more rapidly than clay soils and for this reason a plant must die more quickly in a sandy soil than in one containing an excess of clay. Since the plant is directly dependent upon the soil for its water supply, in times of drought the tenacity with which a soil holds water is a matter of utmost importance in the economy of the plant. The quality at once becomes of the greatest significance in the consideration of the factors which control the limit of water available for the use of the plant. 
Soils in nature always overlie strata of other kinds, and the water contained in them tends to settle by gravity to the stratum below. The rate of percolation depends upon the capillarity of the soil, and upon the impeding action of the hygroscopic water; probably upon even more obscure factors such as are involved in the varying chemical composition of the soil solutions.

According to Briggs" "The water contained in the soil in regard to the manner in which it is retained may be considered to be of three kinds, gravitation water, capillary water and hygroscopic water. Gravitation water is that portion which is in excess of the amount which the soil is able to retain under existing conditions, and is consequently free to drain away. The capillary water is that part which would be retained in the capillary spaces under these conditions, and which is capable of movement through capillary action. The hygroscopic water is that found on the surface of the grains, which is not capable of movement through the action of gravity or capillarity.

"The maximum amount of water which a given soil may contain depends upon the resultant effect of two forcesgravitation and surface tension. The force due to gravity is proportional to the mass of the liquid considered and is always directed vertically downward. In other words, it is the weight of the liquid. This mass of liquid would therefore leave the soil if unopposed by the action of some other force, the vertical component of which is acting along the same line as the force of gravity but must be equal to it and in opposite direction."

From this it may be readily deduced that in sandy soils the amount of gravitation water is at a maximum, and capillary water as well as hygroscopic water would be at a minimum. The clays, being the finest grained, hold the maximum amount of capillary and hygroscopic water, but here the gravitation water would be at a minimum. The other soils in quality vary between clay and sand.

*Briggs, Lyman J., Bull. 10, U. S. Lept. of Agr. Division of Soils, p. 6,1897 . 


\section{Physical Water in Soils.}

The term physical water is used in this discussion to designate the water a soil may retain at any time. This is limited only by the water capacity. The maximum amount of physical water occurs in streams, lakes, etc. The minimum amount is found in epiphytic or xerophytic situations. The per cent of physical water in the soil is not a factor of absolute value, since only the water available for the use of the plant can be a stimulus to its growth.

A plant growing in saline soil requires a higher per cent of physical water in order to live. This is illustrated by the data gathered from plants growing on the salt-bottoms near Lincoln. Hrianthus annuus and Panicum crus-galli were found dying in a saline soil with 14.9 per cent of physical water present, while in loam they begin to die only when the physical water drops to less than 10 per cent. Plants can grow well in sandy soil with even less than 5 per cent and in pure sand do not begin to die until the water content falls below 2 per cent. The whole matter seems to hinge upon the ability of the plant to take the capillary and hygroscopic water from the soil. This is dependent upon the vigor of the plant as well as upon the density of the soll solution. The imbibition of water by the roots of the plant is greatly retarded in soils which contain chemicals detrimental to the assimilatory processes of the plant. The salt marsh plants such as Atriplex argentea seem able to take in a soil solution of greater salinity than is capable of absorption by ordinary plants. The leaves of Atriplex often contain crystals of sodium chloride. May it not be that this is formed as a bye-product and serves as a means of elimination of the injurious substances in the soil? Atriplex grows and thrives where a common ruderal plant would die from the effect of the saline soil.

The welfare of the plant is dependent directly upon the amount of nutriment it may obtain from the soil solution and use in its economy. The soil solution may be so dilute in hydrophytic situations that it is necessary for the plant 
to absorb a much larger amount of water in order to obtain sufficient nutriment. On the other hand in times of drought the soil solution may be too concentrated to be available for the use of the plant. In alkaline and saline soils this takes place with a much higher per cent of physical water present.

\section{Physiological Water in Soils.}

The term physiological water designates that portion of the physical water available for the use of the plant. This being the case it varies directly with the amount of physical water on the one hand, and on the other it is limited only by the plant's ability to draw water from the soil. This is varied by the physical factors which affect the vigor of the plant. The plant prefers an optimum of heat, light, etc. This optimum varies with different species. If a plant is subjected to a stimulus which is greater or less than the optimum it must either adapt itself to the new condition by a slight change in its protoplasm and later in its structure, or, failing to adapt its structure, it becomes less vigorous. in the latter case it can, in times of drought, draw less water from the soil before it dies. As a result the amount of physiological water in the soil is less in this case than in the former. The different causes of the variation of the limit of physiological water will be discussed more fully in the chapter on that subject.

\section{Non-Available Water in Soils.}

The amount of non-available water in soils, as has been Indicated in the previous discussion, is varied widely by the porosity and composition of the soil. Nearly all the water in sand is available for the use of the plant, but the evaporation and gravitation from soils of this nature is much more rapid; as a consequence the dmount which the plant can use is much less than it may obtain from a soil containing clay mixed with sand or loam. The finer particles of the clay hold the water so tenaciously that it is much longer in losing its water content. In times of drought this gives the plant in the clay soil a longer lease of life. 
In an experiment with five species of plants growing in six different soils, the plants were allowed to wilt excessively under very dry conditions for two consecutive days. The amount of water in each soil was determined at the time of wilting and at the close of the second day of wilting. The average of the results from five species is as follows:

Water in Wilting Plants and in the Soils.

\begin{tabular}{|c|c|c|}
\hline SOIL & $\begin{array}{c}\text { SOIL } \\
\text { WATER } \\
\%\end{array}$ & $\begin{array}{c}\text { PLANT } \\
\text { WATER } \\
\%\end{array}$ \\
\hline 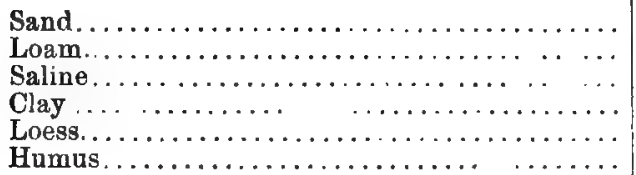 & $\begin{array}{r}043 \\
11.00 \\
14.00 \\
8.50 \\
970 \\
11.20\end{array}$ & $\begin{array}{l}72.6 \\
76.9 \\
81.5 \\
77.7 \\
80.6 \\
74.5\end{array}$ \\
\hline
\end{tabular}

The plants used were Helianthus annurs, a cultivated variety, Hordeum vulgare, Phaseolus vulgaris, Ricinus communis, and Zea mays. Although the limit of non-available water was not quite reached by these plants, the amount of water that they were getting was below the amount requisite for growth. If we arrange the soils in order of the per cents of water they contained at the time of wilting the series is as follows: saline, humus, loam, loess, clay, and sand, the last being the lowest.

The following table gives the average amount of physiological water used by the plants, and evaporated from the soil during the period of drought, and the corresponding loss of water frorn the tissues of the plant:

Water Loss in Plants and Soil during Drought.

\begin{tabular}{|c|c|c|}
\hline SOIL & $\begin{array}{l}\text { LOSS OF SOIL } \\
\text { WATER \% }\end{array}$ & $\begin{array}{c}\text { LOSS OF PLANT } \\
\text { WATER } \%\end{array}$ \\
\hline 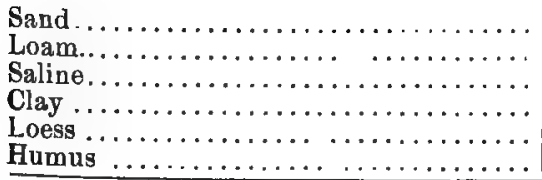 & $\begin{array}{r}10.2 \\
14.4 \\
10.6 \\
10.0 \\
9.0 \\
17.2\end{array}$ & $\begin{array}{r}17.1 \\
13.4 \\
7.2 \\
11.7 \\
87 \\
14.6\end{array}$ \\
\hline
\end{tabular}


It seems almost impossible to determine when plants are wilting in the same degre, that is, when they have lost an equal per cent of water from their weight. Until there is some accurate way of determining this, the results obtained are mere approximations. The whole subject of plant water is a very complicated one since the water content of the plant is varied by so many factors.

The amount of water in the soil non-available for plants in time of drought appears to be affected by the same physical conditions that tend to vary the amount of water in the plant, with the exception that in the soil the variation is more uniform. The tenacity with which the protoplasm of the plant may retain water probably varies not only in species but also in individuals depending primarily upon devices protective against evaporation, and secondarily upon the vigor of the plant. In times of drought, if the process of evaporation be a slow one, the amount of water is increased since the plant has a longer time to adapt itself to the changed condition. On the other hand, a rapid evaporation hastens the death of the plant and tends to raise the limit. The physical factors which increase evaporation are increased heat and light, decreased humidity, and winds. The amount of nun-available water is increased by shade, humid conditions and abundant physical water. The effect of shade upon plant structures is quite noticeable. Down to a certain point of light diminution the size of the leaves is increased, but beyond this point the effect is to decrease the size; at the same time the root system is retarded in its development.

As a result shade conditions affect directly the ability of the plant to exist in times of drought, and directly increase the amount of non-available water. Humid conditions also tend to increase the size of the leaves and at the same time to retard the development of the root system, the effect being almost identical with that of shade. 


\section{THE RELATIVE AMOUNT OF WATER IN THE} PLANT AND IN THE SOIL.

1. The Amount of Water in Healthy Plants and in the Soil in Which They Grow.

Most plants contain a relatively high per cent of water in their tissues. Hydrophytes, as a rule, are the most watery and xerophytes the least so; yet in the latter case there are exceptions among succulents. Mesophytes, as other plants, vary in water content according to the per cent of woodiness or of succulency. Seedlings contain a higher per cent than plants nearer to maturity. The per cent of water usually decreases gradually until maturity is reached, then rapidly to the minimum of the death point.

There are several physical conditions, which may affect the water-retaining power of the plant. Among these are varying humidity and soil moisture, excessive or defioient light, poor food supply, injurious chemicals in the soil, and. injurious gases in the air or soil. Varying soil composition does not seem to affect the water content of the plant unless it causes a lack of food or water, or unless there are present substances which act as poisons to protoplasm.

The following table gives the per cent of water in seedling plants and in the soil at the same time. The plants were three weeks old and were under the most favorable conditions in the plant-house during the month of May. The amount of light received by these plants was considerably less than that received by plants under natural conditions. (For record of physical factors see Comparative r'lot III.) 
The Water Content of Seedlings.

\begin{tabular}{|c|c|c|c|}
\hline PLANT & SOIL & $\begin{array}{l}\text { SOIL } \\
\text { WATER, \% }\end{array}$ & $\begin{array}{c}\text { PLANT } \\
\text { WATER, } \%\end{array}$ \\
\hline Phaseolus vulgaris............ & $\begin{array}{l}\text { Sand } \\
\text { Loam } \\
\text { Saline } \\
\text { Clay } \\
\text { Loess } \\
\text { Humus }\end{array}$ & $\begin{array}{r}83 \\
244 \\
299 \\
169 \\
12.4 \\
19.0\end{array}$ & $\begin{array}{l}90.4 \\
90.1 \\
90.0 \\
89.5 \\
89.6 \\
90.0\end{array}$ \\
\hline Helianthus an uus............ & $\begin{array}{l}\text { Sand } \\
\text { Loam } \\
\text { Saline } \\
\text { Clay } \\
\text { Loess } \\
\text { Humus }\end{array}$ & $\begin{array}{l}12.9 \\
249 \\
20.5 \\
164 \\
199 \\
29.1\end{array}$ & $\begin{array}{l}94.7 \\
92.2 \\
93.6 \\
87.6 \\
93.9 \\
90.2\end{array}$ \\
\hline Hordeum vulgare............. & $\begin{array}{l}\text { Sand } \\
\text { Loam } \\
\text { Saline } \\
\text { Clay } \\
\text { Loess } \\
\text { Humus }\end{array}$ & $\begin{array}{l}119 \\
291 \\
295 \\
18.2 \\
18.7 \\
289\end{array}$ & $\begin{array}{l}89.2 \\
91.3 \\
88.4 \\
93.6 \\
86.4 \\
85.7\end{array}$ \\
\hline 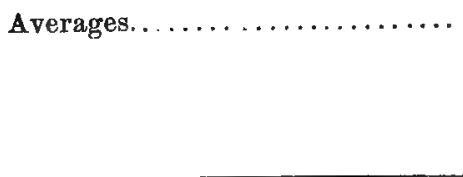 & $\begin{array}{l}\text { Sand } \\
\text { Loam } \\
\text { Saline } \\
\text { Clay } \\
\text { Loess } \\
\text { Hrimus }\end{array}$ & $\begin{array}{l}11.0 \\
261 \\
26.6 \\
17.3 \\
17.0 \\
25.6\end{array}$ & $\begin{array}{l}91.4 \\
912 \\
90.6 \\
901 \\
900 \\
89.6\end{array}$ \\
\hline
\end{tabular}

In obtaining the data in the preceding table, it was noted that Phaseolus and Helianthus plants were more uniform in size and vigor than those of Hordeum. The following table gives the per cent. of water in seedlings at the age of five weeks, plants of the same species buing used, and the experiment being conducted in the plant cases. The following is the record of physical factors:

Physical Record Averages.

\begin{tabular}{|c|c|c|c|c|}
\hline CASE & $\begin{array}{l}\text { TEMPERATURE } \\
\text { AIR, SOYL }\end{array}$ & $\begin{array}{l}\text { RELATIVE } \\
\text { HUMIDITY }\end{array}$ & $\begin{array}{l}\text { MAXIMUM } \\
\text { LIGHT }\end{array}$ & $\begin{array}{c}\text { SOIL } \\
\text { MOISTURE }\end{array}$ \\
\hline 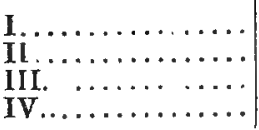 & $\begin{array}{ll}61^{\circ} & 58^{\circ} \\
74^{\circ} & 66^{\circ} \\
76^{\circ} & 70^{\circ} \\
77^{\circ} & 71^{\circ}\end{array}$ & $\begin{array}{l}64 \% \\
72 \% \\
55 \% \\
77 \%\end{array}$ & $\begin{array}{c}110 \% \\
0.5 \% \\
12.0 \\
12.0\end{array}$ & $\begin{array}{l}13.1 \% \\
145 \% \\
14.7 \% \\
16.4 \%\end{array}$ \\
\hline
\end{tabular}


Water in Plants, (in Per Cent of Weight.)

1. Phaseolus vulgaris.

\begin{tabular}{|c|c|c|c|}
\hline \multirow[b]{2}{*}{ CASE } & LOESS & LOAM & SAND \\
\hline & $\begin{array}{l}\text { Water in } \\
\text { Soil, Plant }\end{array}$ & $\begin{array}{l}\text { Water in } \\
\text { Soil, Plant }\end{array}$ & $\begin{array}{l}\text { Water in } \\
\text { Soil, Plant }\end{array}$ \\
\hline 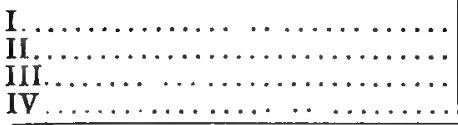 & $\begin{array}{ll}15.5 & 78.7 \\
14.4 & 92.4 \\
11.9 & 904 \\
186 & 90.3 \\
\end{array}$ & $\begin{array}{ll}17.1 & 84.7 \\
18.7 & 95.4 \\
17.7 & 92.0 \\
21.8 & 91.0\end{array}$ & $\begin{array}{ll}1.1 & 80.4 \\
0.7 & 89.7 \\
0.6 & 81.7 \\
1.3 & 85.2\end{array}$ \\
\hline \multicolumn{4}{|c|}{ 2. Zram ys. } \\
\hline 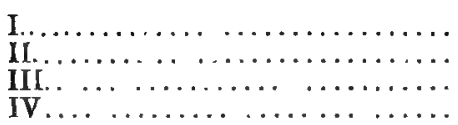 & $\begin{array}{ll}15.5 & 70.4 ? \\
14.4 & 93.3 \\
119 & 879 \\
18.6 & 923\end{array}$ & $\begin{array}{ll}17.1 & 79.5 ? \\
18.7 & 932 \\
17.7 & 94.8 \\
21.8 & 924\end{array}$ & $\begin{array}{ll}1.1 & 85.2 ? \\
0.7 & 91.8 \\
06 & 925 \\
1.3 & 889\end{array}$ \\
\hline
\end{tabular}

3. Hordeum vulgare.

\begin{tabular}{|c|c|c|c|}
\hline 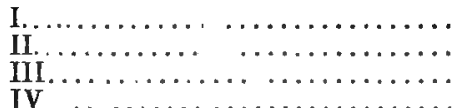 & $\begin{array}{ll}15.5 & 80.0 \\
14.4 & \ldots . . \\
11.9 & 88.8 \\
18.6 & 86.1\end{array}$ & 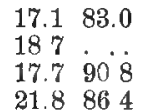 & 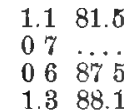 \\
\hline
\end{tabular}

A verages.

\begin{tabular}{|c|c|c|c|}
\hline & $15.5 \quad 76.3$ & 17.182 .4 & 1.1857 \\
\hline II. & 14.4928 & 18.7943 & 07907 \\
\hline & 11.9890 & 17.792 .5 & 0.787 .2 \\
\hline 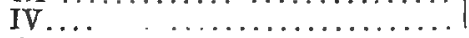 & $18.6 \quad 89.6$ & $21.6 \quad 90.2$ & 1.387 .4 \\
\hline
\end{tabular}

Totals of all Cases.

\begin{tabular}{|c|c|c|c|c|}
\hline & 15.186 .7 & $18.8 \quad 89$ & 9.8 & 0.987 .7 \\
\hline
\end{tabular}

In the preceding table, the plants in Case I had been slightly injured by cold a few days before the determination was made. The plants in sand in two of the cases apparently did not have sufficient water for full turgidity.

Seedlings of the same species have approximately an equal per cent. of water. The most healthy and most vigorously growing seedling may not have the highest per cent of water; in fact the reverse is usually true. The effects of different soils upon the water content of plants is not very marked in the case of seedlings. The effect upon older plants 
may be greater. Turgid plants growing in sand have the highest per cent. of water. This must be due to the lack of nutrient salts in quartz sand. The plants in this soil grew the slowest and were the least vigorous in appearance. Those growing in humus were by far the most vigorous in size and appearance, yet had the lowest per cent of water. The series of soils taken in order of plant vigor from the highest to the lowest is as follows: humus, loess, clay, saline, loam, sand. The fact that parasitic fungi attacked the roots of the plants to a greater extent in some soils than in others slightly vitiates the results obtained. The plants of Zea in Case II were attacked by Fiusarium but the results from the diseased plants were not included in the table. One thing is strongly indicated, namely, that the plant which is most vigorous does not necessarily contain the most water. In fact, the least vigorous plants were the most watery, for example, those growing in the shade in Case II.

2. The Amount of Water in Plants Wilting Strongly under Measured Physical Conditions, and the Corresponding Amount in the soil.

The cause of the wilting or drooping of the aerial portions of the plant is the lack of cell turgidity due to a loss of water. When the protuplasm loses water, it ceases to exert a pressure upon the cell-walls and gradually shrinks in bulk allowing the whole superstructure of the softer portions of the plant to collapse. Any physical factor that increases the evaporation from the leaves to such an extent that the roots cannot furnish a compensatory supply is certain to bring about this condition. Plants growing in natural conditions often wilt considerably during the hotter and dryer portion of the day and recover their turgidity during the cooler and moister hours of the night. Most plants seem able to withstand a succession of such experiences without serious injury if timely rains intervene.

Although many observations were made upon the relative amount of water in wilting plants and in the soils in which they grow, but one experiment was conducted with this end 
in view. This was carried on with the plants in Comparative Plot III. The following data were obtained from five species of plants growing in six kinds of soil.

Water Content of Healthy and Wilting Plants, and Water in Soil at the Time of Wilting.

Helianthus annuus.

Physical Condition at the Time of Wilting. "

Air temperature................................ $90^{\circ}$

Relative humidity .... ....................... $44 \%$

Soil temperature...................................... $104^{\circ}$

Maximum light......................................

\begin{tabular}{|c|c|c|c|}
\hline SOIL & $\begin{array}{c}\text { WATER IN SOIL } \\
\%\end{array}$ & $\begin{array}{l}\text { IN WILTING } \\
\text { PLANT, \% }\end{array}$ & $\begin{array}{l}\text { IN TURGID } \\
\text { PLANT, } \%\end{array}$ \\
\hline 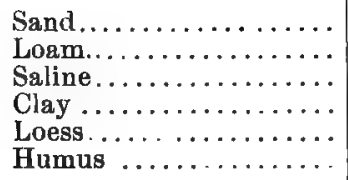 & $\begin{array}{r}0.4 \\
11.6 \\
15.1 \\
9.1 \\
10.9 \\
11.5\end{array}$ & $\begin{array}{l}78.8 \\
87.6 \\
85.4 \\
84.9 \\
81.5 \\
79.2\end{array}$ & $\begin{array}{l}94.7 \\
92.2 \\
936 \\
87.6 \\
93.9 \\
90.2\end{array}$ \\
\hline
\end{tabular}

Hordeum vulgare.

Physical Conditions at the Time of Wilting.

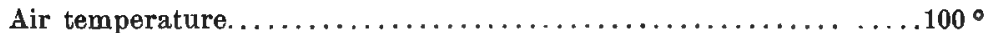

Relative humidity................................ $47 \%$

Soil temperature......................................

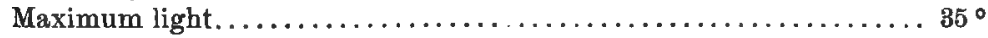

\begin{tabular}{|c|c|c|c|}
\hline SOIL & $\underset{\%}{\text { WATER IN SOIL, }}$ & $\begin{array}{l}\text { IN WILTING } \\
\text { PLANT, \% }\end{array}$ & $\begin{array}{l}\text { IN TURGID } \\
\text { PLANT, } \%\end{array}$ \\
\hline 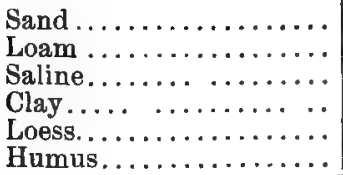 & $\begin{array}{r}0.4 \\
9.8 \\
9.8 \\
6.4 \\
8.1 \\
11.3\end{array}$ & $\begin{array}{l}70.0 \\
52.0 \\
82.2 \\
698 \\
79.4 \\
69.1\end{array}$ & $\begin{array}{l}89.2 \\
91.3 \\
88.4 \\
93.3 \\
86.4 \\
88.8\end{array}$ \\
\hline
\end{tabular}


Phaseolus vulgaris.

Physical Conditions at the Time of Wilting.

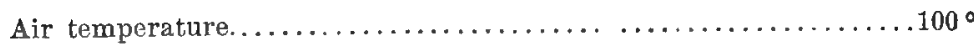

Relative humidity............................. $48 \%$

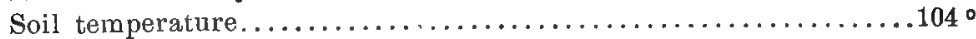

Maximum light...................................... $35 \%$

\begin{tabular}{|c|c|c|c|}
\hline Sort & $\underset{\%}{\text { WATER IN SOIL, }}$ & $\begin{array}{l}\text { IN WILTING } \\
\text { PLANT, } \%\end{array}$ & $\begin{array}{l}\text { IN TURGID } \\
\text { PLANT, } \%\end{array}$ \\
\hline 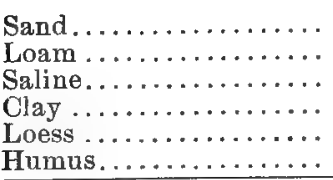 & $\begin{array}{r}04 \\
10.6 \\
17.2 \\
8.5 \\
8.4 \\
9.3 \\
\end{array}$ & $\begin{array}{l}780 \\
80.5 \\
80.0 \\
78.9 \\
856 \\
81.0\end{array}$ & $\begin{array}{l}90.4 \\
90.1 \\
900 \\
895 \\
896 \\
90.0\end{array}$ \\
\hline
\end{tabular}

Ricinus communis.

Physical Conditions at the Time of Wilting.

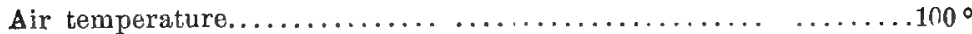

Relative humidity................................46\%

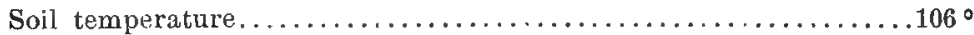

Maximum light...............................

\begin{tabular}{|c|c|c|c|}
\hline SOIL & $\begin{array}{l}\text { WATER IN SOIL. } \\
\qquad \%\end{array}$ & $\begin{array}{l}\text { IN WILTING } \\
\text { PLANT. \% }\end{array}$ & $\begin{array}{l}\text { IN TURGID } \\
\text { PLANT, } \%\end{array}$ \\
\hline 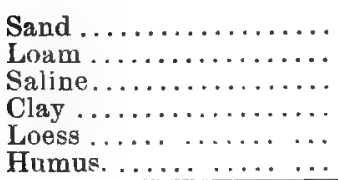 & $\begin{array}{r}05 \\
103 \\
12.0 \\
92 \\
78 \\
12.1\end{array}$ & $\begin{array}{l}632 \\
82.5 \\
76.5 \\
806 \\
743 \\
79.7\end{array}$ & $\begin{array}{l}84.4 \\
84.4 \\
84.4 \\
84.4 \\
84.4 \\
84.4\end{array}$ \\
\hline
\end{tabular}

Zea mays.

Physical Conditions at the Time of Wilting.

Air temperature......................................

Relative humidity............................. 47\%

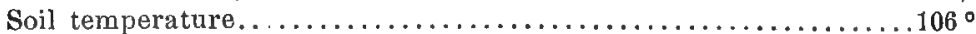

Maximum light. . . . . . . . . . . . . . . . . . . . . . . $35 \%$ 


\begin{tabular}{|c|c|c|c|}
\hline SOIL & $\begin{array}{c}\text { WATER IN SOIL, } \\
\%\end{array}$ & $\begin{array}{l}\text { IN WILTING } \\
\text { PLAN', } \%\end{array}$ & $\begin{array}{l}\text { IN TURGID } \\
\text { PLANT, } \%\end{array}$ \\
\hline 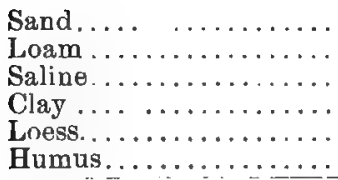 & $\begin{array}{r}0.4 \\
12.5 \\
16.8 \\
9.4 \\
131 \\
11.0\end{array}$ & $\begin{array}{l}72.2 \\
81.7 \\
83.4 \\
75.0 \\
82.4 \\
835\end{array}$ & $\begin{array}{l}92.0 \\
92.0 \\
93.0 \\
92.0 \\
92.5 \\
92.0\end{array}$ \\
\hline
\end{tabular}

Average of Results.

Physical Conditions at the Time of Wilting.

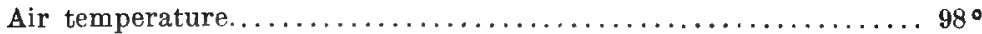

Relative humidity.............................. $47 \%$

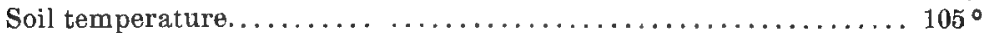

Maximum light. .............................

(Number of plants, 60.)

\begin{tabular}{|c|c|c|c|}
\hline SOIL & $\underset{\%}{\text { WATER IN SOIL, }}$ & $\begin{array}{l}\text { IN WILTING } \\
\text { PLANT, } \%\end{array}$ & $\begin{array}{l}\text { IN TURGID } \\
\text { PLANT, \% }\end{array}$ \\
\hline 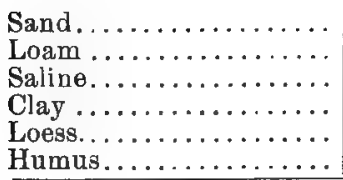 & $\begin{array}{r}0.43 \\
11.0 \\
14.2 \\
8.5 \\
9.7 \\
11.2\end{array}$ & $\begin{array}{l}726 \\
76.9 \\
81.5 \\
777 \\
806 \\
74.5\end{array}$ & $\begin{array}{l}90.2 \\
90.0 \\
89.7 \\
89.4 \\
893 \\
891\end{array}$ \\
\hline
\end{tabular}

If from these data the soils are arranged according to the amount of water they contained at the time the plants were wilting, they make the following series from the highest to the lowest per cent: saline, humus, loam, loess, clay, sand.

The following table indicates the average time the plants in each soil were subjected to drought, the average per cent of water lost from each soil, and the average per cent of water lost from the plants if based on the amount found in turgid plants. 
Per Cent of Water Loss in Plants and Soils.

\begin{tabular}{|c|c|c|c|}
\hline SOIL & $\begin{array}{c}\text { TIME IN } \\
\text { DRYING ODT. }\end{array}$ & $\begin{array}{l}\text { WATER LOSS } \\
\text { OF SOIL. }\end{array}$ & $\begin{array}{l}\text { WATER LOSS } \\
\text { OF PLANT. }\end{array}$ \\
\hline 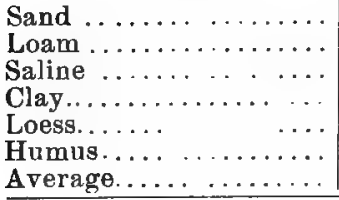 & $\begin{array}{l}6.0 \text { days } \\
6.2 \\
6.8 \\
6.0 \\
5.2 \\
6.0 \\
6.0 \\
\end{array}$ & $\begin{array}{r}10.2 \\
14.4 \\
10.6 \\
10.0 \\
9.0 \\
17.2 \\
11.9\end{array}$ & $\begin{array}{r}17.1 \\
134 \\
7.2 \\
11.7 \\
8.7 \\
14.6 \\
121 \\
\end{array}$ \\
\hline
\end{tabular}

The table above is not accurate as to the time of drying out given for sand, or as to its water loss, because the plants in sand were watered in order to keep them alive until they would die under the same temperature conditions as those in the other soils.

The great difference which these results show in the comparative amounts of water in the soil and in the plant at the time of wilting call for an explanation which at the best must be more or less theoretical. We may assume that the difference of 8 per cent. between the sand and the clay is due solely to a difference in capillarity and surface tension, and to the presence of a greater amount of hygroscopic water in the clay. This explanation may account for the greater portion of the difference between clay and sand. It will not however explain why the plant wilts at a higher per cent. of soil water in loam, humus and saline soils.

The sand used was quartz sand and contained only a trace of soluble salts. On the other hand, the saline soil had the highest per cent. of soluble matter. May it not be possible that the concentration of these salts as the soil gradually loses water is a factor that plays a very important part in checking the interchange which takes place between the solutions in the protoplasm of the root hairs, and the soil solution? There is no doubt that the increasing capillarity and hygroscopicity of the finer soils impedes this important process, either causing it to cease, or bringing about an actual transfer of solutions from the protoplasm of the plant to the soil solution. In the case of 
saline and alkaline soils, the root hairs, being strongly acid in their nature, must suffer from the increasing alkalinity of the soil. This is a fertile field for investigation and it is hoped that these problems will soon be taken up and solved by the plant physiologist.

In mesophytes when wilting, the amount of water lost by the soil nearly equals the amount lost by the plant. It might be inferred that a plant ceases entirely to take water from the soil the moment wilting begins, and from this time on it simply undergoes evaporation. However, it can hardly be true that the plant ceases entirely to take water from the soil until it has lost much from its own weight. The facts in the matter may be obtained by a simple epxeriment. If several plants be taken at the time when they begin to lose turgidity and part of them uprooted, and their roots carefully sealed with wax or cocoa butter, these should lose water more rapidly, if it be true that plants in the soil do not cease to absorb water when they begin to wilt. Both should lose weight equally if the plants in the soil are not obtaining water, and finally, if the sealed plants should lose water more slowly than those in the soil it would be positive proof that the soil is actually taking water from the protoplasm of the roots.

The plant absorbs very little water from the soil after it is wilting excessively. From the very nature of the root hairs, it is quite certain that they perish soon after the plant begins to suffer from drought. This is indicated by observations to the effeet that the roots of many annuals die before the stem and that they commence to lose water about the time the leaves die. Should it be found out later that this is certainly the case, the effect will be to increase the estimates of the limit of the non-available water and consequently to diminish those of the available water. This being the case the tables at the close of this treatise will need revising, but the general results and conclusions of the work will rernain unchanged. 
3. Observations upon the Water Content of Plants Dying from Drought and the Corresponding Water Content of the Soil.

The relative amount of water in plants dying from drought is usually varied by the same causes that vary the amount of water in the healthy plant. This varies with the natural woodiness or succulency of the plant and is greatly modified by the physical conditions under which the plant develops. The discussion of the wilting plant in the previous chapter might be made to include the dying plant, for there can be no definite line of demarcation between the two conditions. The wilting is but the beginning of disturbances of function in the plant which if continued result in its death. Owing to the difficulty of examining the roots of the plant at every stage of the process of wilting and dying, it is almost impossible to tell when a plant ceases to obtain water from the soil, and actually begins to die. So long as this is the case there will be an uncertainty about the results obtained in estimating non-available soil water. The plant must certainly live for some time after it ceases to take water from the soil. The length of this period depends directly upon the store of water in the plant and its ability to hoard it. The ordinary mesophyte seems to be deficient in both regards, yet in a number of species studied, such plants lived even after the roots had died, that is, until the soil apparently aided the roots in extracting water from the resistant living cells of the stems. This happened usually under conditions where the evaporation of water took place very slowly, and the death of the plant was prolonged.

Grasses when turgid contain a lower per cent of water than many other herbaceous plants, and when dying they show a still greater proportionate difference. It was impossible to experiment along all lines, and the data given here are from observations made at various times in connection with the other lines of experimentation. A grass containing 15 per cent of water or less is dead almost beyond a doubt; with from 15 to 20 per cent its condition is uncertain and it is doubtful if it could be revived; above 20 per cent, life usually remains in most grasses. A succulent plant 
apparently dies with a much higher per cent of water than a woody one, although its death usually takes place more slowly. The roots of Coleus and Nasturtium die before the stems, but after the root is dead and dry the plants will not recover although there may be 50 per cent of water remaining in the stems. Lobelia syphilitica usually begins dying at 50 to 70 per cent and is usually dead at 40 per cent.

The following results indicate the difference between some rosette plants of hydrophytic tendencies in the amount of water in the soil and plant when the plant is dying, and the amount in rosettes of xerophytic tendencies. The plants were grown in loam in the plant house.

Lobelia syphilitica.

\begin{tabular}{l|c|c|c}
\hline PHYSICAL CONDITIONS & $\begin{array}{c}\text { PHYSIOLOGICAL } \\
\text { WATER IN SOIL, } \\
\%\end{array}$ & $\begin{array}{c}\text { NON-A VAILABLE } \\
\text { WATER IN SOIL, } \\
\%\end{array}$ & $\begin{array}{c}\text { WATER IN } \\
\text { DYING PLANT, } \\
\%\end{array}$ \\
\hline In Sun, Wet Soil ........ & $15-30$ & 11.4 & 57.9 \\
In Shade, Wet Soil ...... & $15-30$ & 115 & 44.1 \\
In Sun, Dr. Soil ...... & $8-15$ & 103 & 52.0 \\
In Shade, Dry Soil.... & $8-15$ & 7.7 & 68.1 \\
\hline
\end{tabular}

Average with Six Species of Xerophytic Tendencies.

\begin{tabular}{l|r|l|l}
\hline In Sun, Wet Soil........ & $15-30$ & 8.6 & 39.0 \\
In Shade, Wet Soil...... & $15-30$ & 6.1 & 338 \\
In Sun, Dry Soil . . . . & $8-15$ & 6.9 & 314 \\
In Shade, Dry Soil..... & $8-15$ & $\mathbf{6 6}$ & 390 \\
\hline
\end{tabular}

From this and similar results obtained, it was found that hydrophytism tends to make a plant more sensitive to excessive water loss, so much so that it dies with a greater per cent of water in its tissues than in the soil. The hydrophytic plant usually has more water in its tissues when turgid and it is to be expected that it might die with a slightly greater relative per cent of water, both in the plant and in the soil. Other variations of the water in dying plants will be discussed under the next section. The following tables give results from three species, one of xerophytic tendencies, one of hydrophytic tendencies, and a mesophytic grass, all grown in loam in the plant house. 
Variable Water Content in Plants and Soil.

Atriplex argentea (stem stage).

(xerophytic.)

\begin{tabular}{|c|c|c|c|}
\hline TIME IN DTING & $\begin{array}{l}\text { PHYSIOLOGICAL } \\
\text { WATER IN SOIL, } \\
\qquad \%\end{array}$ & $\begin{array}{l}\text { NON·AVAILABLE } \\
\text { WATER IN SOIL, } \\
\text { \% }\end{array}$ & $\begin{array}{c}\text { WATER IN } \\
\text { DYING PLANT, } \\
\not \%\end{array}$ \\
\hline 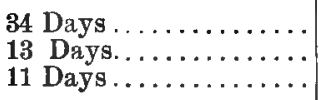 & $\begin{array}{l}13.2 \\
10.4 \\
10.0\end{array}$ & $\begin{array}{l}4.1 \\
4.2 \\
8.9\end{array}$ & $\begin{array}{l}261 \text { dead } \\
27.1 \text { dead } \\
35.6 \text { dying }\end{array}$ \\
\hline \multicolumn{4}{|c|}{$\begin{array}{c}\text { Eclipta alba (flowering stage.) } \\
\text { (hydrophytic.) }\end{array}$} \\
\hline 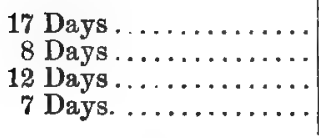 & $\begin{array}{r}2.6 \\
150 \\
20.0 \\
15.0\end{array}$ & $\begin{array}{l}6.5 \\
7.6 \\
7.4 \\
88\end{array}$ & $\begin{array}{l}37.0 \text { dead } \\
43.0 \text { dead } \\
500 \text { dying } \\
50.8 \text { dying }\end{array}$ \\
\hline \multicolumn{4}{|c|}{$\begin{array}{c}\text { Elymus canadensis (bunches.) } \\
\text { (mesophytic.) }\end{array}$} \\
\hline 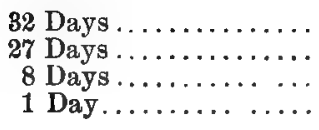 & $\begin{array}{r}15.5 \\
15.0 \\
10.0 \\
2.0\end{array}$ & $\begin{array}{l}4.8 \\
5.1 \\
5.8 \\
5.6\end{array}$ & $\begin{array}{l}15.2 \text { dead } \\
21.6 \text { dying } \\
238 \text { dead } \\
26.5 \text { dying }\end{array}$ \\
\hline
\end{tabular}

The data given in the preceding table were obtained from plants thought to be dying, the determination being made at a time when all the plant's aerial organs appeared to be either dying or dead. The per cent of water in a dying plant as well as in a living growing plant varied with the vegetative stage and age of the plant, depending on whether it is a seedling, a rosette, a stem, or a fruiting plant. The first in the series has the highest and the last the lowest per cent of water. Again the amount of water in a dying plant may depend upon the variation of the nature of the tissues in different species, ranging from succulents with the greatest per cent of water in their tissues to woody plants containing the least.

The following table indicates the effect of different physical stimuli during the lifetime of the plant upon the relative 
per cent of water in the plant at the time of dying. They are taken from Comparative Plot II (See record of average physical factors for this plot). These are the averages from 36 pots of Zea mays and Phaseolus vulgaris.

Effect of Physical Stimuli.

PLANT

NON-AVAILABLE WATER IN DYING WATER IN SOIL. PLANT.

Cas. I. (Reduced temperature).

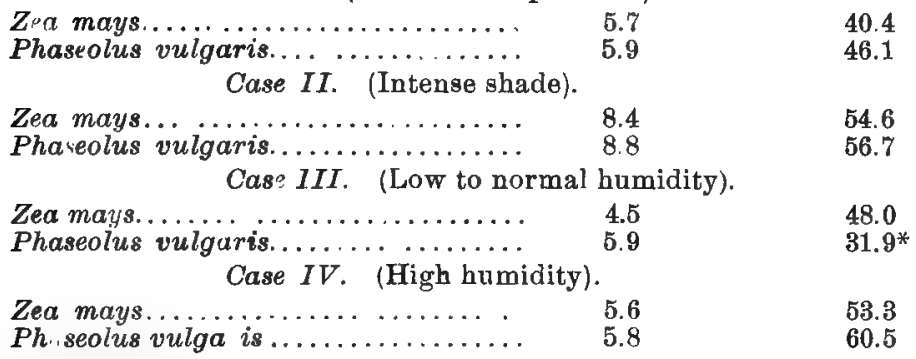

*Partly diseased

In determining the water in dying plants, great care was taken to have the plants near the death point, and this being the case the variations in the results from different conditions may be ascribed partly to differences in plant vigor, and partly to a change in the structure due to adaptation. The intense shade in Case II. was without doubt the cause of the high limit of soil water and the high per cent. of water in the dying plants. The plants in this case were greatly elongated in length. They were etiolated and lacked in fibro-vascular tissues. As soon as they were deprived of a water supply in the soil, they collapsed. The results from Case IV. indicate that high humidity has a tendency to produce the same effect on the limit as shade, but to a less degree.

C. NON-AVAILABLE WATER IN SOILS; HOW VARIED.

1. Effect of Increased Heat (and Light).

In the consideration of the effects of heat, light must be included, because it was inseparably connected with the high 
temperatures of the plant house during the last few days of experimentation. Yet in summing up the results it can hardly be claimed that the light in the plant house at any time was too intense for the best development of the plants. The maximum amount of light was slightly over 40 per cent of the standard of June 22. The temperature however was high, the average maximum for the last twelve days was $100^{\circ}$ for the air, and $101^{\circ}$ for the soil. Plants dying under these conditions had a higher limit of physiological water and of plant water than plants dying under cooler conditions with less light. The following table gives the results from Comparative Plot III. from plants grown in the plant house during May, 1901, in loam, loess and sand.

Comparative Plot III. (Increased heat and light).

Physical factors during the drought period.

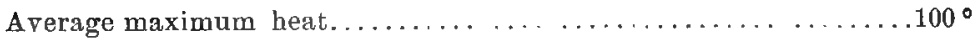

Average minimum humidity ........................ $51 \%$

Average maximum soil temperature....................... ${ }^{\circ}$

Average maximum light. . . . . . . . . . . . . $3 \% \ldots \ldots \ldots$

Phaseolus vulgaris.

\begin{tabular}{|c|c|c|}
\hline SOIL & $\begin{array}{c}\text { NON-AVAILABLE } \\
\text { WATER. }\end{array}$ & $\begin{array}{c}\text { WATER IN } \\
\text { DYING PLANT. }\end{array}$ \\
\hline 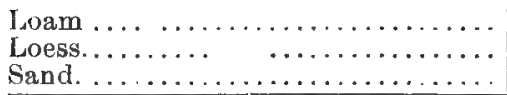 & $\begin{array}{l}8.7 \\
8.7 \\
0.26\end{array}$ & $\begin{array}{l}530 \\
53.8 \\
38.8\end{array}$ \\
\hline
\end{tabular}

Helranthus annuus.

\begin{tabular}{|c|c|c|}
\hline 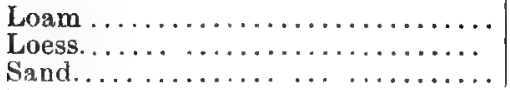 & $\begin{array}{r}11.2 \\
10.2 \\
0.2\end{array}$ & $\begin{array}{l}61.4 \\
55.2 \\
449\end{array}$ \\
\hline
\end{tabular}

Hordeum vulgare.

\begin{tabular}{|c|c|c|}
\hline 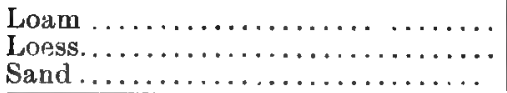 & $\begin{array}{l}8.7 \\
9.1 \\
0.3\end{array}$ & $\begin{array}{l}39.4 \\
50.1 \\
53.0\end{array}$ \\
\hline
\end{tabular}


Ricinus communis.

\begin{tabular}{|c|c|c|}
\hline SOIL & $\begin{array}{c}\text { NON-AVAILABLE } \\
\text { WATER }\end{array}$ & $\begin{array}{l}\text { WATER IN } \\
\text { DYING PLANT }\end{array}$ \\
\hline 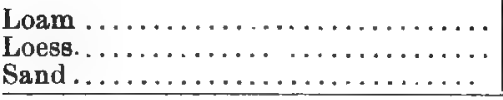 & $\begin{array}{r}9.1 \\
109 \\
044 \\
\end{array}$ & $\begin{array}{l}61.0 \\
49.6 \\
24.3\end{array}$ \\
\hline \multicolumn{3}{|l|}{ Zea mays. } \\
\hline 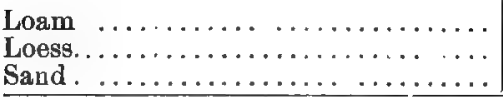 & $\begin{array}{r}10.1 \\
9.3 \\
0.41 \\
\end{array}$ & $\begin{array}{l}10.4 \\
56.1 \\
582 \\
\end{array}$ \\
\hline \multicolumn{3}{|c|}{ Average for Five Species. } \\
\hline 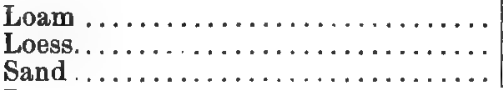 & $\begin{array}{l}96 \\
9.6 \\
0.32\end{array}$ & $\begin{array}{l}61.0 \\
528 \\
438\end{array}$ \\
\hline
\end{tabular}

The plants named in the following tables were grown in the plant house under a lower average temperature and less intense light than those of the previous plot, and the results are given for comparison.

Comparative Plot $I I$.

Physical record while plants were dying.

Average maximum temperature........................9. $94^{\circ}$

Average minimum humidity ............................ $46 \%$

Average maximum soil temperature....................... $90^{\circ}$.

Average maximum light..........................

Phaseolus vulgaris.

\begin{tabular}{|c|c|c|}
\hline SOIL & $\begin{array}{c}\text { NON-A VAILABLE } \\
\text { WATER IN SOIL, } \\
\%\end{array}$ & $\begin{array}{c}\text { WATER IN } \\
\text { DYING PLANT } \\
\%\end{array}$ \\
\hline 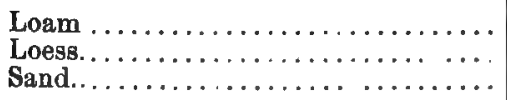 & $\begin{array}{l}6.2 \\
5.8 \\
0.45\end{array}$ & $\begin{array}{l}27.2 \\
22.3 \\
29.6\end{array}$ \\
\hline
\end{tabular}

Helianthus annuus.

\begin{tabular}{|c|c|c|}
\hline $\begin{array}{l}\text { Loam } \ldots \ldots \ldots \ldots \ldots \ldots \ldots \ldots \ldots \ldots \ldots \ldots \\
\text { Loess. } \ldots \ldots \ldots \ldots \ldots \ldots \ldots \ldots \ldots \ldots \ldots \ldots \ldots \ldots \\
\text { Sand } . \ldots \ldots \ldots \ldots \ldots \ldots \ldots \ldots \ldots\end{array}$ & $\begin{array}{l}48 \\
7.3 \\
0.78\end{array}$ & $\begin{array}{l}24.9 \\
49.2 \\
31.8\end{array}$ \\
\hline
\end{tabular}




\section{Hordeum sativum.}

\begin{tabular}{|c|c|c|}
\hline SOIL & $\begin{array}{l}\text { NON·AVAILABLE } \\
\text { WATER IN SOIL, } \\
\qquad \%\end{array}$ & $\begin{array}{c}\text { WATER IN } \\
\text { DYING PLANT } \\
\%\end{array}$ \\
\hline 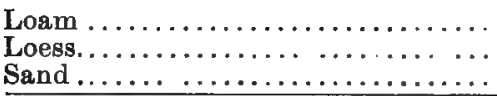 & $\begin{array}{l}3.5 \\
2.6 \\
0.46\end{array}$ & $\begin{array}{l}43.7 \\
23.5 \\
27.0\end{array}$ \\
\hline
\end{tabular}

Ricinus communis.

\begin{tabular}{|c|c|c|}
\hline 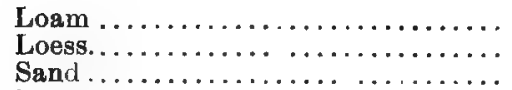 & $\begin{array}{l}5.9 \\
6.4 \\
1.0\end{array}$ & $\begin{array}{l}17.8 \\
291 \\
309\end{array}$ \\
\hline
\end{tabular}

Zea mays.

\begin{tabular}{|c|c|c|}
\hline 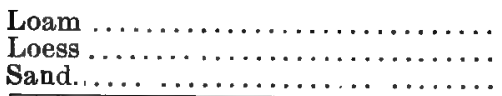 & $\begin{array}{l}3.7 \\
4.7 \\
06\end{array}$ & $\begin{array}{l}29.8 \\
33.9 \\
49.8\end{array}$ \\
\hline
\end{tabular}

Averages.

\begin{tabular}{|c|c|c|}
\hline $\begin{array}{l}\operatorname{Loam} \\
\text { Loess. } \ldots \ldots \ldots \ldots \ldots \ldots \ldots \ldots \ldots \ldots \ldots \ldots \ldots \\
\text { Sand. } \ldots \ldots \ldots \ldots \ldots \ldots \ldots \ldots \ldots \ldots \\
\end{array}$ & $\begin{array}{l}5.0 \\
5.3 \\
0.65\end{array}$ & $\begin{array}{l}28.7 \\
29.6 \\
39.8\end{array}$ \\
\hline
\end{tabular}

Average Increase in Plot III.

\begin{tabular}{|c|c|c|}
\hline 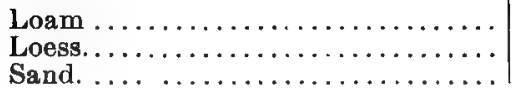 & $\begin{array}{l}4.6 \\
4.6 \\
0.3^{*}\end{array}$ & $\begin{array}{r}32.3 \\
23.2 \\
4.0\end{array}$ \\
\hline
\end{tabular}

*Decrease.

Owing to the lower per cent. of water in the plants in Plot II., it is difficult to get accurate comparisons with those of Plot III. and to make definite conclusions from the results. If the non-available water in each case could have been determined at the time when the plants of the same species had approximately the same amount of water in their tissues, the comparisons might have been made with a greater degree of accuracy. It certainly can be said that the comparison of the results indicate that temperatures even 
slightly above normal at the time of dying increase considerably the amount of non-available water.

Obervations and determinations of water content were conducted with plants of Syntherisma sanguinale in the plant house during a period lasting from March 25 to May 3. The results of these observations are separated into two groups, 19 were made before April 5, and 19 afterward. The results will now be compared.

Physical factors, March 25 to April 15.

Average air temperature. $\ldots \ldots \ldots \ldots \ldots, \ldots \ldots \ldots 1^{\circ}$

Average relative humidity ..........................67\%

Average soil temperature............................ $72^{\circ}$

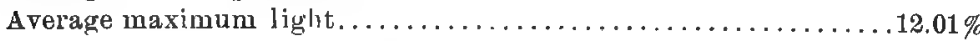

Physical factors, April 15 to May 8.

Average air temperature............................ $6^{\circ}$

Average rela ive humidity ...........................66\%

Average soil temperature......................... $80^{\circ}$

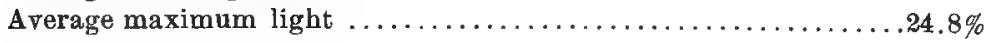

Syntherisma sanguinale.

Average of 19 plants, Mar. 25 to Apr. $15 \ldots$. Average of 19 plants, Apr, 15 to May $3 \ldots . .$.

\begin{tabular}{|l|c} 
LIMIT & $\begin{array}{c}\text { PLANT } \\
\text { WATER }\end{array}$ \\
\hline 6.1 & $\begin{array}{l}40.1 \\
5.1\end{array}$ \\
\hline
\end{tabular}

In the same way the observations on Chaetochloa viridis are separated into two groups, eight taken before April 14, and eight after April 14, the physical factors being quite the same as before.

Chaetochloa viridis.

\begin{tabular}{l|c|c}
\hline & LIMIT & $\begin{array}{c}\text { PLANT } \\
\text { WATER }\end{array}$ \\
\hline Average of 8 plants, Apr. 3 to Apr. 14....... & $\begin{array}{l}7.0 \\
6.1\end{array}$ & $\begin{array}{l}38.8 \\
25.7\end{array}$ \\
\hline
\end{tabular}


It may be well to give the results from two other grasses in addition to the previous ones, the physical conditions being similar.

Panicum crus-galli.

\begin{tabular}{|c|c|c|}
\hline & LIMIT & $\begin{array}{l}\text { PLANT } \\
\text { WATER }\end{array}$ \\
\hline $\begin{array}{l}\text { Average of } 5 \text { plants, Apr. } 5 \text { to Apr. } 17 . \ldots \\
\text { Average of } 5 \text { plants, Apr. } 17 \text { to Apr. } 29 .\end{array}$ & $\begin{array}{l}6.6 \\
6.2\end{array}$ & $\begin{array}{l}29.3 \\
33.1\end{array}$ \\
\hline
\end{tabular}

Chaetochloa glauca.

\begin{tabular}{|c|c|}
\hline $\begin{array}{l}\text { Average of } 6 \text { plants, Mar. } 9 \text { to Apr. } 1 \ldots \ldots \ldots \\
\text { Average of } 6 \text { plants, Apr. } 1 \text { to Apr. } 29 \ldots \ldots \ldots\end{array}$ & $\begin{array}{l}7.5 \\
7.1\end{array}$ \\
\hline
\end{tabular}

The results from these grasses indicate that the increase of light and heat was favorable to the plants, 1ncreasing their ability to take water from the soil. The winter conditions of the plant house were shade conditions, and as the light increased the plants became more and more vigorous; the heat increased at a more rapid ratio than the light, but in case of the grasses did not become excessive while the plants were dying, the physical conditions being similar to those of Comparative plot II.

\section{EFFECT OF TEMPERATURE BELOW THE NORMAL.}

Since the normal or optimum temperature may vary with the habitat of the plant, the results obtained by growing plants of different habitat under a certain range of temperature fluctuations are apt to vary with different plants. Case I contained plants of three species, viz.,Zea mays, Phaseolus vulgaris, and Hordeum vulgare, grown in loam, loess and sand, at a temperature usually varying from $5^{\circ}$ to $20^{\circ}$ below that of the other cases. The following results from Case I and Case III, will show the effects of a slightly lowered temperature as compared with those of the normal in raising or lowering the limit of physiological water. The averages are taken from thirty plants in each case. 


\begin{tabular}{|c|c|c|}
\hline PEYSICAT, FACTORS & CASE I & CASE III \\
\hline Average temperature & 61 。 & $76^{\circ}$ \\
\hline 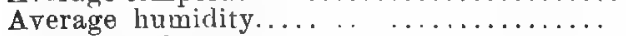 & $64 \%$ & $55 \%$ \\
\hline Average soil temperature................ & $58^{\circ}$ & $70^{\circ}$ \\
\hline 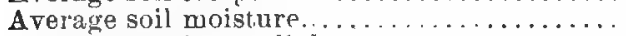 & $151 \%$ & $14.7 \%$ \\
\hline $\begin{array}{l}\text { Average maximum light................ } \\
\text { Results. }\end{array}$ & $11.0 \%$ & $12.0 \%$ \\
\hline Average limit of physiological water........ & $54 \%$ & $48 \%$ \\
\hline Average water of dying plants. ....... & $34.7 \%$ & $30.4 \%$ \\
\hline
\end{tabular}

The colder air retarded somewhat the growth of plants in Case I, and its effect was to increase the average of nonavailable water to the extent of 0.6 per cent. The plants in Case I were longer in dying than those in Case III because in the lower temperature the evaporation took place more slowly. The average time of dying in Case I was 19 days, in Case III 25 days. The slower evaporation of water tended to allow the plant more time for adaptation, lowering the physiological water limit and counteracting the effect of the cooler temperature. This being taken into consideration the difference in the average from the two plants would be the more significant if it were not so small. The difference caused by the cold was not noticeable in the cuse of barley and oats, but was quite striking in the case of corn and beans. This bears out observations of farmers to the effect that the small grains grow well during a cool spring, but corn is retarded.

3. Effect of Increased or Decreased Humidity, when excessive.

The plants grown under the conditions of case IV, were constantly in a humid air, even when the soil was deprived of water in the determination of the physiological water. They grew taller than those in the other cases. The leaves were slightly larger, and the root system less extended than those of the plants in the Cases I and III where the air was less humid. Comparing the results with those from Case III, the case with the normal conditions, the effect of the increased humidity is readily seen. 


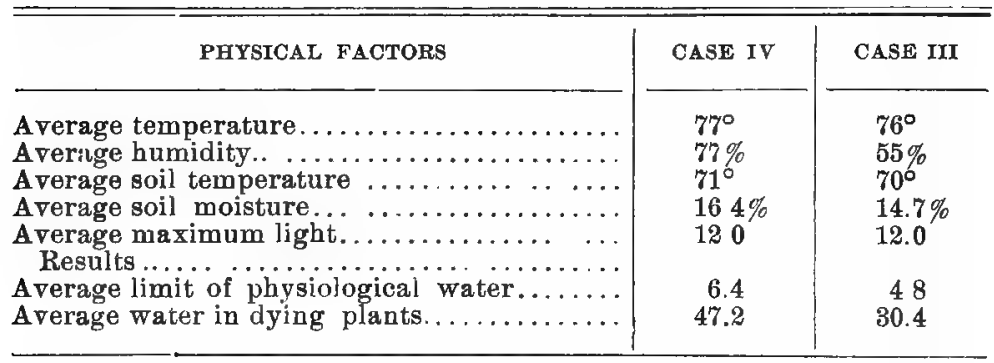

From this experiment it is proven that humid air tends to increase the size of the leaves, and to diminish the root system of the plant. The plant begins to adapt itself to living in such conditions, but in such a way that when the soil dries out even with the air humidity unchanged, it is unable to draw as much water from the soil as a plant growing and dying in less humid atmosphere. The difference in nonavailabe water, 1.8 per cent., is quite marked.

The effect of suddenly transferring plants raised in a moist atmosphere to a dry one was shown by taking two collections of potted plants raised in the plant house and transferring one of them to the xerophytic conditions of a dry laboratory, leaving the other in nearly similar conditions of light and heat in the more moist plant house. The following data show the effect of true xerophytic conditions on plants raised in a more humid atmosphere.

Comparative Physical Record of Plant House and Laboratory.

Average air temperature, planthouse ..................71 ${ }^{\circ}$

Average air temperature, laboratory .................. $72^{\circ}$

Average relative humidity, planthouse.................62\%

Average relative humidity, laboratory . . . . . . . . . . . . . $38 \%$

Average soil temperature, planthouse..................68

Average soil temperature, laboratory.................6.67\%

Average maximum light, planthouse................... 10.5\%

Average maximum light, laboratory.................... $20.5 \%$ 


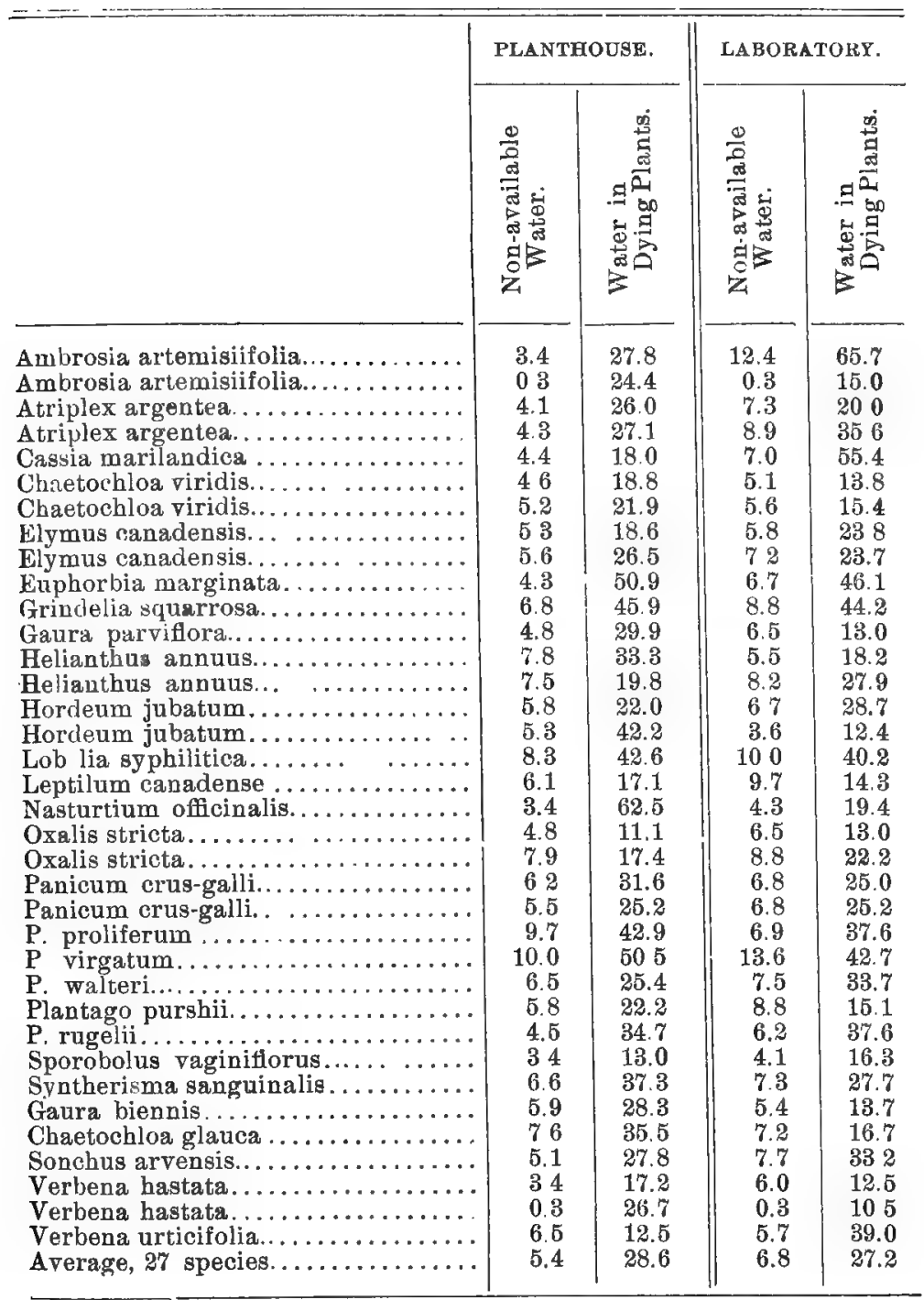


When a plant is suddenly transferred from moist conditions to xerophytic, the rapid change prevents the plant from adapting itself at all to its conditions, and death takes place quickly. This was especially true of the two plots for which data are just given. The plants in the laboratory were all dead by the time the first in the plant house began to die, the average time of drying up was twice as long for the plant house. The difference in the averages is quite pronounced. The plants transferred to the xerophytic laboratory died at an increase of 1.4 per cent in the limit. This indicates that periods of drought following quickly after moist weather and good growing conditions may injure plants more quickly in the fields than a more gradual change from wet to dry weather.

4. The Effect of Increased or Decreased Soil Moisture during the Growing Period.

If a xerophyte is placed under the conditions of a mesophyte, or if the latter is placed under semi-hydrophytic conditions, in either case the plant must adapt itself to the new conditions or become unhealthy and die. Such a plant can rarely fully adapt itself in one generation but manages to exist and reproduce itself though in a condition of lowered vitality. The same thing is true where the order is reversed and the hydrophyte and mesophyte are put under the effect of a lower per cent of soil moisture than that to which they are accustomed.

The following data were taken from plants growing in the plant house in the same physical conditions, with the exception of a difference in the water content of the soil. The results indicate the effect of increasing the water in the soil beyond the amount to which the plant has been accustomed. Most of these plants are mesophytes but several have xerophytic tendencies. The latter show the greatest differences. 


\begin{tabular}{|c|c|c|c|c|c|c|}
\hline \multirow[t]{2}{*}{ n } & \multicolumn{3}{|c|}{$\begin{array}{l}\text { NORMAL SOIL } \\
\text { WATER. }\end{array}$} & \multicolumn{3}{|c|}{$\begin{array}{c}\text { EXCESSIVE SOIL } \\
\text { WATER. }\end{array}$} \\
\hline & 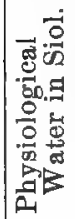 & 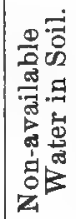 & 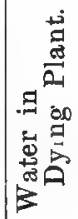 & 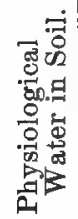 & 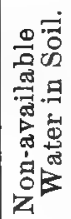 & 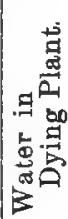 \\
\hline Acnida tamariscina. ...... & 14.0 & 8.6 & 44.4 & 20.0 & 132 & 48.6 \\
\hline Ambrosia artemisiifolia..... & 102 & 5.2 & 174 & 15.0 & 33.7 & 32.6 \\
\hline Aster cordifolius. ......... & 5.0 & 6.9 & 328 & 15.0 & 7.2 & 35.6 \\
\hline Gaura parviflora ..... & 5.1 & 7.4 & 37.5 & 150 & 9.8 & 30.6 \\
\hline 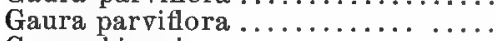 & 6.6 & 5.2 & 19.2 & 15.0 & 9.1 & 46.8 \\
\hline Gaura biennis............... & 84 & 7.8 & 52.5 & 15.0 & 90 & 34.6 \\
\hline Iva frutescens $\ldots \ldots \ldots \ldots \ldots \ldots \ldots$ & 63 & 67 & 39.2 & 14.0 & 83 & 34.3 \\
\hline Lactuca scariola............... & 8.6 & 9.6 & 44.2 & 16.1 & 11.1 & 53.4 \\
\hline 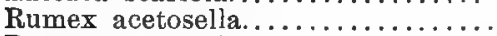 & 8.2 & 8.4 & 394 & 180 & 11.2 & 37.9 \\
\hline 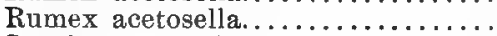 & 100 & 64 & $36 . \tilde{2}$ & 15.5 & 12.2 & 32.6 \\
\hline 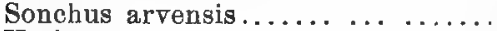 & 5.6 & 5.4 & 27.8 & 15.0 & 8.0 & 43.1 \\
\hline Verbena stricta................ & 105 & 57 & 234 & 17.3 & 6.4 & 265 \\
\hline Average...................... & 8.2 & 6.9 & 34.6 & 15.9 & 95 & 37.8 \\
\hline
\end{tabular}

The plants grown in the excessively moist soil did not develop a root system so vigorous or so extensive as those grown in the dryer soil. The leaves were a lighter green, and the growth of the plant was less rapid. The general effect of excessive soil water was similar to that of a humid atmosphere on a plant which is growing in a soil containing abundant but not excessive soil moisture.

In comparison with the previous results, in the following table are given some data obtained by growing plants of hydrophytic tendencies under the same conditions of light and air in which the former were grown. 


\begin{tabular}{|c|c|c|c|c|c|c|}
\hline & \multicolumn{3}{|c|}{ DRY TO MOIST SOIL } & \multicolumn{3}{|c|}{ VERY WET SOIL } \\
\hline & 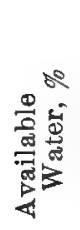 & 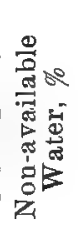 & 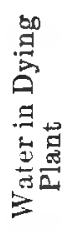 & 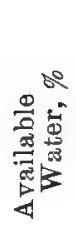 & 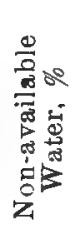 & 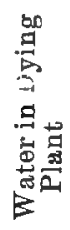 \\
\hline Lobelia syphilitica............ & 5.7 & 7.7 & 68.1 & 12.0 & 11.4 & 57.9 \\
\hline Lobelia syphilitica........... & 20 & 10.3 & 52.0 & 120 & 11.5 & 44.0 \\
\hline Sium cicutifolium......... . & 6.0 & 8.2 & 360 & 160 & 8.5 & 55.5 \\
\hline Sium cicutifolium............ & 7.0 & 8.8 & 514 & 150 & 9.0 & 45.8 \\
\hline Sagittaria latifolia........... & 80 & 13.2 & 52.3 & 14.0 & 21.4 & 45.2 \\
\hline Sagittaria latifolia.......... & 6.0 & 11.0 & 46.0 & 21.0 & 144 & 35.0 \\
\hline 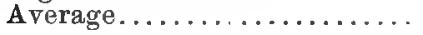 & 6.1 & 9.7 & 50.9 & 15.0 & 12.7 & 47.2 \\
\hline
\end{tabular}

Of the plants in the foregoing table, those in the wet soil were by far the more vigorous, since this is their natural substratum. Those in the dry soil had adapted themselves partially to the conditions by reducing the size of their leaves, and increasing the relative amount of their root systems. Sagittaria, being nearer a true hydrophyte, varied the most. All plants showed more or less adaptation to the dryer conditions. The striking difference of 30 per cent in the limit of available water shows to what extent this adaptation had taken place.

5. The Effect of Decreased Light on Non-available Water in Soils.

Plants were grown in the shade in wet soil in the plot designated as P-I in the planthouse, and in dry soil in plot $P$-II in the same locality. The average maximum light of these plots was only 1.5 per cent of the standard at noon June 22. Another shade plot was that of Case II in the lab. oratory. This had a maximum average of only 0.5 per cent but the average for the whole period of daylight was only about one tenth of the light in the shade plots in the planthouse. 
In the plant house the effect of shade was to increase the size of the leaves of the plant. This was especially the case with rosettes of Ranunculus sceleratus, Gaura biennis and $G$. parviflora.

The leaves of the shade rosettes not only had larger blades and longer petioles but they were elevated to a greater angle with the surface soil. It is hard to account for this fact. It may be due to the stimulus of decreased light causing the leaves to stretch towards the source of light. It undoubtedly increases evaporation from the leaves, thus hastening the transfer of soil nutrients through the plant. A study of the comparative anatomy of sun and shade leaves belonging to the same species, would no doubt show essential differences in the arrangement of the mesoplyll, in the thickness of the epidermal covering and in the comparative number of stomata per unit of leaf surface. The leaves of Ranunculus sceleratus were modified to a greater degree than those of the other plants.

The following table indicates the effect of shade in raising the limit of available water in the soil. The shade plants show an increase of 0.8 per cent of non-available water in the dry soil and of 1.3 in the wet. The plants are put in two groups; those grown in dry loam, plot P-II, and those in wet, plot P-I. The amount of available water in the former varied from 0 to 10 per cent, in the latter from 10 to 30 per cent. 
Plants in Dry Soil Plot P-II.

\begin{tabular}{|c|c|c|c|c|}
\hline \multirow[b]{2}{*}{ PLANT } & \multicolumn{2}{|c|}{ IN SUN } & \multicolumn{2}{|c|}{ IN SHADE } \\
\hline & 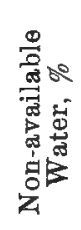 & 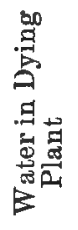 & 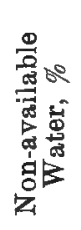 & 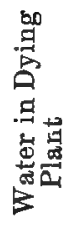 \\
\hline Aster cordifolius................ & 7.2 & 35.6 & 8.4 & 36.0 \\
\hline Gaura parviflora............... & 7.4 & 29.4 & 7.4 & 37.5 \\
\hline Gaura parviflora................ & 6.4 & 35.5 & 52 & 19.2 \\
\hline 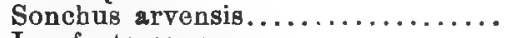 & 8.0 & 43.1 & 9.9 & 45.3 \\
\hline 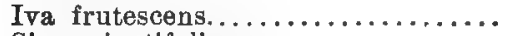 & 6.7 & 39.2 & 7.4 & 23.5 \\
\hline Sium cicutifolium ............. & 8.8 & 51.4 & 8.5 & 55.5 \\
\hline Lobelia syphilitica.............. & 7.7 & 52.0 & 10.4 & 68.1 \\
\hline 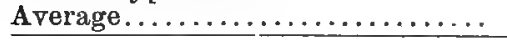 & 7.4 & 40.9 & 8.2 & 40.7 \\
\hline \multicolumn{5}{|c|}{ Plants in Wet Soil Plot P-I. } \\
\hline Aster cordifolius. . . . . . . . . . . . & 7.2 & 35.6 & 84 & 45.8 \\
\hline Gaura biennis $\ldots \ldots \ldots \ldots \ldots \ldots$ & 9.4 & 34.6 & 11.0 & 44.2 \\
\hline Ambrosia artemisiifolia.......... & 5.7 & 19.2 & 8.7 & 32.5 \\
\hline 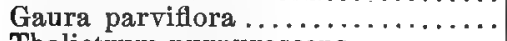 & 9.3 & 39.1 & 9.9 & 300 \\
\hline Thalictrum purpurascens ... . . . . & 8.1 & 24.1 & 9.6 & 32.7 \\
\hline 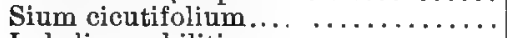 & 82 & 36.0 & 9.0 & 45.8 \\
\hline Lobelia syphilitica................ & 11.5 & 44.1 & 11.9 & 57.1 \\
\hline 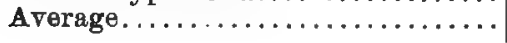 & 6.4 & 30.4 & 9.7 & 41.1 \\
\hline
\end{tabular}

Comparison of Plants Grown under Normal Conditions with those Grown under shade Conditions.

(Plant Cases.)

Case III (Normal).

Physical Record.

Average air temperature.............................. $76^{\circ}$

Average relative humidity .............................55\%

Average soil temperature............................... $70^{\circ}$

A verage soil moisture................................. $14.7 \%$

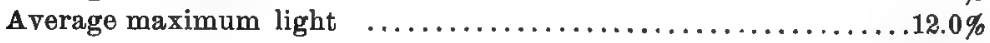

RESUISS

Average 9 plants.

NON-AVATLALE PLANT WATER WATER $4.8 \% \quad 30.4 \%$ 
Case II (Deficient in light, other conditions normal.)

Physical Record.

Average air temperature .......................... $74^{\circ}$

Average relative humidity ........................

Average soil temperature... . . . . . . . . . . . . . . . $66^{\circ}$

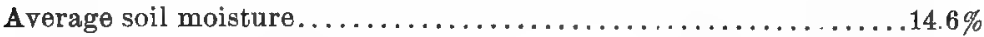

Average maximum 1 ght. . .......................... $0.5 \%$

RESULTS

NON-AVAILABLE PLANT

WATER WATER

Average 9 plants.................. $9.0 \% \quad 41.5 \%$

These give the wide difference of 4.2 per cent in the amount of water non-available for the use of the plant. Such intense shade brought about a pathological condition in Case II.

The plants in plots P-I and P-II were under precisely the same physical conditions excepting that of soil water. They received several times more light than those in the shade in Case II. The latter were in a diseased condition and would never have borne fruit had they been permitted to live. The former adapted themselves in a greater or less degree to their conditions and showed every indication of fruiting had they been allowed to continue their existence.

It cannot be definitely determmed when a plant ceases to be able to adapt itself to its conditions, that is, just when a plant passes from a normal to a pathological condition. If a plant is changed from a sunny situation into one of constant shade there can be little doubt that it suffers from the effects for a time, and during this period must be in a pathological condition. If the shade is not too intense it may so adapt itself to its conditions as to be able to perpetuate its species even when its condition is not one of health.

When one plant is unable to witharaw as much water from the soil during drought as another of the same spceies situated under different air or light conditions, it cannot be safely maintained that this is because the one in the less favorable condition has adapted itself to its new habitat by a change of structure. Although it has a modified structure, 
its protoplasm may not be able to function properly, and assimilation may be so hindered by a lack of proper protoplasmic stimuli that the plant is unable to get and use as much food as it could in a more favorable habitat. It is no doubt true, that after several generations a plant may fully adapt itself to such a change, and then its higher limit of nonavailable water mav be due to certain changes in the function and structures of the organs of the plant.

6. Effects of Soil Texture and Composition on the Limit of Available Water.

Before entering into a discussion of this subject, a summary will be given of all the results of experimentation which bear directly upon this problem. Since the plants compared grew under similar physical conditions in each instance where the different soils are compared, it is not considered necessary to give with any table the data for the physical factors.

The following averages are from five species, viz: Helianthus annuus, Hordeum vulgare, Phaseolus vulgaris, Ricinus communis, and Zea mays, all grown in the plant house.

Comparative Plot III.

Average of Thirty Pots.

\begin{tabular}{|c|c|c|}
\hline SOIL & $\begin{array}{c}\text { NON-AVAILABLE } \\
\text { WATER, } \%\end{array}$ & $\begin{array}{l}\text { WATER IN } \\
\text { PLANT, } \%\end{array}$ \\
\hline 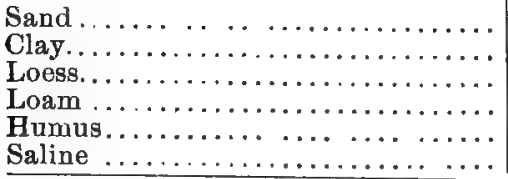 & $\begin{array}{r}0.31 \\
8.6 \\
9.2 \\
9.5 \\
10.7 \\
14.0\end{array}$ & $\begin{array}{l}43.8 \\
51.3 \\
52.7 \\
548 \\
48.4 \\
57.4\end{array}$ \\
\hline
\end{tabular}




\section{Comparative Plot $1 I$.}

Three species, Phaseolus vulgaris, Hordeum vulgare, and Zea mays, grown in the plant cases under four different combinations of physical factors.

\begin{tabular}{|c|c|c|}
\hline SOIL & $\begin{array}{c}\text { NON-AVAILABLE } \\
\text { WATER }\end{array}$ & $\begin{array}{l}\text { WATER IN } \\
\text { PLANT }\end{array}$ \\
\hline 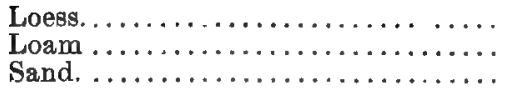 & $\begin{array}{l}8.4 \\
8.4 \\
0.49\end{array}$ & $\begin{array}{l}41.7 \\
45.1 \\
59.2\end{array}$ \\
\hline
\end{tabular}

\section{Comparative Plot I}

(Thirteen species grown in the planthouse.)

\begin{tabular}{|c|c|c|}
\hline SOIL & $\begin{array}{c}\text { NON-AVAILABLE } \\
\text { WATER }\end{array}$ & $\begin{array}{l}\text { WATER IN } \\
\text { PLANT }\end{array}$ \\
\hline 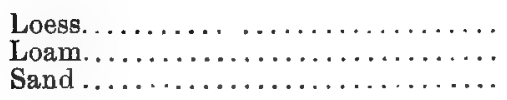 & $\begin{array}{l}5.9 \\
5.5 ? \\
0.65\end{array}$ & $\begin{array}{l}30.1 \\
31.6 \\
32.1\end{array}$ \\
\hline
\end{tabular}

\section{Plot P.I.}

Below are given the results obtained from thirteen species grown in the plant house under similiar conditions except soil.

\begin{tabular}{|c|c|c|}
\hline SOIL & $\begin{array}{c}\text { NON-AVAILABLE } \\
\text { WATER }\end{array}$ & $\begin{array}{l}\text { WATER IN } \\
\text { PLANT }\end{array}$ \\
\hline $\begin{array}{l}\text { Clay } \ldots \ldots \ldots \ldots \ldots \ldots \ldots \ldots \ldots \ldots \ldots \ldots \ldots \ldots \ldots \ldots \\
\operatorname{Loam} \ldots \ldots \ldots \ldots \ldots \ldots \ldots \ldots \ldots \ldots \ldots\end{array}$ & $\begin{array}{l}7.3 \\
8.5 \\
0.8\end{array}$ & $\begin{array}{l}35.2 \\
41.1 \\
29.6\end{array}$ \\
\hline
\end{tabular}

General Average for All Plots.

\begin{tabular}{|c|c|c|}
\hline SOIL & $\begin{array}{c}\text { NON-AVAILABLE } \\
\text { WATER }\end{array}$ & $\begin{array}{l}\text { WATER IN } \\
\text { PLANT }\end{array}$ \\
\hline 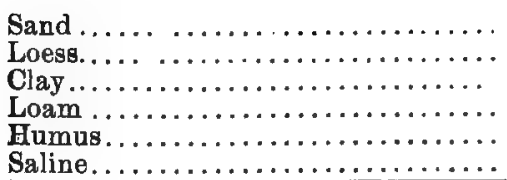 & $\begin{array}{r}0.5 \\
7.8 \\
7.9 \\
80 \\
10.7 \\
14.0\end{array}$ & $\begin{array}{l}42.1 \\
41.6 \\
43.4 \\
43.1 \\
48.4 \\
57.4\end{array}$ \\
\hline
\end{tabular}


From the foregoing tables it is easy to determine the relative positions of loose soils used in a scale of limits. If placed in order of the highest to the lowest, the order is saline, humus, loam, clay, loess, sand.

Sand lowers the limit of available water. All the results from sand averaged 0.3 per cent, it being air dry when the plant was near the death point. The sand used did not contain sufficient silt to hold much hygroscopic water, for it contained less than one per cent of silt. The presence of sand in the soil has a direct effect in lowering the limit as was shown by plants in a few pots which had sand mixed with either loam or clay. It must not be supposed, however, that because a plant assisted by evaporation and gravity might take water out of sand to less than one per cent when it leaves eight per cent in loam under the same conditions, that sand is a better soil in which to grow plants in a dry climate. Sand being so porous evaporates water very readily, the rate of evaporation decreasing with a decrease in the size of the grains. For this reason it evaporates water more rapidly than other soils. It also allows water to pass rapidly through it by gravitation to the stratum beneath, since the forces of capillarity and surface tension are not sufficient to retain the water as in other finer soils. The soil which absorbs water most quickly loses it by evaporation and gravitation most rapidly. It also loses the nutrient soil solutions rapidly by leaching.

The finer soils like the clays hold water the most tenaciously. This quality tends to raise the limit of water by increasing the hygroscopic water. In such soils the forces of capillarity and surface tension as they are designated by soil physicists may be arrayed against the forces of solution operating in the root hairs of the plants, and tend to negate the latter. If it be maintained that the great difference between the limit of the fine clayey soils and the coarse sandy soils is due solely to the relative increase of these two forces in the former, the limit of non-available water in saline, humus, loam and loess should have been lower than that of clay soil and higher than that of sand, for these soils contained 
less silt and clay. Hence these were more porous than clay and the forces of capillarity and surface tension in them must have been less powerful. Since the limit of these soils is higher than that of clay there is evidently another disturbing factor, especially in the case of the saline and humus soils. This must be attributed largely to the increased amount of electrolytes or soluble salts in the soil. The following table of ratios includes a comparison of the soils taking the per cent in loess as a basis and calling this 1.00 in each column. It may be seen that the per cent of soluble salts varies approximately with the increased amount of non-available water. The variation of the other soil qualities shows no uniformity, when compared with each other or with the nonavailable water.

Table of Comparative Ratios.

\begin{tabular}{|c|c|c|c|c|c|c|}
\hline SOIL & 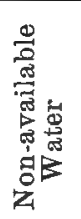 & $\begin{array}{l}\frac{\infty}{\pi} \\
\frac{ \pm}{2} \\
\frac{9}{0} \\
\frac{0}{0} \\
0 \\
\infty\end{array}$ & 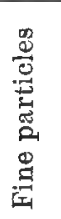 & 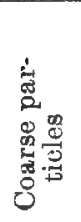 & 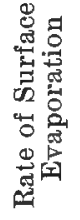 & 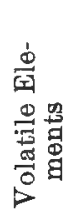 \\
\hline 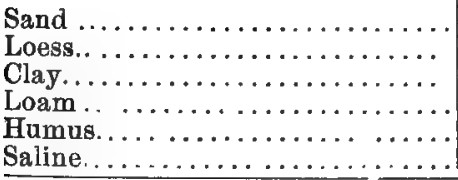 & $\begin{array}{r}4 \\
100 \\
101 \\
104 \\
137 \\
179\end{array}$ & $\begin{array}{l}100 \\
133 \\
125 \\
183 \\
417\end{array}$ & $\begin{array}{r}4 \\
100 \\
107 \\
100 \\
57 \\
104 \\
\end{array}$ & $\begin{array}{r}524 \\
100 \\
33 \\
75 \\
221 \\
88\end{array}$ & $\begin{array}{r}132 \\
100 \\
106 \\
104 \\
90 \\
114\end{array}$ & $\begin{array}{r}12 \\
100 \\
163 \\
293 \\
415 \\
147\end{array}$ \\
\hline
\end{tabular}

After much effort to combine these ratios to get a common ratio which would correspond to that of the non-available soil water, it was found that the ratios of soil solution were the only ones that compared directly with those of the nonavailable water. It was found that if the per cent of solution be taken as a basis, and the square root extracted and multiplied by 20 , a set of ratios or numbers were obtained 
which coincided approximately with the per cent of the nonavailable water in each soil. The following are the ratios:

\begin{tabular}{|c|c|c|c|c|c|c|}
\hline SOIL & 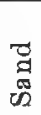 & 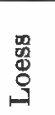 & $\frac{\sigma}{0}$ & 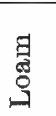 & $\begin{array}{l}\stackrel{\infty}{E} \\
\underset{⿴ 囗 十}{g} \\
\text { 吕 }\end{array}$ & 导 \\
\hline$\%$ of Non-available Water. & 0 & 7.8 & 7.9 & 8.0 & 10.7 & 12.0 \\
\hline $20 \sqrt{\%}$ of Soluble Salts..... & & 70 & 8.0 & 7.8 & 9.4 & 14.1 \\
\hline
\end{tabular}

The amount of soluble salts in the sand was so low that the chemist did not determine it. It was very low, posssibly not much greater than that of the tap water used in watering the plants growing in it. This being the case the same rule would hold as for the other soils, viz., the per cent of non-available water in the soils increased as the square root of the per cent of the soluble salts. Since the per cent of soluble salts in the soil increases directly as the per eent of water in the soil decreases, the increasing density of the soil solution would tend to reduce the amount of available food for the plant, and since the supply of watery foods for it varies with difference in density existing between the solutions in the protoplasm of the root-hairs and that of those in the soil the protoplasm would take less water from the soil solution just at the time the plant needed a greater water supply. The fact that evaporation from the plant is aiways excessive in times of drought would hasten the result. On the other hand the rapid evaporation from the leaves, when a plant is losing more water than it is getting would tend to concentrate the solutions in the plant and increase their os. motic efficiency in taking in water from the soil solutions. These questions are of great interest and importance and should be investigated thoroughly with reference to the plant in its relation to the soil.

The observations made in the field during August 1900, brought out a fact of considerable importance, viz., that herbaceous plants growing in hard soils could not root as deeply as those growing in loose soils. Plants are enabled to grow rapidly only when they can develop large and vigorous root 
systems which may supply them with the nutriment necessary for rapid growth. In hard soil a plant is hindered in the development of its roots even from the beginning. As a result it does not grow so large as a plant in looser soil because it does not have the means to absorb water nor can it draw from such a large and varied soil area. As a result, in periods of drought, it is first to die.

The limit of physiological water for various plants in the field was determined for both hard and loose soils. The average results give a slightly higher limit for plants in hard soils. The results for the herbaceous plants when averaged give 5.9 per cent as a limit in hard soils, and 5.2 for loose soils. Ten species of grasses have an average limit of 6.8 per cent in hard, 6.4 per cent in loose soil. These results may be sligbtly inaccurate because the soils compared were from different localities and probably differed slightly in composition and porosity. The plants were taken from similar conditions of heat, light, and physiography.

The soils used in the comparative plots were of a loose texture, care being taken in filling the pots not to pack the soil, which was allowed to settle and becume denser by gravity. The time of experimentation was short, being less than two months, and none of the soil became hard. This enabled each of the plants to root throughout the pot, and added much to the accuracy of the results.

\section{The Recovery of Plants from Drought Effects.}

When a plant has suffered from loss of water by wilting badly, it may be revived by watering it. One box of potted plants in the plant house, grown under nearly normal conditions, was allowed to dry until the plants were wilted badly, in fact were dying, for excessive wilting is but the condition preliminary to death. Water was then sprinkled upon the soil in sufficient quantity to make it moist enough either to fully or to partially revive the plants. Again the plants were allowed to dry out, this time to the death point. Deter minations of non-available water in these pots showed that 
the plants had never fully recovered from the first drought, for they were unable to withdraw as much water from the soil as similar plants which had been allowed to die without reviving them. The increase of the limit ranged from 2 to 7 per cent, varying possibly with the amount of injury done to the finer roots and root-hairs of the plants. The results indicate that in such instances the root-hairs are probably destroyeu

The following tables of data show the effects of wilting on some of the plants in Comparative Plot III. Under the heading "Wilting Plant" is given tirst the amount of water in the plant at the time it wilted badly from drought, second the per cent of water in the soil at the same time, and under the heading "Dying Plant," first the amount of water in the dying plant, second that in the soil at the same time, taken when the plant was dying after having been partially revived from the first excessive wilting. In the last instance the plants were revived by adding water to the soil. care being taken not to wet the leaves of the plant. 


\section{Average Physical Conditions.}

Air temperature ................................... $98^{\circ}$

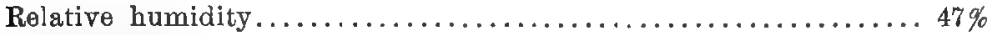

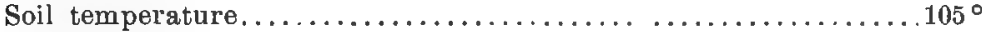

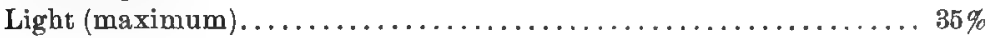

Phaseolus vulgaris.

\begin{tabular}{|c|c|c|c|c|}
\hline \multirow[b]{2}{*}{ sorl } & \multicolumn{2}{|c|}{ WILTING PLANT } & \multicolumn{2}{|c|}{ DYTNG PLANT } \\
\hline & 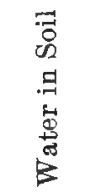 & $\underset{\vec{G}}{\stackrel{\vec{G}}{\vec{G}}}$ & 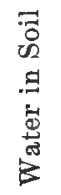 & 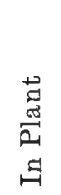 \\
\hline 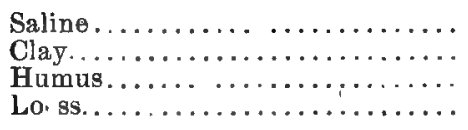 & $\begin{array}{r}17.2 \\
8.5 \\
93 \\
84\end{array}$ & $\begin{array}{ll}80 & 0 \\
78 & 9 \\
810 & 0 \\
85.6\end{array}$ & $\begin{array}{r}19.8 \\
85 \\
11.0 \\
8.7\end{array}$ & $\begin{array}{l}537 \\
51.8 \\
32.1 \\
52.8\end{array}$ \\
\hline
\end{tabular}

Helianthus annuus.

\begin{tabular}{|c|c|c|c|c|}
\hline Loam . . . . . . . & 11.6 & 87.6 & 11.2 & 61.4 \\
\hline Saline $\ldots \ldots \ldots \ldots \ldots \ldots \ldots \ldots$ & 15.1 & 85.4 & 127 & 59.0 \\
\hline Clay. . . . . . . $\ldots \ldots \ldots \ldots$ & 91 & 84.9 & 9.3 & 608 \\
\hline Lowss. . . . . . . . . . . . $\ldots \ldots \ldots$ & 109 & 815 & 111.2 & 55.2 \\
\hline Humus . . . . . . . . . . . & 14.5 & 79.2 & 11.8 & 596 \\
\hline
\end{tabular}

Ricinus communis.

\begin{tabular}{|c|c|c|c|c|}
\hline Saline.......... & 12.0 & 76.5 & 12.4 & 600 \\
\hline 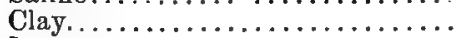 & 9.8 & 806 & 108 & 55.9 \\
\hline 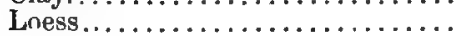 & 78 & 74.3 & 10.9 & 49.6 \\
\hline 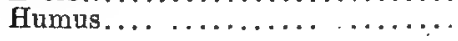 & 12.1 & 797 & 123 & 61.9 \\
\hline
\end{tabular}

These plants were subjected to more severe drought than those first mentioned in this chapter. In each case the limit of physiological water was noticeably increased and the plants died in a per cent of soil water equal to or greater than the soil contained at the first period of excessive wilting. The limit at which they died was higher than the limit at which they die when not revived at any tiwe during the period of drought. 
The fact that after being revived the plants could not reduce the physiological water to as low a limit as before indicates that the absorptive system of the plant had suffered serious injury before they were revived. It must be that the root-nairs and even the finer roots had been killed. If this be the case in nature, a temporary shower could not furnish the plant with sufficient water to grow new roothairs, and the plant would continue to die in spite of the slight addition to the soil moisture.

"From the results it might bo inferred also that in times of drought a shower might be of absolute harm. In some localities in the West many farmers believe that in times of drought a small shower not sufficient to "wet down the soil" is an injury to the crop. But this can hardly be true since the shower checks the rapid loss of water from the plant by evaporation and enables it to live longer and may be the cause of prolonging its life until heavier rains fall.

8. The Limit of Physiological Water; Variations in Different Genera and Species.

The notes from experimentation indicate in a general way variations in the limit of physiological water corresponding to the natural habitat of the plant. The limit for mesophytes for loamy soils falls generally between 5 to 11 per cent.

Mesophytes with xerophytic tendencies may drop below this general average in their limit while, those bordering on hydrophytes in their habitat may have a limit even higher than this. By arranging the limits in a descending series commencing with amphibious plants, or those that root in mud, and passing through the mesophytes to xerophytes, the amount of non-available soil water in loam decreases from 20 per cent for some amphibious plants to an air dry'soil as a limit for intense xerophytes.

Fleshy plants have lower limits than woody ones. Nasturtium and Coleus, although not true succulents, retain water so tenaciously in their stems that the soil becomes almost air dry before they die. 
The following table gives the limit for several typical plants ranging from amphibious plants to mesophytes of xerophytic tendencies.

Table of General Variation.

\begin{tabular}{|c|c|c|}
\hline PLANT & $\begin{array}{c}\text { PHYSIOLOGICAL } \\
\text { WATER, } \\
\%\end{array}$ & $\begin{array}{c}\text { WATER IN } \\
\text { DYING PLANT, } \\
\%\end{array}$ \\
\hline Potamogeton lonchites.............. & 248 & 32.8 \\
\hline Echinodorus cordifolius............. & 19.6 & 48.5 \\
\hline 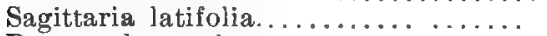 & 15.6 & 44.6 \\
\hline 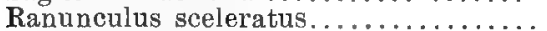 & $10 . \overline{5}$ & 57.1 \\
\hline Lobelia syphilitica. . . . . . . . . . . . . & 10.3 & 30.1 \\
\hline Panicum walteri. . . . . . . . . . . . & 8.7 & 27.2 \\
\hline 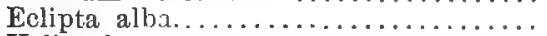 & 7.5 & 45.1 \\
\hline Helianthus annuus. . . . . . . . . . . . . . & 6.5 & 36.5 \\
\hline Ambrosia artemisiifolia.. . . . . . . . . & 5.4 & 24.3 \\
\hline Atriplex argentea. . . . . . . . . & 42 & 26.6 \\
\hline Nasturtium oficinale.............. & 3.7 & 33.4 \\
\hline 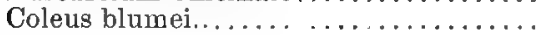 & 3.0 & 70.0 \\
\hline
\end{tabular}

Thus we see that not on'y do plants of different genera vary in their limit but also species of the same genus.

Some instances of specific variation are given in the following table.

Table of Specific Variation.

\begin{tabular}{|c|c|c|}
\hline PLANT & $\begin{array}{l}\text { PHYSIOLOGICAL } \\
\text { WATER, LIMIT }\end{array}$ & $\begin{array}{l}\text { WATER IN } \\
\text { DYNG PLANT }\end{array}$ \\
\hline 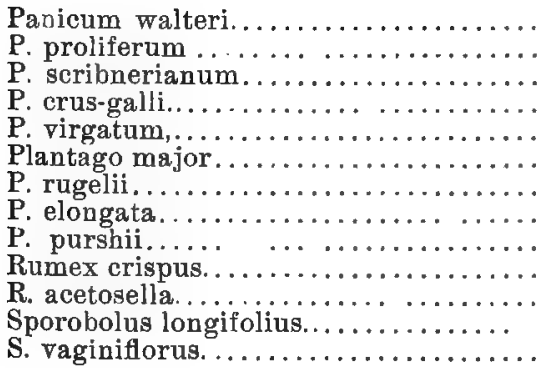 & $\begin{array}{l}8.7 \\
7.8 \\
7.3 \\
7.2 \\
6.8 \\
7.7 \\
5.9 \\
5.8 \\
5.5 \\
8.3 \\
6.6 \\
5.8 \\
4.4\end{array}$ & $\begin{array}{l}27.2 \\
39.4 \\
27.8 \\
35.6 \\
23.0 \\
25.8 \\
27.0 \\
22.2 \\
41.0 \\
354 \\
34.8 \\
20.9 \\
18.9\end{array}$ \\
\hline
\end{tabular}


The few results obtained from hylophytes or plants of the woods, and poophytes or plants of the meadow and prairie, indicate that the former have a higher limit than the latter. The plant house, it should be remembered, approached nearer to hylophytic conditions, and this favored shade-loving plants. Sun-loving plants on the other hand by being placed under the semi-shade conditions of the plant house tended towards a higher limit than if under more natural conditions. The following table gives the results obtained with violets.

\begin{tabular}{|c|c|c|}
\hline PLANT & $\begin{array}{l}\text { NON-AVAIL- } \\
\text { ABLE WATER }\end{array}$ & $\begin{array}{c}\text { WATER IN } \\
\text { DYING PLANT }\end{array}$ \\
\hline 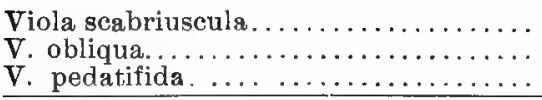 & $\begin{array}{l}9.0 \\
8.9 \\
7.5\end{array}$ & $\begin{array}{l}26.7 \\
397 \\
22.9\end{array}$ \\
\hline
\end{tabular}

The first species grows in protected places, the second in both sunny and shady localities, and the last in the open prairies. These data as well as others indicate that the limit of hylophytes will average higher than that of poophytes. Since shade has the effect of raising the limit of available water it is to be expected that in the structural differences between poophytus and hylophytes the latter have shade adaptations which are of such a nature as will lessen the ability of the plant to withstand drought.

\section{DROUGHT EFFECTS. THE MANNER IN WHICH MANY PLANTS, PRINCIPALLY MESOPHYTES, DIE.}

The plants studied were principally herbs of both monocyclic and dicyclic types. The behavior of the two types in times of drought is slightly different. Certain drought effects are common to all plants. In general, if the epidermal covering of the plant is uninjured at the beginning, death takes place more slowly and the order in which the different organs suffer loss of water is quite constant. If any portion of the plant has suffered mechanical or structural injury, this 
order may be changed since the injured member loses water more rapidly than any other part of the plant and, as a result, is almost certain to die first. If a leaf has been injured by having the epidermis broken by the force of the wind or by the impact of hail, if it has been punctured by sucking insects or lacerated by biting insects, it commences to die from the point of injury, as soon as the plant fails to obtain sufficient water to keep its tissues turgid. The stem may be similarly affected by such injuries. The root is often injured by insects, and even more frequently by the filaments of parasitic fungi. Plants suffering from root injuries are almost certain to die from the root upwards.

When the plant is healthy and vigorous, and has not received any mechanical injuries, the growing point in stems and rosettes is the last part vo die, because the meristem cells hold their vitality more tenaciously than other cells and are able to live to the last. In succulent plants they even seem to be able to draw upon the neighboring cells for water. This may be the case in all plants. When the period of drought is prolonged but not intense, the roots of fibrousrooted plants commonly die before the stem, but after all or most of the leaves have perished. This is the way many monocyclic herbs die; Atriplex argentea, Phasealus vulgaris, Euphorbia marginata, Ambrosia artemisiifolia are good ex. amples; Phaseolus, Coleus, Gaura, Amórosia and a number of other plants partially adapt themselves to slow drovght by dropping their leaves, the oldest first. In this way they check rapid evaporation and soon reduce it to a minimum.

Dicyclic herbs often have more or less fleshy roots which hold water tenaciously in times of drought; especially is this the case when the plant is in the rosette stage. The leaves during this period of the plant's existence die much sooner than the roots.

When a herbaceous plant sends up a stem preparatory to flowering, the growing point of the stem holds water in its culls tenaciously, and where the plant has not suffered from physical injury, the leaves die first, the root next, then gradually the stem dries out and last of all the young 
cells of the growing point perish. But when the plant has reached the flowering stage, the last part of the plant to die is the immature fruit, the order of dying for the other organs being the same as before.

Plants which propagate by means of bulbs and tubers often suffer drought with only a temporary injury. Sagittaria latifolia continues its existence principally by means of tubers. One of the first things a saggitaria plant acomplishes after two or three leaves appear is to send out an underground shoot or stolon which forms at its terminus a bulb. These bulbs are usually mature long before the plant produces flowers and fruit, and when a young plant suffers from a period of drought all the reserve energy of the leaves is apparently transferred to the tuber which matures as the other parts of the plant die. Bulbous plants behave in a similar way: a young bulb is formed which matures as the other portions of the plant dry up from loss of water. Bulbs and tubers are very resistent to the effects of dry soil and may retain their vitality for months under conditions of most severe drought.

Woody plants like trees and shrubs usually have roots which penetrate the earth to a great depth. When such plants die from drought, the root is the last portion of the plant to die. The same appears to be true in case of most pleiocyclic herbs, having either fibrous roots or a woody tap root.

In case of death from sudden drought, such as occurred with the plants placed in the xerophytic atmosphere of the laboratory, in some monocyclic herbs the root was the last organ to die. The excessive evaporation from the leaves hastened the death of the stem and growing point. Drought rarely takes place so suddenly in nature.

The root seems by far the most adaptable part of the plant, and the leaves stand next in order. Plants growing in dryer soil usually have proportionately larger root systems, providing the degree of dryness does not become so excessive as to prevent the development of the plant. In the case of mesophytes in nearly every instance noted, excessive water in 
the soil retarded the formation of the root system. This of course is to be expected for there must be a most intimate connection between the root and the medium in which it grows. The very existence of the plant is dependent upon its food supply as received from the air and soil and circulated through the tissues by means of water received through the roots. Under varying conditions of soil moistures such as those in which the mesophyte finds itself it can not be too conservative in the modification of its root system, and the meager observations made indicate that our ruderal mesophytes have a root system which is varied directly with the conditions of soil moisture and food supply. This is a most interesting and fascinating subject for future investigation.

\section{CONCLUSION AND SUMMARY.}

\section{A. WATER IN PLANTS AND SOILS.}

\section{Water in Soils.}

The amount of physical water in soils varies directly with their water-retaining capacity and the chief known factors affecting the water capacity are gravity, capillarity, and surface tension acting within the soil, and evaporation acting upon the surface of the soil.

The amount of physiological water in soils varies directly with the amount of physical water held in the soil and inversely with the amount of hygroscopic water. The adaptability and vigor of the plant varies its power to draw water frum the soil. The kind, quality, and texture of the soil and the density of the soil solution are important variants in determining the limit of physiological water.

The amount of water in the soil non-available for the use of the plant is dependent directly upon the ability of the plant to withdraw water from the soil. The ability of the plant is measured by its inherent nature as manifested in the form of protective structures, by its adaptability to its changing life conditions, and by the vigor of its protoplasm. Soil composition and soil texture affect directly the ability of the 
plant to withdraw water from the soil. The vigor of the plant is lessened by light, heat, temperature and moisture effects which vary too widely from the optimum necessary for the plant, and such physical factors are indirect causes in increasing or decreasing the amount of non-available water in the soil. The limit of physiological water varies directly with the square root of the per cent of soluble salts in the soil.

\section{Water in Plants.}

The per cent of water in the tissues of the plant is usually the greatest in seedlings, and decreases gradually as the plant grows older. The individual plants containing the bighest per cent of water are not necessarily the healthiest, since the most vigorous growing plants contain a lower per cent than less thrifty ones. Shade plants and plants grown under humid conditions have a relatively high per cent of water, due probably to a poor development of the fibrovascular system. Plants grown in different soils indicate the following series beginning with the soil bearing plants of the highest water content, viz., sand, loam, saline, clay, loess, humus. The most watery plants were more often attacked by fungous plants than those less watery.

Mesophytes apparently lose water when first wilting in almost the same ratio by weight as the soil. This may indicate that the plant gets little or no water from the soil after it begins to wilt excessivuly, and that the root system is already beginning to die at the extremities. Hydrophytes probably lose weight more rapidly than the soil. Xerophytes lose weight very slowly when subjected to drought, and some may retain most of the water in their tissues even after the substratum is air dry. The tenacity with which the water in soils resist the absorbent action of the root hairs of the plant varies in different soils. The soils of experimentation arranged in order of their resistance from the highest to the lowest are: saline, bumus, loam, loess, clay, sand, The chief probable factors of vari- 
ation are capillarity, surface tension, and varying concentration of the soil solution.

The per cent of water in dying plants varies not unly with the species, but also with the individual. Seedlings die with a greater per cent of water in their tissue than older plants, and they usually leave an increased amount of nonavailable.water in the soil. Mature plants when dying contain the minimum per cent of water. Shade plants as well as those grown in humid air die with a higher per cent of water in both soil and plant. Dryness of atmosphere modifies the growing plant so that it may live on a slightly lower per cent of water in the soil. Rosette plants of xerophytic tendencies contain less water in their tissues when dying than rosettes of hydrophytic tendencies, and also may die at a lower per cent of soil moisture. The same observation was made with regard to plants of both groups with immature stems.

\section{B. NON-AVAILABLE WATER IN SOILS.}

Heat (and light) below or above the optimum for the plant affect it unfavorably, decreasing its ability to withdraw water from the soil during drought, increasing the nonavailable water.

Increased relative humidity of the air, when high, increases the size of the leaves and retards the development of the root system. Transferring a plant from a humid or moist atmosphere suddenly into a comparatively dry one hastens the death of the plant, especially when the soil at the same time is deprived of its water supply. In both the former and the latter case the plant dies at a higher limit of non-available water. Excessive soil moisture during the growth of a mesophytic plant tends to retard development of the root system and to raise the limit of non-available water. Where mesophytes of strong hydrophytic tendencies or where hydrophytes are placed in both dry and wet soils the plants seem able to adapt themselves partially to their new soil conditions and die at a lower limit of soil 
moisture than under normal conditions. Such plants reduce the size of their leaves when grown in dry soil.

The influence of shade upon plants accustomed to fuil sun. light is such that they die at a higher limit in times of drought. This fact was shown by the results from both plant house and fields. It cannot be decided as to whether any of the plants in the experiment had fully adapted themselves to shade conditions and whether the consequent change in function and structure was the cause of the increased amount of non-available water in the soil. Since the change of habitat was unfavorable to the plants, there is the possibility that most of them were still suffering from the lack of sufficient light stimuli and the protoplasm as a consequence was unable to function normally. Since shade plants contain a higher per cent of water in their tissues, it is possible that the lessened density of the solutions in the protoplasm of the root hairs lowers the ability of the plant to take water from the soil solution. This tends to raise the limit of non-available water.

The limit of physiological water is varied by several factors involved in the composition and texture of the soil. The great difference in the limit of sand and clay might be attributed to the increasing negative effect of capillarity and surface tension in the latter This would tend to reduce the absorption of water by the root hairs. In case of humus, loess, saline and loam soil there are very evidently other disturbing factors. In these soils a very important factor must be that of the variable density of the soil solution. Diosmotic interchange, aside from other forces, varies directly with the difference in density between the soil solution and the solution in the protoplasm of the root hairs; as a result the plant may obtain less and less water from the soil as it dries out. Finally the solution in the soil may become so concentrated that it actually extracts water from the roots of the plant.

Plants wilting excessively from drought when slightly revived by watering are not able before they die to remove as much water from the soil as plants dying without ever having been revived. This is apparently due to the loss of the 
root hairs in times of excessive wilting. In nature a shower which revives the plant but does not furnish sufficient water to the soil for its gruwth will not enable it to push out new root hairs, and as a result only permits the plant to live longer by retarding excessive evaporation.

The ability of plants to take water from the soil varies in an ascending scale from hydrophytes, through mesophytes, to xerophytes The limit of available water for a plant, in a general way, coincides with the physical conditions of its habitat. This being the case we have variations not only among genera but also among species, and even among individuals, depending upon the physical conditions under which the plant has grown and upon tendencies inherited from its ancestral forms. The relative activity of the protoplasm is a very important factor in deternining the amount of water that a plant may abstract from the soil in times of drought.

\section{DROUGHT EFFECTS.}

Herbaceous plants, both annuals and biennials when affected by slow drought usually die in the following manner. The ulder leaves of the plant perish first in order of their age, the younger leaves living to the last; the finer roots die first, then the coarser roots, tinally the stem and last of all the growing point of the stem, or if the plant is flowering, the immature fruit. If a plant of either type has suffered a structural injury, it is very apt to die from the point of injury, since it loses water more rapidly at this boint.

Trees and shrubs and pleiocyclic herbs lose their aerial organs first, the roots being usually the last part of the plant to die. Bulbous and tuberous plants although immature form bulbs or tubers rapidly when caused to die by drought. Propagation has become the favorite means of continuing the existence of these plants.

The structure of the protoplasm of ; the meristem cells in the growing point of the stems and in the immature fruits of herbaceous plants, as well as in others, must be of such chemical and physical nature as will enable these cells by 
osmotic action or otherwise to take up and retain the watery solutions from older and less vigorous cells. Unless some action of this sort takes place the growing point of the stem would always die first, and not last. 
SPECIES LISTS

AND

SPECIES SHEETS 



\section{SPECIES LISTS-NON-AVAILABLE WATER.}

At the close of this explanatory chapter are two lists of species, the first giving the average results from plants grown under the full light in the plant house, and the second a list of results from plants in the fields about Lincoln. In growing a large number of plants, except in the comparative plots, it was impossible to keep the soil equally moist in all the pots, the amount of water in the soil averaging from 10 to 25 per cent. As a result some of the pots with smaller plants maintained a higher per cent of soil moisture than others with larger plants. The conditions of air and soil temperature were quite uniform. Some of the results obtained from a few plants are placed in the "Species Sheets" immediately following the "Species Lists". Here are given the results of work from both plant house and laboratory. These results vary as might be expected, since the plants are grown in different physical conditions as regards soil, air, temperature and light. One slight source of variation is the injuries received by many plants from the punctures of small insects infecting the plant house; another is that caused by parasitic root fungi. The average results of plant house and laboratory plants is somewhat higher than that for plants in their natural habitat, yet not so great as might be expected, when the greater humidity, the deficient light of the plant house and the effects of the injuries from insects and parasites are taken into consideration. The plants in the field are also subject to injuries from insects and fungi, and in addition are more apt to suffer mechanical injuries from the wind. Where the results seemed to depart widely from the limit of plants of similar habitat but different species, the error was directly traceable to the mechanical or structural injuries received by the plants before or dur- 
ing the period of drought from one of the sources indicated above. Such results were omitted in the general average for each species.

It may be well to question the accuracy of the results obtained in calculating the limit of physiological water, where the water in the dying plant was less than 20 per cent in grasses and less than 25 per cent in other plants, since it is probable that most plants have ceased to obtain any water from the soil by the time there is less than 30 to 50 per cent of water in their tissues. This is a problem for further investigation since the results indicate that there is a variation in the amount of water in the plant when it dies, not only in different species, but also in the different plants even if taken at the same age. Such variation is probably caused by differences in the structure of plants growing under different physical conditions. The whole matter is also inseparably connected with the vigor of the plant and its adaptability to its surroundings. 
LIST OF SPECIES.

(Average results obtained under Plant House conditions, plants growing and dying in loam under similar physical conditions.)

\begin{tabular}{|c|c|c|c|c|}
\hline \multirow[b]{2}{*}{ NAME } & \multirow{2}{*}{ 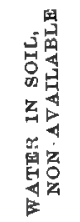 } & \multicolumn{2}{|c|}{ WATER IN PLANTS } & \multirow{2}{*}{ 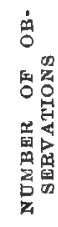 } \\
\hline & & $\begin{array}{l}0 \\
Z \\
a \\
A\end{array}$ & 思 & \\
\hline Abutilon abutilon Gaertn... & 5.6 & 33.3 & & 1 \\
\hline Acnida tamariscina (Nutt.) Wo & 7.4 & 25.1 & 91.0 & 5 \\
\hline Alsine media $L . . . . . . .$. & 7.9 & 29.9 & 850 & 3 \\
\hline Althaea officinalis $[\ldots \ldots \ldots \ldots \ldots \ldots$ & 7.5 & 50.0 & & 1 \\
\hline Amaranthus hybridus $L \ldots . . . . . . .$. & 7.5 & 17.5 & & $\begin{array}{l}1 \\
2\end{array}$ \\
\hline Ambrosia artemisifolia $\mathrm{L}$. & 5.4 & 24.3 & 80.0 & 23 \\
\hline Ambrosia trifida L. . . . . . . . . & 6.4 & 29.1 & 0.0 & 4 \\
\hline Andropogon provincialis Lam.......... & 6.3 & 18.7 & & 1 \\
\hline ca $L \ldots \ldots \ldots \ldots$ & 8.4 & 27.0 & & $\begin{array}{l}1 \\
1\end{array}$ \\
\hline Asparagas officinalis $\mathbf{L} . \ldots \ldots \ldots \ldots \ldots$ & 7.0 & 53.3 & & 1 \\
\hline 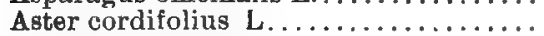 & 6.9 & 32.8 & & 4 \\
\hline Aster paniculatus Lam............... & 8.8 & 139 ? & & 2 \\
\hline Atriplex argentea Nutt... & 4.2 & 26.6 & & 4 \\
\hline$L \ldots \ldots \ldots \ldots \ldots \ldots \ldots$ & 6.2 & 40.1 & 90.0 & 11 \\
\hline Beta vulgaris L. (Sugar beet). . . . . . . . . & 5.8 & 87.7 & 91.0 & 11 \\
\hline Bidens frondosa $L \ldots \ldots \ldots \ldots \ldots$. . . . . & 7.8 & $39 . \%$ & 84.0 & 8 \\
\hline Bouteloua curtipendula (Michx) Torr .. & 4.6 & 44.0 & & 1 \\
\hline Brauneria pallida (Nutt.) Britton ..... & 6.6 & 33.6 & & 1 \\
\hline Brassica nigra (L.) Koch............. & 6.6 & 17.9 & & 1 \\
\hline Brassica oleracea L................ & 58 & 23.1 & & 2 \\
\hline Im (Willd.) Kuntze..... & 7.7 & 42.9 & 87.0 & 4 \\
\hline Cardamine hirsuta L............... & 6.0 & 21.9 & 72.0 ? & 2 \\
\hline Carduus altissimus $\mathrm{L} \ldots \ldots \ldots \ldots \ldots \ldots$ & 6.5 & 19.6 & & 1 \\
\hline Carex straminea Willd.............. & 6.4 & 16.6 & & 1 \\
\hline vulpinoidea Michx............ & 7.5 & 305 & & 2 \\
\hline ista L.... & 6.4 & 187 & & 3 \\
\hline Cassia marilan & 7.0 & 39.5 & & 8 \\
\hline Cenchrus tribuloides $\mathrm{L} \ldots \ldots \ldots \ldots \ldots$. . . & 6.6 & 25.6 & & 5 \\
\hline a (L.) Scribn......... & 7.5 & 31.3 & 82.0 & 15 \\
\hline (L.) Scribn........... & 6.8 & 344 & & 2 \\
\hline Chaetochloa viridis (L.) Scribn......... & 5.3 & 36.7 & 78.0 & $2 \tilde{1}$ \\
\hline Chrysopogon avenaceus (Michx.) Benth & 8.8 & 460 & & 2 \\
\hline 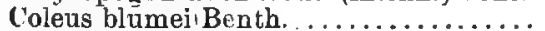 & 3.0 & 70.0 & & 1 \\
\hline miatim & $10.8 ?$ & 18.6 & & 1 \\
\hline bita maxima Duchesne......... & 69 & 50.7 & & 4 \\
\hline Cyperus aristatus Rottb........... & 7.2 & dead & & 1 \\
\hline Draba caroliniana Walt. & 7.8 & 19.7 & & 2 \\
\hline Echinodorus cordifolius (L.) Griseb.... & 19.6 & 48.5 & 87.6 & 2 \\
\hline Eclipta alba (L.) Hassk ............ & 7.5 & 45.1 & 87.6 & 4 \\
\hline Slymus canadensis $\mathrm{L}$. & 5.9 & 180 & 80.0 & 11 \\
\hline
\end{tabular}


LIST OF SPECrES.-Continued.

\begin{tabular}{|c|c|c|c|c|}
\hline \multirow[b]{2}{*}{ NAME } & \multirow{2}{*}{ 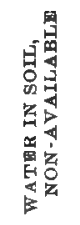 } & \multicolumn{2}{|c|}{ WATER IN PLANTS } & \multirow{2}{*}{ 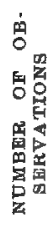 } \\
\hline & & 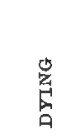 & 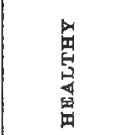 & \\
\hline Elymus virginicus $L \ldots \ldots \ldots \ldots \ldots \ldots$ & 5.6 & 287 & & 1 \\
\hline Erigeron ramosus (Walt.) $\mathrm{B} . \dot{\mathrm{S}} . \mathrm{P}_{\ldots} \ldots$. & 7.1 & 20.2 & & $\begin{array}{l}1 \\
2\end{array}$ \\
\hline Eupatorium ageratoides $L_{\ldots} . . . \ldots \ldots \ldots$ & 6.5 & 23.6 & & 1 \\
\hline Eragrostis purshii Schrad............. & 7.5 & 16.0 & 80.0 & 1 \\
\hline Euphorbia dentata Michx............ & 10.7 & 30.0 & & 1 \\
\hline Euphorbia marginata Pursh.............. & 5.8 & 59.0 & & 3 \\
\hline 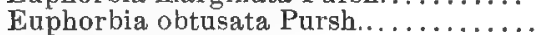 & 6.3 & 18.7 & & 1 \\
\hline Fragaria virginina Duchesne.......... & 6.4 & 23.1 & 77.0 & 2 \\
\hline Falcata comosa (L.) Kuntze............ & 6.9 & 25.1 & & 1 \\
\hline 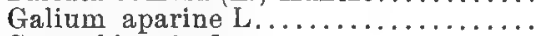 & 10.6 & 42.6 & 72.1 & 4 \\
\hline Gaura biennis L......... & 5.9 & 31.8 & 84.1 & 9 \\
\hline Gaura parviflora Dougl. . & 6.5 & 30,6 & 80.0 & 17 \\
\hline Geum canadense Jacq.. . . . . . . . . . & 80 & 29.3 & & 7 \\
\hline Grindelia squarrosa (Pursh.) Dunal.... & 6.8 & 45.9 & 83.8 & 6 \\
\hline Helianthus annuus 1 . var. ?......... & 6.4 & 28.2 & 80.6 & 4 \\
\hline 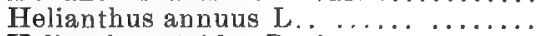 & 6.5 & 36.5 & 89.9 & 19 \\
\hline Helianthus rigidus Desf. . . . . . . . . . & $83 ?$ & 20.0 & 88.0 & 1 \\
\hline Hordeum jubstum L.... & 6.7 & 28.7 & 85.5 & 10 \\
\hline Hordeum vulgare $\mathrm{L} . \ldots \ldots \ldots \ldots \ldots \ldots$ & 6.8 & 37.5 & 90.0 & 27 \\
\hline Ipomoea purpurea Roth............. & 4.1 & 47.6 & & 2 \\
\hline Iva frutescens $\mathrm{L} . \ldots \ldots \ldots \ldots \ldots \ldots \ldots$ & 7.0 & 44.7 & $61.5 ?$ & 10 \\
\hline 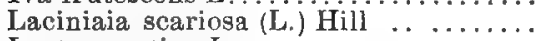 & $9.0 ?$ & 498 & & 1 \\
\hline Lactuca sativa L.......... & 8.5 & 27.5 & 92.0 & 6 \\
\hline Lactuca scariola L. ............... & 9.6 & 44.2 & 88.2 & 9 \\
\hline Lactuca pulchella (Pursh.) D. C.... . . & 9.0 & 54.4 & & 2 \\
\hline Lepachys columnaris (Pursh.) T. \& G.. & 7.2 & 39.2 & 70.0 & 3 \\
\hline Lepidium apetalum Willd............ & 68 & 27.8 & 76.5 & 7 \\
\hline Leptilum canadense (L ) Britton......... & 6.6 & 26.3 & & 6 \\
\hline Lobelia syphilitica L............... & 10.3 & 30.0 & 86.0 & 9 \\
\hline Lotus americanus (Nutt.) Bisch.......... & $9.6 ?$ & 20.0 & & 2 \\
\hline Lycopersicum esculentum Mill.......... & 5.3 & 27.1 & & $\tilde{1}$ \\
\hline Monarda fistulosa $L . \ldots \ldots \ldots \ldots \ldots$ & 6.7 & 34.9 & 82.6 & 4 \\
\hline Nasturtium officinale $\mathrm{R} . \mathrm{Br} . . . \ldots \ldots$. & 3.7 & 33.4 & 91.3 & 7 \\
\hline Nicotiana tabacum L............ & 4.6 & 31.0 & & 1 \\
\hline 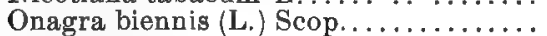 & 6.9 & 35.6 & $\ddot{8} \ddot{2} \ddot{0}$ & 9 \\
\hline Oryza sativa L.................... & 7.0 & 18.9 & $62.5 ?$ & 9 \\
\hline Oxalis stricta $\mathrm{L} . \ldots \ldots \ldots \ldots \ldots \ldots$ & 6.2 & 40.0 & 81.3 & 12 \\
\hline Panicum capillare L...... & $8.2 ?$ & 33.3 & & 1 \\
\hline Paxicum crus galli L........ & 7.2 & 35.6 & $\ddot{7} \ddot{6} \ddot{0}$ & 13 \\
\hline 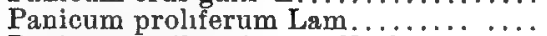 & 78 & 39.4 & 85.0 & 3 \\
\hline Panicum scribnerianum Nash......... & 7.3 & 27.7 & 83.9 & $\mathfrak{z}$ \\
\hline Panicum virgatum L............... & 6.8 & 23.0 & & 4 \\
\hline Panicum walteri Pursh............. & 8.7 & 27.2 & 814 & 4 \\
\hline Pastinaca sativa L................. & $4.3 ?$ & dead & & 1 \\
\hline Penstemon cobrea Nutt............. & 8.33 & 32.2 & & 3 \\
\hline
\end{tabular}


LIST OF SPECIES.-Concluded.

\begin{tabular}{|c|c|c|c|c|}
\hline \multirow[b]{2}{*}{ NAME } & \multirow{2}{*}{ 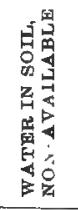 } & \multicolumn{2}{|c|}{ WATER IN PLANTS } & \multirow{2}{*}{ 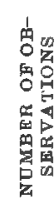 } \\
\hline & & 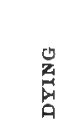 & 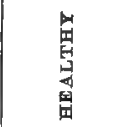 & \\
\hline Phaseolus vulgaris $L \ldots \ldots \ldots \ldots \ldots$. & 7.9 & 44.1 & 90.4 & 36 \\
\hline Physalis heterophylla L............. & 6.8 & 25.4 & & 1 \\
\hline Physalis virgıniana Mill............. & 8.8 & 41.5 & & 2 \\
\hline Pisum sativum $\mathrm{L} \ldots \ldots \ldots \ldots \ldots \ldots \ldots$ & 7.4 & 43.7 & 89.8 & 10 \\
\hline Plantago elongata Pursh... ........ & 58 & 22.2 & & 3 \\
\hline 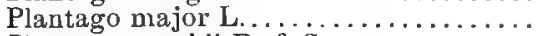 & 7.7 & 258 & 88.5 & 3 \\
\hline Plantago purshii R. \& S........... & 5.5 & 41.9 & & 1 \\
\hline 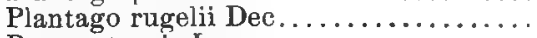 & 6.9 & 27.0 & 84.0 & 13 \\
\hline 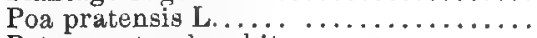 & 6.9 & 391 & & 5 \\
\hline 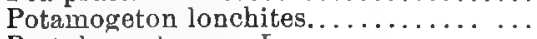 & 24.8 & 32.8 & 86.9 & 1 \\
\hline Portulaca oleracea L. .. & 8.6 ! & 56.0 & & 3 \\
\hline Potentilla arguta Pursh ............. & 8.4 & 31.3 & 78.8 & 3 \\
\hline Potentilla mnnspeliensis L............ & 7.3 & 29.1 & 71.4 & 7 \\
\hline Psoralea floribunda Nutt............. & 7.3 & 48.3 & 83.8 & 1 \\
\hline Quercus macrocarpa Michx........... & 6.6 & 18.9 & 75.5 & 1 \\
\hline Ranunculus abortivus L.............. & 6.5 & 261 & 87.1 & 4 \\
\hline Ranunculus sceleratus L............ & 10.5 & 57.1 & 89.3 & 2 \\
\hline 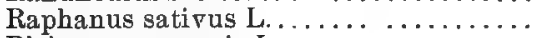 & 6.9 & 39.2 & 93.5 & 19 \\
\hline Ricinus communis L............. & 65 & 21.3 & 84.8 & 5 \\
\hline Roripa sinuata (Nutt.) Hitchcock....... & 8.9 & 33.2 & & 2 \\
\hline Rumex acetosella L............... & 66 & 34.8 & 80.6 & 8 \\
\hline 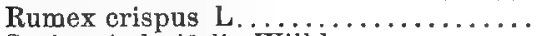 & 8.3 & 354 & 80.8 & 11 \\
\hline Sagittaria latifolia Willd............ & 15.6 & 44.6 & 93.2 & 4 \\
\hline Secale cereale $L . \ldots \ldots \ldots \ldots \ldots \ldots$ & 6.1 & 27.6 & 83.3 & 2 \\
\hline Sium cicutifolium J. F. Gmel... . . . . . . & 8.7 & 40.9 & & 4 \\
\hline Solanum nigrum L.............. & 5.6 & 258 & & 5 \\
\hline Sonchus arvensis $\mathrm{L} \ldots \ldots \ldots \ldots \ldots$ & 5.1 & 27.8 & & 5 \\
\hline Sporobolus longifolium (Torr.) Wood.. & 5.8 & 209 & $65.2 ?$ & 5 \\
\hline Sporobolus vaginiflorus (Torr.) Vasey.. & 4.4 & 18.7 & & 4 \\
\hline Steironema ciliatum (L.) Raf ......... & 7.9 & 25.7 & 80.2 & 2 \\
\hline Strophostyles helvola $\left(V_{4},\right)$ Britton...... & 5.1 & 414 & & 1 \\
\hline Syntherisma sanguinalis (L.) Nash..... & 6.6 & 37.3 & 81.5 & 38 \\
\hline Thalictrum purpurascens L.......... & 8.1 & 241 & & 2 \\
\hline Thelesperma gracile ('Jorr.).......... & 6.7 & 43.3 & & 3 \\
\hline Trifolium pratense L.............. & 5.8 & 30.6 & & 2 \\
\hline Trifolium repens $\mathrm{L} \ldots \ldots \ldots \ldots \ldots \ldots$ & 8.11 & 20.4 & 73.2 & 2 \\
\hline Triticum vulgare Vill.............. & 6.0 & 24.4 & 86.5 & 10 \\
\hline Verbena bracteosa Michx............ & 6.7 & 31.8 & & 1 \\
\hline Verbena hastata L. ............. & 6.5 & 19.5 & & 3 \\
\hline Verbena stricta Vent................ & 6.8 & 29.6 & & 8 \\
\hline Verbena urticifolia L.. . . . . . . . . . & $5.9 ?$ & 24.0 & & 4 \\
\hline Vernonia baldwinii Torr............ & 6.0 & 24.1 & & 3 \\
\hline 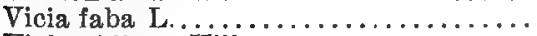 & 7.0 & 32.4 & 90.5 & 5 \\
\hline Viola obliqua Hill.................. & 8.9 & 59.7 & 82.5 & 1 \\
\hline Viola pedatifida Don ..... . . . . . . & 7.5 & 12.9 & 80.0 & 1 \\
\hline Viola scabriuscula (T. \& G.) Schwein... & 9.0 & 26.7 & & 5 \\
\hline Zea mays $\mathrm{L}$. (sugar corn)............. & 6.0 & 58.7 & 90.7 & $\mathbf{3}$ \\
\hline Zea mays L. (Jellow dent). . . . . . . . . & 5.9 & 39.0 & 89.1 & 27 \\
\hline & & & & 743 \\
\hline
\end{tabular}




\section{LIST OF SPECIES.}

(List obtained from plants in fields about Lincoln during a dry period, August, 1900.)

\begin{tabular}{|c|c|c|c|c|c|}
\hline \multirow{2}{*}{ SPEC!ES } & \multicolumn{4}{|c|}{ LIMIT OF PHYSIOLOGIOAL WATER } & \multirow{2}{*}{ 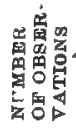 } \\
\hline & $\begin{array}{l}\text { LOOSE } \\
\text { LOAM }\end{array}$ & $\begin{array}{l}\text { HAT:D } \\
\text { LOAM }\end{array}$ & SALINE & $\operatorname{CLAY}$ & \\
\hline Amaranthus graecizans......... & 60 & & & & 4 \\
\hline A. retroflexus $\ldots \ldots \ldots \ldots \ldots \ldots$ & 5.6 & & & & 4 \\
\hline Atriplex argentea............. & 5.0 & & 14.9 & & 7 \\
\hline Chaetnchloa viridis.......... & 5.6 & 8.0 & $\ldots \ldots$ & 5.7 & 12 \\
\hline Chaetochloa italica............ & 5.2 & $\therefore \ldots$ & . $\ldots$. & $\ldots \ldots$ & 4 \\
\hline Chenopodium album .......... & 5.7 & & $\cdots \cdots \cdots$ & . .... & 4 \\
\hline Distichlis spicata............. & & 5.6 & $\cdots \ldots \ldots$ & $\ldots \ldots$ & 2 \\
\hline Eragrostis major $\ldots \ldots \ldots \ldots \ldots$ & 5.0 & 6.5 & 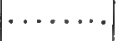 & $\ldots \ldots$ & 1 \\
\hline Euphorbia maculata............ & 5.3 & 6.0 & $\mid \ldots \ldots \ldots$ & $\ldots \ldots$ & 4 \\
\hline E. nutans.... $\ldots \ldots \ldots \ldots \ldots \ldots$ & $\ldots \ldots$ & 6.0 & …..... & $\cdots \cdots \cdot$ & 1 \\
\hline E. serpens $\ldots \ldots \ldots \ldots \ldots \ldots \ldots$ & 5.8 & 7.6 & $\cdots \cdots$ & $\ldots \ldots$ & 5 \\
\hline Grindelia squarrosa ............ & $\ddot{5} \ddot{6}$ & 5.0 & $\cdots \cdots \cdots$ & $\cdots \cdots$ & 2 \\
\hline Helianthus annuus. . . . . . . . . & 56 & 7.6 & 16.4 & $\cdots \cdots$ & 9 \\
\hline & $\cdots \cdots$ & $\begin{array}{l}5.0 \\
6.0\end{array}$ & $\cdots \cdots$ & $\cdots \cdots \cdots$ & 1 \\
\hline 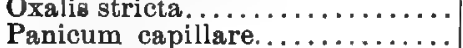 & 6.0 & $\begin{array}{l}6.0 \\
6.2\end{array}$ & & $\cdots \cdots \cdots$ & $\frac{1}{5}$ \\
\hline 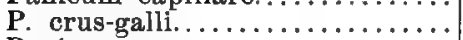 & & 6.7 & $\cdots$ & $\cdots \cdots \cdots$ & 6 \\
\hline 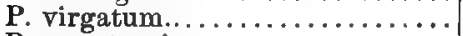 & 6.6 & & & & 1 \\
\hline Poa pratensis. . . . . . . . . . . . & ..... & & & $9.5 ?$ & 2 \\
\hline Polygonum aviculare............ & $\ldots$ & 5.0 & & $\ldots \ldots$ & 4 \\
\hline P. camporum.............. & $\ldots$ & & 16.4 & $\ldots \ldots$ & 1 \\
\hline Portulaca oleracea ............ & $\ldots \ldots \ldots$ & 3.3 & $\ldots \ldots$ & ....... & 2 \\
\hline Sorghum vulgare... ......... & 6.1 & 6.7 & $\ldots \ldots$ & $\ldots \ldots$ & 3 \\
\hline Syntherisma sanguinalis, . . . . . . & $\ldots \ldots$ & 6.7 & $\ldots \ldots$ & $\ldots \ldots$ & 9 \\
\hline Verbena bastata............... & & 5.6 & 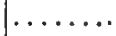 & $\ldots \ldots$ & 1 \\
\hline \multicolumn{2}{|l|}{$\begin{array}{l}\text { Total observations from field... } \\
\text { Total observations from plantho }\end{array}$} & & & & $\begin{array}{l}102 \\
743\end{array}$ \\
\hline \multicolumn{2}{|l|}{ Total......... } & & & & $84 \overline{5}$ \\
\hline
\end{tabular}




\section{EXPLANATION OF THE DATA IN THE FOLLOWING} SPECIES SHEETS AND COMPARATIVE PLOTS.

1. "Date." This is the date of final weighing of the plant, wilting or dead.

2. "Locality, "Under sub-heading. " $\mathrm{A}$ " is the locality where the plant was grown.

Under "B" the locality where the drying took place.

The following abbreviations are used for different localities where plants were grown:

"P.-H." Normal conditions, Plant House.

"P.-I." Wet, Shade, ", ", ",

"P.-II." Dry, Shade, ", ", ",

"P.-III." Wet, Sun, ", ", ",

"P.-IV." Dry, Sun, ", " ,

"C.-I." Cool plant case.

"C.-II." Shade, ", ",

"C.-III.," Normal, ", ,"

"C.-IV." Humus, ", ",

"Lab." Xerophytic conditions, laboratory.

3. "Plant Stage" is the stage of growth of the plant at the time the period of drying began. "Ros." indicates the rosette stage, "Fl" the flowering stage, and "St." the stem forming period, while "Sdl." indicates the immature seedling condition.

.4 "Time". This column gives the number of days it took the plant to reach the limit of available soil water.

5. "Soil Water." Under "a" of this heading is the per cent of availale water in the soil, and under" " $b$ " is the per cent of non-available water in the soil.

6. "Plant Water." In column " $a$ " is the per cent of water in the plant at or near the limit of available water. In column " $b$ " is the per cent of water in a healthy plant of about the same age grown under similar conditions.

7. The letter "e" placed before a per cent indicates that the per cent was not determined by weight, but by estimation. 
SPECIES SHEETS.

Phaseolus vulgaris $L$.

\begin{tabular}{|c|c|c|c|c|c|c|c|c|c|c|}
\hline \multirow{2}{*}{ DATE } & \multicolumn{2}{|c|}{ LOOALITY } & \multicolumn{2}{|c|}{ sort } & \multirow{2}{*}{$\begin{array}{l}\text { PLANT } \\
\text { STAGEE }\end{array}$} & \multirow{2}{*}{$\begin{array}{l}\text { TIME } \\
\text { DYING }\end{array}$} & \multicolumn{2}{|c|}{ SOIL WATER } & \multicolumn{2}{|c|}{ PLANT WATER } \\
\hline & a & $\mathrm{b}$ & a & b & & & at & b & a & b \\
\hline $4-26$. & P-H. & P-H. & Loe. & Ls. & Sdl. & $25 \mathrm{da}$. & 4.8 & 6.3 & 17.6 & e90.4 \\
\hline $4-29$. & 6 & " & " & “" & $" 6$ & 28 " & 5.8 & 5.3 & 26.92 & 90.4 \\
\hline 4-22. & “ & “" & Lm. & "“ & “" & 21 & 3.4 & 6.7 & 29.2 & 904 \\
\hline 4-27. & ، & ، & $"$ & 16 & . & 26 “" & 4.3 & 58 & 853 & 90.4 \\
\hline 4.26 & .. & “ & Sd. & “، & $\because$ & 25 " & 2.3 & 0.5 & 14.8 & 90.4 \\
\hline 4-29. & " & 4 & $"$ & "، & $1 "$ & 28 “ & 2.3 & 0.4 & 44.8 & 90.4 \\
\hline
\end{tabular}

COMPARATIVE PLOT II.

\begin{tabular}{|c|c|c|c|c|c|c|c|c|c|c|}
\hline 5- 6. & C-I. & C-I. & Loe. & Ls. & Sdl. & $26 \mathrm{da}$. & 12.3 & 83 & 56.3 & $\mathrm{e} 90.4$ \\
\hline 4.30. & C-II. & C-II. & " & " & & 20 “ & 6.1 & 13.8 & 54.6 & 92.3 \\
\hline 4-26. & C-III & C.III. & "، & “" & .4 & 16 " & 14.4 & 8.5 & 39.7 & 90.4 \\
\hline 4-23. & C-IV. & C-IV. & “ & " & ، & 19 ، & 138 & 12.0 & 63.6 & 92.3 \\
\hline 4.26. & Lab. & Lab. & " & ، & "، & 16 ، & 4.2 & 7.9 & 55.7 & 87.2 \\
\hline 9. & C-I. & $C-i$. & Lm. & Is. & Sdl. & $29 \mathrm{da}$. & 15.2 & 9.1 & 70.0 & ə90.4 \\
\hline $5=4$. & C-II. & C-IX. & ". & “ & "، & $24 \quad "$ & 10.0 & 12.3 & 65.0 & 93.2 \\
\hline 4-26. & C-III. & C-III. & "، & “" & " & 16 & 131 & 8.9 & 36.4 & 92.0 \\
\hline $4 \cdot 27$. & C-IV. & C-IV. & “" & “" & ، & 26 “" & 20.3 & 111 & 71.6 & 92.44 \\
\hline 5- 4 & Lab. & Lab. & " & " & “ & 24 " & 6.3 & 9.1 & 74.2 & 90.0 \\
\hline $5-3$ & C'-I. & C-I. & Sd. & Ls. & Sdl. & $23 \mathrm{da}$. & 3.1 & 0.3 & 12.0 & $\mathrm{e} 90.4$ \\
\hline $5-1$. & C-II. & C-II. & "6 & $" 1$ & "6 & 21 “" & 4.2 & 0.3 & 60.7 & 93.2 \\
\hline 4.26. & C-III & C-III. & "' & “" & “. & 16 & 5.3 & 0.4 & 8.7 & 92.0 \\
\hline 4.30. & C-IV. & C-IV. & " & " & "4 & 19 ' & 5.7 & 0.5 & 46.3 & 88.9 \\
\hline 4.29. & Lab. & Lab. & " & “، & “" & 13 & 2.3 & 0.5 & 48.8 & " \\
\hline
\end{tabular}


Hordeum vulgare $L$.

\begin{tabular}{|c|c|c|c|c|c|c|c|c|c|c|}
\hline \multirow{2}{*}{ DATE } & \multicolumn{2}{|c|}{ LOCALITY } & \multicolumn{2}{|c|}{ SOIL } & \multirow{2}{*}{$\begin{array}{l}\text { PLANT } \\
\text { STAGH H }\end{array}$} & \multirow{2}{*}{$\begin{array}{l}\text { TIME } \\
\text { DYING }\end{array}$} & \multicolumn{2}{|c|}{ SOIX WATER } & \multicolumn{2}{|c|}{ PLANT W $\triangle T$ TEE } \\
\hline & a & b & a & b & & & $\mathbf{a}$ & b & a & b \\
\hline $\begin{array}{l}5-9 . \\
4-24 . \\
4-30 . \\
5-6 . \\
4.29 .\end{array}$ & $\begin{array}{l}\text { C-I. } \\
\text { C-II. } \\
\text { C-III. } \\
\text { C-IV. } \\
\text { Lab. }\end{array}$ & $\begin{array}{l}\text { C-I. } \\
\text { C-II. } \\
\text { C-III. } \\
\text { C-IV. } \\
\text { Lab. }\end{array}$ & $\begin{array}{c}\text { Loe. } \\
\text { “، } \\
\text { “، }\end{array}$ & $\begin{array}{c}\text { Ls. } \\
\text { “" } \\
\text { “" } \\
\text { ". }\end{array}$ & $\begin{array}{c}\text { Sdl. } \\
\text { ،6 } \\
\text { ، } \\
\text {. }\end{array}$ & $\begin{array}{rr}29 & \text { da. } \\
8 & “ \\
14 & ، \\
26 & ، \\
13 & ،\end{array}$ & $\begin{array}{l}7.6 \\
3.1 \\
4.6 \\
9.2 \\
9.2\end{array}$ & $\begin{array}{r}8.9 \\
11.9 \\
7.3 \\
9.2 \\
9.0\end{array}$ & $\begin{array}{r}21.5 \\
8.3 \\
52.0 \\
31.5 \\
30.5\end{array}$ & $\begin{array}{c}79.7 ? \\
\text { e85.0 } \\
88.8 \\
86.1 \\
91.3\end{array}$ \\
\hline $\begin{array}{l}5-9 . \\
4-27 . \\
4-30 . \\
5-6 . \\
4-29 .\end{array}$ & $\begin{array}{l}\text { C-I. } \\
\text { C-II. } \\
\text { C-III. } \\
\text { C-IV. } \\
\text { Lab. }\end{array}$ & $\begin{array}{l}\text { C-I. } \\
\text { C-II. } \\
\text { C-III. } \\
\text { C-IV. } \\
\text { Lab. }\end{array}$ & $\begin{array}{c}\text { Lm. } \\
، \\
، \\
، \\
،\end{array}$ & $\begin{array}{l}\text { Ls. } \\
\text { ،" } \\
\text { “" }\end{array}$ & $\begin{array}{c}\text { Sdl. } \\
\text { “" } \\
\text { “" } \\
\text { " }\end{array}$ & {$\left[\begin{array}{cc}29 & \text { da. } \\
11 & " \\
14 & ، \\
26 & ، \\
13 & ،\end{array}\right.$} & $\begin{array}{r}11.1 \\
4.0 \\
8.0 \\
12.0 \\
11.9\end{array}$ & $\begin{array}{r}6.0 \\
16.5 \\
9.9 \\
9.9 \\
7.4\end{array}$ & $\begin{array}{l}31.4 \\
11.5 \\
49.0 \\
32.2 \\
47.9\end{array}$ & $\begin{array}{r}83.0 \\
\text { e85.0 } \\
90.8 \\
86.4 \\
91.8\end{array}$ \\
\hline $\begin{array}{l}4-30 . \\
4-24 . \\
4-25 . \\
5.1 . \\
4-30 .\end{array}$ & $\begin{array}{l}\text { C-I. } \\
\text { C-II. } \\
\text { C-III. } \\
\text { C-IV. } \\
\text { Lab. }\end{array}$ & $\begin{array}{l}\text { C-I. } \\
\text { C-II. } \\
\text { C-III. } \\
\text { C-IV. } \\
\text { Lab. }\end{array}$ & $\begin{array}{c}\text { Sd. } \\
\text { ، } \\
، \\
،\end{array}$ & $\begin{array}{l}\text { Ls. } \\
“ " \\
، \\
16\end{array}$ & $\begin{array}{c}\text { Sdl. } \\
\text { "، } \\
\dddot{~} \\
\text { "، }\end{array}$ & $\begin{array}{rr}14 & \text { da. } \\
8 & ، \\
9 & \cdots \\
21 & \cdots \\
14 & \text { ‘ }\end{array}$ & $\begin{array}{l}2.9 \\
1.0 \\
5.5 \\
6.0 \\
2.5\end{array}$ & $\begin{array}{l}0.22 \\
0.5 \\
0.2 \\
0.2 \\
0.46\end{array}$ & $\begin{array}{r}26.0 \\
6.8 \\
5.2 \\
20.3 \\
47.7\end{array}$ & $\begin{array}{c}91.5 \\
\text { e85. } \\
87.5 \\
88.1 \\
91.3\end{array}$ \\
\hline
\end{tabular}

Zea mays L. (Yellow Dent Corn.).

(COMPARATIVE PLOT II.)

\begin{tabular}{|c|c|c|c|c|c|c|c|c|c|c|}
\hline \multirow{2}{*}{ DATE } & \multicolumn{2}{|c|}{ LOCALITT } & \multicolumn{2}{|c|}{ solL } & \multirow{2}{*}{$\begin{array}{l}\text { PLANT } \\
\text { STAGE }\end{array}$} & \multirow{2}{*}{$\begin{array}{c}\text { TLME } \\
\text { DYING }\end{array}$} & \multicolumn{2}{|c|}{ SOIL WATER } & PLANT & WATER \\
\hline & a & b & a & b & & & $\mathbf{a}$ & b & a & b \\
\hline 4-2\%. & P.H. & P-H. & Loe. & Ls. & Sdl. & $16 \mathrm{da}$. & 6.5 & 4.6 & 34.9 & 98.3 \\
\hline $6-26$. & 6 & 4 & 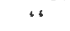 & ". & " & $15 \quad$ “ & 6.3 & 4.8 & 33.0 & 93.3 \\
\hline 4-29. & C-I. & C-I. & a &. & $" 4$ & 19 " & 9.0 & 90 & 43.3 & $78.7 ?$ \\
\hline $4-30$. & C-II. & C-II. & ، & " & "“ & 20 & 6.0 & 11.3 & 60.3 & 92.4 \\
\hline 4-30. & C-III. & C-III. & $"$ & . & “ & $20 \cdots$ & 16.4 & 6.5 & 43.4 & 90.4 \\
\hline $5-3$. & C-IV. & C-IV. & ، & .. & “، & 23 & 12.6 & 7.1 & 57.9 & 90.3 \\
\hline 4-27. & Lab. & Lab. & “ & $\because$ & “ & $17 \quad ،$ & 9.3 & 8.3 & 41.4 & 92.3 \\
\hline 4-27. & P-H. & P-H. & Lm. & Ls. & Sdl & $16 \mathrm{da}$. & 7.0 & 3.1 & 11.3 & 933 \\
\hline 4-27. & & " & $1 "$ & “" & " & 16 '، & 5.9 & 4.2 & 47.3 & 933 \\
\hline 5- 9. & jC-I. & C-I. & “" & " & “" & 29 & 16.7 & 7.6 & 41.5 & 84.7 \\
\hline 4-30. & C-II. & C-II. & " & " & " & 20 & 9.2 & 12.7 & 50.4 & 95.4 \\
\hline 4-29. & C-II1. & C-III. & 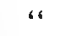 & 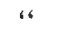 & “ & 19 & 14.6 & 6.1 & 48.2 & 92.0 \\
\hline $5-3$. & C-IV. & C-IV. & .. & “" & " & 23 & 12.6 & 8.8 & 59.9 & 91.9 \\
\hline 4-27. & Lab. & Lab. & “ & “ & $\because$ & $17 . " 6$ & 8.5 & 7.2 & 39.1 & 91.8 \\
\hline $4-30$. & P-H. & P-H. & Sd. & Ls. & Sdl. & 29 da. & 3.8 & 0.6 & 52.8 & 93.3 \\
\hline 4-30. & & & “ & " & $"$ & 29 “" & 3.8 & 0.6 & 36.8 & 93.3 \\
\hline 4-25. & C-I. & C-I. & 16 & "، & $"$ " & 16 “" & 2.3 & 0.45 & 36.3 & 80.4 \\
\hline 4-26. & C-II. & C-II. & .. & " & “ & 16 & 5.6 & 0.1 & 53.3 & 89.7 \\
\hline 4-27. & C.III. & C-III, & ." & $" 1$ & " & $17:$ & 5.3 & 0.4 & 22.5 & 81.7 \\
\hline 5. 1. & C-IV. & C-IV. & 4 & “ & "6 & 21 " & 5.9 & 0.3 & 42.2 & 85.2 \\
\hline $5-2$. & Lab. & Lab. & " & 4 & $"$ & $22 "$ & 4.1 & 0.3 & 29.5 & 91.3 \\
\hline
\end{tabular}


COMPARATIVE PLOT NO. III.

Sand.

\begin{tabular}{|c|c|c|c|c|c|c|c|}
\hline \multirow{2}{*}{ PLANTg } & \multirow{2}{*}{$\begin{array}{l}\text { TIME IN } \\
\text { DYING }\end{array}$} & \multicolumn{2}{|c|}{ GEALTHY PL'T } & \multicolumn{2}{|c|}{ WILTING PL'NT } & \multicolumn{2}{|c|}{ DYING PLANT } \\
\hline & & SOIL & PLANT & SOIL & PLANT & SOIL & PLAN I \\
\hline 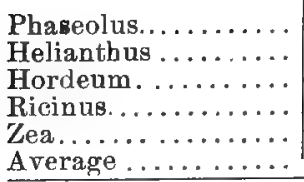 & $\begin{aligned} & 13 \text { da. } \\
& 9 \text { “. } \\
& 17 \text { “، } \\
& 16 \text { ‘ } \\
& 11 \text { “ } \\
& 15 \text { “" } \\
&\end{aligned}$ & $\begin{array}{r}8.3 \\
8.7 \\
11.9 \\
11.5 \\
129 \\
10.6 \\
\end{array}$ & $\begin{array}{l}90.4 \\
94.7 \\
89.2 \\
85.4 \\
93.0 \\
90.5 \\
\end{array}$ & $\begin{array}{l}0.42 \\
0.41 \\
0.41 \\
0.5 \\
0.4 \\
0.43 \\
\end{array}$ & $\begin{array}{l}78.0 \\
78.8 \\
70.0 \\
63.2 \\
72.2 \\
72.4\end{array}$ & $\begin{array}{l}0.26 \\
0.2 \\
0.31 \\
0.44 \\
0.41 \\
0.32\end{array}$ & $\begin{array}{l}38.8 \\
44.9 \\
53.0 \\
24.3 \\
58.2 \\
43.8\end{array}$ \\
\hline \multicolumn{8}{|c|}{ Loam. } \\
\hline 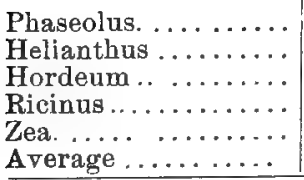 & 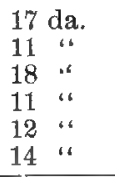 & $\begin{array}{l}24.4 \\
23.8 \\
29.7 \\
23.7 \\
24.9 \\
25.3\end{array}$ & $\begin{array}{l}90.1 \\
922 \\
913 \\
84.4 \\
93.0 \\
90.2 \\
\end{array}$ & $\begin{array}{r}10.6 \\
11.6 \\
9.8 \\
10.3 \\
12.5 \\
10.9\end{array}$ & $\begin{array}{l}805 \\
87.6 \\
52.0 \\
82.5 \\
81.7 \\
76.8 \\
\end{array}$ & $\begin{array}{r}8.7 \\
11.2 \\
8.7 \\
91 \\
101 \\
9.5\end{array}$ & $\begin{array}{l}53.0 \\
61.4 \\
39.4 \\
610 \\
60.4 \\
54.8\end{array}$ \\
\hline \multicolumn{8}{|c|}{ Saline. } \\
\hline 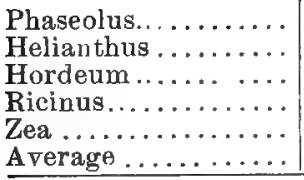 & 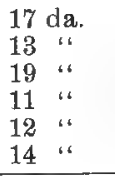 & $\begin{array}{l}28.9 \\
26.3 \\
29.5 \\
28.4 \\
20.5 \\
24.7 \\
\end{array}$ & $\begin{array}{l}90.0 \\
936 \\
83.4 \\
844 \\
920 \\
88.7 \\
\end{array}$ & $\begin{array}{r}17.2 \\
151 \\
98 \\
12.0 \\
168 \\
14.2 \\
\end{array}$ & $\begin{array}{l}800 \\
85.4 \\
82.2 \\
76.5 \\
83.4 \\
81.5 \\
\end{array}$ & $\begin{array}{l}19.8 \\
12.7 \\
119 \\
12.4 \\
13.3 \\
14.0\end{array}$ & $\begin{array}{l}53.7 \\
59.0 \\
53.4 \\
60.0 \\
61.0 \\
57.4\end{array}$ \\
\hline \multicolumn{8}{|c|}{ Clay. } \\
\hline 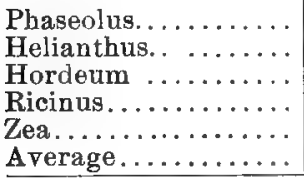 & 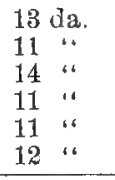 & $\begin{array}{l}16.9 \\
21.0 \\
18.2 \\
20.0 \\
16.4 \\
18.5\end{array}$ & $\begin{array}{l}89.5 \\
87.6 \\
93.3 \\
84.4 \\
92.0 \\
89.4 \\
\end{array}$ & $\begin{array}{l}8.5 \\
9.1 \\
6.4 \\
9.2 \\
9.4 \\
8.5\end{array}$ & $\begin{array}{l}78.9 \\
84.9 \\
69.3 \\
80.6 \\
75.0 \\
77.7\end{array}$ & $\begin{array}{r}85 \\
9.3 \\
7.1 \\
10.3 \\
7.9 \\
8.6 \\
\end{array}$ & $\begin{array}{l}51.8 \\
603 \\
42.2 \\
55.9 \\
48.0 \\
51.7\end{array}$ \\
\hline \multicolumn{8}{|c|}{ Loess. } \\
\hline 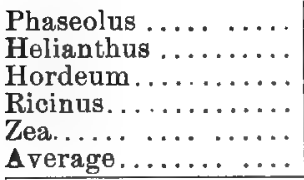 & 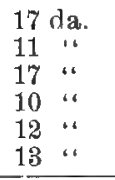 & $\begin{array}{l}12.4 \\
18.0 \\
18.7 \\
24.7 \\
19.9 \\
18.7\end{array}$ & $\begin{array}{l}89.6 \\
93.9 \\
864 \\
84.4 \\
920 \\
89.3 \\
\end{array}$ & $\begin{array}{r}8.4 \\
10.9 \\
8.1 \\
7.8 \\
13.1 \\
10.1 \\
\end{array}$ & $\begin{array}{l}85.6 \\
815 \\
794 \\
74.3 \\
82.4 \\
80.9\end{array}$ & $\begin{array}{r}8.7 \\
10.3 \\
7.1 \\
10.9 \\
9.3 \\
92 \\
\end{array}$ & $\begin{array}{l}52.3 \\
55.2 \\
50.1 \\
48.6 \\
56.1 \\
52.7\end{array}$ \\
\hline \multicolumn{8}{|c|}{ Humus. } \\
\hline 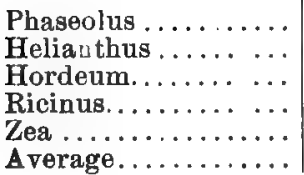 & $\begin{array}{l}17 \text { da. } \\
11 ، \\
17 \text { ، } \\
10 ، \\
11 \text { “، } \\
13 \text { “ }\end{array}$ & $\begin{array}{l}19.0 \\
25.3 \\
28.9 \\
24.7 \\
29.1 \\
25.4\end{array}$ & $\begin{array}{l}900 \\
90.2 \\
88.8 \\
84.4 \\
92.0 \\
89.1\end{array}$ & $\begin{array}{r}9.3 \\
11.5 \\
11.3 \\
12.1 \\
11.9 \\
11.2\end{array}$ & $\begin{array}{l}81.0 \\
79.2 \\
69.1 \\
79.7 \\
63.5 \\
74.5\end{array}$ & $\begin{array}{r}11.0 \\
11.3 \\
9.8 \\
12.3 \\
9.1 \\
10.7\end{array}$ & $\begin{array}{l}32.1 \\
59.7 \\
30.6 \\
61.9 \\
58.0 \\
48.4\end{array}$ \\
\hline
\end{tabular}


MECHANICAL ANALYSIS OF SOILS.

Bv Mr. A. T. Wiancko, Assistant Agriculturist, Agricultural Experiment Station, University of Nebraska.

\begin{tabular}{|c|c|c|c|c|c|c|}
\hline \multirow{2}{*}{ INGREDIENTS } & $\begin{array}{l}\text { sorL } \\
\text { N.). } 1\end{array}$ & $\begin{array}{l}\text { sorl } \\
\text { No. } 2\end{array}$ & $\begin{array}{l}\text { SOIL } \\
\text { No. } 3\end{array}$ & $\begin{array}{l}\text { sort } \\
\text { No. } 4\end{array}$ & $\begin{array}{l}\text { SoII } \\
\text { No. } 5\end{array}$ & $\begin{array}{l}\text { solf } \\
\text { N } 1.6\end{array}$ \\
\hline & SANO & LOAM & SALINE & CLAY & L) BSS & HDMUS \\
\hline Coarse Gravel.... & 13.85 & 0.20 & 0.67 & & 1.27 & 3.88 \\
\hline Fine Gravel ..... . & 18.04 & 1.82 & 1.35 & 0.77 & 1.23 & 7.58 \\
\hline Coarse Sand... . & 21.46 & 3.12 & 2.08 & 1.62 & 1.76 & 8.84 \\
\hline Medium Sand .... & 3644 & 7.30 & 3.60 & 2.88 & 7.18 & 13.30 \\
\hline Fine Sand..... & 5.92 & 1.24 & 8.26 & 0.76 & 9.63 & 7.27 \\
\hline Very Fine Sand... & 2.88 & 9.06 & 14.53 & 10.88 & 15.98 & 13.00 \\
\hline Silt. $\ldots \ldots \ldots \ldots$ & 0.34 & 50.21 & 27.12 & 2809 & 24.13 & 17.37 \\
\hline Fine Silt...... & 0.15 & 11.13 & 20.47 & 7.96 & 24.16 & 10.65 \\
\hline Clay........ & 0.08 & 9.34 & 20.08 & 39.92 & 15.10 & 4.82 \\
\hline Water*.......... & 0.60 & 3.20 & 2.92 & 2.80 & 2.91 & 2.03 \\
\hline Volatile Matter... & 0.33 & 8.2 & 4.12 & 4.58 & 2.80 & 14.42 \\
\hline Loss or Gain $\nmid . . .$. & -0.51 & -1.62 & $\uparrow 2.62$ & -1.94 & +3.24 & $\dagger 1.13$ \\
\hline Soluble Salts .. & $\ldots \ldots$ & 0.1519 & 0.5092 & 0.1574 & 0.1282 & 0.2193 \\
\hline
\end{tabular}

*Water in air dry soil. † 





The Development and Structure of Vegetation 
The WoodrdFF-Collins PRINTING COMPaNy. LINCOLN, NEBRASKA. 


\title{
THE DEVELOPMENT AND STRUCTURE OF VEGETATION.*
}

\author{
Frederic E. Clements.
}

Vegetation exhibits certain phenomena which are characteristic manifestations of the forces which lie at its foundation. Such phenomena are peculiar to it, and are entirely distinct from those primary activities of the individual that are termed functions. This conception will be clearer if we consider vegetation as an entity, the changes and structures of which are in accord with certain basal principles in much the same fashion that the functions and structures of plants correspond to definite laws. But the fundamental phe. nomena of plants as individuals, and of plants united in a complex termed vegetation are altogether different concepts. Thus, functions are properties of individuals, and are fundamental for them alone. They are rhythmic or progressive changes in the individual, and are characteristic of vegetation only in as much as it is a complex of such indi-

*Read before the Botanical Society of America at the Denver meeting, August 1901.

NoTE. The principles here enunciated constitute at once the working basis and the conclusions of a special investigation into the phylogeny and structure of vegetation begun in 1897. These studies have been pursued in the grassland and woodland formations of Nebraska, and throughout the formations of the Rocky Mountains of Colorado. Certain of these principles, association, invasion and zonation, have long been recognized, but no systematic consideration of them has been attempted heretofore. The principle of succession is more recent, and is not so well developed, while alternation is herein defined and analysed for the first time (Cfr. note, Rep. Bot. Surv. Nebr. 5:55 1901). These principles were first formulated in their present relation early in 1898 as working hypotheses, and have been subjected to constant test in the field since that time. Their confirmation has been repeated and conclusive, and they are now submitted as a deflnite and logical basis for research in the development and structure of vegetation. The present paper is intended to constitute the general portion of a detailed report dealing with the physical factors of the prairie formation in Nebraska, and with the structural expression of such factors. The special portion will appear in a later report of the Survey, while the application of the principles to mountain formations will be made in a forthcoming volume upon the vegetation of the Rocky Mountains of Colorado. 
viduals. The latter exhibits within itself peculiar changes, likewise rhythmic and progressive. These changes constitute or produce the fundamental phenomena of vegetation.

From the nature of this primary dependence upon environment, vegetation is essentially dynamic, but, on account of its marked reaction upon the habjtat, it exhibits a constant tendency to become static. Its characteristic phenomena, then, are manifestations of change, in which each successive stage is less dynamic and correspondingly more static. The physical and biological factors which constitute a habitat are so diverse and their interaction so complicated that complete stasis is never attained, though forest and grassland sometimes closely approximate this condition. Even in such formations, however, the facies alone are static, the subordinate species being in constant readjustment to slowly intensifying conditions. Furthermore, in all formations, whether they have attained a degree of stasis by progressive changes or not, there is still a seasonal rhythm of appearance, reproduction and decadence, which remains always and essentially dynamic. Finally, the phenomena of vegetation illustrate this primary distinction between the dynamic and the static. Invasion and succession are completely d.ynamic in their operation, i. e., developmental, while association, zonation and alternation are relatively static, i. e., structural. The further consideration of the dynamics of vegetation will, in consequence, be found under invasion, and, especially, under succession.

The fundamental phenomena of vegetation are association, invasion, succession, zonation and alternation. Of these, association represents the stage to which vegetation has been brought by the cumulative changes of the past. It corresponds essentially to the historical fact in vegetation. Invasion and succession are dynamic forces ro phenomena which are at work modifying the present facts of association. They are initiated by physical factors, and are wrought out by the interaction of physical and biological factors. Hence, they are representative of the physical fact 
in vegetation. Zonation and alternation, on the other hand, are static phenomena, characteristic of the vegetative covering as it is and will be when it becomes more and more static. Thus, the relation of plants to the surface of the globe finds its basal expression in the one great principle or phenomenon of association, for which indeed vegetation is but another name. In its dynamic aspect, developmentally, association is controlled by two laws, that of invasion and of succession, while in its static aspect, structurally, it conforms to the laws of zonation and of alternation.

In the analysis of vegetation. it is imperative that the twofold nature of its origin be kept clearly in mind. Just as a species of today is the result of accumulated ancestral facts modified to a certain degree by existing physical factors, so a particular vegetation of the present time, indeed all vegetation, is the result of a complex physical fact, the habitat or environment, actıng upon a biological or historical fact, the antecedent vegetation. Since the basis of vegetation is twofold, physical and historical, the principles manifested in it must correspond to the one or the other.

In the following treatment, the fundamental facts of vegetation are considered separately in a somewhat detailed and systematic manner. The arrangement under each is the same. The conception of each principle and its relation to the others are first discussed; then the consideration of causes, categories, manifestations and results follows in the logical order. A short statement of the historical development of each subject is given in the proper place. Because of its bearing upon the origin and modification of formations, the neglected doctrine, polyphylesis, is considered in connection with invasion. 



\section{ASSOCIATION}

The principle of association is the fundamental law of vegetation. Indeed, association is vegetation, for the individual passes into vegetation, strictly speaking, at the moment when other individuals of the same kind or of different kinds become grouped with it. It is then (and the same statement necessarily holds for vegetation) the coming together and the staying together of individuals and, ultimately, of species. A concrete instance will illustrate this fact. In the development of the blowout formation of the Nebraska sandhills (Redfieldia-Muhlenbergia-anemodium), association begins only when the first plant of Redfeldia flexuosa is joined by other plants that have sprung from it, or have wandered in over the margin of the blowout. Henceforth, however dynamic the blowout may remain, or however static it may become, association is a settled characteristic of it until some new and overwhelming physical catastrophe shall destroy the associated individuals. It will readily be seen that association does not depend upon particular individuals, for these pass and others take their place, but that it does depend essentially upon number of individuals.

Association involves the idea of the relation of plants to the soil, as well as that of plants to each other. It is synonymous with vegetation only when the two relations are represented, since there may be association such as that of a parasite with its host, which does not constitute vegetation. But it will be seen that the relation of the parasite to the host is practically identical with the relation of the plant to the soil or stratum, and the two concepts mentioned above become merged. From this it follows that association results in vegetation only when the two ideas are distinct. The concept of association contains a fact that is everywhere significant of vegetation, namely, the likeness or unlikeness of the individuals which are associated. In the case of parasite and host, this unlikeness is marked; in vegetation, all degrees of similarity obtain. As will be evident when the causes which lead to association are con- 
sidered, alternate similarity and dissimularity of the constituent individuals or species is subordinate as a feature of vegetation only to the primary fact of association.

In considering the causes which produce association, it is necessary to call in evidence the primary facts of the ontogeny of concrete examples of the working of this principle. These facts are so bound up in the nature of vegetal organisms that they are the veriest axioms. Reproduction gives rise immediately to potential, and ultimately, in the great majority of cases, to actual association. The degree and permanence of the association are then determined by the immobility of the individuals as expressed in terms of attachment to each other or to the stratum, such as sheath, thallus, haustoria, holdfasts, rhizoids, roots, etc. The range of immobility is very great. In terrestial plants, mobility is confined almost entirely to the period when the individual lies dormant in the seed, spore or propagative part, which is alone mobile. In aquatic spermatophytes, the same is true of all attached forms, while free floating plants such as Lemna are mobile in a high degree, especially during the vegetative period. Among the algae and hydrophilous fungi, attached forms are mobile only in the spore or propagative condition, while the motile forrus of the plancton typify the extreme development of mobility. The immediate result of reproduction in an immobile species is to produce association of like individuals, while in the case of a mobile species reproduction may or may not lead immediately to association. We may lay down the general principle that immobility tends to maintain the association of the individuals of the same generation, i. e., the association of like forms, while mobilty tends to sepurate the similar individuals of one generation and to bring unlike forms together. With the mobile algae, separation of the members of each generation is the rule, unless the individuals come to be associated in a thallus, or are grouped in contact with the substratum. Flowering plants that are relatively immobile, especially in the seed state, drop their seeds beneath and about the parent plants, and in consequence dense association of the 
new plants is the rule. In very many cases, however, this primitive tendency is largely or completely negatived by the presence of special dissemination contrivances. which are nearly, if not quite, as effective for many terrestrial plants, as the free floating habit is for algae. From this point, the whole question of mobility belongs to migration, just as the adjustment between the parent plants and their offspring, or butween plants established and the mobile plants to be established belongs to occupation.

If association were determined by reproduction and immobility alone, it would exhibit areas dissimilar in the mass of individuals, as well as areas dissimilar in the kınds of individuals. Some areas would be occupied by plants of a single species, others by plants of several or many species. This tendency of association to show differences is, however, greatly emphasized by the fact that vegetation is fundamentally attached to and dependent upon a surface that exhibits the most extreme physical differences. For this reason, new differences in association appear, due not only to the morphological differentiation of vegetation forms, but also to the changes in the degree and manner of association produced directly by the different habitats. Association might then be defined as a grouping together of plant individuals, of parents and progeny, which is initiated by reproduction and immobility, and determined by environment. It is a resultant of differences and similarities. In consequence, association in its largest expression, vegetation, is essentially heterogeneous, while in those areas which possess physical or biological definiteness, habitats and vegetation centers, it is relatively homogeneous. This fundamental peculiarity has given us the concept of the formation, an area of vegetation, or a particular association, which is homogeneous within itself, and at the same time essentially different from contiguous areas, though falling into a phylogenetic series with some and a biological series with others. From its nature, the plant formation is to be considered the logical unit of vegetation, though it is not, of course, the simplest example of association. 
The term formation was proposed by Grisebach in 1838; he defined the concept as follows: "Ich möchte eine Gruppe von Pflanzen, die einen abgeschlossenen physiognomischen Charakter traegt, wie eine Wiese, ein Wald, etc., eine pflanzengeographische Formation nennen. Sie wird bald durch eine einzige gesellige Art, bald durch einen Complex von vorherrschenden Arten derselben Familie charakterisiert, bald zeigt sie ein Aggregat von Arten, die, mannigfaltig in ihrer Organisation, doch eine gemeinsame Eigenthuemlichkeit haben, wie die Alpentriften fast nur aus perennierenden Kräutern bestehen." Before Grisebach's time, several writers had perceived the essential correspondence between habitat and vegetation, though they did not recognize the fundamental importance of this relation. Linné mentioned a few generalized stations in Flora Lapponica (1737) and Flora Suecica (1745), but in the Philosophia Botanica (1751: 265) he not only distinguished the following twenty-five habitats, but also characterised each briefiy and listed the principal genera: mare, littora, fontes, fluvii, ripae, lacus, stagna, paludes, cespitosae, inundata, uliginosa, alpes, rupes, montes, campi, sylvae, nemora, prata (a. fertilia, b. exsucca, c. humidiuscula), pascua, arva, agri, versurae, culta, fimeta, ruderatae, In addition, he arranged stations in four groups with respect to the kind of soil, viz., humus, arena, (mobilis, farinacea, vulgaris, sabulosa), argilla, creta.

Hedenberg (1754:73) recognized twenty-four stations, naming them after the specific names of characteristic species, and classifying them in five groups as follows: Aquaticae; (1) Marinae (2) Maritimae (3) Lacustres (4) Palustres (5) Inundatae (6) Uliginosae (7) Caespitosae; Alpinae, (8) Aetierea (9) Occlusae; Umbrosae, (10) Nemorosae (11) Sylvaticae; Campestres, (12) Arvenses (13) Cultae (14) Ruderales (15) Pratenses (16) Arenariae (17) Argillaceae (18) Cretaceae; Montanae, (19) Glabretosae (20) Collinae (21) Rupestres; Parasiticae (22) Arboreae (23) Herbaceae (24) Radicales. Each station is described briefly, and the vegetation usually characterised in a few words, e. g., ; "10. Nemorosae. Loca Nemorum sub arboribus 
frondosis, humo laxa, frondibus per hyemem tecta, aestate foliis obumbrata. Plantae fragiles, florentes vere ante frondescentiam." Finally, the species of the Swedish flora are grouped under their proper stations.

Humboldt (1807:17) recognized formations only incidentally, as in the following: "Les bruyères, cette association de l'erica vulgaris, de l'erica tetralix, des lichen icmadophila et haematomma se répandent depuis l'éxtremité la plus septentrionale du Jutland, par le Holstein et le Lune. bourg jusqu'au 52 degré de latitude." Mirbel (1815:583) in his monumental Terminologie Methodique, characterised briefly forty-two stations, indicating a few typical species in each, and grouped them as follows: Terrestres, arenariae, saxatiles, ruderales, argillosau, cretaceae, graniticae. oleraceae, hortenses, vineales, agrestes, arvenses, pratenses, sylvaticae, umbrosae, campestres, collinae, montanae (alpestres, alpinae), glaciales, hyperboreae, salinae, littorales, maritimae; Aquaticae, marinae, lacustres, fontinales, fluviatiles, submersae, emersae, fluitantes, natantes. palustres, uliginosae, torfaceae; Amphibiae; Epiphytae; Parasiticae, epirhizae, corticales, epizyloneae, epiphyllae; Subterraneae, interraneae, cavernariae. That De Candolle (1820: $25,29)$ clearly understood the importance of stations is shown by his statement: "L'étude des stations est, pour ainsi dire, la topographie, et celle des habitations la géographie botanique," and also by his classes of stations. Schouw (1824:153) proposed an arrangement of stations that was far in advance of his time, and exhibits much of fundamental value at the present time. He was the first to distinguish clearly a number of formations (Bestünde), and to apply definite names to them, such as ericetum, rhododendretum, coryletum, pinetum, palmetum, etc. Meyen (1836) merely adopted Schouw's system, without adding anything of especial importance to it. Grisebach's recognition of the station as a biological unit, the formation, made a permanent place for this concept in phytogeography, and the study of vegetation with reference to its constituent formations became more and more frequent. A. DeCandolle 
(1855) ignores formations and treats only incidentally of stations: this is explained by the fact that he wrote of botanical geography, considering the treatment of stations to belong to botanical topography. With the work of Kerner (1863), Grisebach (1872), Engler (1879), Hult (1881,1885), Goeze (1882), Beck (1884), Drude (1889) and Warming (1889), the recognition and delimitation of formations came to constitute the foundation of vegetational research. a relation which the work of the last decade has greally emphasized. At the present time, the formation stands in precisely the same relation to vegetation, and hence to phytogeography, that the species does to the flora, and to taxonomy. In consequence, the primary lines of research are the same for each, and the foundations of phytogeography must be laid upon the development, structure and classification of formations in the same thorough way that the superstructure of taxonomy has been built upon the development, structure and classification of species.

Since the present paper deals with vegetation as a whole, the development of formations will be treated under succession, the structure of formations under zonation and alternation, while their classification will be found under association and succession.

In endeavoring to analyse the causes of association, it must be kept clearly in mind that the concrete examples from which all generalisations must be drawn are often in very different stages of development, and are of correspondingly different ages. For this reason, it has seemed best to consider the primary relations of association in general in this place, leaving the treatment of the effects of invasion, succession, alternation, and zonation to be taken up under these topics.

Various categories of association may be distinguished according to the dominant physical factor concerned, or the point of view taken. These will fall into two series, as we consider the relation of plant to plant with reference to some object or characteristic, or the grouping of plants together in response to some dominant factor. In the first series 
may be placed association with reference to substratum, to the ground (occupation), and to migration: in the second belong light and water content association. It should be noted that these are all actual associations in nature, and not concepts such as the vegetation form, within which plants from widely different associations may be classifed. Naturally, it does not follow that it is not logical or valuable to gruup together those plants, such as hydrophytes, sciophytes, hysterophytes, etc., which have a common relation to some factor, but belong to different formations. ${ }^{1}$

Stratum association (associatio stratalis). Plants manifest independent or dependent association with reference to the stratum to which they are attached and from which they derive food or support. Independent association (associatio contigua) is exhibited by those holophytic species of a formation which are entirely independent of each other with respect to mechanical support or nutrition. It is characteristic of the greater number of the constituent species of formations. Dependent association (associatio mutua) is manifested in the relation between host and parasite, stratum and epiphyte, support and liane. The idea here involved has long been recognized. Humboldt (1806) included liwnes as one of his eighteen vegetation forms. DeCandolle (1820:32) distinguished parasites and false parasites, the latter growing on dead plants, or attached to living plants, Schoww (1823 : 159) made a much more miunte division, in which plantae parasiticae radicantes correspond to parasites, plantae parasiticae superficiales to epiphytes, and plantae eprphytae spuriae, plantae epizoae, etc., to saprophytes. Meyen (1836:70) followed Schouw in the main, but established the group epiphytes to include lichens, and epiphytes in the present acceptation of the term. Drude (1890:64, 68), recognized the four groups, arranging them among vegetation forms. Warming (1895) distinguished six kinds of association, parasitism, helotism, mutualism,

\footnotetext{
1 Clements, F. E. A System of Nomenclature for Phytogeography Engler 31:b1 1902.
} 
epiphytes, lianes and commensalism. Commensalism corresponds to the primary principle of association which has given rise to vegetation. Homogeneous commensalism is the term applied to social exclusive plants, in which the patch is composed of a single species,. Such association is extremely rare in nature, and, if the most minute forms be considered, probably never occurs. On the other hand, heterogeneous commensalism, in which individuals of more than one species are present, is everywhere typical of vegetation. Warming regards saprophytism merely as a specialised kind of parasitism, an opinion that may well be defended. Helotism, however, is also a mere modification of parasitism, if it is not indeed parasitism pure and simple. Mutualism is an altogether vague concept, including parasites, epiphytes, and endophytes of doubtful physiological relation. Pound and Clements (18:8:101) treated lianes, parasites and saprophytes as vegetation forms, relating herbaceous creepers and twiners to the lianes, and dividing the fungi and lichens into nine groups. Whatever the value of these divisions may be from the standpoint of vegetation forms, they represent the same relation between plant and nutritive stratum, and with respect to association should be merged in one group. To Schimper (1898:208) belongs the credit of having been the first to perceive the essential similarity of all such groups from the standpoint of association. He terms these, plant societies (Genossenschaften), retaining the four groups already established, lianae, epiphyta, saprophyta and parasiticae. It is evident that dependent association comprises extremely divergent forms, from the slightly clinging herb, such as Galium, to the most intense parasite. The distinction, however, is a clear one, if restricted to that relation between plants, in which one acts as a mechanical support or stratum, or as a nutritive host for the other.

Ground association (associatio terrena). The first division of formations into open and closed seems to have been made by Engler and Drude(1893 : 55). Open formations were detined as those having incomplete stability and 
heterogeneous composition, while closed formations have a definite uniform stamp. Willkomm (1896:29) made use of the same distinction in classifying the plant formations of Spain, and Pound and Clements (1898:321, 405) related the concept more closely to association. What is true of formations is equally true of vegetation, so that association may be regarded as open or closed with reference to the density and thoroughness with which the plants occupy the ground. In open association (associatio aperta), the ground is slightly or partially occupied, readily permitting the entrance of rew plants without the displacement of those already present. Such an arrangement is characteristic of the early stages of a formation, or of a succession of formations. It produces unstable open formations (sporcadophytia), ${ }^{1}$ which arise, usually after denudation, in sandhills, blowouts, gravel slides, dunes, flood plains, burned areas, etc. In closed as sociation (associatio clausa), occupation of the ground is com. plete, and the invasion of new species can occur only through displacement. Closed association results in stable, closed formations (pycnophytia), such as forest, thicket, meadow and prairie. As open association characterizes the early stages of a succession of formations, so closed association is peculiar to the later or last stages of all such successions. In short, open formations represent certain phases of the dynamic condition of vegetation, while closed formations correspond to the relatively final static conditions of vegetation. It is a fundamental principle of association that every succession from denudation, or from newly formed soils begins with open formations, and ends with a closed formation. The causes leading up to open and closed association are intimately connected with development, and will be considered under invasion and succession.

Species guild association (associatio peregrina). Drude (1885) bas distinguished a kind of association peculiar to invasion, in which there is a successive or concomitant movement of certain species of a formation into another

${ }^{1}$ Clements. F. E. A System of Nomenclature for Phytogeography Engler 31:b1 1902. 
formation or region, resulting in species guilds ${ }^{1}$ (Artengenossenschaften). The association in this case is largely one of community of origin or area, and of concomitance of migration. It is especially characteristic of areas adjacent to formational and regional limits. As it is a direct result of invasion, the species guild will be more fully considered under this principle.

Light association (associatio luminalis.) Schouw (1823: 166) divided plants into three groups upon the basis of light intensity in the habitats, using the terms plantae aphotistae, subterranean or darkness plants, plantae umbrosae, shade plants and plantae apricae, light or sunshine plants. Kabsch (1865: III) distinguished two classes, light plants and night plants, i. e., subterranean, dividing the former into sun and shade plants. Warming (1895: 15) designated sun plants as heliophilous, and shade plants as heliophobous. Pound and Clements (1900:166) applied the term photophilous to sun plants and sciophilous to shade plants, while Clements (1902: 166) has made a threefold division which corresponds essentially to Schouw's, namely, heliophyta, sciophyta and scotophyta.

The constituent species of formations show two fundamentally different groupings with respect to light In the one case, the individuals are on the same level, or nearly so, in such a way that each has direct access to sunlight. Such an arrangement is characteristic of most grassland and her. baceous formations. In the case of desert formations, there is often considerable difference in the height of the plants, but the distance between them is so great as to admit of direct illumination of all. This arrangement may be termed coordinate association (associatio superior). ${ }^{1}$ In forests, thickets, and many herbaceous wastes, the height and density of certain species enable them to dominate the formation. In a dense forest, the trees receive 1900 .

'Pound and Clements. The Phytogeography of Nebraska 2 ed. 175 
practically all the light incident upon the formation, and the shrubs, herbs, fungi and algae of lower habit and inferior position must adapt themselves to the diffuse light which passes through or between the leaves. The same is equally true of dense thickets and wastes, except that the vertical distance is less, and the diffuseness of the light is correspondingly modified. In these formations, the dominant trees, shrubs or herbs, the facies, constitute a primary or superior layer. The degree of $\mathrm{sub}$ ordinate associat i o (associatio inferior), as a result of which inferior layers will arise, will be entirely determined by the density of the facies. In open woodlands, which are really mixed formations of woodland and grassland, the intervals, and usually the spaces beneath the trees, also, are covered with poophytes, showing an absence of subordination due to light. This is the prevailing condition in the pine formation (Pinus ponderosa-xerohylium) of the ridges and foothills of western Nebraska. When, however, the trees stand sufficiently close that their shadows meet or overlap throughout the day, the increasing diffuseness begins to cause modification and rearrangement of the individuals. By photometric methods, the light in a forest is found to be least diffuse just below the facies, while the diffuseness increases markedly in passing to the ground. The taller stronger individuals are consequently in a position to assimilate more vigorously, and to become still taller and stronger as a result. Just as these have taken up a position inferior to that of the facies, so the shorter or weaker species must come to occupy a still more subordinate position. This results, not only because the light is primarily weaker nearer the ground, but also because the taller plants interpose as a second screen. The complete working out of this arrangement with reference to light produces typical subordinate association, which finds its characteristic expression in the layering of forests and thickets. Layers tend to appear as soon as open woodland or thicket begins to pass into denser conditions,and, up to a certain point, at which they disappear, they become the more numerous and the more marked, the 
denser the forest. In the Otowanie Woods (Quercus-Hicoria hylium), layering usually begins at a light value of .1 (1= normal sunshine in the open). Thornber ${ }^{1}$ has found the same value to obtain in the thickets of the Missouri bluffs. In these, again, layers disappear at a value of .005, the extreme diffuseness making assimilation impossible except for occasional mosses and algae. A number of herbaceous plants are present in the spring, but these are all prevernal or vernal bloomers, which are safely past flowering before shade conditions become extreme. In the Fraxinus-Catalpaalsium, all inferior holophytic vegetation disappears between the light value of .004 and that of .003. The spruce-pine formation (Picea-Pinus-hylium) of the Rocky Mountains, with a light value of .01, usually contains but a few scattered herbs, mostly evergreen: in some cases there are no subord. inate plants, other than mosses and hysterophytes. The lodge-pole pine formation (Pinus murrayana-hylium) with light values often less than .005, is nearly or quite destitute of all but hysterophytic undergrowth. Such extremely dense formations are examples of coordinate association merely, since the formation is reduced to a single superior layer, in which the individuals of the facies bear the same spatial relation to incident light. In layered formations, in addition to the subordinate relation of other species to the facies, there is, of course, a kind of coordinate association manifested in each layer.

Water content association (associatio aqualis). De Candolle (1820:14) summarized the characteristics of water plants and dry land plants, without, however, recognising them as two distinct groups. Schouw (1823:157) was the first to give definite expression to the value of the moisture content of the soil in the grouping of plants. He established three groups: (1) swamp plants (plantae paludosae) including also slime plants (plantae limosae), which he regarded as passing readily into aquatic plans (plantae emersae); "plants which grow in moist meadows" (plantae uliginosae, 'Thornber, J. J. The Prairiegrass Formation in Region I. Rep.
Bot. Surv. Neb. 5:36, 461901 
but also termed Bruchpflanzen!; (3) “"plants that love a dry soil (plantae soli aridi). In distinguishing plants with respect to modium, Schouw divided water plants into plantae vera aquatzcae s. plantae submersae, which he termed Hydrophyta, and plantae aquaticae spuriae sive plantae emersae. The groups, plantae marinae and plantae aquae dulcis, are based upon the chemical properties of the water, and the second group is further analysed into plantae lacustres, fuviales, fontanae, stagnariae, etc. Species growing in saline situations are represented by strand plants (plantae littoralis $v$. maritimae), while, in arranging plants according to the chemical nature of the soil, salt plants are designated as plantae salinae, Halophyta. Meyen (1836:50), though in the main following Schouw very closely, includes all aquatic plants under Hydrophya. To Thurmann (1849) belongs the great credit of perceiving, at a time when the chemical nature of the soil was thought to determine the character of the vegetetion, that the physical properties really control the water content, and are hence of the first importance. He divided soils into two classes; eugeogenous, those that disintegrate readily and completely, and hence are water-retaining, and dysgeogenous, those that strongly resist weathering and decomposition, and in consequence lose water readily. Plants growing on ougeogenous soils were termed hygrophilous, and those in dysgeogenous soils, xerophilous, while those which seem to grow indifferently in either were called ubiguitous. Thurmann understood clearly that it was the degree of decomposition and aggregation, and not the chemical nature of the rocks, which determined the last group, as so-called silicious species were found to grow on calcareous soils when these were loose, and calcareous species on silicious soils that had become compact. A. De Candolle (1855:423), without attempting a grouping of plants with reference to water content, which lay outside his field of botanical geography, none the less clearly noticed this fundamental difference in soils, as the following will show: "Ily a des minéraux qui se réduisent plus ou moins facilement en terre ou en sable, et qui offrent des conditions de ténacité particulières: 
come current. Ascherson (1883:784), in a consideration of De Candolle's groups, first employed the word xerophyta, as a substitute for xerophilae. Wiesner (1889:79) made use cf the terms, hydrophyta, hygrophyta, and xerophyta, including halophyta in the latter.

Warming (1895:116) gave a great impetus to the investigation of the ecological aspects of phytogeography by making the fundamental water content classes the basis of his entire treatise. He considered salt plants to be distinct from xerophytes, establishing in all four types of vegetation.

"I. Die Hydrophytenvegetation. Diese ist eine extreme Vegetation, deren Pflanzen entweder ganz oder gröszenteils von Wasser umgeben sind oder in einem sehr wasserreichen Boden wachsen (der Prozentgehalt an Wasser beträgt vermuthlich mehr als 80.)

II. Die Xerophytenvegetation ist das entgegengesetzte Extrem, dessen Pflanzen auf Felsboden oder, jedenfalls während eines längeren Zeitraumes im Jahre, in wasserarmem Boden und in trockner Luft wachsen. Der Wassergehalt kann gewisz, wenn er am geringsten ist, unter $10 \%$ betragen.

III. Die Halophytenvegetation schliesst sich morphologisch an die vorige nahe an, verdient aber, für sich aufgestellt zu werden, eine Meinung, die unter anderem durch Stahl's Untersuchungen bekräftigt wird. Sie ist eine sehr extreme Vegetation, die an Salzboden gebunden ist und deren morphologische Eigenthümlichkeiten ebenfalls durch die Regulierung "der Transpiration verursacht zu sein scheinen.

IV. Die Mesophytenvegetation umfasst die Vereine, die an Boden und Luft von mittlerer Feuchtigkeit angepasst sind, an einen Boden, der sich auch in dera Salzgehalte nicht auszeichnet. Die Pflanzen sind in morphologischer und in anatomischer Hinsicht nicht besonders extrem ausgeprägt."

Pound and Clements (1898:94), rejecting the term mesophytes, established six groups, the first three, hydrophytes, xerophytes and halophytes, corresponding to Warming's, while the last three, hylophytes, poophytes and ruderal plants 
(aletophytes), were formed by dividing the mesophytes, largely upon the basis of light association. Schimper (1898: 3) merged halophytes in xerophytes as only one of several subdivisions of the latter, and termed mesophytes tropophytes, on account of the alternation of wet and dry conditions during their period of growth. In postulating the dictum, "E s muss zwischen physikalischer und physiologischer Trockenheit, bezw. Feuchtigkeit untersch e i d en werd en; letztere allein kommt für das Pflanzenleben, also auch für die Pflanzengeographie, in Betracht," Schimper placed the study of vegetation upon a new basis. Pound and Clements (1900:169) followed the division into the three fundamental groups, hydrophytes, mesophytes and xerophytes, retaining hylophytes, poophytes and aletophytes as subdivisions of mesophytes.

Warming's great contribution to ecology lies in his full recognition of the inherent value of water content association as its basis, and in making a logical and systematic treatise out of the seattered results of many workers. The exceeding timeliness of his work made it practically certain that his views would influence the general character of ecological research for a long time to come. The weak points in his system were the treatment of humic acid (bog) plants under hydrophytes, and the separation of halophytes from xerophytes as a coordinate group. This by no means indicates that Warming failed to see the contradiction involved in the occurrence of xerophytes in apparently hydrophytic situations, or to appreciate the many points of similarity between xerophytes and halophytes. On the contrary, he has summed up (176) in what was for that time a very lucid manner, the status of the relation of swamp xerophytes to their habitat. It remained, however, for Schimper to perceive the essential similarity of this relation, as determined by Johansen for bog plants, with that found in crymophytes by Kihlmann (1890) and Goebel (1891), and in halophytes by himself $(1890,1891)$, and to give expression to the fundamental law of habitats, namely, that it is the physio- 
logical water content, and not the physical, which determines the impress of the plant and of the formation. Accepting the easily demonstrable fact that an excess of salts or acid in the soil water, as well as cold, tends greatly to diminish the available water of the soil, i. e., the physiological water content, it is at once seen why saline, bog and arctic plants are as truly xerophytic as those that grow on rocks or in desert sands. An anomalous case, which, however, physical factor records have explained fully, is presented by many plants growing in alpine gravel slides, strands, blowouts, sandbars, etc., in which the water content is considerable, but the water loss excessive, on account of extreme heat or reduced air pressure. The effect of these conditions is to produce a plant xerophytic as to its aerial parts, and mesophytic or even hydrophytic as to subterranean parts. Such plants may from their twofold nature

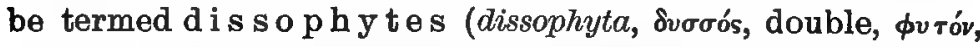
ró, plant): they are especially characteristic of dysgeogenous soils in alpine regions where transpiration reaches a maximum, but are doubtless to be found in all gravel and sand habitats with high water content. With these corrections, the concept of water content association, which owes much to both Warming and Schimper, but is largely to be credited to Thurmann, becomes completely and fundamentally applicable to all vegetation.

The discrepancies in Warming's arrangement which have just been mentioned have led to several attempts to rest a fundamental distinction of groups upon some other factor than that of water content. The most satisfactory of these are the developmental classifications, such as those of Hult, Graebner, Meigen and Cowles (termed physiographic by the latter), and the nutrition content classification of Graebner. Both classifications are valuable, especially the developmental, because they furnish other view points of the many relations of formations, but they have in no wise the fundamental value of the classification based upon water content. The naturalisation and stabilisation of invading species by 
which the developmental series is established are determined in the first degree by the physiological water content of the habitat, while a careful study of the nutrient content groups of Graebner will suffice to show that these depend in the first place upon water, and only in a secondary degree upon nutrition values.

Up to the present time, the general character of the habitat, together with the gross appearance of the plant itself, has been thought sufficient to determine the proper position of a plant or a formation in the water content classification. Such a method is adequate, however, only for plants and formations which bear a distinct impress. For an accurate classification into the three categories, hydrophytes, mesophytes and xerophytes, it is necessary to make exact determinations of the normal physical and physiological water of the habitat, and to supplement this, in some degree at least, by histological studies. Except in the case of saline, acid and frozen soils, the physical water content alone will be a fairly accurate index, especially in habitats of similar soil composition. For an exact and comprehensive classification, however, and particularly in comparative work, the physiological water content must constitute the sole criterion. The physical water content is readily obtained by taking soil samples from day to day, or from time to time throughout the growing season. ${ }^{1}$ These samples are taken from the desired depth, usually from the layers in which the root activities are greatest, in so far as this may be determined, placed in air tight cans and weighed as soon as possible. The cans are then opened and oven-dried at a temperature of $40^{\circ} \mathrm{C}$ for three days, though the time is much less for porous gravels and sands, and is sometimes greater for the most compact clays. When thoroughly dried, the samples are again weighed, the difference between the two weights giving the total amount of water in the soil, i. e., the physical water. Having determined the latter, a second soil sample is taken at a time

'Pound and Clements 1.c. 2 ed. 1671900 
when the species to be investigated is completely wilted, and the amount of water present is determined as before. This is the non-available water. The physiological water content is then obtained by subtracting the amount of non-available water from the physical water content. The one drawback to this method is the difficulty of causing the plant to wilt in nature. This can be done only by cutting the soil in such a way as to destroy the capillarity, and by covering it in such a way during times of precipitation that there is no access of moisture. A more feasible method is to transfer the plant in its own soil to the planthouse, where the physical factors may be accurately controlled, but this can be used only in the case of relatively small plants. As the absorptive power varies widely in different individuals of the same plant, it is imperative that water content experiments be conducted only with normal healthy plants at the time of maturity. Some preliminary work in the accurate investigation of the water content of different habitats has been done by two of the writer's pupils: Thornber (1901) has investigated the comparative water content values of woodland and prairies, while Hedgcock (1902) has made a special study of the physiological water of various soils. The present characterisation of many plants and formations as hydrophytic, mesophytic or xerophytic must be regarded as largely tentative, and the final classification will be possible only after the thorough quantitative investigation of their habitats. ${ }^{1}$

The relation to water content association of the concept underlying the groups, hylophytes, poophytes and aletophytes, established by Pound and Clements in 1898, is a complex one. This idea, which Schimper (1898:173) recognized later in the same year in the corresponding divisions, Gehölz, Grasflur and Wüste, is a valid one; though involving

1 The methods and instruments employed in the exact quantitative determination of water content, as well as humidity, temperature, light, wind, etc., in the various formations of Nebraska and Colorado are treated in detail in a paper entitled, "The Physical Factors in Ecology." This paper was presented before the Botanical Society of America at its Denver meeting in 1901, and will soon be published. 
light association in a certain degree in hylophytes and poophytes, it really represents the fundamental zonation of vegetation with reference to water, in which the first zone, the ocean (hydrophytes), has been ignored. The proper series, then, is ocean, forest, grassland and desert, corresponding exactly to hydrophytes, mesophytes and xerophytes, in which mesophytes, largely because of differences of water content and humidity, but also on account of light, break up into hylophytes (forest) and poophy tes (grassland). The same concept is thus seen to underly both series, the apparent difference in the two arising simply from the fact that the one, ocean, forest, grassland and desert represents the fundamental structure of vegetation, while the other, hydrophytes, hylophytes, poophytes and xerophytes, emphasizes the causative factors.

From the above, it follows that Schimper's so-called climatic formations, forest, grassland and desert, are merely a somewhat incomplete expression of water content association. As to the validity of his division of all formations into climatic and edaphic, there is also room for grave doubt. ${ }^{1}$. While there can be no question of the existence of great formations, or series of formations, which bear a distinctive impress, yet one may well hesitate to apply the term climatic to them. The conditions which have to do with the water supply of a plant are of so much more importance in determining its character than those which regulate the water loss, that all plants, and consequently the formations which they constitute, are primarily influenced by soil, i. e., they are edaphic. In the ultimate analysis, however, species and formations owe their peculiar impress to a certain constant relation between the mean of climatic conditions and the mean of edaphic ones. This relation between the soil and the atmosphere must be as constant in the case of an extensive formation as in that of a restricted one; otherwise, the formation will change character. The difference between the two formations, other than a different 1899 .

${ }^{1}$ Clements, F. E. Schimper's Pflanzengeographie Science 9:747 
relation between soil and climate, is merely one of extent, arising out of the fact that a certain correspondence of soil and climate prevails over a larger or smaller area, as the case may be. Truly edaphic formations are possible only where the soil moisture is so great as to make water loss entirely negligible as a factor capable of producing xerophytic modification or a tendency toward it. This is the case only in hydrophytic situations. Hydrophytic species are cosmopolitan; they are much the same the world over, in a very moist or in a very dry climate, in heat or in cold. Water loss is normally never great enough to seriously threaten the water supply. With mesophytes and xerophytes, it is very different. Here there is constantly a limit to the water supply, and the production of mesophytic or xerophytic vegetation depends upon the nearness with which the water loss approaches the water supply. Hydrophytic formations might, then, be regarded as truly edaphic, in a sense, though mesophytic and xerophytic formations cannot properly be called climatic. Any distinction at all on such grounds is at present gratuitous. As is true of all fundamental problems in phytogeography, it must await the general employment of quantitative methods. While our knowledge of soil factors is in an extremely elementary condition, we have no exact understanding whatsoever of the sum of physical factors, which we term climate. According to Schimper's idea, as well as in the general opinion, edaphic or soil factors are thought to vary greatly, while climatic ones are thought to be constant over vast areas. The investigations of the author during the last five years indicate conclusively that this is erroneous, and that soil factors are relatively, less variable and climatic ones more variable, as would be expected. The whole question awaits the labors of many investigators through terms of years. 


\section{* BIBLIOGRAPHY}

1751 Linné, C. von

1754 Hedenberg, A.

1805 Humboldt, A. von

1806

1815 Brisseau-Mirbel, C. F.

1820 De Candolle, A. P.

1823 Schouw, J. F.

1836 Meyen, F. J. F.

1843 Grisebach, A.

1849 Thurmann, J.

1855 De Candolle, A.

1863 Kerner, A.

1865 Kabsch, W.

1872 Grisebach, A.

1873 Le Grand, A.

1874 Contejean, C.

De Candolle, A.
Philosophia Botanica.

Stationes Plantarum Amoen. Acad. 4:64.

Essai sur la Géographie des Plantes.

Ideen zu einer Physiognomik der Gewächse.

Elémens de Physiologie Végétale, et de Botanique.

Essai Elémentaire de Géographie Botanique.

Grundzủge einer allegemeinen Pflanzengeographie.

Grundriss der Pflanzengeographie

Bericht über die Leistungen in der Pflanzengeographie

Essai de phytostatique appliquée à la chaine du Jura et aux contrées voisines

Géographie Botanique Raisonée

Pflanzenleben der Donauländer

Das Pflanzenleben der Erde Die Vegetation der Erde

Statistique Botanique de Ferez Ann. Soc. Agr. Loire $17: 290$

De l'influence du terrain sur la végétation Ann. Nat. Sci. $5: 20: 266$

Constitution dans le regne végétal de groupes physiologiques applicables à la géographie ancienne et moderne Arch. Sci. Phys. Nat. 1874:5 
1879 Engler, A.

1882 Goeze, E.

1883 Ascherson, P.

1885 Drude, 0.

1886

1888 Kerner, A. 1889 Drude, O.

1889 Wiesner, J. 1890 Drude, $\mathrm{O}$.

Schimper, A.F. W.

Kihlmann, A. O.

1891 Schimper, A. F. W

1892 Goebel, K.

MacMillan, C.

Warming, E.

1893 Engler \& Drude

1895 Warming, E.
Versuch einer Entwicklungsgeschichte der Pflanzenwelt Pflanzengeographie

Von der Verbreitung der Pflanzen Leunis Synopsis $1: 724$.

Die Vertheilung und Zusammensetzung ostlicher Pflanzengenossenchaften in der Umgebung von Dresden Isis

Die systematische und geographische Anordnung der Phanerogamen Schenck Handbuch $3: 487$

Pflanzenleben

Über die Principien in der Unterscheidung von Vegetationsformationen Engler $11: 21$

Biologie der Pflanzen

Handbuch der Pflanzengeog. raphie

Über Schutzmittel des Laubes gegen Transpiration Monatsb. Akad. Berl. 7

Pflanzenbiologische Studien aus Russisch-Lappland

Die indo-malayische Strandflora

Pflanzenbiologische Schilderungen II

The Metaspermae of the Minnesota Valley

Lagoa Santa

Die Vegetation der Erde Engler 17 :b55

Plantesamfund 
1896 Drude, 0.

Willkomm, M.

1898 Pound \& Clements

Schimper, A. F. W.

Graebner, P.

Nilsson, N. H.

Delpino, F.

1900 Pound \& Clements

1901 Cowles, H. C

1902 Clements, F. E.
Deutschlands Pflanzengeographie

Grundzüge der Pflanzenverbreitung auf der Iberischen Halbinsel

The Phytogeography of Nebraska

Pflanzengeographie auf physiologischer Grundlage

Gliederung der Westpreussischen Vegetationsformationen Schrift. Nat. Ges. Danzig $9: 43$

Einiges über die Biologie der schwedischen Sumpfpflanzen Bot. Cent. $76: 9$

Stadi di geografia butanica secondo un nuovo indirizzo Mem. R. Acc. Sci. 5:7:329

The Phytogeography of Neb. raska 2 ed.

The Physiographic Ecology of Chicago and Vicinity Bot. Gaz. 31:73

A System of Nomenclature for Phytogeography Engler $31: \mathrm{b} 1$ 


\section{INVASION}

By invasion (Goeze 1882:109) is understood the movement of plants from an area of a certain character into one of a different character, and their colonization in the latter. This movement may concern an individual, a species or a group of species. From the nature of invasion, which contains the double idea of going into and taking possession of, it usually operates between contiguous formations, but it also takes place between formational zones and patches. More rarely and less noticeably, there may be invasion into a remote vegetation, as a result of long carriage by wind, water, birds, railroads or vessels. Movement or migration, however, represents but one of the two ideas involved in invasion. Migration merely carries the spore, seed or propagule into the area to be invaded. In ecesis, ${ }^{1}$ the spores or seeds germinate and grow, after more or less adjustment and, in case the latter becomes sufficiently complete, the new plants reproduce and finally become established. With all terrestrial plants, invasion is possible only when migration is followed by ecesis, because of the inherent differences of formations or of areas of the same formation. In the case of surface floating forms, such as Lemnaceae, and of the plancton, ecesis is of much less importance, on account of the uniformity of the medium and the lack of attachment, and migration is often practically synonymous with invasion.

\section{MIGRATION}

Migration has been sometimes used loosely as a synonym for invasion, but it is here employed in its proper sense of removal or departure, i. e. movement, and is contrasted with ecesis, the making of a home, the two ideas being combined in invasion, which is a moving into and a taking possession of. An analysis of migration reveals the presence of four factorq, mobility, agency, proximity and topography. Not all of these are present in every instance of migration, as for example in the simple elongation of a rootstalk, but in the great majority of cases each plays its proper part.

\footnotetext{
${ }^{1}$ From the Greek, oikîбts, the act of coming to be at home, henc
} adjustment to the habitat, oikos. 
Mobility represents the inherent capacity of a plant for migration, and in its highest expression, motility, is in itself productive of movement. As a general rule, however, modifications for securing mobility are ineffective in the absence of proper agents, and the effective operation of the two will be profoundly influenced by distance and topography.

The historical development of the concept of migration has been extremely fragmentary, although its recognition dates back to the beginning of phytogeography. The need for a systematic consideration of the subject has been obscured by the greater interest taken in the origin and naturalization of cultivated plants. Linné (1745:2) first mentioned migration briefiy as follows: "Migrarunt ad floram nostram ab Exteris regionibus multae plantae, quae suas in Suecia fixere sedes ad littora praesertim \& oras maritimas". In the Philosophia Botanica (1752:86), he has made an excellent analysis of dissemination, distinguishing wind-distributed parts (Volitantia) as pappose, comate, winged and inflated, mechanically propelled seeds (Elasticae) as contractile, mucronate, fibrillar and creeping, and finally, parts with devices to secure attachment as those having hamate calyx, pericarp or seeds. He indicated, moreover, that animals distribute seeds by deglutition and mastication, while streams, seas, tides, lakes and rains all aid in dissemination. Humboldt (1805:22) was apparently the first to employ the terms migration and mobility, but he did this only incidentally in connection with the enforced migration of cultivated plants. Mirbel (1815:348) treated briefly of dissemination by elasticity of the pericarp and by winds, water, birds, mammals and man, drawing the larger number of his illustrations from Linné. He also made a detailed classification of seeds and fruits $(593,796)$ according to their form and surface. DeCandolle (1820:45) recognized the primary importance of natural barriers (barrières) in gradual migration, considering at some length the obstructive effect of large bodies of water, deserts, mountain ranges and radically different vegetation, 
and he pointed out that plants are able to pass barriers to a greater or less degree by reason of natural or artificial means of transport, such as water, winds, animals and man. Link (1820:263) observed the descent of alpine plants into river valleys, pointing out the agency of mountain streams, and indicating also the activity of ocean currents in bringing about transportation, Schouw's silence in regard to migration would be inexplicable in view of the fact that his book is largely a treatise on distribution, were it not for a chance statement (1823:182) in which he speaks of "Die Anhänger der Migrationstheorie", and a note (184) in which he says: "Diese Theorie ist in der neuesten Zeit von Männern als DeCandolle, Link, und von Buch vertheidigt worden". Meyen (1836:39) considered briefly the currents of the air and ocean as transportive agents, but added little of importance to Link's account. DeCandolle (1832:595) considered the natural dissemination of seeds under the following heads: fruits pseudospermes (achenes, etc.) in which the fructification plays a special part by the elongation of the peduncle, the opening of the involucre or the swelling of the receptacle, or the fruit is itself pappose, barbed, etc.; fruits charnus (fleshy fruits); fruits capsulaires, and des plantes hypocarpogées. Henfrey (1852:50) has discussed the agents of dissemination in an interesting manner, though he did not contribute any new matter, with the exception of a short treatment of barriers. A. DeCandolle (1855:613) considered the causes of transport in a most minute though somewhat unsystematic fashion. His book is a mine of details, especially in regard to cultivated species. He was the first to point out that, as a rule, barriers are obstacles to naturalization rather than to migration. Darwin (1859:624) brought forward in a chapter on the means of dispersal a large number of experiments and observations relating to the transport of seeds by ocean currents, birds and other animals. Kabsch (1865:543) did not attempt a consideration of all transportive agents, but he gave particular attention to the action of water currents and of glaciers. 
Hildebrand (1873) brought together the results of various observers in a treatise on dissemination, which is still the most extensive and the most important in this field. He divided distributive agents into winds, water, animals, dessication, and developmental feutures which effect distribution, such as turgescence, growth and motility, making a minute analysis of the contrivances under each. He classified contrivances for wind distribution as: (a) smallness and lightness, (b) winged structures, (c) hairy and feathery modifications of the seed, fruit, style, peduncle, rhachis or glumes. Adaptations for water distribution are relatively few, consisting mostly of air-containing tissues or organs. Animal-distributed parts are divided into: (a) fleshy fruits, (b) hooked fruits and (c) glandular fruits. The author furthermore discussed the significance of the lack of useless modifications, the distribution of dissemination contrivances in the various groups and their influence upon migration. Grisebach (1877:4) established a fundamental law of barriers as follows: "La loi suprême servant de base a l'établissement persistant de flores naturelles, doit donc être reconnue dans les barrières qui en ont entravé 'ou completement empêché le mélange," and he illustrated its application briefly in a few general cases. He considered the general facts of migration of arctic-alpine plants and of forest and steppe plants, but more with reference to the interchange of species between regions than with respect to the methods and agents ${ }_{y}^{\text {in }}$ dispersion. Comber (1874:248) endeavored to relate modifications for dissemination, such as coma, pappus, spines, etc. to the geographical area of the species of the British flora, but, while this method deserves a more thorough trial, his results are too conflicting to be of much value.

An extremely important contribution to the subject is that of Engler (1879), but as this work deals with invasion as a whole rather than with either of its parts, migration or ecesis, consideration of it will be reserved for another place. Goeze (1882:96) grouped migration agents in two classes, the one including the various aqueous agents, currents, streams, 
rains, etc., and the other comprising winds, animals and man. Hult (1886:bl) andeavored to show that moss spores and, in consequence, the spores of all cryptogams are not disseminated by the wind over great stretches. His evidence, however, is drawn entirely from distribution, with little regard to the unique importance of ecesis, and hence it affords no real support to his contention. He has made much of the fact that the mosses which invade a denuded area are mostly drawn from neighboring formations, but this signifies merely that the wind carries the greater number of spores a short distance rather than a long one. Huth (1887) wrote a comprehensive treatise upon houked and retrorsely barbed fruits, enumerating the examples in a long list of families and indicating their relation to distributive agents. Dingler (1889:331) investigated the comparative transportability of wind-distributed seeds'and fruits, as determined by their velocity in falling. As a result of his experiments, he made the following division of disseminules: (1) dustlike, Micrococcus, spores, Lycoperdon; (2) grainlike, Papaver, Sibbaldia; (3) bladder-inflated, Astragalus, Ostrya. (4) filifurm, Bromeliaceae; (5) disciform, Iris, Ulmus; (6) convex-disciform, Ptelea; (7) parachute-like, Taraxacum, Valeriana, Asclepias, Salix, Dryas, Pulsatilla; (8) winged-turbiniform, Rumex; (9) elongate-lamelliform, Ailanthus, Tecoma; (10) elongate-lamelliform, seed at the side, Bignonia; (11) elongate-lamelliform, seed at the end, Fraxinus; (12) elongate-lamelliform, seed at end but one side also weighted, Acer, Pinus. Wiesner (1889:105) summarized briefly but somewhat comprehensively the subject of migration, while Drude (1890:100) touched it in a more general way.

Kerner (1892:791) has furnished an account of disseminination which rivals that of Hildebrand. The wealth of detail especially is something extraordinary. Disseminules are grouped under two main heads: offshoots, and fruits and seeds, though the author recognizes that whole plants are sometimes distributed. Under offshoots are considered mycelia, protonemata, roots, root-stalks, runners, hormogones, zoogonidia, soredia, gemmae, buds, etc., while their 
dispersal is treated with respect to motility, growth, water, wind, expulsive mechanisms, animals and man. Under fruits and seeds, there is recognized a large class of slingfruits in which propulsion is effected by turgidity, dessication, torsion or elasticity, and a small group of creeping and hopping fruits. With respect to agents, Kerner treats of dissemination by water, including drainag, by water and wind, and by wind alone, distinguishing under the latter, rolling and flying fruits, which are flattened, winged, comate or parachute-like. Migration "by the agency of animals is by means of deglutition, concealment, or attachment, the latter occurring by means of mud, water, mucilage, glandular hairs or barbed processes. Attention is also given to the elongation of peduncles and to other contrivances by which seeds are placed in a position to be more effectively influenced by distributive agents. Excellent monographs of the dissemination contrivances in particular families have been made by Dammer (1893:260) for Polygonaceae and by Buchwald (1895:494) for Leguminosae. Pound \& Clements (1898: 153) grouped contrivances for wind distribution in four classes; (1) comate and pappose seeds; (2) winged seeds; (3) winged fruits; (4) tumbleweeds. Water distribution is by streams or by surface drainage, while dissemmation by means of animals occurs by deglutition, carriage or attachment. Schimper (1898:32) has simply considered the general relation of wind and water to dissemination.

Mobility (mobilitas) denotes potentiality of migration as represented by modifications for this purpose. It corresponds, in a sense, to dissemination, though seed production also enters into it. Its most perfect expression is found in those plants which are themselves motile, Bacteriaceae, Oscillatoria, Volvocacede, and Bacillariaceae, or possess motile propagules, such as most Phycophyta. On the other hand it is entirely undeveloped in many plants with heavy unspecialized seeds and fruits. Between these two extremes lie by far the greater number of plants, exhibiting the most various degrees of mobility, from the motile though almost immobile offshoots of many Liliaceae to the immotile but very 
mobile spores of fungi. It is thus seen that motility plays a relatively small part in migration, being practically absent in terrestrial forms, and that it bears a very uncertain relation to mobility. In analysing the latter, contrivances for dissemination are seen to determine primarily the degree of mobility, whlle the number of seeds produced will have an important effect in increasing or decreasing it. A third factor of considerable importance is also involved, namely, position with reference to the distributive agent, but any exact knowledge of its importance must await systematic experiment somewhat after the methods of Dingler, but with air-currents, etc., of known velocity and direction. The time is not distant when by such methods it will be possible to establish a coefficient of mobility, derived from terms of position, weight, resistant surface, and trajectory for definite wind velocities or for particular propulsive mechanisms.

Plants exhibit considerable diversity with reference to the part or organ modified, or at least utilized, for dissemination. This modification, though usually affecting the particular product of reproduction, may, in fact, operate on any part of the plant and in certain cases, upon the entire plant itself. In the majority of plants characterized by alternation of generations, the same individual may be disseminated in one generation by a reproductive body, and in the other by a propagative one, as is the case in the oogones and conidia of Peronospora, the spores and gemmae of Marchantia, the fruits and runners of Fragaria, etc. Special modifications have, as a rule, been developed in direct connection with spores and seeds, and mobility reaches its highest expression in these. It is, on the other hand, greatly restricted in offshoots and plant bodies, at least in terrestrial forms, though it will now and then attain a marked development in these, as shown by the rosettes of Sempervivum and the tumbling plants of Cycloloma. For the sake of convenience, in analysing migration, all plants may be arranged in the following groups with reference to the organ or part distributed. 


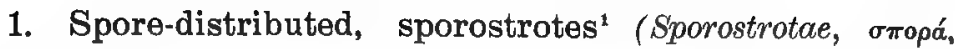
$\dot{\eta}$, spore, $\sigma \tau \rho \omega \tau$ cós, $\dot{\eta}$, óv, strewn.) This includes all plants possessing structures which go by the name of spore, such as the acinetes of Nostoc and Protococcus, the zoogonidia of Olothrix, Ectocarpus, etc., the conidia, ascospores and basidiospores of fungi, the tetraspores of red seaweeds, and the gemmae and spores proper of liverworts, mosses and ferns. These are almost always without especial contrivances for dissemination, but their extreme minuteness results in great mobility.

2. Seed-distributed, spermatostrotes (Spermatostrotne, $\sigma \pi \epsilon_{p} \mu a$, ró, seed). This group comprises all flowering plants in which the seed is the part modified or at least disseminated. The mobility of seeds is relatively small, except in the case of minute, winged or comate seeds.

3. Fruit-distributed, carpostrotes (Carpostrotae, картós, \&, fruit). The modifications of the fruit for distribution exceed in number and variety all other modifications of this sort. All achenes, perigynia, utricles, etc., properly belong here.

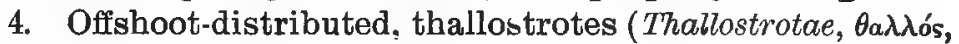
$\delta$, shoot). To this class are referred those plants, almost exclusively cormophytes, which produce lateral, branch-like propagules, such as root-sprouts, rhizomes, runners, stolons, rosettes, etc. Migration with such plants is extremely slow, but correspondingly effective, since it is almost invariably followed by ecesis.

5. Plant-distributed, phytostrotes (Phytostrotae, фvтóv, ró, plant). This group includes all plancton and surface forms, whether motile or non-motile, and those terrestrial plants in which the whole plant, or at least the aerial part, is distributed, as in tumbleweeds and in many grasses.

Any investigation of migration to be exact must confine itself to fixed forms, and for these the degree of perfection

${ }^{1}$ Throughout the present paper, Greek or Latin equivalents have been proposed for practically all terms, because of the conviction that a uniform international nomenciature is as imperative and as certain for phytogeography, as it has been for taxonomy. The English form, however, is given precedence, since it alone will suffice for the majority of English-speaking botanists. 
shown by dissemination contrivances corresponds almost exactly to the degree of mobility, Because of the difficulty of ascertaining the effect of ecesis, it is impossible to deter. mine the actual effectiveness in nature of different modifications, and the best that can be done at present is to regard mobility, together with the occurrence and forcefulness of distributive agents, as an approximate measure of migration. The general accuracy of such a measure will be more or less evident from the following. Of 118 species common to the foothill and sandhill regions of Nebraska, regions which are sufficiently diverse to indicate that these common species must have entered either one by migration from the other, 83 exhibit modifications for dissemination. while 8 others, though without special contrivances, are readily distributed by water, and 4 more are mobile because of minuteness of spore or seed. Some degree of mobllity is present in 73 per cent of the species common to these regions, while of the total number of species in which the mode of migration is evident, viz. 95, 66 per cent are wind-distributed, 20 per cent animal-distributed, and 14 per cent are water-distributed. It need hardly be noted that this accords fully with the prevalence and forcefulness of winds in these regions. Of the species peculiar to the foothill region, many are doubt. less indigenous, though a majority have come from the montane regions to the westward. The number of mobile species is 121 , or 60 per cent of the entire number, while the number of wind-distributed ones is 85 , or 70 per cent of those that are mobile. Among the 25 species found in the widely separated wooded blufi and foothill regions, 2 only, Amorpha nana and Roripa nasturtium, are relatively immobile, but the minute seeds of the latter, however, are readily distributed, and the former is altogether infrequent.

The following groups of plants may be distinguished according to the character of the contrivance by which dissemination is secured.

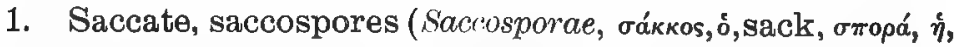
seed, fruit). Here are to be placed a variety of fruits, all of which agree, however, in having a membranous envelope, 
or an impervious, air-containing pericarp. In Ostrya, Physalis, Staphylea, the modification is for wind-distribution, while in Carex, Nymphaea, etc., it is for water-transport.

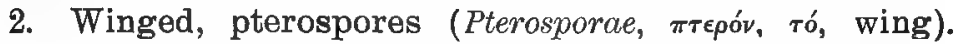
This group includes all winged, margined, and flattened fruits and seeds, such as are found in Acer, Betula, Rumex, many Umbelliferae, Graminaceae, etc.

3. Comate, comospores (Comosporae, кó $\eta$, $\dot{\eta}$, the hair of the head). To this group belong those fruits and seeds with long silky hairs, Gossypium, Anemone, Asclepias, etc., and those with straight capillary hairs or bristles not confined to one end, Typha, Salix, etc.

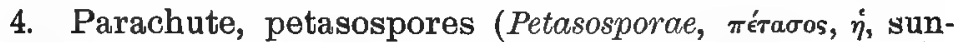
shade, umbel). The highly developed members of this group, Taraxacum, Lactuca, and other Ligulitiorae are connected through Senecio and Eriophorum with the preceding. These represent the highest development of mobility attained by special modification.

5. Chaffy-pappose, carphospores (Carphosporae, кápфos, тó, scale, husk of fruit). In this group are placed those achenes with a more or less scaly or chaffy pappus with slight mobility, as in Rudbeckia, Brauneria, Helianthus, etc.

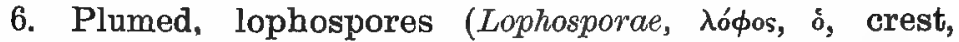
plume). In the fruits of this class, the style is the part usually modified into a long plumose organ, possessing a high degree of mobility as in Pulsatilla, Sieversia and Clematis.

7. Awned, acospores (Acosporae, $\stackrel{\alpha}{\kappa} \eta \dot{\eta}, \dot{\eta}$, point). These are almost exclusively grasses, in which the awns serve for distribution by wind, water or animals, and even, according to Kerner, by hygroscopic creeping movements. The mobility in many cases is great.

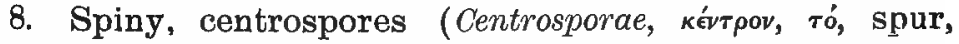
spine). This group contains a few representatives which possess a moderate degree of mobility by attachment, as in Tribulus and Cenchrus.

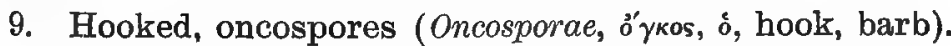
The members of this group are extremely numerous, and 
the degree of mobility as a rule is very high. All exhibit in common the development of hooks or barbs, by which they are disseminated in consequence of attachment, though the number, size and disposition of the hooks vary exceedingly.

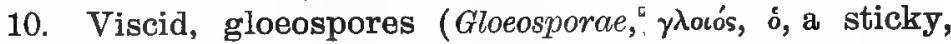
clammy stuff). In these, the inflorescence is more or less covered with a viscid substance, as in species of Silene, or the fruit is beset with glandular hairs, as in Cerastium, Salvia, etc.

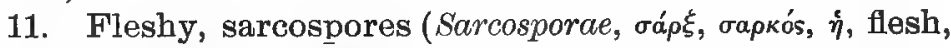
pulp of fruit). These are intended for dissemination by deglutition, largely by birds; the effectiveness of the modifcation depends in a large degree upon the resistance of the seed envelope to digestion. The mobility varies greatly. but the area over which migration may be effected is large.

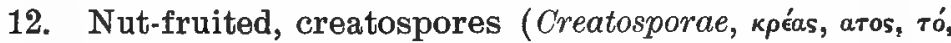
meat). This group includes those plants with nut fruits, which are carried away and secreted by animals for food.

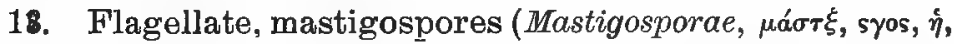
lash). These are plants with ciliate or flagellate propagative cells, i.e. zoogonidia, as in Protococcus, Ulothrix, Oedogoninm, Ectocarpus, etc., or with plant bodies similarly motile, Bacteriaceae and Volvocaceae.

The position on the plant of the organ to be disseminated, i.e., its exposure to the distributing agent, plays a considerable part in determining the degree of mobility. In the majority of plants, the position of the inflorescence itself re. sults in maximum exposure, but in a large number of forms special modifications have been developed for placing the spores or seeds in a more favorable position. In both cases, there are often present also devices for bringing about the abscission of the seed or fruit. It is moreover self-evident that the height of the inflorescence above ground or above the surrounding vegetation is likewise of considerable im. portance in increasing the trajectory. It is yet too early to make a complete classification of contrivances for placing disseminules in the most favorable exposure, but the following will serve as a basis for future arrangements. 
1. In all operculate Discomycetes, and especially in the Ascobolaceae, where the asci project above the hymenium, the spores are raised above the surface by tensions within the apothecium. This might be regarded as dissemination by expulsion, if it were not for the fact that the spores fall back into the cup. unless carried away by the wind.

2. In Gasteromycetes and in certain Hepaticae. the spores are not only elevated slightly above the sporophore by the expanding capillitium or by the mass of elaters, but they are also held apart in such a way that the wind blows them out much more readily.

3. In Bryophyta, the sporophore regularly dehisces by a slit, or is provided with a peristome. Both structures are for the purpose of sifting the spores out into the wind: by reason of their hygroscopicity, they also insure that the spores will not be shaken out in wet weather.

4. In a few grasses, such as Stipa and Aristida, the twisting and interwining of the awns lift the floret out of the glumes, and at the same time constitute a contrivance readily blown away by the wind or carried by attachment.

5. In certain Compositae, the involucral scales are reflexed at maturity, and at the same time the disk becomes more or less convex, serving to loosen the achenes. This result is also secured in certain species by the drying and spreading of the pappus hairs.

6. The scapose Liguliflorae, Taraxacum, Agoseris, etc., are characterized by the elongation of the scape after anthesis, with the result that the head is raised to a considerable height by the time the achenes are mature.

7. Carpotropic movements, though primarily for another purpose, often serve to bring seeds and fruits into a better position for dissemination.

The relation of spore or seed-production to mobility is obvious in the case of mobile species: in the case of immobile ones, it is just as evident that it has no effect, though it may still have considerable influence in increasing migration. In the case of two species with equally effective dissemination contrivances, the one with the largest seed- 
production will be the more mobile. On the basis of the relation of seeds to flower, two groups of plants may be distinguished ${ }^{1}$, one, Polyanthae, in which the flowers are many and the seeds few or single, as in Compositae, and the other, Polyspermatae, Portulaca, Yucca, etc., in which the number of seeds to each flower is large. So far as the actual number of seeds produced is concerned, polyanthous plants may not differ from polyspermatous ones, but, as a rule, they are much more highly specialised for dissemination and are more mobile. The number of fertile seeds is also much greater, a fact which is of great importance in ecesis, and which, taken in connection with mobility, partially explains the supremacy of the composites. Among the fungi and algae, the amount of spore-production in a large degree determines the mobility, since these forms are intrinsically permobile.

In the last analysis, however, the possibility of migration depends upon the action of distributive agents: in the absence of these, even the most perfect contrivance is valueless, while their presence brings about the distribution of the most immobile form. In short, migration depends much more upon such agents than upon mobility, however perfect the latter may be. It is, moreover, evident that the amount and extent of migration will be determined primarily by whe permanence and forcefulness of the agent, as indicated by its ability to bring about transportation. Finally, as will be shown later, the direction and rapidity of migration depend directly upon the direction and intensity of the agent.

Migration results when spores, seeds, fruits, offshoots, or plants are moved out of their home by water, wind, animals, man. gravity, glaciers, growth, or mechanical propulsion. Corresponding to these agents, there may be recognized the following groups.

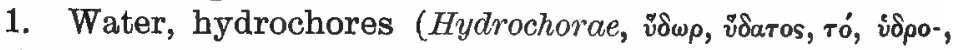
water, $\chi \omega \rho \epsilon \epsilon$, spread abroad). These comprise all plants distributed exclusively by water, whether the latter acts as ocean currents, tides, streams, or surface run-off. In the

1Pound and Clements Phytogeography of Nebraska 841898. 
case of streams and run-off, especially, mobility plays little part, provided the disseminules are impervious, or little subject to injury by water. Motile plants, or those with motile cells, which belong entirely to this group, may be distinguished as autochores (Autochorae, aviós, self), which correspond closely to mastigospores.

2. Wind, anemochores (Anemochorae, $\chi^{*} v \epsilon \mu \circ$, wind). This group" includes the majority of all permobile terrestrial plants, i.e. those in which modifications for increasing surface have been carried to the extreme, or those which are already permobile by reason of the minuteness of the spore or seed. Saccate, winged, comate, parachute, pappose, plumed, and, to a certain extent, awned seeds and fruits represent the various types of modifications for wind-distribution.

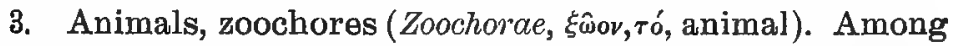
terrestrial plants, dissemination by attachment represents essentially the same degree of specialisation as is found in wind-distributed plants. The three types of contrivances for this purpose are found in spinose, hooked, and glandular fruits. Dissemination by deglutition and by earriage, either intentional or unintentional, though of less value, play a striking part on account of the great distance to which the seeds may be carried. Dissemination by deglutition is characteristic of sarcospores, and distribution by carriage of creatospores.

4. Man, brotochores (Brotochorae, ßporós, ó, a mortal man). Dissemination by man has practically no connection with mobility. It operates through great distances and over immense areas as well as near at hand. It may be intentional, as in the case of cultivated species, or unintentional, as in thousands of native or exotic species. No other disseminating agent is comparable with man in respect to universal and obvious migration.

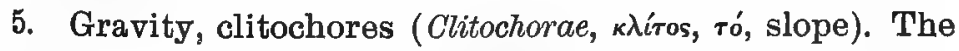
members of this group are ${ }_{3}^{*}$ exclusively colline, montane, and alpine plants, growing on rocks, cliffs, and gravel-slides (talus), etc., in which the seeds reach lower positions merely 
by falling or more frequently, by the breaking away and rolling down of rock or soil masses and particles. Dissemination by this method is relatively insignificant, though it plays an important part in the rock fields and gravel slides of mountain regions, particularly in the case of immobile species.

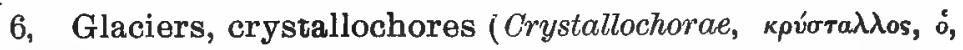
clear ice). At the present time, transport by glaciers is of slight importance, because of the restriction of the latter to alpine and polar regions, where the flora is poorly developed. In the consideration of migrations during the glacial epoch, however, it plays an important point.

7. Growth, blastochores (Blastochorae, $\beta \lambda a ́ \sigma \tau \eta, \dot{\eta}$, growth). The mobility of species disseminated by offshoots is extremely slight, and the annual movement relatively insignicant. The certainty of migration and of ecesis, is, however, so great, and the presence of offshoots so generally the rule in terrestrial plants that growth plays an important part in migration, especially within formations.

8. Propulsion, bolochores (Bolochorae, $\beta$ od $\eta^{\prime}, \mathfrak{\eta}$, a throw). Like growth, dissemination by mechanical propulsion, though operating through insignificant distances, exerts an important effect in consequence of its cumulative action. The number of plants, however, with contrivances for propulsion is very much smaller than the number of blastochores. All bolochorous species agree in having modifcations by means of which a tension is established. At maturity, this tension suddenly overcomes the resistance of sporangium or fruit, and throws the enclosed spores or seeds to some distance from the parent plant. In accordance with the manner in which the tension is produced, sling-fruits may be classified as follows.

(a) Hygroscopicity, pladoboles (Pladobolae, $\pi \lambda a ́ \delta o s, o ́$, moisture, - $\beta$ ódos, thrown). These include the ferns with annulate sporangia, in which the expansion of the annulus by the absorption of moisture, bursts the sporangium more or less suddenly, though the actual propulsion of the spores seems to come later as a result of dessication. 
(b) Turgescence, edoboles (Oedobolae, oídos, тó, swelling). Dissemination by turgescence is highly developed in Pilobolus and in Discomycetes, though in the latter turgescence results rather in placing the spores in a position to be readily carried by the wind. Impatiens and Oxalis furnish familiar examples of fruits which dehisce in consequence of increased turgidity.

(c) Dessication, xerioboles (Xeriobolae, $\xi_{\eta p i ́ a, ~}^{\eta}$, dryness). The number of fruits which dehisce upon drying is very large, but only a small portion of these expel their seeds forcibly. Geranium, Viola, Erysimum, and Lotus illustrate the different ways in which dessication effects the sudden splitting of fruits.

(d) Resilience, tonoboles (Tonobolae, róvos, ó, a stretching or tension). In some plants, especially composites, labiates and borages, the achenes or nutlets are so placed in the persistent calvx or involucre that the latter serves as a sort of mortar for projection, when the stem of the plant is bent to one side by any force, such as the wind or an animal. It will be noticed that two separate agents are actually concerned in dissemination of this sort.

Frequently, two or more agents will act upon the same disseminule, usually in succession. The possibility of such combinations in nature is large, but actual cases seem to be infrequent, except where the activities of man enter into the question. Some parts, moreover, such as awned inflorescences, are carried almost equally well by wind or animals, and may often be disseminated by the cooperation of these two agents. The wind also often bluws seeds and fruits into streams by which they are carried away, but here again, parts adapted to wind-dissemination are injured as a rule by immersion in water, and the number of plants capable of being scattered by the successive action of wind and water is small.

In the present state of our knowledge of migration, it is impossible to establish any definite correspondence between dissemination-contrivance, agent, and habitat. As a general rule, plants growing in or near the water, in so far as they 
are modified for this purpose at all, are adapted to watercarriage. Species which grow in exposed grassy or barren habitats are for the most part anemochores, while those that are found in the shelter of forests and thickets are usually zoochorous, though the taller trees and shrubs, being $\theta \mathbf{x}$ posed to the upper air-currents, are generally wind-distributed. There is then a fair degree of correspondence, in as much as most hydrophytes are hydrochorous, most hylophytes, zoochorous, and the majority of poophytes and xerophytes, anemochorous. Definite conclusions can be reached, however, only by the statistical study of representative formations.

With respect to their activity, agents may be distinguished as constant, as in the case of currents, streams, winds, slope, growth, and propulsion, or intermittent, animals and man. In the former, the direction is more or less determinate, and migration takes place year by year, i.e., it is continuous, while in the latter dissemination is largely an accidental affair, indeterminate in direction, and recurring only at indefinite intervals. The effective conversion of migration into invasion is greatest when the movement is continuous, and least when it is discontinuous, since, in the latter, species are usually carried not only out of their particular habitat but even far beyond their geographical area, and the migration, instead of being an annual one with the possibility of gradual adjustment, may not recur for several years, or may, indeed, never take place again. The rapidity of migration is greatest in the case of intermittent agents, while the distance of migration is variable, being great chiefly in the case of man, ocean-currents and wind, and slight when the movement is due to slope, growth or propulsion. Disregarding the great distances over which artificial transport may operate, seeds may be carried half way across the continent in a week by strong-flying birds, while the possibilities of migration by growth or expulsion are limited to a few inches, or, at most, to a few feet per year. This slowness, however, is more than counterbalanced by 
the enormously greater number of disseminules, and their much greater chance of becoming established.

The direction of migration is indeterminate, except in the case of those distributive agents which act constantly in the same direction. The general tendency is, of course, forward, the lines of movement radiating in all directions from the parent area. This is well illustrated by the operation of winds which blow from any auarter. In the case of the constant winds, migration takes a more or less definite direction, the latter being determined to a large degree by the fruiting period of any particular species. In this connection, it must be kept clearly in mind that the position of new areas with reference to the original home of a species does not necessarily indicate the direction of migration, as the disseminules may have been carried to numerous other places in which ecesis was impossible. The local distribution of zoochorous species is of necessity indeterminate, though distant migration follows the pathways of migratory birds and animals. In so far as dissemination by man takes place along great commercial routes, or along highways, it is determinate. In ponds, lakes and other bodies of standing water, migration may occur in all directions, but in ocean currents, streams, etc., the movement is determinate, except in the case of motile species. The dissemination of plants by slopes, glaciers, etc., is local and definite, while propulsion is in the highest degree indeterminate. Migration by growth is equally indefinite, with the exception that hydrotropism and chemotropism result in a radiate move. ment away from the mass, while propulsion throws seeds indifferently into or away from the species-mass. From the above, it will be seen that distant migration may take place by means of water, wind, animals or man, and, since all these agents act in a more or less definite direction over great distances, that it will be in some degree determinate. On the other hand, local migration will as regularly be indeterminate, except in the case of streams and slopes. The direction of migration then is controlled by these distributive agents, and the limit of migration is determined by the 
intensity and duration of the agent, as well as by the character of the space through which the latter operates.

ECESIS.

By the term ecesis is designated the series of phenomena exhibited by an invading disseminule, from the time it enters a new formation until it becomes thoroughly estab. lished there In a word, ecesis is the adjustment of a plant to a new habitat. It comprises the whole process covered more or less incompletely by acclimatisation, naturalisation, accomodation, eto. It is the decisive factor in invasion, in as much as migration is entirely ineffective without it, and is of great value in indicating the presence and direction of migration in a great dumber of species where the disseminule is too minute to be detected, or too little specialised to be recognisable.

Humboldt (1805:21) touches the subject of ecesis only incidentally in speaking of the lack of sufficient flexibility in any known species of phanerogams to enable it to accomodate itself to all latitudes and altitudes. DeCandolle (1820: 51) deals with naturalisation in a very general way, and cites several instances of its operation in adventive species. Henfrey (1852:51) speaks of the inability of alpine plants to accomodate themselves completely to lowland conditions, in which, however, they often persist in consequence of continuous migration. A. DeCandolle (1855:623) considers naturalisation at length, but almost wholly with reference to results, so that his excellent discussion belongs almost entirely to distribution. He gives a comprehensive though summary account of the obstacles to naturalisation, treats of the latter at small and great distances, and concludes with an account of the origin of cultivated species. Darwin (1859), in his chapter un the struggle for existence, discusses with more or less detail the biological conditions under which ecesis takes place in an invaded formation. He argues for a wide range of adaptability in most organisms, on account of their power of acclimatisation, though he does not produce any evidence of this from the plant kingdom. Grisebach $(1872: 215,264)$ pays especial attention to the inter- 
change of arctic and alpine plants, and formulates the fundamental law of barriers, viz., "les plantes endemiques augmentent en raisun des obstacles qui s'opposent à leur extension." Nägelí (1874:109) lays down an exact procedure for investigating the struggle for existence. His most important conclusion is that a stronger plant rarely drives the weaker one out entirely, but that their numerical relation is an expression of the ratio between their capabilities for existence. Engler (1879: XI) recognizes that the viability of seeds differs widely, and that the chances of ecesis depend directly upon the length of time necessary for migration. He connects ecesis directly with distribution in the principle that "Allmälig verbreiten sich die Formen eines Formenkreises soweit Bodenverhältnisse, klimatische Verhältnisse, und Concurrenz anderer Planzen es gestatten." Warming (1895:351), in connection with commensalism and succession, summarizes in a comprehensive manner the effect of occupation upon ecesis. Schimper (1898:55) treats acclimatisation briefly, taking into consideration adjustment to temperature alone.

The relation of migration to ecesis is a most intimate one: the latter depends in a large measure upon the time, direction, rapidity, distance and amount of migration. In addition, there is an essential alternation between the two, in as much as migration is followed by ecesis, and the latter then establishes a new centre from which further migration is possible, and so on. The time of year in which fruits mature and distributive agents act has a marked influence upon the establishment of a species. Disseminules designed to pass through a resting period are often brought into conditions where they germinate at once, and in which they perish because of unfavorable physical factors, or because competing species are too far advanced. On the other hand, spores and propagules designed for immediate germination mas be scattered abroad at a time when conditions make growth impossible. The direction of movement is decisive in that the seed or spore is carried into a habitat sufficiently like that of the parent to secure establishment, or into one 
so dissimilar that germination is impossible, or at least is not followed by growth and reproduction. The rapidity and distance of migration have little influence, except upon the less resistant disseminules, conidia, gemmae, etc. Finally, the amount of migration, i. e., the number of migrants, is of the very greatest importance, affecting directly the chances that vigorous disseminules will be carried into places where ecesis is possible.

Normally, ecesis consists of three essential processes, germination, growth and reproduction. This is the rule among terrestrial plants, in which migration regularly takes place by means of a resting part. In free aquatic forms, however, the growing plant or part is usually disseminated, and ecesis consists merely in being able to continue growth and to insure reproduction. Here establishment is practically certain, on account of the slight differences in aquatic habitats, excepting of course the extremes, fresh water and salt water. The ease indeed with which migration and ecesis are effected in the water often makes it impossible to speak properly of invasion in this connection, since aquatics are to such a large extent cosmopolitan. In dissemination by offshoots, the conditions are somewhat similar. Here also, ecesis comprises the sequence of growth and reproduction, and invasion, in the sense of passing from one habitat to another, is of rare occurrence, as the offshoot grows regularly under the same conditions as the parent plant. The adjustment of growing plants and parts is so slight, and their establishment so certain on account of their inability to migrate into very remote or different habitats, that they may be ignored in the following discussion.

In accordance with the above, it would be possible to. distinguish three groups of terrestrial plants, (1) those migrants which germinate and disappear, (2) those which germinate and grow but never reproduce, (3), those which reproduce, either by propagation or generation, or both. Such a classification has little value, however, since the same species may behave in all three fashions, depending upon the habitat to which it has migrated, and since 
invasion does not occur unless the plant actually takes possession, i. e., reproduces. From the latter statement, it follows that invasion occurs only when a species migrates to a new place, in which it germinates, matures and reproduces; maintainance by annual invasion simply, in which the plants of each year disappear completely, cannot then be regarded. as invasion proper. On the other hand, though such instances are rare, it is not necessary that the invaders produce fruit, provided they are able to maintain themselves, or to increase by propagation. Furthermore, if a plant germinate,grow and reproduce, it is relatively immaterial whether it persist for a few years or for many, since, as we shall see under Succession, the plants of one invasion are displaced by those of the next, the interval between invasions increas. ing with the stabilisation.

The germination of seed or spore is determined by its viability and by the nature of the habitat. Viability depends upon the structural characters of fruit, seed-coat and endosperm, and to a degree upon the nature of the protoplasm or embryo. The first three affect the last directly, by protecting the embryo against dryness, against injury due to carriage by water or by deglutition and, probably in some cases, against excessive heat or cold. Hegelmeier (1873), Lohde (1874), Bachmann (1881), and particularly Marloth (1883 : 225), have investigated the structure of seed-coats. The latter established the following groups, which are summarized here somewhat fully because of their bearing upon ecesis: (1) seed-coats without protective elements, endosperm absent or rudimentary, Epilobium, Impatiens, Parnassia, Sagittaria, etc.; (2) protective elements lacking or few, endosperm highly developed with thick-walled cells, Liliaceae, Primulaceae, Rubiaceae, etc.; (3) protective cells present in the seed-coats, endosperm little or none, Boraginaceae, Crassulaceae, Cruciferae, Labiatae, Papilionaceae, etc. ; (4) protective elements present, Asclepias, Campanula, Gentiana, Silene, Saxifraga, etc. (5) protective cells present, endosperm thickwalled, Euonymus, Helianthemum, Ribes. The protective cells are of various kinds: (1) epidermal cells strongly cuticular- 
ized, Caryophyllaceae, Crassulaceae, Fumariaceae, Saxifragaceae; (2) parenchyma thick-walled, several-layered, Aesculus, Castanea, Fagus; (3) parenchyma cells with the inner ur radial walls thickened, Campanula, Erythraea, Gentiana; (4) epidermal cells cup-shaped, thick-walled, Cruciferae, Ribes, Vaccinium; (5) parenchyma with thickened, cellulose walls, Geranium, Viburnum; (6) a single row of stone-cells, Labiatae; (7) tissue of stune-cells, Hippuris, Naias, Potamogeton; elongate stone-cells, Coniferae, Cupuliferae, Euphorbia, Linum, Malva, Viola; (9) short columnar thick-walled branched cells, Cucurbitaceae, Datura, Hypericum; (10) prosenchyma with cellulose walls, Clematis; (11) prosenchyma with lignified walls, Fraxinus, Rhamnus, Ranunculus. The seed-coats have a certain influence in determining germination at the proper time, in as much as they make it difficult for the seed to germinate under the stimulus of a quantity of warmth and moisture insufficient to support the seedling. The effect of the endosperm, as well as that of other food-supply in the seed, upon germination and the establishment of the seed. ling is obvious.

The behavior of seed or spore with respect to germination depends in a large degree upon the character of the protoplasm or embryo, though in just what way is at present a matter of conjecture. It is evident that many seeds are not viable because fertilisation has not been effected. and in consequence no embryo has developed. This is the usual explanation of the low germinating power of the seed of some species, especially polyspermatous ones. But even in viable seeds the behavior is always more or less irregular. The seeds of some species will grow immediately after ripening, while others germinate only after a resting period of uncertain duration. The same is true of spores. Even in the case of seeds from the same parent, under apparently similar conditions, while the majority will germinate the first year, some will lie dormant for one or more years. The precise reason why many seeds and spores germinate more readily after being frozen is equally obscure. The period of time for which disseminules may remain viable is 
extremely diverse, though, as would be expected, it is much longer as a rule for seeds than for spores. The greater vitality of seeds in the case of ruderal plants suggests that this diversity may be due simply to variation in the vigor of the embryos. It would seem that under proper conditions seeds may retain their viability for an indefinite period.

The influence of habitat upon germination is of primary importance, though the manner in which its influence is exerted is by no means as evident as might be supposed. In the case of seeds sown in the planthouse, it is almost universally the case that germination is less than in nature, notwithstanding the fact that temperature and moisture appear to be optimum. In nature, the seeds of the species may be carried into a number of different formations, any one or all of which may present conditions untavorable to germination. With respect to probability of germination, habitats are of two sorts, those which are denuded and those which bear vegetation. It is impossible to lay down general propositions with respect to either group, since germination will vary with the character of the invading species, the annual distribution of heat and moisture in the habitat, etc. In a general way. however, it may be stated that the chances for germination are greater in vegetation than in denuded areas, chiefly because the latter are usually xerophytic. On the other hand, the lack of competition in the denuded area tends to make ultimate establishment much more certain. Here, as elsewhere when exact statistical results are desired, the use of the quadrat, and especially of the permanent quadrat, is necessary to determine the comparative germina. tion of the invading species in relation to denudation and vegetation.

The seedling once established by germination, the probability of its growing and maturing will depend upon its habitat form, plasticity and vegetation.form. Even though it may germinate under opposite conditions, a typical hylophyte, such as Impatiens for exaraple, will not thrive in an open meadow, nor will characteristic poophytes, such as most grasses, grow in deep shade. In the same way, 
xerophytes do not adapt themselves to hydrophytic habitats, nor hydrophytes to xerophytic conditions. Many mesophytes, however, possess to a certain degree the ability to adjust themselves to somewhat xerophytic or hydrophytic situations, while woodland plants often invade either forest or meadow. This capability for adjustment, i.e., plasticity, is greatest in intermediate species, those that grow in habitats not characterised by great excess or deficiency of some factor, and it is least in forms highly specialised in respect to water-content, shade, etc. It may then be established as a fundamental rule that ecesis is determined very largely by the essential physical similarity of the old and the new habitat, except in the case of plastic forms, which admit of a wider range of accomodation. The plasticity of a plant is not necessarily indicated by structural modification, though such adjustment is usually typical of plastic species, but it may sometimes arise from a functional adaptation, which for some reason does not produce concomitant structural changes. The former explains such various habitat forms of the same species as are found in Galium boreale, Gentiana acuta, etc., and the latter the morphological constancy of plants like Chamaenerium, which grow in very diverse habitats.

The vegetation form of the invading species is often of the greatest importance in determining whether it will become established. The vegetation form represents those modifications, which, produced in the original home by competition,i.e., the struggle for existence, are primarily of value in securing and maintaining a foot hold. These comprise all structures by means of which the plant occupies a definite space in the air, through which the necessary light and heat reach it, and in the soil, from which it draws its food supply. These structures are all organs of duration or of perennation, such as root, rootstalk, bulb, tuber, woody stem, etc., which find their greatest development among trees and shrubs, and their least among annual herbs. But while the invaders are aided in securing possession by the proper vegetation form, the occupation of the plant already 
in possession is increased by the same means, and the out. come is then largely determined by other factors. To avoid repetition, the bearing of occupation upon invasion will be considered under succession.

\section{BARRIERS.}

DeCandolle (1820:45) seems to have been the first to use the term barrier and to distinguish the various kinds, though Hedenberg (1754:67) clearly saw that stations of one kind were insurmountable obstacles to plants belonging to a very different type. DeCandolle points out that the natural barriers to continuous invasion ("transport de proche en proche") are: (1) seas, which decrease invasion almost in inverse proportion to their extent; (2) deserts; (3) mountain ranges, which are less absolute on account of passes, valleys, etc.; (4) vegetation, marshes being barriers to dry land plants, forests to those that fear the shade, etc. Henfrey (1852:56) discusses the effect of geological and climatic barriers, and recognizes that man and animals often place a limit upon the spread of plants. A. DeCandolle $(1855: 70,207)$ treats of climatic barriers at considerable length, while Darwin (1859) discusses topographical barriers in a more general wav. Grisebach (1872:4), in discussing the effect of barriers upon the constitution of vegetation, lays down the fundamental rule that: "La loi suprême servant de base à l'établissement persistant de flores naturelles, doit donc être reconnue dans les barrières qui en ont entravé ou complétement empêché le mélange." Pound and Clements (1898:18) distinguish the physiographical barriers in invasion as obstructive, i. e., barriers, and conductive, agencies for migration.

Any feature of the topography, whether physical or biological, that restricts or prevents invasion, is a barrier. Such features are usually permanent and produce permanent barriers, though the latter may often be temporary, existing for a few years only, or even for a single season. In this last case, however, they are as a rule recurrent. Barriers may furthermore be distinguished as complete or incomplete with respect to the thoroughness with which they limit 
invasion. Finally, the consideration of this subject gains clearness, if it be recognized that there are barriers to migration as well as to ecesis, and if we distinguish barriers as physical or biological with reference to the character of the feature concerned.

Physical barriers (obices physicae) are those in which limitation is produced by some marked physiographic feature, such as the ocean or some other large body of water, large rivers, mountain ranges and deserts (including ice and snow-fields). All of these are effective by virtue of their dominant physical factors: hence they are barriers to the ecesis of species coming from very different habitats, but they act as conductors for species from similar vegetation, especially in the case of water-currents. A body of water, representing maximum water-content, is a barrier to mesophytic and xerophytic species, but a conductor for hydrophytic ones: deserts set a limit to the spread of mesophytic and hydrophytic plants, while they offer conditions favorable to the invasion of xerophytes; and a high mountain range, because of the reduction of temperature, restricts the extension of macrothermal and mesothermal plants. A mountain range, unlike other physical barriers, is also an obstacle to migration, in as much as natural distributive agents rarely act through it or over it.

Biological barriers (obices bioticae) include vegetation, man and animals, and plant parasites. The limiting effect of vegetation is exhibited in two ways. In the first place, a formation acts as a barrier to the ecesis of species invading it from the formations of another type, on account of the physical differences of the habitats: whether such a barrier be complete or partial will depend upon the degree of dissimilarity existing between the formations. Hylophytes are unable to invade a prairie, though open thicket plants may do so to a certain degree. In the same way, a forest formation on account of its diffuse light is a barrier to poophytes, and a swamp because of the amount and character of the water-content sets a limit to both hylophytes and poophytes. Formations, such as forests, thickets, etc., sometimes act also as direct obstacles to migration, as in the 
case of tumble-weeds and other anemochores, clinochores, etc. A marked effect of vegetation in decreasing invasion arises from the closed association typical of stable formations and of social exclusive species. In these, the occupation is so thorough and the struggle for existence so intense that the invaders, though fitted to grow under the physical factors present, are unable to compete with the species in possession for the requisite amount of some necessary factor. Closed associations usually act as complete barriers, while open ones restrict invasion in direct proportion to the degree of occupation. To this fact may be traced a fundamental law of succession, viz., the number of stages in a succession is determined largely by the increasing difficulty of invasion as the habitat becomes stabilized. Man and animals affect migration directly, though not obviously, by the destruction of disseminules. They operate as a pronounced barrier to ecesis wherever they alter conditions in such a way as to make them unfavorable to invading species, or when, by direct action upon the latter, such as grazing, tramping, parasitism, etc., they turn the scale in the struggle for existence. The absence of insects adapted to insure fertilisation is sometimes a serious barrier to the establishment of adventicious or introduced plants. The presence of parasitic fungi, in so far as they destroy the seeds of plants, acts as an obstacle to migration, and restricts or prevents ecesis in so far as the fungi destroy the invaders, or place them at a disadvantage in the struggle for existence.

Physical barriers are typically permanent in character, while biological ones are either permanent or temporary, depending upon the permanence of the formation and the constancy of the physical factors which determine it. A stable formation, such as a forest, or meadow, which acts as a decided barrier to invasion from adjacent vegetation, may disappear completely, as a result of a land-slide, flood or burn, or through the activity of man, and may leave an area into which invaders crowd from every point. Often, without undergoing marked change, a formation which has presented conditions unfavorable to the ecesis of species of mesophytic character, may by reason of a temporary change 
in climate become sufficiently modified to permit the invasion of mesophytes. On the other hand, a meadow ceases to be a barrier to prairie xerophytes during a period of unusually dry years. A peculiar example of the modification of a barrier is afforded by the defoliation of aspen forests in the mountains, as a result of which poophytes have been enabled to invade them. Nearly all xerophytio stretches of sand and gravel, dunes, blow-outs, gravel-slides,

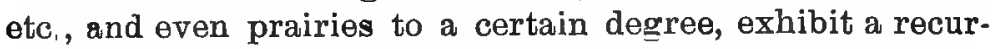
rent seasonal change in spring, as a result of which the hot dry surface becomes sufficiently moist to permit the germination and growth of invaders. which are entirely barred out during the remainder of the year. In an absolute sense, no barrier is complete, since the coldest as well as the dryest portions of the earth's surface are capable, at times at least, of supporting the lowest types of vegetation. Relatively, however, in connection with the natural spread of terrestrial plants, it is possible to distinguish partial barriers from complete ones. Such a distinction is of importance in the consideration of invasions from a definite region, as it is only in this restricted sense that complete barriers have produced endemism.

Distance, though hardly to be considered a barrier in the strict sense of the word, unquestionably plays an important part in determining the amount of invasion. The effect of distance is best seen in the case of migration, as it influences ecesis only in those rare cases where viability is affected. The importance of distance, or to take the converse, of proximity, is readily ascertained by the study of any succession from denudation. It has been established that the contiguous vegetation furnishes $75-90 \%$ of the constituent species of the initial formation, and in mountainous regions, where ruderal plants are extremely rare, the percentage is even higher. The reason for this is to be found not only in the fact that the adjacent species have a much shorter distance to go and hence will be carried in much greater quantity, but also in that the species of the formations beyond must pass 
through or over the adjacent ones. In the latter case, the number of disseminules is relatively small on account of the distance, while invasion through the intermediate vegeta. tion, if not entirely impossible, is extremely slow, so that plants coming in by this route reach the denuded area only to find it already occupied. It is as yet impossible to give a definite numerical value to proximity in the various invasions that mark any particular succession. This will not be feasible until a satisfactory method has boen found for determining a coefficient of mobility, but, this once done, it will be a relatively simple matter, not merely to trace the exact evolution of any succession of formations, but actually to ascertain from the adjacent vegetation the probable constitution of a particular future stage.

From what has been said, it follows that the primary effect of barriers upon vegetation is obstruction. Where the barrier is in the pathway of migration, however, it causes deflection of the migrant as a rule, and sets up migration in a new direction. This is often the case when the strong winds of the plains carry disseminules towards the mountains and, being unable to cross the range, drop them at the base, or, being deflected, carry them away at right angles to the original direction. The same thing happens when resistant fruits and seeds borne by the wind fall into streams of water or into ocean currents. The direction of migration is changed, and what is normally a barrier serves as an agent of dissemination.

\section{ENDEMISM.}

Wildenow (1811:98) supposed that each great mountain range had its own peculiar species, which had wandered down into valleys and lowlands and had produced a corresponding flora. Upon this basis, wholly erroneous as it was, he established a floral region to correspond to each principal mountain range. Brisseau-Mirbel (1815:582) defined indigenous plants as those which are natural to the soil in which they grow, and exotics as those not natural to the country inhabitated; but apparently he referred only to cultivated plant.. DeCandolle $(1820: 51)$ used indigenous in the sense of native 
to contrast with introduced, and employed the word aboriginal to denote peculiar plants. The latter, as he has shown, are due to barriers, and are of primary importance for the limitation of floral regions, of which he distinguished twenty. With respect to distribution, he distinguished endemic genera and families, composed of species growing in a single country, and sporadic genera, which are distributed over the entire world. Schouw (1823:505) extended the use of peculiar species in the recognition of regions, and laid down the following rules: "Um ein pflanzengeographisches Reich zu bilden verlange ich von einem Theile der Erdoberfläche; 1. dass wenigstens die Hälfte der bekannten Arten diesem Erảtheile eigenthümlich gehöre; 2. dass wenigstens $\frac{1}{4}$ der Gattungen entweder völlig eigenthümlıch sey oder doch wenigstens in dem Erdtheile ein so entschiedenes Maximum habe, dass die in anderen Erdtheilen vorkommenden Arten nur als Repräsentanten zu betrachten sind. 3. Dass einzelne Pflanzenfamilien gleichfalls entweder diesem Erdtheile eigenthümlich seyen, oder wenigstens ein entschiedenes Maximum dort haben. Doch dürfte man wohl, selbst wo das letztere Requisit fehlt, den Erdtheil als ein besonderes Reich ansehen, wenn die Verschiedenheiten der Gattungen bedeutend sind." Fenzl (1833:19) proposed to replace the word sporadic, because of its use in migration, by polydemic, and suggested the term pandemic for truly cosmopolitan species. Meyen (1836: 160), while he recognized the value of Schouw's statistical method, emphasized more particularly the importance of physiognomy, i. e. mass, habit and distribution, in determining the divisions of vegetation, a method already employed by Treviranus (1802:85), but without success on account of the rudimentary development of the subject.

A. DeCandolle (1855:476) rejected the terms endemic and sporadic for reasons which do not seem very valid. $\mathrm{He}$ brought together in the chapters on the delimitation and area of species an enormous mass of facts, many of which have a direct bearing upon endemism. Darwin (1859:66) considered at some length the proportion of endemic species in 
oceanic islands, in which the sea is a very obvious barrier to migration. Kabsch (1865:551) defined endemic plants as follows: "Es sind endemische Arten, welche da wo sie heut noch gefunden werden, auch entstanden, und aus Mangel an günstigen Umständen nicht im Stande gewesen sind, sich weiter zu verbreiten". He treated the endemism of oceanic islands in considerable detail and proposed the following rules for the determination of endemic species: "Endemisch im strengsten Sinne des Wortes werden wir eine Pflanzenart für einen Florenbezirk nennen:

(1) Wenn die Pfianze nur in einem bestimmten Florenbezirk und in keinem anderen vorkommt.

(2) Wenn die Pflanze zwar in mehreren Bezirken besteht, in diesem einen aber ihre hervorragendste Verbreitung findet, und dasselbst auch die grösste Zahl ihrer ihr zunächst stehenden Verwandten hat";

Kabsch was the first to perceive that endemism is, from the standpoint of phylogeny, relative and not absolute, a fact expressed in the following postulate: "Scheilt es wahrscheinlich zu sein, dass die Mannigfaltigkeit, die Gestaltenfülle der Pflanzendecke unserer Erde von der Natur hauptsächlichst nur vermöge der Wanderungfähigkeit der Pflanzen hervorgebracht worden, so dass man behavpten kann, die Ureltern aller gegenwärtigen Pflanzenformen seien vor längerer oder kürzerer Zeit naturalisirte Arten gewesen, aus denen dann erst, sei es durch geschlechtliche Vermischung, sei es durch stufenweise Umbildung im Sinne der Transmutationslehre die Typen enstanden, die wir jetzt für endemisch halten müssen." Grisebach $(1872$ : 267) recognized the fundamental relation between barriers and endemism, expressing it in the law that, "Les plantes endemiques augmentent en raison des obstacles qui s'opposent à leur extension". His account of endemism in the vegetation of oceanic islands is by far the most exhaustive that has been made.

Engler (1879) handled the related subjects of endemism and. invasion in such a comprehensive and detailed fashion that his work will for a long time stand unrivalled in these fields It practically amounts to a handbook of these subjects and is 
so extensive that any summary of it is impossible within the limits of this paper. In his "Leitende Ideen", Engler laid down the following principles with reference to endemism: '19. Aus 17 and 18 geht hervor dass in Ländern von hohem Alter, namentlich in gebirgigen Gegenden, deren Vegetation seit langem nicht durch geologische Ereignisse vollständig vernichtet wurde, ein reicher Endemismus herrschen muss." “20. Endemische Formen können aber auch in verhältnissmässig jungen Gebieten reichlich auftreten, wenn nämlich diese Gebiete, wie die asiatischen Steppen, die amerikanischen Prärien, oder die südamerikanischen Pampas, durch ihre Beschaffenheit nur einer beschränkten Zahl von Vegetationformen die nöthigen Existenzbedingungen gewähren".

"21. Der Unterschied zwischen alten und neuen Florengebieten mit reichem Endemismus besteht gewöhnlich darin, dass in den älteren Gebieten die Artenzahl der Gattungen eine geringere, in den neueren die Artenzahl einzelner Gattungen gewöhnlich eine sehr grosse ist."

Buchenau (1881:124), in dealing with the distribution of the Juncacene, has summarized the causes of endemisn in the following brief but excellent statement: "Der Endemismus beruht nicht allein auf der Fähigkeit irgend eines Florengebietes, neue Formen hervorzubringen, sondern auch auf der (activen oder passiven) Unmöglichkeit für die erzeugten Formen sich weiter zu verbreiten. Ein reiches Entstehungsgebiet kann daher ausgeprägten Endemismus zeigen, wenn seine Erzeugnisse entweder unfähig zu weiten Wanderungen sind, oder wenn es von unübersteiglichen Naturschranken umgeben ist: der Endemismus wird dagegen verwischt erscheinen, wenn es den entstandenen Arten möglich ist, sich über weite Fläche auszubreiten." Drude (1890:124) has distinguished endemic plants as corresponding, when the original continuous area of a variable species has been inter. rupted in such a way as to form several smaller areas occupied by subspeeies or new species, and as relict, when a species originally of extensive distribution is able to maintain itself in a limited area only, on account of change $\mathrm{d}$ 
conditions of life. It is quite possible that relict endemics, through the earlier invasions of the original species, may be found in a place in which the latter did not arise: in consequence, there are endemics which have arisen in their present area, and those which have migrated to it. The latter may often be further modified, and would then fall in the first class. The author has further pointed out that the concept of endemism varies with the size of the area, and that it is inapplicable to political divisions, except when these have natural limits. Delpino (1898:356) emphasized the primary importance of endemism in regional limitation, and considered the relative meaning of endemism in groups of various rank and in regions of different extent. Pound and Clements (1900:53) extended the concept of endemism to cover vegetation, in such a way that one may distinguish vegetation elements, e. g., endemic formations, as well as floral elements.

Since its first use by DeCandolle, the term endemic has been employed quite consistently by phytogeographers with the meaning of "peculiar to a certain region." Someconfusion, however, has arisen from the fact that a few authors have made it more or less synonymous with indigenous and autochthonous, while others have regarded it as an antonym of exotic. In its proper sense, endemic refers to distribution, and not to origin. Its exact opposite will be found then in Fenzl's term polydemic, dwelling in several regions. Indigenous (autochthonous) and exotic, on the contrary, denote origin, and are antonyms, indigenous signifying native, and exotic, foreign. As Drude has shown, endemic plants may be either indigenous, as in the case of those species that have never moved out of the original habitat, or exotic, as in the much rarer instances where a polydemic species has disappeared from its original home and from all regions into which it has migrated except one. It is understood that not all indigenous or exotic species are endemic. The proportion of endemic to polydemic species is a variable and somewhat artificial one, depending upon the size of the divisions employed. 
The primary causes of endemism are two, lack of migration and presence of barriers. Since distributive agents are practically universal, lack of migration corresponds essentially to immobility, a fact which decreases the difficulty of ascertaining the immediate causes of endemism in any particular species. Either immobility or a barrier may produce endemism; extremely immobile plants, for example, liliaceous species propagating almost wholly by underground parts, are as a rule endemic, while alpine plants and those of oceanic islands are endemic in the highest degree, regardless of their mobility. When the two conditions act concomitantly upon a species, endemism is almost inevitable. It cannot be supposed, however, that immobility or natural barriers alone, or the concomitance of the two, must invariably give rise to endemic species; the most immobile plant may be carried into another region by unusual or accidental agencies, or the most formidable barrier to migration may be overcome by the intensity of an agent or through the action of man. Endemism is also brought about by the modification of species: new or nascent species are as a rule endemic. Whether they will remain endemic or not will depend upon the perfection of their contrivances for dissemination and apon the presence of barriers to migration or ecesis. Finally, as Drude was the first to point out, the disappearance of a polydemic species in all regions but one, owing to the struggle for existence or to changed physical conditions, will result in endemism.

Endemism is readily recognized by methods of distributional statistics, applied to areas limited by natural barriers to migration or ecesis. For political areas, it has no significance whatever, unless the boundaries of these coincide with barriers. It determines in the first degree the validity of regions, though the latter are often recognized also by the presence of barriers and by the character of the vegetation. Endemism may occur in areas of vegetation of any rank from a formation to a zone. Comparisons to be of value, however, can be instituted only between areas of the same order, i. e. between two or more formations, two or 
more regions, provinces, etc. In the same way, taxonomic groups of the same rank should be used in such compari-

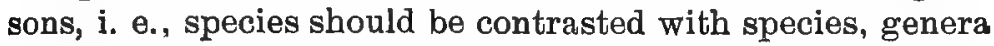
with genera, and families with families, except when it is desired to obtain some measure of the age of the vegetation by the differentiation of the endemic phyla within it. There will be seen to exist a fundamental correspondence between the rank of the floral division and the taxonomic group, though the apparent exceptions to this are still too numerous to warrant its expression in a general law. As a rule, however, formations most frequently show endemic habitat forms and species, more rarely endemic genera; regions and provinces commonly exhibit endemic species and genera, rarely endemic families, while zones and hemispheres contain endemic orders as well as families. This correspondence is readily seen to depend primarily upon the fact that increased differentiation in the taxonomic sense is a concomitant of the increased invasion of endemic species, measured in terms of distance and difference in habitat.

It is too early to decide satisfactorily whether it is proper to speak of formations as endemic. At first thought, it would seem that all formations, with the exception of ruderal ones, were endemic, but a study of almost any transition area between regions would seem to point to the opposite conclusion, viz. that no formations are properly endemic. It is equally impossible at present to distinguish different types of endemics, such as relictae, etc., as any such classification must await the elaboration of a method for determining the phylogeny of a natural group of species by an investigation of their comparative differentiation iu connection with their migration in all directions from the vegetation centre into new habitats. In short, it will not be possible to make a thorough study of endemism and to postulate its laws until modern methods of research 'have been extended to a much larger portion of the vegetation of the globe. The final task of phytogeography is the division of the earth's vegetation into natural areas. It will be at once evident that most plants cennot properly be called 
endemic until the natural regions in which they are found have been accurately defined, a work which has barely begun. In the much simpler matter of distribution, upon which the accuracy of statistical methods depends directly, there are few regions sufficiently well known at the present time to yield anything like permanent results.

\section{POLYGENESIS.}

Before Darwin, the question of the origin of species at two or more places on the earth's surface, either simultan. eously or successively. was merely one of single or multiple creation, and the answer was usually determined by the bias for or against migration. In the Crigin of Species, the question was summarily dismissed, and migration was invoked to explain all the phenomena of distribution. With rare exceptions, this has been the position of most botanists since Darwin. DeCandolle (1820:52), without insisting upon the creation of new species at a single center, seems to have been of this opinion, as the following will indicate: "Si l'on réfléchit maintenant a l'action perpetuelle des quatre causes de transport de graines que je viens d'indiquer, les eaux, les vents, les animaux et l'homme, on trouvera, je pense, qu' elles sont bien suffisantes pour expliquer ce petit nombre de végétaux qu’on retrouve semblables dans les continens divers." Schouw (1824:122), as an opponent of what he termed the migration theory, must have favored the view that species were created at two or more centres, though he failed to express himself definitely, as the following will show: "Ob diese Pflanzen in beiden Theilen der Erde ihre ursprüngliche Heimath haben oder nicht, ist in pflanzengeog̣raphischer Hinsicht gleichgültig." Henfrey (1852:47), discussed briefly the "opinion that species were created in different places, at different centres, either simultaneously or at different epochs," but dismissed it as unsatisfactory because of the possibility of diffusion. A. DeCandolle (1855: 1114) considered the single or multiple origin of species merely with reference to the number of individuals of each species originally created, reaching the conclusion that both methods were probably to be found in nature. Darwin 
(1859:619) rejected unconditionally the idea of polygenesis, and sought to explain all distribution by migration, though he admitted that this was difficult in many cases. He expressed his ideas on this subject in the following statement: "It is also obvious that the individuals of the same species, though now inhabiting distant and isolated regions, must have proceeded from one spot, where their parents were first produced : for, as has been explained, it is incredible that individuals identically the same should have been produced from parents specifically distinct. We are thus brought to the question, which hus been largely discussed by naturalists, namely, whether species have been created at one or more points of the earth's surface. Undoubtedly there are many cases of extreme difficulty in understanding how the same species could possibly have migrated from some one point to the several distant and isolated points where now found. Nevertheless, the simplicity of the view that each species was first produced within a single region captivates the mind. $\mathrm{He}$ who rejects it rejects the vera causa of ordinary generation with subsequent migration, and calls in the agency of a miracle." Palacky (1864, Just '89: 169) was apparently the first, after the publication of Darwin's theory, to lend support to the doctrine of polygenesis, but his work, unfortunately, is not accessible.

Kabsch (1865:552) has asserted most emphatically his opinion that species have single centres of creation and that they proceed from a single individual. In this connection he has made the following statements: "Jede Art besitzt nur einen Punkt auf der Erde, wo sie entstanden und von dem aus sie sich weiter verbreitet hat: es giebt nicht zwei Punkte auf der Erde in einiger Entfernung von einander und hat es $z u$ keiner Zeit gegeben, wo die oft so äusserst zarten und für unser Auge zum Theil noch verborgenen oder nicht erkannten Lebensbedingungen, welche auf die Umbildung der Pflanzen wirken, sich so vollkommen gleich gewesen wären, dass dieselbe Pflanzenform an verschiedenen Punkten zugleich hätte entstehen können. Aehnliche Verhältnisse sind jedoch jetzt und noch mehr in früheren Erdepochen 
zahlreich vorhanden gewesen; aber Aehnliches vermochte nur Aehnliches, nie vollkommen Gleiches hervorzubringen. . . . hat die Natur uns ein untrĭgliches Zeugniss aufbewahrt, dass sie selbst an verhältnissmässig einander nahe gelegenen Orten unter einem Klima, dass für unser Wahrnehmungsvermogen wenigstens vollkommen gleichartig genannt werden muss, niemals vermochte gleiche, sondern immer nur ähnliche Pflanzenformen zu schaffen, dass somit unsere ursprüngliche Annahme, dass jede Pflanzenart nur von einem Individuum ausgegangen, eine nicht wegzuleugnende Begründung besitzt."

Nägeli (1873:189) seems to have had some idea of both polygenesis and polyphylesis, but the fact that he was not dealing primarily with such questions makes it difficult to decide just how clearly he perceived these relations: "2. Eine Pflanzenform $\mathrm{A}$ ist über ein grosses Gebiet verbreitet. Innerhalb dieses Gebietes findet sich cönobitisch mit A die verwandte Form B auf verschiedenen Standorten, welche ihrer Lage nach eine Einwanderung höchst unwahrscheinlich und selbst unmöglich erscheinen lassen. . . . Wenn es nun sicher ist, dass die cőnobitischen verwandten Formen nicht durch spätere Wanderung zusammen gekommen sind, so ergiebt sich die fernere Frage, ob der in ihren Merkmalen ausgesprochene Abstand zwischen ihnen während ihrer Geselligkeit unverändert geblieben ist, oder ob er sich geändert hat. Die Aenderung gestattet wieder eine doppelte Möglichkeit: entweder ist der Abstand grösser oder kleiner geworden. Entweder divergiren die beiden Formen und müssen somit in früherer oder späterer Vergangenheit von einem gemeinsamen Ursprung ausgegangen sein, oder die beiden Formen convergiren und werden in früherer oder späterer Zukunft zusammenfliessen. (201) Eine Pflanzenform bildet Varietäten von denen nicht nur eine sondern zwei sich als existenzfähig erweisen, und nach zwei entgegengesetzten Richtungen hin die Mutterform an Stärke übertreffen . . Diese zweite Art der Speciesbildung, die man die didymogenetische nennen kann, ist von der ersten, der monogenetischen, nicht streng geschieden". 
Engler (1879:318) was the first to deal concretely with the question of multiple origin by extending his observations to species and varieties. His conclusions seem to make the existence of polyphylesis (as he termed it) as certain as is possible without direct experiment. His argument is as follows: "Kann das, was man gewohnlich Art, Gattung, Familie nennt, nur an einer Stelle oder auch an zwei Stellen der Erde und dann selbstverständlich an mehreren entstehen? . . Das gewisse äussere Verhältnisse an verschiedenen Orten eine Pflanze in gleicher Weise umgestalten können wissen wir. Es haben zwar keineswegs die äusseren Verhältnisse, unter denen gewöhnlich eine bestimmte Varietät $b$ existiert, sofort die Umwandlung einer andern Varietät $a$ derselben Art in die Varietät $b$ zur Folge: aber diese Umwandlung kann bei den Descendenten und zwar an verschiedenen Stellen eintreten. Nehmen wir nun an, eine Art $A$ habe in der Natur im Laufe der Zeit ein grosseres Verbreitungsarea] gewonnen, so ist es nach Obigem möglich dass dieselbe an zwei von einander entfernten Stellen $m$ und $n$, welche annäherend gleiche Bedingungen gewähren, dieselbe Varietät $a$ erzeugt. Praktisch haben wir solche Fälle ganz besonders bei Hochgebirgspflanzen wo sehr oft das Areal der böheren Regionen Raum bietet für die Varietäten, welche sich aus einer Art entwickeln, dass die grössere Areal der nächst tieferen Region einnimmt.

Somit können also schliesslich Gattungsgruppen in entfernteren Gebieten entstehen, welche doch $\mathrm{zu}$ einander in einer gewissen verwandtschaftliche» Beziehung stehen.

Nach diesen Erwägungen stehe ich nun nicht an, mich zur Lehre von der Einheit des Ausgangspunktes einer Gattung zu bekennen, jedoch eben nur der natürlichen Gattungen'. Drude (1886:200) accepted the doctrine of the multiple origin of major groups, but he did not extend it to species and varieties. Finally, Delpino (1898:6) has expressed himself very definitely against the multiple origin of species: "Per ogni specie stabilmente concretata e fissa io credo doversi ammettere un centro di formazione ed uno soltanto. E veramente, considerando l'infinita fluttuazione delle forme 
organiche, ammettere che la stessa identica species possa essersi attuata e concretata in tempi diversi, e in due o piú luoghi distinti, é tale improbabilitá che confina coll' assurdo. Se ogni specie deve ritenersi come il prodotto d'un immenso numero di fenomeni antecedenti, eome 1'effelio di una quasi infinita quantitá di adattamenti ad un ambiente infinitamente mutevole, come pu6 un cosifattamente complicato prodotto essersi ripetuto e manifestato due o piú volte, in due o piú tempi distinti, in due o piú luoghi separati? Una cotal congettura accettata da Grisebach e da altri, é per me assurda. Io mi ribello contro essa.".

The idea of polyphylesis, as advanced by Engler, contains two distinct concepts: (1) that a species may arise in two different places or at two different times from the same species, and (2) that a genus or higher group may arise at different places or times by the convergence of two or more lines of origin. It is here proposed to restrict polyphylesis, as its meaning would indicate, to the second concept, and to employ for the first the term polygenesis, first suggested by Huxley in the sense of polyphylesis. The term polyphylesis is extended, however, to cover the origin of those species which arise at different places or times from the convergence of two or more different species, a logical extension of the idea underlying polyphyletic genera, though it may seem at first thought to be absurd. Polygenesis may be formally defined as the origin of one species from another species at two or more distinct places on the earth's surface, at the same time or at different times, or its origin in the same place at different times. Polyphylesis, on the contrary, is the origin of one species from two or more different species at different places, at the same time or at different times. It is evident that what is true of species in this connection will hold equally well of genera and higher groups. Opposed to polygenesis is monogenesis, in which a species arises but once from another species; with polyphylesis is to be contrasted monophylesis, in which the species arises from a single other species. It will be noticed at once that these two concepts are closely related. The 
following diagrams will serve to make the above distinctions more evident :

I. Polygenesis II. Polyphylesis III. Monogenesis (Monophylesis)
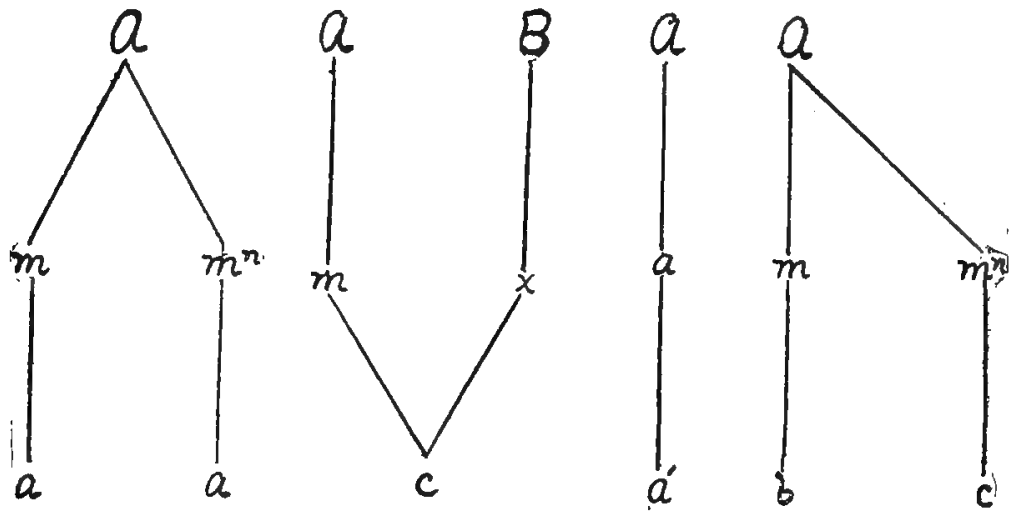

In $\mathrm{I}$, a species $A$, becomes scattered over a large area in a series of places, $m$. . . $m^{n}$, with the same physical factors, in any or all of which may arise the new species $a$. In II, a species with xerophytic tendency, $A$, and one with mesophytic tendency. $B$, in the course of migration find themselves respectively in a more mesophytic habitat, $m$, and a more xerophytic one, $x$, in which either may give rise to the new form, $c$, which is more or less interwediate between $A$ and $B$. In III, the method of origin is of the simplest type, in which a species is modified directly into another one, or is split up into several.

In affirming the probability of a polygenetic origin of species, there is no intention of asserting that all species originate in this way. It seems evident that a very large number of species of restricted range are certainly monogenetic, at least as far as origin in space is concerned: it is possible that any species may arise at two or more distinct times. Polygenesis can occur readily only in species of more or less extensive area, in which recur instances of the same or similar habitat. The relative frequence and 
importance of the two methods can hardly be conjectured as yet, but origin by monogenesis would seem to be the rule.

The arguments adduced by Engler in support of polygenesis are in themselves conclusive, but the investigations of the past decade have brought to light additional proofs, especially from the experimental side. In determining the physical factors of prairie and mountain formations, and especially by methods of experimental ecology, the author has found that habitats are much less complex than they are ordinarily thought to be, since water-content and humidity, and to a less degree light, constitute the only factors which produce direct modification. In addition, it has been ascertained that the minimum difference of water-content, humidity or light, necessary to produce a distinguishable morphological adjustment, is much greater than the unit differences recorded by the instruments. In short, the differences of habitats, as ascertained by thermograph, psychrometer and photometer. are much greater than their efficient differences, and, with respect to their ability to produce modification, habitats fall into relatively few categories. A striking illustration of this is seen in the superficially very different habitats, desert, strand, alkali plain, alpine moor, and arctic tundra. all of which are capable of producing the same type of xerophyte. It follows from this that many more or less plastic species of extensive geographical area will find themselves in similar or identical situations, measured in terms of efficient differences, and will be modified in the same way in two or more of these. In mountain regions, where interruption of the surface and consequent alternation are great, the mutual invasion of contiguous formations is of frequent occurrence, often resulting in habitat forms. ${ }^{1}$ The spots in which these nascent species, such as Galium boreale hylocolum, Aster levis lochmocolus, etc., are found, are often so related to the area of the parent species as to demonstrate conclusively that these forms are the result of polygenesis and not of migration. Naturally,

${ }^{1}$ Clements, F. E. A System of Nomenclature for Phytogeography. Engler 31:b 17 1902. 
what is true of a small area will hold equally well of a large region, and the recurrence of the same habitat form may be accepted as conclusive proof of polygenesis. The most convincing evidences of multiple origin, however, are to be found in what De Vries has called "mutations". It makes little difference whether we accept mutations in the exact sense of this author, or regard them as forms characterised by latent variability. The evidence is conclusive that the same form may arise in nature or in cultivation, in Holland or in America, not merely once, but several or many times. In the presence of such confirmation, it is unnecessary to accumulate proofs. Polygenesis throws a new light upon many difficult problems of invasion and distribution, and, as a working principle, admits of repeated tests in the field. It obviates, moreover, the almost insuperable difficulties in the way of explaining the distribution of many polygenetic species on the basis of migration alone.

In 1898, the author first advanced a tentative hypothesis to the effect that a species homogeneous morphologically may arise from two distinct though related species: during subsequent years of formational study, the conviction has grown in regard to the probability of such a method of origin. Since the appearance of Engler's work, a polyphyletic origin for certain genera has been very generally accepted by botanists, but all have ignored the fact that the polyphylesis of genera carries with it the admission of such origin for species, since the former are merely groups of the latter. I cannot, however, agree with Engler, that polyphyletic genera, and hence species also, are necessarily unnatural. If the convergence of the lines of polyphylesis has been great, resulting in essential morphological harwony, the genus is a natural one, even though the ancestral phyla may be recognizable. If, on the other hand, the convergence is more or less imperfect, resulting in sub-groups of species more nearly related within the groups than between them, the genus can hardly be termed natural. This condition may, however, prevail in a monophyletic genus with mani- 
fest divergence, and still not be an indication that it is arti ficial.

Darwin (1859:186), in speaking of convergence, says: "If two species belonging to two distinct though allied genera, had both produced a large number of new and divergent forms, it is conceivable that these might approach each other so closely that they would have all to be classified under the same genus: and thus the descendants of two distinct genera would converge into one." The application of this statement to species would at once show the possibility of polyphylesis in the latter, and a further examination of the matter will demonstrate its probability. It is perfectly evident that a species may be split into two or more forms by varying the conditions, let us say of water-content, and that the descendants of these forms may again be changed into the parent type by reversing the process. This has, in fact, been done experimentally. Since it is admittedly im. possible to draw any absolute line between forms, varieties and species, it is at once clear that two distinct though related species, especially if they are plastic, may be caused to converge in such a way that the variants may constitute a new and homogeneous species. This may be illustrated by a concrete case at present under investigation. Kuhnistera purpurea differs from $K$. candida in being smaller, in having fewer, smaller and more narrow leaflets, and a globoid spike of purple flowers in place of an elongated one of white flowers; in a word, it is more xerophytic. This conclusion is completely corroborated by its occurrence. On dozens of slopes examined, Kuhnistera purpurea has never been found mingling with $K$. candida on lower slopes, except where an accident of the surface has resulted in a local decrease of water-content. The experiment as conducted is a simple one, consisting merely in sowing seed of each in the zone of the other, and in growing $K$. purpurea under controlled mesophytic conditions, and $K$. candida under similarly measured xerophytic conditions in the plant house.

While the polyphyletic origin of species is in a fair way to be decided by experiment, it receives support from several 
well-known phenomena. The striking similarity in the plant body of families taxonomically so distinct as the Cactaceae, Stapeliaceae, and Euphorbiaceae, or Cyperaceae and Juncaceae, indicates that a vegetation form may be polyphyletic. On the other hand, the local appearance of zygomorphy, of symphysis, and of aphanisis in the floral types of phylogenetically distinct families is a proof of the operation of convergence in reproductive characters. To be sure, the convergence is never so great as to produce more than superficial similarity, but this is because the groups are markedly different in so many fundamental characters: the same tendency in closely related species would easily result in identity. As in the case of polygenesis, the relatively small number of typically distinct habitats makes it clear that two different species of wide distribution, bearing to each other the relations of xerophyte to mesophyte, of hydrophyte to mesophyte, or of poophyte to hylophyte, might often find themselves in reciprocal situations, with the result that they would give rise to the same new form. The final proof of the polyphylesis of species is afforded by the experiments of DeVries in mutation. De Vries found that Oenother a nanella arose from $O$. Lamarckiana, $O$. laevifolia, and O. scintillans: Oenothera scintillans arose from 0 . lata and o. Lamarckiana; Oenothera rubrinervis from O. Lamarckiana, 0 . laevifolia, O. lata, O. oblonga, O. nanella and $O$. scintillans, etc. Whatever may be the rank assigned to these mutations, whether form, variety or species, there can be no question of their polyphyletic origin, nor, in consequence of the connection of mutations with variations through such inconstant forms as $O$. scintillans, O. elliptica and $O$. sublinearis, of the possibility of polyphylesis in any two distinct though related species or genera.

\section{KINDS OF INVASION.}

With respect to the frequency of migration, we may distinguish invasion as contin uous (invasio continua), or intermittent (invasio infrequens). Continuous invasion, which is indeed usually mutual, occurs between contiguous formations of more or less similar character, in which there 
is an annual movement from one into the other, and at the same time a forward movement through each, resulting from the invaders established the preceding year. By far the greater amount of invasion is of this sort, as may readily be seen from the fact that migration varies inversely as the distance, and ecesis may decrease even more rapidly than the distance increases. The significant feature of continuous invasion is that an outpost may be reinforced every jear, thus making probable the establishment of new outposts from this as a centre, and the ultiwate extension of the species over a wide area. The comparatively short distance and the regular alternation of migration and ecesis render invasion of this sort very effective. An excellent illustration of this is seen in transition areas and regions, which are due directly to continuous and usually to mutual invasion. Intermittent invasion results commonly from distant carriage, though it may occur very rarely between dissimilar adjacent formations, when a temporary swing in the physical factors makes ecesis possible for a time. It is characterised by the fact that the successicn of factors which have brought about the invasion is more or less accidental, and may never recur. Intermittent invasion is relatively rare, and from the small number of disseminules affected, it is of little importance in modifying vegetation quantitatively. On the other hand, since a species may often be carried far from its geographical area, it is frequently of great significance in distribution.

When the movement of invaders into a formation is so great that the original occupants are finally driven out, the invasion may be termed comple te (invasio perfecta). Such invasion is found regularly in the case of many ruderal formations, and is typical of the later stages of many successions. It is ordinarily the result of continuous invasion. If the number of invaders is sufficiently small that they may be adopted into the formation without radically changing the latter, the invasion is partial (invasio imperfecta). This is doubtless true of the greater number of invasions, though 
these are regularly much less striking and important than instances of complete invasion.

The permanence of invasion depends upon the success attending ecesis, and upon the stability of the formation. It has already been noticed that under certain conditions plants may germinate and grow, and, if they are perennials, even become established, and still ecesis be so imperfect that reproduction is impossible. Others may find the conditions sufficiently favorable for propagation, but unfavorable for the formation of flowers and fruits. Finally, there are plants which seem to be perfectly established for a few years, only to disappear completely. The latter are examples of temporary invasion (invasio temporalis). It is necessary to draw clearly the line between complete and partial invasion in this connection. The former is temporary in the initial or intermediate stages of nearly all successions, as compared with the ultimate stages, though it is in a large degree permanent in comparison with the partial invasion of species which are able to maintain themselves for a lew years. In a sense, there is a real distinction between the two, inasmuch as a particular stage of succession is permanent as long as the habitat remains essentially the same: a critical study of the species of such stages shows, however, that they manifest very different degrees of permanence. Species which invade stable vegetation temporarily have been termed adventive by $\mathrm{A}$. DeCandolle (1855: 643). Permanent invasion (invasio diutina) occurs when a species becomes permanently established in a more or less stable formation. It is characteristic of the great majority of invaders found in the grassland and forest stages of successions.

Plants which have arisen within a formation or have been a constituent part of it since its origin are indige nous (species indigenae). Contrasted with these are the species which have invaded the formation since it received its distinctive impress: these are derived (species derivatae seu advenae). The determination of the indigenous and derived species of a formation or larger division is of the utmost im. 
portance, as it enables us to retrace the steps by which the formation has reached its present structure, and to reconstruct formations long since disappeared. To render it less difficult, it is necessary to scrutinize the derived elements closely, first, because it is easiest to recognize the indigenous species by eliminating the derived, and second, because this analysis will show that not all derived species have entered the formation at the same time and from the same sources. Watson (1847:63) distinguished plants as colonist, alien, denizen and native, the last corresponding to indigenous. Colonists are weeds of fields and wastes, rarely found outside of places modified by man; aliens are plants which, though now more or less established, have been certainly or presumably introduced from foreign counries, while denizens are species which maintain themselves as though indigenous, but are suspected of being introduced. A. DeCandolle (1855:643) made the following division: (1) adventive, of foreign origin, but only temporarily ustablished; (2) naturalized, established but certainly of foreign origin; (3) probably foreign; (4) possibly foreign; (5) indigenous, of undoubted native origin. Pound and Clements (1900:52) distinguished regional elements as proper (indigenous), derived, and adventicious. Neither of these three groupings gives perfectly definite and consistent divisions, and for this reason the following arrangement is proposed. With respect to their original formation or region, species are either indigenous, native: or derived, foreign. Derived species may be termed vicine (species vicinae), when they are fully established invaders from adjacent formations or regions, and adventicious (species adventiciae), when they have come from distant formations and have succeeded in establishing themselves. Finally, those derived species which are unable to establish themselves permanently are adventive (species adventivae).

THE MANNER OF INVASION.

Since the ecesis of invaders depends in large measure upon the occupation of the plants in possession, the method and degree of invasion will be determined by the presence 
or absence of vegetation. Areas without vegetation are either originally $\mathrm{nak}$ ad (areae nudae) or d e $\mathrm{n} u \mathrm{~d}$ ed (areae denudatae), while vegetation with respect to the degree of occupation is open (sporadophytia), or closed (pycnophytia). Each tope of area presents different conditions to invaders, largely with respect to the factors determining ecesis. Naked habitats, rocks, talus, gravel-slides and dunes, while they offer ample opportunity for invasion on account of the lack of occupation, are really invaded with the greatest difficulty, not only because they contain originally few or no disseminules, but also because of their xerophytic character and the difficulty of obtaining a foothold, on account of the extreme density or instability of the soil. Denuded habitats, blowouts, sanddraws, ponds, flood plains, wastes, fields and burns, usually afford maximum opportunit. for invasion. They invariably contain a large number of disseminules ready to spring up as soon as the original vegetation is destroyed; the surface, moreover, is usually such as to catch disseminules and to offer them optimum conditions of moisture and nutrition. Open formations are readily invaded, though the increased occupation renders entrance more difficult than it is in denuded areas. Closed formations, on the other hand, are characterised by a minimum of invasion, partly because invaders from different formations find unfavorable conditions in them, but chiefly because the occupation of the inhabitants is so complete that invaders are unable to establish themselves.

Invasion takes place by the penetration of single individuals or groups of individuals. This will depend in the first place upon the character of the disseminule. It is evident that, no matter how numerous the achenes may be, the invasion of those anemochorous species with comate or winged seeds or one-seeded fruits will be of the first type, while all species in which the disseminule is a several or many-seeded fruit or plant, as in hooked fruits, tumbleweeds, etc., will tend to pruduce a group of invaders. Occasionally of course, the accidents of migration will bring together a few one-seeded disseminules into a group, or will 
scatter the seeds of a many-seeded fruit, but these constitute relatively rare exceptions. This distinction in the matter of invasion is of value in studying the relative rapidity of the latter, and the establishment of new centres, but it is of greatest importance in explaining the historical arrangement of species in a formation, and bence has a direct bearing upon alternation. It is entirely independent of the number of invaders, which, as we have seen, depends upon seed-production, mobility, distance, occupation, etc., but is based solely upon mode of arrangement, and will be found to underlie the primary uypes of abundance, copious and gregarious. In this connection, it should also be noted that the contingencies of migration, especially the concomitant action in the same direction of two or more distributive agencies, often results in the penetration of a group of individuals belonging to two or more species. This may well be termed mass invasion (invasio solida): it is characteristic of transition areas or regions aud, along valleys or other natural routes for migration, it gives rise to species guilds (Artengenossenschaften, Drude). The movement of species guilds constitutes one of the most complex and interesting problems in the whole field of invasion, the solution of which can be attempted only after the thorough analysis of the simpler invasions between formations. A better understanding of the meaning of invasion by species guilds is imperative for the natural limitation of regions, as at present such groups constitute alien associations in many regions otherwise homogeneous.

The invasion of a formation may occur at three different levels: (1) at the level of the facies, (2) below the facies, (3) above the facies, depending directly upon the relative height of invaders and occupants. The invasion level is an extremely simple matter to determine, except in the case of woody plants, such as shrubs and trees, which attain their average height only after many years. Its importance is fundamental: the level at which invasion occurs not only determines the immediate constitution of the formation, whether its impress shall still be given by the occupants, or 
by the invaders or by both together, but it also decides the whole future of the formation, i. $e$. whether the invaders or occupants shall persist unmodified or modified. The problem is an extremely complex one, but the careful analysis of invasion at each level throws a flood of light upon it The entrance of invaders of the same general height as the facies of a formation results regularly in mixed formations. This is well illustrated by the structure of the transition areas between two formations of the same category, i. e. forests, meadows, etc. It is seldom, however, that the facies and invaders are so equally matched in height and other qualities that they remain in equilibrium for a long period: one or the other has a slight advantage in height, or the one suffers shading or crowding better than the other, is longer-lived or faster-gruwing, with the result that invader yields to occupant, or occupant to invader. It is a well known fact that many mixed formations represent intermediate stages of development.

Invasion at a level different from that of the facies is in. evitably followed by modification. If the invasion takes place below the facies, the invaders will be exterminated gradually, or slowly assimilated. In either case, there is little structural change in the formation, and its stability is affected slightly or not at all. If the invaders overtop the facies in any considerable number, the entire formation undergoes partial or cumplete modification, or in extreme cases it disappears, as is typically the case in succession. A peculiar variation of invasion at a level above the facies is seen where woody plants invade grassland, when the trees or shrubs become more or less uniformly scattered in an open woodland or open thicket. Here the grassland takes on an altogether different appearance superficially, though it is usually unchanged, except beneath and about the invaders, where either adaptation or extermination results. Finally, it should be borne in mind that the invasion of a particular formation, especially in the case of layered thickets and forests, often takes place at two levels, at the height of the facies and below the facies. 
The investigation of invasion furnishes the basis for all developmental and structural study of vegetation. Observational methods, though of great value in indicating the causes, direction, rapidity, amount and results of invasion, are in themselves insufficient, and must at present be supplemented and in time replaced by experimental methods. For this purpose, the writer has extended the application of the quadrat, the use of which was first suggested by Pound and Clements (1898:19) for the accurate determination of abundance. In this connection, the quadrat is now used experimentally to follow in an exact and minute manner the changes produced by invasion. The permanent quadrat makes it possible to record from year to year the incidental invasions on any one spot, or the changes of a succession as the latter passes from one stage to another. If a quadrat list showing the annual increase or decrease in abundance of each species is all that is desired, the list quadrat, which is five meters square, is sufficient. A permanent record of the exact changes in a quadrat area is of much greater value, however; such a record is obtained by the use of the chart quadrat. The size of the latter depends upon the character of the vegetation: in open formations, and in forests and thickets without dense undergrowth, the chart quadrat should be two meters square, but in closed vegetation the accurate plotting of a quadrat is an arduous task, and the area best adapted to this work is a meter square. The permanent record of a chart quadrat is made upon centimeter plotting paper, the scale being one centimeter to each decimeter, and a close-focus photograph is also made of each quadrat charted. In practice, chart quadrats have been made permanent by driving a properly labelled stake at the north east curner of the quadrat.

An invaluable aid to the study of invasion and succession is the experimental or denuded quadrat. A chart quadrat is staked out and plotted in the usual way, and a photograph is made of it. The area is then completely denuded, especial care being taken to remove all perennial parts. Such denuded quadrats are then charted and photographed each 
succeeding year or season, until the area has again become stabilised. Denuded quadrats are of great value in determining the comparative mobility and ecesis of the species of a formation or patch, and they are indispensable for the reconstruction of the early stages of a succession.

A device known as the migration circle is employed to study the amount, direction, and rapidity of movement away from any particular group of individuals, which is essentially a miniature vegetation centre. The circle is used in place of the quadrat, because it is probable that migration will operate in all directions. The migration circle, like the quadrat, may be either temporary or permanent: the latter, which gives by far the more valuable results, is secured by driving a properly labelled stake in the midst of the particular plant group, and recording the radius of the area. The size of the circle varies with the height and mobility of the species studied; the most satisfactory circles are those with one, five or ten-meter radius. The twenty-five meter circle is useful at the margin of denuded areas and in very open vegetation, but the area enclosed is so large as to make it impracticable in closed formations. The choice of a circle depends upon the size of the nucleus of individuals taken as a center and upon the distance between two or more adjacent centers. Because of the large area to be cleared, it has not been found feasible to use denuded circles with a radius larger than one meter. The value of a denuded circle ten meters in radius for the comparative investigation of migration and ecesis would be so great, however, that its use is certain, as experimental methods in the field come to be recognized as more and more imperative. 


\section{BIBLIOGRAPHY}

1745 Linné, C. v.

1751

1802 Treviranus, G. R.

1805 Humboldt, A. V.

1811 Willdenow, K. L.
Flora Suecica

Philosophia Botanica

Biologie

Essai sur ld Géographie des Plantes

Allgemeine Bemerkungen

über den Unterschied der Vegetation auf der nordlichen und südlichen Halbkugel der Erde

1815 Brisseau-Mirbel, C. F. Elémens de Physiologie Végétale et de Botanique

1819 Buch, C. L. v.

Allgemeine Übersicht der Flora auf den Canarischen Inseln

1820 Link, H. F.

Die Urwelt und das Alterthum DeCandolle, A. P.

Essai Elémentaire de Géographie Botanique

1823 Schouw, J. F.

Grundzüge einer Allgemeinen Pflanzengeographie

1832 DeCandolle, A. P. 1833 Fenzl, E.

Physiologie

Versuch einer Darstellung der geographischen Verbreitungs- und Vertheilungsverhälínisse der natürlichen Familien der Alsineen

1836 Meyen, F. J. F.

1843 Grisebach, A.

1847 Watson, H. L.

Grundriss der Pflanzengeographie

Bericht über die Leistungen in der Pflanzengeographie Cybele Britannica: or, British plants and their geographical relations.

1848 Humboldt, A. V. Views of Nature 1852 Henfrey, A. 1855 DeCandolle, A.

The Vegetation of Europe Géographie Botanique Raisonnée 
1859 Darwin, C.

1860 Sendtner, O.

1865 Kabsch, W.

1871 Zimmer

1872 Grisebach, A.

1873 Hildebrand, F.

Hegelmeyer, F.

1874 Nägeli, C.

Lohde, G.

1875 DeCandolle, A.

1876 Drude, O.

Blytt, A.

Hoffmann, H.

1879 Engler, A.

1881 Buchenau, F.
Origin of Species

Die Vegetationsverhältnisse des Bayerischen Waldes

Das Pflanzenleben der Erde

Über Pflanzenwanderungen

Die Vegetation der Erde

Die Schleuderfrüchte und ihr in anatomischen Bau begründeter Mechanismus Pringsheims Jahrb. 9:235.

Der Verbreitungsmittel der Pflanzen Jast '73:224

Ueber Bau und Entwickelung e in iger Cuticulargebilde Pringsheim's Jahrb. 9:286 Verdrängung der Pflanzen. formen durch ihrer Mitbewerber Sitzungsber. Akad. Wiss. Wien 1874: 109

Ueber die Entwicklungsgeschichte und den Bau einiger Samenschalen Just 2:441 Sur les causes de l'inegale distribution des plantes rares dans la chaîne des Alpes Just 3:663

Anwendung physiologischer Gesetze zur Erklärung Vegetationslinien Just 4:674 Essay on the Immigration of the Norwegian Flora Just '76: 693

Über Accommodation Just '76: 952

Versuch einer Entwicklungsgeschichte der Pflanzenwelt Monographie der Juncaceen Engler Jahrb. 1: 124 
Hildebrand, F.

Bachmann, E. T.

1882 Goeze, E. Ascherson, $\mathrm{P}$.

1883 Hildebrandt, $H$.

Marloth, R.

1884 Warming, E.

1886 Drude, O.

1887 Hult, R.

1887 Huth, E.

1889 Dingler, $\mathrm{H}$.

Wiesner, J.

1890 Drude, O.
Die Lebensdauer und Vegetationsweiqe Engler Jahrb. 2:51 Just ' $81: 302$

Darstellung der Entwicklungsgeschichte und des Baues der Samenschale der Scrofularineen Just '81: 494

Pflanzengeographie

Von der Verbreitung der Pflanzen

Einige Beobachtungen über den Witterungs-Einfluss auf die Lebensdauer und Vegetationsweise der Pflanzen Engler Jarhb. 4:1

Über mechanische Schutzmittel der Samen gegen schädliche Einflüsse von aussen Engler Jahrb. 4: 247

Über Sprossbau, Überwinterung und Verjüngung Engler Jahrb. 5: 56

Die systematische und geographische Anordnung der Phanerogamen

Moosfloran i traktan mellan Aavasaksa och Pallastunturi Engler Jarhb. 8:1 b Die Klettpflanzen mit besonderer Berücksichtigung ihrer Verbreitung durch Tiere Just' $87: 432$

Die Bewegung der pflanzlichen Flugorgane

Biologie der Pflanzen Handbuch der Pflanzengeographie 
1891 Kerner, A.

1892 MacMillan, C.

1893 Sernander, R.

Dammer, U.

1895 Warming, E. Buchwald, J.

Sernander, R. \& Kjellmark, $\mathrm{K}$.

1897 Costantin, J.

1898 Pound, R. \& Clements, F.E.

Delpino, F.

1900 Pound, R. \& Clements, F. E.
Pflanzenleben

Metaspermae of the Minnesota Valley

Die Einwanderung der Fichte in Skandinavien Engler Jahrb. $15: 1$

Die Verbreitungsausrüstungen der Polygonaceen Engler Jahrb, $15: 260$

Plantesamfund

Die Verbreitungsmittel der Leguminosen der tropischen Afrika Engler Jahrb. $19: 494$

Eine Torfmoor Untersuchung aus dem nördlichen Nerike Bull. Geol. Inst. Ups. $2: 4$

Accommodation des plantes aux climats froids et chauds Bull. Soc. Bot. Fr. $31: 490$

The Phytogeography of $\mathrm{Ne}$ braska

A Method of Determining the Abundance of Secondary Species Minn. Bot. Studies $2: 19$

Studi di geografia botanica secondo un nuovo indirizzo Mem. R. Accad. Sci. V. $7: 329$

Schimper, A. F. W. Pflanzengeographie auf physiologischer Grundlage

The Phytogeography of Nebraska 2nd edition 
1901 Thornber, J. J.

1902 Jaccard, P.
The Prairiegrass Formation in Region I Rep. Bot. Surv. Nebr. $5: 29$

Gesetze der Pflanzenvertheilung in der alpinen Region Flora $90: 349$
1820 DeCandolle, A. P.

1823 Schouw, J. P.

1852 Henfrey, A.

1855 DeCandolle, A.

1859 Darwin, C.

1864 Palacky, J.

1865 Kabsch, W.

1872 Grisebach, A.

1873 Nägeli, C.

1879 Engler, A.

1886 Drude, 0.

1898 Delpino. F.

1901 DeVries, H.
Essai Elémentaire de Géographie Botanique

Grundzüge einer Allegmeinen Pflanzengeographie

The Vegetation of Europe

Géographie Botanique Raisonnée

Origin of Species

Pflanzengeographische

Studien Just'89:169

Das Pflanzenleben der Erde

Die Vegetation der Erde

Das gesellschaftliches Entstehen never Species Bot. Mitth. $3: 365$

Versuch einer Entwicklungsgeschichte der Pflanzenwelt Die systematische und geographische Anordnung der Phanerogamen

Studi di geografia botanica secondo un nuovo indirizzo Mem. R. Accad. Sci. V. $7: 329$

Die Mutationstheorie 


\section{SUCCESSION.}

In tracing the :historical development of the principle of succession, a difficulty constantly arises because of an unavoidable tendency to interpret the statements of earlier authors in the light of later knowledge. Previous to 1880 , relatively few observations of the operation of this principle had been made, and these were more or less incidental, except in the case of some writers upon forestry, who made no endeavor to connect the process with phytogeography. The first recorded mention of succession seems to have been that of Biberg (1749:78), who indicated in a very general way the changes of vegetation on rocks from the initial lichens to the ultimate forest. DeCandolle (1820:27), in discussing the struggle for existence, spoke of the cultures of the dunes of the "Landes", in which the rapidly growing Genista, after having served as a "cover" for seedlings of Pinus, was finally driven out by the latter. The first use of the term succession was by Steenstrup in studying the changes of the forest vegetation of Zealand (fide Grisebach (1844:16). Berg (1844) studied the successive modifications of the deciduous forests of the Harz in connection with their gradual disappearance ,before the pines. Humboldt (1850:10) evidently recognized the presence of succession in vegetation, though he mentioned it but incidentally: "All sea coasts afford evidence of the hundreds, or even thousands of years, which must pass before the moving sand can yield a firm basis for the roots of herbaceous plants in those hot and rainless regions where neither Lecideae nor other lichens can grow." "In northern regions, the absence of plants is compensated for by the covering of Baeomyces roseus, Cenomyce rangiferin$u s$, Lecidea muscorum, Lecidea icmadophila, and other cryptogamia which are spread over the earth and which may be said to prepare the way for the growth of grasses and other herbaceous plants. In the tropical world, where mosses and lichens are only observed to abound in shady places, some few oily plants supply the place of the lowly lichen'(125). 
"Thus one organic tissue rises, like strata, over the other and as the human race in its development must pass through definite stages of civilisation, so also is the gradual distribution of plants dependent upon definite physical laws. In spots where lofty forest trees now rear their towering summits, the sole covering of the barren rock was once the tender lichen: the long and immeasurable interval was filled up by the growth of grasses, herbaceous plants and shrubs." (214)

Henfrey (1852:56) has dealt briefly with the changes in vegetation due to the influence of man: "It is certain that the appropriate stations of many plants would be destroyed with the removal of forests, and new conditions of soil created for the habitation of immigrants from other regions. But the modification of the surface so as to alter the physical condition of the soil, is by far the most important change brought about in reclaiming land for cultivation. The banking out of the sea changes by degrees the vegetation of its shores: bare sand-dunes, where scarcely a plant could maintain a precarious footing, are by degrees covered by vegetation: sandy inland wastes are rescued from the heath and firs, and made to contribute at first by Coniferous woods, such as the larch and when the soil has become by degrees enriched, by the plants requiring a better nourishment, to the general stock of wealth; and in these changes many species are destroyed, while others naturally making their way into a fitting station, or brought undesignedly by the hand of man, grow up and displace the original inhabitants." Vaupell (1851:12) confirmed the earlier observations of Steenstrup on the Danish forest in general, but determined the succession to be Pinus, Quercus, Betula, Fagus. A. DeCandolle (1855:472) cited the obsorvations of de la Malle (1848) and Maguy (1850) upon the succession of different forest formations in France and Germany, but he failed to perceive the essential identity of this phenomenon with what takes place when a forest is cut or burned down, for he mentions the latter only to insist upon the difference between the two. He further held the erroneous idea that 
such changes were peculiar to social species, and hence concerned only a few plants and only certain localities or countries. Hoffmann (1856:189) found the raspberry to be the first invader in burns in the Ural Mountains: this was followed successively by the service-berry, alder, birch, and other deciduous trees, and these were finally replaced by the pines and other conifers. Hill was apparently the first to call attention to a general law of succession in burns or cuttings, viz., that the second growth is always of genera different from those found in the original vegetation. Stossner (1859), according to Drude (1902:15), described in a detailed manner the conversion of a fallow field covered with blue and yellow pansies into a mountain meadow.

Middendorff (1864:641) recognized the wide occurrence of succession ("Wechselfolge"), but considered the unlikeness of the different stages which had been noted by Hill to be exceptional to the general rule. "Es traf sich gerade, dass, wo ich im Bereiche der grossen Urwaldungen Gelegenheit hatte ältere Brandstätten und das Emporschiessen neuen Waldes auf ihnen zu beobachten, ich fast ausschliesslich die früheren Nadelholzarten von Neuem dieselben Flecken bedecken sah. Kiefern, Lärchen, siberische Edeltannen sab ich in schönstem Schlusse, nicht selten in vorllig ungemischten Beständen als schlanken Stangenwald aus der Asche ihrer Väter hervorgehen, und das selbst dort, wo in den umliegenden Wäldern Birken sich gern eingesprengt zeigten.

"Betrachten wir also die Sache näher, so ergibt sich, dass keineswegs die jetzt vielberufene Wechselwirthschaft der Natur in Betreff der Waldungen vorwaltend herrscht, sondern vielmehr dass Gegentheil. Wie sollte dem auch anders sein? Findet man denn nicht sogar dieselben Gräser und Blumen, findet der Botaniker uicht seine Seltenheiten stets an demselben Orte wieder? Es liegt keineswegs in der Natur der Dinge, dass ein solcher Wechsel der Baumarten stattfinden müsse. Eine jede Baumart macht gewisse Ansprüche an den Bođen, den Untergrund, die Feuchtigkeit, an Licht, an den Schutz, sowohl vor Wind und Wetter als 
vor Sonnenschein, vor überwuchernden Pflanzen und "dergleichen mehr. Eine jede Baumart gedeiht und vermehrt sich, alle anderen verdrängend, bis zur Alleinherrschaft, dort wo sie ihr eigenthümlichen Ansprüche am best zu befriedigen vermag.

"Will ich etwa damit sagen dass dort wo jetzt Urwälder stehen, auch schon seit Jahrtausenden stets dieselben Baumarten in regelmässig auf einander folgenden Generationen sich ausgesäet haben, aufgewachsen sind, alterten, vermoderten, wiederum aufwuchsen? Wenn es sich darum behandelt, eine Regel aufzustellen, so dürfte, glaube ich, diess allerdings im Urzustande die Regel sein, nämich für Nord-Asien und Nord-Europa. Wie erklären sich aber dann die häufigen Ausnahmen, die man beobachtet? Ich schreibe sie vorzüglich dem Eingriffe des Menschen in das Getriebe der Natur zu."'

Kabsch (1865:75) pointed out the primary rôle of lichens in the origination of succession on rock surfaces: "Die Flechten sind die wahren Pioniere der Vegetation, sie graben sich in den weichen Kalk wie in den härtesten Basalt, zersetzen das Gestein und mischen die befreiten Bestandtheile desselben mit ihren eigenen Zersetzungsprodukten, um so in der Vereinigung von organischem und unorganischem Material die Möglichkeit zum Gedeihen einer höheren Pflanzenvegetation vorzubereiten." Engler (1879; IX) has written a comprehensive treatise upon the great successions of the past, but these can, of course, be painted only in the large, and have in consequence only a remote connection with the changes of present successions. Hult (1881, 1885) was the first to investigate succession in a systematic fashion, and to him also belongs the credit of having laid down the general principles upon which the developmental study of formations must be based. He recognized the fundamental fact that most formations are merely transition stages to a few ultimate formations. He has traced the developmental history of each type of intermediate formation through its successive stages in his region, and has studied the substitution of facies in the ultimate forests. 
In 1887, Hult investigated the invasion and substitution of one formatiou by another in the alpine regions of Lappmark. He found that in the dryer places Cladineta and Alectorieta finally replaced all other associations, while in moist places grass and herbaceous formations passed into formations of dwarf shrubs, the universal rule being a transition from hygrophilous to more or less xerophilous conditions.

Treub (1888), after the destructive eruption of Krakatau in 1883 , found that Cyanophyceae were the first plants to appear on the new soil: these were followed by an exclusive vegetation of ferns, in which a few phanerogams, especially composites, began to appear by the third year. Senft (1888) traced the development of vegetation on naked xerophytic slopes of the Horselbergen. The pioneers were lichens, especially Parmelia, followed soon by mosses, Hypnum, Barbula, and after a few years by Festuca ovina, which appeared first in the rock clefts and then spread into a thick sod. The next invaders were all grasses, Koeleria cristata, Briza, Melica, and Brachypodium, which almost entirely replaced Festuca in three years: the grasses were then invaded, though not completely replaced, by herbs, Helianthemum, Verbascum, Lactuca scariola, Agrimonia eupatoria, Anemone, Gentiana, etc., and by a few shrubs, Juniperus communis, Viburnum lantana, and Crataegus. Later appeared Ligustrum, Cornus and Rhamnus, and still later, Cotoneaster, Crataegus, Prunus and Rosa, forming a thicket twolve years after the inception of the succession. The last stage was a forest of Sorbus, Corylus. Fagus, Alnus, Tilia and Acer. Krassnoff $(1883,1886)$ found that forest clearings in the Altai were recovered partly by persistent forest plants, and partly by invading steppe plants, Paeonia, Bupleurum, Aconitum, Chamaenerium, Delphinium, Geranium, Thalictrum, etc. In certain places where fire had destroyed the forest growth, the ground was still covered after a lapse of many years with a tall herbaceous growth of Helleborus, Aconitum, Thalictrum, Paeonia, Pedicularis and Rosa. It seems highly improbable, 
however, that this is an ultimate formation, as Krassnoff and Warming indicate.

Fliche (1888) investigated the behavior of forest plantations in lower Burgundy, and reached the following conclusions, many of which are of general application. The original plantations, chiefly birch and exotic cultivated species, were overgrown by the indigenous oaks and beeches, except on poor ground. Shrubs, bushes and most herbs disappear as the growth of the trees renders the light more and more diffuse, In new plantings, the grasses and field weeds disappear the first year, and are replaced by the weeds of fallow fields. The latter are overgrown the very next year by bushes, with which shrubs soon associate themselves, until finally the invading trees drive out all but a few shade-loving species. Migration into these areas is chiefly by birds and winds, the latter bringing by far the larger number of seeds, though the former are really more effective because of the greater chance of ecesis. The definitive ecesis of a species is independent of its mobility, as is seen by the fact that the immobile Fagales completely replace the very mobile birches and pines. From his work, the author derives two general rules of reforestation, which also bold in large part for similar natural successions. "Erstlich ist es fehlerhaft, Moose und Gestrüpp (Juniper"us, Genista, Prunus,) auf gleiche Stufe zu stellen mit den Ackerunkräutern und darum auszurotten: beide verbessern den Boden, bereiten ihn für den Wald vor, fördern die Keimung der Samen, und schützen, wie an mehreren Beispielen gezeigt wird, die aufschiessenden Bäumchen: sind diese erstarkt, so verschwindet das Gestrüpp von selbst; das Moos aber leistet noch weitere Dienste. Sodann gedeiht der Wald dann am besten und regeneriert sich auch ohne Zuthun des Menschen von selbst, wenn er die Arten, und in dem Verhältnis enthält, wie sie in der jeweiligen Gegend einheimisch, und eben den bezüglichen Wachstumsbedingungen am besten angepasst sind; und eben dieses Verhältniss stellt sich in einem sich selbst überlassenen Walde immer wieder her." 
Douglas (1889) used the term succession in combating the then prevalent idea, that, when a forest is once destroyed, a similar forest could never again be established on the same spot. He pointed out that the period necessary fur such reestablishment would depend largely upon the mobility of the trees concerned, but that jt was finally to be determined by the persistence of the species. Warming (1890) found in the marshy regions along the east coast of the North Sea that Zostera filtered out and retained the fine particles of soil in the deeper water, resulting in the formation of mud banks, while algae and Salicornia herbacea filled the same office in shallow water. As the ground became higher and dryer, Glyceria replaced Salicornia, and was accompanied by Triglochin, Suaeda, Plantago, Glaux, Atriplex, etc.; this vegetation was then in its turn driven out by Juncus, Hordeum, Festuca, Lepturus, Armeria, Artemisia, etc. Kihlmann (1890) studied the gradual drying up and disappearance of the Sphagnum swamps, a phenomenon of frequent occurrence in Russian Lapland. Sphagnum is slowly replaced by the more xerophytic mosses and by lichens. The earlier fruticulose forms are followed by a crust of Lecanora tartarica, in which grow feebly Empetrum, Ledum, and Vaccinium myrtillus. The succession is then closed, except where the lichen crust is destroyed by some accidental agency. Kihlmann further found that young dense growths of birch, in which no coniferous seedlings appeared, arose where the forests of red fir had been burned off, and he concluded that this was due to the difference in the time necessary for the ripening of their seeds. The fact that the formation was still young and dense would seem simply to indicate that the fir had not yet found the proper conditions for invasion, conditions which sooner or later must occur.

Kerner (1890:267,895), without paying attention to particular formations, summed up in a graphic though somewhat general way certain main facts of succession. "This mechanical retention and storage of dust by rock-plants and of mud by aquatic plants is of the greatest importance in determining the developmert of the 
earth's covering of, vegetation. The first settlers on the bare ground are crustaceous lichens, minute mosses and algae. On the substratum prepared by them larger lichens, mosses and algae are able to gain a footing. The dead filaments, stems and leaves pertaining to this second generation arrest dust in the air and mud in the water, and thus prepare a soft bed for the germs of a third generation, which on rocks consists of grasses, composites, pinks and other small herbs, and in water of pond weeds, water-crowfoots, hornwort and various plants of the kind. The second generation is produced in greater abundance than the first, and the third developes more luxuriantly than the second. The third may be followed by a fourth, fifth and sixth. At all times and in all places, we see younger generations displacing the older and building upon the foundations laid by their predecessors. The first settlers have a hard fight with uncompromising elements to seize possession of the lifeless ground. Years go by before a second generation is enabled to develop in greater luxuriance upon the earth prepared by the first occupiers; but there is no cessation in the productive and regulative effects of vegetable life, and its energy and aptitude in the work result in the erection of its green edifices over wider and wider areas. New germs are established upon the smouldered dust of dead races, and others on the plant forms adapted to the altered substratum, and so, for hundreds and thousands of years, the changes go on, until at length the tops of forest trees wave above a black and deep soil, the battlefield of a number of bygone generations. At leasu three successive series of settlers may thus be traced upon every spot, and not infrequently the number is four or five. Now, if each of these groups corresponds to a particular community, which is as a matter of fact the case, the phenomenon described must produce the same impression as though the communities became transformed into one another in the course of time. It is therefore necessary to recognize the existence of the incipient and decadent stages as well as that of predominance. In the incipient stage, relics of the community which previously occupied 
the same spot are still to be found, and in the stage of decadence the first pioneers of the community that is to succeed make their appearance."

Korschinsky (1891:473) opposed the view that modifications of vegetation were entirely dependent upon changed "physico-geographical" relations, and brought forward the development of the oak forests of middle Russia as evidence in support of this view. He concluded "dass die Eichen. wälder des mittleren Russlands, welche in Gestalt eines ununterbrochenen Grenzstriches das Steppengebiet von dem der Coniferenwälder trennen, auf besagtem Wege entstanden sind, d. h. dass sie inmitten der freien Wiesensteppen anfangs in Gestalt strauchartigen Eichengehölzes hervorwuchsen, letzteres sodann mehr und mehr um sich griff und zu jungen Eichenwäldchen wurde, um sich schliesslich in compacte Waldungen umzubilden: dass also diese Eichenwälder alle die Stadien durchliefen, wie wir sie jetzt auch noch an verschiedenen Punkten des Steppengebietes zu beobachten Gelegenheit haben. Hieraus aber folgt, dass dort, wo wir jetzt Eichenwälder oder deren Spuren vorfinden, zu einer früheren Zeit Steppen existiert haben, die folglich etwas weiter gegen Norden erstreckten, als wir es jetzt sehen. Jedoch können die Veränderungen der Vegetation auch ganz selbstständig, abgesehen von allen klimatischen Einflüssen erfolgen, indem sie ausschliesslich durch die Lebenseigenschaften der concurrierenden Formen, durch das Erscheinen neuer Eindringlinge, überhaupt durch die socialen Bedingungen der Pflanzenwejt an und für sich herbeigeführt werden." Warming (1891) traced the building of dunes on the Danish coast and the development of vegetation upon them. The mobile dunes begin simply as heaps of sand formed by tides, waves and wind, the particles of which are as a rule less than one third of a millimeter in diameter. The further growth of such dunes is made possible by sandbinders, Psamma arenaria, Elymus arenarius, Carex arenaria, Agropyrum junceum, Alsine peploides, etc. The last two are found only on the lower dunes, and are sooner or later driven out by Psamma and 
Elymus, which are especially adapted to the building of high dunes, because of their ability to push up through a cover of sand. Other plants, algae, lichens and mosses, and lowgrowing spermatophytes, find their way in among the shoots of Elymus and Psamma, and, as the sand becomes more and more fixed, slowly conquer the intervening spaces. The dune gradually becomes more stable, and is finally spread with a thick low gray-green cover, before which the two original sand-binders disappear. Finally, the stable dune ma.y pass over into a stable Calluna heath. Sernander (1892:9) has made the following observations in regard to the invasion of the pine in Scandinavia: "Auf entblösstem Boden entsteht bekanntlich rasch eine bunte Ansiedlung einer Menge verschiedenartiger Pflanzenformen. Bald vereinigen sich diese zu einer geschlossenen Decke und damit ist ein verhängnisvoller Kampf zwischen den verschiedenen Ansiedlern eingetreten. Sie ringen mit einander um Licht, Raum und Nahrung; grosse Massen unterliegen mehr oder weniger rasch, während eine verhältnismässig geringe Anzahl sich über die Leichen der Besiegten ausbreitet. Aus diesem rücksichtslosen Kampf geht eine im Verhältnis zu der mehr zufällig zusammengekommenen Ansiedlung nach bestim. mten Gesetzen gebildete Pflanzengesellschaft; $d$. h. eine geschlossene Pflanzenformation hervor. Damit hat aber die Entwicklung nicht aufgehört. Die relative Gleichgewichtslage, in welche die die Formation zusammensetzenden Elemente jetzt gekommen zu sein scheinen, ist selten eine dauernde. Nicht nur dass gewisse Mitglieder der Gesellschaft sich noch immer auf Kosten anderer ausbreiten, sondern auch neuen Ansiedlern aus der Nachbarschaft gelingt es, festen Fuss unter den älteren auf dem Platze zu fassen, und nun fängt zwischen diesen ein mehr oder weniger heftiger Streit an. Die Entwicklung der Vegetation'schlägt neue Wege ein, und gewisse relative Ruhestadien während dieser Entwicklung zeigen sich als distinkte Formationen. deren gegenseitige Folge einen der wichtigsten und nächstliegenden Gegenstände der modernen biologischen Pflanzengeographie ausmacht. Schliesslich 
muss jedoch ein wirkliches Gleichgewicht eintreten. Man hält dieses Gleichgewicht für erreicht wenn sich die überlebenden Pflanzenformen in bestimmten Proportionen verjüngen und keine neuen Elemente weiter eindringen können. Die sogenannte Schlussformation ist nunmehr gebildet."

Krause (1892, 1893) studied the origin of the heaths and meadows of northern Germany, reaching the conclusion that the former had for the most part arisen from forests by reason of cultural changes, while the latter also had resulted from changes wrought by man in swamp and moor. Flahault and Combres (1894) found Arthrocnemum macrostachyum to be the pioneer on the low saline alluvium at the mouth of the Rhone. Afterwards, Salicornia, Atriplex and Aeluropus established themselves and were in their turn finally dis. placed by a very different vegetation consisting of Juniperus and Pinus. Wojeikow (1894) gave a very complete summary of the way in which man affects vegetation, considering especially the results of those activities which destroy plant coverings and consequently initiate succession.

Warming has not only contributed very largely to the investigation of succession, but he has also made (1895) the first comprehensive summary of the many scattered results obtained in this field. He has considered the changes of vegetation under three heads, which correspond closely to different types of succession: "(1) Neuer Boden (2) Durch langsamer Veränderungen auf bewachsenem Boden hervorgerufene Vegetationsveränderungen. (3) Vegetationsveränderungen ohne Veränderungen im Klima oder im Boden." The following statement constitutes the first attempt to deduce from various sources the general rules underlying the principle of succession. 'Es ist schwierig, schon jetzt etwas Allgemeines über die auf einem neuen Boden auftretende Vegetation zu sagen, weil es darüber wenige eingehende Untersuchungen giebt: jedoch scheint folgendes aus diesen hervorzugehen (vgl. Hult, Grevillius, u. a.).

1. Die erste Vegetation ist offen. Es vergeht immer einige Zeit, bevor sich eine zusammenhängende Vegetationsdecke 
bildet. Die Individuen stehen anfangs sehr zerstreut, aber allmählich wird ihre Menge grösser.

2. Die Artenzahl ist anfangs gering, wächst jedoch und ist nach Verlauf einer gewissen Zeit grösser als später, indem viele Arten anfänglich einen günstigen Platz finden, aber später verdrängt werden, wenn sich die Decke schliesst und sich tyrannischere Arten eingefunden haben. Verschiedene Teile des neubewachsenen Geländes werden sich oft sehr ungleichartig mit Pflanzen bedecken. Allmählich wird die Vegetation gleichartige und artenärmer,

3. Sehr oft werden ein-und zweijährige Arten zuerst viel zahlreicher als später sein, indem sie auf dem offenen Gelände günstigere Bedingungen finden als auf dem bedeckten: viele werden der Unkrautflora der Gegend angehören. Darauf werden die mehrjährigen Kräuter oder die Holzpflanzen überwiegen.

4. Die zuerst einwandernden Arten werden die sein, welche in der Nähe vorkommen und die besten Mittel für die Verbreitung durch Wind oder Vögel haben. Die Geröllhalden der Alpen werden zuerst von Arten mit fliegenden Samen besiedelt (Kerner). Wird in Norwegen ein Nadelwald zerstört, so wandern zuerst Birken und Happel (leichter fliegende Früchte und Samen) nebst Sorbus (Beeren) ein (Blytt, Hult).

5. Handelt es sich um die Einwanderung von Bäumen, so werden die Lichtbäume oft vor den Schatten ertragenden erscheinen; das Umgekehrte kann nicht stattfinden.

6. Die Ausbildung zu ausgeprägten Vereinen geht allmählich vor sich. Die ersten, miteinander gemischten Individuen gehören in Wirklichkeit zu verschiedenen natürlichen Vereinen, die sich erst nach und nach auf die passendsten Standorte vertheilen. Man kann demnach von Anfang s Übergangs -, und Schlussvereinen sprechen".

Graebner (1895:500) has made a careful study of the origin of the heath formation in northern Germany. In the development of the heath upon the litoral dunes, the strand plants are replaced to some degree by annual sandbinders, Jasione, Eriophila, and Spergula, between which appear Aira, 
Arubis, Solidago and Chrysanthemum. In the protection of the individuals of these species develop colonies of lichens, Cladonia, Cetraria islandica, Baeomyces roseus, etc. and mosses, Bryum argenteum, Dicranum scoparium, Ceratodon purpureus, ttc., while here and there spring up little plantlets of Calluna and Empetrum. During rainy seasons, a tenacious crust of algae, largely Cyanophyceae, covers the sand everywhere; this crust serves to hold the sand particles together, and upon dying produces the first humus. The mosses also play an important part in fixing the sand, in as much as many species, when more or less covered by the blowing sand particles, send up new shoots, forming a tuft. The lichens especially are humus builders. Finally, in a locality modified in the way above, the seedlings of flowering plants are able to maintain themselves in large quantity. Calluna and Empetrum enter more and mure abundantly, Jasione, Leucanthrmum and Solidago decrease in number, while Hypnum schreberi spreads steadily and covers the moist places with a thick turf. In a similarly detailed fashion, Graebner has traced the development of the heath moor and, more generally, the modification of forest, and heath moor into heath, as well as the changes exhibited by the latter, especially under culture. Grevillius (1895) investigated the vegetation of the islets of the Hjelmar sea, which Callmé had studied in 1886. He found 215 species in place of the 115 observed by the latter. These were more uniformly distributed over the island, and the vegetation had become much more closed. The typical structure of the vegetation was as follows: (1) a strand-zone of grasses and sedges, (2) a zone of shrubs, mostly Salix, below which grew small strand plants, (3) a central nucleus of dense young forest, mostly of Betula verrucosa, Populus tremula, and Alnus glutinosa. Pines were found sparingly on a few of the islands. Meigen (1896:212) found that, in the vineyards of Saxony, which had been destroyed to exterminate the Phylloxera, $73 \%$ of the invaders of the first year were annual, 13\% biennial, ruderal plants, $13 \%$ were perennials, while none were woody plants. The changes of the second jear con- 
sisted in the disappearance of some of these, but especially in the entrance of new invaders. Of the former, the annuals were the first to disappear, while of the latter, perennials were in the majority. After six years, vegetation was still largely ruderal. From his work, Meigen has deduced the following conclusions: "Bei gleichen oder wenig verschiedenen climatischen Verhältnissen hängt die Folge der Formationen in erster Linie von der Bodenbeschaffenheit ab. Zunächst kommt es also darauf an, festzustellen, was für verschiedene Standorte innerhalb des zu untersuchenden Gebietes vorkommen, wobei sich eine Gliederung der Standorte ergeben muss aus der physikalischen und chemischen Beschaffenheit, sowie aus ihren Beziehungen zu Wärme, Licht und Feuchtigkeit, also den Faktoren, die vor allen den Charakter der Vegetation bestimmen. Dann ist auszugehen von einem völlig von Pflanzen und Vegetation freien Boden und nun zu ermitteln, welche Formationen sich hier auf den verschiedenen Standorten ansiedeln. Auf festem Fels, auf losem Sand, in stehendem Wasser, in Bächen und Flüssen, überall finden wir besondere Gemeinschaften, die in ihren Gliedern und ihrer Gesamtheit für den besonderen Standort auch besonders organisiert sind. Jede dieser Einzelformationen muss dann für sich weiter verfolgt worden, es sind die Änderungen zu untersuchen, die sie erleidet und die schliesslich zur Herstellung einer Folgeformation führen. Daraus ergeben sich dann Reiben von Formationen, die unter sich aufs engste zusammenhängen, weil jede folgende sich aus der vorhergehenden entwickelt hat. So wird man endlich zu einer Formation gelangen, die nun, sich selbst überlassen, keine Änderung mehr unterliegt, also die Schlussformation der Reihe bildet, womit das Gleichgewicht an dem betreffenden Standort hergestellt ist. Nun sind die gegenseitigen Beziehungen dieser Formationsreihen zu bestimmen. Daraus wird sich dann ergeben, ob die Schlussformation einer Reihe durch Berührung mit Formationen aus andern Reihen noch weitere Veränderungen erleiden kann. Es kann auch eine Formation Schluss. formation mehrerer Reihen sein, so dass diese convergieren, 
während andere parallel neben einander laufen. Auch schon vor Ausbildung einer Schlussformation trifft nicht selten eine Reihe mit einer anderen zusammen und geht in deren Richtung weiter."

Pound \& Clements $(1898: 216,259)$ traced the development of the blowout formation and of waste formations on denuded areas. They distinguished formations as primitive or recent with respect to origin; the latter alone are to be considered in the study of existing successions. Recent formations arise by nascence, as in the case of areas destitute of a floral covering, or by modification, when a patch or formation is changed by the invasion of other species. Cowles' work (1899:95) on the dune vegetation of Lake Michigan, modelled after Warming's studies on the Denmark coast. was the first special account of succession to be published in this country. The author has classified dunes as embryonic, wandering and established, and has followed the detailed development of vegetation in each of these. His conclusions form an excellent résumé of the stages in dune successions. Nilsson (1899:89) followed in a detailed manner the development of vegetation on the cliffs and in the swamps of middle Swoden. The cliff succession is especially interesting on account of the full treatment of the lichen stages. Cowles (1901:73) has applied his methods of successional research to the study of river-valleys and shorelines with very instructive results, and he has summed up his investigations in a developmental classification of much value. Whitford (1901:289) has made a study of the forests of northern Michigan along the same lines. Weber $(1901,1902)$ has made further contributions to the developmental history of moor and forest, Graebner (1902) to the phylogeny of the heath vegetation and Clements (1902) to the succession of talus formations.

Succession is the phenomenon in which a series of invasions occurs in the same spot. It is important, however, to distinguish clearly between succession and invasion, for, while the one is the direct result of the other, not all invasion produces succession. The number of invaders must 
be large enough, or their effect must be sufficiently modifying or controlling to bring about the gradual decrease or disappearance of the original occupants, or a succession will not be established. Partial or temporary invasion can never initiate a succession unless the reaction of the invaders upon the habitat is very great. Complete and permanent invasion, on the other hand, regularly produces successions, except in the rare cases where a stable formation entirely replaces a less stable one without the intervention of other stages Succession depends in the first degree upon invasion in such quantity and of such character that the reaction of the invaders upon the habitat will prepare the way for further invasion. The characteristic presence of stages in a succession, which normally correspond to formations, is due to the peculiar operation of invasion with reaction. In the case of a denuded habitat, for example, migration from adjacent formations is constantly taking place, but only a small number of migrants especially adapted to somewhat extreme conditions are able to become established in it. These reach a maximum development in size or number, and, in so doing, react upon the habitat in such a way that more and more of the dormant disseminules present, as well as those constantly coming into it, find the conditions favorable for germination and growth. The latter, as they in turn attain their maxinum, cause the gradual disappearance of the species of the first stage, and at the same time prepare the way for the individuals of the succeeding formation. It is at present impossible to determine to what degree this substitution is due to the struggle for existence between the individuals of each species and between the somewhat similar species of each stage, and to what degree it arises out of the physical reaction.

It is evident that geological succession is but a larger expression of the same phenomenon, dealing with infinitely greater periods of time, and produced by physical changes of such intensity as to give each geological stage its peculiar stamp. If, however, the geological record were sufficiently complete, we should find unquestionably that these great 
successions merely represent the stable termini of many series of smaller changes, such as are found everywhere in recent or existing vegetation.

The fundamental causes of succession are invasion and reaction, but the initial causes of a particular succession are to be sought in the physical or biological disturbances of a habitat or formation. With reference to the initial cause, we may distinguish normal succession (successio normalis), which beyins with nudation, and ends in stabilisation, and a nom alous succession (successio anomala), in which the facies of an ultimate stage of a normal succession are replaced by other species, or in which the direction of movement is radically changed. The former is of universal occurrence and recurrence; the latter operates upon relatively few ultimate formations. In the origin of normal successions, nudation may be brought about by the production of new soils or habitats, or by the destruction of the formation which already occupies a habitat. In a few cases, the way in which the habitat arises or becomes denuded is not decisive as to the vegetation that is developed upon it, but as a rule the cause of nudation plays as important a part in the development of a succession as does the reaction exerted by the invaders. The importance of this fact has been insisted upon under invasion. New soils present extreme conditions for ecesis, possess few or no dormant disseminules, and, in consequence, their successions take place slowly and exhibit many stages. Denuded soils as a rule offer optimum conditions for ecesis, as a result of the action of the previous succession, dormant seeds and propagules are abundant, and the revegetation of such habitats takes place rapidly and shows few stages. The former may be termed primary succession (successio primitiva), the latter, secondary succession (successio secuta). PRIMARY SUCCESSIONS

These arise on newly formed soils, or upon surfaces exposed for the first time, which have in consequence never borne vegetation before. In general they are characteristic of mountain regions, where weathering is the rule, 
and of lowlands and shores, where sedimentation or elevation constantly occur. The principal physical phenomena which bring about the formation of new soils are: (1) elevation, (2) volcanic action, (3) weathering, with or without transport.

1. Buccession through elevation. Elevation was of very frequent occurrence during the earlier, more plastic conditions of the earth, and the successions arising as a result of it must have been important features of the vegetation of geological periods. Today, elevation is of much less importance in changing physiography, and its operation is confined to volcanic islands, coral reefs and islets, and to rare movements or displacements in sea coasts, lake beds, shore lines, etc. There has been no investigation of the development of vegetation on islands that are rising, or have recently been elevated, probably because of the slow growth of coral reefs and the rare appearance of volcanic islands. On coral ruefs, the first vegetation is invariably marine, but as the reef rises higher above the surf line and the tide, the vegetation passes into a xerophytic terrestrial type adapted to an impervious rock soil, and ultimately becomes mesophytic. In volcanic islands, unless they are mere rocks over which the waves rush, the succession must always bogin with a xerophytic rock formation. The best known example of a rising coast line is found in Norway and Sweden, where the southeastern coast is rising at the rate of five or six feet a century. There can be little question that such changes of level will produce marked changes in vegetation, but the modification will be so gradual as to be scarcely perceptible in a single generation. It is probable that the forests of the Atlantic coastal plains are the ultimate stages of successions initiated at the time of the final elevation of the sea bottom along the coast line.

2. Succession through volcanic action. The deposition of vulcanic ashes, and flows of lava are relatively infrequent at present, occurring only in the immediate vicinity of active volcanoes, chiefly in or near the tropics. Successions of this sort are in consequence not only rare, 
but they are also relatively inaccessible to investigaturs. They have been studied in a few cases, for example, those of Krakatoa by Treub, but this study has been confined to the general features of revegetation. Ash fields and lava beds are widely different in compactness, but they agree in having a low water and nutrition content. The pioneer plants in both will be intense xerophytes, but the soil differences will determine that these shall be sandbinders in the former, and rock-weathering plants in the latter.

Practically all primary successions start on soils produced by weathering: this is also true of coral or volcanic islets and of lava beds, for no terrestrial vegetation can secure a foothold upon them until the surface of the rock has been to some extent decomposed or disintegrated. Weathering, as is well known, consists of two processes, disintegration and decomposition, which usually operate successively, though they are sometimes concomitant. Disintegration usually precedes, especially in rock masses, and, unless it is soon followed by decomposition, results in dysgeogenous soils. Decomposition often goes hand in hand with disintegration, or it takes place so ravidly and perfectly that it alone seems to be present. In either case, the resulting soil is eugeogenous. The relation of decomposition to disintegration determines the size and compactness of the soil particles, and upon the latter depend the porosity, capillarity and hygroscopicity of the soil. These control in large degree the character of the first vegetation to appear on the soil.

Another point of fundamental value in determining revegetation is the disposition of the weathered rock. If it remains in situ, it will evidently differ in respect to compact. ness, homogeneity, nutrition content, water content, disseminules, etc., from weathered material which has been transported. An essential difference also arises from the fact that a rock may be weathered a long distance from the place where the decomposed particles are finally deposited, and in the midst of a vegetation very different from that found in the region of deposit. The disposition of the weathered material affords in consequence a satisfactory basis for the 
arrangement of primary successions. The following classification is proposed, based upon the soil groups established by Merrill (1897: 300).

3. Succession in residuary soils. Residuary soils are always sedentary, i. e., they are formed in situ. They show certain differences dependent upon the rock from which they originate, which may be mixed crystalline shale, sandstone or limestone, but the thoroughness of decomposition causes these differences to be comparatively small. Residuary soils are typically eugeogenous : their successions in consequence usually begin with mesophytes, and consist of a few stages. The soluble salt content is comparatively low, since all soluble matters are readily leached out. Successions in these soils are especially characteristic of shale, sandstone and limestone ledges or banks. Cumulose deposits, like residuary ones, are sedentary in character, but, as they are produced by the accumulation of organic matter, they will be considered under reactions of vegetation upon habitat.

4. Succession in colluvial soils. Colluvialdeposits owe their aggregation solely or chiefly to the action of gravity. They are the immediate result of the disintegration of cliffs, ledges and mountain sides, decomposition appearing later as a secondary factor. The masses and particles arising from disintegration are extremely variable in size, but they agree as a rule in their angular shape. The typical example of the colluvial deposit is the talus, which may originate from any kind of rock, and contains pieces of all sizes. Gravel slides differ from ordinary talus in being composed of more uniform particles, which are worn round by slipping down the slope in response to gravity and surface wash. Boulder fields are to be regarded as talus produced by weathering under the influence of joints, resulting in huge boulders which become more and more rounded under the action of water and gravity. This statement applies to those fields which are in connection with some cliff that is weathering in this fashion: otherwise, boulder fields are of aqueous or glacial origin. The character of the successions 
in talus will depend upon the kind of rock in the latter. If the rock is igneous or metamorphic, decomposition will be slow, and the soil will be dysgeogenous. Successions on such talus consist of many stages, and the formations are for a long time open and xerophytic. In talus formed from sedimentary rocks, especially shales, limestones, and calcareous sandstones, decomposition is much more rapid, and the successions are simpler and more mesophytic.

5. Succession in alluvial soils. Alluvial soils are fluvial when laid down by streams and rivers, and litoral when washed up by the waves or tides. They are formed when any obstacle retards the movement of the water. decreasing its carrying power, and causing the deposit of part or all of its load. They consist of more or less rounded, finely comminuted particles, mingled with organic matter and detritus. Alluvial deposits are especially frequent at the mouth of streams and rivers, on their terraces and flood plains, and along silting banks as compared with the erosion banks of meanders. The filling of ponds by the erosion due to surface drainage, and of lakes by the deposition of the loads of streams that enter them, results in the formation of new alluvium. A similar phenomenon occurs along coasts, where bays and inlets are slowly converted into marshes in consequence of being shallowed by the material washed in by the waves and tides. Such paludal deposits are invariably salt water or brackish. Contrasted with these, which are uniformly black in consequence of the large amount of organic matter present, are the sandbars and beaches, which, though due to the same agents, are light grey or white in color, because of the constant leaching by the waves. Two kinds of alluvial deposits may accordingly be distinguished: (1) those black with organic matter, and little disturbed by water, and (2) those of a light color, which are constantly swept by the waves. The successions corresponding to these are radically different. In the first, the pioneer vegetation is hydrophytic. consisting largely of amphibious plants. The pioneer stages retard the movement of the water more and more, and correspondingly 
hasten the deposition of its load. The marsh bed slowly rises in consequence, and tinally the marsh begins to dry out, passing firstinto a wet meadow, and then into a meadow of the normal type. A notable exception to this sequence occurs when the swamp contains organic matter or salts in excess, in which case the vegetation consists indefinitely of swamp xerophytes, or halophytes. The first vegetation on freshwater sandbars is xerophytic, or, properly, dissophytic, unless they remain water-swept, and the ultimate stages of their successions are mesophytic woodlands composed of water-loving genera, Populus, Salix, etc. It seems certain, however, that these will finally give way to longer-lived hardwouds. Maritime sandbars and beaches are always saline, and their successions run their short course of development entirely within the group of halophytes, unless the retreat of the sea, or freshwater floods change the character of the soil. The chemical action of underground waters also produces new soils, which might be classed as alluvial. These soils are essentially rock deposits, travertine, silicious sinter, etc., made by iron and lime springs, and by geysers, and they must be changed by decomposition into soils proper to be comparable with alluvial soils.

6. Succession in a eolian soils. The only windborne soils of geological importance at the present time are those which form dunes, both inland and coastal. Aeolian deposits consist largely of rounded sand particles, which are of almost uniform size in any particular dune, but vary greatly in dunes of different ages. The reaction of the pioneers on dunes plays an important part in building the latter, but the immense dunes of inland deserts, which are entirely destitute of vegetation, seem to indicate that its value has been overestimated. The first stages in dune successions are dissophytic, i. e., the plants grow in a soil of medium or high water content, but in an atmosphere that is extremel.y xerophytic. The ultimate stages vary widely in accordance with the region in which they occur: they may be xerophytic heaths, or mesonbytic meadows and forests. $\mathrm{Be}$ cause of their striking character and economic significance. 
dunes have received much attention, with the result that their successions are the most thoroughly known of all. Prairie and steppe formations are probably to be regarded as the ultimate stages of successions established on windborne losss, and it is possible that the same is true of sandhill vegetation in the prairie province.

7. Succession in glacial soils. The formation of glacial deposits is at present confined to alpine and arctic regions. Recent successions in such soils are localised in these regions, and are in consequence relatively unimportant. There can be little question, however, that the thorough investigation of succession in and near the moraines of existing glaciers will throw much light upon the successions of the glacial period. Moraines, drumlins, eskars and alluvial cones represent the various kinds of glacial deposits. They agree in being heterogeneous in composition, and are covered today with ultimate stages of vegetation, except in the immediate vicinity of glaciers.

\section{SECONDARY SUCCESSIONS}

Generally speaking, all successions on denuded soils are secondary. When vegetation is completely removed by excessive erosion, it is an open question whether the resulting babitat is to be regarded as new or denuded. Erosion is rarely so extreme and so rapid, however, as to produce such a condition, even when it results from cultivation or deforestation. It is, moreover, especially characteristic of newly formed soils, and, in studying succession in eroded babitats, it is fundamentally important to determine whether erosion has produced denudation, or has operated upon a new soil. The great majority of secondary successions owe their origin to floods, animals, or the activities of man, and they agree in occurring upon decomposed soils of medium water content, which contain considerable organic matter. and a large number of dormant migrants. These successions consist of relatively few stages, and are rarely of extreme character.

8. Succession in erodedsoils. Eroded soils show considerable differences, as they arise in consequence of 
erosion by water, or by wind, though the initial stages of revegetation derive their character more from the aggregation of the soil than from the nature of the erosive agent. Eroded soils are as a rule xerophytic. In the case of erosion by water, dysgeogenous soils are readily worn away in consequence of their lack of cohesion, as in sanddraws, etc., while eugeogenous soils are easily eroded only on slopes, as in the case of ravines, hillsides, etc. In the former, the extreme porosity and slight capillarity of the sand and gravel result in a low water content: in the finer soils, the water content is also low, on account of the excessive runoff, due to compactness of the particles and to the slope. The erosive action of winds upon soils bearing vegetation is not very general : it is found to some extent in more or less established dunes, and exists in a marked degree in buttes, mushroom rocks and blowouts. The first two are regularly xerophytic, the last as a rule, dissophytic. The early stages of successions in eroded soils are composed of xerophytes. In loose soils, these are forms capable of binding the soil particles together, thus preventing wash, and increasing the accumulation of fine particles, especially of organic matter. In compact soils, the effect is much the same: the pioneers not only decrease erosion, but at the same time also increase the water content by retarding the movement of the run-off.

9. Succession in flooded soils. The universal response of vegetation to floods is found in the amphibious plant, which is a plastic form capable of adjustment to very different water contents. Floods are confined largely to river basins and coasts: in hilly and mountainous regions, where the slope is great, any considerable accumulation of flood waters is now impossible, although of frequent occurrence when land forms were more plastic.

In all streams that have become graded, the fall is insufficient to carry off the surplus, water in the spring when snows are 'melting rapidly, or at times of unusual precipitation. These waters accumulate, and, overflowing the banks, spread out over the lowlands, resulting in the form- 
ation of a well-defined flood plain. This is a periodical occurrence with mature streams, and it occurs more or less regularly with all that are not torrent-like in character. The effect of the overflow is to destroy or to place at a disadvantage those plants of the flood plain that are not hydrophytes. At the same time, a thin layer of fresh silt is deposited upon the valley floor of sand or alluvium. Flooding is most frequent and of longest duration near the banks of the stream. It extends more or less uniformly over the flood plain, and disappears gradually or abruptly as the latter rises into the bench above. Floods destroy vegetation and make a place for secondary successions by drowning out mescphytic species, by washing away the aquatic forms of ponds and pools, and by the erosion of banks and sandbars. They affect the amphibious vegetation of swamp and shore to a certain extent, but, unless the period of flooding is long, they tend to emphasize such formations rather than to destroy them. The stillwater formations of many cutoff and oxbow lakes owe their origion to a river which cuts across a meander in time of flood. This result is more often attained by the alternate silting and erosion of a meandering river by which it cuts across a bend in its channel. The usual successions in flooded lands are short as a rule: amphibious algae, liverworts and mosses soon give way to ruderal plants, and these in turn to the original mesophytes of meadows, or dissophytes of sandbars. In the case of ponds and pools, the process of washing-out or silting-up merely removes or destroys the vegetation, without effectively modifying the habitat, and the secondary successions that follow are extremely short.

10. Succession by subsidence. Subsidence is a factor of the most profound importance in changing vegetation. It operates over vast areas through immense periods of time. For these reasons, the changes are so slow as to be almost imperceptible, and the resulting successions can be studied only in the geological record. Extensive subsidence is confined today to coastal plains, as in Greenland, the south Atlantic coast, and the region of the Missis- 
sippi delta, where its effects are merged with the paludation of tidal rivers, and the wave and tide erosion of the sea shore. Such successions are unique in as much as the denuding force operates very slowly instead of quickly, and the first pioneers of the new vegetation appear before the original formation has been destroyed. In all cases, the succession is from mesophytic or halophytic formations to paludose, and, finally, marine vegetation. In small areas of subsidence, such as shore slips along lakes and streams, sink holes and sunken bogs, the succession is usually both short and simple, mesophytes giving place to amphibious and ultimately to aquatic forms.

11. Succession in landslips. Landslips occur only in montane and hilly regions, and here they are merely of local importance. In many respects, they are not unlike talus: they show essential differences, however, in that they are not sorted by gravity, and in that they destroy vegetation almost instantly. The succession arises as a rale, not upon the original soil, but upon that of the landslip, and, as pointed out elsewhere, might well be regarded as primary.

12. Succession in drained, or dried soils. In geological times, the subsidence of barriers must often have produced drainage and drying-out, just as elevation frequently resulted in flooding and lake formation. At the present time, the drying-out of lakes and ponds is the result of artificial drainage, or of climatic changes. The former will be considered under successions brought about by the agency of man. Climatic changes when general operate so slowly that the stages of such successions are perceptible only whun recorded in strata. More locally, climate swings back and forth through a period of years, with the result that in dry years the swamps and ponds of wetter seasons are dried out, and the vegetation destroyed or changed. If the process be gradual, the succession passes from hydrophytic through amphibious to mesophytic, and, in dry regions, xerophytic conditions: when the process of drying out occurs rapidly, as in a single summer, the original for- 
mation is destroyed, and the new vegetation consists largely of ruderal plants. A peculiar effect of climate occurs in regions with poor drainage, where the result of intense evaporation is to produce alkaline basins and salt lakes, in which the succession becomes more and more open, and is finally represented by a few stabilized halophytes, or disappears completely.

13. Succession by animal agency. Successions of this class are altogether of secondary importance, the instances in which animals produce denudation being relatively few. Such are the heaps of dirt thrown up by prairie dogs, and uther burrowing animals, upon which ruderal plants are first established, to be finally crowded out by the species of the original formation. Buffalo wallows furnish examples of similar successions in which the initial stages are subruderal, while overstocking and overgrazing frequently produce the same result with ruderal plants.

14. Succession by human agency. The activities of man in changing the surface of the earth are so diverse that it is impossible to fit the resulting successions in a natural system. While man does not exactly make new soils, he exposes soils in various operations, mining, irrigation, railroad building, etc. He destroys vegetation by fire, lumbering, cultivation and drainage, and, if he cannot control climate, he at least modifies its natural effects by irrigation and the conservation of moisture. The operations of man extend from sea coasts and swampy lowlands through mesophytic forests and prairies to the driest uplands and inlands. Since the adjacent formations determine in large degree the course and constitution of a succession, it will be seen that the effects of any particular activity upon vegetation will differ greatly in different regions. For convenience, all classes of successions arising from the presence and activity of man will be considered in this place, though, as indicated above, some might well be regarded as pruducing primary successions, while others produce anomalous ones. 
(a) Succession in burned areas. It will suffice merely to point out that "burns" may arise naturally through lightning, volcanic cinders, lava flows, etc., but the chances are so slight that these causes may be ignored. The causes of fires are legion, and, as they have little or no effect upon results, they need not be considered. From their nature, fires are of little significance in open vegetation, deserts, polar barrens, alpine fields etc., since the area of the burn can never be large. In closed formations, the extent of fires is limited only by the area of the vegetation, and the effect of wind, rain and other forces. Forest fires usually occur during the resting period, except in the case of coniferous forests. In grassland, the living parts are underground during autumn and winter, when prairie fires commonly occur. As a consequence, the repeated annual burning of meadow or prairie does not result in denudation and subsequent succession. On the contrary, it acts in part as a stabilising agent, in as much as it injures the typical vegetation forms of grassland much less than it does the woody invaders. All formations with perennial parts above ground, viz. thicket, open woodland, and forest, are seriously injured by fire. A severe general fire destroys the vegetation completely: a local fire destroys the formation in restricted areas, while a slight or superficial burn removes the undergrowth and hastens the disappearance of the weaker trees. In the latter case, while the primary layer of the forest remains the same, succession takes place in the herbaceous and shrubby layers. These successions are peculiar in that they are composed almost wholly of the proper species of the forest, and that they are very short, showing only a few poorly defined stages. A local fire initiates a succession in which the pioneers are derived largely from the original formation, particularly when the latter encloses the burned area more or less completely. The constitution of the intermediate and ultimate stages will depend in a larger degree still upon the size and position of the burn. When a particular formation is destroyed wholly or in large part, the first stages of the new 
vegetation are made up by invaders from the adjacent formations. In the most perfect types of succession, this dissimilarity between the new and the old vegetation continues to the last stage, in which the reappearance of the facies precedes that of the subordinate layers. In many forest successions, however, the general physical similarity of the ultimate stages permits the early reappearance of the herbaceous and shrubby species, and the final stages affect the facies alone. Successions in burned areas operate usually within the water content groups. The reconstruction of a mesophytic forest takes place by means of mesophytes; of the rarer xerophytic and hydrophytic forests, through xerophytes and hydrophytes respectively. This is due to the fact that the alteration of the soil is slight, except where the burning of the vegetation permits the entrance of erosion, as on mountain slopes.

(b) Succession in lumbered areas. Commercial lumbering, especially where practised for woodpulp as well as for timber, results in complete or nearly complete destruction of the vegetation by removal and the change from diffuse light to sunlight, or by the action of erosion upon the exposed surface. In the first place, short mesophytic successions will result: in the second, the successions will be long and complex, passing through decreasingly xerophytic conditions to a stable mesophytic forest. Where a forest is cut over for certain species alone, the undisturbed trees soon take full possession, though the causes effective in the beginning will ultimately restore the original facies in many instances. Such successions are anomalous, and will be treated under that head.

(c) Succession by cultivation. The clearing of forests and the "breaking" of grassland for cultivation destroy the original vegetation : the temporary or permanent abandonment of cultivated fields then permits the entrance of ruderal species, which are the pioneers of new successions. This phenomenon takes place annually in fields after harvest, resulting in the secondary formations of Warming, in which practically the same species reappear 
year after year. In fields that lie fallow for several years, or are permanently abandoned, the tirst ruderal plants are displaced by new-comers, or certain of them become dominant at the expense of others. In a few years, these are crowded out by invaders from the adjacent formations, and the field is ultimately reclaimed by the original vegetation. unless this has entirely disappeared from the region. The number of stages depends chiefly upon whether the final formation is to be grassland or woodland. Other activivies of man, such as the construction of buildings, roads, railways, canals, etc., remove the native vegetation, and make room for the rapid development of ruderal formations. In and about cities, where the original formations have entirely disappeared, the chance for succession is remote, and the initial ruderal stages become more or less stabilised. Elsewhere the usual successions are established, and the ruderal formation finally gives way to the dominant type. In mountain and desert regions, where ruderal plants are rare or lacking, their place is taken by subruderal forms, species of the native vegetation capable of rapid movement in them. These, like ruderal plants; are gradually replaced by other native species of less mobility, but of greater persistence, resulting in a short succession operating often within a single formation. From the nature of cultivated plants, succession after cultivation generally operates with. in the mesophytic series.

(d) Succession by drainage. Successions of this kind show much the same stages as are found in those due to flooding. They proceed from aquatic or swamp formations to mesophytic termini, either grassland or woodland. When drainage takes place rapidly and completely, the pioneer stages are usually xerophytic: cases of this sort, however, are infrequent.

(e) Succession by irrigation. Irrigation produces short successions of peculiar stamp along the courses of irrigating canals and ditches, and in the vicinity of reservoirs. These are recent, as a rule, and are usually found in the midst of cultivated lands, so that their complete 
history is still a matter of conjecture. The original xerophytes are forced out not only by the disturbance of the soil, but also by its increased water content. A few of them often thrive under the new conditions, and, together with the usual ruderal plants and a large number of lowland mesophytes and emphibious forms derived from the bunks of the parent stream, constitute a heterogeneous association. This is doubtless to be regarded as an initial stige of a succession, but it is an open question whether the succession will early be stabilised as a new formation, or whether the original vegetation will sooner or later be reestablished under somewhat mesophytic conditions. From the number of mesophytes and from the behavior of valleys, it seems certain that the banks of such canals will ultimately be occupied by a formation more mesophytic than bydroph.tic, into which some of the surrounding xerophytes of plastic nature have been adopted.

Anomalous successions are those in which the physical change in the habitat is relatively slight. resulting in a displacement of the ultimate stage, or the disturbance of the usual sequence, merely, instead of the destruction and reconstruction of a formation, or the gradual development of a now series of stages on new soil. In nature, the ultimate grass or forest stage of a normal succession is often replaced by a similar formation, especially if the facies be few or single. It is evident that certain trees naturally replace others in the last stages of a forest succession, without making the latter anomalous. The last occurs only when a normal stage is replaced by one belonging properly to an entirely different succession, as when a coniferous forest replaces a deciduous one in a hardwood region. The presence and development of such successions can be determined only after the normal types are known. The interpolation of a foreign stage in a natural succession, or a change of direction, by which a succession that is mesotropic again becomes hydropbytic, is easily explained when it is the result of artificial agents, as is often the case. In nature, anomalous 
successions are commonly the result of a slow backward and forward swing of climatic conditions.

A normal succession will regularly be perfect (successio perfecta); it passes in the usual sequence from initial to ultimate conditions, without interruption or omission. Imperfect succession (successio imperfecta) results when one or more of the ordinary stages is omitted anywhere in the course, and a later stage appears before its turn. It will occur at any time when a new or denuded habitat becowes so surrounded by other vegetation that the formations which usually furnish the next invaders are unable to do so, or when the abundance and mobility of certain species enable them to take possession before their proper turn, and to the exclusion of the regular stage. Incomplete successions are of great significance, in as much as they indicate that the stages of a succession are often due more to biological than to physical causes, the proximity and mobility of the adjacent species being more determinative than the physical factors. Subalpine gravel slides regularly pass through the rosette, mat, turf, thicket, woodland and forest stages: occasiunally, however, they pass immediately from the rosette, or mat condition to an aspen thicket which represents the next to the last stage. Such successions are by no means infrequent in hilly and montane regions: in regions physiographically more mature or stable, perfect successions are almost invariably the rule.

It may be stated as a general principle that vegetation moves constantly and gradually toward stabilisation. Each successive stage modifies the physical factors, and dominates the habitat more and more, in such a way that the latter seems to respond to the formation rather than this to the habitat. The more advanced the succession, $i$. e., the degree of stabilisation, the greater the climatic or physiographic change necessary to disturb it, with the result that such disturbances are much more frequent in the earlier stages than in the later development. Constant, gradual movement toward a stable formation is characteristic of continuous succession (successio continua). Contrasted 
with this is intermittent succession (successio intermissa), in which the succession swings for a time in one direction, from xerophytic to mesophytic for example, and then moves in the opposite direction, often passing through the same stages. This phenomenon usually is characteristic only of the less stable stages, and is generally produced by a climatic swing, in which a series of hot or dry years is followed by one of cold or wet years, or the reverse. The same effect upon a vast scale is produced by alternate elevation and subsidence, but these operate through such great periods of time that one can not trace, but can only conjecture their effects. A normal continuous succession frequently changes its direction of movement, or its type, in transitiun regions or in areas where the outposts of a new flora are rapidly advancing, as in wide mesopbytic valleys that run down into or traverse plains. Here the change is often sudden, and grass and desert formations are replaced by thickets and forests, resulting in abrupt succession (successio abrupta). Species guilds are typical examples of this. More rarely, a stage foreign to the succession will be interpolated, replacing a normal stage, or slipping in between two such, though finally disappearing before the next regular formation. This may be distinguished as interpolated succession (successio interpolata).

The apparent terminus of all stabilisation is the forest, on account of the thornughness with which it controls the habitat. A close examination of vegetation, however, will show that its stable terms are dependent in the first degree upon the character of the region in which the formation is indigenous. It is obviously impossible that successions in desert lands, in polar barrens, or upon alpine stretches should terminate in forest stages. In these, grassland must be the ultimate condition, except in those extreme habitats. alpine and polar, where mosses and lichens represent the highest type of existing vegetation. Forests are ultimate for all successions in habitats belonging to a region generally wooded, while grassland represents the terminus 
of prairie and plains successions as well as of many arcticalpine ones.

\section{CAUSES AND REACTIONS.}

The initial cause of a succession must be sought in a physical change in the habitat: its continuance depends upon the reaction which each stage of vegetation exerts upon the physical factors which constitute the habitat. A single exception to this is found in anomalous successions, where the change of formation often hinges upon the appearance of remote or foreign disseminules. The causes which initiate successions have already been considered: they may be summarised as follows: (1) weathering. (2) erosion, (3) elevation, (4) subsidence, (5) climatic changes, (6) artificial changes. The effect of succeeding stages of vegetation upon a new or denuded habitat usually finds expression in a change of the habitat with respect to a particular factor, and in a definite direction. Often, there is a primary re. action, and one or more secondary ones, which are corollaries of it. Rarely, there are two or more coordinate reactions. The general ways in which vegetation reacts upon the habitat are the following: (1) by preventing weathering; (2) by binding aeolian soils; (3) by reducing run-off and preventing erosion: (4) by filling with silt, and plant remains; (5) by enriching the soil; (6) by exhausting the soil; (7) by accumulating humus; (8) by modifying atmospheric factors. The direction of the movement of a succession is the immediate result of its reaction. From the fundamental nature of vegetation, it must be expressed in terms of water content. The reaction is often so great that the habitat undergoes a profound change in the course of the succession, changing from hydrophytic to mesophytic or xerophytic, or the reverse. This is characteristic of newly formed or exposed soils. Such successions are xerotropic

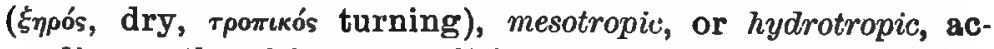
cording to the ultimate condition of the habitat. When the reaction is less marked, the type of habitat does not change materially, and the successions are xerostatic, (orarcós stopping) mesostatic, or hydrostatic, depending upon the 
water content. Such conditions obtain for the most part only in denuded habitats.

(1) Succession by preventing weathering. Reactions of this nature occur especially in alpine and boreal regions, in the earlier stages of lichen-moss successions. They are typical of igneous and metamorphic rocks in which disintegration regularly precedes decomposition. The influence of the vegetation is best seen in the lichen stages, where the crustose forms make a compact layer, which diminishes the effect of the atmospheric factors producing disintegration. In alpine regions especially, this protection is so perfect that the crustose lichens may almost be regarded as the last stage of a succession. There are no recorded observations which bear upor this point, but it seems certain that the pioneer rock lichens, Lecanora, Lecidea, Biatora, Buellia and Acarospora, cover alpine rocks for decades, if not for centuries. Ultimately, however, the slow decomposition of the rock surface beneath the thallus has its effect. Tiny furrows and pockets are formed, in which water accumulates to carry on its ceaseless work, and the compact crustose covering is finally ruptured, permitting the entrance of foliose forms. The latter, like the mosses, doubtless protect rock surfaces, especially those of the softer rocks, in a slight degree against the influence of weathering, but this is more than offset by their activity in hastening decomposition, and thus preparing a field for invasion. Rocks and boulders (petria, petrodia, phellia) furnish the best examples of this reaction: cliffs (cremnia) usually have a lichen covering on their faces, while the forces which produce disintegration operate from above or below.

(2) Succession by binding aeolian soils. Dunes (thinia) are classic examples of the reaction of pioneer vegetation upon habitats of wind-borne sand. The initial formations in such places consist exclusively of sandbinders. plants with masses of fibrous roots, and usually also with strong rootstalks, long, erect leaves and a vigorous apical growth. They are almost exclusively perennial grasses and sedges, possessing the unique property 
of pushing up rapidly through a covering of sand. They react by fixing the sand with their roots, thus preventing its blowing about, and also by catching the shifting particles among their culms and leaves, forming a tiny area of stabilisation, in which the next generation can establish a foothold. The gridual accumulation of vegetable detritus serves also to enrich the soil, and makes possible the advent of species requiring better nourishment. Blowouts (anemodia) are almost exact duplicates of dunes in so far as the steps of revegetation are concerned: while one is a hollow, and the other a hill, in both the reaction operates ppon a wind-swept slope. Sandhills (amathia) and deserts (eremia) show similar though less marked reactions, except where they exhibit tynical inland dunes. Sandbinders, while usually classed as xerophytic or halophytic, are in reality dissophytes. Their roots grow more or less superficially in moist sand, and are morphologically mesophytic, while their leaves bear the stamp of xerophytes. The direction of movement in successions of this kind is normally from xerophytes to mesophytes, i. $\theta_{.}$, it is mesotropic. In sandhills and deserts, the succession operates wholly within the xerophytic (dissopnytic) series. Along sea coasts, the mesophytic terminus is regularly forest, except where forests are remote, when it is grassland.

(3) Succession by reducing run-off, and preventing erosion. All bare or denuded babitats that have an appreciable slope are subject to erosion by surface water. The rapidity and degree of erosion depend upon the amount of rainfall, the inclination of the slope, and the structure of the surface soil. Regions of excessive rainfall, even where the slope is slight, show great though somewhat uniform erosion: hill and mountain are deeply eroded even when the rainfall is small. Slopes consisting of compact eugeogenous soils, notwithstanding the marked adhesion of the particles, are much eroded where the rainfall is great, on account of the excessive run-off. Porous dysgeogenous soils, on the contrary, absorb most of the 
rainfall; the run off is small and erosion slight, except where the slope is great, a rare condition on account of the imperfect cohesion of the particlıs. In compact soils, the plants of the initial formations not merely break the impact of the rain-drops, but, what is much more important, they delay the downward movement of the water, and produce numberless tiny streams. The delayed water is largely absorbed by the soil, and the reduction of the run-off prevents the formation of rills of sufficient size to cause erosion. As in dunes, such plants are usually perennial grasses, though composites are frequent: the root system is, however, more deeply seated, and a main or tap root is often present. On sand and gravel slopes, the loose texture of the soil results generally in the production of sandbinaers with fibrous roots. Unlike dunes, such slopes exhibit a large number of mats and rosettes with tap roots, which are effective in preventing the slipping or washing of the sand, and run little danger of being covered, as is the case with dune-formers. In both instances, each pioneer plant serves as a center of comparative stabilisation for the establish. ment of its own offspring, and of such invaders as find their way in. From the nature of these, slopes almost invariably pass through grassland stages before finding their termini in thickets or forests. Bad lands (hydrotribia) furnish the most striking examples of eroded habitats. The rainfall in the bad lands of Nebraska and South Dakota is small (300 $\mathrm{mm}$.); yet the steepness of the slope and the compactness of the soil render erosion so extreme, that it is all but im. possible for plants to obtain a foothold. Their reaction is practically negligible, and the vegetation passes the pioneer stages only in the relatively stable valleys. Mountain slopes (ancia), and ridges and hills (lophia) are readily eroded in new or denuded areas. This is especially true of hill and mountain regions, which have been stripped of their forest or thicket cover by fires, lumbering, cultivation or grazing. Where the erosion is slight, the resulting succession may show initial xerophytic stages, or it may be completely mesostatic. Excessively eroded habitats are xerostatic, as 
in the case of bad lands, or, more frequently, they are mesotropic, passing first through a long series of xerophytic formatiuns. Sandbars (cheradia, syrtidia) should be considered here, though they are eroded by currents and waves, and not by run-off. They are fixed and built up by sand-binding grasses and sedges, usually of a hydrophytic nature, and pass ultimately into mesophytic forest.

(4) Succession by filling with silt, and plant remains. All aquatic habitats into which silt, wash, or other detritus is borne by streams, currents, floods, waves or tides are slowly shallowed by the action of the water plants present. These not only check the movement of the water, thus greatly decreasing its carrying power, and causing the deposition of a part or all of its load, but they also retain and fix the particles deposited. In accordance with the rule, each plant becomes the center of a stabilising area, which rises faster than the rest of the floor, producing the well-known hummocks of lagoons and swamps. All aquatics produce this reaction. It is more pronounced in submerged and amphibious forms than in floating ones, and it takes place more rapidly with greatly branched or dissected plants than with others. In pools (tiphia) and lakes (limnia), debouching streams and surface waters deposit their loads in consequence of the check exerted by the still water and the marginal vegetation, and delta-like marshes are quickly built up by filling. Springs (crenia) likewise form marshes where they gush forth in sands, the removal of which is impeded by vegetation. The flood plains and deltas of rivers show a similar reaction. The heavily-laden flood waters are checked by the vegetation of meadows and marshes, and deposit most of their load. The banks of streams (ochthia) and of ditches (taphria) are often built up in the same fashion by the action of the marginal vegetation upon the current. The presence of marginal vegetation often determines the checking or deflecting of the current in such a way as to initiate meanders, while natural levees owe their origin to it, in part at least. Along low sea coasts, waves and tides hasten 
the deposit of river-borne detritus, causing the water to spread over the lowlands and form swamps. They often throw back also the sediment that has been deposited in the sea, the marsh vegetation acting as a filter in both cases. Successions of the kind indicated above are regularly mesotropic. Where the soil is sandy, and the filling-up process sufficiently great, or where salts or humus occur in excess, xerophytic formations result. In certain cases, these successions appear to be permarently hydrostatic, changing merely from floating or submerged to amphibious conditions, but this is probably due to the slowness of the reaction. As a rule, the accumulation of plant remains is relatively slight, and plays an unimportant part in the reaction. In peat bogs and other extensive swamps, the amount of organic matter is excessive, and plays an important rôle in the building up of the swamp bed. Its greatest reaction, however, results frum its partial decomposition under water. This process sets free a number of so-called humous acids, which inhibit the absorption of water, and cause the development of xerophytes in what are apparently hydrophytic habitats.

(5) Succession by enriching the soil. This reaction occurs to some degree in the great majority of all successions. The relatively insignificant lichens and mosses produce this result upon the most barren rocks, while the higher forms of later stages, grasses, herbs, shrubs, and trees, exhibit it in marked progression. The reaction consists chiefly in the incorporation of the decom. posed remains of each generation and each stage in the soil. A very important part is played by the mechanical and chemical action of the roots in breaking up the soil particles, and in changing them into soluble substances. Mycorrhizae, bacterial nodules and especially soil bacteria play a large part in increasing the nutrition content of the soil, but the extent to which they are effective in succession is completely unknown. The changes in the color, texture and food value of the soil in passing from the initial to ultimate stages of a normal succession are well-known, and 
have led many to think them the efficient reactions of such successions. It seems almost certain, however, that this is merely a concomitant, and that, even in anomalous successions where facies replace each other without obvious reasons, the reactions are concerned more with water content, light and humidity than with the food content of the soil.

(6) Succession by exhausting the soil. This is a reaction not at all understood as jet in nature. A number of phenomena, such as the "fairy rings" of mushrooms and other fungi, the peripheral growth and central decay of lichens, Lecanora, Placodium, Parmelna, and of matforming grasses, such as Muhlenbergia, and the circular advance of the rootstalk plants, indicate that certain plants at least withdraw much of the available supply of some essential soil element, and are forced to move away from the exhausted area. It is probable that the constant shifting of the individuals of a formation year after year, a phenomenon to be discussed under alternation, has some connection with this. It will be impossible to establish such a relation, however, until the facts are exactly determined by the method of quadrat statistics. So far as native formations are concerned, there cannot be the slightest question that prairies and forests have existed over the same area for centuries without impoverishing the soil in the least degree, a conclusion which is even more certain for the open vegetation of deserts and plains. With culture formations, the case is quite different. The exhaustion of the soil by continuous or intensive cultivation is a matter of common experience in all lands settled for a long period. Calcium, phosphorus, and nitrogen compounds especially are used up by crops, and must be supplied artificially. The reason for this difference in reaction between native and culture formations seems evident. In harvesting, not merely the grain, but the stews and leaves, and in gardening often the root also, are removed, so that the plant makes little or no return to the soil. In nature, annual plants return to the ground every year all the solid matter of routs, 
stems, leaves and fruits, with the exception of the relatively small number of seeds that germinate. Perennial herbs return evervthing but the persistent underground parts: shrubs and trees replace annually an immense amount of material used in leaves and fruits, and sooner or later by the gradual decay of the individuals, or by the destruction of the whole formation, they restore all that they have taken from the soil. This balance is further maintained to an important degree by the activity of the roots, which take from the deep-seated layers of the soil the crude materials necessary for the formation of leaves and fruits. Upon the fall and decay of these, their materials are incorporated with the upper layers of the formation floor, from which they may be absorbed by the undergrowth, or find their way again into the lajers permeated by the tree roots. From the universal occurrence of weeds in cultivated regions, the pioneers in impoverished or exhausted fields are uniformly ruderal plants. As is well-known, the seed production and ecesis of these forms are such that they take pussession quickly and completely, while their demands upon the soil are of such a nature that the most sterile field can rapidly be covered by a vigorous growth of weeds. As indicated elsewhere, ruderal formations ultimately yield to the native vegetation, though in regions so completely given over to culture that native formations are lacking or remote, it is probable that successions reach their final stage within the group of ruderal plants.

(7) Succession by the accumulation of hum us. This is the characteristic reaction of peat bogs, and cypress swamps (oxyilia), in which the accumulation of vegetable matter is enormous. The plant remains decompose slowly and incompletely under the water, giving rise to the various humic acids. These possess remarkable antiseptic qualities, and have an injurious effect upon protoplasm. Like alkalies, they hinder the absorption of water by the root hairs, though this is also due in part to poor aeration, and in consequence call forth xerophytic modifications. The same tendency exists in practically all inland 
marshes and swamps, but the quantity of decomposing vegetation in many is not great enough to produce an efficient reaction. The characteristic plants of peat bogs and humus swamps are xerophytes, which possess a superficial root system: structurally, they are almost identical with dissophytes. The occurrence of zerophytes in situations typically hydrophytic is no longer a puzzling contradiction of the unique value of water content in the control of vegetation. It is readily explained by the reduction of the available water, and by the lack of proper aeration in the presence of humic acids. Formations of this type usually start as freshwater swamps. The succession is consequently xerotropic, but no thorough study of its stages has as yet been made.

(8) Succession by modifying atmospheric f a c t o r s. All layered furmations, forests, thickets, many meadows and wastes, etc., show reactions of this nature, and are in fact largely or exclusively determined by them. The reaction is a complex one, though it is clear that light is the most efficient of the modified factors, and that humidit. temperature, and wind, while strongly affected, play subordinate parts. In normal successions, the effect of shade, i. e., diffuse light, enters with the appearance of bushes or shrubs, and becomes more and more pronounced in the altimate forest stages. The reaction is exerted chiefly by the facies, but the effect of this is to cause increasing diffuseness in each successively lower layer, in direct ratio with the increased branching and leaf expansion of the plants in the layer just above. In the ultimate stage of many forests, especially where the facies are reduced to one, the reaction of the primary layer is so intense as to preclude all undergrowth. Anomalous successions often owe their origin to the fact that certain trees react in such a way as to cause conditions in which they produce seedlings with increasing difficulty, and thus offer a tield favorable to the ecesis of those species capable of enduring the dense shade. Successions of this kind are almost invariably 
mesostatic, as it is altogether exceptional that layered formations are either xerophytic or hydrophytic.

\section{LAWS OF SUCCESSION.}

The investigation of succession has so far been neither sufficiently thorough nor systematic to permit the postalation of definite laws. Enough has been done, however, to warrant the formulation of a number of rules, which apply to the successions studied, and afford a convenient method for the critical investigation of all successions upon the basis of initial causes, and reactions. Warming has already brought together a few such rules, and an attempt is here made to reduce the phenomena of succession, including its causes and effects, to a tentative system. At present it is difficult to make a thoroughly satisfactory classification of such rules, and they are here arranged in general conform. ity with the procedure in succession.

I. Causation. The initial cause of a succession is the formation or appearance of a new habitat, or the efficient change of an existing one.

II. Reaction. Each stage reacts upon the habitat in such a way as to produce physical conditions more or less unfavorable to its permanence, but advantageous to the invaders of the next stage.

III. Proximity and Mobility.

(1) The pioneers of a suceession are those species nearest at hand that are the most mobile.

(2) The number of migrants from any formation into a habitat varies inversely as the square of the distance.

(3) The pioneer species are regularly derived from different formations, as the latter nearly always contain permobile species capable of effective ecesis.

(4) The plants of the initial stages are norwally algae and fungi, with minute spores, composites and grasses, which possess permobile fruits, or ruderal plants, on account of their great seed production.

IV. Ecesis.

(1) All the migrants into a now, denuded, or greatly 
modified habitat are sorted by ecesis into three groups: (1) those that are unable to germinate or grow, and soon die; (2) those that grow normally under the conditions present; (3) those that pass through one or more of the earlier stages in a dormant state to appear at a later stage of the succession.

(2) Wherever ruderal vegetation is present, it contributes a large number of the pioneer species of each succession, on account of the thorough ecesis. In other regions, this part is plajed by subruderal native species.

(3) Annuals and biennials are characteristic of the early stages of secondary successions, on acount of their great seed production and ready ecesis.

(4) In layered formations, heliophytes appear before sciophytes: they ultimately yield to the latter, except where they are able to maintain a position in the primary layer.

(5) Excessive seed production and slight mobility lead to the imperfect ecesis of individuals in dense stands, and in consequence usually produce great instability.

(6) Each pioneer produces about itself a tiny area of ecesis and stabilisation for its own offspring, for the disseminules of its fellows, or of invaders.

(7) Species propagating by offshoots, or producing relatively immobile disseminules in small number, usually show effective ecesis, as the offspring appear within the area of the reaction of the parent forms.

V. Stabilisation.

(1) Stabilisation is the universal tendency of vegetation.

(2) The ultimate stage of a succession is determined by the dominant vegetation of the region. Lichen for. mations are often ultimate in polar and niveal zones: grassland is the final vegetation for plains and alpine stretches, and for much prairie, while forest is the last stage for mesophytic midlands and lowlands, as well as for subalpine regions. 
(3) Grassland or forest is the usual terminus of a succession: they predominate in lands physiographically mature.

(4) The limit of a succession is determined in large part by the progressive increase in occupation, which unakes the entrance of invaders more and more difficult.

(5) Stabilisation proceeds radiately from the pioneer plants or masses. The movement of offshoots is away from the parent mass, and the chances of ecesis are greatest near its edges, in a narrow area in which the reaction is still felt, and the occupation is not exclusive.

\section{General Laws.}

(1) The stages, or formations, of a succession are distinguished as initial (prodophytia), intermediate (ptenophytia), and ultimate (aiphytia).

(2) Initial formations are open, ultiwate formations are closed.

(3) The number of species is small in the initial stages. It attains a maximum in intermediate stages, and again decreases in the ultimate formation, on account of the dominance of a few species.

(4) The normal sequence of vegetation forms in succession is: (1) algae, fungi, mosses; (2) annuals and biennials; (3) perennial herbs; (4) bushes and shrubs; (5) trees.

(5) The number of species and of individuals in each stage increases constantly up to a maximum, after which it gradually decreases before the forms of the next stage. The interval between two maxima is occupied by a mixed formation.

(6) A secondary succession does not begin with the initial stage of the primary one which it replaces, but usually at a much later stage

(7) At present, successions are generally mesotropic, grassland and forest being the ultimate stages, though many are xerostatic or hydrostatic. If 
erosion continue until the sea level is reached, the ultimate vegetation of the globe will be hydrophytic. Should the heat of the sun decrease greatly before this time, the last vegetation will be xerophytic. i. e. crymophytic.

(8) The operation of succession was essentially the same during the geological past as it is today: from the nature of their vegetation forms, the record deals largely with the ultimate stages of such successions.

CLASSIFICATION AND NOMENCLATURE.

New or denuded babitats arise the world over by the operation of the same or similar causes, and they are revegetated in consequence of the same reactions. Similar habitats produce similar successions. The vegetation forms and their sequence are usually identical, and the genera are frequently the same, or corresponding in regions not entirely unrelated. The species are derived from the adjacent vegetation, and, except in alpine and coast regions, are normally different. The primary groups of successions are determined by essential identity of habitat or cause, e. g., aeolian successions, erosion successions, burn successions, etc. When they have been more generally investigated, it will be possible to distinguish subordinate groups of successions, in which the degree of relationship is indicated by the similarity of vegetation forms, the number of common genera, etc. For example, burn successions in the Ural and in the Rocky mountains show almost complete similarity in the matter of vegetation forms, and their sequence, and have the majority of their genera in common. A natural classification of successions will divide them first of all into normal and anomalous. The former fall in to two classes, primary and secondary, and these are subdivided into a number of groups, based upon the cause which initiates the succession.

The need of short distinctive names of international value for plant formations is obvious: it has become imperative that successions also should be distinguished critically, and designated clearly. From the very nature of the case, it is 
impossible to designate each formation or succession by a single Greek or Latin term, as habitats of the same char. acter will show in different parts of the world a vegetation taxonomically very different. It may some day be possible to use a binomial or trinomial for this purpose, somewhat after the fashion of taxonomy, in which the habitat name will represent the generic idea as applied to formations, and a term drawn from the floristic impress the specific idea. Such an attempt would be futile or valueless at the present time: it could not possibly meet with success until there is more uniformity in the concept of the formation, and until there has been much accurate and thorough investigation of actual formations, a task as yet barely begun. At present, it seems most feasible as well as scientific, to designate all formations occupying similar habitats by a name drawn from the character of the latter, such as a meadow formation, poium, a a forest formation, hylium, a desert formation, eremium, etc. A particular formation is best designated by using the generic name of one or two of its most important species in conjunction with its habitat term, as Spartina-Elymus-poium, Picea-Pinus-hylium, Cereus-Yucca-eremium, etc. Apparently a somewhat similar nomenclature is adapted to successions. The cause which produces a new habitat may well furnish the basis for the name of the general groups of successions, as pyrium (literally, a place or a habitat burned over), a burn succession, tribium, an erosion succession, etc. A burn succession consists of a sequence of certain formations in one part of the world, and of a series of quite different ones, floristically, in another. A particular burn succession should be designated by using the names of a characteristic facies of the initial and ultimate stages in connection with the general term, e. g., Bryum-Picea-pyrium, etc. A trinomial constructed in this way represents the desirable mean between definition and brevity. Greater definiteness is possible only at the expense of brevity, while to shorten the name would entirely destroy its precision. The follow-

${ }^{1}$ Clements, F. E. A System of Nomenclature for Phytogeography. Engler 31:b5 1902. 
ing classification of successions is proposed, based upon the plan outlined above. The termination -ium (-غîv) has been used throughout in the construction of names for successions, largely for reasons of euphony. If it should become desirable to distinguish the names of formations and successions by the termination, the locative suffix -on(-wv) should be used for the latter. The terms given below would then be hypson, rhyson, hedon, sphyron, prochoson, pnoon, pagon, tribon, cluson, repon, olisthon, xerasion, theron, broton, pyron, ecballon, camnon, ocheton, ardom.

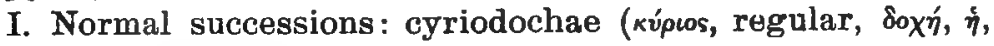
succession)

a. Primary successions : protodochae ( $\pi \rho \hat{\omega} \tau$ os, first, primary)

1. By elevation: hypsium (ưuos, ró, height, elevation, -ยî́ov, place)

2. By volcanic action: rhysium ( $\dot{\rho} v \sigma i s, \dot{\eta}$, flowing, especially of fire)

3. In residuary soils : hedium ("'́os, ró, a sitting, base)

4. In colluvial soils : sphyrium ( $\sigma \phi \dot{p} \rho o v$, tó, ankle, talus)

5. In alluvial soils: prochosium ( $\pi \rho x_{\chi} \omega \sigma t s, \dot{\eta}$, a deposition of mud)

6. In aeolian soils : pnoium ( $\pi v \eta^{\prime}, \dot{\eta}$, blowing, blast)

7. In glacial soils: pagium ( $\pi \dot{\gamma}$ os, $\delta$, that which becomes solid, i. e., a glacier)

b. Secondary successions: hepodochae (

8. In eroded soils : tribium ( $\tau i \beta \omega$, wear or rub away)

9. In flooded soils: clusium ( $\kappa \lambda$ v́ $\iota \iota$, ${ }^{\circ}$, a drenching, flooding)

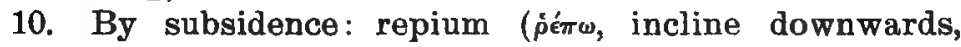
sink)

11. In landslips : olisthium (ö $\lambda \iota \sigma \theta o s, \delta$, slip)

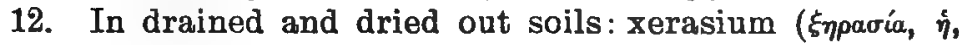
drought)

13. By animal agencies : therium ( $\theta \dot{\eta} p, \dot{o}$, wild animal)

14. By human agency : brotium (Bporós, $\delta$, a mortal)

a. Burns: pyrium ( $\pi \hat{v} p$, ró, fire)

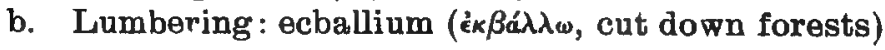



c. Cultivation : camnium ( $\alpha a ́ \mu \nu \omega$, cultivate)
d. Drainage: ochetium (ỏxerós, ó, drain)
e. Irrigation : ardium ( $\alpha \rho \delta \omega$, irrigate)

II. Anomalous successions: xenodochae ( ḱvos, strange, unusual)

The following series will illustrate the application of this system of nomenclature to particular successions, and their stages, or formations.

Thlaspi-Picea-sphyrium : pennycress-spruce talus succession

Thlaspi-Eriogonum-chalicium: pennycress-eriogonum gravel slide formation

Elymus-Gilia-chalicodium : wildrye-gilia half gravel slide formation

Quercus-Holodiscus-lochmodium : oak-fringewood dry thicket formation

Pinus-xerohylium : pine dry forest formation

Picea-Pseudotsuga-hylium : spruce-balsam forest formation

Bryum-Picea-pytium : moss-spruce burn succession

Bryum-telmatium : moss meadow formation

Aster-Chamaenerium-poium : aster-fireweed meadow formation

Deschampsia-Carex-poium : hairgrass-sedge meadow formation

Salix-Betula-helolochmium : willow-birch meadow thicket formation

Populus-bylium : aspen forest formation

Picea-hylium : spruce forest formation

Lecanora-Carex-hedium : lichen-carex residuary succession

Lecanora-Gyrophora-petrium : crustose lichen rock formation

Parmelia-Cetraria-chalicium : foliose lichen gravel slide formation

Paronychia-Silene-chalicodium : nailwort-campion gravel slide formation

Carex-Campanula-coryphium : sedge-bluebell alpine meadow formation

Eragrostis-Helianthus-xerasium : eragrostis-sunflower drainage succession

Eragrostis - Polygonum - telmatium : eragrostis - heartsease wet meadow formation

Helianthus-Ambrosia-chledium : sunflower-ragweed waste formation

INVESTIGATION OF SUCCESSION.

The study of succession must proceed along two fundamental lines of inquiry. It is necessary to investigate 
quantitatively the physical factors of the initial stages, and the reactions produced by the subsequent stages. This should be done by automatie instruments for humidity, light, temperature and wind, in order that a continuous record may be obtained. Water content and precipitation readings are taken daily or even less frequently, while soil properties, and physiographic factors, altitude, slope, surface and exposure, are determined once for all. It is equally needful to determine the development and structure of each stage with particular reference to the adjacent formations, to the stage that has just preceeded, and the one that is to follow. For this, the use of the permanent quadrat is imperative, as the sequence and structure of the stages can be understood nnly by a minute study of the shifting and rearrangement of the individuals. In the examination of successions, since cause and effect are so intimately connected in each reaction, it is especially important that general and superficial observations upon structure and sequence be replaced by precise records, and that vague conjectures as to causes and reactions be supplanted by the accurate determination of the physical factors which underly them.

The period of time through which a primary succession operates is usually too great to make its complete stady possible within a single lifetime. Secondary successions run their course much wore quickly, and a decade will sometimes suffice for stabilisation, though even here the period is normally longer. The longest and most complex succession, however, may be accurately studied in a region, where several examples of the same succession occur in different stages of development. In the same region, the physical factors of one example of a particular succession are essentially identical with those of another example in the same stage. If one is in an initial stage, and the other in an intermediate condition, the development of the former makes it possible to reestablish more or less completely the life history of the latter. The same connection may be 
made between intermediate and ultimate stages, and it is thus possible to determine with considerable accuracy and within a few jears the sequence of stages in a succession that requires a century or more for its complete development. In the Rooky mountains, gravel slides (talus slopes) are remarkably frequent. They occur in all stages of development, and the alternating slides of different ages furnish an almost perfect record of this succession. This method lacks the absolute finality which can be obtained by following a succession in one spot from its inception to final stabilisation, but it is alone feasible for long successions, $i$. e., those extending over a score or more of years. When it comes to be universally recognized as a plain duty for each investigator to leave an exact and complete record in quadrat maps and quadrat photographs of the stages studied by him, it will be a simple task for the botanists of one generation to finish the investigation of the successions begun by their predecessors.

BIBLIOGRAPHY

1749 Biberg, I. J.

1820 De Candolle, A. P.

1842 Steenstrup, J.

1844 Berg, E. v.

1850 Humboldt, A. v.

1851 Vaupell, C.

1852 Henfrey, A.

1855 De Candolle, A.

1856 Hofmann
Oeconomia Naturae. Amoen. Acad. 4:1

Essai Elémentaire de Géographie Botanique 27

Geognostisk-geologisk-Undersögelse i det nordlige Själland

Das Verdrängen der Laubwälder durch die Fichte und Kiefer. Grisebach Berichte $1844: 15$

Views of Nature 125, 213

De Nordsjaellandske Skormoser

The Vegetation of Europe 67 Géographie Botanique Raisonnée 743

Der nördliche Ural und das Küstengebirge Pae-Choi 
1858 Hill

1859 Stossner, A.

1863 Kerner, A.

1864 Middendorff, A.

1865 Kabsch, W.

1871 Müller, P. E.

1879 Engler, A.

1881 Hult, R.

1884 Grōnlund, C.

1885 Hult, R.

1887

Callmé, A.

1888 Treub, M.

Senft, F.

Krassnoff, A.

Fliche, M. P.

1889 Tanfiljew, G.
Reise in Sibirien

Vegetationsverhältnisse von Annaberg und Umgebung Das Pflanzenleben der Donauländer

Die Gewächse Sibiriens

Das Pflanzenleben der Erde Bjärgfyrren (Pinus montana) Tidskr. pop. Fremst.

Versuch einer Entwicklungsgeschichte der Ptlanzenwelt

Forsok till analytisk behandlung af formationerna Medd. Soc. Fenn. 8

Planteväxten paa Island. Nat. For. Festschr. 1884-90 Blekinges vegetation. Just '85 : 312

Die alpinen Pflanzenformationen des nordlichstens Finnlands. Engler $11:$ b61 Om de nybildade Hjelmaroarnes vegetation. Bihang Sv. Vet. Akad. Handl. XII Notice sur la nouvelle flore de Krakatau. Ann. Jard. Buit. 7

Der Erdboden. Just '88: 45 Bemerkungen über die Vegetation des Altai. Engler $9: 53$

Un reboisement, étude botanique et forestière. Engler 11: 104

Über die Sümpfe des Gouv. Petersburgs. Engler $24: 68$ 
Douglas, $\mathbf{R}$.

1890 Warming, E.

Kihlmann, A. C.

Blinge, J.

Kerner, A.

1891 Korschinsky, S.

Warming, E.

1892 Weber, C.

Krause, E. H. L. Blytt, A.

1893 Sernander, R.

Grevillus, A. Y.

Krause, E. H. L.
Succession of Forest Growth. Gard. Chron. '89: 10. Jugt '89: 172

Fra Vesterhavskystens Marskegne. Vid. Medd. Foren. 1890

Pflanzenbiologisch Studien aus Russisch-Lappland

Über den Einfluss der mittleren Windrichtung auf das Verwachsen der Gewässer. Engler 11 : 264 Pflanzenleben. 1: 267, 4: 895 Über die Entstehung und das Schicksal der Eichenwälder in mittleren Russland. Engler 13: 471

De psammofile Vegetationer in Danmark. Vid. Medd. Foren.

Über die Zusammensetzung des natürlichen Graslandes. Engler 16: b56

Die Heide. Engler 14: 515

Über $z w e i$ Kalktuffbildungen in Gudbrandsdalen. Engler 16: b1

Die Einwanderung der Fichte in Scandinavien. Engler $15: 1$

Om vegetationens utreckling pa de nybildade Hjelmaroarne. Bot. Cent. Beih. 5: 36

Beitrag zur Geschichte der Wiesenflora in Norddeut. schland. Engler 15: 387 
Stebler, F. G. \& Schröter, C.

1894 Wojeikow

Flahault, C. \& Combres, $P$. Weber, C.

1895 Warming, E.

Graebner, $\mathrm{P}$.

Meigen, F.

Grevillius, A. Y.

1896 Nilsson, A.

Meigen, F.

MacMillan, C.

1897 Merrill, G. P.
Beiträge zur Kenntniss der Matten und Weiden der Schweiz. Engler 17: b17

Die Einwirkung des Menschen auf die Natur. Engler 24: 69

Sur la flore de la Camargue. Bull. Soc. Bot. Fr. 41: 37

Über Veränderungen in der Vegetation der Hochmoore unter dem Einflusse der Cultur. Engler 23: b15

Plantesamfund, Grundtrok af den oekologiske Plantegeografi. $352,363,370$

Studien über die norddeutschen Heide. Engler $30: 500$ Beobachtungen über Formationsfolge bei Freyburg a. d. Unstrut. Deut. Bot. Monat. 13

Die erste Pflanzenansiedelung auf den Reblausherde. ib.

Studier ofver vegetationens sammansättning. Bver. Geol. Unders. 144.

Om örtrika barrskogar. Tids. Skog. 93

Die Besiedlung der Reblaus. herde in der Provinz Sachsen. Engler 21: 212

On the Formation of Circular Muskeag in Tamarack Swamps. Torr. Bull. 23: 500

Rocks, Rock-weathering and Soils. 
1898 Pound, R. \& Clements, F. E.

Graebner, P.

1899 Cowles, H. C.

Nilsson, A.

1900 Gerhardt, J.

Meigen, F.

1901 Cowles, H. C.

Elmore, C. J.

Whitford, H. N.

Weber. C. A.

1902 Clements, F. E.
The Phytogeography of Nebraska.

Über die Bildung natürlichen Vegetationsformationen in norddeutschen Flachlande. Bot. Cent. 77: 212

The Ecological Relations of the Sanddunes of Lake Michigan. Bot. Gaz. 27: 95

Nagra drag ur de svenska växtambällenas utvecklingshistoria. Bot. Cent. $1900: 370$

Handbuch des deutschen Dünenbaues.

Beobachtungen über Formationsfolge im Kaiserstuhl. Deut. Bot. Monat. 18: 145

The Physiographic Ecology of Chicago and Vicinity. Bot. Gaz. 31 : 73

Flora of a Dried-up Millpond. Proc. Nebr. Acad. Sci. $7: 29,69$

The Genetic Development of the Forests of Northern Michigan. Bot. Gaz. 31 : 289 Über die Erhaltung von Mooren und Heiden Norddeutschlands in Naturzustande. Abh. Nat. Ver. Bremen 15: 263

Herbaria Formationum Coloradensium.

A System of Nomenclature for Phytogeography.

Engler 31 : b1 
Drude, $O$.

Graebner, P.

Weber, C. A.
Der Hercynische Florenbezirk

Die Heide Norddeutschlands. Über die Vegetation und Entstehung des Hochmoores von Augstumal. 


\section{ZONATION}

The recognition of vegetation zones dates from Tournefort (1717), who found that, while the plauts of Armenia occupied the foot of Mount Ararat, the vegetation of the slopes above contained many species of southern Europe: still higher appeared a flora similar to that of Sweden, and on the summit grew arctic plants, such as those of Lapland. Linné (1751) generalised this idea in the dictum, "Planta diversoe indicant altitudinem perpendicularem terrce." Raimond (180I) studied the vegetation of the Pyrenees, and established the characteristic zones. A forest belt of Quercus robur occurred on the lowermost slopes. This was bordered by a zone of Fagus silvatica, and this in tarn by Abies taxifolia, and Taxus communis, which were replaced above by Pinus silvestris and $P$. mugho. Above the forest ran a border of thickets, consisting of Rhododendron, Daphne, Salix, etc., and beyond these, reaching to the snow cap, lay a zone of herbs and lichens, Gentiana, Primula, Saxifraga, etc. Humboldt and Bonpland (1805:37) employed the word region to designate the zones of mountains, restricting zone to the vegetation belts determined by latitude: they were not entirely consistent in their use of these terms, however. The results of their study of the vegetation of the Andes were graphically represented upon a chart of Chimborazo and Cutopaxi, in which were indicated the regions, the principal species of each and the variations of temperature, pressure and humidity. Schouw (1823:169) followed Humboldt and Bonpland in the use of zone and region, and further distinguished the formur as zones of latitude, and zones of longitude. A. De Candolle (1855:248) discussed at length the question of the upper and lower limits of mountain plants, first considering in a general way the influence of humidity, air and soil temperatures, exposure, pressure, soil composition, eta., and then in a more detailed manner the distribution of the 
representative species. His conclusion was that temperature is the principal cause of zonation upon mountains, and that dryness and insolation are secondary causes. Kabsch (1855:303) distinguished twelve zones of vegetation, corresponding exactly to the temperature zones of the earth, and recognised eight regions in altitude, designatng them according to the type of vegetation. Engler (1879:334) used the term zone for the primary divisions of provinces: these do not correspond to vegetation zones, but represent zonally disposed regions or districts for the most part. Grisebach (1872:129) established continental zones upon the busis of dominant trees. Köppen (1884:215) laid down the principle that the influence of temperature upon vegetation is best measured by the length of the period in which the temperature lies between certain limits. On this basis, he proposed the following zones: 1 , tropical zone, temperature above $20^{\circ} \mathrm{C}$. for all months; 2, subtropical zones, above $20^{\circ} \mathrm{C}$. for 4-11 months; 3 , temperate zones, a, constantly temperate, $b$, surnmers hot, c, summers temperate, winters cold; 4, cold zones, temperate for 1-4 months, the other months cold; 5 , polar zones, below $10^{\circ} \mathrm{C}$. for all months. Drude (1887:3) characterised and charted the following zones of vegetation: 1, nördliche glacial zone: 2, nördliche winterkalte zone; 3 , nördliche sommerheisse zone; 4, tropen zone; 5 , südliche sommerheisse und gemässigte zone; 6 , südliche kalte zone.

Raunkiaer (1889) noted the zonal arrangement of subformations in the dune valleys of Jutland, where, in passing from the dry margin of the valley to the moist center, the following plants appear in zones: Calluna vulgaris, Erica tetralix, Myrica gale, and Aira uliginosa. Magnin (1893:241,303) gave a new trend to the study of zones by his thorough investigation of the zonation of the Jura lakes. He found that the majority of these lakes regularly show the same series of zones: 1, a litoral zone of amphibiuus plants, Phragmites, Scirpus; 2, an interior zone of floating plants, Nuphar; 3, a central zone of floating or somewhat submerged plants, Potamogeton: 4, a center of clear water, where Ceratophyllum, Naias, Chara, Nitella, etc., grow submerged. 
Each zone was named from its facies, giving the series, caricetum, phragmitetum, scirpetum, nupharetum, potamogetonetum, and charetum. $\mathrm{He}$ discussed the influence of the general physical conditions of the lakes and of the structure of the various species upon zonation, but in such general terms that he was unable to give them definite expression. MacMillan (1896:500) studied the zonation of the sphagnum moors of Minnesota, and expressed his results in an important generalisation. "It would seem that there are two principal types of plant arrangement in their habitats. These are: (1) Zonal and (2) Azonal. The first is connected either with environmental conditions as a principal factor, as in the case of the zonal distribution upon dome-shaped islands, upon roches moutonnées, and on a larger scale upon mountain peaks and isolated ranges, along lake or ocean strand, and surrounding moors, or it may depend more particularly upon the character and habits of life of the plants themselves, as, for instance, in the case of the "fairy-ring" fungus. The mauter may be summed up in a sentence. Generaliu, when there is well-marked radial symmetry in the trpographic feature upon which a group of plants is distributed, zonal arrangement is the response of the plant population to these symmetric physiognomic conditions, but when the topographic feature is devoid of such well-marked radial symmetry the plants dispose themselves according to the aronal type. Talus-heaps, flat extended meadows, highly irregular bills, shallow marshy ponds, and other such localities may servo as illustrations of asymmetrical habitats. A variety of conditions determines whether the distribution upon a given area be zonal or azonal. And it is worthy of note that the same formation may in one case arise by zonal, in another by azonal distribution". In a later paper, (1897:949), MacMillan classified an extensive series of shore formations as zonal and azonal, including in the latter talus, boulder, rock and humus shores, in which there is often a mure or less rudimentary zonation. Clements (1897:968) described the lateral zones of subruderal plants. which border the old trails of the Great Plains. Pound and Clements (1898:220) 
pointed out that bilateral symmetry in the habitat was an important cause of zonation, and insisted upon the fundamental nature of zonation, as a universal feature of vegetation. Clements (1902:b19) distinguished three types of tcpographical symmetry, viz., radial, bilateral, and unilateral, to which correspond radial, bilateral and unilateral zonation.

As the historical summary shows, the concept of zonation is the oldest in phytogeography. Notwithstanding this, it has never been clearly defined, nor has there been any detailed investigation of the phenomenon itself, or of the causes which produce it. Zones are so common, and often so clearly marked, that they invite study, but no serious attempt has heretofore been made to analyse zonation, or to formulate a definite method of investigating it. Zonation is the practically universai response of plants to the quantitative distribution of physical factors in nature. In almost all habitats, one or more of the physical factors present decreases gradually in passing away from the point of greatest intensity. The result is that the plants of the habitat arrange themselves in belts about this point, their position being determined by their relation to the factor concerned. Close investigation will show that there is hardly a formation that is entirely without zonation, though in many cases the zones are incomplete or obscure for various reasons. Zonation is as characteristic of vegetation as a whole as it is of its unit, the formation, a fact long ago recognised in temperature zones. A continental climate, however, often results in the interruption of these, with the consequence that these belts of vegetation are not always continuous.

\section{CAUSES OF ZONATION}

The causes that produce zones are either biological, or physical: the first have to do with some characteristic of the plant, the second with the physical features of the habitat. Biological causes arise from the method of growth, from the manner of dissemination, or from the reaction of the species upon the habitat. The formation of circles as a 
result of radial wowth is a well-known occurrence with certain plants, but it is much more common than is supposed. In the case of agarics, this phenomenon has long been known under the name of "fairy-rings". It is found in a large number of moulds, and is characteristic of early stages of the mycelium of the powdery mildews. It occurs in nearly all maculicole fungi, and is exhibited by certain xylogenous fungi, such as Hysterographium. Among the foliose lichens, it is a common occurrence with the rock forms of Parmelia, Placodium, Physcia, and Lecanora, and with the earth forms of Parmelia and Peltigera. The thalloid liverworts show a similar radial growth. The flowering plants, and many mosses also, furnish good examples of this sort of growth in those species which simulate the form of the mycelium or thallus. These are the species that form mats, turfs, or carpets. Alpine mat formers, such as Silene acaulis, Paronychia pulvinata, Arenaria sajanesis, etc., are typical examples. Xerophytic, turf-forming species of Muhlenbergia, Sporobolus, Bouteloua, Festuca, $P o a$, and other grasses form striking ring-like mats, while creeping species of Euphorbia, Portulaca, Amarantus, etc., produce circular areas. Rosettes, bunch-grasses, and many ordinary rootstalk plants spread rapidly by runners and rhizomes. The direction of growth is often indeterminate in these alsc, and is in consequence more or less bilateral or unilateral. Growth results in zonation only when the older central portions of the individual or mass die away, leaving an ever-widening belt of younger plauts or parts. This phenomenon is doubtless due in part to the greater age of the central portion, but seems to arise chiefly from the demands made by the young and actively growing parts upon the water of the soil. There may possibly be an exhaustion of nutritive content, as in the case of the fungi, but this seems improbable for the reason that young plants of the same and other species thrive in these areas. It must not be inferred that these miniature growth zones increase in size until they pass into the zones of formations. Growth contributes its share to the production of these, but there is 
no genetic connection between a tiny plant zone, and a zone of vegetation.

Radial, and bilateral growth play an important part in formational zones in so far as they are related to migration. The growth of the runner or rhizome itself is a very effective means of dissemination, while the seeding of the plants thus carried away from the central mass is most effective at the edge of the newly occupied area. This holds with equal force for plants with a mycelium or a thallus. The circular area becomes larger year by year: sooner or later, the younger, more vigorous, and more completely occupied circumference passes into a more or less complete zone. This will result from the reaction of the central individuals upon the habitat, so that they are readily displaced by invaders, or from their increasing senility and dying out, or from the invasion of forms which seed more abundantly and successfully. This result will only be the more marked if the radiating migrants reach a belt of ground especially favorable to their ecesis. In this connection, it must be carefully noted that vegetation pressure, before which weaker plants are generally supposed to flee, or bv which they are thought to be forced out into less de. sirable situations, is little more than a fanciful term for radial growth and migration. It has been shown under invasion that disseminules move into vegetation masses, as well as away from them, the outward movement alone being conspicuous, because it is only at the margin and beyond that they find the necessary water, light and room for growth.

Certain reactions of plants upon habitats produce zonation. The zones of fungi are doubtless caused by the exhaustion of the organic matter present, while in lichens, and mosses, the decrease in nutritive content has something to do with the disappearance of the central mass. In the mats of flowering plants, the connection is much less certain. The reaction of a forest, or thicket, or even of a tall herbaceous layer is an extremely important factor in the production of zonation. The factor chiefly concerned bere 
is light. Its intensity is greatest at the edge of the formation, and just below the primary layer: the light becomes increasingly diffuse toward the center of the forest, and toward the ground. In response to this, both lateral and vertical zones appear. The former are more or less incomplete: and are only in part due to differences in illuminatiun. The vertical zones or layers are characteristic of forest and thickets, and are caused directly by differences in light intensity.

The physical causes of zonation are by far the most im. portant. They arise from differences in temperature, water, and light. In the large, temperature differences are the most important, producing the great zones of vegetation. In a particular region or habitat, variations of water content and humidity are controlling, while light, as shown above, is imporant in the reactions of forest and thicket. Physical factors produce zonation in a habitat or a series of babitats, when there is either a gradual and cumulative, or an abrupt change in their intensity. Gradual, slight changes are typical of single babitats, abrupt, marked changes of a series of habitats. This modification of a decisive factor tends to operate in all directions from the place of greatest intensity, producing a characteristic symmetry of the habitat with reference to the factor concerned. If the area of greatest amount is linear, the shading-out will take place in two directions, and the symmetry will be bilateral, a condition well illustrated by rivers. On the other hand, a central intense area will shade out in all directions, giving rise to radial symmetry, as in ponds, lakes, etc. The essential connection between these is evident where a stream broadens into a lake, or the latter is the source of a stream, where a mountain ridge breaks up into isolated peaks, or where a peninsula or landspit is cut into islands. The line that cunnects the points of accumulated or abrupt change in the symmetry is a stress line or ecotone (oikos, ó, habitat, tóvos, ó, stress). Ecotones are wellmarked between formations, particularly where the medium changes: they are less distinct within formations. It is 
obviuus that an ecotone separates two different series of zones in the one case, and merely two distinct zones in the other.

The physical symmetry of a habitat depends upon the distribution of water in it, and this is profoundly affected by the soil and the physiography. The influence of precipitation is slight or lacking, as it is nearly uniform throughout the habitat: the effects of wind and humidity are more localised. Differences of soil rarely obtain within a single habitat, though often occurring in a zoned series. The strikingly zonal structure or arrangement of habitats is nearly always due to differences in water content produced by physiographic factors, slope, exposure, surface and altitude. The effect of these upon water content and humidity is obvious. Wherever appreciable physiographic differences occur, there will be central areas of excess and deficiency in water content, between which there is a symmetrical modification of this factor. Peaks are typical examples of areas of deficiency, lakes and oceans of areas of excess. When these areas are extreme and close to each other, the resulting zonation will be marked; when they are moderate, particularly if they are widely separated, the zones produced are obscure. Asymmetry of a habitat or a region practically does not exist. Central areas of excess and deficiency may be very large and in consequence fail to seem symmetrical, or the space between them so great that the symmetry is not conspicuous, but they are everywhere present, acting as foci for the intervening areas.

The response of vegetation to habitat is so intimate that physiographic symmetry everywhere produces vegetational symmetry, which finds its ready expression in plant zones. The reaction of vegetation upon habitat causes biological symmetry, typical of growth zones and light zones. From these facts, it is clear that zonation will be regularly characteristic of the vegetative covering. The zonal arrangement of formations is usually very evident: the zones of a formation are often obscured, or, where the latter occupies a. uniform central area of excess or deficiency, they are 
rudimentary or lacking, as in shallow ponds. Zones are frequently imperfect, though rarely entirely absent, in new soils, such as talus. They are rendered obscure in several ways. In the initial stages of a succession, as well as in the transitions between the various stages, the plant population is so scattered, so transient. or so dense as to respond not at all to a degree of symmetry which produces marked zonation in later formations. The alternation of conspicuous species not only causes great interruption of zones, but often also completely conceals the zonation of other species, such as the grasses, which though of more importance in the formation, have a lower habit of growth. Furthermore, the ecotones of one factor may run at right angles to those of anuther, and the resulting series of zones mutually obscure each other. Finally, such a physiographic feature as a hill may have its symmetry interrupted by ridges or ravines, which deflect the zones downward or upward, or cause them to disappear altogether, while the shallows or depths of a pond or lake may have the same effect. An entire absence of zones, i. e. azonation, is exceptional in vegetation. Almost all cases that seem to exhibit it may be shown by careful examination to arise in one of the several ways indicated above.

Two kinds of zonation are distinguished with reference to the direction in which the controlling factor changes. When this is horizontal, as with water content and temperature, zonation will be lateral: when it is vertical, as in the case of light, the zonation is vertical. There exists an intimate connection between the two in forests, where the secondary layer of small trees and shrubs is continuous with a belt of trees and shrubs around the central nucleus, and the lower layers of bushes and herbaceous plants with similar zones still further out. This connection doubtless arises from the fact that conditions are unfavorable to the facies, outside of the nucleus as well as beneath it. Floristically, each layer and its corresnonding zone are distinct, as the one consists of shade, the other of sun species. Lateral zonation is radial when the habitat or physiographic 
feature is more or less circular in form, and it is bilateral, when the latter is elcngated or linear. Vertical zonation is unilateral.

Radial zonation is regularly characteristic of elevations and depressions. From the form of the earth, it reaches its largest expression in the girdles of vegetation corresponding to the zones of temperature. The zones of mountain peaks are likewise due largely to temperature, though bumidity is a very important factor also. Mountain zones are normally quite perfect. The zonation of islands, hills, etc., is due to water content. In the former, the zones are usually quite regular and complete; in the latter, they are often incomplete or obscured. Prairies and steppes are not zoned as units, but are complexes of more or less zonal hills and ridges. Ponds, lakes and seas regularly exhibit complete zones, except in those shallow ponds where the depth is so slight that what is ordinarily a marginal zone is able to extend over the entire bottom. The line between an elevation and a depression, i. e., the edge of the water level, is the most sharply defined of all ecotones. It separates two series of zones, each of which constitutes a formation. One of these is regularly hydrophytic, the other is usually mesophytic. The line between the two can rarely be drawn at the water's edge, as this is not a constant, owing to waves, tides, or periodical rise and fall. There is in consequence a more or less variable transition zone of amphibious plants, which are, however, to be referred to the bydrophytic formation. Nearly all forest formations serve as a center about which are arranged several somewhat complete zones. As a rule, these merge into a single heterugeneous zone of thickets.

Bilateral zonation differs from radial only in as much as it deals with linear elevations and depressions instead of circular ones. Wiih this difference, the zones of ranges and ridges correspond exactly to those of peaks and hills, while the same relation is evident between the zones of streams, and of lakes and ponds. The ecotones are identical except as to form: they are linear in the one, and circular in the 
other. Incompleteness is more frequently found in bilateral zonation, though this is a question of distance or extent, rather than one of symmetry.

Vertical zonation is peculiar in that there is no primary ecotone present, on either side of which zones arrange them. selves with reference to the factor concerned. This arises from the fact that the controlling factor is light, which impinges upon the habitat in such manner as to shade out in but one direction, i. e., downward. Vertical zones appear in bodies of water, on account of the absorption of light by the water. In a general way, it is possible to distinguish bottom, plancton, and surface zones, consisting almost wholly of algae. There is little question that minor zones exist, especially in lakes and seas, but these await further investigation. The must characteristic vertical zones occur in forests, where the primary layer of trees acts as a screen. The density of this screen determines the number of zones found beneath it. In extreme cases the foliage is so deuse that the light beneath is insufficient even for mosses and lichens. As a rule, however, there will be one or more zones present. In an ordinary deciduous forest, the layers below the facies are five or six in number: (1) a secondary layer of small trees and shrubs; (2) a tertiary layer of bushes; (3) an upper herbaceous layer of tall herbs; (4) a middle herbaceous layer; (5) a lower herbaceous layer; (6) a ground layer of mosses, lichens, other fungi and algae. The upper layers are often discontinuous, the lower ones are more and more continuous. As a forest becomes denser, its layers disappear from the upper downward, the ground layer always being the last to disappear because of its ability to grow in very diffuse light. A vertically zoned formatiun shows a complex series of reactions. The primary layer determines the amount of heat, light, water, wind, etc., for the subordinate layers in general. Each of these layers then further determines the amount for those below it, the ground layer being subject in some degree to the control of every layer above it. This accounts probably for the definiteness and permanence of this layer. The 
degree to which the lower layers influence the upper by reacting upon the habitat is not known. It is evident that this infiuence must be considerable by virtue of their control of the water supply in the upper soil strata, by virtue of their transpiration, their decomposition, etc.

The ecotone between two formations is never a sharp line, but it is an area of varying width. The edge of this area which is contiguous to one formation marks the limit for species of the other. Both formations disappear in this transition zone, but in opposite directions. The overlapping which produces such zones arises from the fact that the physical factors tend to approach each other at the line of contact between formations, and that many species are more or less adjustable to conditions not tco dissimilar.

As a fundamental expression of progressive change in the amount of heat and water, zoaation is the most important feature of vegetation. It constitutes the sole basis for the division of continental as well as insular vegetation. The continent of North America furnishes striking proof of the truth of this. Conforming to the gradual decrease of temperature and water content northward, three primary belts of vegetation stretch across the continent from east to west. These are forest, grassland, and polar desert. The first is further dirided into the secondary zones of broadleaved evergreen, deciduous, and needle-leaved forests. At right angles to this temperature-water symmetry lies a symmetry due to bumidity alone, in accordance with which forest belts touch the oceans, but give way in the interior to grasslands, and these to deserts. It is at once evident that the mutual interruption of these two series of zones bas produced the primary features of North American vegetation, i. $\Theta$. tropical forests where heat and water are excessive, deserts where either is unusually deficient, grassland when one is low, the other moderate, and deciduous and coniferous forests, where the water content is at least moderate, and the temperature not too low. Such a simple yet fundamental division has been modified, however, by the disturbing effect which three continental mountain systems have 
had upon humidity, and upon temperature symmetry. The two are intimately interwoven. The lowering of temperature due to altitude produces the precipitation of the windborne moisture upon those slopes which look toward the quarter from which the prevailing winds blow. A mountain range thus makes an abrupt change in the symmetry, and renders impossible the gradual change from forest to grassland and desert. The Appalachian system is not sufficiently high to produce a pronounced effect, and forests extend far beyond it into the interior before passing into prairies and plains. On the other hand, the influence of the Rocky mountains and the Sierra Nevada is very marked. The latter rise to a great height relatively near the coast, and condense upon their western slopes nearly all of the moisture brought from the Pacific. The Rocky mountains have the same effect upon the much drier winds that blow from the east, and the two systems in consequence enclose a parched desert. This series of major zones thus becomes, starting at the east, forest, grassland, desert, and forest, instead of the more symmetrical series, forest, grassland, desert, grassland, forest, which would prevail were it not for these barriers. This actual series of major zones undergoes further interruption by the action of these mountain systems in deflecting northern isotherms far to the south. This action is greatest in the high ranges, the Rocky mountains and the Sierras, and least in the lower Appalachians. Its result is to carry the polar deserts of the north far southward along the crests of the mountains, and to extend the boreal coniferous forests much further south along thair slopes. In the Appalachians, this means no more than the extension of a long tongue of conifers into the mass of deciduous forests, and the occassional appearance of an isolated peak. In the western ranges, it produces two symmetrical series of minor mountain zones, forest, alpine grassland or desert, and forest, to say nothing of the foothill and timber liue zones of thicket.

There seems to be no good reason for distinguishing the zones of mountains as regions. The term itself is inappli- 
cable, as it has no reference to zonation, and is used much more frequently as a term of general application. Its use tends to obscure also the essential identity of the so-called vertical zones of mountains with the major continental zones, an identity which can not be insisted upon too strongly. For the sake of clearness, it is important to distinguish all belts of vegetation as zones, though it is evident that these are not all of the same rank. The following division of the vegetation of North America is based upon the fundamental principles of continental symmetry, and the community of continental and mountain zones.

I. Polar-niveal zone-zona polari-nivalis

II. Arctic-alpine zone-zona arctici-alpina.

Arctic province-provincia arctica.

Alpine province-provincia alpina.

III. Boreal-subalpine zone-zona boreali-subalpina.

Alaska province-provincia alaskana.

Cordilleran province-provincia cordillerana.

Ontaria province-province ontariensis.

IV. Temperate zone-zona temperata.

Atlantic province-provincia atlantica.

Appalachian province--provincia appalachiana.

Nebraska province-provincia nebraskensis.

Utah province-provincia utahensis.

Coast province-provincia litoralis.

Pacific province-provincia pacifica.

V. Subtropical zone-zona subtropicalis.

Florida province-provincia floridana.

Mexican province-provincia mexicana.

VI. Tropical zone-zona tropicalis.

Antilles province-provincia antilleana.

Andean province-provincia andeana.

The investigation of zones rests upon the instrumental study of the factors of ecotones, and upon the quadrat examination of contiguous zones and of the transition area between them. As the methods are essentially the same as for alternation, their detail will be found under the discussion of this principle. 


\section{BIBLIOGRAPHY}

1717 Tournefort, J. P.

1751 Linné, C. v.

1791 Swartz, O.

1801 Ramond, L. F. E.

1805 Humboldt, A. v. \& Bonpland, A.

1813 Wahlenberg, G.

1823 Schouw, J. P.

1836 Meyen, F. J. F.

1855 De Candolle, A.

1865 Kabsch, W.

1874 Contejean, C.

1879 Engler, A.

1883 Krasan, F.

1884 Köppen. W.

1887 Drude, $O$.

1889 Raunkiar, C.
Relation d'un Voyage du Levant

Philosophia Botanica

Observationes Botanicæ

Voyages au Mont-Perdu et dans la partie adjacente des Haut-Pyrénées

Tableau Physique des Regions Equatoriales

De Vegetatione et Climate Helvetiæ Septentrionalis 5,57

Grundzüge einer allgemeinen Pflanzengeographie. 169, 292

Grundrisz der Pflanzengeographie. 51, 93, 161

Géographie Botanique Raisonnée. 1238

Das Pflanzenleben der Erde. 303

De l'influence du terrain sur la végétation. Ann. Nat. Sci. V: $20: 266$

Versuch einer Entwicklungsgeschichte der Pflanzenwelt

Über die Bedeutung der gegenwärtigen Verticalzonen der Pflanzen, etc. Engler 4: 266

Die Wärmezonen der Erde. $1: 215$

Atlas der Pflanzenverbreitung Vesterhavets Ost-og Sydkysts Vegetation. Bot. Cent. 41: 361 
Wiesner, J.

1890 Drude, O.

1893 Magnin, A.

Flahault, C.

Coville, F. V.

1894 MacMillan, C.

Pieters, A. J.

1895 Warming, E. Kurtz, F.

1896 MacMillan, C.

1897 Clements, F. E.

MacMillan, C.

1898 Pound, R. \& Clements, F. E.

Schimper, A. F. W. Pflanzengeographie auf physiologischer Grundlage.

Biologie der Pflanzen. 203 Handbuch der Pflanzengeographie. 69

Recherches sur la végétation des lacs du Jura. Rev. Gen. Bot. 5 : 241, 303, 515 Bas-Languedoc et les pays voisins. Bull. Bot. Soc. Fr. 40: 36

Botany of the Death Valley Expedition. 16

On the Occurrence of Sphagnum Atolls in Central Minnesota. Minn. Bot. Studies 1: 1

The Plants of Lake St. Clair. Bull. Mich. Fish Com.

Plantesamfund

Die Flora des Chilcatgebietes im südostlichen Alaska. Engler 19: 327

On the Formation of Circular Muskeag in Tamarack Swamps. Torr. Bull. $23: 500$ Peculiar Zonal Formations of the Great Plains. Am. Nat. 31: 968

Observations on the Distribution of Plants along Shore at Lake of the Woods. Minn. Bot. Studies. 1: 968 The Phytogeography of Nebraska. 220

227
Les zones botaniques dans le 


\section{ALTERNATION.}

The term alternation is used to designate that phenom. enon of vegetation, in which a formation recurs at different places in a region, or a species at separate points in a formation. Although it is a fundamental feature of vegetation, it has not been recognized heretofore, and has no history as a concept. A degree of historical interest attaches, however, to one or two ideas that contain something of this principle. Schouw (1823:168), in dealing with the geographical areas of species, distinguished them" as interrupted or continuous, interrupted distribution containing, at least by inference, some idea of alternation. In his concept of substitution (221). Schouw has expressed a kind of alternation found in related species or genera, which occur in different regions. Meyen (1836:89) adopted the ideas of Schouw in regard to interrupted areas, and definitised them in such a way that interruption comes to be identical with the simplest type of alternation, i. e., that in which a given species recurs in separated but similar stations. A. De Candolle (1855:472) spoke incidentally of sparse plants, which "changert un peu de place, par une loi générale d'alternance", but he did not carry the idea further. In the vicarious or representative forms of Drude (1890:124) and others, there is a hint of this principle, but it was not elaborated. Thornber (1901:55) discussed the general features of the alternation of species in the prairie grass formation. Jaceard (1901:547), in his statistical studies of Alpine vegetation, has laid especial stress upon the floristic diversity of different examples of the same formation. He has sought to use this as an index of the physical character of the habitat, however, and has not developed it further.

Alternation is the response of vegetation to the heterogeneity of the surface of the earth. It is in sharp contrast to zouation in as much as it is directly caused by asymmetry in the topography. In consequence, it deals with the subdivisions of zones, arising from physical differences within the symmetrical area. It deals with vegetation areas of 
every rank below that of, major zone, with the habitat and geographical areas of species, and in a certain way, with the correspondence of vicarious genera. The breaking un of vegetation into formations is a striking example of alternation. The same phenomenon occurs in every formation, producing associations and minor plant groups, and everywhere giving variation to its surface and structure. The essential idea involved in this prisciple is the recurrence of like formations, asscciations, or groups, which are more or less separated by formations, associations, or groups differing from them. It is an exact expression of the primary law of association that heterogeneity of structure varies directly as the extent and complexity of the habitat, or the series of habitats. Vegetation is made up of what are superficially homogeneous formations, but upon analysis these are seen to contain associations. The latter, though more uniform than formations, break up into groups, each of which still shows a characteristic heterogeneity arising from the varying number and arrangement of its coustituent species.

The primary cause of alternation is physical asymmetry, which is everywhere present within the symmetrical areas which produce zones. This is influenced so strongly, however, by migration and plant competition (phyteris') that the consideration of this subject will gain in clearness, if thess are treated as separate causes. The essenital relation between them must not be lost sight of however. Migration carries disseminules into all, or only some of the different areas of a formation, or into different formations, with little respect to the physical nature of these. The physical character of these asymmetrical areas determines that some of these plants shall be established in one series of places, and some in another, while the competition between the individuals in the various areas determines the numerical value of each species as well as its persistence. These three causes are invariably present in the production of alternat-

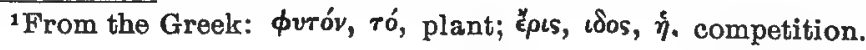


ing areas, and originally, i. e., in new or denuded soils, the sequence is constant, viz., migration, ecesis in asymmetrical areas, and competition.

With respect to the different portions of an asymmetrical area. migration will have one of three effects: (1) it will carry disseminules into both favorable and unfavorable areas: (2) into favorable ones only, or (3) into unfavorable ones alone. From the radial nature of migration, the first case is far the most frequent: it is typical of sporostrotes, and the highly specialized spermatostrotes and carpostrotes. The effect of migration is uniform here, and alternation arises in consequence of the selective power of ecesis. It is evident that migration does not have even an indirect effect, when the disseminules are carried into none but unfavorable situations. Where the movement is into favorable places alone, alternation is the immediate result. The intermittent operation of migration, and the presence of barriers are responsible for the absence of plants in situations favorable to them, and in consequence bring about a certain alternation between corresponding species.

The selective operation of physical factors upon the disseminules carried into the different parts of an asymmetrical area is the usual cause of alternation. Asymmetry alone is universal within the more conspicuous structures termed zones, down to the smallest areas which a group of plants can occupy. The difference between contiguous areas, particularly within the same habitat, is often small. It sometimes seems inefficient in the initial stages of a succession when a single species is present, but even in extreme cases its effect will be recognisable in the size and density of the individuals. Asymmetry is clearly evident in vegetation where two symmetrical series cross each other, or when a symmetry is interrupted by barrier-like elevations or depressions. Within formations, it arises trom differences, often very slight, in slope, exposure, elevation, from irregularities of surface. differences in soil structure, or composition, in the amount of cover, and in the 
reactions of the living plants. At the last point, it is in direct, connection with piant-competition.

Much uncertainty, as well as diversity of opinion, seems still to exist in regard to the precise nature of the competition between plants that occupy the same area. It has long been admitted that the phrase, "struggle for existence", is true of this relation only in the most figurative sense, but the feeling still prevails that, since plants live in associations, there must be something mysterious and vitalistic in their relation. No one has been able to discuver anything of this nature, but nevertheless the impresssion remains. Such a direct relation exists only between parasites, epiphytes and lianes, and the plants which serve to nourish or support them. In the case of plants growing on the same stratum, actual competition between plant and plunt does not occur. One individual can affect another only in as much as it changes the physical factors that influence the latter. Competition is a question of the reaction of a plant upon the physical factors which encompass it, and of the effect of these modified factors upon the adjacent plants. In the exact sense, two plants do not compete with each other as long as the water content and nutrition, the heat and light are in excess of the needs of both. The moment, however, that the roots of one enter the area from which the other draws its water supply, or the foliage of one begins to overshade the leaves of the other, the reaction of the former modifies unfavorably the factors controlling the latter, and competition is at once initiated. The same relation exists throughout the process: the stronger, taller, the more branched, or the better rooted plant reacts upon the habitat, and the latter immediatel. exerts an unfavorable effect upon the weaker, shorter, less branched or more poorly rooted plant. This action of plant upon habitat and of habitat upon plant is cumulative, however. An increase in the leaf surface of a plant not merely reduces the amount of light and beat available for the plant near it or beneath it, but it also renders necessary the absorption of more water and other nutritive material, and correspondingly de- 
creases the amount available. The inevitable result is that the successful individual prospers more and more, while the less successful one loses ground in the same degree. As a consequence, the latter disappears entirely, or it is handicapped to such an extent that it fails to produce seeds, or these are reduced in number or vitality.

Competition in vegetation furnishes few instances as simple as the above, but this will serve to make clear the simplest case of ordinary competition, i. e., that in which the individuals belong to a single species. The various individuals of one species which grow together in a patch shuw relatively slight differences, in beight. width, leaf expanse or root surface. Still, sume will have the largest surfaces for the impact of water, heat and light. while others will have the smallest: the majority, perhaps. will occupy different places between the extremes. 'The former will receive more than their share of one or more factors. The reaction thus produced will operate upon the plants subject to it inversely as the amount of surface impinged upon. The usual expression of such competition is seen in the great variation in height, branching, etc., of the different individuals, and in the inability of many to produce flowers. This is particularly true of annuals, and of perennials of the same generation. In the competition between parents and offspring of the same perennial species, the former usually have so much the advantage that the younger plants are often unable to thrive or even germinate, and disappear, leaving a free space beneath and about the stronger parents. This illustrates the primary law of competition, viz., that this is closest when the individuals are most similar. Similar individuals make nearly the same demands upon the habitat, and adjust themselves least readily to their mutual reactions. The more unlike plants are, the greater the difference in their needs, and some are able to adjust themselves to the reactions of others with little or no disadvantage.

In accordance with the above principles, the competition is closer between species of like form than between those 
that are dissimilar. This similarity must be one of vegetation or habitat form, not one of systematic position. The latter is in fact of no significance, except where there is a certain correspondence between the two. Leaf, stem and root characters determine the outcome, and those species most alike in these features will be in close competition, regardless of their taxonomic similarity or dissimilarity. This is as conclusive of the competition between the species of the same genus, as it is between those belonging to genera of widely separated families. From this may be deduced a second principle of competition viz., the closeness of the competition between the individuals of different species varies directly with their similarity in vegetation or habitat form. This principle is of primary importanc $\theta$ in the competition which arises between occupants and invaders in the different stages of succession. Those invading species that show the greatest resemblance to occupants in leaf, stem and root form experience the greatest difficulty in establishing themselves. The species, on the contrary, which are so unlike the occupants that they come in at a clear advantage or disadvantage, establish themselves readily, in the one case as a result of the reaction, in the other by taking a subordinate position. This principle lies at the base of the changes in succession which give a peculiar stamp to each stage. A reaction sufficient to bring about the disappearance of one stage can be produced only by the entrance of invaders so different in form as to materially or entirely change the impress of the formation. Stabilisation results when the entrance of invaders of such form as to exert an efficient reaction is no longer possible. $I^{n}$ forests, while many vegetation forms can still enter, none of these produce a reaction sufficient to place the trees at a disadvantage, and the ultimate forest stage, though it may change in compositiun, can not be displaced by another.

It is obvious that the vegetation forms and habitat forms of associated species are of fundamental importance in determining the course and result of competition. Identity of vegetation form regularly produces close competition, and 
the consequent numerical reduction or disappearance of one or more species. Dissimilarity, on the other hand, tends to eliminate competition, and to preserve the advantage of the superior form. Species of trees compete sharply with each other when found together: the same is true of surubs, of rosettes, etc. The relation of the shrubs to the trees, or of the rosettes to the shrubs of a formation is one of subordination rather than of competition. The matter of height and width often enters here also to such a degree that the tallest herbs compete with the bushes and shrubs, and rosettes with mats or grasses. The amount and disposition of the leaf surface are decisive factors in the competition between species of the same vegetation form, in so far as this is governed by light. In those plants in which the leaves are usually erect, notably the grasses and sedges, the competition between the aerial parts is relatively slight, and the result is determined by the reactions of the under. ground stems and roots.

The position of the competing individuals is of the greatest importance. The distance between the plants affects directly the degree of competition while their arrangement, whether in groups according to species or singly, exerts a marked influence by determining that the contest shall be between like forms, or unlike forms. Position is controlled primarily by the relation existing between seed-production and dissemination. It is of course influenced in large measure by the initial position taken by the invaders into a nudate area, but this is itself a result of the same phenomena. The individuals of species with great seed-production and little or no mobility usually occur in dense stands. In these, the competition is fierce, for the two reasons of similarity and density, and the result is that the plants fall far below the normal in height and width. This is an extreme example of the group arrangement. When the seed-production is small, the mobility may be great or little without seriously affecting the result. The individuals of a species of this kind will be scattered among those of other species, and the closeness of competition will 
depend largely upon the similarity existing between the two. The arrangement in such cases is sparse. A species with great seed-production and great mobility usually shows both kinds of arrangement, the position of the individuals, and the competition between them varying accordingly. This is due to the intermittent action of distributing agents, making it possible for the seeds to fall directly to the ground during the times that winds, etc., are absent. The three types of arrangement indicated above are termed gregarious, copious, and gregario-copious. They furnish the basis for the investigation of abundance, ${ }^{1}$ which deals essentially with the number and arrangement of the individuals of competing species. The effect of distance, i. e., the interval between individuals, upon competition is fundamental. The competition increases as the interval diminishes, and the reverse.

The view here advanced, i. e., that competition is purely physical in nature, renders untenable the current conceptions of vegetation pressure, occupation, etc. Masses of vegetation are thought to force the weaker species toward the edge, thus initiating an outward or forward pressure. As has been shown above, no such phenomenon occurs in vegetation. This movement is nothing but simple migration, followed by ecesis, and has no counection with "weaker" species, or the development of a vital pressure. The direction taken by the migrating disseminules is essentially indeterminate. Migration seems to be outward, or away from the mass, merely because the ecesis is greater at the edge, where the increased dissimilarity between plant forms diminishes the competition. The actual movement is outward, but it takes place through the normal operation of competition. In this connection, it should be pointed out that the common view that plants require room is inexact, if not erroneous. This is difficult of proof, as it is impossible to distinguish room as such from the factors normally present, light, heat, water and nutrient salts, but it seems obvious that the available amounts of these will

${ }^{1}$ Pound and Clements. Phytogeography of Nebraska 2 ed. 591900 
determine the space occupied by a plant, irrespective of the room adjacent plants may allow it. The explanation of competition upon physical grounds likewise invalidates the view that plants possess spheres of influence, other than the areas within which they exert a demonstrable reaction upon the physical factors present.

Competition plays a very important role in alternation. It produces minor examples of alternation in the physical units of an as.pmmetrical series. Its greatest influence, nowever, is exerted in modifying the effects of asymmetry. The reaction of occupants emphasizes, or reduces the effect of asymmetry, and has a corresponding action upon alternation. This result of competition is typical of succession, in which the sequence of stages arises from the interaction of occupant and invader.

Alternation involves two ideas, viz., the alternation of different species or formations with each other, and the alternation of uhe same species or formation in similar but separate situations. This is the evident result of asymmetry, in response to which contiguous areas are dissimilar and remute ones often similar. Individuals of the sume species, or examples of the same formation may be said to alternate between two or more similar situations, while different species, or formations are said to alternate with each otber, occurring of course in situations different in character. From the nature of alternation, the two phenomena are invariably found together.

It is possible to distinguish three kinds of alternation: (1) of a formation, association, layer, facies or species in similar situations; (2) of similar or corresponding formations, species, etc., in similar situations; (3) of facies and other species with respect to number. The last two are merely variations of the first, arising out of slight differences in the physical factors of the alternating areas, the adjacent flora, or the course of competition. For this reason, they are not sharply set off, and it is undesirable to distinguish them by actual terms. The alternation of different examples of the same formation is a significant feature of greatly 
diversified areas, such as mountains. It is naturally much less characteristic of lands physiographically more uniform. A xerophytic formation will alternate from ridge to ridge, a mesophytic formation between the intermediate valleys: aquatic vegetation will alternate from pond to pond, or stream to stream. The appearance of new or denuded soils upon which successious establish themselves is the most important cause of the alternation of formations. The weathering of rocks in different areas of the same region produces in each a sequence of similar or identical formations. The same statement is true in general of other causes of succession, such as erosion, flooding, burning, cultivation, etc., wherever they operate upon areas physically similar and surrounded by the same type of vegetation. The areas of more or less heterogeneous formations characterized by major physical differences are occupied by associations. In an extensive formation, the arme association alternates from one to another of these areas that are similar. When the formation is interrupted and occurs here and there in separate examples, an association often alternates from one to another of these. An association regularly derives its character from the fact that one or more of the facies of the formation is more intimately connected with certain areas of the latter than with others. This explains why the alternation of associations and facies are usuallv identical. Layers sometimes alternate botween different examples of the same forest or thicket formation, when they are suppressed in some by the diffuseness of the light.

The alternation of species is a typical feature of formations: it is absent only in those rare cases where the latter consist of a single species. The areas of a habitat which show minor physical, or historical (i. e., competitive) differences are occupied by groups of individuals belonging to one or more species responsive to these differences. Each of these groups will recur in all areas essentially similar, the intervals being occupied of course by slightly different groups. Such groups are constituted by gregarious or 
copious species of restricted adjustability. Sparse plants like ise alternate, but they necessarily play a much less conspicuous part. In habitats not too heterogeneous, a large number of species are sufficiently adjustable to the slight differences that they occur throughout the formation. Often, to be sure, they show a characteristic response, expressed in the size or number. This is illustrated by the facies and many of the principal species of the prairie formation. Festuca, Koelera, Panicum, and Andropogon occur throughout, except in the moist ravines which are practically meadows. Astragalus, Pooralea, Erigeron and Aster grow everywhere on slopes and crests, but they are much more abundant in certain situations. Other plants. Lomatium, Meriolix, Anemone, Pentstemon, etc., recur in similar or identical situations upon different hills. Lomatium alternates between sandy or sandstone crests, Meriolix and Pentstemon occur together upon dry upper slopes, while Anemone alternates between dry slopes and crests.

Owing to the accidents of migration and competition, similar areas within a habitat are not occupied by the same species, or group of species. A species found in one area will be replaced in another by a different one of the same, or a different genus. The controlling factors of the area render imperative an essential identity of vegetation and habitat form, though in systematic position the plants may be very diverse. Such genera and species may be termed corresponding. The relation between such plants is essentially alternation: it should, perhaps, be distinguished from alternation proper as corresponsive. The prairie formation furnishes a good example of this on exposed sandy crests, upon which Lomatium, Comandra and Pentstemon alternate. Formations exhibit a similar correspondence.

All species that alternate show a variation in abundance from one area to another. Frequently, the difference is slight, and may be ignored, except in determining abundance. Very often, however, the variation is so great that a facies may be reduced, numerically, to the rank of a principal species, or one of the latter to a secondary species. 
This phenomenon is distinguished as numerical alternation. It arises from the fact that the similar areas are sufficiently different to affect the abundance, without producing complete suppression. It is probable that this result is due almost entirely to competition. Astragalus crassicarpus grows on all the slopes of the prairie formation, but on some it has the abundance of a facies, while on others it is represented by a few scattered individuals This difference is much more striking in separate examples of the same formation, particularly when a normal facies is reduced to the numerical value of a secondary species This is a matter of great importance in the study of formations, for it has doubtless often resulted in mistaking an association for a formation.

Alternation furnishes the logical basis for what may be called comparative phytogeography. The latter is of much broader scope than the old subject of geographical distribution, for it treats not only of the distribution of formations and associations as well as of species, but it also seeks to explain this by means of principles drawn from the relation between habitat and vegetation. Jaccard (1901:547) has given a fruitful trend to distributional studies. When the latter come to be fully based upon physical factor investigations, and upon the effects of migration and competition as shown'in alternation, the comparative study of formations will represent the highest type of phytogeographical'uctivity.

The investigation of the physical factors which produce alternation concerns itself primarily with the conditions in the nucleus of the alternating areas, except in those cases where the areas are rather sharply delimited by an ecotone. Here, as "elsewhere, the water content, and the physiographic factors which modify it, seem to be of greatest importance, though"humidity doubtless has a more important effect than is ordinarily ascribed to it. In layered formations, light is of course the controlling factor. The influence of migration is best determined by the use of the migration circle described under invasion. The effects of competition 
and of the shifting brought about by physical changes from year to year are measured and recorded by means of permanent quadrats, and quadrat photographs. These methods are held to be indispensable to the scientific investigation of alternation, as well as invasion, succession, and zonation. The wealth of detail connected with the description, plaeing and operation of physical factors instruments, with the form of records and curves, and the application of the various quadrat and photographic methods, has made it necessary to refer these to another paper.

BIBLIOGRAPHY

1823 Schouw, J. P.

1836 Meyen, F. J. F

1855 DeCandolle, A.

1874 Nägeli, C.

1890 Drude, $\mathrm{O}$.

1901 Thornber, J. J.

1901 Jaccard, P.
Grundzüge einer allgemeinen Pflanzengeographie

Grundrisz der Pflanzengeographie

Géographie Botanique Raisonnée

Verdängung der Pflanzenformen durch ihre Mitbewerber. Sitzungber. Akad.

Wiss. München 3:205

Handbuch der Pflanzengeographie

The Prairiegrass Formation in Region I. Rep. Bot. Surv. Nebr. 5:55

Etude Comparative de la Distribution Florale dans une portion des Alpes et du Jura. Bull. Soc. Vaud. $37: 547$ 






W.

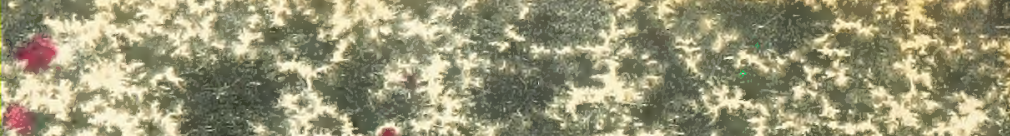

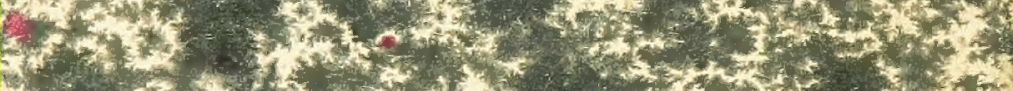

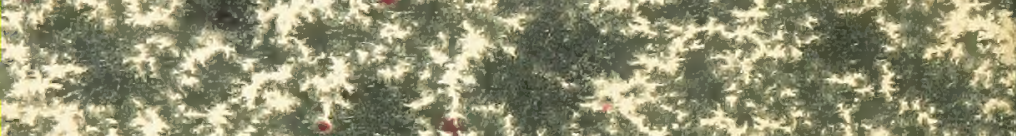

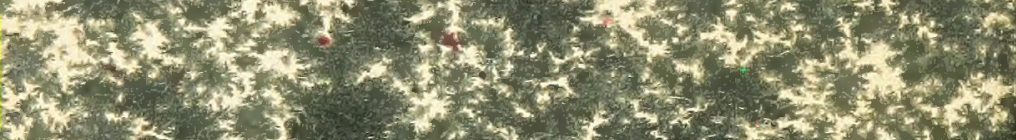
W.

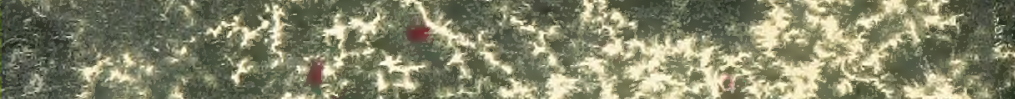

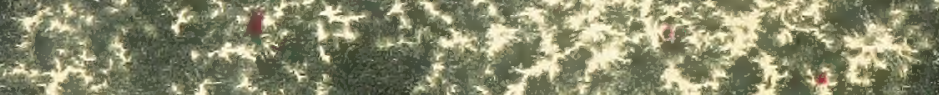

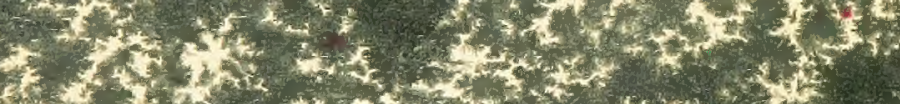

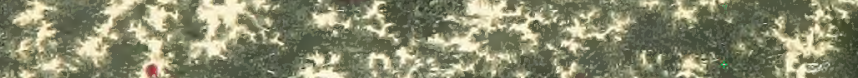

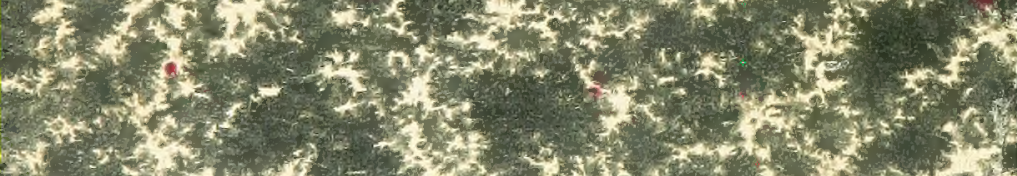

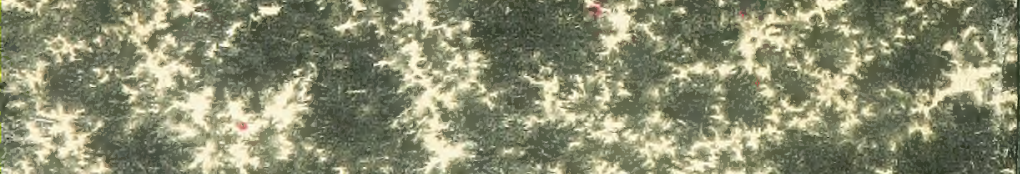

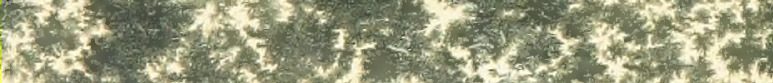

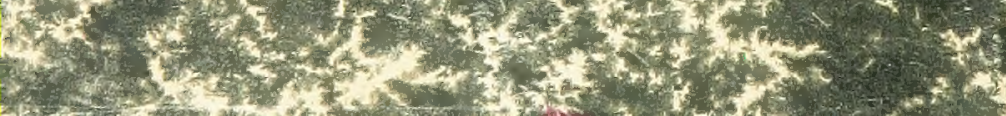

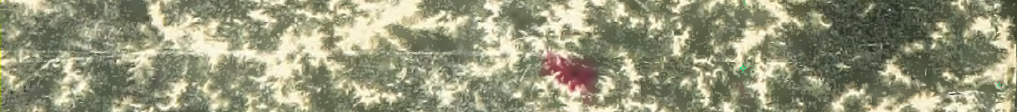

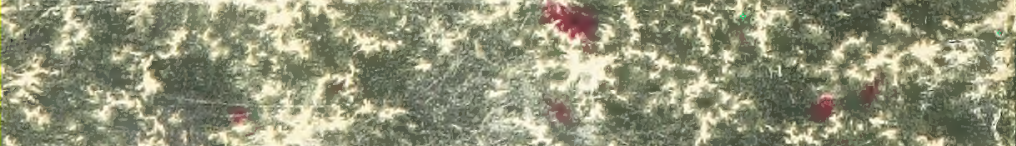
n.t.

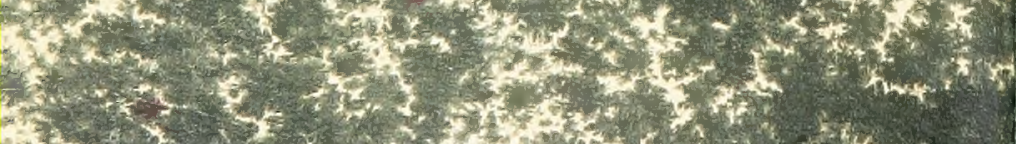

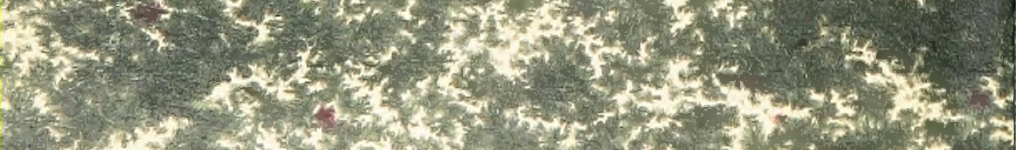

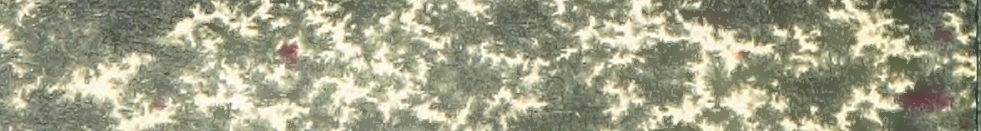

